$$
\angle B L-91-\operatorname{Rev}(9 / 96)
$$

LBL-91 Revised

UC-414

September 1996

\title{
CURRENT EXPERIMENTS IN PARTICLE PHYSICS \\ Particle Data Group
}

\author{
H. Galić
}

Stanford Linear Accelerator Center, Stanfond University, Stanford, CA $9 \$ 305$ USA

F. Lehar

Centre d'Ettdes Nucleaires de Saclay, F-91191 Gif-sur-Yvette, France

V.I. Klyukhin, Yu.G. Ryabov

Institute for High Energy Physics, RU-142884 Protvino, Moscow Region, Russia

S.V. Bilak, N.S. Ilarionova

Instikute of Theoretical and Experimental Physics, RU-117259 Moscow, Russia

B.A. Khachaturov, E.A. Strokovsky

Joint Jnotżtute for Nuclear Research, RU-141980 Dubra, Moscon Regrion, Russio

C. M. Hoffuman

Los Alamos National Laboratory, Los Alomos, NM 87545 , USA

P.-R. Kettle

Paul Scherrer Inotitute, CH-5232 Viligen PSI, Swizerland

A. Olin

TRIUMF, 4004 Wesbrook Mall, Vancouver BC V6T 2As, Carado

F.E. Armstrong (Technical Associate)

Particle Data Gronp, Lawrence Berkeley Nationel Laboratorys Berkeley, CA $947 \%$, USA

\begin{abstract}
This report contains summaries of current and recent experimente in Particle Physics. Included are experiments at BEPC (Beijing), BNL, CEBAF, CERN, CESR, DESY, FNAL, Frascati, ITEP (Moscow), JINR (Dubna), KEK, LAMPF, Nowosibirsk, PNPI (St. Petersburg), PSI, Saclay, Scrpukhov, SLAC, and TRIUMF, and also several proton decay and solar nettrino experiments. Excluded are experimente that finished taking data before 1901. Ingtructions are given for the World Wide Web (WWW) gearching of the conputer database (maintained under the SLAC-SPIRES gystem) that contains the summaries.
\end{abstract}

The publication of this report is supported by the Director, Office of Energy Reatuch, Office of High Energy and Nuclear Physics, the Division of High Energy Physics of the U.S. Department of Energy tunder Contract No. DEAC03-76SF00098, and by the U.S. National Science Foundation under Agreement No. PHY-932055t. Partint funding to cover the cast of the publication is also pravided by an implementing arrongement between the povermments of Japan (Monbusho) and the United States (DOE) on cooperative research and development. $H$. Galic is supported by the U.S. Department of Erergy ander Contract No. DE-ACOS-76SF00515. 
DHSCLAIMER

This report wa prepared as an ecoount of watk sponsoted by in arency of the Uaiced Stites Govetnimeat. Neithos the United States Goverament aor any agency thereof, nor any of their employees, makes any wirtenty, expess or implied, of

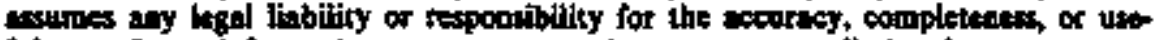

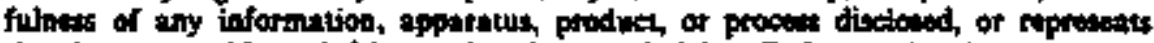

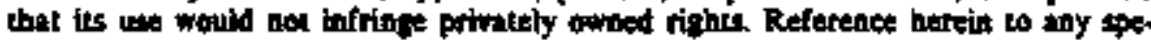
dific commercial modust, process, or servioe by trode ande, trademark, manolac-

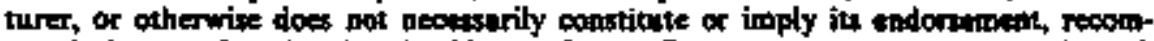
mendation, ar fevoring by the United Stutes Govenmient or nny agency thereof. The vitws and opinions of sulbors exprested hereis do not aecestertly state or reflect those of the Lnited States Covernenem or any agency theseof. 


\section{DECLANER}

Portions of this document may be illegible in electronic image produets. Images are produced from the best avilabie ariginal docoment. 


\section{TABLE OF CONTENTS}

Introdnction $\ldots \ldots \ldots \ldots \ldots \ldots \ldots \ldots \ldots \ldots \ldots$

Searching the EXPERIMENTS computer database $\ldots \ldots \ldots$. 3

Spokesperson index $\ldots \ldots \ldots \ldots \ldots \ldots \ldots \ldots$

Abbreviations used in the summaries

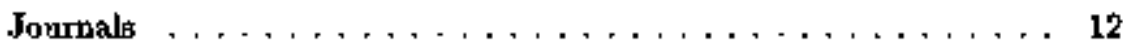

Kinematic variables $\ldots \ldots \ldots \ldots \ldots \ldots \ldots \ldots$

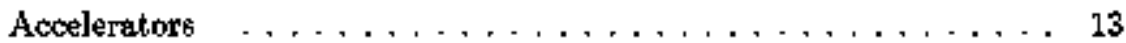

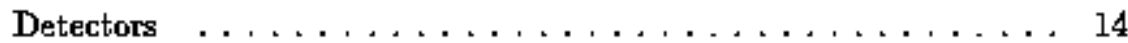

Summarles of experiments

BEPC (Beijing) $\ldots \ldots \ldots \ldots \ldots \ldots \ldots \ldots \ldots, 17$

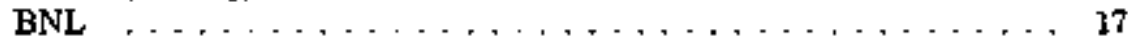

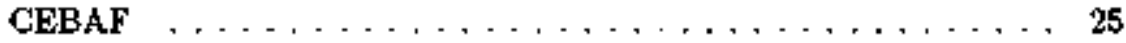

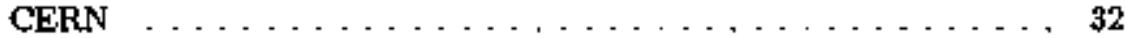

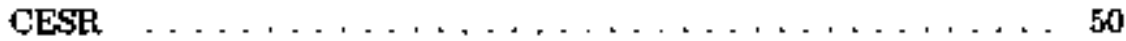

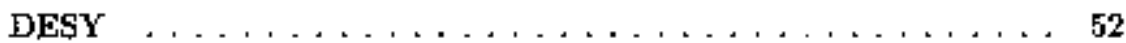

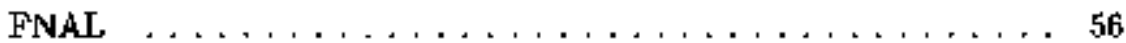

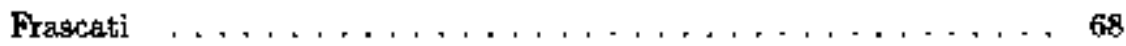

JTEP (Moscow $), \ldots \ldots \ldots \ldots \ldots \ldots \ldots, \ldots \ldots, \ldots \ldots$

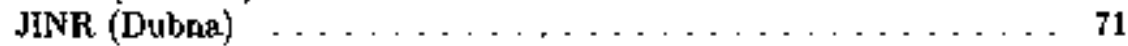

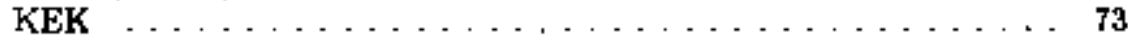

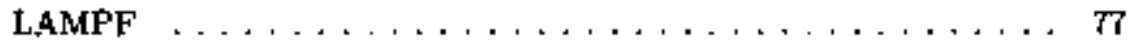

Nowosibirsk . . . . . . . . . . . . . . . . 80

PNPI (St. Petersburg) $\ldots \ldots \ldots \ldots \ldots \ldots \ldots \ldots \ldots \ldots \ldots$

PSI $\ldots \ldots \ldots \ldots \ldots \ldots \ldots \ldots \ldots \ldots \ldots \ldots \ldots, \ldots 2$

Saclay $\ldots \ldots \ldots \ldots \ldots \ldots \ldots \ldots \ldots \ldots \ldots \ldots$

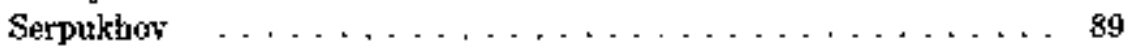

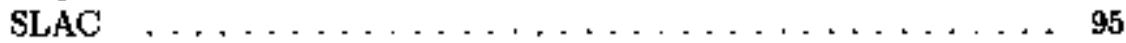

TRIUMF $\ldots \ldots \ldots \ldots \ldots \ldots \ldots \ldots \ldots \ldots \ldots$

Undergronnd/Underice/Underwater $\ldots \ldots \ldots \ldots \ldots 10 \ldots$ 


\section{INTRODUCTION}

This report contains full summaries of 180 approved current and recent experiments in elementary particle physics. The focus of the report is on selected experiments which directly contribute to our better understanding of elementary particles and their properties such as masses, widths or lifetimes, and branching fractions. This year's report is, in a way, a companion volurne to the main Particle Data Group publication, Review of Particle Physics. Experiments at the following laboratories ave included:
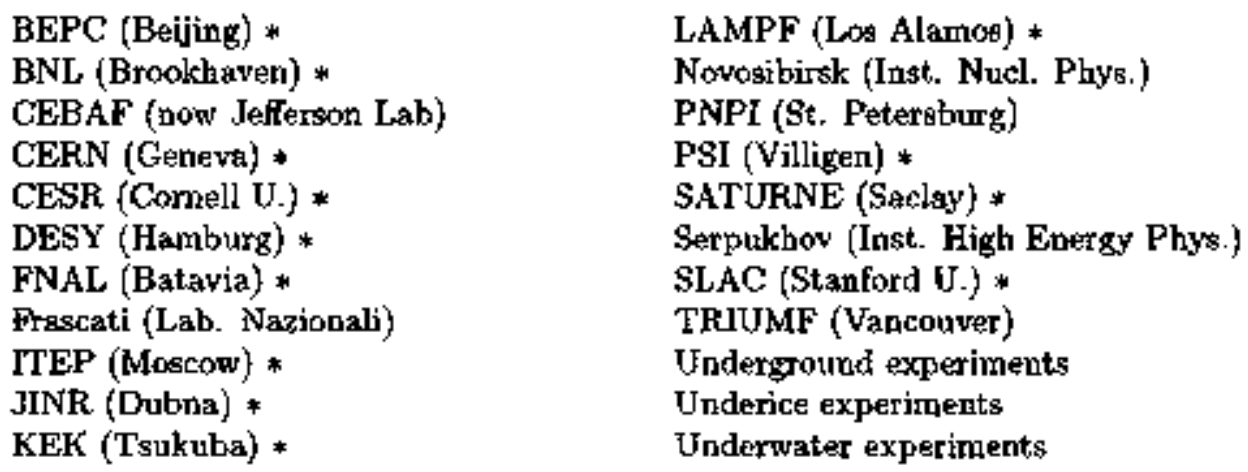

For the institutions marked with an asterisk, the corresponding summaries section also contains a brief description of the particle physice plans at the laboratory till the end of the century.

We also list 263 other experiments at the above laboratories. Although, to the best of our knowledge, these experiments do not measure directly the properties of particles listed in the Review of Particle Physics, they still may be of considerable interest to the particle physics community. These additional experiments only have brief entries in the printed version of the report, but often have a full description in an online-accessible database from which the report is produced. The readers are encouraged to find more information about those experiments in the database (see below).

We exclude from this year's report the experiments for which the data collection was completed before 1991. We generally do not cover the experinents dealing primarily with nuclear levels or other nuclear-structure properties. As to the choice of institutions, we try to include those in which particle physica experiments top the list of priorities at the laboratory.

Sources of information - Our first information about an experiment usually comes from the propweal for the experiment. Subsequently, we follow the progress of the experiment as best we can in laboratory reports such as Eaperiments at $C E R N$. Finally, a few months before an edition of this report is to appear, we send copies of the summaries of the experiments to the spokespersons for checking and updating. If a reply is received - as was the case for $90 \%$ of the experiments there is $a$ " next to the spokesperson's name. Since current experiments are often in flux, we rely heavily on these replies to be up to date; no $r$ by the spokesperson means the summary may be inaccurate or incomplete. (For a handful of experiments, we verified our information with a senjor member of the experiment, not the spokesperson, but for simplicity put a $v^{\prime}$ by the spokesperson. For experiments with more than one spokesperson, all the spokespersons are checked even if only one of them replied.)

Computer database - This report is produced from a computer database maintained at SLAC under the SPIRES database management system. The database, azmed EXPERIMENTS, also contains information from earlier editions of this report, going back to about 1975, and including older experiments at Argonne, INS, IUCF, and Rutherford. See page 3 for a guide to using the EXPERIMENTS database via the World Wide Web (WWW). 
Summaries - Each summary lists several dates related to the experiment: the date of the proposal, the approval date, and when the data-taking began and was completed. The title of the proposal and the most recent list of participants are given. The detector used in the experiment is identified either by a generic name (e.g., counter) or by a widely known acronym (e.g., SLD). The most important reactions and particles studied and the beam energy or momentum are listed where known. A brief comment describing the apparatus and the main goals of the experiment may follow. A summary ends with a list of any journal articles on results or instrumentation of the experiment. Related experiments, similar either in methods used or in subject of study, are also listed. Where known, an e-mail contact address and the WWW uniforn resource locator (URL) are given.

Abbreviations - To keep the summaries brief, abbreviations are used to indicate journals, kinematic variables, accelerators, and detectors. The abbreviations are usually obvious but are also defined near the beginning of the report. The abbreviated forms are needed for searching the EXPERIMENTS database online.

Acknowledgments - P. Yamin (BNL), D. Buckle (CEBAF), M. Draper and B. Powell (CERN), and J. Parker (FNAL) kindly provided computer files with data on experiments from thejr respective institutions. Valuable additional information was obtained from $P$. Yamin (BNL), G. Fraser (CERN), P. Drell (CESR), P. Folkerts (DESY), R. Rubinstein (FNAL), M.V. Danilov (ITEP), H.K. Walter (PSI), and D.W. Leith (SLAC). We thank F.E. Armstrong (LBL) for her help with the final processing of the manuscript. We particularly thank the hundreds of spokespersons who took the time to reply to our inquiries.

Comments and requests - We invite comments pointing out omissions, obscurities, ontof-date information, and errors. We also encourage spokespersons to send us proposals and letters of intent for their future experiments. Comments and other material should be sent to:

EXPERDMENTS ( $\% / 0$ H. Galić)

SLAC Library, MS 82

P.O. Box 4349

Stanford, CA 94309, USA

-mail: expbase@slac. stanford .edu

To order additional copies of the Current Experiments from North and South America, Australia, and the Far East write to:

CURRENT EXPERIMENTS

Particle Data Group, MS 50-308

Lawrence Berkeley National Laboratory

Berkeley, CA 94720, USA

e-mail: pdg@ıbl, gov

Requests from all other areas should go to:

CERN Scientific Information Service

CH-1211 Geneva 23

Switzerland

http: / / rowas, cern.ch/library/

(select - Ordering CERN Publications) 


\section{SEARCHING THE 'EXPERIMENTS' DATABASE VLA WORLD WWE WEB}

The summarjes of current and many earlier experiments related to particle physics are contained in a computer database called EXPERLMENTS, maintained at SLAC under the SPIRES database management system. Note that even the experiments which ouly have a brief description in this report may still have a full description in the database. You can acces the EXPERIMENTS database most easily vis the World Wide Web (WWW). The first part of this section describes a simple database search based on the Web forms. This is followed by a few examples of a more adranced search technique. The concluding part lists some otber coroputer-reachable sources of interest to experimental particle phyaicists.

General information - If your computer is linked to the Internet, you should be able to access the World Wide Web. The Web was brought to life in the early nineties by Timothy Berners-Lee apd collaborators at CERN. Free WWW software is ayajable for various platforms and various needs, from PC's to large computer systems, from simple line-mode browsers to sophisticated full-screen navigators. Names of some of the popular software packages that enable the Web access are Lynx, Netscape, and Mosaic, but the selection is by no means restricted to those three. Learn from your local computing center where and how to obtain the Web software most appropriate for your computer.

EXPERIMENTS home page - The EXPERMMNTS database and many other SPIRES-based SLAC Library databases are searchable via WWW. Find the home page for the EXPERMMENTS database at:

http://uru-spires. slac stanford .edu/find/experiments

When you open this docturnent, you find a simple fonm which allows you to fill in one or several elements which beat describe your search criteria. In the old days, when the database was only accessible through e-mail, you had to send a request written in the SPIRES search language to the SPIRES - mail server. The Web form eliminates this step, and even a novice can immediately begin a searching session. Furthermore, a Web search presents much more than the old e-mail search method. The e-mail result was a text, while the WWW result is a hypertext. The marked hypertext leads to related links, and you can, for example, find a more thorough bibliographic description of journal articles corresponding to a given experiment (from the HEP database), and often read or print a postscript verion of such articles. Sirilarly, if an experiment bas a Web bome page, you will be able to get there easily, and you may also find a direct link to the database entries of the experiments related to the selected one.

Simple form searching - Forms, in general, are simple to use and don't require mucb explanation. Explore them on your own, or use the following bints to get to the expected result evep sooner. The EXPERAMENTS form allows you to find a result by a titlo-word search, by a search for a participant in an experiment, or by the experiment's official code number. You can also find all the experiments in which a certain institution is involved, or your search could be based on, for example, the particles atudied in an experiment. You fll in the entries you know, leaving the others blank. The 'result' of a search is a database entry which fully describes an experiment, including the complete list of authors, a title, a description of the experiment's goals and methods, a list of resulting journal articles, etc.

To find all the experiments in which the atthor Johnson has participated, type the author's last name (surname) into the appropriate box. If an initial is added, separate it by a comma, e.g., johrson. g. Keep in mind that somebody you may know as Betty could officially be Elizabeth, Bob may be signing his papers as Robert, etc. If you are not sure what the right first name is, just do not use the initial. The last name can be truncated, c.g., leder\# for Lederman. To deal with a combination of several authors, use the words and, or, ant and not, e.E' johnson or smith will yield experiments by either physicist, jojneson and sulth will find experiments in which both bave participated, whereas johnson and not smith will yield experiments by Johnson so long as Smith is not involved. If a special character appears in a name, such as the ü in the German spelling of Miller, try alternate spellings, such as nuller or nueller. If you happen to knotr that anthor Johnson is from SLAC, for example, use botb the participant and the affiliation boxes. If you are only interested in experiments which author Johnson did at Fermilab, type in the name (johngon), and also select Fermilab in the list of accelerators. To initiate \& search, press the Perform Search button. Do not forget to use the Clear All Fields button between two searches. 
If you are interested in the experiments that study the CP violation, for example, try the title-word search. Enter any or all expected words from the title in any order. A truncated search may be used, e.g., cp\# in the title-word box yields titles containing the word CP standing free (as in CP violation), and also all the titles in which CP is hyphenated (as in CP-violating). If you type aolar ventrino into the title-word box, you will find all the experiments with tbese two words (any order) in the titles. You can combine any number of other elements with your title-word search. For example, to find rome of the recently approved $\mathrm{CP}$ violation experiments, type csp: in the titleword box, and select 'After' and '1990' in the two pull-down menus for the date-approved search element.

To find all the experiments in which people from a given institution participated, type the institution's pame (exactly as it appears in this report's summaries) into the affiliation box. Similarly, type the experiments' official code number, as it appeane in this report, iwto the exp-number box (e.g., type cern-na-048 to fand more about the NA48 experiment at CERN). For many experiments you can also use a nickname instead of the complete code number. For example, if you type opal in the exp-number box, you will find the CERN-LEP-OPAL entry, while do or dzero yields the FNAL-740 description. (You can also find the OPAL and Do entries by selecting the corresponding detectors in the list of detectors). To find both Do and CDF entries, use dzoro or cdf, and to find all fotur LEP experiments, type cern-1ep-; into the exp-number box.

To use the studied-particle box, you must know the database-adopted spelling of various particles. We use $\mathrm{kt}_{-}$, for example, and not kplus for $K^{+}$, and $\underline{p i 0}$, not pizero for $\pi^{0}$. An antiparticle name in the database is uftally formed by adding the suffix bar to the corresponding particle name. Thus the antiproton in the studied-particle box should be written as pbar. An extensive list of the 'correct' particle names can be found on the EXPERJMENTS belp page. (Note that the particle names in the studied-particle search are strictly standatdized. In a title search, to the contrary, particle names are somewhat variable in their spelling and severs forms should be tried).

If you are interested in finding all the experiments at a given accelerator, select the appropriate labozatary in the list of accelerators. Similarly, if you know a detector's name, try the list of detectors. The defaults are 'Any Accelerator' and 'Any Detector'. The date-approved search is useful if you know the approval year. You can also eliminate older experimenta from your earch result if you select the date-approved carefully. The default for the date-approved is 'Don't Know'.

Advanced searching - For the old hands and for those who want to have better control over searching, the form has a separate part named 'Make yotur own search'. This part ouly has one soarch window into which you type a complete SPIRES seasch command. To learn more about the SPIRES search language, check the 1994 and earlier editions of Current Experimerts. Here we give a few exampleg of what could be typed into the \$PIRES search window. We use lower case, but a search is case insensitive, and in an actual search you may use both lower and upper case characters.

Important note: whenever a term you wish to find contains characters, ' $)$, ' $>$ ', ' $<$ ', or '(', the entire search value must be enclosed in double quotes (see examples below)

FIND, SHOW and BROWSE are some of the command verbs you can use in SPIRES searching. You can formulate a variety of search commands by using these three verbs. For example,

ghow indexes

(Shows the available search terms, e.g., AUTHOR, PAPER, EXP, etc. Use these terms in your search request to BROWSE and FIND, e.g., BROWSE AUTHOR ..., or FIND PAPER ... , FIND EXP ..., etc.)

browse author trilling

(Displays values in the author-name index surrounding the stated value.)

bridtise_exp

(No search value for EXP is given, SPIRES returns several randon values of experingent codetumbers.) 
brouse exp disar

(Disptays values in the code-number index alphabetically surrounding the specified value. Useful if you do not know the exact form or speiling of a search value.)

Find author rubbia, $c$ or

Itnd author $c$ rubbia

(Finds experiments in which the stated anthor has participated. The first-name initial io optional: 11nd_author_rubbia will also work fine.)

find exp anturne-758

(Finds the record corresponding to the specified value.)

find paper "PR_D37 (1988) 1131"

(Finds the experiment reported in the stated reference. Note that the reference has spaces but no commss between the elcments. Double quotes are obligatory, see the bor above.)

Ilad reaction " $\theta+\theta-\cdots m+m u-x^{\prime \prime}$

(The 'arrow' is composed of two minus signs and a 'greater-than' sigo. Note the obligatory quotes.) find ac kek-trítan

(Finds experiments using the stated accelerator.)

tind de eryetal-ball

(Finds experiments using the stated detector.)

find title ep phase difference

(Finds experiments with the words CP, PHASE, and DFFERENCE in the title. The arder of title words in a search command is unimportant.)

find af ratgers $\mathrm{u}$

(Finds experiments in which people from the stated institution participated.)

find af rutzer*

(Finds experiments with the stated affitation. "\#" represents any remaining characters.) find particle d+

(Finds experiments studying the specified particle.)

The following searcli commands are also allowed:

\section{find author profix patters}

(Finds authors whose last names begin with the string PATTERS, e.g. Patterson)

find af prefix northwest

find reaction prefix pit n

find exp gld

(For most of the large experimental collaborations you may type just the collaboration name instead of the full experimental number)

Compound searching is not only possible, but also debirable, because it keeps search results sualler:

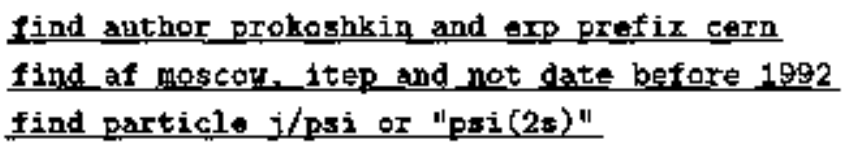

Occasionaliy a sastch may fail. With some care you can reduce unstaccessful searching to a minimum: (i) Remember that any searcl value containing any of the apecial characters $),\rangle,\langle,($, must be enclased in double quotes. See the examples above for the PAPER, REACTION, and PARTICLE searches; (ii) Une the 'correct' form of a seatch value: a database-adopted particle name (e.g., $K+$, not Kplus), proper experiment code-name (e.g., FNAL-761, not FNAL-E761), etc. To find the correct forms, use the BROWSE command for the index you are searching. 
Other SLAC-SPIRES databases - Several other SLAC Library databases of interest to experinental physicists are available via. WWW. They are all listed on the SPIRES home page:

ht tp://uvp-spires slac stanford.sdu/find/spires.btml

(1) HEP-PREPRINTS database is a joint project of SLAC and DESY libraries, and contains more than 320,090 bibljograpbic entries on particle physics papers (preprints, journal articles, reports, theses, conference papers, etc.); (2) BOOKS contains bibliograpbic summaries of more than 20,000 textbooks, conference proceedings, monographs, etc., covering high-energy physics and related topics; (3) CONFERENCES database liste past and future conferences, schools, and meetings of interest to the particle-physics conumunity; (4) HEPNAMES contains more tban 30,000 e-mail addresses of people working in high-energy and medium-energy physics. (By the way, you can retrjeve the HEPNAMES data without leaving the EXPERIMENTS search form: to get the author Jobsson's e-mail address, for example, just type the command query johnson into the 'Make your own search' window); (5) Datababe INSTITUTIONS lists about 5,000 addreses (and, often, phone and fax numbers) of institutions related to high-energy physios.

Other computer accessible sources - Clones of the EXPERIMENTS database are maintained for local users at the Yukawa Institute (Kyoto-SPIRES), Durham University (Durharn-RAL BDMS), and IHEP (Protvino BDMS), The Durharn version cen also be found at bttp://ept1. duI ac.uk/HEPDATA/ExP

A Web document called Experiments Online is a guide to home pages of various high-energy physics collaborations. It is updated weekly. To add your experiment's home page to the document, write to expbaseofislac stanford. edu. Find Experiments Online at:

$$
\text { http: / / ww-spires. slac. stanford. edv/Lind/expliat . btml }
$$

We urge all active experimental collaborations to establish their home pages on the Web. Such pages keep the collaboration members better informed, and should also serve to represent the group to the world. At the very minimum, each home page should list the current members of the collaboration and ideptify the spokesperson, give the objectives of the experiment and describe the experimental apparatus, and list the journal articles, conference papers and preprints which have resulted from the collaboration's work.

Experimental physicists are invited to post their papers to the hep-ex eprint archive. To receive detailed instructions on kubritting and retrieving papers; send a blank e-mail message with the subject help to: hep- $\times x(0 \times x x$. lanl gov . Email listings of the experimental physics titles and abstracts submitted to the archive can be received daily by sending a blank e-mail with the subject subscribe <your-name> to hep-ex. The listings and papers can also be accessed through the Web link http://xxx.1anl.gov/

You will hind a well-documented list of other computer accessible sources of interest to particle physicists in the latest edition of the Review of Particle Physics, Physical Review D54 (1996) 1-720. The list is also posted at:

$$
\text { http: } / / \text { wow slac, stanford edu/libtary } / p d g / \text { hepinfo,htmI }
$$

The Review itself is available over the Web. Check the Berkeley Particle Data Group WWW server at http: //Pdg.2bl.gov/ 
AEE, K. (KEK, Trukubs) KEK-TE-0OS ABEGG, $\mathrm{R}$. (TRIUMF) TRIUMF.482 AMMOSOV, V.V. (SErpdkhow, IFVE) SERPUKHOV-145 ANGHINOLFI, A. (INFN, Genos) CEBAF-93-03I APPEL, J.A. (FerTaItab) FNAL-791

ARMSTRONG, D.S. (LBL, Berkeloy) TRIUMF-570 ARNOLD, R.G. (A mericsn U) SLAC-E-I43 ARNOLD, R.G. (Ametkan U) SLAC-E-155 ASANO, Y, (Toukebs U, Inst Appl Phys ) KEK-TE-001 ASHERY, D. (Tel Avr U ) TRIUMF-445 AVIGNONE, F.T. (Sonth Carabna U) ITEP.g96 AVIBNONE, F.T. (South Caroling U) ITEP-912 AYRES, D.S. (ArgonRE) FNAL-B75 AZHGIREY, L.S. (Dubne, JINE) JINR-LHE-0941-3 AZUELOS, G. (Montreal U) TRIUMF-45?

BADERTSCHER, A. (Zurich, ETH) PSFR-86.05 BAGHAEI, H. (VnEIDIS U) CEBAF-8,-015 BALDIN, A.A. (Dubne, JINR) JINR-LHE-1010 BALL, J. (Saclay') SATURNE-22S

BALTaY, C. (Yale U) \$LAC-SLC-SLD BARABASH, A.S. (MOSç, I'TEP) ITEP-876 GARAB ASH, A.S. (MoBcow, ITEP) ITEP-896 BARISH, B.C. (CAl Terb) UNDERGROUND.MACRO BARNES, P.D. (los Algmog) BNL-813 BARNES, P,D. (Los Alaraco) BNL-B36 BARWICK, 界. (UC, Irvine) UNDERICE-AMANDA BECK, D+H. (Jlinals $U$, Urbans) CEBAF-91-017 BEJER, E.W. (Penn U ) UNDERGROUND-SUDBURY BEISE, B. (Maryland U) CEBAF-gl-OOA4 GELLETTINI, Q. (Pra U and SNS, Plas) FNAL-741 BeLostoTsKY, S.L. (St Petersburg, JNP) SATURNE-145 BELYAKOV-BODIN, V.I. (Mogcaw, ITEP) SERPUKHOV-171 BERNSTEIN, R.H. (Ferratib) FNAL-B15 BERTIN, P. (Cletrmant-Ferrand U) CEBAF-94-002 BEFTINI, R. (Saclay) SATURNE-213

BHANG, H.C. (Stoul Nationd U) KEK.307 BING, O. (Strasbourg, CFN) SATURNE-174 BJRCHLLL, J, (Mailoto U) TRIUMF-497.287 BJOFKEN, J.D. (SLAC) FNAL-864 BLANPIBD, G. (Solath Gatules U) SATURNE-2OS BODEK, A. (Rochetster W) SLAC.E-140X BOGG1LD, H. (Bohr InEt) CERN-NA-044 BOGUSLAVSKY, I.V. (Dubn,, JINR) SERPUKHOV-161 BOLOTOY, Y.N, (MoSCOW, INR) SERPUKHON-166 BONNER, B.E. (RHe U ) BNL-61? BONNER, B.E. (R.ce U) BNL-\$54 BOUDARD, (Sarlay) SATURNE-14S BOWLES, T+J. (LOS Alamo?) UNDERGROUND-SAGE BOWMAN, J.D. (LoB Alemas) LAMPF.1188 GOYARD, J.L. (Otzay) SATURNE-190 BOYARD, J.L. (OTaAy) SATURNE-251 BOYAFD, 3.Z. (Otsay) SATURNE-278 ERACK, J.T, (TRIUMF) TRFUMF-645 GRADAMANTE, F. (Triegte U) CERN.PS-206 BRAUN-MUNZINGER, P. (StNNY, Story Brook) BNL-814

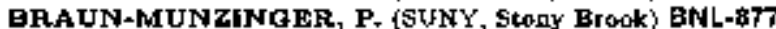
BHEIDENBACH, M. (SLAC) SLAC-SLC-SLD BRESSANI, T. (TLIN U \} FRASCATLDAPHNE-FINUDA BR1scon, W.J. (Goorge Washington U) BNL.909 BRISCOE, W.J. (George Washugton v) CEBAF-94-103 BRODZINSKI, R.L. (Batlelle Mem Ingt) ITEP-912 BROOKS, W.K (CEBAF) CEBAF-94017 BRYMAN, D.A. (TRIUMF) BNL-787 BUNJATOV, S.A. (DUbEa, JLNR) SERPUKHOV-136 BURKE, D.L. (SLAC) SLAC-E-144 BURKERT, V.D. (CEBAF) CEBAF-Bg-037 BURKEAT, V.D. (CEBAF) CEBAF-B9-039 BURKERT, V.D. (CEEAF) CEBAF-89.042 BURKERT, V.D. (CEBAF) CEBAF-91-002 BURK ERT, V.D. (CEBAF) CEBAF-91-023
BURKERT, V.D. (CEBAF) CEBAF-91-024 BUAKERT, V.D. (CBBAF) CEBAF. $\$ 3.006$ BURCESON, G.R- (New Mexico State U ) LAMPF-1878 BURLESON, G.R. (New Mexico \$tate U) LALtPF-1266 BURLESON, G.R. (New Mexro Sta1e U ) LAMPF-126? BUTLER, J.N. (Formulab) FNAL-687

CALDWELL A (Nevnt Labs, Columbin U) DESY-HERA-ZEUS CAEVETTI, M. (Flozence UI and INFN, Florence) CERN-NA-048 GARITHERS, W. (LBL, Berkeley) FNAL-741

CARAOLL, A.S. (Braokbaven) BNL-650

CASON, N.M. (Notre Dame U) BNL-652

CATES, G.D. (P (1) Doton U) CEBAF-94-010

CATES, O.D. (Princeston U) LAMPF-1231

CESTER, R. (TUIn U) FNAL-740

CESTER, R. (Turın U) FNAL-\$35

CHANG, C.C.G. (WIaryland V) CEBAF-89-033

CHAEMAN, C. (Brookhavan) BNL-866

CHAS'CELER, R. (Duke U) CEBAF-93-036

CHATTER.JEE, L. (Jadovpar U) FNAL-802

CHIB A, J, (KEK, Taukuba) KEK-257

CHRJEN, R.E. (Brookheven) BNL-674

CHRIEN, H.E. (Brtokhaven) BNL-890

CHRIEN, R.E. (Broukhaver) BNL-905

CHRIBN, R.E. (Eroolbaved) CEBAF-69-009

CHRistraN, D.C. (Fermalab) FNAL-862

CHUNG, S.U. (Btontharen) BNL-B52

CLAJUS, M. (UCLA) SATURNE-258

CLEMENT, H. (Tubingen U) PSI-R-85-13.3

CLEMENT, H. (Tubrigen U) PSI-R-89-03

COLE, B.A. (Columbls U) BNL-910

COLE, P.L. (George Washnugton U) CEBAF-94-109

COMFORT, J.R. (Arrona State U' LAMPF-1178

COMFOFT, J.R. (AN30Ra State † ) LAMPF-1256

CONETTh, S. (VIrGTII U) FNAL-TT1

CONNELLY, J.P. (Gerrge Wakhongtan †) CEBAF-94-109

COOPER, M.D. (LOA AlImes) LAMPF.969

COOPER, M.D. (Lod Alamor) LAMPF-1240

COOPER, P.S. (Permulab) FNAL-781

CORCORAN, M.D. (Ruce U \} FNAL-683

COUSINS, R.D. (UCLA) BNL-BEg

$\operatorname{COX}$, B.B. (VIEINIA U) FNAL-TI 1

CRABB, D.G. (Vugima Џ) CEBAF-91-023

CRAWFORD, H.J. (UC, Berkeley, Space Sn) BNL.978

CRAWFORD, H.J. (UC, Berbelfy, Spers Sel ) BNL-896

CUMALAT, J.P. (Colorado U ) FNAL-68?

CUMALAT, J P. (Colorado U) FNAL-831

CUMMING, J.B. (Brookhaven) BNI-B44

CUMMINGS, w. (Sเmon Fraatr U) TRIUMAF-623

DATz, S. (OAk Ridge) CERN-WHA-099

DATZ, S. (OBk Rıdige) CERN-WA-099.2

DAUM $_{+}$M. (PSI, Villigen) P5I-R-95-03

DAUM, M. (PSI, VLligen) PSI-R-96-05

DAVTS, C.A. (Mnntoba U and TRJUMF) BNL-8E5

DAVIS, C.A. (TRTtiMAF) TRIUMF-49日

DAVIS, R, JR, (Penn U) ЏNDERGR-HOMESTAKE-CHLORINE

DAVISON, N E. (Mantobe U) TRUMMF.372

DAY, D.B. (Yizgne v ) CEBAF-89-008

DAY, D.B. Virgula U CEBAF-93-026

DE MARCOO, N. (Turn U and INFN, Turı) SATURNE-23?

DE SANCTIS, E. (Frascati) CEBAF-93-017

DEHNHARD, D. (Mmmeots U ) LAMPF-1267

DEMIDOV, V.S. (M०SCOW, ITEP) ITEP-\$21

DEMIDOV, V.S. (Moacow, ITEP) ITEP-922)

DENISOV, S.P. (STrpukbov, TWVE) SERPUKHOV-1\$2

DENNIS, E.c. (Flonds State サ) CEBAF-89-043

DEUTSCH, M. (MIT) BNL-7eI

DI LELEA, L. (CEFN) CERN-WA-096

DJCELEZ, J.P. (OISRY, IPN) SATURNE-121

DIOELEZ, J.P. (OIJAY, IPN) SATURNE-209

DIEBOLD, G. (Yale U) BNL-886 
DOLGOLENKO, A.G. (Moscow, ITEP) ITEP-913 DOMOGATSKY, G.V. (MOBEOW, INR) UNDERWAT-BAIKAL DUKES, E+C. (VITRInLA U) FNAL-B?] DYTMAN, G.A. (PItLbnrgh U) CEBAF-89-039 DZIERBA, A- (1ndana U ) CE,BAF-94-016 EGGER, J.P. (Nenchatel U ) PSI-R-86-05 EICHLER, R.A. \{Zutueb, ETH\} DESY-HERA-H1 ELOUADRHIFI, L. (Chrlatopher Newport U) CEEAF-94005 ELSEN, E. (DESY) DESY.HERA.H1 EWYO, H. (Kyoto U) KEK-325

ERMOLOV, P.F. (MOes Dtase U) 5ERPUKHON-161 EVANS, D. (Bırmingham U) CERN-WA-085 EWAN, G.T. (Queen U , Kuggoton) UNDERGR-SUDBURY FA.ESSLER, M.A. (Munk.h TI) CERN.PS-197 FETSCHER, W, (Zurkh, ETH) PSHR-94-10 FICENEC, J. (Vurguna Tecb) CEBAF-94-103 FILIPPONE, B.W. (CB] Tris) CEBAF-B9-D00 FILIP PONE, B.W. (Cxl TEtb) SLAC-NE-18 FIN'ser, M. (Charles U apd Dubra, JINR) P\$l-R-95-00 FINN, J.M. (Willaam and hary Coll ) CEBAf-g1-010 FLYAGEN, V.E. (Dubna, JINR) SERPUKHOV-167 FONTAINE, J.M. (Saclay) SATURNE-225 FFaNKLIN, G.B. (Oskuegle Mellon U) BNL-013 FRANKLIN, G.B. (Carnegle Melion U) BNL-836 FRANKLIN, G.B. (Carnete Mellon UV ) BNL.8Q5 FRANZLNI, P. (Rome U) FRASCATI-DAPHNE-KLOOE FRASCARIA, R. (Oray, IPN) SATURNE-121 FRASCARLA, R. (OrRay, IPN) SATURNE-220 FRJEDMAN, E, (Hebrew U) TRIUMF-596 FROECH, R. (PSI, Villget) PSI-R-67-01 FRULLANI, S. (Rome U, Tortergats) CEBAf-g1-011 FUKUDA, T. (Takyo U , INS) BNL-906 PUNSTEN, H.O. (Whlliam and Mary Coll ) CEBAF-89-043 FUNSTEN, H.O. (W'Hllam and Mary Coll) CEBAF-91-024 FUNGTEN, H.O. (W'illam and Mary Coll) CEBAF-93-022 FURGET, C. (Greboble U) SATURNE-290

GAAFDE, C. (Copenbagen V ) SATURNE-190 GABRIELSE, G. (Harvard U ) CERN-PS-196 GAGLIARDI, C. (Texat A and M) TRIUMF-614 GAI, M. (Connackicut U ) CEBAF-B9-039 GAO, H. (Wnos U, Urbans) CEBAF.94-104 GAJCON, M. (Saclny) SATURNE-25B GAVRSN, V.N. (MOBCOW, INR) UNDERGROUND-SAGE GEER, \$. (Fermitab) FNAL-a61 GEER, S. (Fermlab) FNAL-668 GHOSH, D. (Jadavpur U ) CERN EMU-014 GHOSH, D. (Jadsvpor U) FNAL-802 GIACOMFILI, G. (BOLGGa U) CERN-EMU-018 GIACOMELLI, G. (Bologas U) UNDERGR-MACRO GLLL, D.R. (TRILMF) TRIUMF-614 GLLMAN, R. (Rutgat U ) CEBAF-94-012 GIOVANETTI K. (Jamea Madison U) CEBAF.89-039 CLAGOLEV, V.V. (Dubna, JINR) JINR-LHE-0941-5 GLASHAUSSER, C. (Hutgere V) CEBAF-89-033 GLASS, G (TPX:S U) LAMPF-1300 GOL,EIN, G D. (Illunbis U, Ufbans) FNAL-773 GOMEZ, J. (CEBAF) CEBAF-93-024 GORRINGE, T,P, (Kentucky U) TRIUMF-B12 GOTTA, D. (Juhach, Forachungazentrum) CERN-PS-207 GOTTA, D. (Julich, Forschungazentrum) PSLR-94-01 GOULD, H. (LBL, Berkeloy) BNL-ag2 GHAM, P.A.M. (Loo Alsmos) LAMPF.1310 GRANNIG, P.D. (SUNY, Stoü' Brooly) FNAL-740 GRECHKO, V,E. (MTOBCOW, JTEP) ITEP-892 GRECHKO, V.E. (Mfoocow, ITEP) ITEP-Bg? GREENIAUS, LC. (Alberta II) TRIUMAF-369 GRJON, N. (Treste Uf) TRIUMF-624 GRION, N (Trueste U) TRIJMF- 653 GUTBFOD, H.H. (Darmgtadt, GSI) CERH-WA-098
HAEUSSER, O.F. (TRICThF and Simon Fraber U) LAMPF-1267 HAEUSSER, O.F. (TRIUMP and Gmono Frader U) TRIUMF-557 HAEUSSGEF, O.F. (TRIUMF and Sımon Framer U) TRIUMA-566 HAFFTER, P. (Rapel t) ) P\$1-2-B9-06

haguenaUER, M. (Ecole Pobytech, LPNHE) CERN-UA-004-2 HALLMAN, TA. (UCLA) BNL-396

HALZEN, F, (W'GconsIn U, Madron) UNDERICE-AMANDA HAMAGAK, H. (Tokyt U, IN\$) BNL-BE6

GARSHMAN, D.R. (ATdT Bell Lobs, Morray Hiil) BNL-849

HARTMANN, F.J. (Manmch, Teth U ) P\$1-R-91-08

FASHJMOTO, O. (Takyo U, INS) KEK-140A

HASHIMOTO, O. (Tokyo U , INS) KEK-336

HASINOFF, M.D. (Britssh Colunbus U) TRIUMF-4S2

HALSSER, O.F. (Sumo Fther U and TRIUMF) TRIUMF-683

HE, Y, (UC, Rerkeley) CERN-WA-101

HEDDLE, D. (Chrtatophet Nevport U) CEBAF-94-00S

HEINRFCH, W. (Sregen U ) CERN-EMUU-17

HEPPELMANN, B. (Penn SIste U) BNL-850

HERSMAN, F.W. (New Haingshire U ) JINRR-LHE-CA41-1C

HESS, R. (Gernos $\theta$ ) SATURNE-144

HEUER, R.D. (CERN) CERN-LEP.OPAL

HICKS, K.H. (Oho U ) BNL-89?

HICKS, K.H. (Ohno U ) CEBAF-98-030

HICKS, K.H. (Ob,o U1) TRIUMF,633

HLLL, J,C. (IOWE Statie U ) CERN-NA-053

HOLT, R.J. (Illons U, Urbans) CEBAF-89-012

HOLT, R.J. (IItrnols U, Urbene) CEEAF-94-012

HOLT, R.J. (Illumol U, Urbana) CEBAF-94-104

HOLT, R.J. (IIlumole U, UrbBDS) SLAC-NE-17

HOLZSCHEITER, M.H. (Los Alsmod) CERN-PS-200

HOURANI, E. (OTEAy, IPN) SATURAE-209

HSIUNG, Y.B. (Farmbb) FNAL-832

HUGHES, E.W. (SLAC) SLAC-E-142

HUGHES, E.W. (SLAC) SLAC-E-154

HUGHES, V.W. (Yale U ) BNL-621

HUGHES, V.W. (Yale U ) CERN NA-047

HUGHES, V.W. (Yale U) LAMPF-1054

HUNGERFORD, E.V. (Houstan U) BNL-774

HUNGERFORD, E.V. (Houktan V) BNL-B74

HUNGERFORD, E.V. (IIOAGLOT U) BNL-907

HUNGERFORD, E.V, (Hovaton U) CEBAF-89-009

HUSSEIN, A.H. (Northern Brutish Columbie U) LAMPF-1236

HUTCHEON, D.A. (TRIUNP) TRIUMF-643

HYDEWRIGHT, C.E. (Old Dommen U ) CEBAF-91-014

IEIRI, M. (KEK, TeukLibs) KEK-251

IEIFI, M. (KEK, Teukuba) KEK-289

IMAI, K. (Kyoto If ) BNL-886

IMAL, K. (Kyoto U) KEK-224

IMAZATO, J. (KEK, Trukuha) KEK-246

SVANOV, Y.M- (St Petergbarg, INP) SERPUKHOV-177

IWASAKI, M. (TokYo U, INS) KEK-228

JACKSON, G.P. (Fermilsb) FNAL-853

JACKSBON, H.E. (Argonne) CEBAF-91-D03

JAHN, R. (Gonk U) SATURNE-2Z2

JAIN, P.L. (SUNY, Bufalo) BNL-875

JAIN, P.L. (SWNY, Buflalo) GERN-EMU-D11

JANOUECH, M. (ZuFk, ETH) PSI-R-45-09

JASTRZEBSKI, J, (Wharsass U, Heavy Jon LAt) CERIY-PS-209

JELLEY, N.A. (OXFIA U) UNDERGROUND-SUDBURY

JENKINS, D.A. (Wurgewta Tecb) CEBAF-94-103

JOHNS, K.A. (Arizana IT) FNAL-\$00

JOHNSON, R.R. (Brtigh Columbes U T TRJUMF-624

JOUFDAN, J. (Basel U) PSI-Z-Bg-02

JULLIAN, S. (Orssy, LAL) NEMO-2 (Hsted under ITEP)

JUNGMANN, K- (Heidelberg ti, Fhys Inst) PSI-R-89.06 JUNGMANN, K, (Gindelber U U Pbys Int) PSI-R-92.08 KAJIKAWA, R (Nagaya U) KEK-TE-D02

KAZBFLE1SCH, G.R. (Okloboma U) FNAL-855

KANAVE,S, V.P. (Moscow, ITEP) ITEP-g14 
KANE, J.R. (William and Mary Coll) ENIL-871 KAPLAN, D.M. (NortherA Illoror U) FNAL-769 KAWABATA, S. (KEK, Tqukuba) KEK-TE-0@? KAWAL, H. (Chrb U) KEK-248

KEK ELIDZE, V.D. (Dubna, JINR) SERPUKHON-159 KHACHATUROV, B.A. (OUBNA, MNR) JINR-LHE-094]-4 KHACHATUROV, B.A. (DubDs, JINR) SATURNE-225 KHAN, H.A. (Panitech, Jalamabad) CERH-EMU-D19 KHAN, H.A. (Purtech, Islsmaland) CERN-EMU-020 KHAZIN, B.1. (Novosibrsk, IYF) NOVOSIBIRSK-CMD-2 KHRYKIN, A.G. \{Dubrs, JIINR) JINR-LNP-09 KILLAN, K. (Julach, Forsthuageantrom) CEFN-PS-185-2 KIM, C.O. (Koree U) FNAL.843

KINSON, J.B. (Birmingham I) CERN-WWA-094

KIRK, A. (CEPN) CERN-WA.VII

KIRK, A. (CERN) CERN-WA-102

KIRP1CHN1KOV, I.V. (hfoscow, ITEP) ITEP-861 KIRPICHNIKOV, LV. (WLAcow, ITEP) ITEP.g12 KIRPICHNTKOV, t.V. (Koecow, JTEP) ITEP-921 KIRSTEN, T. (Heidelberk, MPI) UNDERGR-GALLEX KISELEV, Y.T. (Moscow, ITEP) ITEP-873 KISEEEV, Y.T. (Moscow, ITEP) ITEP.911 KISELEV, Y.T. (Moordw, ITEP) ITEP-941 KISELEV, Y.T. (Modcow, JTEP) ITEP-951 KISHIMOTO, T. (Osaken U) KEK-278 KLANNER, $\mathbf{R}$. (DESY) DESY.HERA-ZEUS KLEIN, S.R. \{LBL, Berkeley) SLAC-E-146

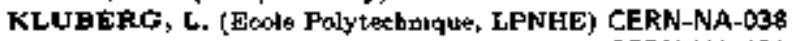
KLUBERG, E. (Ecole Polytex/hnlque, LPNHE) CERN-NA-050) KLUBERG, L. (Ecole Polytechrigue, LPNHE) CERH-NAA-DS1 KLUGE, W. (Karkisulhe U , IEKP) PSI-R-85-13-3 KNAPP, B.C. (Columber L') FNAL.690

KORKMAZ, E. (Nortberm Brttrh Fodumba U ) TRIUMF-704 KOgSOV, M.V. (MMEOW, ITEP) CEBAF-99-012

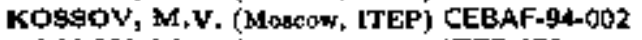
KOSSOV, M.V. (MOBCOW, ITEP) ITEP-853 KOTELNIKOV, K.A. \{Lebedev lngt \} CERN-EMU-015 KOVABH, M.A. (Kentucky U) KEK-235

KOVASH, M.A. (Kentucky V, TRIUMF.643 KOVA\$H, M.A. (Kentucky U) TRIUMF. 661 KOX, S. (Grenoble U) SATURNE-166 KOX, 8 . (Orenoble U) SATURNE-235 KOX, s. (Grewatk U) SATURNE-253 KOX, s. (Gronoble U) $\$$ ATURNE-290 KRISCH, A.D, (Michigan U) SERPUKHOV.UNK.0OI KRUGLOV, S.P. \{St Petersburg, ]NP) BNL-913-914 KRUGLOV, S.P. (Si Patergburg. INP) ITEP-9I4 KRUQLOV, S.P. (\$L Paterebutg, INP) PNP4-SC-124 KRUTENKOVA, A.P. (Moscow, ITEP) ITEP-901 KRUTENKOVA, A.P. (Mascow, JTEP) ITEP-923 KRYSHKIN, V.I. (Serpukhov, WVE) SERPUKHOW-155 KRYSHKIN, V.I. (Serpukbov, IFVE) SERPUKHOV.175 KUHN, S.E. (Old Damianon if ) CEBAF-93-D09 KUNNE, R.A. (Saclag) SATURNE-27B KUREPIN, A.B. (Moscon, INR) JINR-LHE-0969 KWIATKOWSKI, K. (Indiana U') BNL-900 KYCIA, T. (Brookhaven) BNL-913-914 LAGET, 3.M. (SAGAY) CEGAF-93-031 LANDE, K. (PEDU U \} LAMPF-1213 LANDE, K+ (PEnn U) UNDERGR-HOMESTAKE-CHLORINE LANDE, K. (Penn U) UNDERGR-HOMESTAKE-IODINE LANDSBERG, L.G. (\$ErpakhoY, IFVE) SERPUKHOV.169 LANDSBERG, L.G. (SESPrkhow, IFVE) SERPUKHOV 178 LARSON, B- (Siman Fraser U) TRIUWF-557

LE BORNEC, Y. (Orsay, IPN) SATURNE-196 LE BORNEC, $\mathbf{Y}$, (Orssy, IPN) SATURNE-280 LEARNED, J.G. (HawsI U) UNDERWATER-DUMAND LEDOUX, R.J. (MIT) BNL-859 LEHAR, F. (S-ClyY) JINR-LHE-0941-4 LEHAR, F. (Seclay) SATURNE-144
LEKSIN, G.A. (MOBCOW, ITEP) ITEP-853 LEKSIN, G.A. (Mobcow, ITEP) ITEP-BQ4 LEKSTN, G.A. (Mo日Gow, ITEP) ITEP.895 LEPJKHIN, Y.B. (Moocow, ITEP) ITEP-B⿺5 LL, 3. (Beijinc. Inst Hich Energy Phys) BEPC.BES LINDENBAUM, 5.J. (BNL and CIty Coll, N Y ) BNL-830 LINDENBAUM, S.J. (BNL and City Coll, N Y ) BNL-881 LITTENBERG, L.S. (Brookhaven) BNL-787 LITVINENKO, A. (Dubna, JINR) JINR-LHE-0063-4 LOPATIN, I.V. (St Paterabute, INP) PNPI-\$C-147 LORD, J.J. (Wrohington U, SeattIe) FNAL-793 LOUIS, W.C. (Los Alamos) LAMPF.1173 LOURLE, R. (Vurguns U) CEBAF-91-011 LUK, K.B. (LBL, Berkeley and VC, Berkelay) FNAL-871 IUNDBEAG, B.G. (Fermleb) FNAL.872 LYTKIN, L. (Dubrs, JINR) SATURNE-258 LYTKIN, L. (Dubna, JINR) SATURNE-280 LYUBIMOV, V.A. (MOECO\%. ITEP) ITEP-832 MACK, D.J, (CEBAF) CEBAF-93-021

MACRI, M. (Gedos U and INFN, Gemas) CERN-PS-202 MADEY, R. (Hampkon U) CEBAF-93-090

MAGAHIZ, R- (Carneq1* Mellon V) CEBAF-95-003 MA.JKA, R.D. (Yale $\mathrm{b}$ ) ENL. 864

MALAKHOV, A.[, (Dubng, JINR) JINRLLHE-OST]-1 MALAKHOV, A.I. (Dubna, JMR) JINR-LHE- $0973-2$ MANLEY, D.M. (Kent State U) CEBAF-9I-024 MAFCHAND, C. (SAclay) CEBAF-93-031

MAFSHAK, M.L. (Nmmota U ) UNDERGROUND-SOUDAN-2 MAFTELLOTTI, G. (Fome Us ind INFN, Rome) CERN-WA,084 MARTINGKA, C. (Koplate U) JINR.LHE-0941-5 MASSCARENHAS, N. (CAI Twch) CERN-NA-055 MA\$\$ON, G.S. (BSOQI UI) PSI-Z-BQ-OT

MASUDA, Y. (KEK, ToukubB) KEK.291

MATHA, w.E. (Regina U) TR1UMF-506

MATSUI, T. \{KEK, TBukubł KEK-TE-001

MATTHIAS, B. (Headelbarg D , Pbya Iwat) PSI-R-92-08

MAY, M. (Brookhaven) BNL-TB1

MAY, M. (Broohthaven) BNL-EBS

MAYKR, B. (Saclay) SATURNE-19B

MAYER, B. (Saclay) SATURNE-246

MAYTAL-BECK, S. (Tha Aviv U) TRIUMF-445

MCCARTHY, J.s. (Vrging U) SLAC-E-155

MCDONALD, A.B. (Quetns V , Kugatan) UNDERGR-SUDBURY

MCDONALD, K.T. (Princeton U) SLAC-E-144

MCGAUGKEY, P.L. (LAR Alamos) FNAL-866

MCNAUGHTON, M.W. [LoB AlBmos] LAMPF-a76

MCNAUGHTON, M.W. (La Alswos) LAMPF-1234

MONAUGHTON, M.W. (LOF Alamos) LAMPF-1293

MEASDAY, D.F. (Britsh Golumbar U ) TRIUMF.537

MECKING, B.A. (CEBAF) CEBAF-89-045

MECKING, B.A. (CEBAF) CEBAF-91-024

MELIgSINOS, A.C. (Rocheater U) BNL.840

MELIVSINOS, A.C. (Racheotor U) SLAC-E-144

MESTAYER, M. (CEBAF) CEBAF-93-030

MEZIANI, Z _.E. (Temple tf) CEBAF-94-0to

MIGNEREY, A, (Maryland US) BNL-917

MLLLS, A.P., JR. (MTKT Bell Labs, Murray Hit) BNL-849

MILNER, RG. (MIT, LNS) CEBAF-91-007

MILNER, R G. (MIT, LNS) DESY-HERA-HERMES

MELNER, R.G. (MIT, LNS) SLAC-NE-18

MENEHART, R-C, (VITMU, U) CEBAF-89.037

MINEHART, R.C. (VIrgInE U) CEBAF-89-038

MINEHART, R.C. (VIrgibus U) CEBAF-69-042 MINEHAFT, RCC (Varguis U ) CEBAF-91-023 MINEHAFT, R.C. (Virgina U ) CEBAF-93-036 MISCHKE, R.E. (Los Alamos) LAMPF-1240

MISKIMEN, R.A. (Masasatbuedts U Amherat) CEBAF-94-015 MOISEEV, A.M. (Serpukhav, JFVE) SERPUKHOV-161 MOLZON, W, R (UC, Irvine) BNL-371

MONTGOMERY, H.E. (Fermuab) FNAL-740 


\section{SPOKESPERSON INDEX}

MORAtes, A. (Zaragora U) ITEP-912 MORLET, M. (OTFaY, IFN) SATURNE-253 MORONI, L. ([NFN, Mitan) FNAL-831 MOREIS, C.L. (Los A lamab) LAMPF.1190 MOR\&CH, H.P. (\$aclay) SATURNE-220 MORSCH, H.P. (Saclay) SATURNE-2S1 MORSE, W.M. (Brookharen) BNL-A21 MURPHY, C.T. (Fotcmlab) FNAL-853 MUTCHLER, 0.3. (RtCe U) CEBAF-89-024 NAGAE, T. (Tokyo U , INS) BNL-905 NANDA, 3, (CEBAF) CEBAF-39-033 NAPOLITANO, J.J, (Renkedmer Poby) CEBAF,93-033 NAPOR[TANO, J.b. (Rehezelder Foly) CEBAF-94-014 NAPOLTANO, J.J. (Reptselse Poly) CEBAF-94-016 NARASIMHAM, V.S. (Tata Last) UNCERGROUND-KGF NAYILIAT-CUNCJC, O. (Zurich, ETH) PSI-Z-90.07 NEFKENS, B.M.K. (UCLA) BNL-890 NEFKENS, B.M.K. (UCLA) BNL-913-914 NEFKENS, B.M.K. (UCLA) SATURNE-198 NEFKENS, B.M.K. (UCLA) \$ATURNE-246 NEMENOV, L.L. (Dubna, JINR) CERN-PS-212 NEMENOV, L.L. (DubHA, JINF) SERPUKHOV 174 NIETO, M.M. (Los Alistos) CERN-PS-200 NTORADZE, M.Q. (Thblas Itata U ) JINR-LHE-0941-5 NOLTE, E. (Mumich, Tech U) CERN-NA-054 NOZDFACHEV, V.N. (MonçW, ITEP) SERPUKHOV-173 NUMAD, T. (TRUUMF) TRIUMF-703

OELERT, W. [Julıch, Forachungazentrum\} CERN-P5-210 OLSEN, S.L. (Hawain U ) KEK-TE-003 OPPER, A.K. (Alberta U) TRIUMF-704 OREAR, J, (Cornell U, LNS) FNAL-611 OTTERIUND, I. (LuRd J) BNL.363 OTTERIUND, 1. (Lund U) CERN. EMVU-012 PAGE, \$.A. (Menitoba U) TRIUMF-497-287 PANEGRATSEV, Y.A. (Dnbng, JINR) JINR-L.HE-J01I-1 PAOLONE, V. (UC, DaYS) FNAL-8̈72 PAUL, S. (Heldelberg, Max Planck taat) CERN-WA-089 PAVAN, M.M. (Erttmb Columbia U ) TFUUAF-645 PAVLOPOULOB, P. (ERel U) CERN-PS-195 PENG, J.C (Ler A lomos) BNL.a90 PENG, J.C. (Loa Alamos) BNL-907 PENG, J.C. (Lo日 Alenoos) FNAL-769 PERDRISAT, C.F. (WUllom \& Mary Call) CEBAF-93-027 PERDRISAT, C.F. (William \& Mary Coll) SATURNE-202 PERDFISAT, C.F. (Willom \& Mary Coll) SATURNE-240 PERROOT, F, (Saclay) SAT URNE-144

PETERson, A,J. (Colorado U ) BNL-874

PETERSON, H.J, (Celorado U ) KEK-352

PETRATOS, G.M.C. (Kent State U) CEBAF-91-026

PETRATOS, G.M.G. (Kent Stale U) CEBAF-93-024

PIILONEN, L.E. (VirginLe Tथch) LAMPF-J240

PILE, P.H. (Brookbaven) BPL-886

PINFOLD, J.L. \{Alberse U \} CERN-LEP-OE

PISKUNOV, N.M. (Dubns, JINR) JINR-LHE-09d1-1A to -1D PISKUNOV, N.M. (Dubas, JINR) JINR-LHE-094t-4

PLATNER, E.D. (Brootbaven) BNL-810

PLATNER, ED. (ETookheven) BNL-991

POCANIC, D. (Virgmta U) LAMPF-1179

POCANIC, D. \{Virgnis Ut \} PSH-R+B9-01

POLING, F.A. (Minnenota U) CESR-CLEO

PREEDOM, B. (South Caroing U) CEBAF-94-002

PREEDOM, B. (South Carolne U) SATURNE-121

PRETZL, K. ('Berd U) CERN-NA-052

PRETZL, K. (Bern U) P\$1-Z-90-12

PFICE, P.B (UC, Berkeley) BNL.882

PROKOFIEV, A.N, (St Felergburg, INP) PNPA-SC-120

PROKOSHKJN, Y.D. (Serpulhow, IFVE) CERN-NAA-012-2

PROKOSHKIN, Y D. (SArptakho, IFVE) SERPUKHOW-172

PUNJABI, V. (Norlol'k State U) CEBAF-93-02?

PUNJABI, V. (Norfolk \$tate U) SATURNE-249
PUROHIT, M.Y, (South Caroline U) FNAL TO1 GUERCIGH, E. (CERN) CERTN-WWA-D97

RACAZZI, \$. (Milan $U$ and INFN, MIJa) CERN-NA-056

RAI, G. (LBL, Berbeloy) BNL-895

RAMEIKA, H.A. (Fomiab) FNAL-800

FAPIN, D, (Gonevs U) SATURNE-225

REAY, N.W, (Kangs State U) FNAL-803

REBKA, G.A., JR (Wyoling U) LAMPF-1310

REKALO M.P. (KharkDN, FT T) JINR-LHE-0941-3

REMSBER G, L.P. (Brookhaven) BNL-859

RIPANI, M. (INFN, GeaOX) CEBAF-93-006

RISTINEN, R.A. (Colorado U) LAMPF-119D

RITCHEE, B.G. (Amzons Strle U ) CEBAF-91-008

RITCHLE, B.G. (ArJaons State U) CEBAF-94-008

RITCHIE, J.L. (Twast U) BNL-871

ROBERTS, E.L, (Eapton U) BNL-821

HOBERTS, D.A. (Xichıgu U) LAMPF-1310

FOBERTS, J.B. \{RAce U) BNL-817

ROCK, 9.9. (Amerugan U) \$LAC-E-140X

ROEHRICH, K. (Jvluch, Forschangwentram) CERN-PS-185-2

FOERRICH, $\boldsymbol{K}$ (Julıch, Forbchungszentrum) CERN-PS-185-3

FOLANDI, L (OERN) CERN-LEP-ALEPH

HONDON, O.A. (VIrginıs U) SLAC-E-143

ROSSI, L. (Genos U and INFN, Gtoos) CERN-WA-OQ2 ROSSI, R- (Francati) CEBAF.93-017

ROTONDI, A. (Pava $U$ and 1NFN, Pavis) CERNAPS-202 RUBBIA, C. (CERN) CERN.PS-2t1

RUBBLA, C. (CERN) UNDERGROUNDICARUS

RUBIN, P.D. (Richmond U) CEBAF-93-022

RUN, R. (Trieste U) TRIUMF-508

RU1, R. (Triegte U ) TRIUMF-624

RUI, $R$ - (Trueste U ) TRIUMF-653

RUss, 5 s. (Carnegue Mellon i) FNAL-781

SADLER, M.E. (Abslepe Christian v) BNL-913-914

SAOLER, M.E. (Akltone Chruttan U) LAMPF-J26B

SAKAGUCHI, H. (Kyoto U) KEK-269

SANDWEISS, J. (Yale U) BNL-864

SAFYCHEVA, E.J. (MO\$COW SLate U) JINR-LHE-0971-1

SARYCHEVA, L.I (MOAOOW Stake U) JINR-LHE-0971-2

SASAO, N. (Kyoto U) KEK-162

SAWAFTA, $\boldsymbol{R}$ - (Brookbeven) BNiL-887

SCHELLMAN, H.M. (Narthwestern U) FNAL-665

SCHMIT', H. (Freiburg U) PSI-R-87-12

SCHMTTI, H. (Fretbnrg U) PSH-R-95-OB

SCHROEDER, H. (DESY) DESY-DOR|S-ARGUS

SCHUMACHER, R.A. (Carnegre hiellon $v$ ) CEBAF.89-004

SCHWARTZ, A.J. (Princaton U ) BNL-886

SEALOCK, R. (VIrgula U) CEBAF-89-017

SELIVANOV, V. (Kurchstor Inst, Moscow) TRtUMF-614

SEREDNYAKOV, S.I. (NovdouburG, JYF) WOVOSIBIRSK-SND SETO, R.K. \{UC, Furexalde) BNL-917

SEVIOF, M.E. (Btitub Golumbis U) TRIUMF-561

SEVror, M.E. (Erilsh Columbit U ) TRUAMF-624

SEYBOTH, P. (Mudub, Mnx Planck Innt) CERNE. NA-035

SHAEVITZ, M.H. (Columbra U) FNAL-B15

SHAROV, V.I. (Dubna, JINR) JINR-LHE.0941-A

SHEINKMAN, V.A. (MOLCOW, ITEP) ITEP.011

SHEINKMAN, V.A. (NogCow, ITEP) ITEP-94I

SHEINKMAN, V.A. (Noscow ITEP) ITEP-951

SHMMANSKIY, \$.S. (DUbna, JINK) JINR-LHE-1011-1

SHINGHARA, A, (NBgoYa U) KEK-262

SIEBERT, R. (Orgay. IP (N) SATURNE-222

SIEMLARCZUK, T. (Watshw, INR) JINR-LHE-094)-5

BITNIK, I.M. (Dubna, JINR) JINR-LHE-0941-1A and -1D

SITNIK, I-M. (Dubas, JINF) JINR-LHE-0941-3

SITNIK, I-M. (DubEs, JINR) SATURNE-249

GLATTERY, P.P. (Rochester U ) FNAL-706

SMIANITSKY, A.V. (Moocaw, ITEF) ITEP-895

SMITH, A.y.S. (Frinceton V) BNL.797

SMITH, E. (CEEAF) CEBAF-93-022 


\section{SPOKESPERSON INDEX}

SMITH, G.R. (TRחUAF) TRIUMF-560 SMITH, G.R. (TRIUMF) TRIUNF-719 SOBER, D.I. fOakbulk U \} CEBAF-91-015 SOKOLOVSKY, V,V, (Mog6OW, ITEP) SERPUKHOV-147 SOLOVIANOV, V.t. (SEPukhor, IFVE) \$ERPUKHOV-UNK solMOV, S.V. (Mogeow Phys Eng [nat) SERPUKHOV-126 SOUDFI, P.A. (SYraCuश Џ) CEBAF-91-010 EOUDER, P.A. (Syracube U) LAMAPF-1231 SP[NKA, F. (Argoune) BNL-9]3-914 BPINKA, H. (Argonne) JiNRR-LHE-0941-4 SPINK A, H. (Argonne) SATURAE-225 STEADMAN, B.G. (MIT) BNL-B66 STEVENSON, N.R. (Sakkatcherran U) TRIUMF-502 STOCK, R. (Frankfurt U) CERN-NA-049 STOLER, P. (Rentaklaer Poly) CEBAF.91.002 BTROKOVSKY, E.A. (Dubna) JINR-LHE-0941-1A and -IB STROKOVSKY, EA. (Dubnt) SATURNE-27g \$TEOOT, J,P. (Etnsebla U, JISN) CERN-NA-012-2 STRUNOV, L.N. (DubL, JINR) JINR-LHE-O941-1E STRUNOV, L.N. (Dubna, JWNR) JINR-LHE.0941-4 SUTORMIN, A.I. (Moscow, [TEP) ITEP.942 TATUTI, M. (JNFN, GenOa) CEBAF-g1-t002

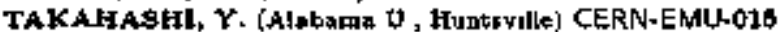
TAMURA, H. (Tokyo U) KEK-218 TAMURA, H. (Tokyo U) KEK-2B? TAMURA, H. (TakYo tf) KEK-326 TANNER, N.W. (ONford U) UNDERGROUPD-SUDBURY TAQQU, D. (PS1, Valtigen) PSI-R-91-08 TATISCHEFF, 由. (Orsay, [PN) SATURNE-244 TAYLOR, C.C. (Choe Wegtern Reserve U) FNAL-E64 TEREKHOV, Y.V. (Lícacow, ITEP) ITEP-873 THIBAULT, C. (Ormy, CANAM) CERH-PS-189 TIKHONOV, Y.A. (Novosuburk, TYF) NOVOSIBIFSK-KEOR TING, s.C.C. (MIT) CERH-LEP-L3 T1PPENS, W.E. (UCLA) BNL.909 TIPFENB, W.B. (UCLA) BNL.913-914 TOKI, W. (Colorado Stat* U) BEPC-BES TOMASI-GUSTAFSSON, $\mathrm{R}$. (Saclay) SATURNE-253 TOTSUKA, Y. (ToKYo U , ICRR) UNDERGR-KAMIOK TOTSUKA, Y. (TOKYO I , ICFR) UJNDERGR-SUPERKAMIOK TRETLLE, D. (CERN) CERN-LEP-DELPHI TRIBELE, R. (Texas A And M) TRIUMF-614 TROYAN, Y.A. (Dubne, JINR) JINR-LHE-0936-3 TSERRUYA, I. (Wermann Inst') CERN-NA-045 TSERRUYA, I. (WoIsmanin last) CERN-NA-045-2 TYAPKIN, A.A. (Dubns, JINR) SERPUKHOV-157 UGGERHOJ, E. (Aarbu' J) CERN-NA-043-2 UGGERHOJ, E. (Aarbus U) CERH-PS-194-3 USOV, $\boldsymbol{Y}, A$. (Dubü, ITWR) JINR-LNP-23 VAN DER SCHAAB, A. (Zunth U) PSI-R-87-03 VAN OERS, W.T.H. (Manttobs U) TRIUMF-36g VAN OERS, W.T.H. (Nanitobs U) TRIUMF-497-287
VAN ORDEN, J,W, (CEBAF) CEBAF-B9-033

VASILIEV, A.N. (Serpukhov, TFVE) SERPUKHOV-149

VENUE, W. (Rtutharfard) CERN-LEP-DELPHI

VERCELLIN, E. (TUII V \} SATURNE-237

VINEYAFD, M,F, (R.thmond U) CEBAF-93-OOB

VINEYARD, M.F. (Puthmond U) CEBAF-94-01?

VIOLA, V.E. (Indiaga Џ) BNI,-900

VLASSOV, A.V. (Moscow, ITEP) ITEP.894

VODOPIANOV, A.S. (Dubna, JINE) JINR-LHE-1010

VON EGIDY, T. (Mun]ch, Tech Џ) CERN-PS-203

VOVENKO, A.S. (Serpukbow, JFVE) SERPUKHOV.136

WADDINGTON, C.J. (Minnesets U) BNL-868

WADDINGTON, C.J. (Manлt해, U) BNL-869

WAF, Y.W. (Chicago U) FNAL-799

WANG, K. (VIrgIUIZ i) CEBAF-94-015

WELlER, H.R. [Duke U \} CEBAF-93-036

WENDER, GA. (LOA Alamos) LAMPF-1208

WEYER, G. \{ Aarhax U \} CERN-IS-300

WHITNEY, RLL (CBEAF) CEBAF-94-109

WHITXAL, D.M. (\$Imon Frawer Ut \} TRUUMF-630

WTLDENHAJN, P. (PAOD U) UNDERGR-HONESTAKEIODINE WTLHELM, I, (Charles U) JINR-LNP-23

WTLKES, R.J. (Washington U , Senttke) BNL-863

WILLIS, N. (Oraay, IPN) SATURNE-280

WINSTEIN, B.D. (ChIIAgo U ) FNAL.B32

WINTEL, K. (Huraboldt U, Berlin) CERH-WA.079

WINTER, K. (Humboldt U, Barlin) CERN-WA-095

WO.JCICKL 1 \$.G. (Stanford U) BNL-87)

WO.J ClCKt, \$.c. (Stanford tI) FNAL-875

WOLTER, W, (Cracow, INP-EXP) CERN-EMU-013

WRIOHT, D.H. (TRIUNA) TRIUMF-592

WURZINGER, R. (Saclay) SATURNE-280

YaMANNAKA, T. (Onnke U) FNAL-7o9

YAMAZAK1, T. (Tukyo Џ, INS) CERN-PS-205

YEGNESWARAN, A.S. (CEBAF) CEBAF-94-015

YONNET, J. (Saclay) SATURNE-202

ZAITSEV, A.M. (Sexpukho, 1FVE) SERPUKHOV-164

ZAJC, W.A. (Columbia U) BNt-a59

ZAFUEIN, A.V. (DubBs, JINR) JINR-LHE-094I-IE

ZAVATTINI, E. (Trute U) PSA-R-93-05

ZEIDMAN, B. (Argannt) CEBAF-91-016

ZELDOVICH, O. $\Psi$. (MOHCD, JTEP) ITEP-B3?

ZELLER, M.E. (Yale U ) BNL-Bib5

ZGHICHE, A. (Strasbonrs, CFN) SATURNE-2BO

ZCHICHt, A. (Eologaz í) UNDERGROUND-LVD

ZIEMINSKI, A. (IRdiaga U) FNAL-672A

ZJTLMANN, B. (Bage[ Us) P\$1-Z.91-02

ZOLIN, L (Dokna, JINR) JINR-LHE-0983-4

ZU PUTLITZ, G. (Kadelberg t) , Phys Jnat) LAMPF-1054

ZUPRANSKI, P. (Sollan Ingt) SATURNE=190 


\section{ABBREVIATIONS USED IN THE SUMMARES}

\section{JOURNALS}

Following ore abbreqiations for journals listed in the gummaries:

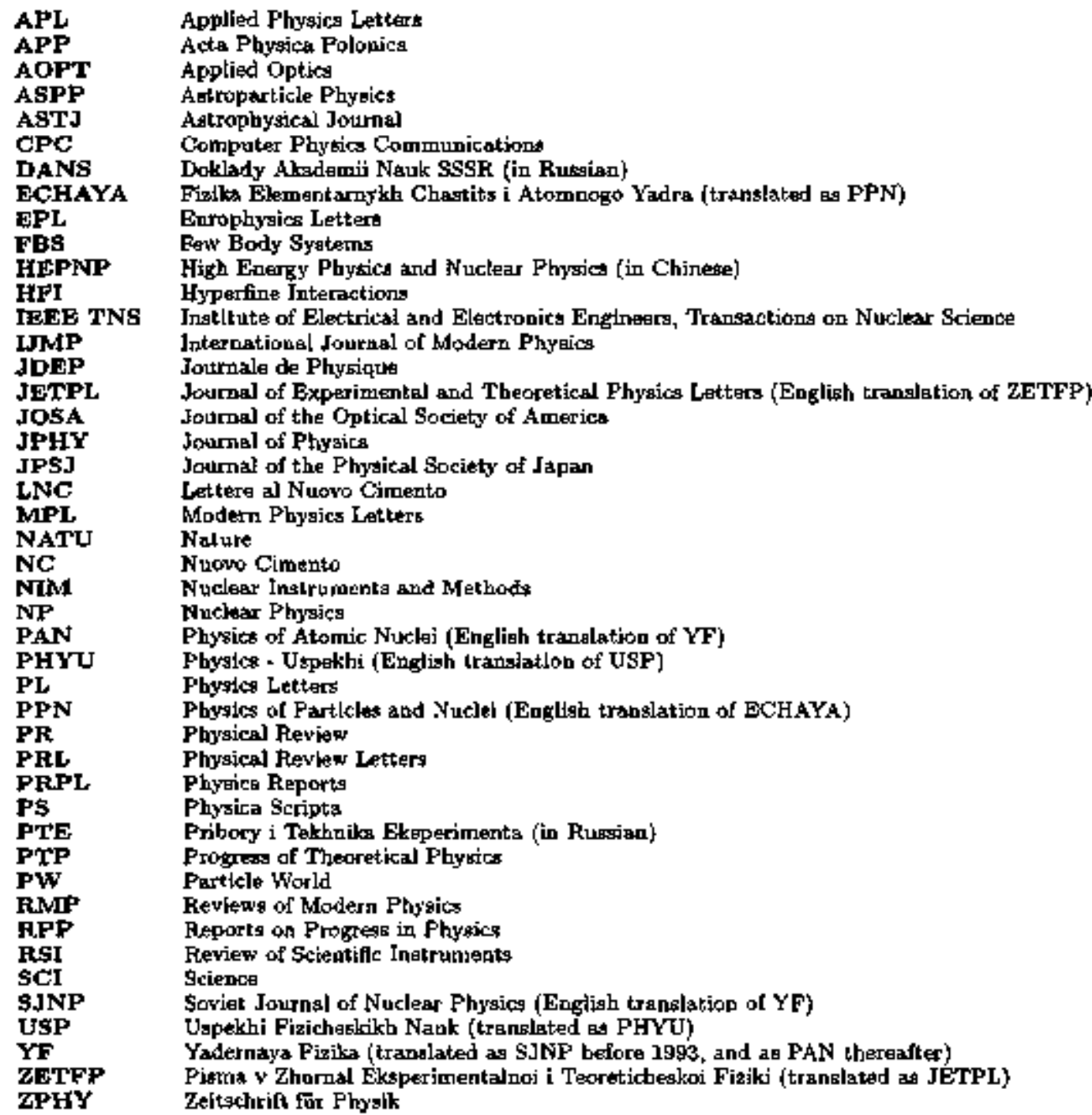




\section{KINEMATIC VARIABLES}

Following are abbrepiations used with reactions to indicate the moments of energies at whech they are studied:

PLAB beatn momentum in the lab frame

TLA B best kinetic anergy in the lab frame

ELAB beant totel energy in the lab frame

PLAB/N boam momentum per nucleon in the lab frame

TLAB/N beam kinetic energy per macleon in the lab frame

ELAB/N baam total energy per nucleon in the lab frame

ECM lotal enery in the cim. Urame

\section{ACCELERATORS}

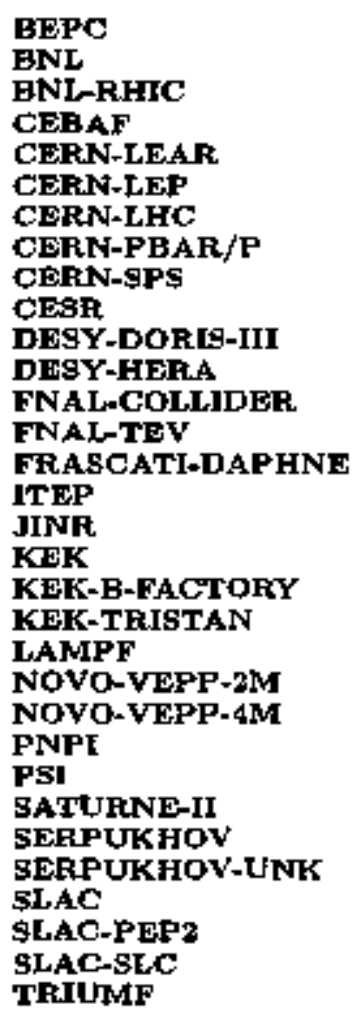

Beijing $\mathbf{e}^{+} \varepsilon^{-}$collider (3.t $\mathrm{GeV} \mathbf{E c m}$ )

Brookhayen AGS proton synchrotron (31 GaV/c Plab)

Brookhawen relativistic heavy ion collider (100 GeV/n per beam)

CEBAf linear accelerator with continuons $\varepsilon^{-}$beam (4.0 GeV Ecm)

CERN Low-Energy Antiproton Ring

CERW Large Electron-Positron collider (ana-180 GeV Erm)

CERN Large Hadron Collider (I4 TBV Ekm)

CERN pp collider (900 GeV Esm)

CERN Super Froton Synchroton (450 GeV/e Plab)

Cornell Electron-positron Storage Ring (16 GeV Ecs)

DESY DOPUS electron-poeitron double ring, 1981 upgade

DESY IIERA electron ( $27 \mathrm{GeV})$ - proton ( $(220 \mathrm{GeV})$ collider

FNAL $\bar{p}$ tollider $(2000 \mathrm{GeV}$ Ecm)

FNAL fix target Tevation (1000 GeV)

$e^{+} e^{-}$etorage ring and $\phi$ factory (1 GeV Erm)

ITEF Moscow proton synchrotron (7 GQV/c Plab)

JINR (Dubna) proton synchrotron (10 GeV/c Plab)

KEK protoll sytuchrotron (I2 GeV/c Plab)

KEK two-ring asymmetric $\mathrm{E}$ factory

KEK electron-potitron storaye ring (60 GeV Ecm)

Los Alamos Meson/Proton Factory (1460 MeV/c Plab)

Novasibhrak VEPP-2M electron-positron storage ring (1-4 GeV Ecmo)

Nowodibirsk VEPF-4M ecteron-positron storage ting (7 GeV Eem)

Sytrchrocyclotjon at 3t. Petershurg Inst. Nuc]. Phys. (1 GeV Tilab)

Cyckolron at Pau! Schereer Institute (590 MeV Tlab)

Saclay Satutno-lI p, $\alpha$, and Hee syachrotron

Serpinkhow pratos aynchrotron (76 GeV/c Pleb)

Serpukhow multi.TeV proton machipe

Stanford electron linear accelerator (40 GeV/c Plab)

New \$LAC Positron-Electron Project (asymmetric B factory)

SLAC Linear $e^{+} e^{-}$Collider (100 GeV Eem)

Cerpadian, TRIangle Uniweraity Meson Facility (s20 MeV Tlab) 


\section{DETECTORS}

In sarcbing the EXPERHMENTS database (SLAC/SPIRES) from which this report is taken, use the following abbreriations for gexieral kinds of detectors:

\begin{tabular}{|c|c|}
\hline GALO & calorimeter \\
\hline CNTR & counter(s) \\
\hline COMB & combination of various elements \\
\hline DAS & doubje-arm spectrometer \\
\hline DRIFT & drift chauber \\
\hline GMUt & emulsion \\
\hline IONIZATION & detector looking for ionization \\
\hline MWPC & multiwire proportional chamber \\
\hline PHDTON & photon spectrometer surk as $\mathrm{NaI}$ oc Ge detectors \\
\hline PEASTIC & Lexan, ętc., ugesd like emulsion \\
\hline PMT & various photornultiplier tubsos \\
\hline RICH & ring-imaging Cerenkow detector \\
\hline SAS & aingle arw घpectrometer \\
\hline SCINT & seintillator \\
\hline SPEC & spertiometer syatem \\
\hline \$TRC & streamer chamber \\
\hline TRACK & tradking system \\
\hline WIRE & wire chantber \\
\hline
\end{tabular}

We use the following acronyms for speitle detectors (both in this Report and in the SLAC/SPIRES database):

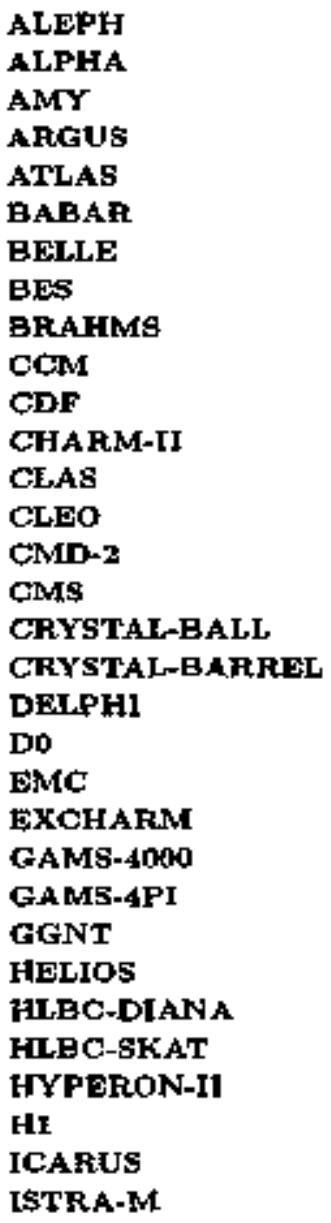

CERN-LEP detectar

JWN magnetic apectrometer aystum

KEK-TRISTAN high-resolution lepton detector

DESY-DOFUS.II datector

CERN-LHC proposed detector

SLAC-PEP2 proposed detector

KEK-B-FACTORY proposed detector

BEPC deteclor

BNL-RHLC proposed Broad Ranga Hadron Magenetic Spęctroneter

FNAL-TEV Chicago Cyclotron Magnst epectrometer

Collider Detector at Fermilab

CEAN-SPS upgrave of CHARM nentrino detector

CEBAF Large Accoptance Spectrometer

CESR spectrometer systent

Nowosibirsk upgrade of CMD detector

CERN-LHC propoesed detector

SLAC-PEP, DESY-DORIS, BNL Crystal Ball large-golid-angle neutral detector

CERN-LEAR large-solid-angle detector

CERN-LEP detector

FNAL_COLLIDER deteclor

CERN-\&PS European Muon Collaboration detector

Serpukhov detector, upgrade of BIS-2M

CERN-SPS $64 \times 64$ cell Pb-glass array

Serpulkhov garnma spectrouneter

Bakkan Gallium-Germagiunm Neutring Telegcope

CERN-SPS detector

ITEP leavy liquid bubble chamber

Sarpukhow hasay liquid bubble chamber

Serpukhor tiagle arm magnatic spectrometar

DESY.HERA detector

Gran Sesso bubble chamber detector

Serpukhov detectoor 


\section{DETECTORS}

\begin{tabular}{|c|c|}
\hline JETSET & CERN-LEAR compsct general purpose detector \\
\hline KEDR & NOVO-VEPP-2M universal magnetic detector \\
\hline KLOE & Fragcati DA $\Phi$ NE detector \\
\hline LAE-E & FNAL-TEY carget-calotiateler muon-Bpectrometer detector tor neutrino phyaics \\
\hline LAMBDAMETER & ITEP detector \\
\hline LSND & LAMPF Liguid Scintillator Neutrino Detectot \\
\hline Ls & CERS-LEP detector \\
\hline MACRO & Gran Sasbo deteçtor \\
\hline MEGA & LAMPF array of electron and photon spectrometers \\
\hline MIS & Serpukbov multiperticle spectrounter \\
\hline MIS-3 & Barpukhov upgrad or MIS \\
\hline MP5 & BNL MultiParticle Spentrometer \\
\hline MPS-II & BNL upgrade of MPS \\
\hline MTS & JTEP detector \\
\hline NEPTUN & Serpukhow-UNK jel target detector \\
\hline NMS & LAMPF Neutral Meaon Spectrowneter \\
\hline OMEGA & CERN, CERN-SPS spectrometer syatem \\
\hline DMEGAPRIME & CEFN-5PS spectrometer system \\
\hline DPAL & CERN-LEP detector \\
\hline PHENIX & BNL-RHIC photon, alectron, and liadron delector, under construction \\
\hline PHOBOS & BNLRHIC two arm mulliparticle spectrometer, under construction \\
\hline PINOT & Saclay bigh resolntion pil and eta detector \\
\hline QUARTZ & Serpukhov crystal-difitantion spectrometer \\
\hline SINDFUM & PSl large-solid-angle magnetic detector \\
\hline SINDFUM-II & PSI upgraded large-angle solenold detector \\
\hline SLD & SLAC.ȘLC detector \\
\hline SND & Norosibirate Spherical Neutral Detector \\
\hline SPES-0 & Saclay modular lead-glage Cerenkov detector \\
\hline SPES-II & CERN, CERN-LEAR high-resolution spectrometer \\
\hline SPES-III & Saclay bigh-resolntion epectsometer \\
\hline SPES-IV & Saclay bigh-resclution spectrometer \\
\hline SPES4-PI & Saclay high-reaolution apectrometęr \\
\hline SPHINX & Serpukhoy detector, also known as SFINKS \\
\hline STAR & BNL-AHIC solenoidal detector, under coostruction \\
\hline TOKIWA & KEK.PS вpectrometar \\
\hline TOPAZ & KEK-TRISTAN solenoidal spectrometer with TPC \\
\hline TPS & FNALl Tagsed Photon Spectrometer \\
\hline VENUS & KEK-TRIBTAN Versatile Economical and Noved Universal Spectronteler \\
\hline VES & Serpikbow magnetic VErtex Spectrometer \\
\hline ZEUS & DESY-HERA detector \\
\hline
\end{tabular}




\section{BEPC Experiments}

\section{BEPC-BES}

(Began date-taking 1991, Le progrebs\})

\section{MEASUREMENT OF THE + LEPTON MASS WITH} THE BEIJING SPECTROMETER (BES)

BES COLLABORATION

BELJING, DHEP - J Z RBL, S M Chen, 5 J Cben, Y Q Cheng 2 D Chase, 五 C Cul, X Z Cur, H L Ding, Z Z Du, C Fang, $M L$ Gad, $S Q$ Gao, $w \times$ Gab, Y N Gso, J H Gu, \$ D Gu, W X Gv, Y N Gud, Y Y Gun, Y Han, J He, G Y Hu, H B Hu, T Hn, D Q Huang, Y $Z$ Huang, 0 H Jhang, $Z$ I Jang, Y $F$ Ls,

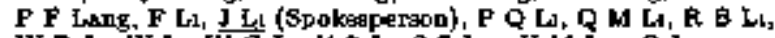
W D L $, W L_{1}, W G L, Y \$ L_{1}, S Z$ Lin, $H$ M $L_{14}, Q$ Lit, R G Ltu, Y Lu, J G Lu, D H Ma, E C Ma, J M Ma, F S Mao, Z P Mao, X C Meng, H L Ni, L J Pan, N D Q , Y K Que,

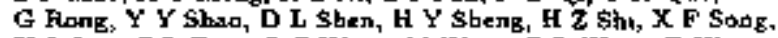
H S gon, G L Tong, $L$ Z Wang, M Walg, P L Wang, P Wang,

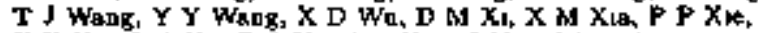
$X \times X_{1 q}, \&$ \& $X_{u}, z Q X_{u}$ \& $T X_{u e}, J$ Yon, $w$ G Yan, $C Y$ Yang, $C M$ Yang, $B$ B Yso, of $H$ Ye, 5 Z Yo, $Z Q Y u$, B Y Zhang, C C Zhang, D H Zbang, A Y Zhang, H L Zhank. J W Zhang, 1 \& 2bang, \$ Q Zhang, Y Zhang, $D \times$ Zhoo,

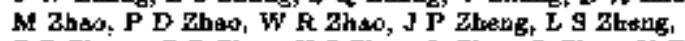

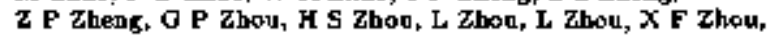
Y H Zhou, Q M Zhu, Y S Zbu, Y C Zhu

BOSTON U .. J A Coller, A 5 Johnoon, J Stank, J S Whataker

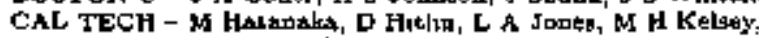

J H Panelta, F Porter, E N Prablokar, X Sht

COLORADO STATE U - J Chan, Q F Ja, W Tok

[Spokesperson], If J Wilsab

HAWAII U - A Breakutone, F Harns, S Olonen, D Palusell

MIT, LNg - O Bardon, R Gowan, M Foro, I Quigley, E Torrence,

F. K Yomamoto

SLAC - R A Becker-Szendy, W M Dumpodie, H Marsiske

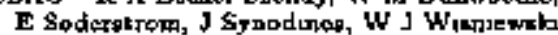

TEXAS U, DALLAS - I Biun, J \$ Campbell, P G rarton, J M [zen, X Lon, B Lowery, J Stendifird

UC, [RVtWE - A I Laniford, Mt Mandelkern, M seberuau.

B Schmid, I Schultz, A Smuth, D P Stoker, G zioulas

WASKUNGTON U, SEA']"CLE - T Bornett, $K$ Young

Accolerator BEPC Detector BES

Reactions

$$
c^{+} E^{-} \rightarrow+^{+} \tau^{-} \quad 36 \mathrm{GoV}\left(E_{e m}\right)
$$

Partinlen studied T

Brtef descruption Uses noth-collunear 2-prong et events with both to and a identified Moseures the threshold behaver of the crote-gection by scanning a very narrow range of entergles, $3504<E_{\mathrm{mm}}<3569 \mathrm{GeV}$ The BES delectar conath of a Muou Counter, TOF Coututera, a Barrel Showter Counter ard the mejn Drift Chamber A major apgrade of the detector will be complated in the Fall 1908, sad dats-testing conturued

Jotrpal papers NTM A308 (1091) 816, PRL 68 (1092) 302L, PL B955 (1995) 374 (erratum FL B\$63 (1995) 287), PRL 71 (1955) 459y, PR D52 (1995) 3781, PF. D53 (1996) 20, avd PR. D54 (1906) 1221

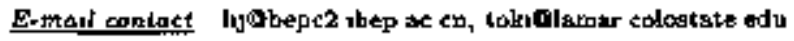
WWW Hone poge

http / / www-bes alac stanford edu/beswww/bes himl

\section{BEPC Future Plans}

The apgeade of the BES opectiometer, which began in 1903, un Dearing its completion, which promare new Imporkant physyes

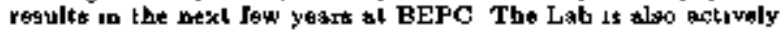
inwolved in the dessgn of the Beylag Teu Charm Factory (BTCF) phuch got positive revew, at home and nbrond BTCF was the maib topic of a recint wotkshop in Bejging

\section{Selected BNL Experiments}

\section{BNL-7\$}

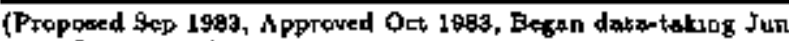
Lags, In progress)

\section{A STUDY OF THE DECAY $K^{+} \rightarrow x^{+}{ }_{y} \bar{\nu}$}

BFOOKHAVEN - 9 Adler, M S Aliys, I H Chang, M DIwan, I 9 Frank, E Garber, IS Haggerty, S H Kettell, T F Kycan, K K L1, L E Littenberg ( $\checkmark$ Spokaperson), C F Ng.

A Sambanathi, A J Steveng, R C Strabd, C Witgig, Y Zhan TOKYO U, JNS - M Kazumon, T Konataubars, M Kursk,

T Monmpto, N Moramaksu, it Okuno, K Orata, A Otono, S Sngimato, K Tarahi, K Uta

KFK - M Aoki, T Intgaku, 5 Kabt, M Kobayalbi, Y Knno, T Sato, T Shinkawa, $Y$ Yoshmura

OSAKA U - Y Kishi, T Nakeno, T Sasaki

PRINCETON U - D Akenb, M Ardebale, M Convery, M M to,

D R Markw, R MePherson, P D heyers, W Sands, M A Selen, F C Shoethaker, A J Smuth ( $v$ 'spokespetston), J R Stone TRIUMF - P Bergburch, E W Blatsmora, D A Brymas

( $\checkmark$ Spokegperaon), M Bturke, J V Gregswell, A Daviel, S Daviel, P Krtching, A Kosaks, M LeNoble, J A Medeagld, J Muldersberger, T Numan, P Padley, I M Pontiseou, R Futiliapon,

G Redlenger, J Foy, MA Rown, R Solutr, A S Turcot

Accelecintor BNL Befector Spectrometer

$$
\begin{aligned}
& \text { Reacliotis } \\
& K^{+} \rightarrow \pi^{+} \mu \bar{V} \\
& \mathrm{~K}^{+} \rightarrow \mathrm{I}^{+} \mathrm{jL}^{+} \mathrm{H}^{-} \\
& \mathrm{K}^{+} \rightarrow \mu^{+} \mu^{+} \mu^{-}{ }^{+r} \\
& \mathrm{~K}^{+} \rightarrow \mu^{+} \mathrm{Na}_{2} \\
& K^{+} \rightarrow x^{+} \text {huggs } \\
& \mathrm{K}^{+} \rightarrow \mathrm{x}^{+} \mathrm{y}_{\mathrm{j}} \\
& \mathrm{K}^{+} \rightarrow \mathbf{x}^{+} \mathrm{X} \\
& K^{+} \rightarrow \pi^{+} \pi^{*} \gamma \\
& x^{0} \rightarrow \nu \bar{y} \\
& \pi^{0} \rightarrow y x \\
& \pi^{0} \rightarrow \sim \vee \bar{y}
\end{aligned}
$$

$$
\begin{gathered}
0 \mathrm{SeV} / e\left(\mathrm{P}_{\mathrm{lab}}\right\} \\
" \\
" \\
" \\
" \\
" \\
205 \mathrm{MeV} / \mathrm{C}\left(\mathrm{P}_{\operatorname{lab}}\right)
\end{gathered}
$$

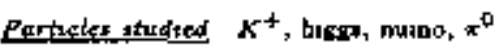

Brof description A seneitivity down to a level of $1 \times 10^{-10}$, nr better, is expected for $\boldsymbol{K}^{+} \rightarrow \pi^{+} \nu \bar{V}$ A pepseurement at this leved delermines $\left|V_{t d}\right|$. given that $m_{*}$ is kwown An obeervation fipalficanthy above this level would indicate a fourth generation of quatka and keptons, the presence of nuatod, or other betw phenominas A sumultaneons moakurement of $\mathrm{K}^{+} \rightarrow \mathrm{x}^{+} X$ to a sengitivity of about $2 \times 10^{-11}$ is alo expected Thus prober the exustence of acuons, famulans, hyperphotong, or other new particles Other processes probe the exabtence of higgs, majoron, mosswe nentrimos, and other bypothetucal portucles The flrat run was completed in Jume 91 Approved for 3000 additional hours with an upgraded beam and detector The new beam ( $750 \mathrm{MoV} / \mathrm{C} K^{+}$, stopped w apectrometer) was comomissoned in 1992, the modified detector in 1994, and dats was alresdy collected with the new sexup in 195b fot Dats Faking is scheduled to reguse in arly 1987

Journal papery NIM A279 (1989) 180, PFU 69 (1949) 2177 , PRL 64 (1990) 21, FRL 65 (1990) 1186, NP (PROC gUPPL) 13 (1990) 568, PRL 66 (J日9) 2189, NIM A32] (1892) 129, PRL 69 (1992) 733. PRL 70 (1893) 252I [erratam PRL 71 (1903) 30s], PR D4t (1009) 1, PR DAs (1903) 1225, and PTL 76 (I998) 1421

Reisted expenmerts $\mathrm{N} / \mathrm{A}$

Exmas contact luttenbeobal gov, smithopnphep pruceton edu doug tomles triunf $\Leftrightarrow$

WWW Home-tage bttp / /ww phy bnl gov/e787/e787 htwl 


\section{BNL-821}

(Proposed Sog 1985, Sep 1980, Approved Nov 1986, Lo prepara. trop)

A NEW PRECISION MEASUREMENT OF THE $q_{\mu}-2$ VALUE AT THE LEVEL OF 0.SE PPM

MUON (G-2) COELABORATION

BOSTON U - D H Erown, R M Cakey. W Earle, E Eletgrhuadia, E 5 Haten, B J Hughes, F Krienes, J P Miller, O Rind, B L Roberts ( $V$ Spokesptriton), L R Sulak, W A Worktell BROÓKHAVEN - I T Bensnte, H N Brows, g Bunte, I R Colkn, \& T Danhy, C R Gardiner, J Id Geller, H Beeuh, J W Jackson, L- Js, $\mathbf{R}$ Lorben, Y Y Ees, R E Meier, W Meng.

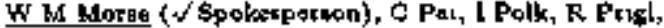

S Aankonribx, J Ssulberg, Y Semertzidı, R Sbuit, L Gnydactup, A Soukas, A Stultros, T Tallence, F Thompoon, F Toldo, $K$ Woodlo

CORNELL U - T Kunashets, Y Orbor

FAIRFIELD It - D W $W_{1}$

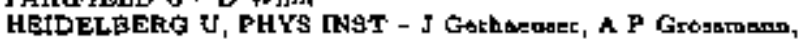
K Juncroand, P yon Woltex, G iu Putlitz

HEIDELBERG, MAX PLANCK INST - U Bwoberled

ILLINOIS Џ, URBANA - P T Debeyec, W Deningor,

D W Herttog, $\$$ A Stedytah, D C Utuer

LEL \& BROOKHAVEN - M A Green

MINNESOTA U - P B Gurhmad, 5 O Gron, J M Kundem,

DE Maxan, D E Miller, C Timmeximsar, D Zuniperman

NovosipLfsk, TYF - L M Barkav, D N Gingorev, B I Khazin,

E A Kurnev, Y M Sbatnnov, E Soladov

TOKYO U - K Naghthibe

BCIENCE U, TOKYO - Y MnUmich

KEK - K Endo, If tirpbayabbi, \& Ichil, S Kurohnwa, T Sato A rampingto

WAKO, RIKEN - K Jibide

YALE U - S K Dhawan, F J M Farley, M Groseo-Perdehorap, V W Hughe: (V Spokespersoon), D M Kawall, S I Redun

Acceleratgr BNL Detectigr Othet

Reschions Polareed beam

$$
\text { muton } \rightarrow E^{ \pm} \nu F \quad 309 \mathrm{GoV} / \mathrm{c}
$$

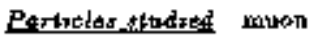

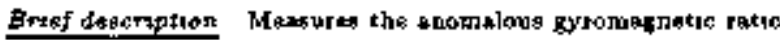
of the muon and provides a dreat teat of the Standerd Model Usex n 7 -meradute toperfetrue moon starage ring with a 1 15.

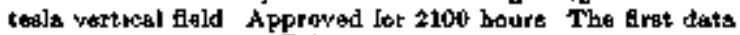
collection *xpested נת FY I997

E manf centact rabertsobu edn, marsejobnl gor, sernos bugheagyale edu

WWW Home-page ittp //Www pby bal gov/gzmnon/home btal

\section{BN1-840}

(Prapcerd Jal 1987, Approvtad Ocl 1987, Beggan datm-takıng Jul 1989, Complalod dala-tahing Doc 1901)

SEARCH FOR THE COHERENT PRODUCTION OF LIGHT SCALAR AND PSEUDODCALAR PAFTICLES

ROCHESTER U - R Csmeron, G Cautatore, $A$ O Melwosnos (V Spokespereon), J T Rogers, G Roobo, Y K Semartzrdis BRDOKHAVEN - H Hatama, D Lazarus, A G Prodell FERACILAB - F A Nęrick

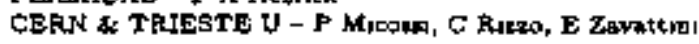

Acctierater NONE Detector Othat

Parincles stendied axros

Bref descriptzat The delector used two CBA superenducting dipoles Searched for lagbl talar or parudosealar particles that couple to the electromagater Reld Laoked for optical rotation of a polanzed laser besch traversung in vacium the $35 \mathrm{~T}$ magnetic fiekd The kenutuvny of $10^{-10} \mathrm{rad}$ corresponds to a limn on the caupling $g_{0 \gamma}$ of $4 \times 10^{-7} \mathrm{GeV}^{-1}$ Did aot reach Delbruck acattengig (reb photons from ysftual photona) below the $e^{+} e^{-}$thresbold
Jourtial papers PRL 64 (1900) 2988, JOSA Es (1991) 520, PL A157 (1991) 125, PRL 69 (1992) 2393, ZPHY C58 (1992) 506. and PR. D47 (1993) 3707

Retated expernmante BNL-B05

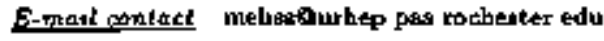

\section{BNL-852}

(Froposed Jan 1989, Approved Mar 1989, Began data-takjug Jun ids3, In progrea)

SEARCH FOR MESONS WITH UNUSUAL QUANTUM NUMBERS

BROOKHAVEN - \$ U Chang ( $\checkmark$ Spokespersop), R W Hackenburg, K Oichanaki, D P Weygand, g J Willukzks INDLANA U - B B Brabson, R A Critcenden, A R Dalerba,

P C Piglioss, J L Gubter, B Kern, R Lindenbubeh, M McKeriey, D R Rutt, E B Scott, P T Smith, T Sulanke, 5 Tengt

MASSACHUSETTS U, DAFTMOUTH - Z Bar-Yam,

J Curmminga, J Dowd, P Eugenio, W Kern, E Kus

MOSCOWW S'TATE U - E Y A noghins, L I Beber, V A Eodysgn,

A Domianow, A M Gribuabin, O L Kadolove, V L Kototkikb

M A Kosin, N Krutlow, A I Oatrovidov, A \& Proskuryatory,

L I Sarycheva, N B Suev, I N Vardanyan, A A Yershow

NOTRE DAME U - T Adams, J M Bishopr N M CAnDM

( $\sqrt{ }$ Spokesperaon), E I Iranoy, J M LoBecco, IJ J Mamak,

A Sonjarl, W D Shephatd, D L Stienike, 3 A Tatgar,

D R Thempaon

NORFHWESTERN U - D 9 Brown, T Podlar, $\mathrm{K}$ K Seth, J $W_{\text {late, }}$ D Zhew

SERPUKBOV - S Denison, A Dushkun, V Kocheckow, V Lupsey, I Shein, A soldatov

REVSGPLAER FOLY - O Adsms, J Kuhb, J Napolntano,

M Nort, I Smoh, D B White, M Witkowski, A s Wright

Accelerntor BNL Detector MPS

\section{Beactions}

$$
\begin{aligned}
& \pi^{-} p \rightarrow n+\pi^{\circ} \\
& \vec{x}^{-} \boldsymbol{P} \rightarrow \forall+n \\
& \pi=p \rightarrow p h H^{-} \\
& \pi^{-} y \rightarrow n^{\prime} x^{-} \\
& \pi^{-} p \rightarrow p \eta \pi^{-} \pi^{+} \pi^{-} \\
& x^{-} p \rightarrow p \pi^{-} \pi^{+} \pi^{-} \\
& x^{-} p \rightarrow n \eta x^{0} *^{0} \\
& \pi^{-} p \rightarrow n \pi \pi^{+} x^{-} \\
& \pi^{-} p-q \pi n^{\prime} \\
& \pi^{-} p-\pi K^{0} K^{0} \pi^{0} \\
& \pi^{-} D-\pi K^{0} \frac{K^{0}}{\pi^{0}} \pi^{0}
\end{aligned}
$$

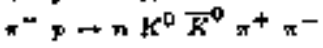

$18 \mathrm{GaV} / \mathrm{c}$

n

4

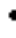

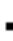

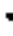

Purticltesefludted exotic-mezon, gluebell, hybred

Bnef description Looke in purticular tor furtber uridence of an

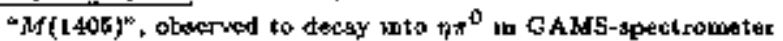

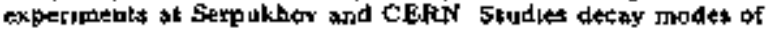
metons, with multiphotons and 0,1 , or 2 charged particles The detcetor is bult around MPS An $\mathrm{H}_{2}$ terget wo rurrounded by a Cal vest Olher parte of the apporatus are therged particle detector, s Cereskov coubter, and a \$ototelemeut lead glass calorineter Approved for a total of b000 hopta Takjag data (July 96)

Journal papers NTM A332 (1993) 4 19, A342 (1994) 398, NTM A857 (1995) 95 and NIM Aa6s (1906) 617

Rejated experaments SERPUKHOV-163, CERN-NA-012-2

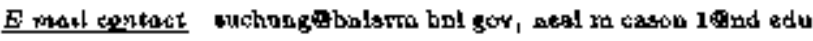
WWW Home-page

http //lomend phy bal gov/-e\$52/homa_eos2 html 


\section{SUMMARIES OF BROOKHAVEN EXPERIMENTS}

\section{BNL-865}

(Propased May 1960, Approwod Jup 1990, Began data-takun, 1995, [c progress)

\section{IMPROVED SEARCH FOR $\mathrm{K}^{+} \rightarrow \pi^{+}{ }_{\mu}{ }^{+} e^{-}$}

\section{ESA5 COLLABORATHON}

BASEL U - G Backenetoks, W Menzol H Wayt

BROOKHAVEN - D Lazarus, L Laupuner, If Ma, P Rebal

MOSCOW, INR - G S Atoyen, Y V [azkoy, D Kacavkehey,

A. A Pabjeguev, Y Poatoev, I N sesnopiouk

NEW MEXjCo if - B Eatazalleck, \& W Eslerts, I Lowe, D Wolfe PSI, VILLIGEN - J Egker, W D Herald, H Kappr. I Mientreer PJTTSBURGH U - R APpel, N E Chent, C A Felder,

H M Gech, D E Ktaus, I G Ober, P A Yominmowak, A Shet, I A Thompen

TB1LtSI sTATE tj - Y \& Bagaturis, D Mazevie, \& V Melitaun, TM Sekbolasbvila

YALE U - D R Berginan, H D Do, I A Locane, W A Majld,

M E Zeller (Y Spokeagerman)

zUFICH U - S Pralak, P Rohmonn, P Trwoel

Acarierstor BNL Detertor Spectrometer, Gatorimeter

\section{Reactiegn:}

$$
\begin{aligned}
& \mathrm{K}^{+}=\mathrm{m}^{+} \mathrm{\mu}^{+} \mathrm{e}^{-} \\
& K^{+} \rightarrow \pi^{+} e^{+} e^{-} \\
& \boldsymbol{K}^{+} \rightarrow \pi^{+} \mu^{+} \mu^{-} \\
& K^{+} \rightarrow \pi^{+} x^{0} e^{+} e^{-} \\
& \mathrm{K}^{+} \rightarrow \pi^{+} \mathrm{x}^{-} e^{+} \\
& x^{\mathrm{D}}-e^{+} e^{-} \\
& \text {(4) } 0 \text { GeV/c }\left\{P_{\text {lab }}\right\}
\end{aligned}
$$

Parlaties sturiated $K^{+}, \star^{\circ}$

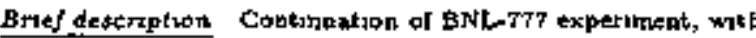
a Pactar of approximstely 70 improved endituvily the main purtace is to obaerve, or place a limit of a Eew tiraeg 10-12, on the brewehing retro of the murn uumber violsterg dacesy $\mathrm{K}^{+} \rightarrow \mathrm{\pi}^{+} \mathrm{H}^{+}{ }_{e}^{-}$U日es a maknetic apectromelet syolam with vary higb rate, and good partucle identificalion Almo at achreving mgnificant impravapents on menurementop of rates and kriematic dutrobutions of otber rare dectys $\left(B R<10^{-1}\right.$ ) Tekang dats (July, of)

\section{Retoted expetrments BND-7T7}

Exmarl contact muthel zellertibule edu WWW Hothe. papte

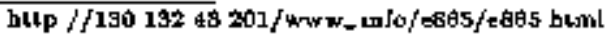

\section{BNL-871}

(Propoed Sep 1900, Approvod Nov 1990, Began datartatang Fôb i995, In progrets)

\section{A NEW SEAFCH FOH VERY RARE $K_{L}$ DECAYS}

UC, IRVINE - Y Abadrey, a G Bachmen, D F Connor, P DeCecca, R A Dpilibarev, N Kaptmatsu, R K Lee,

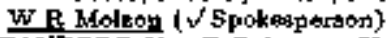

STANPORD $U$ - C G Arroyo, K W Hartmagn M I Hebert

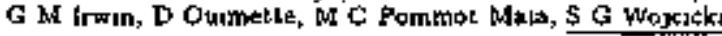
( $Y$ Spókesperson)

TEXAS U - D Ambrom, S C Groesgle, K Lahz. A Midder. J L Ritchie ( $V$ Spaketpernag): V Varakstopoulos

WILLIAM AND NAKY COLL - M Eckhnume, A D Hapcpock, C Hoff, J R Kane ( $/$ Spokespreon) Y Kuang, R D Martin, R E Welkh, EJ Wolli

RLCHMOND U - P D Ruba

Atesiltratar BNL Detefior Spectromet.er

Reactrons

$$
\begin{aligned}
& K_{L} \rightarrow \mu^{-} \\
& K_{L}-e^{+} \\
& K_{L} \rightarrow \mu^{+} \\
& K_{L} \rightarrow \mu^{+}
\end{aligned}
$$

Particiles studsed $K_{L}$
Bnef descrotion $A$ maxh for the decays $K_{L} \rightarrow$ the and $K_{L} \rightarrow$ es with o seasitsyity of $10^{-12}$, and a measurentent of the branching fraction for $K_{L} \rightarrow \mu \mu$ ENL-37L improves an the nenmtivity of BNL-791 by more than \& factor of ter The detector conalote of two dipoles, straw and wire difit

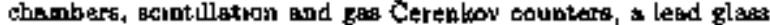
nhower sounter, and a moon rangelobder A novel festure of the experimept as a sbjelded turgaten basm stop unsode the

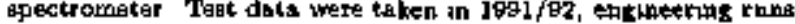
were done in $1993 / 94$, and the act tat dats takung bogan weth a 5-month run in 1995 Approvad for 7,000 hours In progress (1998)

Related expertments BNL-791

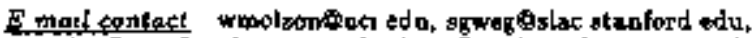

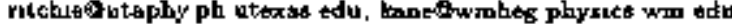

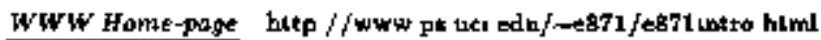

\section{BNL-881}

(Propored Jul 1991, Approved Aug J99\%, In progress)

UTHLFING \& SPECTROSCOFY TO SEARCH FOR EXOTIC GLUEBALLS, EXOTIC HYBRID, OR EXOOTIC MULTIQUAFK STATES

BFOOKHAVEN $t$ CITY COLL. NY - S J LAndentbanm

(Spakenperana)

BHOOKHAVEN - R W Hackenturg, $\mathbf{R}$ S Langacre

CITY COLL, NY - C S Chsn, E Efatathiadis, A A Krsmer. $K$ Zhas Y Z Z

CONNECTICUT U - M Gat

FEAMILAB - K VERIT

RENSSELAFR POLY - G Adam, C Lardberg, A M Porry, B Wojteakhowkk

Acseleretar BNL Detector MPS-II

Reactuotis

$$
\begin{aligned}
& \pi^{-} p \rightarrow \infty \\
& \pi^{-} \boldsymbol{B} \rightarrow \boldsymbol{\phi K}^{+} \mathrm{K}^{-} \mathrm{n} \\
& \pi^{-} p \rightarrow K^{+} K^{-} K^{+} K^{-} n \\
& K^{-} P \rightarrow \phi \Lambda \\
& K^{-} p \rightarrow \phi \Sigma \\
& K^{-} \rightarrow \phi K^{+} K^{-} A \\
& K^{-} \boldsymbol{y \rightarrow \phi K ^ { + } K ^ { - } \Sigma} \\
& K^{-} \boldsymbol{p} \rightarrow \boldsymbol{K}^{+} \boldsymbol{K}^{-} \boldsymbol{K}^{+} \boldsymbol{K}^{-} \Lambda \\
& K^{-} y \rightarrow K^{+} K^{-} K^{+} K^{-} \text {I } \\
& \bar{p}_{p \rightarrow \phi} \rightarrow \star^{\circ} \\
& \bar{p} \rightarrow \mathrm{K}^{+} \mathrm{K}^{-} \pi^{\mathrm{a}} \\
& \bar{p} p \rightarrow K^{+} K^{-} K^{+} K^{-} \pi^{0}
\end{aligned}
$$$$
\begin{gathered}
\& \mathrm{GeV} / \mathrm{C}\left(\mathrm{P}_{\text {lab }}\right) \\
\text { " } \\
" \\
" \\
" \\
" \\
" \\
= \\
= \\
= \\
=
\end{gathered}
$$

Particles studied xldoball, $f_{2}(2010), f_{2}(2300), f_{2}(2840)$

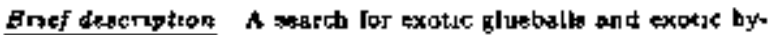
bnds Uoes the MPS lasality and the MESB beam at $\mathrm{B}$ GoV/c, tuned to contan $\pi^{-}, K^{-}$, and 7 Lowering the momentum to 8

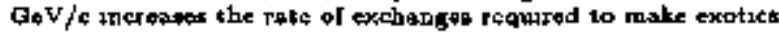
Currestly pousang (May a6)

E mat contact lodehbsumblandag bnl gov WHW Home page

bttp //Www phy bn! gow/-e\$B1/welcome ats ht mi

\section{BNL-909}

(Proposed Oat I 994, Approyed Nor 1994 Eagun dala-taking Apr 1995, Completed dacs-takıng Mday 1995)

ETA PHODUCTION AT THRESHOLD IN THE REAC TIONS $\pi^{-} p-\eta \pi$ AND $K^{-} p \rightarrow \Lambda n$

ABLENE CHRISTTAN U - L D Isenboner, A A Roke, M Spdker

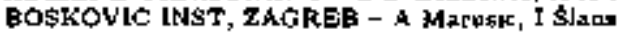

BROOKHAVEN - R Chren, R ŜLt:er

DUBNA A A Elendiev 


\section{SUMMARIES OF BROOKHAVEN EXPERLMENTS}

GEORGE WASHINGTUN U - 2 Benghold, W J Bntcos

( $\checkmark$ Spoleperion), T W Marruan, Z Papsadreor, S A Phupr,

R Pratt, A Shaf

HOUSTON U - R Sawafta

RENSSEL AER POLY - D B Whate

ST PETERSBURG, JNP - V V AbreV, V BthreneV

ST PETERSBURG, JNP - N Korlenk

UCLA - M Clsyus, 3 C McDonald, B M K Nedrets, W B Tuppena

( $\checkmark$ Spokeserent)

Accedernetar BNL Detectior Spectrometer

Beactions

$$
\begin{aligned}
& \pi^{\top} D \rightarrow \text { th } \\
& k^{-} p \rightarrow n A \\
& 680-760 \mathrm{MeV} / \mathrm{C}\left(\mathrm{P}_{\mathrm{lab}}\right)
\end{aligned}
$$

Particles rtudied N(1545 $\left.S_{11}\right), \mathrm{N}\left(1 \mathrm{1070} \mathrm{S}_{01}\right)$

Bref descroption Mastities the totat erdososction, wo well as abgular dastribution for $\eta$ production from thresbold (P $=685$

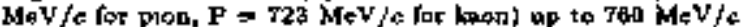

The of parlicle are deteched via the 27 defay morle wing the imptoved $n$ apectrometer Data anblysts in ptogresa al GwU (I

Related experiment, BNL-800, BNL-913, BNL-B14

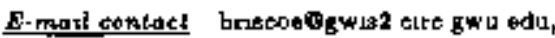

btippensfacdapp phynce ucle adu

WWW Howse-page

betp //uclas phy bnl gow/htdoes/e900/e000 htm

\section{BNI-918-914}

(Proposed Mar 190s, Approved Sep (905, In preparation)

BARYON AND NEUTRAL HYPERON \$PEC. TROSCOPY WITH THE CRYSTAL BALL

CRYSTAL-BALL COLLABORATION

ABLENE CHRISTIAN U - B Draper, I Huddretion,

I D Ieenhower, 2 Mulkey, M E Sadlef ( $\checkmark$ Spokesperasn)

ARGONTE - H Spinks ( $V$ S pokesperson)

ARTZONA STATE U J R Comfort, K Greng

BDSKOVIC IN5T, ZAGREB - M Batınk, I Slavs, I Supek,

A Suarc

BROOKHAVEN - T Kyele ( $\checkmark$ Spokeopetzon)

COLORADO U - R J Pelerson

GEORGE WASHINGTON $\mathrm{v}-\mathrm{c}$ Bennhold, W J Braceo

KARLSFUHE $U$ $\rightarrow$ M Staudenmsier

KENT STATE U - D M Maniay

ST PETERSBURG, INP - V V Absev, V 8 Bekranev.

$N G$ Xoslenko, S F Krujtov (V/5poketaperwon), 1 V Lopstıa,

A G Starant

REGINA U - G J Loloe, Z Papandriou

UCLA - M Cls jux, A Marusk, \$ \& MeDorald, B M K Nefkers

( $\checkmark$ Spokcopereon). W B Tippers ( $\checkmark$ Spokosperson $\}$

VALPARA ISO U, INDIANA - D Gromick, D D Kontke,

R W Manweiler, S Stanulaus

Acetterater BNL Delector CRYSTaL-BaLI

Rnactariss

$$
\begin{aligned}
& \pi^{-} p \rightarrow n \rightarrow \\
& x^{-} \rightarrow x^{0} n \\
& \pi^{-} p \rightarrow \pi^{0} \pi^{0} \pi \\
& \pi^{-} p \rightarrow n \\
& \pi^{-} p \rightarrow x^{D} \pi n \\
& \pi^{-} p \rightarrow \pi \\
& K^{-} p \rightarrow \Lambda_{\gamma} \\
& K^{-} p-\Lambda \pi^{d} \\
& K^{-} p \rightarrow \Lambda 2 z^{0} \\
& K^{-} \rightarrow A_{H} \\
& K^{-} p-\Sigma^{D} \text {. } \\
& K^{-} p-\Sigma^{0} x^{0} \\
& K^{-} \rightarrow \Sigma^{0} 2 \pi^{\circ} \\
& K^{-} \mathrm{p} \rightarrow \Sigma^{0} \mathrm{i}
\end{aligned}
$$

400-1500 MeV/s

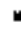

685-1800 $\mathrm{M} / \mathrm{V} / \mathrm{c}$ $1000-1800 \mathrm{M} \times \mathrm{V} / \mathrm{c}$ $1100-1800 \mathrm{MeV} / \mathrm{c}$ 600-1SOC MeV/C

$$
1
$$

T20-1 $1000 \mathrm{hdeV} / \mathrm{c}$ $600-1800 \mathrm{iseV} / \mathrm{c}$

890-18to MeV/c

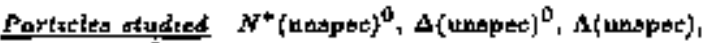 \\ $\Sigma($ u \\ Brtel destriptront Mescares abeolute difterential crast-gections

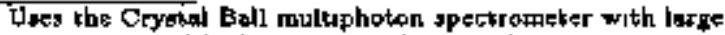

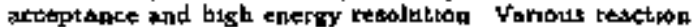 \\ thantels are tdentihed by reconstructing the tryanath mass of \\ the produced $\mathrm{J}$ rays The experment hat two distinet parts \\ The gosk of BNL-913 is a better dotermination of the masses,

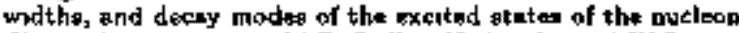 \\ The spoketpersonn sie M E Sadker, if Spiokn sud W E \\ Tuppent BNL-9L4 Btudiet the $\Lambda^{*}$ and $\Sigma^{*}$ resonances vla their \\ bevirsal decays The incident basm momentum is varied in slepe \\ of $25-50 \mathrm{MeV} / c$ from $600 \mathrm{MeV} / c$ in the C-luse to the maximum

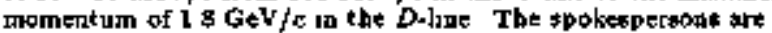 \\ $T$ Kycix, \& P Kruglow and B M K Nellont Approwed for 1000 \\ hours Schedulad to fun in FY 1989 \\ E titail' comilact

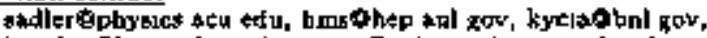 \\ kruglovalops epb to, btippens luelapp phy'buck ucla edu, \\ boeknuseuclapp physice ucla adu \\ wiw Nome-page

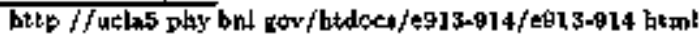

\section{BNL-RHIC-BRAHMS}

(Propused 1992, Approved Apr 1995, In pteparatiob)

\section{THE BRAHMS BXPERMMEN AT RHTC}

\section{BRAHMS COLLABOFATION}

Aecelerator BNL-RHIC Deteetor BRAHMS

Bruef descraptron RHIO prowides an opportunity foe experumenta IDregtigaliag both baryon poer guart gluon plasms in the findtapudity reglon and baryob rich planm it the fragmentation rezions of rapdity The most basuc information avallable for undergtandage the pbepopneds that atcur in heavy Iop collitiond comeg from the momentum spectrs and yields of the ysrous emitted particler as a function ol transyerse momentum and rapidty One of the rowls of the expen meat it to messute the apectra of well edentsied charged badrous over a wide range of rapdity and transverse momenta at a function of the

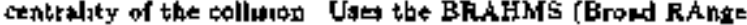
Hodron Magnetic Spoctrometera) detector, consibtseg of a magnetce forward angle hadron spectrometer and a midrapidity spectrameter PAC spproved in Apr 95 For furlater detsils, please coritact the Spokesperson, Dr Fleriming Vidabsok [BNL], ar get in touch with Dr Dans Beavis f [ENL]

Ermeif contact videbsek

WWW Horte page

http //rsgl0l rhic bol gow/exportl/brahms/WWW/brahms htm!

\section{BNL-RHIC-PHENIX}

(Froposed 1989, In preparatton]

PHOTON-ELECTRON NEW HEAVY ION EXPEFU. MENT

\section{PHENIX COLLABOEATION}

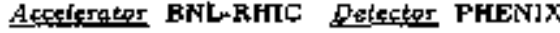

Bnef desctiption Studes simultaweously varpue sugnatures of

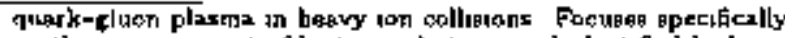
on the beasurement of isptons, photone, and sdeatifred bedrous, and should be capable of exploitwg the heghest lumurogitieg envinconed for RFTC \$tudies also axymmetres of photous a art leptanic dectys of woak bobols in polstized proton restions The FHEN]X detector bystem is bered on an axied field magnest in which the contral rapidity inlerval ix cowered by twa

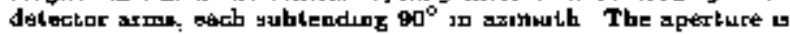
Jnstrumented to delect electrong, photous, snd hadrons Two

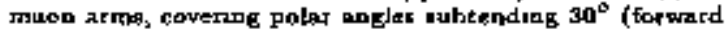
and backward), have a good acceptrance far muon paurs and sllow also alectron-rawon coinc|dence measurearents A4 event multuplicity array to cover over hve rapidity units is bied for 
crent characterization Under conetraction (July 26) The collaboration consusts of over 420 scientists, engneers, sad

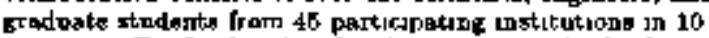
tountrite For further detsils, please contact the Spakesperson,

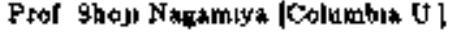

En-moti contacti nagoney WWW Home-page

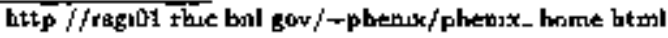

\section{BNL-RHIC-PHOBOS}

(Prapoed Jap 1092, Approved Nov 1902, In preparstion) PEOPOSAL TO BTUDY VERY LOW $p_{\perp}$ PHENOMENA AT FHIC

\section{PBOBOS COLLABORATION}

\section{Accelerefior BNLRHIC Deteritor PHOBOS}

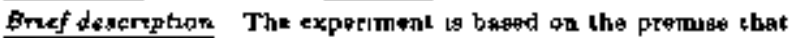
phase tranaitions may be algnaled by large fluctustions in the avorell miltupltcity or aggulas distribution of cbercend particles

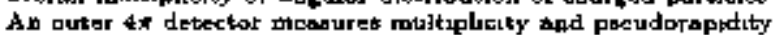
A small subast of particleg around $y=0$ is then studiad in detal by otwonim multipartide npectromenter weth good momentum resalution and partrele identification The deterctor is based on stecon techaology and allows unbassed tnggertng at very bigh rater In preparation (July Q6) For further det suls, please conteast the \$pokegperwo. Prof Wit Bugsa [MITT]

$E$-mail contact bugratomil edu

WWW Hotine-page

hetp //ww whe bnl gov/phobos/common/WWw/phobot html

\section{BNL-RHIC-PP2PP}

(Propoed Jan 1994, Approved Mar 1994, In preparatson)

TOTAL AND DIFFERENTIAL CROSS-SECTIONS, AND POLARIZATION EFFBCTS IN pP ELASTIC SCATTERING AT RHIC

\section{PP2PP COLLABORATION}

\section{Accstertor BNL-RHIC Detectar ?}

Brwef descroption Studiet systemstreslly the pp elatic seatcering firden $\sqrt{4}=60 \mathrm{GeV}$ to $\sqrt{3}=500$ GeV, in two kneratical regions is the Coutomb Nuclest Latarference (CNI) region for [i] betwean 00000 and $012(\mathrm{GeV} / c)^{2}$, mescures (1) the

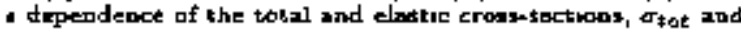
of 1 (2) the ratio of the real to the instipary port of the forward elastic scattering amplitude, and (3) the suclear slope patameter of the pp elartic acativering, b th the intermeduale $|c|$ region, $|z| \leq 15\left(\mathrm{GeV} / \mathrm{c}^{2}\right.$, stadies the swolution of the dip atructure with s, and the and $|t|$ dependence of $b$ The postoblaty of baving polatleted proton beams at RHIC would sllow measurements of (1) the difference in the total crossthetions ss s fonction of imitial trenswerte spin states, (Ii) the analyzing ponver, $A_{N}$, ezpoesally in the dep zegion whero a pronounced structure was found at fixed-target expertments. and (3i) the traneverse spin correlahon parsmeter, $A_{N S}$ Under canstruction (July 4 ) Por further dctouls, plane cantact the Spokesperanon Dr Wlodek Gutyn [BNL]

$E$ matt contatit guryabbal gov

\section{BNL-RHIC-STAR}

(Proposed 1990, Approyed Aug 1991, In preparation)

SOLENOIDAL TRACKER AT RHIC (STAR)

STAR COLLABOFATION

Accelerator BNL-RHUC Detector \$TAR

Bries description STAF th degigned to pearch Jor grgnaturea of quark-gluad plasena formation and to Jnvegtugate the beharior af strangly Interacking matiter al ligh epercy density The thaphas it on the measurement and cortelation of ciobol otrervable on an event-by-event bata sald the ute of hard scettenng of partons to probe the properties of bugh density nuclesr matter The event-by-gvent messurement af globsl abervablex a pasabla becense of the very hich charged particle

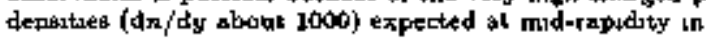
nucleve-nuelaus colturane at RHIC To lulfill the phymit

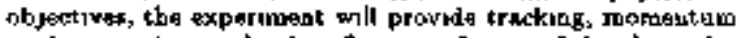
analyss, and partscle identsfication of moat of the chargad

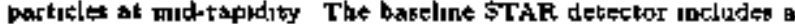

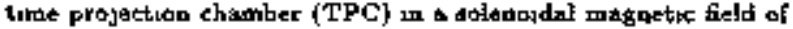

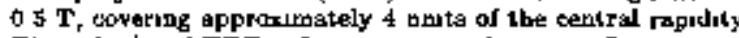
The cylundrted TPC is four metera in deanuater Ionnsusen charge prodpced along particle trajoctones a drifted to tbe iwo fud plohes, where induced gignals sad errival timess bge resd out on 160,000 cathode $D$ ods Particle identjfication will be possible vis $\mathrm{d} E / \mathrm{d}$ a in the $1 / \beta^{2}$ region Upgridea being thaveloped for STAR molude sn array of TOF coumters to extend the PID

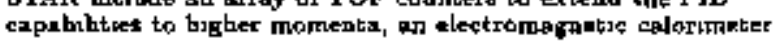
lo provide far the menurement of pentral eperog, and a Mlicon vartex detector which uses postuon berlatuve aulicon davices vith a dreft-time mesourement tmonique $A$ further uporade mang TPG's in the forward derection will extend the particle trackjng coverage to a peendoraprdity of approximately \pm 4 PAC approved August 91 la proparation (July 96) For forther

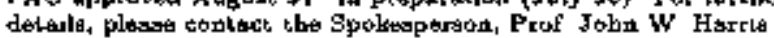

YYale U] Project Drector is Dr Jay N Misr [LEL, and

Deputy Spolcepersob Dr Tymashy J Hallman (BNL]

Joutnal poperis NP A56s (1995) 2770

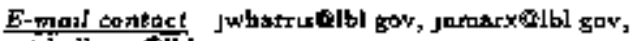

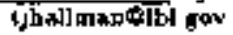

WWW Home-page

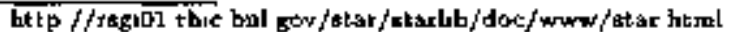

\section{Other BNL Experiments}

Listed hete are tome olter BNL expertments of interest to the particle phynes commanaty Find more detasls atoput those propeces online, th the SLAC's EXPBRIMEN'T' dstabase (xise p 3), or contsct the Gpokespersong Chesk also the gnnun Brookbortin repart 'AGS Exporimente' (BNL-34518), or viest the Web atteg http / /ww rhtehore bol gov/AGS/ (for the AGS experiments), or betp $/ /$ www the binl gav/ (for ihe FHIC exporimedte)

BNL-774 (Completed dats-taking 198I)

SEARCH FOR \& HYPERNUCLEAR LEVELS IN He

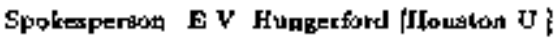

E-meal contact hongentouk edu

ENL-761 (Completed data-takıng 1992)

SPIN DEPENDENCE OF THE $A$ - NUCLEUS INTERACTJON DETERMINED BY OBSERVATION OF HY. PERNUCLEAR $\rightarrow$ R.AYS

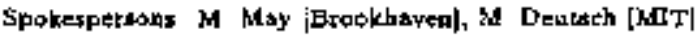

$E$ thati contact may20bol gov, deutbch

BNL-żó (Completed datartaking 1992)

A SEARCH FOR QUARK MATTER (QGP) AND OTHER NEW PBENOMENA UTILIZING HEAVY ION COLLISIONS AT THE AGS

Spokatpertons E D Platzer |Btodkhsven', \& J Lindenbsum [Brookhavea and City Coll, $\mathrm{N} Y$ ]

Emazi contact platnerobpldag bol gav londenbsumbubldag bol goy 


\section{SUMMARIES OF BROOKHAVEN EXPERIMENTS}

BWL-81s (Completed dats-takung Jun 1995)

SEARCH FOR A STRANCENESS - 2 DIBAFYON

Spokesporsons o B Fralutin [Carnegue Mellun U ], P D Earnes [Lod Alemot!

E-matt cotriact franklineernest phys cmu edu, pubarnesteral gor

BNL-914 (Completed data-takın 1992)

STUDY OF EXTREME PERIPHERAL COLLISIONS AND OF THI TRANSITION FROM PERIPHERAL TO CENTRAL COLLISIONS IN REACTIONS INDUCED BY RELATTVESTIC HEAVY IONS

Spokepperson Patex Braun-Munzinger [SUNY, Stony Brook]

E-mal tantact pbinotakipper physict surysb edo

BNL-81T (Taktng date)

POLARIZATION TRANGFER IN HYPERON PRODUCTION

Spoke*peraopt Billy E Bonner, Jay B Foberts [Fuce U|

E-moul condect bonnertphyers, nce edu

BNL-836 (Gompleted datakaking Jul J日84)

SEAFCH FOA A STRANGENESS -2 DIBARYON USING A ${ }^{3}$ He TARGET

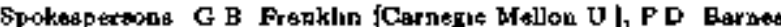
LLos Alspose

E-mult cortact frankloneteraest phys crmu edu, pdharpeosilanl gov

\section{BNL-844 (In preparation)}

MEASUREMENT OF ANGULAR DIETFIBUTIONS

FOR FEAGMENTS IN THE TARGET RAPIDITY

FEGLON

Spoketpetgon James B Curwolng [Btockbaven|

Es meat contact cummigetobnl goy

BNL-849 (Completed data-kakıng 1992)

BEARGH FOF MUDNIUM TO ANTIMUONIUM CON. VERSION

Spokespersone Dale Ruchsrd Kexshmen, A P Mulls. Ir |A T ayrd T Bell Labe, Marray H,Il]

BNL-860 (Tskine data)

EVA, A SOLENOIDAL DETECTOF FOR LARGE ANGLE EXCLUSTVE REACTIONS: PHASE I - DETERMINING COLOR TRANSPARENCY TO $22 \mathrm{GeV} / c$

\$pokcopersons AS Carroll [Brookhaven], S Happeimann [Penn State U I

E-rusu contact cerrollabnl zov, heppetephys peu edu,

heppelmansogbnldeg bal gav

WWW Home pagte

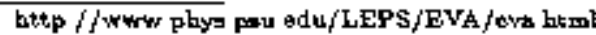

BNL-As4 (Completed dale-takung Jun 1991)

ANTIPROTON-NUCLEUS INTERACTIONS AT 5-10

GeV/e

Spokexpersan B E Bander (Ruce Uy

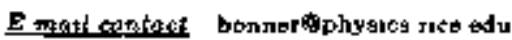

BNL-859 (Completed dels-tatong 1992)

STUDIES OF HIGH DENGITY BAFYON MATTER FFOM HXTENDED MEASURBMENTS OE PAFTICLE MOMENTUM DISTFIBUTIONS AND FROM HIGH PRECISION TWO-PAFTLCLE CORRELATIONS

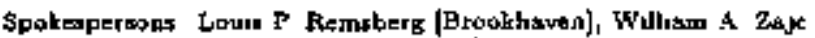
[Columbes U], Robert ] [etdruxx [MITT]

E-meil contact remsbergonct chm bal gov, zBjebinovis neyis colvmbis edu

BNLeses (Conpleted datstakng Sep 1993)

PARTICLE PRODUCTION AND NUCLEAR FRAG.

MENTATION IN COLLISIONS OF HEAVY IONS IN NU. CLEAR TARGETS AT AGS ENERGIES By EMUU0] Collaborntion

Spokespersous Ingvar Ottetlund [Lund $\mathbf{U}$ ], R Jeffrey Wilkes [Wasbingtion U, Senttle]

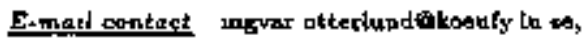

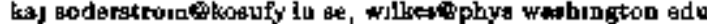
WW'W Home-page hstp //marce phys warbington edto/emwil $/$

BNL-a6-4 (In proparation)

PRODUCTION OF FARE COMPOSITE OHJETS IN RELATTVISTIC HEAVY ION COLLISIONB

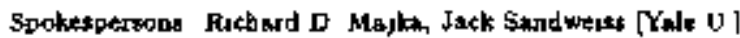

E mat contact pacts asadwessogyole odu

WWW Hotre.page

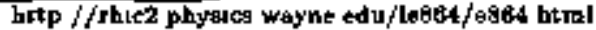

BNL-8e6 (Coripleted dats-takuag Jan 1996)

ITUDIES OF PARTICLE PRODUCTION AT HIGF'

BARYON DENSITY USING THE AU BEAM

By E-802 Collaburation

Spokespersons Chellis Chasmap [Brobakhored], Steve G Sieadman [MTT], Hudekı Hamagakı [Tokyo UU, INS|

E-matil contact chasmsingho hurg bnl gow, satemitho mit edu. hamagetcrinsuly uns u-takyo ate $\mathrm{JP}$ WWW Hotte-page

hotp //mnis m:E edw/nerver/ebas/2868Mann html

BNL-tats (Completed date-takeng Dec 1996)

INTERACTION\$ OF 14.1 GV/NUCLEON NUCLEJ PROM ${ }^{16} 0$ TO ${ }^{197}$ AU IN LIGHT AND HEAVY TARGETS By FLMM Collaboration

Spokesperson if Woddingtop [Munnasats U]

E-mal continct waddingtongishn ape urep adv

BNL-860

MEABUREMPNT OF FRAGMENT YTELDA FOH 1 GoV/NUCLEON At $+\not x$ COLLISIONS By VHIC Collatroration

Spokesperran o Jaks Waddingtan [Minoesous U ]

E.mratl contect Waddingtontoubn aps ume edu WWW Home-page hatp / /ww' ard caltach exdu/uhuc/

BNL-874 (Completed data-takıx Jun 1993)

KAON-NUCLEUS QUASIELASTIC AND ELASTIC BCATTERING

Spokemerzons Robert E Chrea (Breokhaven), Foy Jerome Petergon icolorado U], B V Bongerford (Hotraton U)

E-mat contact chryenobul gov, petersonfopect colot3do edu. hubgersub edu 


\section{SUMMARIES OF BROOKHAVEN EXPERIMENTS}

BNL-87's (Completed data-laking Aug 1999)

STUDY OF PARTICLE PRODUCTION AND NUCLEAR FRAGMENTATION IN RELATIVISTIC HEAVY-ION

COLLIRIONS IN NUCLEAR EMULSIONS

Syokesperson PL Jal [sUNY, Buffalo]

Erranit contact phyjarngabvmm oc buffalo edn

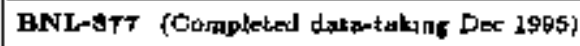

STUDY OF RELATIVTSTIC NUCLEAR COLLISLONS WITH HEAVY BEAMS USING THE EO14 CALORIMETRY AND MODIFIED FOFWARD SPECTROMETER

Spokedperson Petex Beaum-Munzıgger [SUNY, Stony Brock]

E.man cantect pbobukmpert physes sunysb edu WWW Home.page

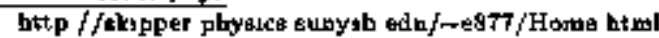

BNL-st8 (Completed datz-taking Oct 1993)

LNVESTJGATION OF ANTINUCLEUS PRODUCTION AND SEARCH FOR NEW PARTLCLES IN NUCLEUSNUCLEUS COLLISIONS AT THE AGQ

Spoknperion H J Crawford [UC, Berkelcy, Space Sc1]

E-maxi contact hucrawtord\$lbl gav, crawlardebaldag bol gov

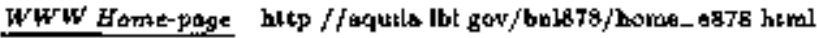

BNL-8ad (Corupleted deus-taknog Oet 1984)

SEARCH FOR PARTICLES WITH $|Z|>3$ AND

NBGATIVE CHARGE OR LAFGE $A / Z$ PRODUGED IN

CENTRAL NUCLEUS-NUCLEUS COLLISIONS

Spakexparton P Buford Proe [UC, Beckeley]

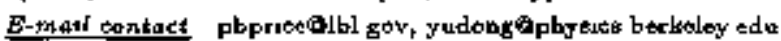

BM1-kas (In preparatios)

EXPERIMENT TO DETECT AA HYPERNUCLEI

Spokespersone M May [Brookhswen], O B Franklan |Carnegue Biellon U], Charlos A Davis |Minitobs U and TRTUMF]

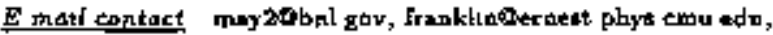

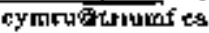

BNL-896 (Completed datantakng Ots 1993)

GEARCH FOR NEW PARTICLES IN NUCLEUSNUCLEUS COLIISIONS

Spakespersoue Kemch Iras !Kyoto U i, Phلp H PLe [Brookbaven], 6 Dhobold [Yale U]

E-molizantact imbakekvax kek Jp, pilefibrl got

BNL-897 (Completed satartaksng 1995)

DO NARROW I HYPERNUCLEAR STATES EXLST'

Spoksepergons Rayad Sawafta [Brookhawei], Kemath H Hulke |Óhio U|

E maut conlact tawaftadoraldag bul gor,

hickestouvaxe cate ohlou edu

BNL-888 (Completed dato-takıng Jul 1992)

SEARCH FOR THE $H$ DIBARYON

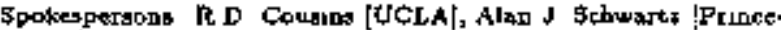
$\operatorname{con} \mathrm{U}$

E-mat cotstest cougingobaldeg ba] goy,

sctrwartztopuphep princeton odv
BNL-890 (Completed datso-takung Jvת 19\%b)

TEST OF CHARGE SYMMETRY IN \# PRODUCTION ON DEUTERUUM

Spokesparsons Roburt E Chrien [Broblbaven], J C Peng [Los hilomod, Bethard a $K$ Keflathe [UCLA]

Ermari contact chruenQbal gov, pentolanol gov, bnefikesterouclapp physuca ucls odt

WWW Hane-page

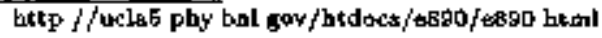

ENL-891 (Ln preparation)

A. SEARCH FOR QUARK MATTEF (QGP) AND

OTHER NBW FHENOMENA UTLLIZNG AU AU COL-

LISIONS AT THE AGS

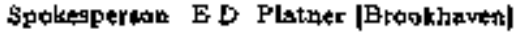

E-mati coneret piatnorobaldag bul gov

WW W IJomespage hetp / wwe phy bnl gov

fant/people/eg91/doc/www/welcone_ 4891 html

BNL-892 (In peeparation)

ELECTRON CAPTURE FHOM PAIF PRODUCTION

Spokepertod Harky Goulda [LEL, Berkeley]

E matl centuset hagouldoflbl gor

WWW Hame page bitp //ww age binl gov/ cagr/

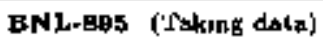

EXCLUETVE BTUDY OF NUCLEAR COLLISIONS AT THE AGS

Spokesperson Gulshan Rat [LBL, Berkeley]

Email contact graiblal gor

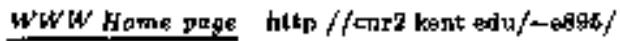

BNL-496 (In preparation)

SEARCH FOR A SHORT-LTVED $H_{0}$ DIBARYON AND SHORT-LIVED STRANGE MATTER, AND STUDY OF HYPERON PRODUCTION IN 11.6 A GeV/C AU AL COLLLIONS

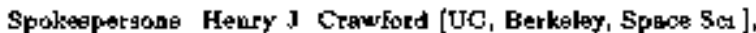

Tim J Hallmen [UCLA]

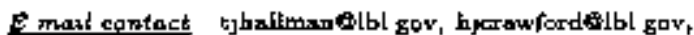
cratwiordobalday bal gow

WWW Hare page hte //aquila lbl gow/bn 1896/harpe_e896 htsol

BNL 900 (Completed deta-takivg Apr 1906)

ENERCY DI\$SIPATION AND MULTIFRACMENTATION IN p + A REACTIONS BETWEEN 2 AND 24 GeV/c

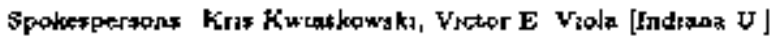

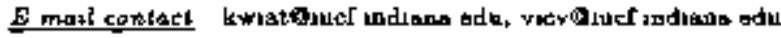

BNL-905 (Taking date)

SEARCH FOR A $\Sigma$ HYPERNUCLEAR BOUND STATE IN ${ }^{4} \mathrm{He}\left(K^{-}{ }^{ \pm}\right.$) HEACTIONS

Spokezparion Tómoím Nagae [Tolyo U, INS]

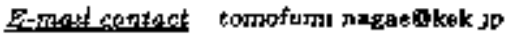




\begin{tabular}{|c|}
\hline BNL-90) (In preparation) \\
\hline $\begin{array}{l}\text { EXPERIMENT TO DETECT DOUELE-A HYPER- } \\
\text { NUCLEI BY OBSERVING CHAFACTERISTIC *- } \\
\text { MESONIC DECAY }\end{array}$ \\
\hline 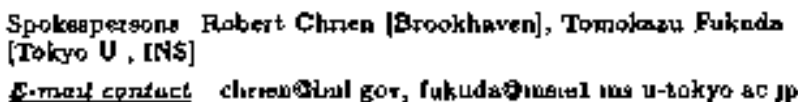 \\
\hline
\end{tabular}
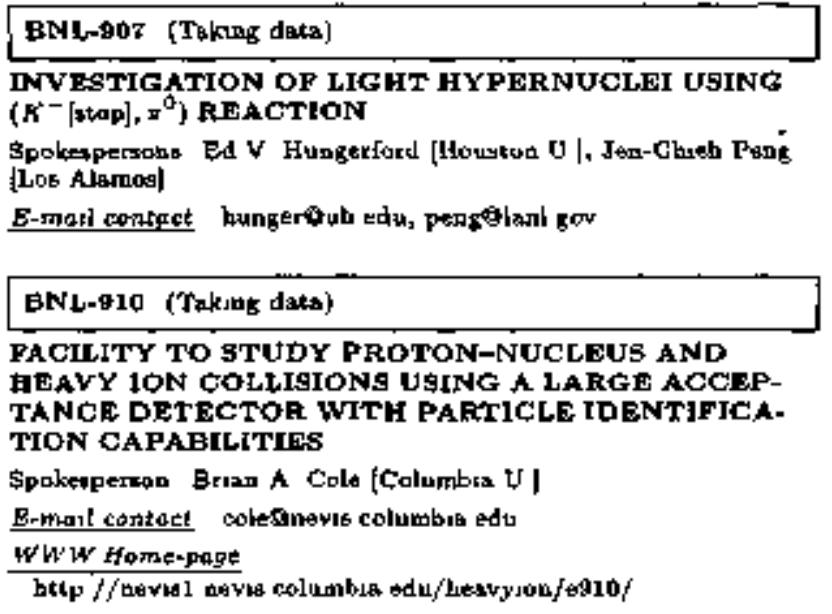

ENL-617 (W praparation)

HIGH DENAITY BARYON MATTER USING RARE PRÓ日ES

Spotrezpersons Alıce Mignerny (Maryland U), R K Seto (UC, Ruveraide!

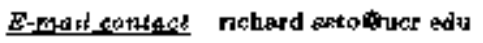

\section{BNL Future Plans}

The physis proxrom ax the tab w centered around the AGS and EHIC facslities Fot the bexi telr years, the AGS proton pqparatr wall contwue to eropharise the sesteh for rare and [orbidder $F$ decoys, the treasurement of $g-2$ for the mugn, and atudies of exotic mesona w the Multr-Particle Spectrometer The heavy ton program wll amplenre the search for the (gtrsngenns 2) $H$ dibsryon sild dearches for arrante boryonic fratments with anmalous thorge/mant ratiod (atrangelets) An AGS-2000 Workatiop tadk place in the spring of 1996 Itn focis what on the competitive, leading-edge phyalcs which can makse use of the $10^{14}$ probant per palse wheb wil be avoulable al the AGS Anuan: the experipenta under caritederation were a atindy of $K^{\theta} \rightarrow \pi^{\phi} \nu \bar{\nu}$,

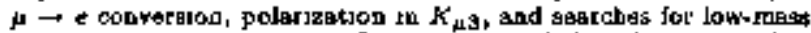

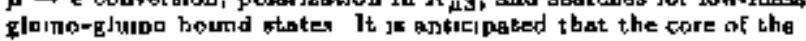
AGS program beyond tho year 2000 wilt davelop trom these and other Workesop effork The RHIC construction propect w well nederwny The cursent schedule falls for the firtt. curculaling and

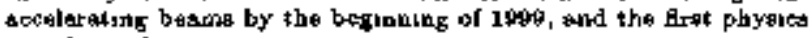
Tuas later that year 


\section{SUMMARIES OF CEBAF EXPERIMENTS}

\section{Selected CEBAF Experiments}

\section{CEBAF-89-024}

(Propased I987, Approved Is89, In preparation)

\section{FADIATTVE DECAYS OF EOW-LYTNG HYPERONS}

CLAS COLLABOFATION

RICE U - \$ Ahmad, B E Boaver, G S Mutthler ( $V$ Spokesporgou), 5 Taylor

WILLJAM AND MARY COLL - M Eelshause, A D Hanoock,

I H Kane, Y N Krank, H. E Welsh

Accelerator CEBAF petector CLAS

Feactions

$$
\gamma^{P}-K^{+} Y^{- \text {(ungpec) }} \quad 140-302 \mathrm{GeV}\left(E_{\mathrm{l} a b}\right)
$$

Patteles atudied $h\left(1405 \$_{01}\right), \Lambda\left(1520 D_{03}\right), \Sigma\left(1395 P_{13}\right)^{0}$

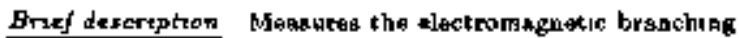

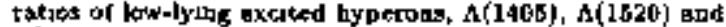
$\mathbf{S}^{0}(1385)$ Uost the CLAS detertor, a supereponduct)as torondal spectrometer with drift chambers and TOF acintillators Determine the fovr-momentum of the sxceted hyperon from the energy of the tagged photon and $K^{+}$momentum The

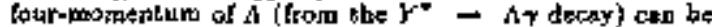
recoustructed from the peotan and $x^{-}$momentum A gaod mane reaclution with CLAS allowra the supprassion of the background due to $\pi^{0}$ decays Uous a togged photen beam abd an LH2 taret Approved for 60 days of rugning in Hall $\mathrm{E}$ Expected to tur is Summer add Fil] 1907

Relaled expertmetrt CEBAF-89.004, BNL-811

E-miatl contect mutchleriphyate ruce edu

\section{GEBAF-89-038}

[Propued Otc 1989, Approved May J日90, In preparation)

MEASUREMENT OF $p\left(e, e^{\prime} \pi^{+}\right\} n_{1} p\left(s, e^{\prime} p\right) \pi^{0}$, AND $n\left(e, e^{t} x^{-}\right) p$ IN THE SECOND AND THIRD RESONANCE REGIONS

\section{N* COLLABORATION}

CEBAF - W Brodk日, V D Burkert (V Spokesperwon), D Joyce, B A kiecking, M Nestajer, B B Niczyporpk. E Sintth A Yexpeswarat

CHRTSTOPHER NBWPORT U - D Doughly, D Heddle, 2 J L DUKE U = H R Weller

FLORID A STATE, U - L C Dennw, P Dragovitach, $K$ W Kemper

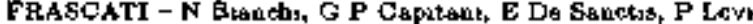

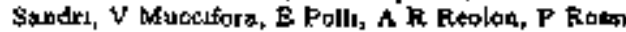

INFN, GENOA - MA Anghinalf, P Carvinero, G Gervino,

L Meamedilu, V Mokreav, G Ricco, M Rupani, M Santone,

M Tafuti, A 2ucchtart]

GEORGE MASON UT - E J LuEb

JKMES MALDLSON U - K GJovanett,

KENT GTARE U - D Kene, D M Mapley

PITTSBURGH U - \$ A Dytman

RENGGELAER POLY - G I Adtarn, N C Mukbopodhyay, P Sweter

VTRGINIA U - D B Day, J S MeCarthy, R C Mtmehart

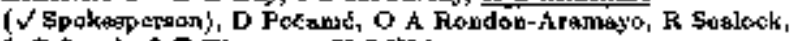

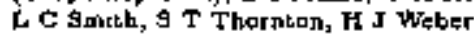

VIRGINIA STATE U C E Strongch

VTRĞINLA TECH F A AfDdt, D A Jasking, L D Ropar

WILLIAH AND MARY COLL - C E Curlson, H 9 Funsten,

TY TUת:

CONNECTICUT U - M Gar ( $\left./ S_{\text {pokesperana }}\right)$

Acctietafor CEBAF Defector ClAS

Reactions

$$
\begin{aligned}
& e^{-} p \rightarrow e^{-} p x^{D} \\
& e^{-} p-e^{-} n x^{-t} \\
& E^{-} p \rightarrow e^{-} p x^{-} \\
& 20,40\left(\mathrm{geV}\left(\mathrm{T}_{\mathrm{Inb}}\right)\right. \\
& \text { • }
\end{aligned}
$$

Particlen studied $N\left(1535 S_{11}\right), N\left(1520 D_{13}\right), N\left(1680 F_{15}\right)$, N(1440 $\left.P_{11}\right)$

Brtel descripiton The expenment whll meagara tranortion form. Factors to anclean excted states in the mase region from 1350 to J800 MeV/c $/ c^{2}$ Schedvled to run is Hall B in 1997

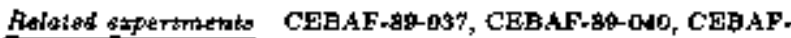
89-043, CFEAF-89-043, CEBAF-91-092, CEBAF-93-038

E-mati contact burkartecebaf gov, minehartovirgubis edu, gaviofuconnym uconn edy

\section{CEBAF- $\operatorname{sen} \theta \dot{\theta} \theta$}

(Propoesd Oet 1989, Approved 1948, It preparatesn)

AMPLITUDES FOR THE $N\left(1535 s_{11}\right)$ AND $N\left(1710 P_{11}\right)$ RESONANCES FROM THE tp $\rightarrow$ EP GCATTERING

\section{CLAS COLLABORATION}

CEBAF - V D Burkert, D Corde, D Joyce, B A Mecting.

M Mertayec, B B Nicryporuk, E Sruth, A Yogneawaran CHFISTOPHER NEWPOET U - D Doukbty, L Elonadrbits, D Heddle

DWKE L L - R Chasteler, D R TuLby, H Weller

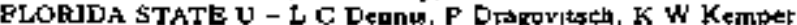
GEORGE MASON U - B J LIED

FRASCATI - N Branchi, O P Capitani, E De Sanctw, P Levi-

Sandru, V Mucte, Eora: E Polli, A R Reolon, F Rosen

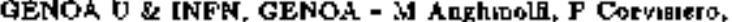

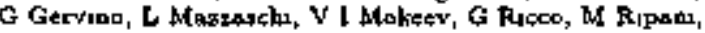

M Ssprone, M Tyut, A Zucchisztr

JAMES MADISON $U-K$ Growanntin ( $\sqrt{ }$ Spokenperson)

KENT STRTE U - D M Yantey

FITTSBURGH U - S A Dytmen ( $\sqrt{ }$ spoke日person), J Mastler,

D Tedeschu, R Thompeon

AFNSSELAER POLY - G 3 Adam, it 0 Mukbopadbyay, J Nipolutano, J Pnce, F Stoler

VIRGINIA U - D B Day, R Maxthall, I S McCartby,

R C Minebart, D Potanles, O A Roudop-A ramaya, R Seralock, S T Thotnton, H W Weber

VIRGINIA STATE $U-C$ E Stronach

VIRGINIA TBOH - A A Ardt, D A Jankin, $L$ D Roper

WILLIAM AND MARY COLL * C E Carloon, H O Funsted,

T Y Tung

YALE U - M G.

Acoeterator CEBAF Detector CLAS

React tons

$$
{ }^{-} \mathrm{p} \rightarrow e^{-} \mathrm{p} \text { य } \quad 2,40 \mathrm{~V}\left(\mathrm{~T}_{\text {lsb }}\right)
$$

Partagles studied $N\left(1635 S_{11}\right), N\left(1710 P_{1}\right), N^{*}$ (unspec)

Bnef detcrzation Covers the tatal ceptermal-mase eqnergrea, $W$, from 135 to I $9 \mathrm{GaV}$ tar the second resonence regron, and from

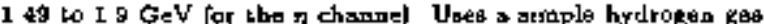
target Scheduled to run in Hall B

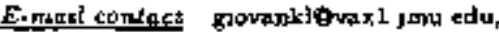
dytmanorsas cis putt edu

WWW Home.page http //Nww cebst gov/clay/CLAS himl

\section{CEBAF-89-048}

(Propaled Oot [989, Approved 1959, In preparation)

MEASUREMENTS OF THE ELECTROPRODUCTION OF THE $\Lambda, A(3520)$, AND $f_{0}(975)$ VIA THE $K^{+} K^{-} p$ AND THE $K^{+} \pi^{2}$, FINAL STATES

ABUENE CHRISTLAN U - D Isephowar, M Sadker MIT, BATES LINEAR ACCELEFATOF - 1 Ghedre CEBAF - V D Burknrt, D Joya, B A Meckjn $Z_{1}$ M Mestayer,

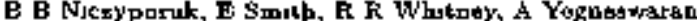
CARNEGIE MELLON U - A A Schumarher CARISTOPHER NEWPORT U - D DNorgbty FLORIDA STATE U - L C Dentis (Spokespourgon), K W Kemper GEORGE MASON U - B J LER

GEORGE WASHINGTON U - A Mokhta

JAMES MADISON II - K Glovanett. 
KANSAS STATE U - T R Donoghut

KENT STATE 0 - D Kełne, D M Manley

MJT - W Y Kım

PITTSBURTic u - S A. Dytman

RENSSELAER POLY - G S Adtuns, N C Mukbapadhyzy, P Stolet

VIRGINLA U - D 1 Day, R Mrrahal, J S MCCartby,

R C Mimehort, 0 A Rundon Aramayo, R Sealock, $\$$ T Thornton, H J Weber

VIROINLA STATE U - C E Stranach

VIRGINIA TECH - F A AIndt, D A Jepkins, L D Rloper

WILLIAM AND MARY COLf - E E Corhon, H. Fungtan

(spokespernon), C F Perdriast

YALE U - M GÁ

Afceitrator CEBAF Delector CLAS

Reactions

$$
e^{-} \rightarrow e^{-x} \quad 4 G e V\left(T_{l a b}\right)
$$

Partailes studied fo $(960), \mathrm{N}\left(1530 D_{\mathrm{aJ}}\right)$

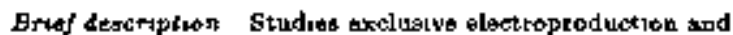
charged hadronk decay of the $f_{\theta}(930)$ reacnance and how-mas A's Schaduked to ran in Hall $B$

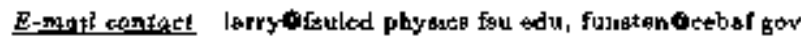

\section{CEBAF-9k=003}

(Fropoes Sep 1991, Approwed Now 1991, Jan 2996, In preparetwon)

THE GTUDY OF EXCITED BARYONS AT HIGH MO. MENTUM TRANSFEF WITH THE CLAS SPECTHOMETER

\section{N" COLLABORATION}

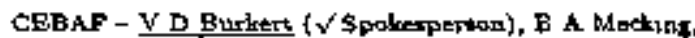

M Mestayer, B B Niczyporuk, E Smitb, B Wojtoekbowakl,

A. Yerneswatam

CHRISTOPHER NEWPORT U - D Doughty, D Heddle, z J h

CONNECTICUT U - M GB

DUKE U - R Cbsgteler, D R TLky; H R Weller

FLOADA STATE U - L $C$ Dembie, P Dragourtsch

FRASCAT1 - N Bianchu, G P Caplladi), E De Samctig, P Levr

Sandri, V Muccifora, E Poll, A P Reolon, P Faseg,

INFN, GENOA - M Anghinoli, P Corynierd, G Gervino,

L Miameschi, V Makeer, G Recep, M Fupapi, M1 Sanzone,

M Tyyts ( $v$ Spokfaperton), A Zutchalt

GEORGE MASON U - B I Lueb

HAMPTON $G$ - K Beard

JAMES MADISON $\mathbf{U}-\mathbf{K}$ GrovandttL

KENT STATE U - D M Manloy

PJTTSEURGH $v$ - S A DylmeD

RENSSELAER POLY - G S Adamg, N C Mukhopadhray,

I Nopolitano; P Srolert (V Spoketperaos)

VIRGINIA U - D Crabb, D B Day, R. Marshalt, I S Mecharthy,

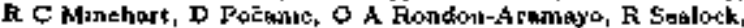

L C Sintb, S T Thornton, H Wober

WILLLAM AND MARY COLL - C E Carison, A Colaman,

H O Funten, T Y Tung

Ascolerator CEBAF Delectat CLAS

Repctions

$$
\begin{array}{cc}
e^{-} p \rightarrow e^{-} p \pi^{*} & \text { e G GeV }\left(T_{\text {lab }}\right) \\
e^{-p} \rightarrow e^{-} p x & \text { - }
\end{array}
$$

Partictes siudied $A\left(1232 P_{33}\right), N\left(1 \$ 40 P_{11}\right), N\left(1535 S_{11}\right)$, $N(10060)$

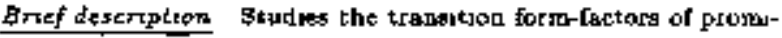
ment resobnnces, $A\left(I_{232} P_{33}\right), N\left(1440 P_{11}\right), N\left(1535 S_{11}\right)$, and $N\left(1680 F_{15}\right)$ at high mementurn trangfers, in the trangition re gron where constituent-quark models are expected to become less relerant and glange asd cuscent-quarks are betueved to play

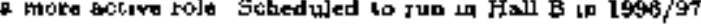

Related arpetitnetth CEBAF-94-01d

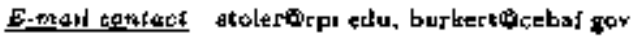

\section{CEBAF-91+008}

(Froposed Sep 1991, Approved Jan r992. In preparstion)

PHOTOPRODUCTION OF $\eta$ AND ${ }^{r}$ MESONS

CLAS COLLABORATION

AFIZONA STATE $v$ - B G Rulche ( $V$ 'Spokesperson) CATHOLIC $\mathrm{V}-\mathrm{H}$ Cranutell, J T O'Bukn, D I Sobet

CEBAF - B A Mreckang

FLORIDA STATE U - L C Denaus

GEORGETOWN U - J Lambert

GEORQE WASHLVGTON U - B L Berman, W J Bruca,

K Dhuga W F Dodge

BOSKOVIC INST, RAGREE - I Slaus

SOUTH CAROLINA U - C D płada, B M Pteedom, A Tan,

S Whosnant

UCLA - B M K Nefkeds

Acceterator CEBAF Detector CLAS

Reestions

$$
\begin{array}{cc}
\gamma P \rightarrow J P & 065-225 \mathrm{GeV}\left(\mathrm{E}_{\text {lab }}\right) \\
\gamma P \rightarrow J^{\prime} p & \sim
\end{array}
$$

Particles studed 7. 7

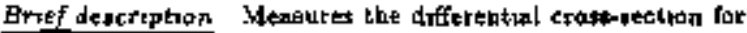
Lhe photoproduch or of of and of mespons ueng the Hall-B bretabstrahlung photon tsgger and the CLAS apectrometer The target i 2 liquid hydjagen cell Identificetion of tho in and $\eta^{\prime \prime}$ Je made by detection of the rocoll protion in the CLAS Studies the propertiea of $\pi_{1} d^{f}, N\left(J 535 S_{12}\right)$. and $\left.N(17) F_{11}\right)$ Schoduled to run in Fall $\mathrm{B}$

Felafed expermerts CEBAF-89-039, CEEAF-89-045, CEBAF93.CDQ, CEDAF.

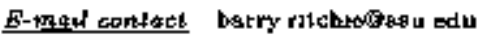

\section{CEBAF-91-011}

(Propased Del 1991, Approved Dec 1993, In preparalion)

HIGH PAECISION SEPAEATEON DF POLARIZED STRUCTURE FUNCTIONS IN ELECTROPRODUCTION OF THE $\triangle$ AND ROPER RESONANCES

CAL STATE, LA - M E Eptern

CEBAF - J P Chen, J I LeRase, I H Mitchel], S Nanda, A Saha INFN, HOME - E Cusbent, R do LeD, F Ghio, M Jodret,

G M U U

INFN, LECCE - R Perrino

MARYEAND U - J J Kolly, F Markowet,

MilT - W Eertoxza, D Dole, S Gilad, A Sarty

NIKHEF, AMSTERDAM - H P Blok

OLD DOMINION U - F E Ulper, C E Wepatein

RUTGERS U - R Gilruan, C Olesbaudeger, G Kumbarinks, F. Rangome, P M Rust

SACLAY - J Y Mouge;

NEW HAMPSHIRE $\boldsymbol{U}$ - J Calarco

ROME U. TORSEHGAYA - Ş Frullen ( $V$ Bpokespereon), F Garibeldi

VIRGINIA U - D II Barkbuti, R Loune ( $\checkmark$ pookespereon),

B Mfilbrath, 9 Var Vergt

WILLIAK! AND MARY COLL - J M Fwn, M Jons, C F Perdrieat

Acoeteritor CEBAF Deterler Spectrometer

Reactions Polenzed beam

$$
e^{-} p \rightarrow e^{-} p \pi^{D} \quad 32 \mathrm{GeV} / \mathrm{c}
$$

Pertictes stadied $\Delta\left(1232 F_{33}\right)$

Brif descrption Studes the six atructure functions in the $N-\Delta$ tranaztion as a function of $\theta_{c}$ The metritement it performed st $Q^{2}=10(G e V / c)^{2}$, at $6 \mathrm{~cm}$ angles, whoch a لlows a slardalone sultupale anslygse of the amplutudes Utes htgh. resolution spoctrometars and a foced plage polarsmeter The target to Lquid bydrogen scheduled to tum in Hall A

Reduted raperaments CEB,AF-39-037, -08-042, -93-036

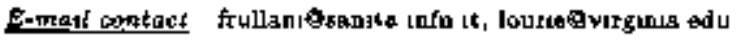




\section{SUMMARIES OF CEBAF EXPERIMENTS}

\section{CFBAF-61-023}

(Propred Oct 1991, Approved May 1992, Jas J995, Ja preparation)

MEASUREMENT OF POLARIZED STRUCTURE FUNCTIONS IN INELASTTC ELECTRON-PROTON SCATTERING USING THE CEBAF LARGE ACCEP. TANCE GPECTROMETER

N" COLLABORATIOK

CEBAF - W Bropks, Y p Burkett ( $\checkmark$ spakepecron),

A Froyberger, B A Macking, M Mestayer, B B Nucsyporuk,

E Smath, A Yernegwaran

CHPLSTOPHER NEWPOKT U - D Daughty, L ENoundrhır',

D Heddle

DUKE U - M Spraeker, H R Weller

FLORIDA STATE U - L $c$ Denans, P Dregovitach, K W Kenipar

FRASCATI - N Biench, G P Capitant, E De Sanctis. P Leys

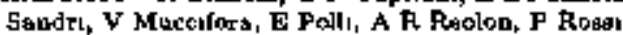

INFN, DENOA - M Anghinolfi, P Corvysuero, G Gervino,

I Matrathil, V Moketv, G Eleco, M Rupant, M Stprane,

M Taubl, A Zucehis ll

HAMPTON $U-K$ B BesRd

JAMES MADISON $U$ - K Grovatest

KENT STATE U - D Keape, D M Kaploy

OLD tOMTNLON tU - $\mathbf{s}$ Kuhn

PITTSBURGH U - $S$ A Dytman

RENSSELAER POLY - G S Adams, N C Mukhopothyoy, P Stoles

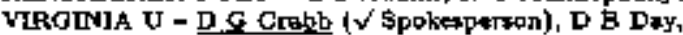

J S MCCarthy, F C Minehart ( $/$ Spokespertan), O A Rondon-

Arameyo, R Scalack, ì $\bar{C}$ S muth, $\$$ T Thomson, H I Wieber

VIRGINIA STATE U - C E Stropach

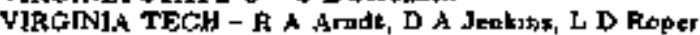

WILLIAN AND MARY COLL - A Colennan, H O Fumbten

T Y Tung

YALE U - W Gat

Acceletutor CEBAP Delectar CLAS

Reactyogs Polarized beam agd target

$$
t^{-} p \rightarrow e^{-} X \quad 12-40,600 \mathrm{eV}\left(T_{\mathrm{lBh}}\right)
$$

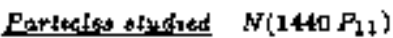

Btwef description Mesoured the indurjve polarized structure functions, $A_{1}$ and $A_{2}$, in the range $02 \leq Q^{2} \leq 25$ (GeV/G) ${ }^{2}$ and $11<W<35 \mathrm{GeV} \mathrm{U}_{\text {sed polarited }} \mathrm{NH}_{3}$ torget Sebedulted co run in Hall B in 1998

Related experiments CEBAF-93-000

Etmal contact burkert Orebal kov, dcrabbowarsmia edu, menehortovirgutia edu

\section{CEBAF-91-024}

(Proposed Oct 1981, Approved Dec 1991, In preparahion)

SEARCH FOA MISSING RESONANCES IN THE ELECTROPRODUCTION OF w MESONS

N* COELABOEATION

CBBAF - WW Erookt, V D Brikgrc (\$pokespersor), D J oyce,

B A Mescking (Spokespersan), M Mestayor, B B Niczyporuk,

E Snith, A Yegeswaran

CHRISTOPIIER NEWPORT U . D Doughty, D Heddle, 2 J Ll

FLOHLA STATE U - L Q DeDan

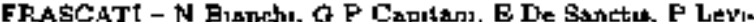

Saudri, V Muecifora, E Pollt, A R Reolon, P Fases

INFN, GENOA - M Anghundfi, P Corvisiero, G Gervino,

L Mrezeschi, V Mokesy, G Rtece, M Ripani, M Sanzone,

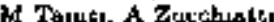

GEORGE MASON U - B J LJeb

HAMPTON U - K B Beard

JAMES MADTSON U - K Grovanett

KENT STATE U - D M Mdanley (Spokesperaon)

PITTSEURG U U

RENSSELAER POLY - G S Adaros, N C Muthopadbyay, P Stoler

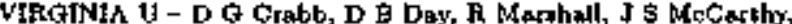

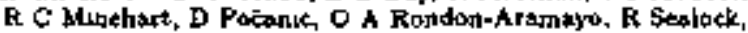
L C 5moth, S T Tbornton, H J Weber

VRGINIA STATE U - C E Stronhch

WTLLLAM AND MLARY COLL - C E Carleon, H O Fansten

(Spokesperson), T Y Tang

YALE $U$ - $M$ G

Acatervitor CEBAF Detectót CLAS

Eractions

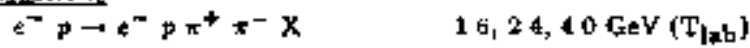

Brtef deseription The expernment is aumed at aesrehung for 'ruhsslag' 3-quark bayyon stated in the maxts rapge from 17 to $22 \mathrm{GeV} / c^{2}$ in the $p w$ decay channed scheduled to rus in Hall B w $1906 / 97$

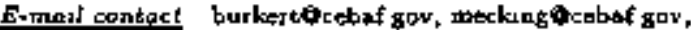

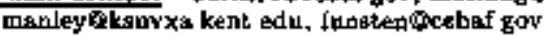

\section{CEBAF-88-00B}

(Propated Apt 1993, Approved Jun 2993, In preparation)

\section{TWO PJON DECAY OF ELECTROPRODUCED LIGHT} QUARK BAFYON RESONANCES

\section{$\mathrm{N}^{*}$ COLLAEQRATTON}

CEBAF - W Erooks, V D Burkert ( $V$ Spokenperson)

B A Meckjng, B B Neryporuk, E 5mith, A Yagnetwaran CHEISTOPHEF NEW POFT U - D Doughty, D Haddle

DUKE U - R Cbsteker, H R. Welter

FLORIA sTaTE U - \& C Deabu, P Dragovitach

FRASGATI - N Bisnchu, G P Coptragu, E tha Sanctis, P Leve

Sandrc, V Mucctions, E Polls, A R. Reokon, P Rasss

INFN, GENOA - M Anghinol6, P Corvistero, V Motosev, C RHCCO,

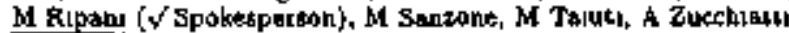

GEORGE MASON U - B J Lab

HAMPTUN $U-K$ B BeBCd

JAMES IAADISON $U$ - $K$ GIOvanett:

KENT STATE U - D M Marley

NORTH CAROCINA STATE U - D R Tillay

PITTSBUI RGH $\mathrm{y}$ - \$ A Dytman

RENSSELAER POLY - G 5 Adame, N C Mukhopadhyay,

J Nepolatsmo, P Stoler

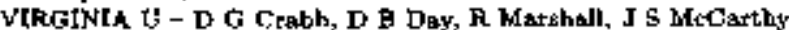

R. C Minehart, D Pozants, O A Fondon-Azamaya, R \$ealock.

L C Smich, S T Thomton, H J Waber

WILLLAM AND MARY COLL - C E Carlood, A Coleman.

H O Fungten, $\boldsymbol{T} Y$ Tung

YALE U - M Ga

Acceterator CEBAF Detector CLAS

Renctions

$$
\begin{array}{ll}
e^{-} n-e^{-} \text {aucloon pion pron } X & 16,24,40 \text { GeV } \\
e^{-} n \rightarrow e^{-} \text {nucleon pron pron } X & \left(\tau_{1 \times b}\right)
\end{array}
$$

Bref descraptors Studies gome sapects of baryon spectia in the Lanstrainge ector, o $q$, the form-Factors of some poorly known states [t atso lookg for highly exclted nucleon statea

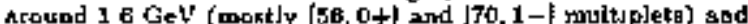
the 'masems' states around $2 \mathrm{GbV}$ (nostily $[86,2+]$ and $[70,2+]$ meltiplets] predicted by quark models Suct stat es would at rengly dectsy through $\Delta \pi$, and oN thennede, both grvong a fad state with imo pron Appraved Ior 900 hours with hydrogen tarket and 400 hours with dequtersum target Expected to run to Hall B th 1996/67

Related experements CEBAF-91-02d; CEBAF-01-002, CEBAF.

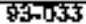

E-math contact burkertocebal gov, npaniogenova in in it

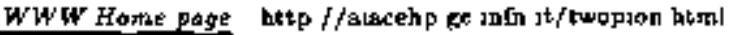




\section{SUMMARIES OF CEBAF EXPERIMENTS}

\section{CEBAF-93-0BO}

(Proposed Apr 1993, Appruved Jun 2093, In preparation)

MEASUREMENT OF THE STRUCTURE FUNCTIONS FOR KAON ELECTROPRODUCTION

\section{CLAS COLLABORATTON}

CEBAF - M Mobtayec ( $\checkmark$ Spokegperson), E Smith

CARNEGIE MELLON U - R Msgahrz, F A Sthumbacher

CHAISTOPHER NEWPOFT U - D Daughty

FLOFIDA STATE $U$ - $\$$ Capatick

GEORGE WASHINGTON U - O Benubald

OHIO U - T Adsmi, R. W Finlay, 5 Grimed, K H Hicket

( $\checkmark$ Spaktaperson), A Kumer, D 5 Oniey, I Rapeport,

L E Wright

PITTSBLtRGH U - S A Dytmbit

AICHMOND U - P D Rubin

BOUTH CAROLINA U - A TAN

WLLLIAM AND MARY COLL - H O Fungten

Accelenotor CEBAF Defegler CLAS

Reactions

$$
\begin{aligned}
& e^{-} p e^{-} K^{+} A \\
& c^{-} \rightarrow E^{-} K^{+} \Sigma^{0}
\end{aligned} \quad 24,32,40 \mathrm{GeV}\left(\mathrm{T}_{\mid \mathrm{X} b}\right)
$$

Partecien fendued $N^{*}$ (uncpec)

Ertel descraption Mesautes $L, T, L T$, and $T T$ atructure fubctione for $\sigma^{2}$ between 1 and $2(\mathrm{GeV} / \mathrm{c})^{2}$ and $w$ between 18 and $22 \mathrm{GeV}$ Mestures losply dependance by compantes $\Lambda$ and $\Sigma$ productions Studies produstion ratw of hyperons up to the $A(1520)$ Measures polsrization of $A$ Seareher for $N^{*}$ resonances which dexny to byperon- $K^{+}$lins! states sebredulod to reת in Hall $B$

Jourtal pogers NLM A323 (1092) J91, and [EEE TNS 39 (1992) 6809

E.mali contact menlsyrertice baf gov

lucksouvars catg obou odu, kheckal Wobuou tod

\section{CEBAF-99-083}

(Proposed Apr 1903, Approved Jun 1993, in proparatjon)

A SEARCH FOR MISSING BARYONS FORMED IN $\gamma p-\not x^{+} *^{-}$USING THE CLAS DETECTOR

CIAS COLLABORATION

RENSSELAER POLY - $\mathrm{G} S$ Adsmb, J Napolitaho

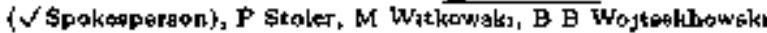
CARNEGIE MELLON $U$ - R Schumacher

RICE U - G Mutchler

KENT STATE O - D M Madey

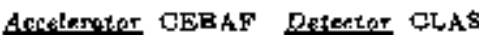

Resctions

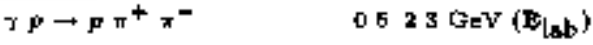

Paricles stadied $N^{*}$ (unepec)

Brut description Dtea taggad photoha and haqud hydrogen

target Scheduled to run w Hall B in $1996 / 9$ ?

Related expertments CEBAF-gB-004, CEBAF-89-024

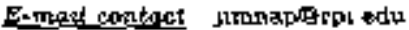

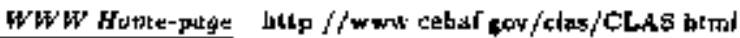

\section{CEBAF-93-086}

(Proposed Apr 1993, Approved Jan 1993, In preparation)

MEASUREMENT OF SINGLE PION ELECTROPRO. DUCTION FROM THE PROTON WITH POLARIZEE BEAM AND POLARIZED TARGET USING CLAS

$N^{*}$ COLEABORATION

CEBAF - W Brouks, V D Burkert, D Joyce, B A Meckıng,

B B Niczyporuk, E S Smith, A Yegueswatan
CHRISTOPHER NEWPORT U - D Doughty, D Hoddle, $z \mathbf{J}$ L, DUtkE i - B. Chasteler ( $\checkmark$ Spolesperson), H R Weller

( $V$ Spoketparion)

PLORIDA STATE U - L O Denur, F Dregovitech

FRASCATI - N Bianchi, G P Croptam, E Di Snctu, P len-

Sandri, V Mucciford, E Polly, A R Reolon, P Rosyi

INFN, GENOA - M Anghinglf, P Corvistero, G Gervino,

L Matstachi, V Mokeov, G Rucco, is Ripani, M Sanzone,

M Tautı, A Zuchiattı

GEORGE MASON $U$ - I J Lle巾

HAMPTON U - K B BeBrd

JAKIES WADISON U - K G1OYametti

KENT STATE U - D N Manlay

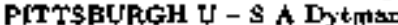

RENSSELAER POLY - G \$ Adarn, N C Mukbopadhyay, P Stoler VLRGINLA U - D G Crabb, D B Dey, J S MeCerthy,

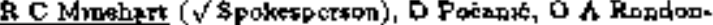

Aramoyo, Fi Sealeck, L C \$muth, 3 T Thornton, H J Weber

VIRGINIA STATE U - C E Stronach

WILLLAM AND MARY COLL - C E Carkon, H O Fungten

YALE U - M Ga

Acrelerator CEBAF Detectur CLAS

Reactions Polerized beans and target

$$
\begin{array}{cc}
e^{-} p \rightarrow e^{-} \nabla^{+} & 12-40 \mathrm{GeV}\left(\mathrm{T}_{\mathrm{lab}}\right) \\
e^{-} p \rightarrow e^{-}{ }^{\circ} & -
\end{array}
$$

Partulfs ftudied $N\left(1440 P_{11}\right), N\left(1520 D_{19}\right), \Delta\left(1232 F_{39}\right)$

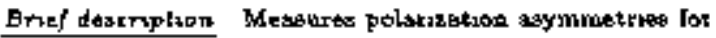
exclnsive sluglepon channels Provides information on the Foper rabonance Schodulad to run in Hell E in 1998/on

Refoted expertarents CEE-4F-99-037, CEBAF-89-038, CEBAF-

自口-010, CEBAF-83-062, CEBAF-62-043, CEBAF-91-003

E.mot contact bobbaratunl tunl dake edu,

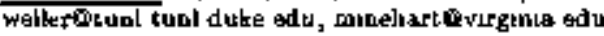

\section{CEBAF-94-008}

(Proposed Apr 1894, Approved Jur 1994, In preparation)

\section{PHOTOPRODUCTION OF $\eta$ 9Ed $\eta^{\prime}$ MESONE PROM} DEUTERUUM

\section{CLAS COLLABORATION}

ARIZON A STATE U - E Rtuche ( $\checkmark$ Spokegperbon)

CEPAF - B A Mackın

GEORGETOWN U - J Lambert

RICHMOND W - G P Gilfoyls, R W Major, M F Yineyard

SOLTH CAROLINA V - C Djank, B Preedam, S Whumbt

Acceleretor CEBAF Detertor CLAS

$$
\begin{aligned}
& \text { Repactigns } \\
& \rightarrow \text { deut } \rightarrow \text { ideut } \quad 063-152 \text { Gov }\left(E_{\text {lab }}\right) \\
& \rightarrow \text { deut } \rightarrow \pi^{\prime} \text { daut }
\end{aligned}
$$

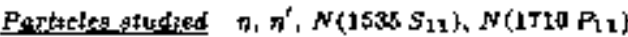

Brtef description Messures the differentisl cross-section for the

cohereat and taroberent photoyrodutwou of th and $\eta^{\prime}$ mesons on the deuteron for photan energles from 063 to $1 \mathrm{B2} \mathrm{GEV}$

Unen a liquid deuterium targes cell and a tagsed pbaton bean Provideg informstion on the properties of $\eta_{1}$, $p^{\prime}$ and enves maight into the strvet ure of related sucleon resonances Studies the bebavor of the mesons in the ligbuly baund two-nucleob

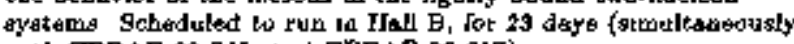
with CEBAF-89-045, and CEBAF-93-0(T)

Related expertiments CEBAF-\$9-03s, OEBAF-39-045, CEBAF. 93-008, CEBAF-93-nIT, CEBAF-91-009

E-mad contect barry ritchieotsu edu

\section{CEBAF-94-016}

(Propobed Apr 1994, Approved Jan 1985, In preperation)

MEABUREMENT OF RARE RADJATVE DECAYS OF THE \& MESON 


\section{SUMMARIES OF CEBAF EXPERIMENTS}

CATHOLIC U - H Crannelt, J O'Bnen, D I Sqber

CEBAF - V D Burkert, B Niexyporuk, E S Smuth

INDIANA U - B Brsberon, R Crictenden, A Duletba

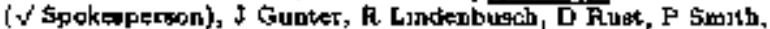

$S$ Tenge

MOSCOW, ITEP - M V KOSBOW

NOTRE DAdsE U - J J Kolata, I Loseco, A H S snjen,

W D Sbephard

FLCE to - 3 Ahmad, B E Eonner, G Nutchler

RICHMOND U - G P Giloyle, P Rubin, $M$ F Yineyard

RENSSEEAER POLY - G S Adsms, I I Napolteane ( $\checkmark \mathrm{Co}$

epokesperann', M $T$ Witkowale

VIRGINIA U - E Ftreī, D Pọ̆ant

WILLIAM AND MARY COLL - D ATmELrong

Accelertor CEBAF Detector Calotsmeter

Refactangs

$$
\checkmark P \rightarrow \text { ग } P \text { GeV (E }
$$

Portacies alvedted \&, $\rho_{1}$

Bruf deacroption Used the $4 \mathrm{GeV}$ waged pbotion beari and a lesd glate detertor to study decays of the $\phi$ mito ell-photon ingl gtates Expects to achieve the branching ratid sensitivity of thent $70^{-5}$, wheh should be enopgh to obereve the (y-d urobservod) decayd $\phi \rightarrow f_{0}(990) \gamma, \phi \rightarrow a_{0}(900)>$, and $\phi \rightarrow f^{\prime} \gamma$, and to improve the measuremeth of ather bracoching ratiod of

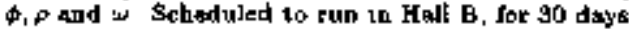

Related expertmenti BNL-852

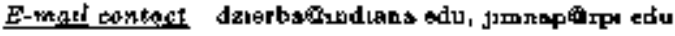
WWW Home-page

bttp $/ /$ www cebar gơv/physica/radphl/radphl btrl

\section{CEBAF-84-109}

(Froposed Dec 1994, Approved Jan 1995, In preparalion)

PHOTOPRODUCTION OF THE a MESON FROM THE PROTON WITH LINEARLY POLARIZED PLOTONS

ARIZONA STATE U - R Alsrcon, J Comfort

CATHOLIC U - H Crannell, S Matthawg, J Q:Bnen, D I Saber

CEEAF - R R Whitpey (V Spoketperson)

GEORGE WASEINGTON U - $\mathrm{A}$ I Barman, W J Erroge,

F 1 Cole ( $\sqrt{ }$ Spokesporson), I P Connelly ( $\checkmark /$ Spokesperson),

K S Dbugs, L Morphy, S Rugarı

KENT STATE U - D M Mauley

OLD DOMINION U - C Hyde-hiright, W Roberts

RENESEL AER POLY - J J Napolitamo

SACLAY G Audit, M Gudal, F Kanne-Perrot, J is Laget.

$C$ Marchand, B Saghat

FLORIDA STATE U, \$CRI - \$ C Cnpstnde

VIRGINIA TECH - J Ficenec, D A Jemkint

Acceleratgr CEBAF Detector CLAS

Reactions Polarised beam

$$
\begin{aligned}
& \text { Tp } \cdots \rho^{0} p \quad 10-1,5 \text { GeV }\left(\mathrm{E}_{\mathrm{lab}}\right) \\
& r p \rightarrow \rho^{+} \\
& \gamma \rightarrow p^{0} p \\
& \gamma_{p} \rightarrow p^{+} \text {r } \\
& 14-2 \text { \& GV }\left(E_{1 / b}\right)
\end{aligned}
$$

Parluctes gludied $N^{*}$ (unspec), $\Delta$ (unspec)

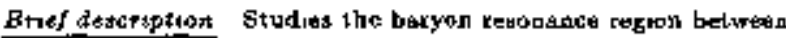

$16 B^{3}$ and $222 \mathrm{GeV}$ center of -mass energy in the oN channel

Juer a lizearly polerized taefed photon boam produced by

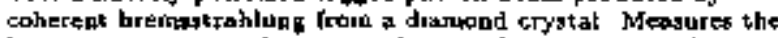

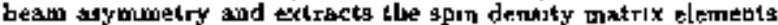

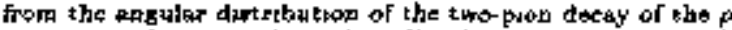
messon as a function of and $t$ thes facilutate the search for baryon retonance contributsons and provides unformation on

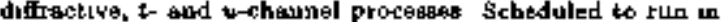

Hall B, Ior II daye at $4 \mathrm{GeV}$, and 9 daye st $6 \mathrm{GeV}$

Relateil expertment; CEBAF-P3-033

E.mall cortact coleounterol cebas gov

\section{Other CEBAF Experiments}

Leted here are some other Jefierson Lab's (Jormerly CEBAF) experiments af interest to the particle physics community Find more detall about these profects online, in the SLAC's EXPEFIMENTS datsbase (aee p 3), or contact the apokespersons The regularly publisbed Progtam Advitory Commillet (PAC) repotls often list all the epproved experiments al the Latb For the coples of these reports contakt uers

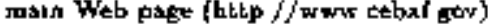

CEBAF-89-0OA (In prepstadion)

ELECTROMAGNETIC PRODUCTION OF HYPERONG By CLAS Collaberation

Spokesperson Fernbard A Sehomacter JCarnegse Mellon U |

E mas contect reubardournest plys coul edu

CEBAF-89-009 (In preperation)

INCLUSIVE BCATTERING FOR NUCLEI $A T *>1$ AND HIGH $Q^{2}$

Spokeppereon* Bradley W Fulppone [Cal Tech], Donal B Day [Vurgemes U]

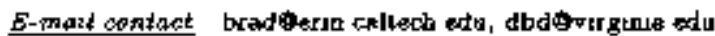

CEBAF-ab-BOG (ID prepstakiou)

INVESTIGATION OF THE SPIN DEPENDENCR OF THE AN EFFECTIVE INTERACTION IN THE P SHELL Spokespersobe Roborl E Chtien [Broolkssvan], Ed Hungarford [Houpton U] ]

Emant contenat chriengibnl gow, hupgersuh edu

CEBAF-80-m12 ('Taküg dats)

TWO-BODY PHOTODISINTEGRATION OF THE

DEUTERON AT FORWARD ANGLES AND PHOTON ENERGIZS BETWEEN 1.6 AND 4.0 GeV

Spokexpermon Foy J Holt [Argonne]

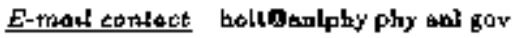

CEBAF-S9-015 (In preparatian)

STUDY OF COINCIDENCE REACTIONS IN THE DIP AND $\triangle$-RESONANCE REOIONS

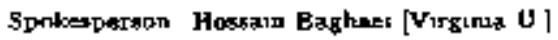

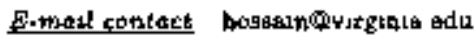

CEBAF-89-017 (In preparation)

ELECTROEXCITATION OF THE A (1292) IN NUCLEI

Spokaspersion Ruchard Saslock [Virgivis U]

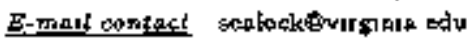

CEBAF-BQ-D3S (In proparati,as)

MEASUREMENT OF FECOIL POLARIZATION IN THE ${ }^{16} O\left(\vec{a}, e^{\prime} \vec{p}\right)$ REACTION WITH 4 Gov ELECTRON\$

Spokespersons Sinxh Nanda, J Wallace Van Orden [CEBAF|,

Chus Cheh (Goorex) Chang [Maryitand II , Cluseles Glashsusger

|Rusgers U |

Esmat contact nondagrcelbaf gov; vanordentional gov,

thang 


\section{SUMMARIES OF CEBAF EXPERIMENTS}

CEBAF-80-0s7 (In prepsration)

ELECTROPKODUCTION OF THE $\triangle\left(1392 P_{33}\right)$ RESO-

NANCE

By $N^{-}$Collaboration

Spokipgernons Volker D Burkert |CBBAF|, RAlph C Mrnebart [Yurginta U]

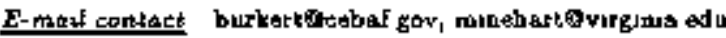

CEBAF-89-04J (In preparstion)

MEASUREMENT OP THE ELECTRON ASYMMETRY

IN THE $p\left(e, e^{\prime} p\right) \pi^{\circ}$ AND $p\left(c_{1} \varepsilon^{\prime} \pi^{+}\right) n$ IN THE MASS

FEGION OF THE $\triangle(1232 P \$ 3)$ FGR $q^{2} \leq 2(\mathrm{G} * V / c)^{2}$

By $\mathrm{N}^{*}$ Collaboralion

Spokespersovs Volker D Burkert [CEBAFl, Ralph C Minehart [Vireluas U ]

E-mistl cofttact burkert Qcebal gov, mianbartevurgemia edu

CEBAF-Bo+045 (In prepatation)

STUDY OF KAON PHOTOPRODUCTION ON DEUTERAUM

Spokesperson Bernhard A Mechng [CEBAF]

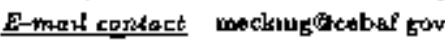

CEBAF-91-003 (In preparation)

A STUDY OF LONGITUDINAL CHARGED PJON

ELECTROPRODUCTION IN ${ }^{2} \mathrm{D},{ }^{3} \mathrm{He}$, AND ${ }^{4} \mathrm{He}$

Spokesperson Horold E Jackson [Argonne]

Esmati aontact helotenl gov

CEBAF-Ól-004 (In praparation)

MEASUREMENT OF STRANGE QUARK EFFFCTS USING PARITY VIOLATING ELASTIC SCATTERING FROM ${ }^{4}$ Ho AT $Q^{2}$ a 0 (GoV $\left./ c\right)^{2}$

Spokesperton Betay Besbe [Marylaud U]

Ematicontact bescotienp umd edu

GEBAF-91-007 (In preparation)

MEASUREMENT OF THE NUCLEAR DEPENDENCE AND MOMENTUM TRANSFER DEPENDENCE OF QUASLELASTIC $\left(c, p^{\prime} p\right)$ SCATTEAING AT LARGE MOMENTUM TRANSFEF

Spokeperson Rechatd \& Milor (M1T)

E-maut cantants mulner6imrtlss mut edv

CEBAF-91-610 (It proparation)

PARUTY VIOLATION IN ELASTIC SCATTERING FROM THE PROTON AND ${ }^{4} \mathrm{He}$

Spakesperaons Paul A Souder [Syraeuse U], J M Finn [Willam sud Mary Coll I

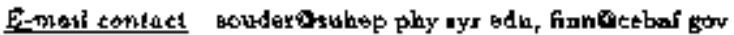
WWW Name-page http//wwe cobsl gow/halla/parity htwl

CEBAF-9l-014 (IU preparation)

QUASIFREE STRANGENESS PRODUCTION IN NUCLEI

Spakceperzon Cherles E Hydo-Wight fOld Domentan U]

E-mant contacet hydeacebal gov
CEBAF-9l-olB (In preparatjon)

HELCITY STRUCTURE OF PION PHOTOPRODUCTION

By CLAS Colleboration

Spokeserson Daniel I Sober [Catbolic U]

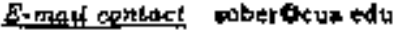

CEBAF-92-010 (1n preparation)

ELECTROPRODUCTION OF KAONS AND LIGHT HYPERNUCLEI

Spokesperson Besjamin Zeadman [Artomna]

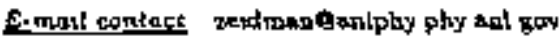

CEEAF-91-017 (In pręparacion)

MEASUREMENT OF $G^{\circ}$, THE FLAVOR SINGEET

CHARGE FORM-BACTOR OF PROTON

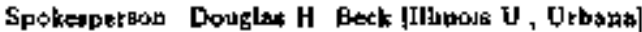

E-mair cantact beckouiphls npl wuc edu

CEDAF-01-026 (In pTeparation)

MEABUREMENT OE THE ELECTRIC AND MAG-

NETIC STRUCTURE FUNCTIONS OF DEUTERON AT LARGE MOMENTUM TRANSFERS

Spokefperaon Getagstmon (Makds) G Pelratob |Kent State U।

Eumati eqriactit petratodaksuyxd kent adu

CEBAF-9a-gOB (In prephation)

DNCLUSIVE क PHOTOPFODUCTION IN NUCLEI

Sputerpergon Muchael F Vireyard [Richmond U I

E-majt cantact vagyardouryex usich odw

CEBAF-8a-00G (Lh preptration)

THE POLARJZED STRUCTURE FUNCTLON $G_{1 n}$ AND

THE $Q^{2}$ DEPENDENCE OF THE GERASIMOV-DFELI

HEARN SUM RULE FOR THE NEUTAON

By $\mathrm{N}^{4}$ Collabaration

Spokesperton Sebastien E Kuhn [OHd Domumon U]

E mail contact kuhnercebst gor

CEBAF-as-019 (In preparation)

ELECTAOPRODUCTION OF LIGHT QUARK MESONS By CLAS Cattabotation

Spokesperson Mikball V Korsov [M [apow, ITEP]

E.mazl conlact kotrantike baf gov

CEBAF-9\%-017 (In preparatpon)

STUDY OF $r d \neg p n$ AND $\gamma d \rightarrow \Delta^{0}$ REACTIONS POR

SMALL MOMENTUM TRANGFERS

Spokespertons Enzo De Sanctib, Pattiza Roeal [Fraecati]

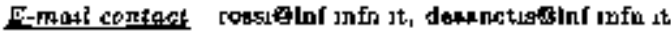

CEBAF-93-üI (In preparstion)

THE CHARGED PION FORM-FACTOR

Spoterapergon Dovjd J Mods |CEBAF|

E-moti contart mackocebef gov 


\section{SUMMARIES OF CEBAF EXPERIMENTS}

CBBAF-93-022 (In prepstation)

MEASUREMENT OF THE POLLARIZATION OF THE ф IN ELECTHOPRODUCT1ON

Spotespersona Eiton Smath [CEBAF], Phulip D Rubsn [Ruchmond

U I. Hertezt O Fupeten [W, Llian and Mary Codl]

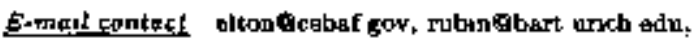

ianftenfictbal goy

CEBAF-93-024 [In pteparation]

MEASUREMENT OF THE MAGNETIC TORM-FACTOR OF THE NEUTRON AT LARGE MOMENTUM TRANS-

\section{FERS}

Spokesperauss Jav]er Gomes [CEBAF], Garesol-

mot (Makii) G Patration [Kant Skate U]

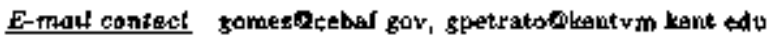

CEBAF-BS-D26 (In preparation)

THE CHAROE FOKM-FACTOR OF THE NEUTRON

Spaksipertion Domal B Day [Virgiaia U |

E-matl conset dbdawirgals adu

CEBAF-0S-027 (In proparation)

BLECTRIC FORM-FACTOR OF PROTON BY RECOIL POLARIZATION

Spokesperaong Vina Punjabe |Norfolk State U J, Cbartes F Per. drtast [Witlom and Mary Coll ]

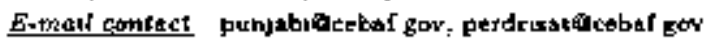

CEBAF-93-081 (In preparalian)

PHOTOPRODUCTION OF VECTOR MESONS AT HIGH $t$

Spokespereang Marco Anghuolfi [INFN, Gebros],

Jean Marc Laget, Cleude Marchand [Saclay]

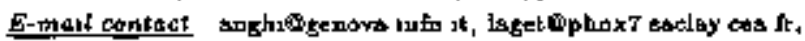
marcband gphnx.7 saclay cea fr

GEBAF-as-oss (in preparation)

THE ELECTRIC AND MAGNETJC PORM-FACTORS DF THE NEUTHON FROM THE $d\left(\bar{z}, e^{\prime} \bar{n}\right) p$ REACTION Spokesperowh Rechard Madey jHanplon U।

E-math contace marley oicelar gow

CEBAF-94-DO2 (In preparation)

PHOTOPRODUCTION OF VECTOR MESONS OFF NUCES!

Spoksaparmong Pitre Bertiu [Cletwont-Fetrand $U$ ।,

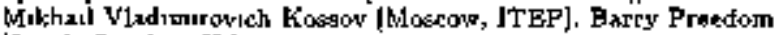

|Sateb Caroluna U |

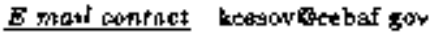

CEEAF-94-00s (LI prepsetion)

DETERMINATION OF THE NA AX,1AL VECTOR

TRANSTTION PORM-FACTOR G ${ }^{A} A$ FROM THE ap $-\varepsilon^{\prime} \Delta^{++} \pi^{-}$REACTION

Spolespersons Latifa Elouadrhtro, Davd Heddle [Christopher Newpert U!

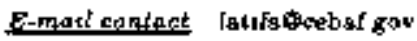

CEBAF-94-D10 (fo preptration)

MEASUREMENT OF THE NEUTRON ( ${ }^{3} \mathrm{HE}$ ) SPIN STRUCTURE FUNCTION AT LOW $Q^{2}$, $\lambda$ CONNEC. TION BETWEEN THE BJORKEN AND DFELI HEARN-GERASIMOV SUM RULES

Spokesprssons Gordon D Cates [Princeton U ], Zew* Eddut Mexiapy [Temple UI

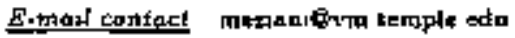

CEBAF-04-012 (In prepazation)

MEASUREMENT OF PHOTOPROTON POLARIZATION IN THE $\mathrm{H}(\mathrm{\gamma}, \vec{p}) x^{0}$ REACTION

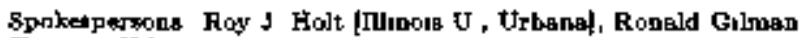
[Rutgore U]

E-man contact gimonomathep rutgers adu

CEBAF-04-014 (In praparatron)

The $\Delta(1233)$ FORM-FACTOR AT HIGH MOMENTUM TRANSFER

Spokesperaon James I Napothano [Rensaclaer Poly]

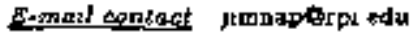

CEBAF-94-b15 (In prepatalion)

STUDY OE THE AXIAL ANOMALY USING THE $\gamma \pi^{+} \rightarrow$ $\pi^{\circ}$ REACTION NEAR THFESHOLD

Spokespetiont Amrit 3 Yegatowaran [CEBAF?, Rory A Mukn

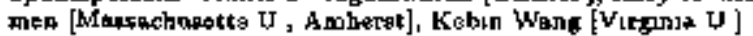

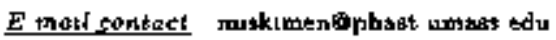

CEBAF-p4-017 (In preparatron)

THE NEUTRON MAGNETIC FORM-FACTOR FROM PRECISION MEAGUREMENTS OF THE RATIO OF QUASIELASTIO ELECTRON-NEUTRON TO ELECTRON-PROTON SCATTERING IN DEUTERUM Spukespersong William K Bropks [CEBAF], Mhchafl F Vineyard [Fuchmond U ]

토-man' contact brookum acebal' gov

CEBAF-B4-103 (Iи prepatsuon)

THE PHOTOPRODUCTION OP PIONS

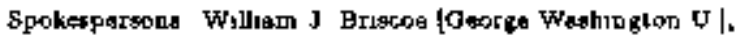
Joha Ficenec, David A Jenkiar (Vurama Techi)

E-meid contact fllceneoourt edu

CEBAF-B4-104 (In prepatacion)

THE FUNDAMENTAL $\rightarrow \pi \rightarrow \pi^{-} p$ PROCESS IN ${ }^{2} \mathrm{H},{ }^{4} \mathrm{ll}$, and ${ }^{12} \mathrm{C}$ IN THE 1.2-6.0 GeV REGION

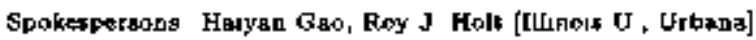

Esmaid contact zeoturipla npt urpc edu

CEBAF-85-00s (In preparscion)

MEASUREMENT OP $\mathrm{K}^{\circ}$ ELECTROPRODUCTION

\$potrepersogn Rehard Magabix [Carnege Mellon U]

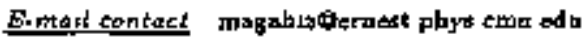




\section{Selected CERN Experiments}

\section{CERIN-IS-300}

(Propoted Sep 1991, Approved Sep 1991, In preparation)

\section{A SEARCH FOR AXIONS AND MASSIVE NEUTRINOS}

IS300-1SOLDE COLLABORATION

AARHUS U - P Hornohoy, H L Nielsen, J w Petersen: K Rusager, [S Wyer ( $\checkmark$ Spokgperson)

CEAN - A De Rujula, H E Ravn

CHALMERS UNIV TECH - B Jonaon, G Nyman

Aecelerator GERN-PS Detector Spectrometer

Partictes atisuted axion, th

Brief descripltar A search for axions and b heary neutiwo

zelyeg on a strong, hugh purtty gourec of rediasctive 1251 A possible $17 \mathrm{keV}$ neut man emiscion in the electron tapiure detay of ${ }^{125}$ I could be obearred by atudying the intarnel

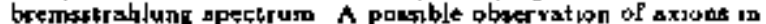
the 355 keV M11 teantition of the ${ }^{125} \mathrm{Te} d$ aughtet is boged on an apalogne of the Mossbauer effect, i $e$ the axion reonssos absorplion tor the ${ }^{125}$ Ta resonsmee abgorber Awsting for a otoggered bearn fram the PS Bonater, expbcted to be avalsble in Sprics o7

Brmasl conflact helgo ravaluctuch

\section{CEFN-LEP-ALEPH}

(Fropoyed 1982, Approyed Nov 1982, Eezan data tatung Aug 1989. In proqre\%s)

THE ALEPH DETECTOR (APPARATUS FOR LBP PHYSICS)

\section{ALEPH COLLABORATION}

ANNECY - D Buskulue, I De Barma, D Decatrp, P Ghez, C Goy, I P Lees, $A$ luoble, is N Bluserd, $\Gamma$ Oder, B Puetrzyk BAFCELONA AUTONOMA U - M Gbmetesan, I I Grespo,

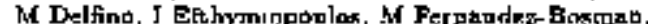
E Fetrandox L Gatrido, A Juts, M Martsnoz, $S$ Oxiou, A Pucheco, C Padilla, F Palla, A Paxciul, J A Perlas, I Fuv, F Sancher, F Teubert

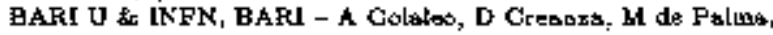
A Farilla, G Gelao, M Girone, 0 leasell, G Maggi, M Magra, N Maribell, S Natal, 5 Nuzxo, A Rabier, G Faxo, F Fomano,

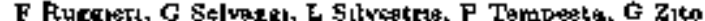

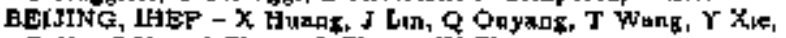
R. Xu, S Xue, J Zhang. L Zhang, W Zhro

CERN - R Alsmahy, A O Banatko, G Bamvejar, M Cotitanarb, P Conias, F Coyle, H Dreverraaun, A. W Forty, M Ftank, A Hagelbarg I Harvey, A. Jacobsen, P Jandt, $B$ Jost. E Katinger, J Knoblach, I Lehrane, E B Martin P Mate, A hinten, R Mrquet, I M Mir, L Moneta, T Oeas, $P$ Palszaz, I R Pater, I F Fuszteszen, F Runjerd, P Renging, L. Rollandi ( $r$ Spokespergon), D Schlsiter, M Schmellung, O Sehuejder, W Tojessy, I R Tomeln, A Venturt, $H$ Waclismuth, A Wagres, $T$ Wildab, W Witrelın, J Wotschack

CLERג1ONT.FERRAND U - $Z$ A jaltoun, A Barres, C Boyer, A Falvard, $P$ Gay, $O$ Guncheney, $P$ Henrard, J Jousyet, B Michel, S Montel, I C Montret, D Pelli, P Psrret, F Podlyskı. J Pronid, J M Rosanghol

BOHR INST - T Fobrnley, J B Haveen, J D Hangen, I R. Eansen, F 4 Haosen, B $\$$ Nllsson, A Wasnanen

DEMOCRITOS NUCLEAR RESEARCH CENTER A KYruakL,

C Marbou, E Simopoutou, I \$letu, A Vayakı, K Zachariadou ECOLE POLY'T ECENIQUE - A Blondel, G Bondesud,

P Bourdan, J C Brent, A Rouge, M Rumpf, R. Tanakr, A Valassi, M Verders, H Videsu

EDINBURGH if $-D$ J Cundlin, $M$ I Prrons

FLorENCE U $z$ INFN, Florence - E focardi, G Parrial FLORIDA STÁrE V - M Corden, C Georgrapoulob, D E Jofío FRASCATI - A Antonell, G BencivenaI, i Bologna, F Bossi, P Compano, G Capon, D Cesper, v Chiarelle, G Feluci,
P Laurell, G Msnnocehl, F Murtas, G P Murtse, L Psgakacqua. M Pepe-Altarejll

GLASGOW U - L Curts, I J Dorrt, A W Halloy, I G Knowles,

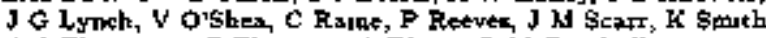

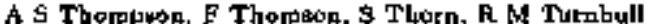

HEWELBER' U - I Boxker, \& Grwentger, o Graele, P Hanke

$\checkmark$ Happ, E E Ktuge, A Putere, B Fengech, M Schemedt,

J Sommer, H Stentel, K Titetel, S Wernet, $M$ Wubsch

IMPERIAI COLL - D Abbstito, R Betsedenck, D M Binnte,

W Cameron, P J Dornan, A Moutoutal, J Nash, G San Martw, J K Sedgbear, A M Stacay, M D W Lhams

[NNSBRJJK $\mathrm{V}$ - G Disgentort, P Girtkr, D Kvha, G Rudolph

LANCASTER U - $C$ K Bowdery, T J Brodbeck, P Colearu,

G Crewtord, A J Finch, F Foster, G Huxhes, T Sloon,

E F Whalan, M I Willam.

MAENZ U, INST FHYS - A Gallo A M Greene, K KSleinkaechs,

$G$ Quasi, B Fenk, E Rohue, H G Sandet, P yan Geromeren,

R Wanko, C Zejlntt2

MAR8EILLE, CPPM - J J Aubert, A M Bencheskb, G Benchonk, A Bonisbent, \& Buprss, D Cilvet, 1 Carr, 0 Dhneonu, F Etienne, N Koutantinudu, D Nieod, P Payre, D Rouseesu, A Sadouki, M Talby, M Thulasidas, K Trabelat

MUNIGH, MAX PLANCK INST - I Abt, R Asermann, C Bener, W Btara, I Dietl, I Dydak, \& Gonse, C Gotzhein, K Jakoto,

H Kroht, G Lutjens, G Lutz. W Mcspner, H G Moter, R Ruchter, A Rosado-Sehloeger, S Schse], R Sett]er, H Seywerd, R St Denib,

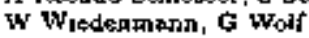

ORsAY, LAL - J Buturdet, O callot, A Condjer, M Daver,

L Drofol. J F Gyiyz, P Heusase, M Jacopet, D W KIm. F Le Diberder, J Lefrapcais, A is Luta, I Nikahe, it J Park, I C Park, $M$ H Srbmant, S Simion, J J Vellet, I V'ldesu

PISA U \& INFN, PISA \& PISA, SCUOLA NORMALE SUPERIORE - P Axzurn, G Bagliest, G Batignam, S Bettarim, C Bossh, G Calderint, M Garpinellh, M A Ciaç, V Ciulli, R. Dell'Oros, R Fautech, I Fertante, L Fos, F Forti, A Gisti, M A. Giargi, A Gregoria, F Ligabus, A L4piar, P \& Marocachapd, A 3.jeszmeo, G Ruso, G Sangubettı, A Sciabs, F spagnolo, J Stamberger, R Tenchini, G Tonelih, $\mathrm{C}$ Vannul, $P$ G Verdini, J Walsh

ROYAL HOLLOWAY - GEDFOAD COLL - A P Betcarndge. G A Blarr, E M Brysht. F Cerntti. I T Chtmbers, Y God, M G Oroen, D L Jahpean, T Mediedr, P Perrodo, J A Strong, J H von Wumersperz-Toller

RUTHEFFORD - D R Boiten]l, R W Clıft, T R Edgecock, $S$ Flaywood, P Malcy, $P$ R Norton, J C Thompens

DAPNIA, SACLAY B Elocb-DevBux, F Colas, $S$ Emery,

W Koannecter, E Ladent, M C Lemaire, E Locci, B Marx, P Perez. J Ravder. I F Ranardy, A Rouggane, J P Sehuller: I Schuriadling, A Trabetst, B Village

UC, SANTA CRUZ - R P Johnem, FY Y Kım, A M Latke. M A Melienl, G Taylor

SHEFFIELD U + A Beddall, C N Booth, $\mathrm{E}$ Borwell, $C \mathrm{~A}$ I Brew, S Cartwright, $F$ Combley, A Kakend, M Letha, W M Newton, C Rankin, J Reveve, L F Thompton

SIBGEN U - A Boltrer, S Brandt, V Buacher, G Covar. E Felgk, O Grupen, G Lutters, J Minguet-Rodrigues, F R,vera, P Sorinyt, L Gmolk, F Stephsn

TRIESTE U, IST FIS \& INFN, TRIESTE - M Aleppo

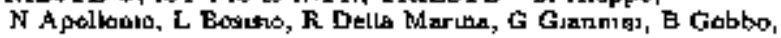
G Muroline, $F$ Rargasa

WASFTNGTON U, SEATTLE - J Rothberg, S Wagrerbaect

WISCONSIN U - S R Armstrong, L Eedlentoni, P Elmer, Z Feng. D P S Ferguton, Y S Gao, S tonzales, J Grabil, T C Greenung, J I Harton, O J Heyes, H Hu, P A McNamara, II], J M Nache. man, W Orejudos, Y B Pan, Y Gaxd, M schmett, I 1 Scott $V$ Sharms ID Turk, A M Wolth, S $\perp$ Wu, $x$ Wu, J M Yar marting, M Zhong, G Zobernig

Accilerator CERN-LEF Deteftor ALEPII

Aleactions

$e^{+} e^{-} \quad<200 \mathrm{GeV}\left(\mathrm{E}_{\mathrm{cta}}\right)$

Partactes studiud $w^{+}, W^{-}, z^{0}$, bvy-fleyor, byy-leptont, huggs, B-purticle

Brief descreption $A$ in detector designed 10 give at much det alled unformatuan as posenble abaut complex events in hugh-

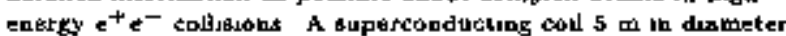
and 6 ro long products a noform 15 tegla field in the beam direction Injide the coll, is order of iscressing radivg, thete 
4t a micfistrip solyd \$late derick, an Inner Tracking Chamber (ITC) uring drift wres, a Time Projection Chamber (TPC),

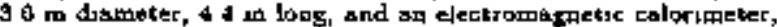
of $2 \mathrm{~mm}$ lead sheets whth proportionsl wire sampliag Otatide of tho colt, a $12 \mathrm{~m}$ thick Fo roturn patb it weed as a hadron calorimeter; and a double Jayer of dirft tubes adds in the muon identrfication Strotic posats of the detector are a precision of noomentum medsuramebtu for charged porticlex, dua to p bigh megnetre fiekd and a TPC, a good mestufertion of alactrons and muons even when they are ummeraed in jets, and a Buskis

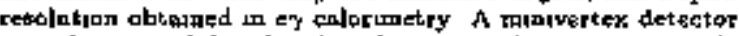
provides a cepsbullty for identifybo becondary veruces, and a

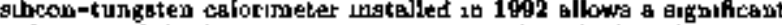
reduction of the lumenanty errar Trking dato (Jably 96)

Joyrnal popers NIM 21T (1983) 305, NIM 217 (1983) 317, NIM A225 (1084) 481, NIM A226 (1984) B2, IEEE TNS 32 (1985) EDS, NIM A22S (1985) 32T, NIM A234 \{1985) 47, NIM A23S (1085) 296, NIM A239 (1965) 192, NIM A244 (1966) 519, NIM A247 (1086) 438, NIM A251 (1986) 419, NIM A252 (1986) 982. DIIM A252 (1986) 399, NIM A252 (1956) 403, JEEE TNS 34 (1987) 133, CPC 45 (1987) 229, CPC 45 (1987) 283, OPC 45 1987) 433, NL31 A267 (1987) 697, JEEE TNS 95 (1908) 316 NFM A263 (J988) 48, NIM A2f3 (J988) 58, NIM A268 (1988) 144, NTM A271 (1988) 448, CPC 57 (1989) 401, [EEE TNS 31 (1989) 1450, IEEE TNS 36 (1989) 1164, IEEE TNS 36 (1\%89) 1514, NIMA A2T7 (2889) 358, NIM A.279 (1989) 212. NIM A283 (1989) 579, PL E23I (1989) 519, L.TMP C1 (1990) 147, IEEE TWS 37 (1900) 1210, NIM A286 (1990) EL, NIM A289 (1990)

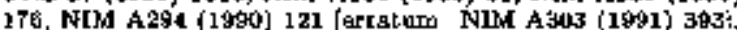
NIM A297 (1900) 153, NIM A297 (1980) 390, HEPTP 14 (1960) 986, PL B234 (1980) 209, PL B234 (1900) 389, PL B23\$ (1920) 399. PL E236 (1950) 36. PL B236 (1990) 235, PL B236 (1960) 511, PL B276 (1990) 5J1, PL B237 (1990) 291, PL B24\} (1990) 141, PL B241 (1990) 623, PL B241 (1990) 635, PL B244 (1900) 541. PL B244 (1990) 551, PL B245 (1890) 2B9, FL B246 (1990) 306, PL B250 (1090) 172, ZPHY CAS (1900) 965, NIM A306 (19日1) 446, NP (PROC SUPPL) 238 (1991) 291, PL E255 (1991) 623, PL B257 (19\$1) 474. PL B257 (1991) 492, PL B258 (J991) 236, PL B250 (1991) 377, PL B282 (J991) 139, PL B2A3 (1991) 112, PL Eas3 (1991) 325, PL B2ed (1991) d76, PL B785 (1991) 430, FL B26s (1941) 45, FL B266 (1891) 218, PE B2T (1991) 181, NIM A315 (2992) 121. NIM A320 (1992) 1'T, NIM A323 (1992) 213, PL B276 (1997) 247, PL B278 (1992) 2009, PL B279 (1902) 4l1, PL B254 (1992\} 151, PL B254 (1992) les, PL B284 (1902) 177, PL B285 (1902) 309, PL B292 (1982) 2to PL B294 (1962) 145: PL B295 (1992) 174, PL B295 (1992) 396, PL B297 (1992) 432, PL B297 (1992) 449, PL B297 (1992) 459 PRPL 2L6 (1992) 253, ZPHY C53 (1992) L ZPHY C53 (1992) 21 ZPHY CS3 (1992) 375, ZPHY C54 (1992) 75, ZPHY C54 (I992) 211, ZPHY C56 (1992) 209, PL 19290 (1999) 479, PL E303 (1993) 168, PL E307 (1693) 187, PL E307 (J943) i94, PL B307 (1903) 2009. PL B30s (1993) 425, PL B\$11 (1999) 425 [Frtalum PL R916 (1998) 631], FL Ba13 (1993) 299, PL B313 (1993) 312, PL E313 (1993) 498, PL B913 (1993) 509, FL B913

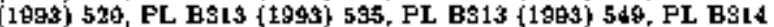
(1993) 469, ZPITY C57 (1093) 17, ZPFY C59 (1993) 216, ZPHY C59 (1998) 369, ZPHY C60 (1993) 71, IEBE TNS 41 (1994) 236, NIM A346 (1994) 661: PL B 321 (1964) 168, PL B322 (1984) 275, FL B322 (1984) 441, PL B332 (1904) 201, FL B332 (1994) 219, PL B334 (1984) 244, PL B335 (1994) 999, ZFHY 662 (1994) 1. ZPHY C62 (199d) 179, ZPHY C62 (1994) 639, ZPHY C64 (I994) 3E1, NiM A360 (jess) 4al, NF (PROC sUPPL) 39EC (1945) 322, PL B343 (1945) 444, PL B44b (1925) 103, PL B340

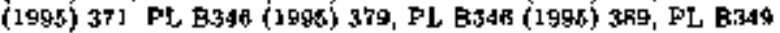
(1995) 209, PL B349 (1995) 585, PL B352 (1995) 474, PL B355 (1995) 381, PL Bas5 (1995) 595. PL B366 (1995) 409, PL B357 (19g5) 4AT (erratum PL B364 (1995) 247], PL Bx57 (1995) 655 PL B35? (1985) 699. PL B350 (1995) 235, PL B351 (1906) 221, ZPHY Co6 (199b) 3, ZPHY O66 (1900) 35, ZPHY CA9 (1996) 15, PL 8365 (1996) 437, PL. B389 (1998) 151, PL B373 (1998) 246, PL B374 (1996) 319, ZPHY C69 (1996) 1\$3, ZPHY C69 (1996) 365, ZPHY C69 (1996) 379, ZPHY C89 (1996) 393, and ZPHY CER (1996) 585

\section{E-mati cortact lugi rolsudiocern ch}

WWW Harre page bttp //alephwww cers ch/WWW/

\section{CERN-LEP-DELPHI}

(Prapused 1982, Approved Nuy 1982, Begtan data-tadeníg Ang 1969, In progress)

\section{THE DELPHI DETECTOR (DETECTOH WTTH} LEPTON PHOTON AND HADRON JDENTHFICATION)

\section{DELPHI COLLABORATION}

ANTWRFP U \& ERUSSELS U, IIHE \& MONS U - D Batekand,

C Bricman, F Cro, M Chen, A Deghoran, S De Brabaudere,

O De Clereq, $P$ Herquet, $\$$ Hoorslbeke, $K$ Huet, $V$ Lefebure,

3 Lemonoe, $A$ Towaradte, $C$ Vander Velde, $W$ K Van Dopunck.

F Verbelure, J H Wicktbs

FOWA STATE U - It B Crawley, D Edsall, A Firestone, L Gorn, T S Hotk, J W Lamkt, D WW Lonte, C K Leqan, R Mc KBy.

W T Meyor, E I Rotanters

ATHENS U - S Katsanbras, C Kourkoumelss, F Nocoladion

L K Rervans

BERGEN U - G Eigen, A G Froderen, R Keramed, A Klovaing, B stugu

BOLOGNA U a INFN, BOLOGNA - A C Benwenut1.

F R Cavallo, $F$ L Navarria, A Perrotta, T Ravell, G Valenti,

FIO QE JANEIRO, CBPF \& RLO DE JANEtRO PONT UNTV

CATOLICA \& RIO DE JANEIRO STATE U - M BegalL,

hi Gandolmen, 2 M MTundrr, M E Pol, R C shotlard, D sonzs Santos

COMENTUS U - P Cbochula, R Jank, F Kubwec, B Sitar

COLLEGE DE FEANCE - P Bellioro, J M Enatet, C Deforx, I Dolbrau, P Freukre], o Trutram

CERN - U Amald, P Antilozos, A Augugtidus, P Bullon,

$Y$ Bolokapytov, $C$ Boqrderar, $R \subset A$ Artwp, A Buyt,

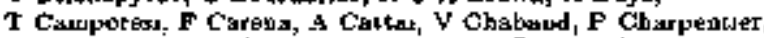

$\checkmark$ Cborow

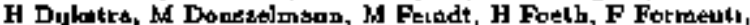

C Gaspar, P Gnvillet, F Hahn, H Herr, 13 Hilke, C Joram,

H Klew. M Korateiros, J C Marin, M Me Cubbrn.

Mi Paganami, L Pape, D Rakt, E Raber, F Strchelbaut,

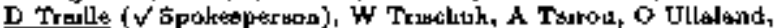

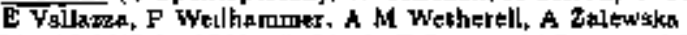

STRASBOURC, CRN $D$ Blach. F Dyams, is Dracot, J P Eagel,

P A F Fseher, D Gete, J P Getber, P Jujllot, V Nikoleenho,

P Pacea r Strub, T Todorav, STodarova, M Wister

DEMOCRJTOS NUCLEAR RESENRCH CENTER -

K Karafapoulas, E Kar uqlor, P Kokkınjax, D Loukad, A Markeu,

K Papageor kiou. E Zevgolatikor

PRAGUE, INST PHYS S Nemecek, M Navsk, J Prmes,

J Rudky, Y Vibso

GENOA U \& INFN, OENOA - M Bozza, M Canepa, C Cson,

B Contri, O Crosett, F Fontenejl, V Gracco, O Konznetsow,

N R Monge, P Moretizas, F Parod, A Petrolied, G Pians,

I Roureaglolo, of Ssnowa, $S$ Squaress

GRENOBLE U - M L Andrqux, R Barate, F Ledrat, F Naraght, L Hoos, O Sahr, $G$ Sajot

HELSINKI U - M Batteglis, R A Brenser, S Cuelisr, K Kurvinen, R Lauhokargss, F. Orays, K Osterberg, H Saxrikka

DURNA - O D Alckseev, D Y Gardin, M S Blerky, G A Chelkow B A Khomenko, W N Ktrovanski, z Krumgtaln, V Molychev, A G Oleheveks, V Poudmakoy, N Pukheeva, A Sedovgky.

Y Sedykh: A N Sunatuan, L G Tkptchev, I A Tyophin,

L s Vertogration, a S Vodopyenov, IN I Zimin

KARLSRUHE U - W D Aped, W De Boer, R Ehret, D C Fries, M Kawer, C Kreutcr, \& Machlum, B Meyer, H Mudler, W Oberschulte-Beckmann, O Podobnn, M Schimmelpiesnเg,

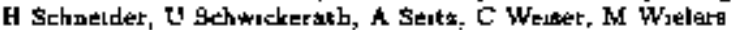

CRacOW P Brutkman, z Hayduk, P Jalochs, K Kotcy],

W Krupinsk, W Kuchwicr, T Losiak: 自 Murya, H Palks,

G Polok, K Rybuckı, M Witek

ORSAY, LAL - F Bambode, B Bouquet, J L Contretas, G Cosme. B Dalmsgne, F Fulda-Quenzar, G Grosdidzer, I Jean-Marie,

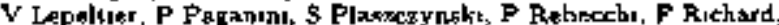
P Roudoos, A Slotebl, A Trombini

La:NCAsTER U - P N Ratod, P Sebger

LISBON, LIP - P Abreu, F Baras, M Espurito Sianto,

R Henragues, A Maio, $A$ Onafre, 1 Peralts, id Pimenta,

1 spassow, B Tame

LIVERPOOL U - P P Allport, P S L Booth, T J V Bawcock,

C Carroll, J H Cowall, A Gallom, M Gibbo, C Green

M Houldeu, J N Jackson, B King, S Marti I Garcis R Mc Nolky. I Riehardgon, 5 Tramatras 
PARIS, CURIE UNIV VI - M Baubular, P Blloir, w Da Sulva, C De la Yasestere, 8 Fucher, F Kopuate, R Pann, I P Tavernet

LUND U - S Almebed, O Barring. E Falk, V Hedberg, C Jerlokos,

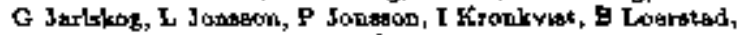
U Myoernmark, O smirnowa, to Trenttomer

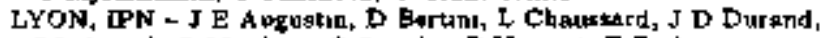

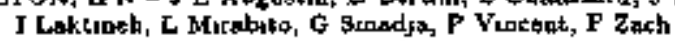

MADRID U - J A Barrio, I S AJchat

MARSEILLE, CPPM - P Delpietre, A Tilqukn

MLLAN $U$ \& INFN, MILAN - A Andreasz, M Bonean,

W Bonvento, M Cacsis, M Calv, Y tour, S Gumanyuk,

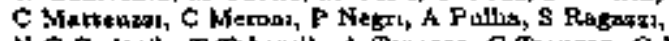

$N$ G Redaell, T Tabsuell, A Tonezzo, C Troncon, G Vegha

BOHR IN\$T - E Dabl-Jenseó, M Dam, G Damgard, N J KJaer,

F Maceller, B 9 Nieloen

CHARLES U - A Laltner

NIKHEF, AMSTERDAM - E Ag*si, E Bondinow, W Hao,

D Halthured, P Klmt, B Koene, M Merk, M Nheurenliuren, 3Y Fuckstuhl, I Siccoma, J Timmermans, D Z Toet, G W Vep Apeldorar, F Van Dam, 1 Vab Eldik

DEMOCRITOS MUCLEAR RESEARCH CENTER -

M Dre, D Fustaulotia, T A Filuppas, E Fokıtio, E N Gase, E E Eeteoufie, T D Papodopaulou, H Rahmap!

OSLO U - L Bugge, T Buran, A L Reand, T E Skaxly, S Stapneg OVIEDO $v$ - J Cuevas Meegtion

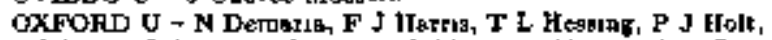

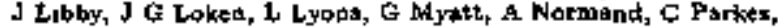

D Radojicre, $\mathbf{P}$ B Rention, A M Segat, K Steveneon, J Thomes,

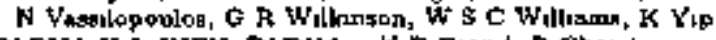

PADUA U $\boldsymbol{E}$ INFN, PADUA $-K$ B Brand, $P$ Checthia,

A De Min, U Gasparinu, A Lipntacks, 1 Luppi, M Markoul,

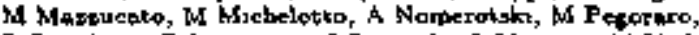

P Roncheze, F Surocnetio, I Staricake, I Ventura, M Varlato, G Zumerls

AUTHERFORD - T Adye, M I Bate, $D$ Crennell, P D Dauncy, B Franeh, G Gopal, J Guy, W J Murray, H T Fhilips,

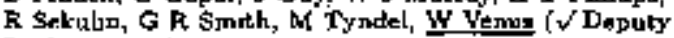
Spokesperson]

FOME U. TORVERGATA \& INFN, ROME - Y Canale,

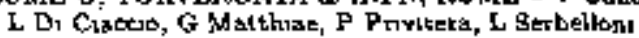
DAPNTA, SACLAY - J Bsudat, M Besoocon, T Bdogncese, G Bortsoy, C De Sesnl-Jean, P Gris, P Jarry, J P L, tugret, P Luts, A Ouraou, F Puerre, I Rupp, V Ruhlmaran-Kleider, Y Socquin, P Siegrite, R Silvestro, S Sumonetk, ML L Turluer, D Vtknows, $\mathrm{H}$ Zto

ROME, ISS \& INFN, ROME - A Baroncedl, C Вово

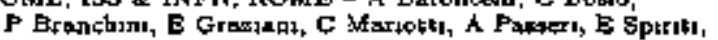
C Stomtoci: L Tortors

SANTANDER U - a J Camacho Foxas, I Garcta, I he topez, I Marco, R Marco, C Marilack-R,verd, F Matorras, A Rujz SERPUKHOV - 1 Ajinenko, M Chepkin, P Cblsopnikor, A. Fenynk, V Kontioukbion, V Lapin, V Obraxtisov, A Ortankoy M Petravyck, N Smiknov, O Trbukiley, V Uvarov, E Vlasow, A Zaikser

STEFAN INST, LJUBLJANA. L LUUBLUAA U - V CIndF,

B Erzen, B Golol, D Zarranuk, D Zontst

STOCKHOLU U - B Aвmah, K Cankocsk, G Ekspang,

F Gunnaraw s O Holmgren, K Hultquast, $\mathbf{R}$ Jacobeson,

E K Jobangson, M Karlsuon, T G M Malmeren, T Mos, P Nus,

C Walck, G C Zuç,holl

TURIN TS is TNFN, TL:RIN - F Bizmah, M Big, R Chence,

D Gamba, E Higliore, G tinauda, A Fomero, $G$ Selolla

TRIESTE U, IST F'LS L INFN, TEÜESTE \& UDINE U -

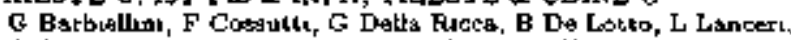

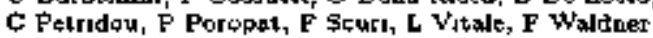

FUل DE JANEIRO U - 5 Amato, M Barbn, M Eerggran. L De Pzuls, $B$ Marecha'

UPPSAL Q U - Q Eotner, T Elzlof, M G J Medbo, K Wiosehage

VALENCLA. U - R. Alematy, 8 Cabrers, MV Gatillo Guments, E Corcine, A Ferrer, I Fubter, C Gercua, J J Hernander,

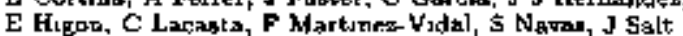

VIENNA: DAW - W Adkm, W Bart, R Frobwrth, J Hiubac, M Krammer G Ledes, D Luko, J MacNaughton, F Mardl, W A Mttaroff, N Nieutuesstar, H Petuegger, M Permieles. MA Regletr $j$ Strieusa

WARSAW, INR - K Doroba, R Gokiln, M Gorrk, K Grzekak, K Nawrich, R Scanowgki, M Szctekpwikı, M Szeptydta, P Zalewsk
wUPPERTAL $U-K$ it Bepki, he Blume, T Brenke,

T Burgsmacller, P Bpechmann, \& Daliru, a Drees, K A Eloes, W Elaing, A Grefrath, S Hahn, K Hamacher, O Klapp, P Langt -

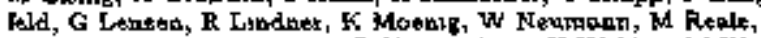
M A E Schyns, P sponholx, B Uteberrobaer, H Wahlen, MT Weper. stall, $D$ Wicke

Accerenator CERN-LEP Detcclor DELPH]

fieactions

$\mathrm{e}^{+} \mathrm{e}^{-} \quad<200 \mathrm{GeV}\left(\mathrm{E}_{\mathrm{cm}}\right)$

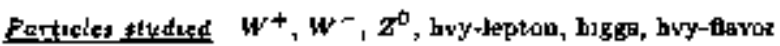

Bref dejersptson A ganeral purposo LEP detector tor physucs on and abpve the $Z^{0}$, offeturg 3-dumentional uformation on curvature and energy deposition with fine spatsal gramularity. ar well as identificition of leptons and hariant over mott of the solid angle A suptroonduceng çal provedes a 12 T solanondal fleld of bigh upiformily Tracking relues on a merovertex deteclor, an inuer detector, \& Time Projpction Chamber (TPC),

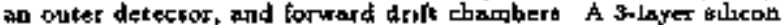
mictrovichrtex dettector allows a precision msasurement of the joteraction vortex and decry vartices of ghortalived particlas nuth an bottolr and charm badrons and + leptons Electrónaguetic ghowert are messured in the barrel whth high granuilarity by the High Denojty Prapection Chamber (HPC) ind in the eudtapt by $j^{\circ} \times 2^{\circ}$ projective towars compoied of lead glase as ab aclive naterual and phototriode readont Hadron identilication is provided manily by liquid and gas ring insiging Cerenkow

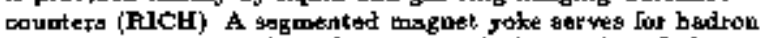
colorimetry and as a filter for mughe which are identzfled in two drift bumber lavert In sddision, gesbtillator ayatems are implazented in the boriel and for ward requgns $A$ staall angle Shashlul-type calormeter (STIC) us ubed for the lvminosity determusen Takun deta (July t6)

Jourmal popers pilM 225 (1984) it?, NIM A228 (1984) 606, NIM A296 (1986) 310, NTM A241 (1986) 429, NRM A 243 (1986) 77, NJM A243 (1980) O1, NIM A2A8 (2986) 317, NIM A252 (1966) 188, NIM A262 (1986) 113, NIM A252 (1986) 413, NIM A252 (1998) 435, NIS A353 (1986) 521, NIM A252 (1986) 573, NIM A25. (1987) 111, NTMI A256 (1967) Ho, NIM A256 (198T) 267, NIM A 257 (1967) 499, N1M A260 (1987) 124, IEEB TNS 34 (1987) 227, NIM A26s (1988) 215, NIM A265 (1988) 218, NDM A269 (1984) 652, NTM A270 (1986) 393, NIM A273 (1983) 053 NIM A275 (1989) 565, NIM A27s (1988) 8d1, NFN A273 (1988) BA 7 , IEEE TNS \$6 (1959) \$90, NIN A275 (1989) 49, NIM A.77 (1969) 154, NIM A277 (1989) 160, NMW A27T (1999) 338, NTMI A27T (Ins9) 347, NIM A279 (1989) 17S, NIM A27b [1989) 51k, NLM A26s (1989) 502, NIM A253 (1680) 867, NLM A283 (1989) T92, PL B23L (1989) 539, NIM A2a9 (1990) 400, NTM

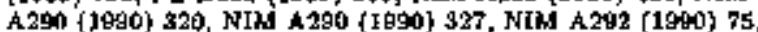
NIM A202 (1990) 359, NIM A202 (1990) BE1, NIM A294 (1000) 424, PL B240 (1990) 2T], PL B24l [1900) 425, FL B24l (1990) 455, PL B2d1 (1990) 449, PL B242 (1890) 536, PL B245 (1990) 275, PL B247 (1990) L37, PL B247 (1900) 14E, PL B24T (1990) $15 \%$, PL B247 (190) 167, PL E262 (1\%50) 140, PL B252 (1930) 149, NP B342 (1900) J, IEEE TNS 38 (19\%) 8Bl, N1M A303

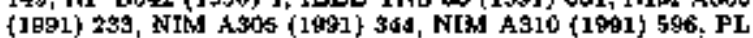
B255 (1991) 464, PL E260 (1091) 340, FL B267 (1991) \$22, PL B26 (199t) 296. NP B\$67 (1991\} 611, ZPHY C50 (1991) 185 ZPHY CE1 (199h) 26, ZPHY QS2 \{1991\} 271. IEEE TNS 39 (1992) Fes. NIM A3I5 (1992) 163, NIM A\$15 (1962) 393, NIM A325 (1492) 209. NIM A923 (1992) 351. NIM A323 (1992) 3653, PL B274 (1992) 230, PL B274 (1992) 499, PL B2TL (1992) 227, PL B275 (1902) 231, PL B276 (1992) 24?, PL B276 (1992) 254, PL B275 (1992) 636. PL B277 (1992) 371, PL B281 (1992) 383

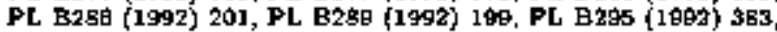
PL B296 (1992) 236, PL B206 (1992) 247, NP B373 (1992) s, NP B368 (1992) 471, ZPHY Cא3 (1992) 41, ZPHY CE\$ (1992)

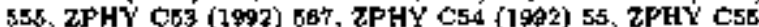
(1962) 565, ZPHY C5B (1962) 4T, ZPHY CS6 (1902) 63, NIMI A328 (1903) 147, NIM A339 (1993) 284, PL B301 (1993) 145, PL B302 (1993) 358, PL B307 (1993) 157 PL E307 (1903) 221,

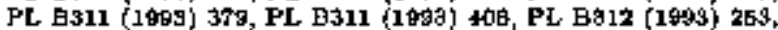
PL B316 (1993) 620, PL B318 (1998) 249, NP B403 (1993) $\theta_{1}$ ZPHY Cs7 (1993) 1B1, 2PHY C59 (1993) 21: ZPHY C59 (1993) 357, 2PHY Csg (1093) 533 lertotum ZPHY C的 (1945) 709], PL B322 (1904) 45 , PL B323 (1994) 242, PL BS24 (1994) 500 , PL B327 (1004) 386, PL B332 (109d) 488, PL B33山 (190\$) 435, PL E338 (1994) 406, PL B34l (1994) 109, NP B417 (1t94) s 


\section{SUMMARIES OF CERN EXPERIMENTS}

[ertakum NP Be26 [1994) 24d], NP Bd1s [1964] 103, NP B421 (1994) 8, ZPHY C61 (2994) 407, ZPFT C62 (1994) 357, ZPFY Ces (I994) 3, ZPHY C63 (1994) 17, ZPHY C64 (1994) 183, NFM A362 (1995) 361, PL B342 (1985) 402, PL B345 (1995) 698, PL B34J (1695) 447, PL B355 (1995) 4) 6, PL B3557 (1995) 255, PL B357 (I995) 715, PL B359 (1995) 411, PL B361 (1965) 207, NP B444 (1925) 3, ZPHY Ces (1995) 555, ZPHY Ces (1965) 569, ZPHY C6S (1996) 58T, ZPEY C65 (1995) 603, ZPHY C66 (1905) 323, 2PHY C66 (1995) 34F, ZPHY CE7 (1995) 1, ZPHY Car (1965) 64, ZPHY C67 (1995) 189, zPHY C67 (1995) 549 , ZPHY DEs (1965) 13, ZPHY C68 (1995) 353, ZPHY CB (1955) 363, ZPHY C68 (1995) 375, ZPHY C68 (1905) 541, ZPHY C69 (2965) I, NIM A368 (J日90) 314, PL B365 (1908) 448, PL 6372 (1996) 172, ZPHY C69 (1906) 223, ZPHY C64 (1096) 561. ZPHY C69 (1905) 675, ZPHY C70 (1906) 179, and ZPHY Cro (1906) 371

E-mat cortact damel tresletarar th WWW Home-page hotp / / delinfo cern th/Delph/Wolcome htwl

\section{CERN-IEP-L3}

(Proposed 2982, Approved Nov 1989, Eegar dath-takng Aug 2989, in progress)

\section{LS EXPERIMENT}

\section{LS COLLARORATION}

AACHEN, TECH HOCHSCH, $I$ PHYS INST $\&$ AACFEN, TECH ILOCHSCH, III PIIYS INST - R Botk, A Bohm, $\mathbf{H}$ Fezefeldt, K Hangerter, B Hartmonn, W Kreme, K Lubelameyer, S tifuller, A Nuppe, D Pandoulne, C Paut, Y J Pei, S Rohther, 5 Ratb.

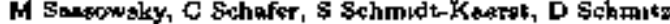

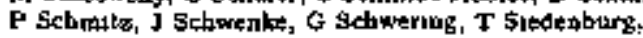

A Straesgner, M Tonutel, M van der Mey, W Wallraft, A Weber, S Wybboff, $\boldsymbol{Y}$ zens

NIKHEF, AMSTERDAM - $9 \mathrm{~J}$ Bobbirk, A P Colyn, P Dunker,

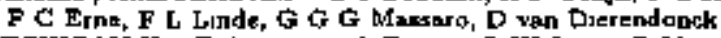

MICHIGAN U - T Aromoan, A Button, $L$ W Jonel, $R$ Moore, K Hudr, B P Roe

ANNECY - O Corliss, X Chereall, G Cougnet, A Degre,

D Ducheanesn, Y Kacyotakib, D Perret-Gedlux, S Roerer-Later, M Vivargent

JOHNS HôkINS U - G Y Chren, A Gauga, G Hu, D Kum, A Pergicr

BASEL $\downarrow-L$ Touscher, $M$ Wadluwa

LOUIStANA STATE U - R R McNal

BEIJLFG, HHEP - G Chen, G M Chen, it \& Chen, B N Jim, 2 A Lu, $\gamma \$$ Lu, $X$ W Taut, $K$ L Tung, $O$ G Yang, $X Y$ Yso, Z Zhang, O Y Zho

HUMBOLDT U, BERLIN - M W Gruentwald, T Hebbeker, S Petrak

BOLOGNA U \& LNFN, BOLOGNA - F Anualma, D Abtreajyad,

M Bsule, o Cara Rameo, F Cindala, D Hotrifatiador

TATA TNST - T Aziz, S Bantrjee, S Dutte, S N Gengull, A Glato, M Marty, G Majumdtr, 8 Mancla, K Masumedar, R Raghsver, s Sarker, K Sudhakar, S C Tonwar

DOSTON U - S Ablen, J Goldatern, A Marın, J Xu, B Zhou

NORTHEASTERN U - G Alverson, \& 5 Gau, T Paol, S Rewcroft, L Taylor

BUCHAREST U - T Aneglegen, F Cotoroba, N Gheordemegcn, A Mihal

BUDAPEST, CRIP - A Collung, D Kis, E Nagy, J Toth, o veszlergomb

HARVARD U - K Strateh

MIT - U Beckes, I Bergea, E Epambills, I D Burger, X O C.44, M Capell, I Gare, II Clare, $T$ S Du, F de Jong: F J Eppling,

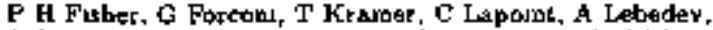

D Luckey, S Nabn, H Postarmin, J Rodın, B Sm1h. W Steuer,

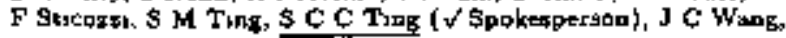
$Y$ F biag

FLORENCE U \& INFN, FLORENCB - O Adnami, F Becattınt,

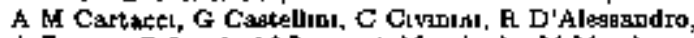
\& Favara, $G$ Landr, $M$ Ianti, A Maccbjoto, M1 Mexchini,

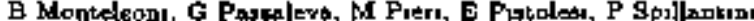
CERN - J Allaby, R Garillere, B Bertuch, M Bragim, J J Blasing, A Herve, V Innovente, J Kurkby, M Lebesu, $P$ Leeod,

J M Le Coff, D Merially, J Mned, D Peach, J A Rabio,

J Solrelo, U Uwer, F Wittyenstein, A Zucbich

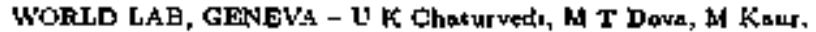
R A Khan, G Sultenow, J D Swain, S X Wu

GENEVA U - G Ambtor, M Bantgurn, W J Burget, C Cecchi, $P$ Extermann, I H Field, L Fred, H Boor an, is N KieneleFocacti, F Muhtera, N Prodult, D Sciarnno, G F Susinpo

HEFEI, CUST - H F Chen, $Z \mathrm{~F}$ Gong, $C \mathrm{~L}_{\mathrm{I}}$, W G Ma, $\mathrm{L}$ S Sn,

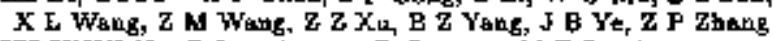
HELSINKI U - P Lannkajnen, R Datonen, MS E Saratsinos

LAUSANNE ti - G Alemenn, A Bay, S Goldfatb, A Kasser, $Y M_{1}, P$ Rusielet

LECCE U - F Cesaton!

LOS ALAMOS - J 5 Kapustunsky, W W Kunnon, J Shukds

LYON, IPN - M Cbemarin, H EI Mamouni, J P Ernenwein, J Fay. P Lebord, J P Martın

MADRID, GIEMAT - M Agulsr-Beniloz, J Alcarae, $J$ Bordugo, J Cessau, M Cerrade, M Ghammeo, N Colino, B de la Crus,

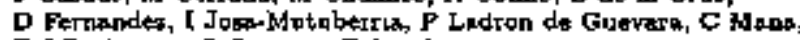
F I Rodrigutes, L Roumero, E Sapchex

MILAN U \& TNFN, MILAN - M ACciarm, A Baschirot to, R Cegteito, C Furatte, \& Fensotti, P G Rancouta, M RetteggI MOSCOW, ITEP - A Arefior, Y Galaktionow, A Klomentor,

I Korolks, V Koutrenko, A Kunip, A Maluner, V Plyankin,

Y Pojtdasv, Y 8koutko, E Shnoslow, I Vetlutaky, I Vorobsev

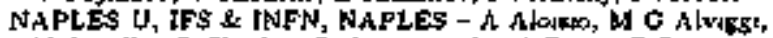

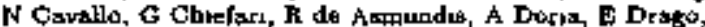

L Lesta, 5 Male, L Merols, M Naproltajo, P Psoluch,

s Patricelli, D Piccolo, e sciboce

CYPRUS U - A Hasan, P Raze, A Vorvolakos

NIKHEF, NIJMEGEN \& NIJNAEGEN U - A Buytenhul,

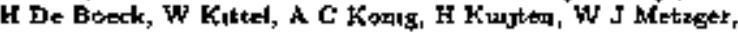

D J Schrotanus, F T Vau de Walle, W C van Houk,

A J $W$ var $\$$

OAK ATDGE - H O Cohn, Y Efremenko, Y Kamythloor, K Read

CAL TEDIt - G Gratto, A Kurkby, D Kirkby, W Lu, R Afount,

B Newman, 5 Shevebenko, A Shvorob, R Y Zhu

PERUGIA U \& TNFN, PERIGIA - B Alpat, P Bertalın,

R Battuglon, G M Bites, M Caris, 5 Easo, E Fisandra, M Penduzar, A Santocchie, L Servol

CARNEGE MELLON U - 9 C Blyth, I C Brock, A Engler.

T Ferguon, $\vec{F}$ Filthaut, $R$ W Kratmex, H K Park, $J$ \& Pinto,

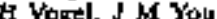

PRINCETON U - P Denes, V K Gupta, P A Piraue,

D P \$tuckland, H Store, C Tully

ROME U kr INFN, ROM1E - P Bagnato, L Batone, B Borgas, F Gavallax1, S castedtini, F DeNotaristefaal, M Diemox,

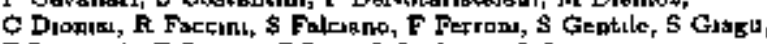
E Leonardi, E Longo, C Luci, L Ludovici, L Leminari,

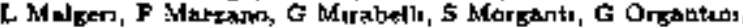

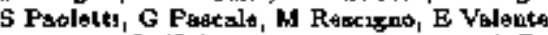

ST PETERSBU AG, INP - V P Andres, O Fedin, F tevtcbeako

D Prokofity, V Sebegelaky. A A Vorobyov. A Zalite, Y Zalite SALERNO U - L Giarell

UC, SAlS DIEGO - I G Hranson, A Domingubz, G kaven SANTIAGO DE COMPOSTELA U - I DUR

SOFTYA, AUTOMATION SCI INSTRUM LAB - N ShIYFTOY,

B Sloysmor

KOREA INST BCL, TAEJON - M T ChOl, 3 K KIm, $S$ C Kum,

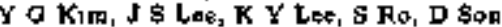

ALABAhA U - L Baksy, J Bnsenta, D DiBitonto, H Tuchscherex

UTREOHT U - A BnIf, T Yan Rhor, W van Rapanm

PURDUE U - K Banco, I J Gutay, 自 C fuemers

PSI, VLLLIGEN - K Deitera, M Fabre, W Lustermann

DESY.IFH, 2EUTHEN - P Kapina, R. Lesote, E Lueb,

W Lohmsen, $B$ Nowsk, S Rtemann, B Schoutuench, A Sopezak, $F$ Tonisch, It Vort, R Volkert

ZUAICH, ETH - H Anderhub, F Behoer, B L Betev, A Biland, D Boumlkov, Y Eugljevic, M Gampanetk, F Di Lodovica, M Duttmar, M Feicint, K Freudenreich, H Hafer, K Latsila-

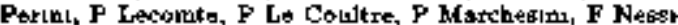
Tedaldr, F Paus?, M Pobl, G RabarCallot, D Ren, A Robolion, H Fylaczewski, N Scholz, H Suter, J Ulbencht, G Viertel, P Zemp

HAMBURG U - H Schopper

TAIWAN, HEP GROUP - A Cban, Y H Chand, A Chen,

SR Hord, W T Lin, I C Stan, SC Yel

Acteleretor CERN-LEP Delecior L3

Regations

$e^{+} e^{-} \quad<300 \mathrm{GeV}$ (E更) 
Partectes atudicd $z^{0}, w^{ \pm}$, \}, quark, gluon, loptant, hvy-lepton, husqu, eparticle

Erref description The detector conssits of a high-voluma low

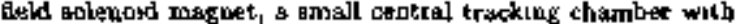
very bigb spotial retolution, a bigb-retoldoton tectromagnatic calormeter enceppulating the central detector, a hadron calanmeter actipg alao as a mpon filter, and high-precinad muon Iracking chandbers The detector is designed to mensure entrgy and prostion of leptons with the hicheat obtsubable prectaron allowngg a maka resolution $\Delta m / m$ smallex than $2 \%$ in dilepton

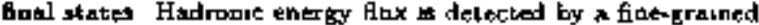
colorimeter, which slso Berves as a muov filter and a trackeng device The outer boondary of the detecror is given by the iran return-yoke of a conventiposl maknet The fifld $\mathrm{s}$ (1) $5 \mathrm{~T}$ Oyet a leagth of 12 m The maon morrentum meajoremeat performed by three oets of deft chsmbers in the central delector refion A forrard-backward muon detection aystem extends the polar adgle cowerage to 22 degrees in the forwaed region Redislly imparda is a combined hedron colórimeter and mupr absorbes The electromagnetic sDergy flow is determened by approximetely 11000 crystats of BGO Full electromegnetic

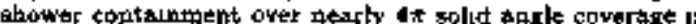
schreved Surrounding the $10 \mathrm{~cm}$ diatneter beam pipe, a high.

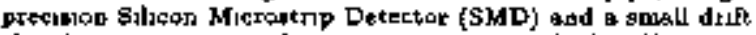

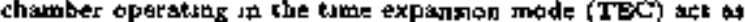
charged perticles vertex detectors Taking dats (July 96)

Jeurnal papers NIM 214 (1293) \$25, NIM A226 (1984) 498, NIM A228 (1985) 294, NIM A235 (1985) 464, NIM A251 (J986) 258 NDM A252 (1086) 304, CPC 45 (1097) 391, NIM A253 (1886) 15, NLM A254 (1887) 535, NTH A256 (1987) 28, NIM A257 (J987) 125, N1M A257 (1987) E28, HEPNP 12 (1987) 587, NIM A256 (1987) 68, NIM A263 (1988) J4, NLM A263 (1988) 343, NIM A265 (1988) 60, NFM A265 (1988) 252, NIM A270 (1888) 397, NIM A272 (1988) 713, NIM A273 (1988) \$71, NIM A273 (1988) 91\$, NIM A271 (1689) 113, NIM A275 (18B9) 71, NIM A275 (1989) 8), NIA A27T (L969) ]87, NIM A27S (1099) 699, NIM A279 (1989) 671, NLM A280 (1984) 25, N/S A283 (1989) 799, NIM А.295 (1959) 403, PL E23k (1989) 509, PL B233 (1989) 590, MPL A (1990) 35, NIM A289 (1906) J03, NIM A289 [1990) 335, NIM A200 (1990) 114, PL B23e (1990) 109, PL B237 (1990) 136, PL B23B (1990) 122, PL B241 (1990) 1]E, PL B247 (1980) 1T7, PL B247 (1900) 473, PL B248 (1990) 203, PL B248 (1900) 227, PL B24\& (1900) 464, PL B24 (1890) \$41, PL B250 (1990) 183, PL Ba50 (1900) 190, PL B260 (1990) 205, PL B251 (1900) 311, PL B251 (1990) 32), PL B252 (1990) 511, PL B242 (1990) 518, PL B252 (1980) 625, PL B252 (1990) 70S, PL B252 (1990) 713, NIM A302 (1991) 53, NIM A306 (1990) 150, NLM A309 (1991) S1B, FL B257 (1991) 450, PL B257 (199) 469, PL B259 (1901) 199, PL B261 (1991) 169, PL B281 (1891) 177. PL B242 (1961) 155, PL B2E3 (18日1) 651, PL E265 (1991) 45I. PL B270 (1991) IIL, PL B271 (1901) 463. PL B2T1 (1901) 461, 2PHY C51 (1991) 179, PL B275 (1997) 209، PL B276 (1902) 247, PL B283 (1902) 454. FL B286 (1992) 171, ['1 B256 (1962) 403, PL B28B (1909) 395, FL B2BS (1992) 404, PL B288 (1992) 4]2, PL B2962 (1992) AS4, PL B262 (1992) 469, PL B202 (1982) 472, PL ER94 (1902) 447, PL B204 (1902) 466, PL P245 (1982) 337, PL B295 (1902) 371, PL B297 (1992) 469, ZPHY CS6 (1992) 39, PL B301 (1993) 136, PL B 303 (1993) 391. PL E306 (19日3) 187, PL B307 (1993) 18\%, PL E3ar (1993) 23T, PE B300 (1993) 461, PL E313 (1993) 326, FL B315 (193ㄱ) 494, PL Ba1s (1993) 427, PL B317 (1999) 467, PL E317 (1989) 474, PL E317 (1998) 637, PL B318 (1999) 675. PEPL 236 (1993) 1, ZPHY C57 (1903) 355, NTM A340 (1994) 396, NITS A343 (1994) 456, NHM A344 (J994) 135, NIM A344 (1994) 212, NMM A344 (1994) 521, NMM AJ48 (1994) d31. NTM A348 (r994) \$S6, NTA A351 (1994) 300, IEEE TNS 41 (1994) 772, PL B331 (1994) 283, PL B338 (19日d) 273, PL B332 (1994) 201. PL B335 (1984) 542, PL B311 (1991) 245. ZPHY C62 (1964) 551 , PL B345 (1895) 7t, PL 1345 (1995) 83, PL 9345 (1905) 689, PL B945 (1986) 909, PL B346 (1955) 190, PL B.550 (1995) 209, PL B35] (1995) 376, PL B362 (1995) 48T, PL B353 (1985) 136, PL B353 (1995) 145, PL 1363 (1995) 118, PL B383 (1995) 12T, PL E353 (1995) 137, PL B370 (1996) 195, PL B370 (1996) 21L, PL B371 (1996) 126, PL B371 (1996) 137, and Pt B374 (1996) 391

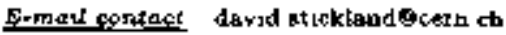

WWW Hotre-page htip //holstoliz cern ch/

\section{CEAN-LEP-OPAL}

(Proposed 1982, Approved Noy 1982, Began datataking Aug 1989, [n progress)

\section{THE OPAL DETECTOR (AN ONNI FURPOSE} APPAFATUS FOF LEP)

\section{OPAL COLLABORATTON}

AACHEN, TECH HOCHSCH, IIJ PHTS INST - J Besthtluft,

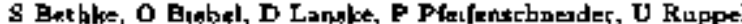
ALBEtitA U - D Gingrich, J Kewlett, J McDunald, S Muliın, J Plufold, H Przyeseznlak, N Rodnugg, P Routenburg, J Schospman, W Springtr

EIPMINGIIAM U - A N Eell, I I Bloodworth, J Blopmer, D G Charlton, S I Hullier, R J Homer, M Jimack, P Jovanovic, T I Mc Mabon, \& W O'Nasla, M Pestce, D Red, D Rugby, S D Talbot, P A Watking, A T Watton, J A Witgon

BOLOGNA U \& INFN, BOLOGNA - S Arcell, P Caprluppi, M Cuffiant, o M Daliavalle, F Fabbri, M Ferrert, M Fierro, G Giaconnell, E Graconelt, C Granda, J Ler16, \$ Marcellın, A Muchelin, A Montsnent, F Odorkel, B Pol, A M Roses, C. Sharra, G P Suralı

BONN U - R Burtoldus, K Detich, A Byrunz, H M Fustorer, C Gerch-Gimbel, S Grose, C Hartmann, T P Kolrott, P Maettig. C Mrarkus, S Menke, if o cureller, i Nelleq, A Parthaus, F Scharf, P Scluetz, M Sclowmacher, A Stabl, B Stockhsutenen, $E$ yon Toerns, N Wernes

BUDAPEST, CRLP - C Hadju, D Horvath, G Paxtor

CAMBRiDGE U - N Atuelamp, 3 R Batley, J R Certer.

$V$ Gibean, M J Goodnck, CM Hawkes, JC Hill,

D E Hulcherolk, C R Jonts, R Shaw, C P Ward, D R Ward, s wolton

CARLETON U - R K CArmegle, C Chartegworth, P E Eatabrook, R J Hemlugway, M Joues, D Karlen, D Koetke, P Krieger. 5 Toww $P$ therber

CERN - E Barberio, T Bebuke, $\mathbf{S}$ Bantroleen, Y Blobel, If I Burakthark, C Burgard, L Del Pozo, A Puertjes, Gleanng, M Gruets, M Guttor, $R$ Hammetolrum, Mi Hanaroul,

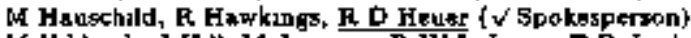
M Haldrekb, $J$ Fill, $M$ Jimente, F W Jone, $T$ R Jutak,

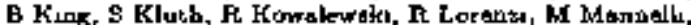

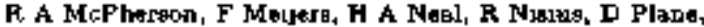

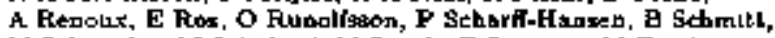

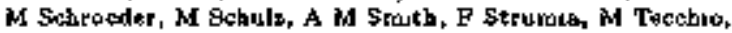
M Thombon, M Turnar-Wation, P Weth, K Zankel, M Znoy CHICAGO U - K J Anderson, R. L Armalroug, 7 Evans,

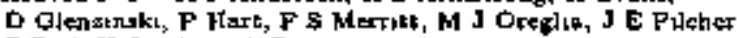
E Pod, H Sandete, A Tuteot

DEBRECEN, INST NUCL FES - J Pollonks

DUKE U - \$ Lavlenschlager, $A$ tac

FREIEUAG $U-P$ Berlach, M Bobıntkı, A Buerked, G Herten,

T Hulge, D Joos, M Kobel, F Kolpin, M Kolrep, J Ludwis. W Mohe, I Patt, H Roeser, K Rumge, KS Sachs, H SchulteConlon, S Sactdner-Rembold, M Thierger, M Vorzoçchi, H C Weber, B Wilkens

TECHNION - S Dodo, J Goldberg, D Lamac, N L upd, Y Roser, S Tarem

DESY \& HAMBURG Uf - J Meyer, S Petzold, C H shepherd. Themistocleolut, A Sittler, J Gletierder, A Whagar, G Wilton AEIDELBERO V, IHEP - P Bock, A M Bosh, P Fath, P IgoKemened, U Jont, T Kren, A Schmits, V Soergal, M Steiert, P Utzac, J vou Krogh, P vod Walter

INDIANA U - S Anderson, B Bouwens, 5 Bra1bsnt, $S$ De Jong, E do Couts e Suva, F Fuedler, G Hannou, H O Ogren, D R Rutt. M Starks, R var Kooton

KOBE v - K Kawagpe, $\mathrm{H}$ Takedn

INIVERSITY COLL, LONDON - B E Anderson, D I Attree, A Charalsmbous, P E L Clarke, J E Contey, R Granficld, M Cresswell, D A Hrayes, J w Lauber, C Lewn, E A Xackigury, D J Muller, T Rooke, P Sharword, A Sktlmen, I I Ward BRUNEL U - K Ametewee, P Habsen: D C Imaie, W Matihews, R Rylleo

QUEEN MAFY - WRSTFIELD COLL - 0 A Beck, A A Garter, MD Evans, W R Gibson, M Hapke, $P$ Kyberd, \& L Lloyd A J Martio, A I McNab, J Morrs, T W Pritcherd, S A Roburs MANCAESTER U - J Allron, $R$ J Barlow, O Beeston,

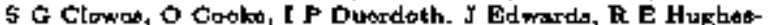
Jones, G D Lafferty, F K Lonbinger, B Nubar, N J Oldersbary, J Pater, P D Pbilips, K Stephens, E Vokurka T R Wyall 
MARYLAND U - A Bell, R Bard, C Y Chang, C Dallapacenom D Fong, M Fouther, $S$ Gascon-Shotkin, A Jawsthery, R G Kellags, F Lohnarm, G Loug. G Bartwaz, A Skgja, G A Snow

MONTREat 11 - G Azuelos, G Besudoin, Mesulseu, s Doucet. J Guteon, if Jeremie, A Joly, E Lolobure, J.P Martin, A Trifrout, P Taras, P V leag, V Zecelk

MONICH U - M Boutemenr, O Duckeck, A D Schale, O Schate OREOON $v$ - W-P LM, D Strom

CRPF, OTTAWA - MS Dixit, I Gagnan, C K Hararove, M J Lonty, H Ma, P G Oakhinn, N K Wetaon

UC, RIVErisIDE - D Chrwman! S L Chu, J W Gary, P Glacomel], W Gorn, J G Layter, P Sehent, A C'shen

RUTHERFORD - K W Bell, R M Brown, N I Geddes, T Gerglrs, F K Jacab, P W Jafirzys, B W Kenaedy, i N Patrick. W G Scoth, M Sprototon, P Stymaviaks

DAPNLA, SACLAY - A Gaidot, F-X Gantit H Lofolux, A Malik, G Vasser

TEL AVIV U - G Alexander, G Bellm, I Cohen, E Sarkisyan, E Taur

TOKYO U S Asxa, K Ishu, J Kengahu, T Kawamoto, T Kobayash, S Komnuyye, T Mashrwo, T Morl, M Mort

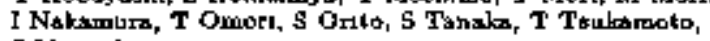
S Yamashisa

BRITISH COLUMBIA U - D A AXen, R Howard, J Kutk, J MiKtona

VIOTORIA U - D Destrich, A Honms, E. K Ketler, I Lawsion, P Poftenberger, S Robertson, is J Foney, M Robvicls, T J Smuth, R Sobne, M Vinetet, J Whate

WEIZNANN LNST - E Duehorn, R. Fulmsu, E Gross, D Lellouch, L Levinun, G Mtkepbere, R Mir, K NgRal, M Shpa, G WoE, G Yokutiels

Accelerver CERN-LEP Detector OPAL

Eecantions

$$
e^{+} e^{-} \quad<200 \mathrm{GeV}\left(\mathrm{E}_{\mathrm{imm}}\right)
$$

Parictes studted $Z^{0}, W^{+}, W^{-}, T, T$, hsy-llovor, glton, begg, s-particle

Brtef descraptron OPAL is a general purpose detectar debobed to study a wide rakge of unexplorod phyoscis at LEP Among the central pbysica lsures are the ntudy of the $Z^{0}$ and $W^{ \pm}$bosons (e g, the deterinanatjon of ther exact maeses and widtht, sad couplings to leptons and qusiks\}, the physecs of heavy flavora (smch th the spectroscopy of $d$ quarks and the Aetermination of the muxing and lifet mes of $B$ states), and yanols QCD topics A geasal search for new particles, in particnlar the Huggs berart, ss being made The mash componeats of the apparatus, in ordar of mcreseing dietanee from the interaction pount, are a slicor mugrevertex detector, centra] deterters conasut of $n$ vertex and a jet chnmber, and $\mathrm{a}$ barrel of $\mathrm{Z}$ chambers, a warm conductor nolepond providing a uniform magnete field of 0 \& $\mathrm{T}$, a TOF stuulilater barrel detector complimented by a scintulating tile endcap detector, \& it lead gless alectromagnatic calorimater, a hadron cqlonmeter tastrumented by gtreatrer

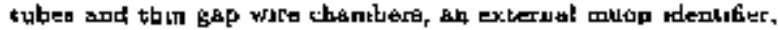
and a forwerd deteckor which includer a arw sroall-sngle siliconturgsten calorimeter Taking datz (July

Jaurnat popprs NHM A23E (1985) 234, JEEE TNS 32 (1995) 736, NIM A242 (1998) 247, NIM A244 (1968) 416, NDS A250 (1996) 603. NHG A252 (1980) 331, NIM A252 (1968) 511, EEEE TNS 34 (1985) 240, CPC \&7 (1987) 55, NiM A260 (1987) 139, NTM A26D (1987) 328, NIM A266 (1988) 11, NMM A265 (1986) 445, [EEE

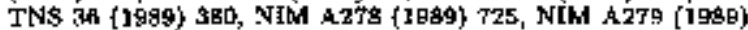
238, NIM A27 (1986) 523, NDM A283 (1989) d92, NLM A2ES (1989) 515, NIHI A2B3 (1989) 650 PL B23] (1989) B30, JEEE TNS 37 (1990) 1584, NEM \&28E (]990) 99, NEN A28B (1990) 107, NLM A286 (1990) 117, NIM A290 (1990) 76, NIM A293 (1990) 145, NIHI A294 (1990) 431, FL B235 (1990) 379, PL B235 (1899) 389, PL B236 (1900) 224, FL B236 (1990) 36d, PL B240 (1990) 250, PL B240 (1990) 261. PL B240 (2990) 497, PL B241 (1990) 133, PL B242 (1990) 298, PL B244 (1990) 135, FL B246 (10日0) 285, PL B2d7 (1990) 448, PL B267 (1990) d58, PL B247 (1990) 617, PL B24a (1990) 211, PL B261 (1920) 211, PL B252 (1990) 159, PL B252 (1990) 290, ZPHY C47 (1990) 505. NIM A302 (1991) 434, NIM ASOS (1991) 275, NIM A31O \{1991) 527. PL 日253 (1991) 611, PL B254 (1991) 298, PL E267 (1981) B31, PL B261 (1991) 334, PL B262 (1991) 341, PL B262 (1991) 351, PL B263 (1991) 123, PL B263 (1991) 311, PL B264 (1991)
219, PL B264 (1991) 467, PL E\%65 (1991) 462, PL Bytse (1991) 201. PL B266 (1691) 485, PL B26T (1991) 143. PL B268 (1991) 122, PL B273 (1991) \$38, PL 1273 (1991) 356 , ZPHY C49 (1991) 1, ZPHY CAg (198]) 49, ZPHY CA9 (198]) 375, ZPHY C50 (1993) 373, ZPHY C52 (1991) 175, ZPHY C52 (1991) 54\$, NIM A313 (1992) 103, NIM A214 (1992) 74, NIM A317 (1992) 4. KIM A320 (1892) 183, NIM A323 (1982) 169, PL E274 (1992) 513, PL E276 (1092) 247, PL B276 (1992) s79, PL B27e (1902) E47, PL E274 (1992) 485, PL B281 (1992) 994, PL B281 (1992) 405, PL 1287 (1992) 399. FL B287 (1992) 401, PL B288 (1992) 373, PL B791 (1992) 503, PL B294 (1992) 438, PL B295 (1992) 3A7, PL B295 (1992) 357, ZPIIY CSS (1992) 539, ZPHY CS4 (1902) 193, ZPFY CS6 (1902) 1, ZPHY C55 (1992) 19], ZPHY O68 (1992) 521, NIM1 A321 (1903) 34, NIM A325 (1893) 129, NIM As25 (1993) 271, NIM A325 (j893) 494, NIM A33a (1993) 330, PL B298 (1903) 456. PL B302 (1993) 523, PL E305 (1903) 407, PL P305 (1999) 416, PL B307 (1993) \$87, PL B307 (1933) 247, PL D311 (L993) 39t, PL EsL2 (1993) 501, PL B313 (1993) 343, PL B31日 (1903) 615, 2PHY C58 (1993) 207, ZPHY C5B (1893) 219, ZPHY C5\& (1093) 387. ZPHY C5d (L9e3) 409, ZPKY CEs (J993) 523, 2PHY C59 (L998) L, ZPFY CHO (1993) 1\%, ZPHY C60 (J日4s) 19, ZPFY C6t) (1998) 199, 2PHY C60 (1693) 217, ZPHY C80 (1993) 397, ZPHY C60 (1993) 579, ZPIIY C6O (1983) 593, ZPHY CE0 (1993) 601, NLM A348 (1944) 409, NIM AS48 (1904) 421, NIM A348 (1994) 424, PL Ba20 (1994) 4IT, PL B327 (1994) 397, PL B327 (1994) 41 I, PL B 328 (1994) 20T, PL B338 (1994) 585, PL B35T (1994) 198, PL Bs37 (199d) 207, PL Bs37 (1964) 393, PL B358 (1894) 497, FL B3a9 (1994) 273, NC 107A (1994) 2007, NC 107A (1994) 2066, ZPFY CBI (1994) 18, ZPHY CQ1 (1994) 189, ZPHY C6I (1994) 209, ZPHY C61 (1994) 367, ZPHY CB3 (1994) 181, ZPHY C63 (1994) 197 , ZPHY CE3 (1994) 363, 2PHY CEA (1994) 1, FL Bs50 (1925) 273, PL B352 (1995) 176, PL B355 (1925) 102, PL B359 (1995) 598, PL E358 (1995) 182, PL B364 (1995) 93, ZPHY Cés (1995) 1, ZPHY Ces (1995) 17, ZPHY C65 (1295) 3), ZPHY CBS (1996) 47, 2PHY CES (1895) 183, ZPIIY CB5 (1995) 367, 2PHY CB6 (1995) 19, 2PHY C68 (1995) 31, 2PHY CEA (1995) 543. ZPFY CE6 (1995) 565, 2PHY C67 (1995) 16. ZPHY C67 (1965) 27, 2PHYY CEF (1925) 46, ZPHY C67 (1985) ET, ZFHY CeT (1995) 203, ZFHY CeT (J905) 305, 2FHY Cब7 (1965) 379, ZPFY C87 (1995) 389, ZPHY C67 (1995) 555, ZPHY Ce6 (1995) 1, ZPHY C68 (1055) 17\%, ZPHY C68 (1995) 20S ZPFY CES (1995) 51日, ZPHY CA8 (L995) 531 ZPHY C6.5 (1995) 555. PL B368 (1996) 244, PL B369 (1996) 183, PL B370 (I906) 174, PL B370 (1\%96) 196, PL B374 (1990) 341, PL B376 (1996) 232, PL Bs7a (1996) 315, ZPHY CA9 (1996) 195, ZPHY C69 (1966) 543, ZPHY C70 (1996) 197, ZPHY C70 (2906) 357. and ZPHY C71 (1908) 1

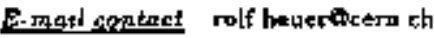

WWW Home-page http //wrowl cern ch/Opalf

\section{CERN-LEP-06}

(Propded 1986, Approved Sep J1899, Began dete-takmg 1990 Campleted dotartalcang Feb 1992)

THE SEARCH FOR HIGHLY IONIZING PAFTKCLES IN $e^{+} e^{-}$COLLISIONS AT LEP USING MODAL

L6-MODAL COLLABORATION

ALBRRTA U - J L Pinjald ( $\checkmark$ Spakemersan)

BOLOGNA U \& INFN, BOLOGNA - G Giscomell, F PatruZli,

F Prodiert, $P$ Serra

HARYARD U - K KInoAhita

Acceiceratar CEFN-LEP Detertor Plascie

Reactans

$\mathrm{e}^{+} \overline{\mathrm{e}^{-}} \rightarrow \quad 90 \Leftrightarrow \mathrm{e}\left(\mathrm{E}_{\mathrm{cm}}\right)$

Particies stidied monopole

Brsef descraptipat The MODAL (MOnvpole Detector At LEP)

detector ti desitgued to weateh for monopoles, dyons, and other busbly tobizang particle it is farmed Irom lexsob/CR3 duelecterc track detextor modules arranged us a polybedral configuration around the intersection repon

Jourwat pagers PR D46 (1992) 861

Enati coninat james pinlold Acern th 


\section{CERN-NA-012-2}

(Fmpotsed Alug 1985, Approved Feb 1986, Began dats-tsking OcL 100 , Completed datartaking Noy 1903)

SEARCH FOR MESONS AND GLUEBALLI DECAYING INTO MULTIPHOTON FINAL STATES PRODUCED IN CENTRAL HADRON COLLISIONE AND STUDY OF INCEUSTVE PRODUCTION OF HEAVY QUARK MESONS

CAMS COLLABOFATION

ANNECCY - T Kinstbu, J P Pelgrentx, M Poulet

KEK - 5 Lnabe, M Kobayahi, T Tauru

LOS ALAMOS - D Alde, E A Knopp

PLSA U Zz [NFN, PLSA - R Belliszzm, A Btez, S M Magsal,

M R Torqual,

SERPUKHOV - S V Donikar, A V Inyakin, G Y Khanatov, A V Kulsk, A A Ledney, S A Polovweleov, V A Folyakoy,

Y D Prokodbkin (V Spokooperana), 5 A Stdovsky

V D SErnoykenko, P M Shagin, A V \$htannikov, A V s sngovzky, $\checkmark \mathrm{P}$ Sugonyarv

BRUSSELS U t BRUSBRLG U, ILSN .- F Bron, I P Strot

( $\checkmark$ Spokesperono)

CHIBA U - H Kawn!

MTYAZAKI U - T Nokeamuten K TAkamptou

YAMAGATA $U$ - H Shimm

CERN - M Boutemert

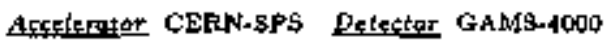

\section{Aractions}

$$
\begin{aligned}
& \pi^{-} \mathrm{p} \rightarrow p \pi^{-2} \operatorname{si}\left(\gamma^{3}\right) \\
& \pi^{-} p \rightarrow 2 \gamma\left(\gamma^{\prime}\right) x \\
& x^{-} n \rightarrow n \pi^{-2} \gamma\left(\gamma^{\prime} 0\right) \\
& { }^{-} n \rightarrow 2 \gamma(\gamma / s) \mathrm{X} \\
& p p \rightarrow 2 p 2 \gamma(\tau ' b)
\end{aligned}
$$

$300 \mathrm{GoV} / \mathrm{c}$

$$
\text { " }
$$$$
\text { it }
$$

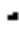

$450 \mathrm{G} \cdot \mathrm{V} / \mathrm{c}$

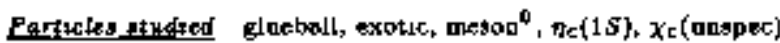

Bref descriptian Sesrches for noutral mesona, excetcs like glueballa, hybrids, and many-quark atakes producad in central hadron-prokut collationg Situdes the Jatluarve badrokic production of ueutral heavy quark mesong Usest the s092-cell electrocoagnetse calorimeter GAMS-d06l supplemented with a forwerd magnetic spectrometer and ancrobt tip kot chambers Dala andyout in progkets (July 96)

Jourmat pupers NIM A2B8 (1988) J12, N1M A269 (1988) 101, PL B201 (1988) 160, YF d7 (1988) 1279, YF 47 (1988) 1639, NLM A276 (J日89) B82, YF 49 (1989) 712, ZPHY O\$3 (1980) 641. DANS $\$ 16$ (1991) 900, NIM A315 (1992) 2), NIM A 342 (1994) 389, PL B340 (1994) 122, NC A 107 (1994) 18BT, NC A107 (1924) 1911, 2PHY CAI (1994) 35, YF 59 (1695) 662, DANS 344 (1995) 169, ZPHY CA6 (1996) 375, and ZPHY C 66 (1996) 379

Retated expertments CERN-NA-012, CERN-WA-081, CERN. WA-102

E.mantscantact prokoshkın jeanpyerre atrastacakn th

\section{CERN-NA-045-2}

(Propeed Jan L694, Approved Apr 1905, Began date-taking Nov 2895: In progrear)

STUDY OF LOW-MASS ELECTRON PAIR AND PHOTON PRODUCTION IN LEAD-LEAD COLL1SIONS

CERES COLLABORATION

BROOKHAVEN - P Holl, H Kraner, P Rehak

CERN - J Schmoraft

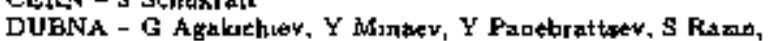

\$ Shimanrkk, V Yureusch

DARMSTADT: GSI - F Draun-Mumanger, D Mrakowiec

HELDELEERG, MAX PLANCK INST - F Ceretth,

U Faschinghaufe, $C$ Fuch, M Hembargar, O Nix, J $P$ wiutat

HCIDELEERG U, ThEP R Baur, C Bormann, h Brees,

S Estw, P Glastitl, C H Jung, B Lenkest, M Mester, A Pfeuffer, H J Specht, J Stachot, T $\$$ Ullmeh, c Vargt, JP Whaskls
MLAN POLYTECHNIC - E Gakt, M Sampletso

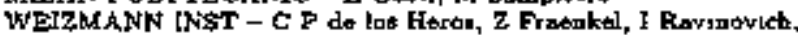
E Spcol, \& Tel+Znr, I T werraya ( $\sqrt{ }$ Spakexperzon)

Acceterator CERN-SPS Detector Ring 1 ndaythe Cerenkow,

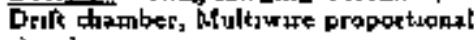
chamber

Rosctions

${ }^{207} \mathrm{~Pb}$ nucleus $\rightarrow e^{+} e^{-\mathrm{X}}$

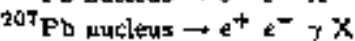

$160 \mathrm{GeV} / \mathrm{e}\left(\mathrm{P}_{1 \mathrm{sb}} / \mathrm{N}\right)$

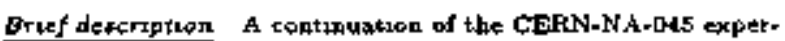
unent dedusted to the necanurespent of $e^{+} e^{-}$parfs and direct photons ptoduced un uucteut-mucleus collisions The min gosl

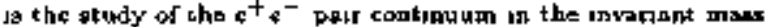
tegron Irom $50 \mathrm{BleV} / \mathrm{c}^{2}$ up to $2 \mathrm{GeV} / \mathrm{c}^{2}$ in a gearch for therrod

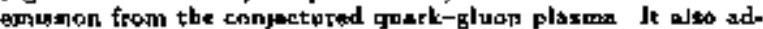
dreases the question of chiral aymametry restiorstion in bigh den91ty matter through lhe study of the vector metsong $\rho$, in and $\phi$ Tha CERES gpectrameter has bean uperaded to cope with the hagher moltiplicitie, and background of the tentral Pb-nucleus

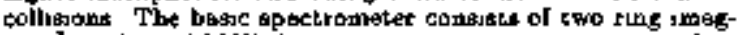

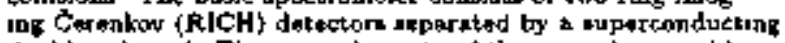
double solenold The matn efoments of the upgrade are soddt. tronal detectors, two sulicon radial-drift chembers inatesd of one ID the orjginal setwp) and a pad chambar (a targe NWPC with pad readout) located behind the aptecrameter They allow resl tracking and belp the paktern recognition of the RlCH detectors, by provediag an a prion knowledge of the reg location ip the

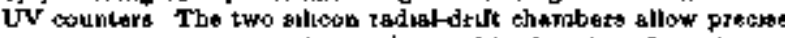
yertex recanstruction and provide rapadity density mIormation They ales play. together with the frat. RICH, a major role in the repectuon of $\gamma$ converanous and $\pi^{\circ}$-Dralita decays The upgrade include atso a dew DAO system with a rate tapability hither by

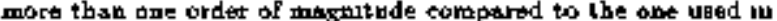
CERT-NA-040 Takong dala (Jw) 96)

Joturnal papery Includas the lut of papers by the CERN-NA.005 experiment IEEE TNS 35 (1986) 4DA, IEEE TNS 35 (1988) 432 , NTM A279 (108B) 798, IEEE TNS 37 (1900) 241, TEEE TNS 39 (1992) 619. NTM A 1616 (1992) 259, NIM A32 (1983) 273. NF A553 (1993) R57c, NMM A343 (1966) 87, NIM A36S (1994) 231 NP AS68 (1964) 879, NP A6*8 (1094) 347e, PL B332 (1994) 471 NIM A366 (1995) 329, NP A590 (1995) 1035, and PFL 75 (1995) 1272

Felated epertmęnte CERN-NA-UA6

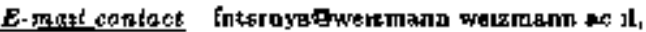
itshisk toerruyatictern ch

WWW Home.pagte betp //ceters pbyst tursheldelberg def

\section{CEFN-NA-047}

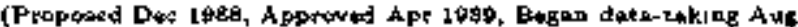
1991, L jrogrest?

MEASUREMENT OF THE SPIN DEPENDENT GTRUC. TURE FUNCTIDNS OF THE NEUTRON AND PROTON

SF1N WUON COLLABORATION (5.MC)

NIKHEF, AMETERDAM - I de Oroet, T J Ketcl, $\mathbf{E}$ Kok,

M Lutmasth, J E J Oberster, H Postran, E P Sichtermang, R ven Dantzug, G van Bliddelkoop

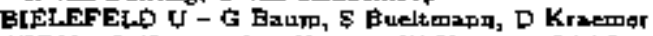
CERN - P Hautle, C A Heuxch, W Kraeger, J-M Le Gof,

T O Nuoukogki, U St, teggler, $F$ Voss

DUBNA - A Karer, Y KLesoley, V Krnvokhjııne, K Medred,

A Nayajcey, D Fobekhonov, D Page, I Savia, G Sminow

FREIBURG If - U Eandgraf, A Wiltmann

GKSS, OEESTHACHT - H Stuhrmant, R Wilumet, J Zhan HELSINKT $U$ OF TECH P Berglund, 3 Kyynarbunen, J Ylogtalo BOGAZICI U - E Arik, T Cubadir, E Gul mite

ISTANDUL, TECH U - T Akdotan, H Guvan, C Oxben, I Reybagcen, $G$ Uael

MAINZ U - E M Kabuns, G K Mallot, I Prets, R \$mitz,

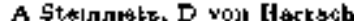

MONS $U-\mathbf{R}$ Wind molders

MIJNICH tI - L Bertav, A Stande, I vogt 


\section{SUMMARIES OF CERN EXPEAIMENTS}

NAGOYA U - T Hagegawa, N Hayesbu, N Horlowa, $S$ Jabuntoto, T J Jaste, T Kageye, A Kishu, T Matguda, K Norl, A Ogawa NORTHEASTERN $U$ - J Moromerato, E von Goeler

NORTHWESTERN U - D Funding, D Milke, R Bosel,

P Shanahan, is Velaeco

RICB U - B E Babner, J Cranshsw, S Etebblatt, T Gartirap, M Lawe, J B Roberts

SAClaAY - N do Botton, E Burtin, A de Lenquen, F Feinstem,

B Fron, F Lehar, A Magnop, F Marie, I Martiva, F PerrotKunne, S Platenkov

SANTLAGO DE CONPOSTELA U - B Aders, C Feroandez,

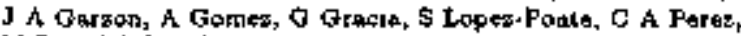
M Plo, 1 Sabarido

TEL AVIV U - J Lichtenstadt, I Sabo

TRUESTE U, IST FIS \& INFN, TRIESTE -. R Btro

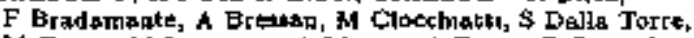

M1 Gargi, M Lamanos, A Marim, A Pemzo, F Puntaferre

P S.chisvon, F Simeom, F Teorstotio, A Zenexts

UCLA - B Derro, G Dulya, G Igo, C Whattem

HOUSTON U - B Mayex: L Pinsky, i Pythk, R Welngte!n

UPPGala U - A Arvidgon, P Bjorkbolm, A Dyring, M Flodiıguex

VIRGLNLA TECH - D Crabb, J MaCerthy

WARSAW, INST NU/CL STUDIES - B Bndelak, J Kiryluk, IT Kurat, J Polec

SOLTAN INST, SWLERK - J Nasadsk, E Rond1o, A Sandace, id $S_{2} \operatorname{lop}_{0}, \mathrm{~W}$ tW

YALE U - A Desbpanda, S Dhawau, VW Hugher (Spakenpersan),

R Plegala

Accelerator CERN-SPS Detector EMAC

Reactions Polanged bear and larget

$$
\text { moon } y \rightarrow \text { muon } X \quad 100-200 \text { GoV }\left(E_{\text {lat }}\right)
$$

muou deut $\rightarrow$ muon $X$

$$
\text { , }
$$

Particles strdied $p, n$, deut

Biref descrotion deasores the apun-dependent ayminetries $A_{1}$ and $\lambda_{2}$ in deef uneinatuc xcotterusy of longitindusally polarized muons by Jongitudinally and trangyersely polarized protane awed deaterons Thents the mucleon spin struct or and Ellis Jates and Bjorken awe rules The apparstus is the upgraded forward ipecteometer which wal ured angurelly by the European

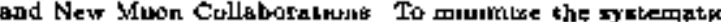
ancertsunties the tsrget contsins two opposttely polarized celle. which are axposed to the mutn bearn timultancounly The polarmed lerget hae a high cooting power and freld homogenenty and long target cells Il wat uted with proton (butanol beoda) and deuteron cornet materiste in previoleg yesets, and with proton materis (amrwna) in 19\%0 Takjog data (Jwe 46)

Jowrnal payere PL B902 (1903) 633. NIM A343 (1994) 400, NIM A349 (1994) 33d, PL B320 (1994) 400, PL B324 (1994) 399, Pl

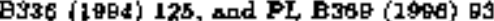

Reinted experaments CERN-NA-0012, CERN-NA-009, CERN-NAO29, CERIN-NA-03T

$E$-mand conlact varnou bughogeysle odu

WWW Home page htep / /astaunos cets ch/waleame html

\section{CERN-NA-048}

(Propoeed Jul 1990 Apprited Nov 1991, in preperation)

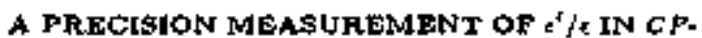
VIOLATING $K^{D} \rightarrow 2 \pi$ DECAYS

NA\$S COLLABOFATION

SACLAY - J Alluti, S Anvar, D Bedterde, F Eergon, J.B Chere, M De Becr, P Dobu, J L Follou, A Givernaud, H Lo Ptowost,

F Louls, E Mazzucato, A Mighlorr, M Mur, B Peyaud, $S$ Srhanne, G Tarte, R Turlay, B Vallage

CERN - F Bal, G D Barr, G Bocquel, P Buchboliz, D Cubdy, N Doble, F Forment1 W Funk, L Gatignon, A Gondec, P Grafkt ram, a Hallgrev, W Iwangki, G Kexceler, A Lacourt,

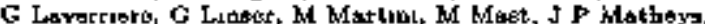
A Norton, P Ponting, D Schmzel, W Sindl, 11 Soxzl, 1 Taureg. $P$ Vende Vyvre, is Velakro, O Voesnack, $H$ W $J$ Wetaring $\mathbf{M}$ Ziolkowgks

CAGLIARI JJ \& INFN, CACLIARI - Y Fan"1, A La, L Muss, A Nappl, P Randarcio, B-G Setal
CAMBRIDGE U - \$ Katvars, R Woore, D J Munday, A Parker, $S$ P Takach, TO White

DUBNA - Y D Kalsgin, A M Kalmun, M N Kapiabth,

V D Kekelrdze, P Khrorav, D A Kiriloy, I A Kojevw kor, $Y$ K Potrebenikov

EDINBURGit U - L Bertolotta, O Boyle, a Hay, A hast,

N McKay, G Oaklatd, K I Ptach, E Veftech, L L J Vıck, A Walker

FERAARA U \& INPN, FERRARA - D Betton, R CAlabrer, F Dalpiaz, I Duclos, P Ferretti Dalpegz, P Frabettt, A Giaboh, $\checkmark$ Guld, E Luppi, F Petruca, L Piemontete, F Rossi, is Savme FLORENCE U \& INFN, FLORENCE - A Buztut, MCalvekts

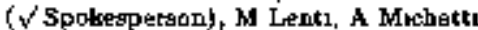

MAINZ U, INST PHYS - H-G Becker, T Bener, H Bluemer,

B Bockkolt, $C$ Ebersberger, \& Kalber, K Kletnknecht, H C Knoeppler, U Koch, L Koeples, S Luitz, B Renk, J Scheadt.

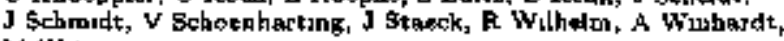
M IVIttgen

VIENNA, OAW - H Dibat, M Jentler, is Markytan, I Mikulet, o Neuhofer, M Perweks, A Tautak. C E Wulz

ORSAY, LAL - J-N Alberl, E Auge, G Earrad, J-E Campagne,

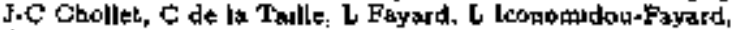
c Martin-Chasostr, A Sthofer, G v val

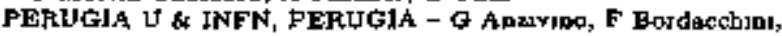
P Cenc1, Del Ragso, P Labrabo, M Pape, M Puntuto, \& Talamont:

PISA U \& INFN, PISA - A Bgat, I Dertank, A Elg, P Gelshura R Carosi, C Cerr1, F Cosiarlini, F Fantechi, F Fudectaro,

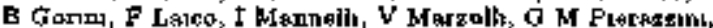
F Reffastl.

TURIN $-Q$ Bnno, A Ceocuci, R Cester, F Mass, F Marchetto, E Mentchetti, R Murs, S Folextin!, N Past rone

SIEGEN V - I Augustan, M Bendsr, G Gilleaben, M Hobler W Otto M Forachangar, C Schmitz, B Schofer, R Werthenbach, 5 winkkr

WAFSAW U A Chlopik, $z$ Guzak, J Naqualski, E Rondio, M Saleper, W Wistets

Acredertior CERN-SPS Detector Calortmatar, Bpoctrometer

Partreles studacd $K_{5}, K_{L}, h$

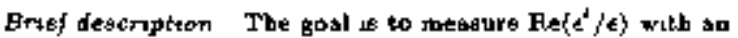
sceurscy of $2 \times 10^{-d}$ The expenmeot thes the $450 \mathrm{GeV} / \mathrm{t}$ SPS proton besm to produce two besely collingar $K_{S}$ and $K_{L}$

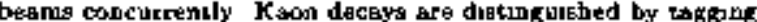
the pratons genetating the $\boldsymbol{K}_{S}$ component $T$ he detector 18 optumiced for the detection of $\pi^{+} \pi^{-}$and $\pi^{0} \pi^{0}$ final states From neutcal kBons orth momenks between 70 and $170 \mathrm{GeV} / c$ Charged deseys are masesurced in a magnetec ppectromoter with a cential dipole Juagnet and two gocs of large and highprecuevon deife chanebers an esch side Neutral decays are

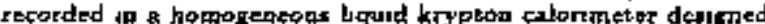
for lugh pate capability, good energy and aptce rewalution, sind snb-ngnosecoud time resolution This configuration petulta collectung all fone modes concurraptly with mumal eystematic error Ouber curppocents of the apparatus include a protod taggar, a hadron calorimeter, and counkers to veto muons and partickes outgide the detoptor accoptance The firgt hargod kron

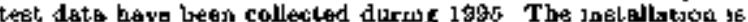
beng completed with the positioning of the LKr colorineter

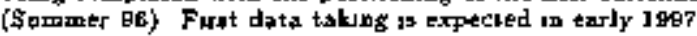

Journal paperg NIM A316 (1892) 1, NIM A323 (1992) 393, N1W A.344 (190d) 48T, NIM A344 (1994) 50T. NIM A360 (1905) 224. NIM A361 (1995) to6 NIM A365 (1995) 26B, NTM A3BT (1995) 283, MIM A370 (1996) 413, and NCM A37s f1996) 213

Retated expertments CERN-PS-195, FNAL=832

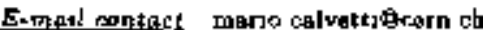
WWW Home-page http //WWwl carn ch/NA48/Wekone hteol

\section{CERN-NA-050}

(Froposed Nor 1991, Approved Facb 1998, Began datarakug Nor 1996, In progress)

STUDY OF MUON PAIRS AND VECTOR MESONS PRODUCED IN HICH ENERGY PL Sb INTERACTIONS NA5O COLLABOEATION 


\section{SUMMARIES OF CERN EXPERIMENTS}

ANNECY - C Baglan, \& Butavere, V Capony, R Kobsakewski EUCHAREST, IAP - C Alema, V Doldea, S Conetantincesu, $S$ Dits

CAGLIARI U 2 ENFN, CAGLJART - M Cerw, C Cualo.

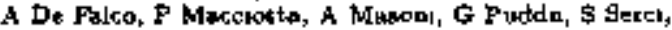
$P$ Temmkov, G Usan

GLERIONT-PERRAND U - A Bstdrt, J Castor, T Chambon, I Cherrol, A De vaux, B Espagnon, J Fatgalx, P Force,

$G$ Landand, L Luqun, 5 Mlontgues, P Ssturmin]

GRAGOW, INST PHYS NUCL TECH - W Dahrowke,

GERN - $G$ Lourenco, F Shabouan, P 5ondereggter

LISBON, LIP - M C Abreo, P Bordalo, S Ramos, S S1lvs

MOSCOW, INR - Y K Gavrikov, M G Golibevs, M Gotrs,

T L Kewartherd, A B Kutepin, $N$ s Topilskays

ORSAY, IPN - I Antrou, C Gerschel. D Joldan, Y Le Bornec, $X$ Tarrago, $N$ Willue

ECOLE POLYTECHNIQUE - A Barban;, B Chaurand, F Fleuret,

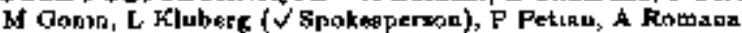
STPASBOUTG, CEN - P Gorodetzhy, C Races

TURIN U \& INF', TURDN - 9 Aleasandro, 5 Beole, G Bonezzols

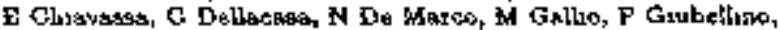

$P$ Gqaita, M Idzik, A Marzarl Chets, M Masera, A Musso.

A Piecotti, W L Prado ds Silva, L Ramello, P Rato Mendas,

L kecati, E \$compariv, E Vertellin

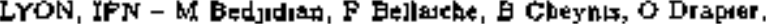

J Y Grossiord, A Gulchard, F Haroutimian, M Jacquin. $F$ Mislek, I $R$ Pinas

YEREVAN FHYS INST - A A Grigeryan, H R Gollanyan,

A S Hakobyan, S S Mebraby's

Accalerator CERN-SPS Detector Calonmeter, Spectrometer

Reaclions

$$
\text { Pb nucleus } \rightarrow \mu^{+} \mu^{-} \mathrm{X} \quad \mathrm{X} \quad \mathrm{GeV}\left(\mathrm{T}_{\mathrm{lab}} / \mathrm{N}\right)
$$

Partictes studiad vmeoon

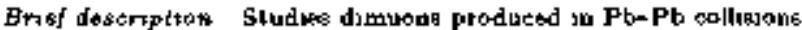
as the Ilacloon-tucleon $E_{\mathrm{c}} \mathrm{m}$ of $19 \mathrm{GeV}$ 'Che gecup is optumized for a muss rang* which indurtes segnals probiag $Q G P$ (Qutark and Gloon Plasma), namely $\phi, J / \psi, \psi^{\prime}$, and (unseparated) $p$ and we vetor mesong it slso covare Drell-Yan dimolon which serve se s normalizshion The delector s an improved varsion of the CERN-NA-03s set.qD, with geutral energy and prullupliaty detectora Pollowsthg the sarget, the Zoro Degrees quartz fiber hadron eslonmeter (ZDC) embedded in the hadron durp, and a

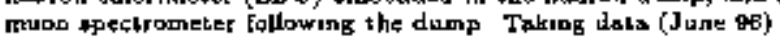

fiejated expermments DEFN-NA-OSB

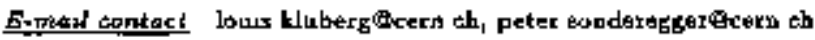
WWW Home page bitp / WWw cern th/NA50/

\section{CERN-NA+051}

(Proposed Apr 1992, Approved Apr 1992, Eegan dsta-taking Jun 1992, Completed dsts-laking Jut 1992)

\section{DRELL-YAN STUDY OF GEA ISOSPIN SYMMETRY}

NA51 COLLABORATION

CERT - P Sonderegger

CLEEMONT-FERHAND U - A Baldit, C Barriere, J Castor,

T Chambon, A Devallx, B Enpaginton, I Fargetix, F Force,

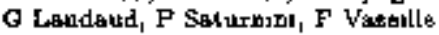

LlsBON, LIF - M C Abreu, P Bocdalo, F Ferretra C lourenos,

$S$ Ramas, S Silva, J Varela

ORSAY, TPN - C Geractel D Joman. X Turrago

ECOLE POLYTECHNique - B Chaurand, L Kiuberg

(V/ Spokespereota), A Romans

STRASBOURG, CRN - P Gocodetzky, D Lazic, R Mazinu, C Rucea

TURIN U \& LNFN, TUTUN - D Alesandro, E Chjavases.

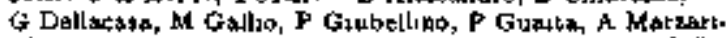

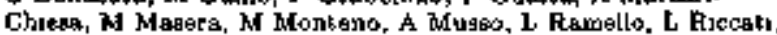

E Seamparis, E Vercellin

LYON, IFN - IA Bed]adian, D CoDtarda, E Dekcrowx, D Drapter,

I $r$ Grograord, A Gutcherd, F Horoutubian, F Malek,

f. Mandry, J R P Pzal

Acctierator CEEN-SPS Detector Spectrametter
Beactions

$$
\begin{aligned}
& p P \rightarrow \mathrm{s}^{+} \mu^{-} \mathrm{X} \\
& p \text { deat } \rightarrow \mu^{+} \mu^{-} X \\
& 450 \text { GeV/c (P) }
\end{aligned}
$$

Brtef description The purpore of the expocimont is to study

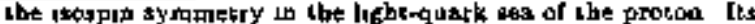
volation ts one potable explsuation of recent wexpected muon deep iwelastix scistering txperimental teaults whwh disagres with the Gottfried sum rule The experiment makes wete of the brae acemptanre mulon spectrometer ured previonsly by CERN.

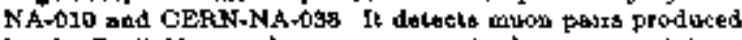
by the Drell-Yan mechantam un of and pod reactions $A$ beam

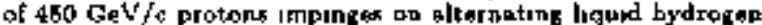
and deutertum targots The aim as bo mesaute the cross-efcion retw at dimuon masseg above $4 \mathrm{Get} / c^{2}$, which ig a seagitive probe of the relative content of light antiguerks $\bar{y}$ and $\bar{d}$ th the proton set

Jossmal papers PL Ba32 (1906) 244

Relut ed epptimerts FWAL-28E, FWAL-T13

Ermad contact lous telutergotern ch

WWW Home-page hetp //yyounfo m2p3 fr/eaxp/nasl htm]

\section{CERN-PS-185-2}

(Propotad Jan 1982, Approved Noy 1992, Began data-takjng Ju 1994, Completed data-taking Jos 1995)

HIGH PRECISION MEASUREMENT OF $\overline{p p} \rightarrow \bar{\Lambda} h$ CROSS-SECTIONG IN THE MASS REGION AROUND 2Js2 $\mathrm{MeV} / \mathrm{c}^{2}$

CEAN -. N Hamasn, (deceased)

CARUEGTE MELLON $U$ - $G$ Franklu, a MeCrady, $C$ A Meyer, B Quinn, $R$ shomacher

ERLANGEN U .. H Donnart, W Eynch, J Houffa, F Stinzing

F helBUEG U - D Alzah, J Franz, I Rauch, E Roseste, M Rah, D Ruachroeter, H Schmutt, $\mathrm{H}$ Todenhagen, T Walter, $\mathrm{H}$ Wuth

JULLCH, FORSCHUNOSZENTRUM - R Broeders, R Ocyar,

K Kilian ( $\checkmark$ Spohtrepersoul), W Oelert, Ki Roehtiel

(V Spokespergon): K Sacks, T Sefrecl

LÓs At AM ÓS - P D Barme:

ILLINOIG U, URBAiNA - R A Euganytein, P Harts, D Hertxag,

T Jones, R Tayloe

NEW MEXICO $\Psi$ - 11 Fucher

UPPSALA U - T Jabanegon, E Traneus

Acreligrator CERN-LEAR Detscter Wire chamber

Reartons

$$
\bar{D} \rightarrow \bar{\Lambda} A \quad I 435-1450 \mathrm{GeV} / \mathrm{C}\left(P_{\mathrm{Lab}}\right)
$$

Brecef description $T$ the aym of the experiment we to verify a posable structure th the exatatyon function of the reacison $\overline{F P} \rightarrow \bar{\Lambda} \Lambda$ undicated by the recent threshold thats of the CERN-PS-185 collaboration at an invariant mesg of about $2232 \mathrm{LfEV} / \mathrm{c}^{2}$ Studies the total aod differestial crove-xections As well as polertestrons and spid correlstrong, withe of few MeV sround the reactson threshold Utes an upgraded vetwop of the PS.185 detector The full $\vec{A} A$ bxemakice u recongtructod from tracks in a $30 \mathrm{cul}$ long atsck of MWPO's and drift chambers The productoon vertex is recongtructed by makings the of four plante of $\mu$-atrip caunters upstream the $\mathrm{CH}_{\mathrm{z}}$ sandwich tarzet Hyperon and antihyperon ars ditunguished by mesos

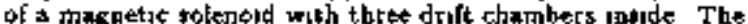

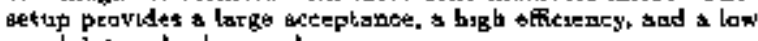
annbulatpon back ground

Reloted expethinents CERN.PS-185, CERN.PS-185-3

E-matl cantatet klaus rohtutbacern th WwW Horre-poge

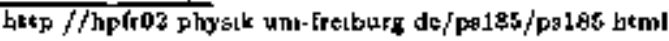

\section{CERN-PS-195}

(Froposed Jan 1965, Approved Sep 1985, Bagan date-taking 1981, In prograss'

TESTS OF CP VIOLATION WITH $\bar{K}^{*}$ AND $K^{0}$ AT LEAR 


\section{SUMMARIES OF CERN EXPERIMENTS}

CPLEAR COLLABORATION

ATHENG U - A Augelopouka, A Apostolakis, E Rozak, L Sskelluou, K Sarigannnts

BASEL U - P Kokktast, F Lęmgruber, P Pavloppulos

( $\checkmark$ 'Spokentersan), G Polivkex, R Euckenbach, T Sehretinger, L Taucher, S Vlachou

BOSTON U - M Cbertok, D Frapels, J F Moller, B L Roberts, D Zıminer mak

SACLAY - G Chardin, M Dejuardis, I Defre, D Garrets, C Guyot C Korhowsk, G Marel, P Schume, A Sastes, C Yeche

CERN $F$ Bloch, M Fidecaro, T Ruf, A Schopper, 8 Touramans

COLMBRa U - J Carvelho, R Ferreira-kakques, E Machado,

J Plnto da Cunha, E Yon Beveren

DELFT ULIV TECH - it tW Hollander, $R$ Krouget.

CW E Var Euk

FRIBOURG U - F Blane, L Furavet, $L$ A \&challer

IOANNINA U .. I Eutangotou, N Monthot, F Truantis

LIVEFP OOL U - A Benell, M Caroll, E Cawley, A Cody,

I R Fry, E Gabatholer, R Gamet, A Hagelded, P J Hayman

LJUBLJ ANA Ut - A Filuperc, I Mandic, M Mikuz, D Zavrtanik

ORSAY, CSNSM - C ThibखI

P\$I, VIELIGEN - P F Kettlo, T Nakada, o W

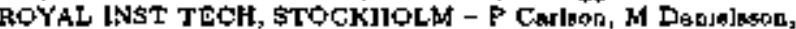

A Go, K Jon-kint

THESS ALONIKI U - S Charalamboue, S Dodoutess,

C Electheriadis, A Liolıos, I Papadopouko

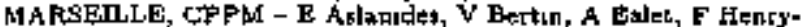

Cousonsez, B Hubert, R Le Goc, F Miontanet, F Touchard

zURICH, ETH - O Behnke, W Fetracher, H J Gerber, B Pargele,

M Schaffer, $P$ Weber, iM Wolter

Aarelerator CEFW.LGAR Detector Spectronteter, Galonmeter Reactions

$$
\begin{array}{cc}
\bar{p} p \rightarrow K^{0} \mathrm{X} & 0 \mathrm{GeV} / \mathrm{c} \\
\bar{p} p \rightarrow \bar{K}^{0} \mathrm{X} & - \\
\text { Partucies sturkeded } & K^{0}, \bar{K}^{0}
\end{array}
$$

Bref description Messums time-dependent $K^{0} \overline{K^{6}}$ decay raite

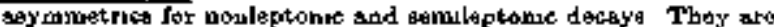
sensitive to $C P$ and $T$ violetion in difiersent and complementary ways, and also provide sennitive teats of $C P \gamma$ A beta nf $10^{6} \bar{p} /$ sec provided by LEAR is brought to rest in a hydrogep ges terget, producheg $K^{D}$ and $\bar{K}^{0}$ through the reaction $\bar{p} p$ $\rightarrow K^{ \pm} \pi^{\mp} K^{0}\left(\bar{K}^{0}\right)$ Dernys of the $K^{0}$ and $\vec{K}^{0}$ are recorded undor the asme oporahing conditions, ingude a maghetsc field sind uang tracklng chambers and a gas eampling electromagrotic exlorimeter The strangeness of the veutral kann as tagerd by doteching the eagh or secompanyug charged kaon stentifod by Cerenkovs and gcint:llatpre Herdware processors are used to

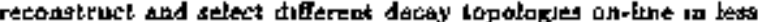
than 25 moctobeconds Taking date (July 96)

Jourtal yopors NIM A279 (1089) 286, NIM A279 (1989) 305, NIM A279 (1989) 317, NIM A283 (1989) 484, NC 102A (1969) 12T, NIM AR9T (1990) 12E, NIT A301 (1991) 424, NP (PROC

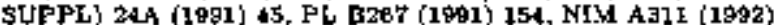
7\%, NFM A321 (1902) 458, NIM A323 (1692) 511, KP (PROC SUPPL) 27 (192) 285, PW 3 (1992) 40, PL B286 (1902) 180, SJNP 55 (1992) 840, NIRt A393 (1993) 93, NIM A330 (1993) 513, NP (PROC SUPPL) 31 (1993) 108, NP A558 (1993) 43c, NF A558 (1993) 437c, NP A558 (1998) \$49c, PAN 57 (1994) 1772, ZPHY Ce3 (1994) 641, PL L36a (1995) 237, PL B369 (1995) 243, PL B364 (1996) 239 ZPHY C65 (1995) 299, NIM A3AB (1906) 666, PL B369 (1896) 367, PL BS70 (1996) 167, PL B374 (1996) 313, aDd ZPHY C70 (1996) 211

Related espertments CERN-NA-048, FNAL-621, FNAL-832

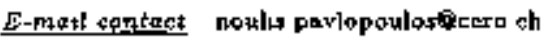
WWW Home page http/Wwwl sarn ah/eplew/Woicome ht ml

\section{CERN-PS+19B}

[Froposed Mar Igsí, Approved Noy 1960, In progrese)

PRECISION COMPARISON DF $\bar{P}$ AND $p$ MASSES JN A PENNING TRAP

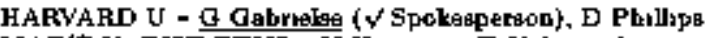

MACNZ U, INST' PBYS - K Hermanan, H Kalwoveky

SEOUL NATLONAL U - W Jhe

Accoterater CERN-LEAR Detector Other

Partictes stadized $\bar{p}$

Eruef descruption Compazes $p$ and $\bar{y}$ mabers to ar accuracy of one part in $10^{10}$ wishin $s$ amedl volume of an lou trep, and do velogs trapping and coolung techniques to allow the productios and itudy of low enerzy antipratons and antihydrogen Antiprotone have bath trepped bolow $3 \mathrm{keV}$ Electrob collog from heV to $<10^{-3} \mathrm{eV}$ has been observed in the trap Also measurst the sntuprotan atorage lifetime Furt attempt to make cold antuby-

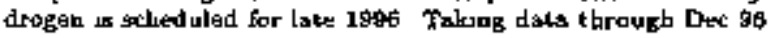

Jouraxl papers PEL 57 (1986) 2504, Fist 58 (1987) 2197, PL A129 (1086) 38, PRL 63 (1989) 1360, PFL 65 (1900) 1317, PRL 74 (1996) 3\$44, PRL 75 (1996) s06, and PRE T7 (1906) (tin prear)

Emealt contget gabnelsenbusle harvard edu

\section{CERN-PS-197}

(Propoed Oet 1985, Approved Apr 1996, Began data-lsting 1989, il prugress'

THE CRYSTAL BARREL MESON SPECTROSCOPY AT LEAR WITH A 4T DETECTOR

CFYSTAL BAFREL COLLABORATION

RUHR U, BOCHUM - K Beuchert, T Desener, H Koch, M Kunse, J L wedemas,, II Matthasy, K Petecs, H Stpeck

BUNN U - B Barnett, R Hackmann, M Hers, fi Kalınowsky,

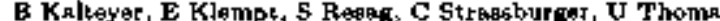

UUDAPEST, CRIP - P FIIA

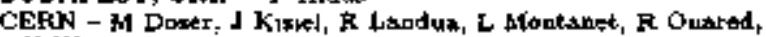
U Whodne:

CARNEGIE MELLON U - A BeTdoz, R McCrady, $D$ A Meyer

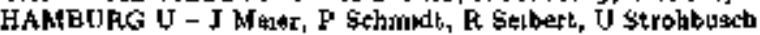

KARLSRUHE U - $\mathrm{S}$ Burchaf, F Bluem, D Eagelbardt,

Q Holtzbausas, MI Techhususer

Lel, - D A rmatrong, T Case, K M Crowe, F H Herasius, P Kantal, M Lakas

QUEEN MARY - WESTFIELD COLL - D V Bugg, I Scott, B Zou

HUNICH U - K Braunt, O Crsmer, N D paodabll, W Duennuraber, MA Paessler (V' 5 pokesperson), N $P$ Hessey, D Jompik,

C Rekterfut, W Roet hel, C Voelcker, D Walther. C Zupancic

NORTHW ESTERN $\boldsymbol{y}$ - B Kaemmle, $K$ Seth

PARIS, CURIE UNIV VI \& PARIS, UNIV VII, LPNHE M. Beqayoun

RUTHERFORD - C A Baker, CI I Batty, C Punder

GTRASBOURG, ORN - M SUTER

UCLA - R P Heddock

ZURICH U - C Amelat, P Gractta, F Outd-Saede, S Spanar, $S$ wou Dombrowskı

Aecelerator CERN.LEAR Detecior ChYSTALBARREL

Reacteong
$\overline{\mathbf{p}} \boldsymbol{p}-$ anmhll
$\overline{\mathbf{p}}$ N - annibul
$0-\operatorname{con} \mathrm{MeV} / \mathrm{s}$

Eartecifs storiugel xluebsli, menen

Brief descriptzon The spectrometer is desigaed 10 provide complete and precuse information on nearly every final state prodaced in sp and gd appihulations ot low enorgy and bo tolleck bigh statestice deta samples The phyesce goal is to identify all light mesons in the jpass ranga from o rd to $23 \mathrm{GeV} / \mathrm{c}^{2}$,

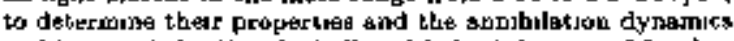
and to search for the gloeball and hybrid degress of froedom The apperatan congste of 1 barrel fhopod electromagnetic calorineier with 13BD CeI(T) crystabis read out by phorodindes, a cyludricel jek drilt chamber, a enlicon w-6trip vartex detector, and sulucen hodoucopes. The wholc dotector in embedded th a

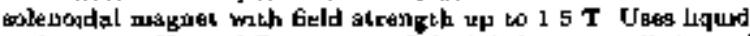
and gaseous $\mathrm{H}_{2}$ and $\mathrm{D}_{2}$ targets Scheduled to run till the end of 1996 
Jourkat papers NP (FROC SUPPL) \& (1989) Bs, PL B260 (1991) 249, NP A527 (1991) 461c, JEEE TNS 39 (1922) 826, NIM A321 (1992) 60, PL B261 (1992) 347, PL E204 (1892) 481, PL B297 (1992) 214, \$JNF 55 (1992) 76T, FL B9J (1993) 362, PL 8311 (1993) 371, PL B319 (1993) 873, ZPHY C5s (19日3) 175, PAN 57 (1994) 1711, PL B322 (1994) 431, PL B323 (1994) 233, PL B327 (1990) 425, PL BJ33 (1994) 277, PL B333 (1994) 277, PL B340 (1994) 259. PAN 57 (1994) 1465 = YF 57 (1994) 1542, Ne 107A (1994) 1815, 2FHY A351 (1994) 325, PL B342 (1905) 433, PL B346 (1095) 203, PL B346 (J955) 363. PL B352 (1996) 187, PL B333 (1945) 5T1, PL B355 (1996) 425, and PL D368 (1995) 399

Reclated erpertrients CERN-PS-201

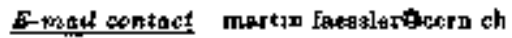

WWW Hame-pags bitp//2yww phys cmu adu/cb/eb beml

\section{CERN-PS-201}

(Proposed Jan 1886, A pproved Sep 1986, Eegan dato-taking Aus 1990, In progrees)

STUDY OF ANTINUCLEON ANNIHLLATIONB AT LEAR WTTH OBELIX, $A$ LARGE-ACCEPTANCE AND HIGH RESOLUTION DETECTOR BASED ON THE OPBN AXIAI FIELD SPECTROMETER

OBELLX COLLABORATIONN

BOLOGNA U \& INFN, BOLOONA - A Bertin, M Brosch, M Ceppona, I D'Antone, 9 De Chetra, A Farretl, D Gall,

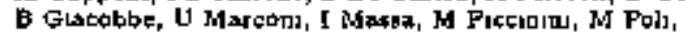

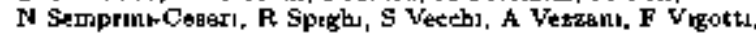
M Villa, A Vitale, A Zoceolt

BRESCIa U a LNFN, BRascen - G Belli, M Corradial, A Donxella, E Lodi' Fizzini, L Veqturell, A Zenou

CAGLJARI U \& INFN, CAGLIARI - A Adama, C Ctcalo, A Lai, A Margoll, L Mrsa, G Puddu, S Serci, P Temnikov, O I Usal

DUBNA - O Y Dealsov, O E Garchzkow, V P Nomekanov, S N Prakhoy, A M Rostideetweraky, M G Saposbrilor, VI Trelyak

FFASCATI - P Ganoten, C Guaraldo, A Lanaro, V Locluerin. $F$ Nochatiu, C Petrabcu, A Roses

LEGNARO - V G Ab|tze \& Cavion, U Gastald, L Lombarti,

G Maron, R A Ricel, $L$ Vannuce, $G$ Vedowato

PADUA If \& INFS, PADUA - A Auderghetto, M Morando

PAVIA U \& INFN, PAVIA - G Bepdisciol, V Fippini,

A Fontana, C Maresano, P Montsgns, A Rotondr

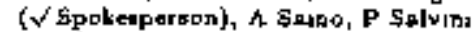

TURIN POLYTECHNIC \& INFT, TURIN - M Agotlio, F Iamze, B Mupeti]

TURIN U $\ell$ INFN, TURIN - F Bzlestra, G $C$ Bonezzola

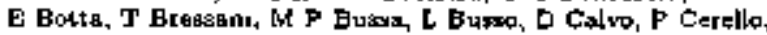
5 Cowte, D D'Isep, L Fays, A Fellciello, L Ferrero, A Fulippl, R. Gorfagnum, A Gratco, A Maggiore 5 Marcallo, D Fanmeri, D Pareza, E Rossetto, F Togello, G Zoel

TRLESTE $t$, IST FIS \& INFN, TRIESTE - $G$ Margaglotth, G Foub, 5 Teraro

UDINE If \& INFN, UDINE - I Ssut

Accelemetor CERN-LEAR Detector Spectrometer

\section{Reflateans}

$$
\begin{aligned}
& \bar{p} \bar{D}-\text { annibul } \\
& 7 \text { dous } \rightarrow \text { annihik } \\
& \bar{p} \text { nucleus }-4 \text { anrubul } \\
& \overline{7} \text { - annwhl } \\
& \bar{n} \text { nucheus } \rightarrow \text { usnıbit } \\
& \begin{array}{c}
0-02 \mathrm{GeV} / \mathrm{c}\left(\mathrm{P}_{\mathrm{lab}}\right) \\
= \\
0-01 \mathrm{GeV} / c\left(\mathrm{P}_{l_{a b}}\right) \\
=
\end{array}
\end{aligned}
$$

Particles stadted roesons

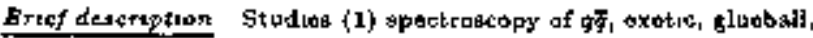
and by brid memong, (2) dynsmice of $N \bar{N}$ interactions, (3) atoric physice with $\bar{p} s_{1}$ and (4) $\bar{p}$ ennlhulations onto mor than ore nucleon A Spiral Projection Chamber (SFC) if used at vertex detector The maknet and the jet drill closuber of the Open Axid Field Speeltometer (AFS) are used for charged particles

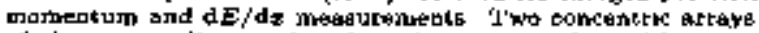
of plaktic seintilletors Jdentify and trigger on charged katan. Garnma shower" aro reconytructed in three dimenosons with gas semplang edarimeter modulı Takng date (July 96) Scheduled to complete the data laking by the end of 1996

Journat papery IEEE TNS 38 (1991) 331, JEEE TNS 38 (1991) 397, [ELE TNS 38 (1991) 399, NIM A306 (1901) 305, PL B256 (1991) 349, NFM A323 (1992) 523, PL B264 (1998) 448, PL B285 (1992) 15, PL B287 (3992) \$6B, 5JNP 55 (1922) 806, N5M A325 (1963) 417, NIM A334 (1903) 391, NP A553 (1993) 651c, NP A658 (1993) 13c, NF A558 (193s) 137c, NP ASSE (1990) 369c NP A558 (1993) 665 $c$, NP A562 (1993) 617, PR A4T (1993) 4517, PL B326 (1994) 40' 7 , PL B334 (1994) 237, PL B337 (1994) 226, PAN 57 (1994) 1614 = YF 67 (1994) 1684, PAN bT (1994) $1716=$ YF 67 (1994) 1787, PAN 57 (1994) 1745 = YF 57 (1994) 181日, NC A1O' (1991) 943, NC A10? (1994) 1325, NC AlD7 (1994) 2837. NP AG69 (1954) 761, NIM A350 (1996) 370. PL B361 (1996) 187, PRL TA (1995) 371. NP A5SS (1995) 577 . NP A694 (1905) 375, and PI B380 (1996) 77

Fitated permests CERN-PS-195, CERN-PS-197, CERN-PS202, BNL

E-mati confect alberto rotondigcetu ch, rotondi9vecern carn ch

\section{CERN-PS-202}

(Proposed 1986, Approved Fab 198?, Beigan dale-tsking Jul 19g1, Completed data-taknig Sep 198d)

JETSET: PHYSICS AT LEAR WITH AN INTEANAL GAS JET TAROET AND AN ADVANCED GENERAL PURPOSE DETECTOR

PS202 COLLABORATTON

BARI U a INTN, BART - C Evarialiats, A Palano

GEFN - D Drused, is Ferro-Luses, R Jones, $B$ Movellec,

J M Perreani, M J Price

ERLANGEN U - W Eyrich, $\mathbf{R}$ Cheyer, S Pomp, F Stinzing

FREJeURG U - H Fisther, J Frane, E Roessle, H Schnoth, M Tscheulm, H J Urbsn, H Wittb

CENOA U \& INFN, GENOA - A Bureo, M Lowatere, M Macri

(V Spokesperson), of Marmelli, S Passaggo, M G Pia, A Pozzo, E Robuti, A Sartronj

ILLINOIS b, URBANA .. P Dsbevec, R A Easerstath, P Hartis,

D Fitrizox, 5 Huchet, F Reumer, J Ruter

KERNPORSOHUNGSANLAGE, JTLICH - K Kiltan, W OAkFt.

K Roshruch, M Fook, O Sternksinp

OSLO U - H Korsmo

UPPSALA U - T Jahangeon

Acreterator CERiN.LEAR Detector JETSET

Reactions

$$
\begin{aligned}
& \bar{p} P \rightarrow \phi \phi \\
& \bar{p} \rightarrow K^{+} K^{-} K^{+} K^{-}
\end{aligned}
$$

Particies stadied glueball

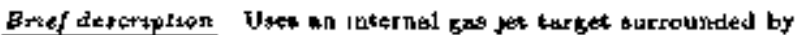
an advanced, compact, noamagaetic deteclor Aun lo a searth for glueball (gg or $g g g$ ) and hybrids ( $(\rho \bar{q})$ over the mage tange

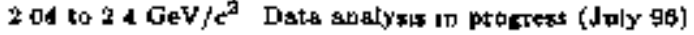

Jotsmat popere NP (PROC SUPPL) \& (1989) 69, SJNP 55 (1992) $827, \mathrm{~N}$ JM Aa35 (1993) 255, NP $A 558$ (1903) 27c, NIM A.44 (1964) $5 \%$, NC 107A (1994) 2829, PAN 57 (1994) 1503= YF 57 (1994) 1578, and PL B345 (1995) 925

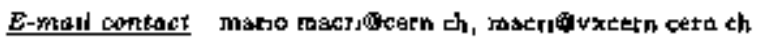

\section{CERN-UA-004-2}

(Approved Jul 1900, Corapleked datistaking)

A PRECISE MEASUREMENT OF THE REAL PART OF THE ELASTIC SCATTERING AMPLETUDE AT THE spps

GENOA UI INFN, GENOA - M Baxzo, G Sette, M ZJto ECOLE POLYTECHNIQUE - C A ugier, D Eernerd, J Bourotto. M Floguenauer (Spotossperaon) 
PRAGUE, INST PHYS - V Kundral, B Nernectk, M Novak, M Sinizangk

ROMB U, TORVERGATA \& INFN, ROME - F Cardarell, L Gerrito, G Matthiae, F Nalalı

YALGNCIA U - F Alted, R Caees, E Samchis, J Velateco Acotetator CERN-PEAR/P Detector Wire chamber

Akactions

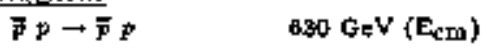

Brtaf deteruption Meaturer the total crass-sact:on and the tatio of af the rowl to the imeginary part of the [orwerd elastic destterng smplitude Tha set rp it eorapowed al two psurs ol

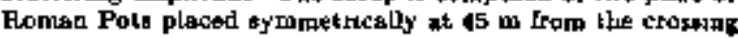
pount The horizontel gcattering siggle is measured by dret chimpern and the vertical conrdisate 18 obtaned by uang \& hodascope Bata andynis in progress (Inne 98)

Jotitnal paptry PL B315 (1908) 500, PL BS16 (1993) 4d8, and PL BS11 (1905) 451

Retated experaments CERN-UA-004

E-mezi contact baguentiolrepn $11 \mathrm{in} 2 \mathrm{~g} 3$ ir

\section{CEAN-WA-079}

(Proposed Apr 1983, Apptoved Jun 1983, Began dats-taking Aug 1986, Completed datowoking d ug logt)

\section{STUDY OF NEUTRINO-ELECTRON SCATTERING AT} THE BPS

CHARAR-Il COLLABORATION

BRUSSELS V, IIHE - P Vilan, G WLquet

CERN - R Beysr, W Flagel, $H$ Grote, T Mouthuy, H Overas,

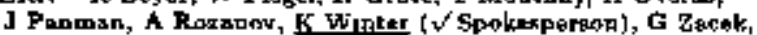
$\checkmark$ Zacek

HAMBURG U - F W Butser, C Foos, L Geriand, T Loyda, F Niebergall, of Radef, P Stshelts, T Vosg

LOUVAIN U - D Favart, G Gregonre, E Knoopa, V temattre

MOSCOW, JTEP - P Gorbunow, E A Grugoriey, V D Khovanaky

A Mralenakav

MUNICH U, EXP PHYS - W Lippoch, A Nathnneh, A Stande, J vort

NAPLES U, IFS \& INFN, NAPEES - A G Coce, A Ereditato. G Florillo, F Marcholti-Stses, v Palladine, P Strolin

INFT, ROME - A Capone, D De Podu, U Dore, A Fronkel-

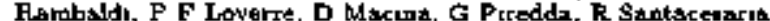

FERRARA U \& INFN, FEFRARA - E D: Copus, 5 Ruchard, B Sints:

MIDDLE EAST TECH U, ANKARA - B Akkin, E Ank, $M g_{\text {entr }}$

Zeyteh, R Savor, P Tollan

BERLIN-ZEUTHEN ADW - K Hillar, E Nahnhaun, b E Rolof Acceterator CERN-SPS Detector CHAfUM-il

Reactions

$$
\mathrm{H}_{\mathrm{p}} \mathrm{t}^{-} \quad \mathrm{S}-\mathrm{HoO} \mathrm{GeV} / \mathrm{e}
$$

$\bar{\nu}_{\mu} \varepsilon^{-}$

$$
\text { " }
$$

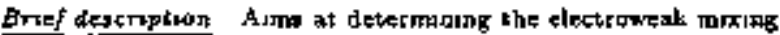
angle $d_{w}$ and the ratio $S_{A} / g V$ frop the ratio of $\nu e^{-}$and

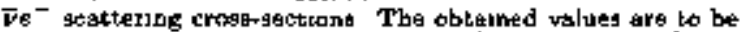
cumpared to thase determined wist $30^{6}$ topas lar cer $Q^{2}$ at LEP The CHARM-II neutting delectot consisti of a risasive, fae. gralued, and low-dengty electronic calorinoter. Eollowed by a

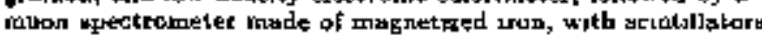
end drift chambers as ective elernents The neesurements are performent in the barn-fincuged wide band nentring besm

Journal papers NIMI A252 (1986) 443, NIM A260 (1987) 368, NIM A263 (1988) 109, PL B213 (1988) 5B4, NIM A27T (1909) 63. NIM A27? (LQ89) 170. NIM A278 (1988) 670. PL B231 (1989) 317. PL B232 (1899) 539, PL B245 (1980) 271 PL B247 (1960) 131, NP (PROC SUPPL) Ir (1981) 30e, PL E259 (1991) 499, PL B281 (1992) 169. PL B3/2 (1993) 351, PL B B09 (1993) 463, PL BSL3 (1993) 267, PL B320 (1993) 203, FL BS32 (1894) 465, PL Ba35 (1994) 346, ZPHY C64 (I994) 539, PL B343 (1995) 453, PL. B345 (1995) 115, and PL B364 (1996) 121

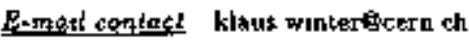

\section{CERN-WA-085}

(Propased Oct 198d, M4r J987, Approved Apr 1987, Began datso takıng Oet 19at, Completced data-taking Sep 1991)

STUDY OF HIGH ENERGY NUCLEUB-NUCLEUS INTERACTIONS USLNG THE $\Omega^{\prime}$ SPECTROMETER EQUIPPED WITH A MULTIPARTICLE HIGH D। DETECTOR

WABE COLLABORATION

ATHENS U - \$ Abatzix, G Voswlasdic.

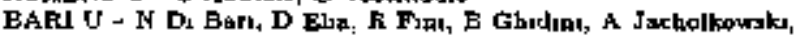

Y Lenti, R A Loconsole, V Manasart. F Nevach

BERGEN U - H Helstrup

BIRMINGHAM U - R Barae, A C Exyr, J N Carney

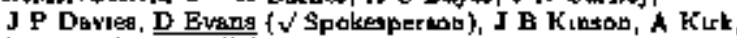

M Venables, O Villalobot-Ballut, MA P Yotrubs

CERN - A Andagtretto, F Antinan, W Beuxch. I P Dhifey,

B R Fremch, A. K Holme, K Knodson, I C Lasnajle, E Quercigb,

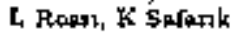

MADRTD, CIENAT - B de ln Cruz

COLLEGE DE FRANCE - M Benzyoun, J Kahabe, P Lerusta,

J I Narjoux M Sene, R Sene, A Volle

OSLO ए - J A Lien, T Storas

Acalferator CERN-Sr's Detector OMEGA-PRIME

Resettonte

D Wi $\quad 200 \mathrm{GeV}\left(\mathrm{T}_{1 \mathrm{sb}}\right)$

${ }^{32} \& W t$

$200 \mathrm{Ger}\left(\mathbf{T}_{\mathrm{lab}} / \mathrm{N}\right)$

Bartioles studed $K^{0}, \Lambda, \bar{\Lambda}_{1} z^{-}, \bar{\Xi}^{+}, n^{-}, \bar{n}^{+}$

Brief description An exploratory experiment to look for new phystic, and ptritcularly for evidepce of a guart-qlion plasma, through an increase in atrange perticle and artipartich

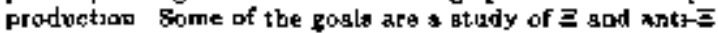

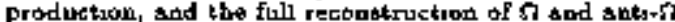
hyperops Also compures the strange and multictrang" particle (ants-particle) productions in $p$ W and $\mathbf{S} W$ interactsons Dats andyoss in progress (Juby 96 )

Joamel papers NP Ad9g (1989) 369c, PL B2dd (1990) 130. NP (P'OC SUPPL) 16 (1990) 406, PL E259 (199) 508, PL B270 (1991) 123, NP A52S (1901) 441e, NP A525 (1961) 445c, NP

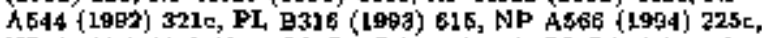
NP Abrte (1994) 491c, FL B347 (1995) 158, PL B35s (1956) 401, PL B 559 (

Related eqPeTmerts CERN-WA-094, CERH-WA-097

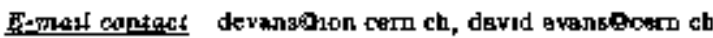

\section{CERN-WA-089}

(Propased Aur 1987, Ang 1987, Approved Feb 1988, Beyn datto talung 1990, Complated dats-tsking Sap 1994)

\section{NEW HYPERON BEAM EXPERIMENT AT THE} CERN-\$PS USING THE OMEGA PACILTY

BR;\$TOL U - D Newbridt, V Smit.h

CEFU - W Beusch, W Klempt.

GENOA U \& WNFN, GENOA - D Barberks, L Pooss

GR ENOBLE U - C Berat, M Buenerd, F Charignod, J Cbatum.

A Foutuber, P Martin, is Rey-Campanwolla, E Vasis

HEIDELBERG, MAX PLANCK INST - E AIbETTOD,

$M$ Beck, $S$ Brans, WW Brueckater, E Baexther, U Dersich,

P Dropminn, S O Gerassimov, M Godbertas, T Haller,

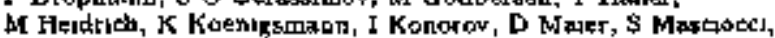

R Mirthalk, C Newaom, S Paul (Spaketpetroa), B Porh, Z Ren,

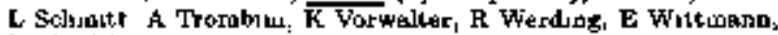
Mi Zaclubersef

HEIDEL BERG U - M Bass, P Leqnert, K NArtens. H Pueteber.

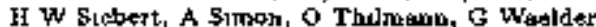

MAINZ U, INST KERNFHYS - E Chudtabov, U MUledier,

$G$ Roener, H Rudolph, B Volkemer 'T Wajcher

LEBEDEV INST - Id I Adqangukh, Y A Alexandtov,

MV avertyatr

HUTGERS U - R Raneome

Acceterator CERN.SPS Detecter OMEGA 


\section{SUMMARIES OF CERN EXPERIMENTS}

\begin{tabular}{|c|c|}
\hline$\Sigma^{-} \mathrm{Cu}$ & $330 \mathrm{GeV} / \mathrm{c}\left(\mathrm{P}_{\mathrm{Iab}}\right)$ \\
\hline$z^{-c}$ & - \\
\hline$\Xi^{-} \dot{c n}$ & 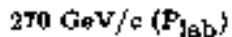 \\
\hline$\Xi^{n} \mathrm{C}$ & 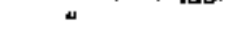 \\
\hline$\pi^{-}$ & $*$ \\
\hline$R=C$ & 4 \\
\hline
\end{tabular}

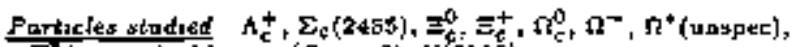
$\Xi$ (uspec), diberyon(s $x-2), E(3100)$

Brted descruption The ang are (1) to study charmed strange baryors, (2) to see if the $U(\$ 100)$ actually exipts, (3) to stody

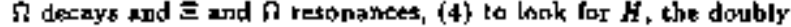
atranta dibaryon, (5) to measure gemilcplone decays of ehormed particlas, $(\theta)$ in study hyperon polarization phemomens, and (7) to kady $\Sigma^{-} e^{-}$elastic scalteras Uess the npgraded OMEGA teicisy and a byperon beam ingisled at the eud of the H1 beamline Data analysia un progress (July 96)

Jotrat popets NIM A313 (1992) 203, NIM A313 (1992) 345. ISTM A3L3 (1992) 429, N2M A323 (1992) $37 \%$, NJM A398 (1994) 3In, NIMI A342 (1996) 364, NIM A363 (1094) 60, NIML A343 (1904) 258, NIM A343 (1954) 279, NIM 4348 (1994) 444, NIM A355 (1995) 351, FIM A357 (1995) 2T4, PL B358 (1095) 151 , ZPHY A350 (1965) 379 . NIM A371 (1966) 27, snd NDM A371 (1996) 192

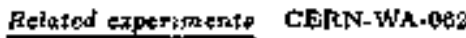

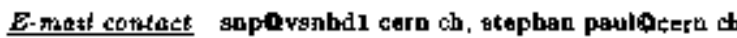
WWW Harte-page hip//vophal cerp th/

\section{CERN-WA-091}

(Propoged Jan 1090, Approwçd Apr 1090, Began dals-taking Jü iggr, Completed dadsmbing Jur 19gd]

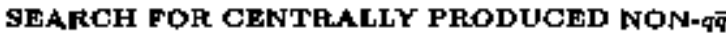
MESONS IN PROTON-PROTON INTERACTIONS AT 450 GeV f BY USING THE CERN R SPECTHOHETER

WA91 COLLAEORATION

ATHENS U - S Abstas, G Vaduladi.

BARl U th INFI, BARl - N DI Barı, F Fını, B Ghudet, V Lebl, A Lacontale, V Maneari, F Navach

BIRMINCHAM U A C Bayea, I N Carney, s Glewer, J P Davit, C J Dodenhof, J B Kinpor, K Norjas. O Vhilalobon-Bsulle, M F Votrubs

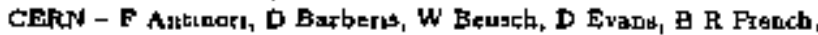
A Jacholkowrkt, A Kurk ( $\gamma$ Spokesperaon), K Kmudoon, J C Laspalle, E Quarcigh

DUBNA - Y Kukhitsky, 5 Maljukow, I Minasbyill, Y Romsnovgky, N Fuserkovich, A Semenov, A Solowsev, G Tehlstehud te

COLLEGE DE TRANCE - M SEne, R SEne

Acceterator CERN-SPG Detector OWEGA.

Beactront

$$
P P \rightarrow P F X
$$

Partictesgatuded mesnse

Brief dexcription A pearch lax aew, noasg states in the wited roglon, $\mathrm{kJtb}$ at least 10 times the stakigtics of the CERN-WA. off experiment Uses a liquid hydroger target Date ana]yar in progreas (July 96)

Jotrnal popers FL B324 (1994) 609, JlC J0TA (1994) 1667, and PL $\overline{6353(1985)} 589$

Related etperiments CERN.WA.07A, CERM.WW-102

E-mati centagt andrew kmktgctm ch

\section{CERN-WA-OB2}

(Froposed Mar 1990, Approved Jul 1990, Began datartakinx Jun 1992, Completed data-taking Sep 1993)

MEASUREMENT OF BEAUTY PAFTICLE LIFETIMES AND HADROPYODUCTION CROSS-SECTIONS

BEATRICEE COLLABORATTON

BOLOONA U \& INFN, BOLOGNA - A Formo, R Gesserod, L Malferran, P Meszant1, A Quaren

CERN - F Anienon, W Beumb, J P Dufry, P Furthaust,

B R Freach, A Kirk, J C Láasalle, M Pasasaeo, V Fyzaboy, G Schulet

DUBNA - S Maljukrov, I Murahvilı, N Rusakovich, A Semenov, A Soldovier

GENOA U \& [NFN, OENOA - M Adimolf, D Barbers,

M Damerı, Q Darbo, R Hurat, P Martınengo, B Deculatı, L. Rrmsi (V Sprkespernan), C Salvo

tMPERAL COLL - D Barray, I Batter, A Dusne, N Jluspoda, D MC Webodale

LEBEDEV INST - M Adsmovicb, Y Alexandron, P Nechaevs, $M$ Znwertyasv

PISA U \& INFN PISA - C Angellm, A Cardin, V Flandano, C Laznerodi, $\mathrm{C}$ Rods

ROME U \& INFN, ROME - C Bnech, F Ceradini, G Capett, A Frenkel, K Harnson, F Lactiva, i Martellated, A Niłall, D Otelsod, G Pentod, E Petrolo, L Pontecorvo, M Torell, S Venezlano, M Vertochbl, I Zenello

ROME U, TOKVERGATA \& INFN, ROME - R Cardar해],

A Di Clactio, $\mathbf{R}$ Samtonico

SOUTHAMPTON US - J G MCENEO

Accelerator CERN.SPS Detartor OMSEGA.PFUMF

Resctions

$\pi^{-}$nocleus $\rightarrow B \bar{B} X$

Particles studized bottom, charm

Briseldexcription An expotimeintal bearch for bosuly particles produced in fxed target hadronve mtaractsons Uates a bugh prapinan Decay Datector and a fast socondary vertax trigger

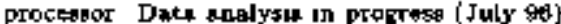

Journol papers N1M A\$15 (192) 95, NP (PROC SUPPL) 2T (1992) 251, NIM A337 (1994) 225, NIMS A351 (1994) 222, NIM A251 (1994) 225, NIM A361 (1995) B06, NIM A368 (1905) 186, NP (PROC guPPL) A4 (1895) 455, Pl. B948 (1995) 256, and PL Bs5a (1965) 563

Reloted experaments FNAL-653, FNAL-T71

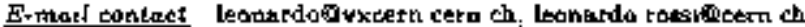

\section{CEEN-WA-094}

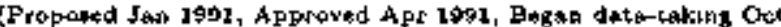
1991, Cotppleted datakaking Nov 1293)

STUDY OE BARYON AND ANTIBARYON GPDCTRA IN SULPHUF-GULPHUR INTERACTIONS AT IOO GaV/F PER NUCLEON

WA9A CCLLABORATION

ATHENS U - 5 A batats, G Vassiliatia

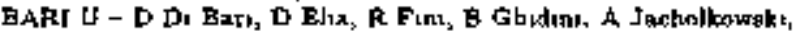
$\checkmark$ Leiuti, R A Locenaile, V Manziri, F Navach

BERGEN U - E Andersen, K Fanebust H Helstenp, $G$ Loyhosden,

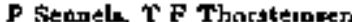

BIRMINGHAM U - J It Carady, 3 P Davies, D Evank,

J B KInson ( $\checkmark$ Spokesperson), A Kırk, K Normen, M Venables,

O Villalobos-Ballie. M F Votruba

COMENIJUS U - J Fiacoute, R Luetars

CERN - F Antinoti, W Beubch, B R French, A K Holtoe,

K Knudgon, J C Lagralle, M Pr5:2800, E Quercigh, K Sararik

KOSICE, IEF - I Boehm, I Kralık, K Fiska, L \$andor, I Urban,

P Zurad

LEGNARO - R A Rre

MADHJD GIEMAT - B de le GTuz, P Ladion do Guerrio

PADUA U1 L INFN, PADUA - A Andrighetto, N Carrar

hi Motando, F Pellogrini, G Segato 


\section{SUMMARIES OF CERN EXPERIMENTS}

CoLlEgE DE FRANCE - M Benayoun, J Kababe, P Leruste, J L. Nerponx, M Sen, R Sene, A Volte SERPUKHDV - V A Kachanev, A V Sangovgky

STRASBOURG, CRN - T Kachelhefier, M E MIchalon-Mentzer, A Mucbilon

TAISSTE U \& INEN, TRIESTE - A Brayar, A Ptazo

Accatatotor CERN-SPS Detector OHIEGA

Reactiots

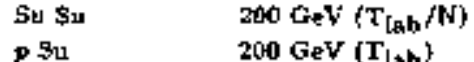

\section{Particles styditell otrange}

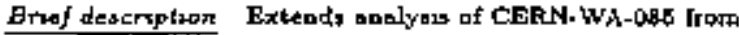
$\$$ w to S S interactions For tbe $\mathbf{3 9 9 2}$ cun the apparalus hos been modified to moarure charged particle spectra (in particulat $p$ and p) with particle idencificsuon wiug an attay of sheon microstrip detectore and a newly upgraded ring imsing Careakav detector

Jourhal papers NP A566 (1994) 499, PE B354 (1995) 178, NP A580 (1805) 317, and NIM A3T1 (1\%6) 22

Retated expertmenls CERN-WA-080, CERN-WH-097

E-mard cortustr jbkghep ph blasin ac ak

\section{CERN-WA-095}

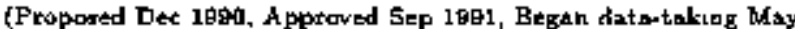
199\$, In progrese)

\section{A NEW SEARCH POR $v_{4} \rightarrow \mu$ OSCLLATIONS}

CHORUS COLEABORATTON

CUKUAOVA U - E Eskut, \& Kays, G Onengut

NIKHEF, AMSTEFWAM - J Kanı, $\mathrm{F}$ G C Otdeman,

J W E Unerwilk, R van Dautzig, C A F J ven der Poel

MHDDLE EAST TECH U, ANKARA - A A Aym, E Pegr,

M Sertn-Zeyrak, R Gever, P Tolun, 31 T Zeyrels

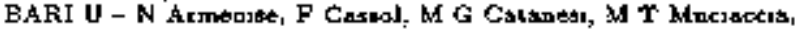

E Radrcion, 9 Simone

HUMBOLDT U, BERLIN - P Lendermann, A Meyer-Sievers, T Pateak, K Winter ( $\checkmark$ Spolrteparion $\}$

BRUSSELS U, IIHE - P hols, M Rruwe, C Mommedt,

M Van der Dancti, P Vilnop, G Wilquet

FERFARA U \& INFN, FERRARA - E DI CRDAs, $C$ LuppI,

S Ituçciard, G Satia

TOHO U - T Kawamura, M Kazuno, S Ogaws, H Shubuye

CERN - R Beyer, J Brubder, M de Jong, J P Fubre, A. Ferterts,

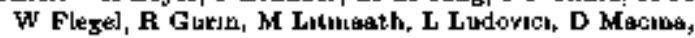
R Meujer Dress, H Metnhard, E Nut, H Dveras, I Panmen.

F Rlctard, A Rosavóy, D Saltebet ó, G Stefanum, R Tzenó,

$C$ Weinbeumer, H Wong, P Zuceliellı

HAIFA U - J Galdberg, K Hapinet

KINKI Uं - H Chilsws

BOCAZICI U - E Arik, I Byrol, A A Meslow

GYEONGSANG NATIONAL U - C H Hahn, H J Jag, DC Kan,

I to Psck, M s Perk, I s Song, C \& Yonn

AICH] U OF EDUCATION - K Kodsma, N Uahids

KOBE L - S Aokt, T Hara

LoUValN U - G Bromjmang, D Favatl, G Gregore, I Herin, V Lemulre

MOSCOW, ITEP - A Artamenow, P Gorbugdv, v Khqvaqdky, V Sbamanor, V Strturnitgky

MUNSTER U, - D Eoneknmper, D Frekers, D Roadenhagen T Halff

NAGOYA U - K Hosbino, M Kobsysalt, Y Kotates, T Kosakı. M Nakamura, T Nakno, K NIn, K Nıwa, Y Obnyanhi, Q Sata, T Toshito

NAPLES U, IFS * INFN, NAPLES S Buontempo, A Ccoco,

A Eredutato, i Fiarlle, F Garuh, F Marcbetri-Staw,

P Mrglozat, V Palladitid, $P$ 5trolin

CSAKA CITY U - K Nakarausa, T Okusgme, T Yoshids

ROME U \& INFW, ROME - A Capore, D DN Peds, S Dn Liberto.

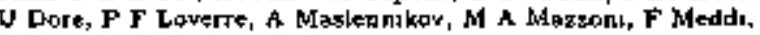

G Piredda, P Fighou, R Sablacesarua

SALERNO U \& INFT, SALEINO - A d, Dartoloweo, G Giella,

G Fomano, G Fosta

UTgUNOMTYA U - Y SBio, I Tezuks

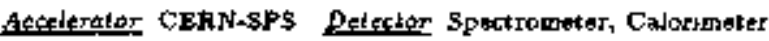

Benctens

$$
\text { or nucleon } \longrightarrow+^{-} \mathrm{X} \quad 25 \mathrm{GeV} \text { (Elab) }
$$

Brief descraptisn The netup connsts of a tarpet rapon, an arcore mascet, a high-precusion calonmeter, and a muon apectrometer Naclapr cmulnop atadk Iorm the \$00-kg mase of the fiducgal target voitume Depayy of shorthlyed particles, such

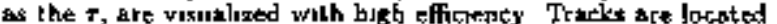
in the omulaun wath bugh-procielan sadilistung flber trackers, and readout with optoslectrontc image intensefiers coupled to CCD comeras, thus permitting tompotersaksured scaning Thb hexagonal arcore magnet pravdes the meaurament af the charge-aign of low energy badrona and muong The bighprecusion salonmets, which is baged on spaghett texhoology. tags the $\tau^{-}$decay by its transwerse momentum imbalance The spectrometer identilies muons and measures thesr momentom and charga $A$ second phose of data taking with new ermulkign stocks is under w'By (July 96 )

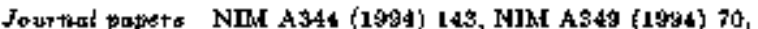
IJMP C5 (1994) 835, NIM A357 (1995) 243, and NIHF A367 (19日5) 367

Related experimants CEFN-Wh-006, FNAL-B09

Ermad contact klana wiaterígern ch

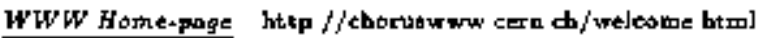

\section{CERN-WA-090}

(Proposed 2991. Approwad 1991, Eagan data-taking Apr 1994)

SEARCH FOR THE OSCILLATION $\nu_{\mu} \leftrightarrow \nu_{\mathrm{z}}$

NOMAD COLLABORATION

ANNECY - G Easmompuerre, J M Gullard, M Gouanere,

I P Mordiburu, L Mogsux, t Feseard, D Sillou, D Verkindt

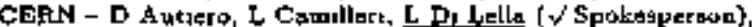

D Ferrere, A Geter, J J Tomea-Cedenas, A Grant, W Huta

L Linnsen, P Nadelec, \& Place, B Pope, C Fods, A Rnbba,

P Stefon, E Tsermeles. F Wtatas

CALABFJA U - L La Fotonda, M Valdeta

DEkTMUND $U$ - D Goppert, C Goessing, D Nardminn,

D Pollowan, B Schmodt, T Wessye, $\mathbf{K}$ Zlaber

DUENA - S Bunystov, O Klımoy, Y Nefedov, B Popov,

S Tereshebenko. S Valuev

FLORENCE U \& INFN, FLORENCE - G Codforto, G Grasisu

巨i Incopial, M Kirsanov, A Lupl, A MarchjonлI, F Martell,

E Penuacchio, C Ruecs, M Vehr

HARYARD U - A Buen, T Dignan, G Foldman, P Hutat, S Mishro

IOHNS HOPKIN'S U - B Elumenteld, I Lang, D M Slede

LAUSANWE U - K Bonams, I Brrd, I Dagaudeas, C Jueph,

M C Ngayen, I P Perzovd, 0 5ozzi, M Steureger, M T Tran,

I M Vieira, M Wharlen

MELBOURNE Џ N fiyet, G Moarhead, C Poulden, M Gevior,

G Taylor, 5 Torey, L Winton

Moscow, INR - 9 Gninenko, A Kovzolev, A Toropin, s Volkon

PADUA U \& INFN, PADUA - M Baldo-Ceolın, F Bobreut,

fi Collastuol, 11 Constalbriso, D Gıbla, A Gugliejmi, 8 Lacaprars,

M Leveder, M Merzetto, G Muat. A Scouza, M Vegonn.

P Zuccon

PARIS, CURIE UNIU V]\& PAFSS, UN]V VII, LPNHE -

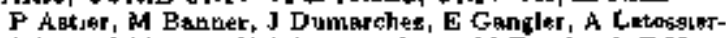

\$çlvop, J-M Eevy, K \$chamaneche, A M Toucherd, F Vandulea

PAVIA If b INFN, PAVIA - P Cattaneo, C Conta, F. Fertari,

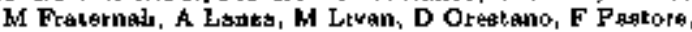

F Pettl, G Poleatlo, A Fumoldi, F Salvatore, V Vercel।

PISA U d INFN, FISA - C Anrelent, V Cavaunn, T Del Prete,

A De Samlo, V Flamulo, C Lazzeron, $G$ Alentom

DAPWLA, SACLAY - A Bsiduseri, J Buruches, J Goset

C Hagner, X Mechain, J P Mleyer, A Pluquet, $P$ Rathoujt.

$T$ Stolarcrik, M Vo, $\mathrm{H}$ Zactone

SYDNEY, ANBTQ - I J Donnelly, K Varrel]

SYDNEY U - S Boyd, L Peate, P Saler, J Ulicha, B Yabaley

UCLA - A Dardeci, R Colatne, T Vinogradove, F Webet

MLSSACHUSETTS U, AMHERST - J D Heraando

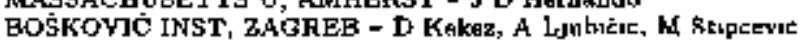




\section{SUMMARIES OF CERN EXPERIMENTS}

\author{
Acceleratos CERN.SPB Delector Spectrometers \\ Reactione \\ $\begin{array}{lc}y \text { nucleon } \rightarrow+\mathrm{K} & 450 \mathrm{GeV} / \mathrm{x} \\ \mathrm{V}_{\mathrm{r}} \text { nucleon } \rightarrow \tau \mathrm{X} & -\end{array}$ \\ Particies attstied \\ Brref descrzption Searche for the oscillation $\nu_{\mu} \curvearrowleft \nu_{r}$ u a \\ Fide band, 10-200 GeY neutrino best Aims at detecting \\ $v_{T}$ harged-ciucrant jaterections by shserving the prodectuon of \\ the $T$ through its varjune decay anoda by taatos of kinematicel \\ criterls The detector recongtructe the event kinemstics \\ It whe the CERN-UA-001 magrat The tartst consicts of \\ 44 drilt chamber planes with a tatal mans of 2 日 tone over \\ a fiducual volume of $26 \times 26 \times 4 \mathrm{~m}^{3}$ It us followed by \\ transition radiation detecipat, by additiopal trackins chambers \\ and by an electromsgatic calorimeter conessting of $87 \mathrm{~b}$ lesd \\ glaeg blockg aud tacluding a preghower detector A badrontc \\ calorumeter rande of iren and geintillator a locatod behupd \\ che electromaxnetuc caldormeter ousiode of tha maghetsc fiek \\ volume $A$ muod detector has two stations of larytares dnft. \\ tube shsmbers on bath urdes of an iron sbaprber The sensitivity \\ of the detector to $(\Delta m)^{2}$ is larger than $1 \mathrm{eV}^{2}$ Taleing data \\ (3aby 96) Expecled to coutimue date takist unt:l the and or \\ 1907 \\ Joursal porpers NIM A372 (1996) 565, and NIM A373 (1905) \\ Related experments CERIN-WWh-095, FNAL-803 \\ Emati corstact lungi di letelasern ch \\ WWW Hame-page htsp / inomadinfa cern h/
}

\section{CERN-WA-102}

(Proposed \$ep 199d, Appraved \$op 1904, Bagan datartakung Ap 1904, In progress)

* SEAFCH FOA CENTRALLY PRDDUCED NONA4 MESONS IN PROTON PROTON INTERACTIONS

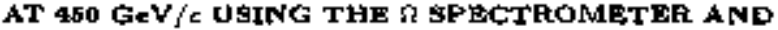
GAMS-4000

WA $1 O_{2}$ COLLABORATION

ANNECY - J P Peugneux, M Pouled

ATHEKS U - M Spyraponlou-Stansuakt, G Vaysuadis

BERGEK U - K liyklebost, I M Olsen

BIRMINGHAM U - J N Caruey, J P Darres, D Evari,

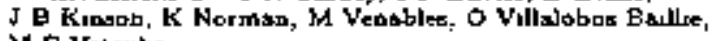
M F Yotruba

BRUSSELS U, IHsN - F G Binon, J M Frere, J P Strod

CERN - W Beusch. B R Froneh, A Jacholkowekl, A K ytk

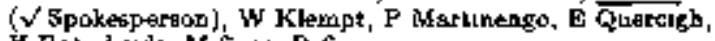
if Rotecheidu, M 'sepe, $\mathbf{R}$ Sent

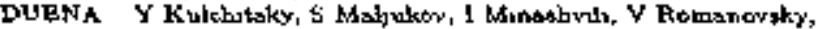

$\checkmark$ Haumientsev, N Fussakovich, A Semenov, A Soloviev, $G$ Tehlatchidge

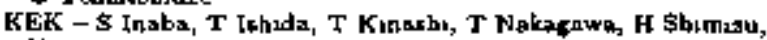

K Takanlab6u, T Tuurv, Y Yayu

UNIVERSITY COLL, LDNDON - G CRone, M EAten

LOS ALAMOS - D Alde, E A Knapp, T Loper

MANCHESTEF U - I Dustdoth, N Lumb, G Snów, $R$ Thounpern

OSLO U - K Danielsan, T Jacobsen

SERPUKTHOV - A V Dalgapalov, S V Dontkav, A V layakin,

G Vhustor, V Kolossov, A A Kondeabov, A A Lednev,

V A Polovilkov, $\$$ A Polyakov, $Y$ D Prokoshkin, $\$$ A \$adovaky,

V D Samoylebko, F id Stage, A V Stannukav, A V Smgorsky,

$V \mathbf{P}$ Sugonyaev

Accelemer CERN-\$PS Detector OMEGA, GAMS4000

Reacteons

$$
\mathrm{D} \rightarrow \mathrm{P} \rightarrow \mathrm{X} \quad \text { 450 GeV/C (P ]ab) }
$$

Partectes aludied meson日

Brtef actcraption Prapases to perIarm two 100 day Jung combung tha multiphntan detection of GAMS-1000 with the chafiged particle detaction of the Omega Spectrometer to wexch for don-4y mesors in the raxtion po $\rightarrow P_{f} X^{D} p_{0}$ at $450 \mathrm{Gev} / \mathrm{c}$ Here, of and p, otand lor the faskst and the glasedt portucle
Lh the lab syotem Particular attention will be patd to the las utstes decaysng 00 no, ph and of $\eta^{\prime}$ The physucs goal is a better understanding of non-perturbetive QCD Taking dats (Jnly 9A)

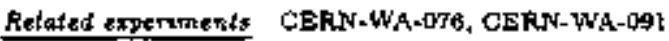

E-magl centact andraw karkegera cb

\section{CERN-LHC-ATLAS}

(Proposed Oct 1992, Dec 1994, Approved Jan 2906, I preparstron)

\section{A TOROIDAL LHC APPARATUS - ATLAS}

\section{ATLAS GOLLABORATION}

Acoeterator CER.V.LHC Delector ATLAS

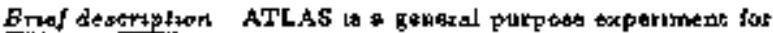
recording the protap-protob collablons at LHC The detectot

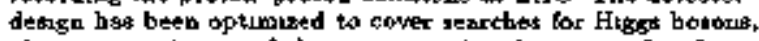
alcernative echemat of the symaretry braking, seerches for

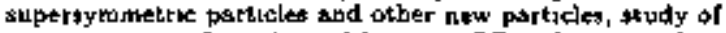
conpoustenegg of quarke and leptont, $C P$ violation in the a vector, measuremepte of the propertic of the third family of quartes, abd more The spparatus inciudte an butio tracking

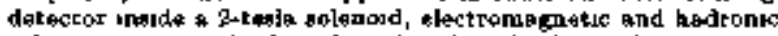
ctorimetere outande the solonwit and In the forward reglons, and brekel and andcap sur-core-torord rquop specteonatet Under conelructian (July 96) The collabarstian conkst. ol mare

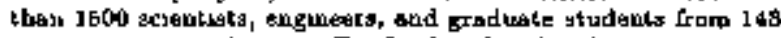

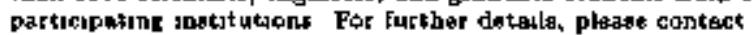
the Spokesperson, Dr Peter Jenni [CERNy, der the Deputy Spoksosperson, Dr Tarsten Akęsopn [Luad U]

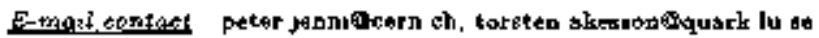
WWW Home page bitp //arlasinfo cern ch/Atlas/Wolcone htjol

\section{CERN-LHC-CMS}

(Fropored Oct 1992, Approved Jas 1906, In prepasation)

THE COMPACT MUON SOLENOID - CMS

\section{CMS COLLABORATION}

\section{Acceletotor CERN-LHC Detector CNS}

Bruf degcripton CMS it a general purpose detector desgued to run at the highest Iuminosity at the LHC It has beek opt I razed for the aearch of the Standerd Model Higgs boaon over a mose range from 90 GeV to 1 TeV, bot it also allows datection of a wide range ol posspble eigretures from sllerbatsve electroweak symmetfy breaking mochamsms Furtbertoore, it J6. well edapted for the study of top, beatyly and tad physics at lower lumiagatieg and will cover soverel binportesu sepscte of the hesry un phyescs pratram CMS ideatilies and mesaures muon: photons and olectrons with high precisson At the cort of the detectot sits a large soperconduct|ng soienrod genassting a uniform magnetk field of $d T$ The acrong magathe field allows a compact desigh for the mugn spect rometer wishout compromising the momentum resolution The central tracker

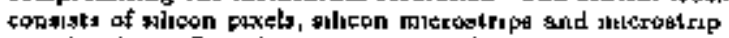

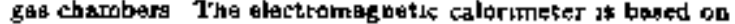

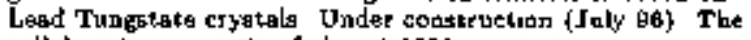
tallaboration convitts of almoth 1500 gchentsts, englneers, and

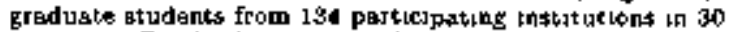
covotries. For further detsts, please contact the Spokepperson,

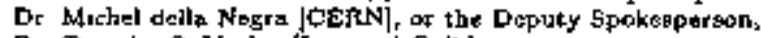
De Tejuder $S$ Virdee [Impensil Coll

E mati gontact michel della neqraficera ch, tepunder virdeequern ch

WWW Home page

bttp $/ /$ cmstafo aera ch/cmsinto/Wolcome htmal 


\section{Other CERN Experiments}

Luted hare are tomo athar CFRN mptrubante of poterent so the particle physics communty Fud more detalla aboul these propects dolune; is the SLAC's EXPERUMENTS dalabase (see p 3), or contuct the spokespereors Chack also the anmuse] report 'Expenments at CBR.Y', whuch lists all the current CERF expr|mant and R\&D praject Find additional information at

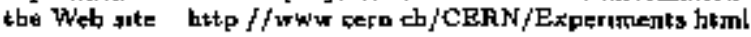

CERN-EMU-0I 1 (Takins dtata)

STUDY OF PARTICLE PRODUCTION AND NUCLEAR FRACMENTATION IN RELATIVISTIC HEAVY ION COLLISIONS IN NUCLEAR EMULSION

spokepperson PL Jain [SUNY, Bufalo]

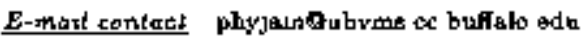

CERN-EMU-012 (Taking Asts)

PARTICLE PRODUCTION, DENSITY FLUCTUATIONS, AND BREAK UP OF DENGE NUCLEAR MAT. TER IN CENTRAL Pb $A$ \& AND Pb Pb INTERACTIONS AT 60-160 A G V

\$potespersan I Otterlubed |Luad U|

E maxt cuntact mgar ollerluudohosufy lu os

\section{CERN-EMU-019 (Campleted dato-taking)}

INTERACTIONS OF 100 GAV/NUCLEON 20\% PB MUCLEI IN EMUL녕 CHAMBERS WITH COPPER AND LEAD TARGETS

By KLMM Colloboration

Spokeqperuen W Waiter [Cracow, INP.Exp]

E-mat contect wwalterwivelel iff edu pl

CEFW-EMU-014 (Taking dats)

STUDY OF MULTIPLIETTY AND ANGULAR CHAR. ACTEFISTICS IN Pb + AINTERACTION AT 200 A $\mathrm{GeV} / \mathrm{c}$

Spokegperson D Ghosh [Jadaypur U|

CERN-EMC-015 (Taking data)

INVESTIGATION OF CENTFAE Pb PB INTERAC-

TIONS AT ENERGLS OE 160 GEV/NUCLEON WITH THE HEEP OP THE HMULSION MAGNETIC CHAMBER

Spskaparson K A Kotalnakov [L towdev Inat]

E-mat contact kotelntiach han mak su

\section{CERN-EML-016 (Talking dat $x$ )}

I INTERACTIONS

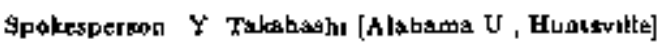

$E$ ment contect iakahashugrabl msfo nasa gov

CERY-EMU-0I7 (Coupleted data-taking Dec 1094)

FRAGMENTATION OF Pb PROJECTILES AT SPS ENERGIES

Spokegpersen wolfzeng Heinrach [Shgen II।

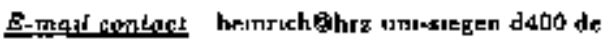

CERN-FMU-O18 (Takjap data)

EXPOSURES OF CRSg STACKS TO LEAD IONS AT THE CERN-SPS

Spoksperson G Giacomell [Bologat U and INFN, Balognal

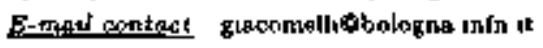

CERN-EMU-01日 (Takug dasa)

NUCLEAR FRAGMENTATION INDUCED BY RELATIVISTIC PROJECTILES \$TUDIED IN THE 4T CONFIGURATION OF PLASTIC TRACK DETECTOHS

Spokeaperson H A Khan [Fustech, Ialsmabadj

CERN-EMU-020 (Takug data)

p-INDUCCBD FISSION \$TUDLES WITH PLASTIC

TRACK DITECTORS USINQ 4\% GBOMETRY

\$pokepermon H A Khan |Finaler.h, lslamabad|

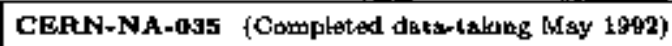

STUDY OF RELATIVISTIC NUCLEUS-NUCLEUS COLLISIONS

By NA36 Collaboration

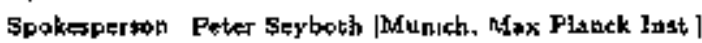

E-mot contact posedroumpirh mppmu mpg de

WwW Fome page bttp//hppat9-1 cem ch/nass haml

CERN-NA-0as (Completed datamtaking May 1992)

STUDY OF HIGH-ENEREY NUCLEUS-NUCLEUS

INTERACTIONS WITH THE ENLAKGED NALO DIMUON SPECTROMETER

Spokeperson Lank Klaberg [Ecole Polytechaque. LPNHE]

E-mal contact lovis klubergticern ch

WWW Horde page batp //www cekn ch/NA3s/

CEFW-NA-ads-2 (Taking dats)

INVESTIGATIONS OF THE COHERENT HARD PHOTON YIELDS FROM BO-a00 GeV/c $e^{ \pm}$IN STRONG CRYSTALLINE FEELDS DF DLAMOND, SI, AND Ge CRTSTALs

Spokesperaon EcLt Uggerho] [Aarhus U I

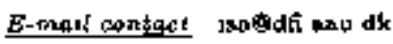

CERTN-NA-044 \{Taking data\})

A FOCUSSING SPERTROMETER FOR ONE AND TWO PARTICLES

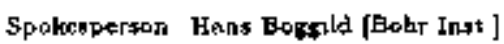

E mati cortact bogtaldobivax ubi dk, hane baggldacern th

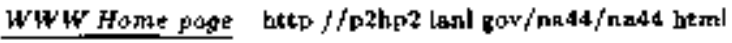

CERN-NA-04B (Completed datastaking Apr 1995)

STUDY OR LOW-MASG ELECTRON PAIR PRODUC. TJON IN HADRON AND NUCLEAR COLLISIDNS AT THE CERN פPS

By CEREs dollaboration

Sprokespersan torhak T'setruys [Wedmann Inst ]

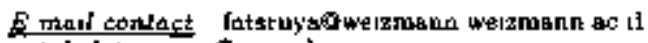

itzhak toersuygbora ch

WWW Hame-poge http //cteresb physi trol-heldelberg def 


\section{SUMMLARIES OF CERN EXPERIMENTS}

CERN-NA-049 (Twkınk dats)

LARGE ACCEPTANCE HADRON DETECTOR FOR AN INVESTIGATION OF PB-INDUCED REACTIONS AT THE CERN SPS

Spokespergon Reinhard Stock |Frankfurt v|

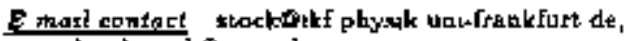

rembard stock focern th

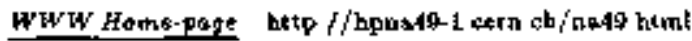

CERN-NA-OS2 (Tukng data)

STRANGELET AND PAFTICLE SEARCH IN Pb Pb COLLISIONS

Spokespermon Ktaut Pretzl [Bern U]

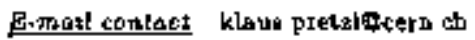

WWW Homectgage bitp $/ /$ wwew lhep unite ch/newmases/

CERN-NA-0ss (Takjng data)

ELECTROMAGNETLC DISSOCIATION OF TARQET

NUCLEI BY ${ }^{208} \mathrm{~Pb}$ PROJECTILES

Spokerperson J C Rill |lowsa Stata U ]

Emati contlact fullatitaseale adu

CERN-NA-0S4 (Taking datB)

DETERMINATION OF CROSG-SECTIONS OF FAST. MUON INDUCED REACTIONS TO COSMOGENIC RADIONUCLIDES

Spokesperson E Nolle [Munch, Tecb U |

CERN-NA-0SS (in proparation)

INVESTIGATION OF FAST NEUTRON PRODUCTION BY 100 TO 250 GOV MUON INTEFACTION ON THIN TARGETS

Spokesperron N Mascatenhera [Cal Tech]

CERN-NA-056 (ID pemparation\}

MEASUREMENT OF PION AND KAON FLUXES BELOW GD GRV/e PRODUCED BY 450 GWV/C PROTONS ON A BERYL LIUM TARGET

By SPY Collaboration.

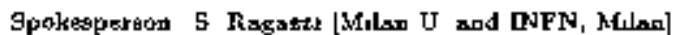

WWW Home-poge http //Ww' cern chis / ASB/

CERAN-PS-185-3 (In preparation)

A MEASUREMENT OF DEPOLARIZATION AND SPIN TALNSFEA IN $\bar{p} p \rightarrow \bar{\lambda}$

Syokesperson

Klaus Roehrich (Juldch, Forachungszentrum] WWW Honte page

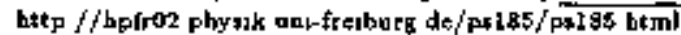

CEAN-PS-189 (Completed dat8-taking Dec 1992)

HIGH PRECISION MASS MEASUREMENTS WITH

A RADIOFREQUENCY MASS SPECTROMETER APPLICATION TO THE MEAGUREMENT OF THE $p \bar{p}$ MASS DIFFERENCE

Spokeperson Catherune Thubaull |Orsay, CSNSM|

Emeal contast catherne thasultacess ah
CERN-PS-194-s (Taking data)

MEASUREMENT OF STOPPING POWERS AND SINGLE IONIZATION CFOSE-SECTIONS FOR ANTIPRO. TONS AT LOW ENERGIES

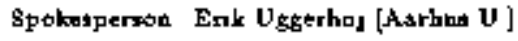

CERN-PS-200 (Thklig detn)

CAPTURE, ELECTRON COOLING, AND COMPRESSION OF ANTIPROTONS IN A LARCE PENNING TRAP AND PHYSICS EXPERIMENTS WTTH AN ULTRA-LOW-ENERGY EXTRACTED ANTIPROTON BEAM

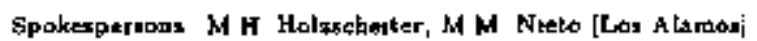
E-mati contact mhborland gov

CERN-PS-30S (Compieted data-takidg Dec 2993) ANTIPROTON INDUCED FISSION AND FRAGMEN. TATION OF NUCLEI

Spokseppersion $T$ vop Egidy [Murach, Tech U]

E-masi contocf th $\times$ egady sphyalk turmperchen de

CERN-PS-20\$ (Taking data)

LASER SPECTROSCOPY OF ANTIPROTONIC HE. LIUM ATOMS

By PS208 Collaboration

Spoktopergou T Ysmataku [Tokyo U, LNS]

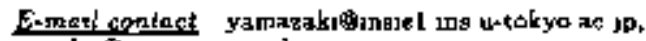
esdataryecra cern eh

WWW Home page http / / wwe cern ch/LEAR_PS205/

CERN-PS-200 tCompletod dato-Laknos Sep 1993)

MEASUREMENT OF THE $\bar{p} p \rightarrow \overline{\bar{\Omega}} \mathrm{T}$ CHARGEEXCHANGE DIFFERENTIAL CROSS BECTION By Cex Collaboration

Spekeeptracn Frateo Brbdatonte !Trebte U sid INFW, Theatel Famad contact franco bradabiante gern th

CERN-PS-207 (Takıng dsta)

PRECISION MEASUREMENT OF THE ENERGLS AND LINE SHAPES OF ANTIPROTONIC LYMAN AND BALMER TRANSITIONS FROM HYDROGEN AND HELIUM ISOTOPES

Spokesperwn D Gotta [Julseh, Forgcheogenentrum]

CERN-PS-200 (Taking datck

NEUTRON HALO AND ANTIPROTON-NUCLEUS POTENTLAL FROM ANTIPROTONIC $x$-RAYS

Spoke日person J Jastrzebskı |Warsar U, Heavy lou Labl

CERN-PS-210 (Taking dala)

ANTIHYOROGEN PRODUCTION IN D Z INTERACTION

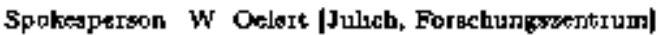

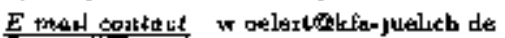

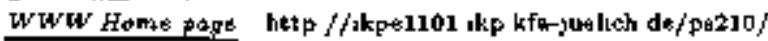


CERN-PQ-211 (In preparation)

EXPERIMENTAL STUDY OF THE PHENOMENOLOGY OF SPALLATION NEUTRONG TN A LARGE LEAD BLOCK

Spokesperzon C Rubbia [CERN]

E-mati contact carlo nubbatarn fb

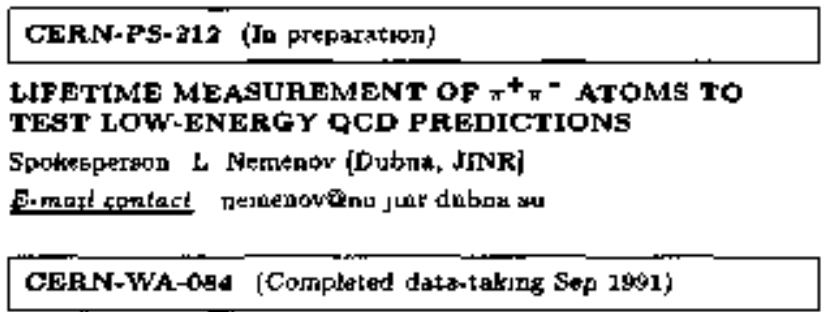

STUDY OF THE PRODUCTION AND DECAY PROPERTIES OF BEAUTY FLAVORED HADRONG

Spokespereon Cuseppe vartellotti JRome U and INFN, Roma!

E.maxt sontact martellothioromal info it

CERN-WA-00T (T'kkine data)

STUDY OF BARYON AND ANTIBARYON SPECTRA IN Pb Pb INTERACTIONS AT $160 \mathrm{G}$ V/C PER NU.

CLEON

Spokesperson Emanuele Quercigh [CERN?

E.mint! contact emlemuele quereyghticerb ch

$$
\text { CEFT-WA-deB (Takung dats) }
$$

LARGE ACCEPTANCE MEABUREMENT OF PHO-

TONS AND CHARGED PARTICEES IN HEAVY ION

REACTLONS

Spokseperaon Haw H Golbrod (Darmatedt. GSI)

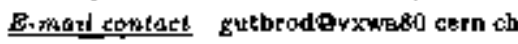

WWW Home pogte http / www cern ch/WA9g/Welcome btml

CERN-WA-099 (Completed dato-taking Apr 1996)

MEASUREMENTS OF PAIR PRODUCTION AND

ELECTRON CAPTURE FROM THE CONTINUUM IN

HEAVY PAFTICLE COLLISIONS

Spokesperion S Datz [Oak Rridge]

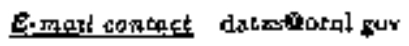

$$
\text { CERIN-WA-096-2 (Takıng data) }
$$

CHARGE CHANGING COLLISLONS, ENERGY LOSS, AND EM NUCLEAR REACTIONS OF 1\$0 GAV A $200 \mathrm{~Pb}$

Spokekpersan \$ Datr [Osk Rudga]

E.montcontact datzationl gov

$$
\text { CERN-WA-101 (Taksog data) }
$$

BTUDY OF VARIOUS PROCESgES WITH A $160 \mathrm{GEV} / \mathrm{c}$ PER NUCLEON PB BEAM

Spokegpereon Yudonk He [UC, Betkeky]

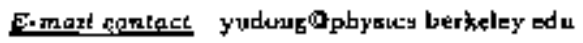

\section{CERN Future Plans}

CERN's main project for the statt of the next mellennium is the LHC proton-protion colluder in the 27-kuldinter LEP tunnel, curreply orchedulad to begin experimenta un 2005 Beewdes the LHC, the ongong program also contsnues CERN'в tradition of

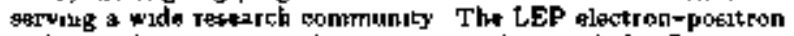
colluder, after six yearis of rusning at and around the $\boldsymbol{Z}$ resouance,

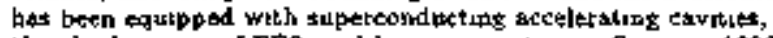
thereby becoming LEP2, and began operating in Sum 1996 Bt 8 collasion naetgy of $J 61 \mathrm{GeV}$, the threshold to produce paurs of $w$ bosons The oflitsion enefsy of LEl'z it being furlher increased a sdduthons auperconducheg cavities are added The collidet ta echredulect to operete till the ond of 1999 AC the SPS proton

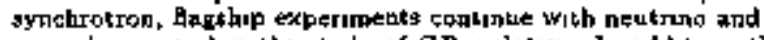
muen beame and in the siudy of $C P$ violalion In addition, the besivy on program at the SPS serves a large range of experments for a sepsrate resesrch community Whale th LEAR low energy

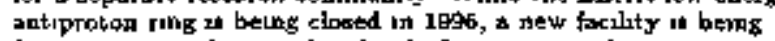
foreseen to supply $\mathrm{a}$ modest level of antiprotons for experrments on 'trapped' astipartides The ISOLDE on-line Lsotope separator, sterved by the 1 GeV Eoogter aynehrotion, cetere to yes suothar group of regeswehers 
SUMMARIES OF CORNELL EXPERIMENTS

\section{CESR Experiments}

\section{CESR-CLEO}

(Proposed 1976, Approved 1877, Began data-katurge Oct t879, In progretst)

\section{THE CLEO EXPERIMENT AT CESR}

\section{CLEO COLLABORATION}

CAL TECH - B Barisb, M Chadha, S Chan, o Ejgen, I S Milled, C OrOrady, M \$schmidtker, J Urhoum, A J Wetntitero,

$P$ Wutrthwe्tin

CARLETON U - K W Edwards

COLORADO U - R Baleat, B H Behrems, $K$ Cho, W T Fotd, H Park, P Rankin, I Foy, If G Smith

CORnELL U - J P Alexender, C Bebek, E E Berger

$K$ Bertkeltran, K Bloom, D o Cannel, H A Cho, D M Colfman,

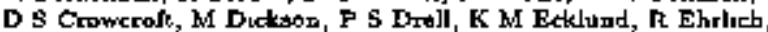
R. ElıB, A Folased, P Gaddarev, \& S Gedlk, B Gittelman,

SW Gray, D L Hartıll, B K Hells]ey, P Hapton, S L Toner

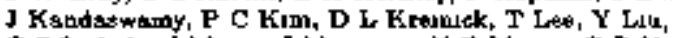

G 5 Ludwig, J Masur, J Mevured, N E Mistry, C R Ng.

E Nordberg, M O Ogx, I R Patterson, D Petersañ, D R R ley, A Soffer, B Va]ent-Spaght, O Werd

FLORJDA I - M Athadas, P Avery: C D Jose, M Lohner, C Frobeot1, J Yaltom

HARVAFD U - G Brandenburg, F A Ertere, Y Gas. D Kım R Wilson, H Yemampkr

HAWAII U - T E Browder, F Lו, Y L $\rfloor_{1}$ J Rodnguet

ILLINOIS U, URBANA - T Bergfeld, E I Eigenstetb, J Emst, G E oladding, O D Golltn, R A Hoos, E Johnyon, M Marab, M Pallmer, M Selep, J J Thistar

MCGILL U - A Bellerive, D 1 Btitton, IR Jasicek,

D [ MacFarlene, $K$ W MeLeen, $F$ M Pate]

iTHACA COLL - A. I Gador

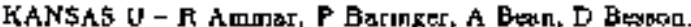

D Coppage, C Dariog, R. Dav's, N Hancouck, S Kotov, I Kravthenkn, N Kwats

MINNESOYA U - S Apderton, Y Kubota, M Lattery, S Eee,

I I O'Nerli, s Petton, RA Poing ( $/$ 'spoke日person). T Riobite, $\checkmark$ Seyirov, A Smitb

SUNY, ALBANY - MC S Alsm, B Atbar, Z Lthg, A H Mphmegd, I] Severin, 9 Timm, $F$ Wuppler

OHIO 5TATE U - A Ansglassow, J E Dubosecd, D Fujino, K K Gan, T Hart, K Fanscheld, it Kagan, R Kasp, J Lee.

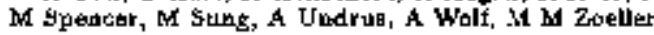

OKLAHOMA U - B Nemat, \& J Rachleh,, W R Rons, P Skubre, M Wood

PURDUE U - M Bishai, J Fast, E Gerrdi, I W Hiason, N Menon, D H Milker, E I Shousla, I P I Shupeey, M Yurko

ROCHESTER U - L Gibbod, S D Johneob, Y Kwan, S Roharts, E H Thorndike, L Tie्r

SLAC - C P Jeagop, K Linge], H Marakka, M L Per]

S F schnfinar. R schindler, D Ugalın, $R$ Whag, $X$ Xrou

SOUTHERN METHODIST U - T E COAD, V Fudoyer,

I Koralkow, Y Maravin, I Narsky, V sbelkov, I Staeck. R Stroynowsti, I Volobues, I Ye

SYRACUSE U - M kitusto, A Rfingr, P Frascom, M Gao bi Coldberg. R Grewna, D Ho, N Horw/t2, S Kopp, G C Monet, F Mountern, Y Mukhin: S Schuh, T Skwarajkkl, S Stoue, $G$ VIethaser, $X$ Xing

UC, SAN DIEGO - D M Aner, D W Blus, W S Prower, G Maetk H P Paay, V Sbarma, lis Slyerta

UC SANTA BARBARA - I Gionberg, R Kutschke, D J Lange,

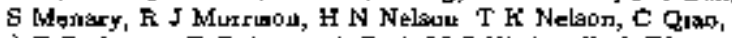
I Rrchmen, D Robert6, A Ryd, M S hijtherell, A Wu

VANDERBILT U - J Bartedt, S E Goarue, V J Jud, 9 Markas

VIRGINIA TECH - R Godang, K KtnoBhita, T C La,

$P$ Poraranowski, S Schreak

wayne Siate U - G Bobverm, D Cinabro, L Pereta, G sun, G J Zhou

Acosterator CESh Eetector CLEO

\begin{tabular}{|c|c|}
\hline$e^{\frac{1}{4}} \varepsilon^{-} \rightarrow$ hadreng & $90-120 \mathrm{G}=\mathrm{V}$ \\
\hline$t^{+} e^{-} \rightarrow t^{+} t^{-}$ & " \\
\hline$e^{+} e^{-} \rightarrow \mu^{+} \mu^{-}$ & - \\
\hline $\begin{array}{l}e^{+} t^{-} \rightarrow e^{+} e^{-} \text {bodrons } \\
e^{+} e^{-} \rightarrow \tau^{+}{ }_{T^{-}}\end{array}$ & - \\
\hline
\end{tabular}

Earteles stadited $\mathrm{Y}(15), \mathrm{T}(2 S), \mathrm{Y}(3 S), \mathrm{T}(4 S), \theta_{1}, D^{+}, D^{6}$. $D_{s}^{+}$, chatmed-baryou

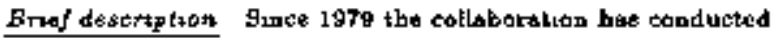
studies of $b, c_{1} r$ and $\gamma 7$ phyejer in $e^{+} e^{-}$interactions pens $10 \mathrm{GeV}$ Current topscs include determination of the CKM pBrameters and the Standard Model teats in decays of hexvy Hawors, as woll as CLD tests in a vancty of precenten Surcessive

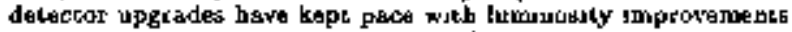
to the Cornell Electron Stotage Rung (CESR), which has deliv. eresd aver $6 \mathrm{f}^{-1}$ or integrated lumposity to date The CLEOII detector (propased 1863, spprowed 1954, operational ance 1980) consista of drilt chambets for trackubs tharged particles and measursug d $5 / \mathrm{d} x$, sime-of-flight counters, o 7800 -element

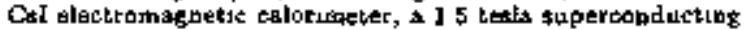

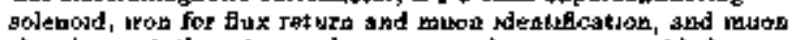
chambers $A$ three.|ayer slicon vertex detector was added Ia Lte Fall of 1995 Taking datn (July 96) A major upprade, the

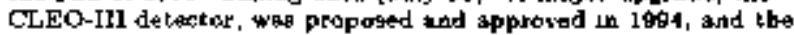
enstallation 4 planned for 1998

Jowrthat popers PRL 44 (1980) 1I08, PRL 45 (1980) 219, PFL 46 (168) 34, PRL 46 (1981) 8B, PRL 48 (1981) 1181, PRL 49 (1982) 1970, PRL 49 (1932) 367, PRL 48 (1982) 610. PRL 48 (1962) 617, NTM 211 (1983) 47, PL B122 (1983) 317, PRL 50 (1983) 807, PRL 50 (1983) 877 , PRL 50 (1089) B8I PRL 51 (1983) 347, PRL 51 (J983) 634, PRL 51 (1989) 1139, PRL 51 (198s) 1145, PR D2T (1283) 475, PR D27 (1983) 1665, PL B 137 (1984) 2JT, PRL 52 (1984) 789, PRL 52 (1984) 1084, PRL B3 (1894) 26, PRL 53 (1864) 1200, PR Dag (1984) 1285, PR D30 (1984) 1433. FR D30 (1984) 1990, PR D30 (1984) 2278, PRL 54 (1985) 381, PRL 54 (1945) 1894, PRL 55 (1985) 923, PRL 55 (1985) 1248 PR DaI (1985) 2161, PR DS1 (1985) 2586, PR D92 (1985) 2294, PR D32 (1985) 2468, PRL 56 (1968) 601, PRL 56

(1986) 1222, PRL 58 (1986) 1593, PRL 56 (1986) 2676, PRL 56 (1096) 2781. PR DSt (1986) 300, PR D34 (198A) 805, PR D34 (1986) 3279, PL B183 (1987) 429, PL B191 (1997) 319, PRL 58 (1987) ] 193, PRL 58 (1987) 30?, PRL 55 (1987) 1814, PRL 59 (1987) 22, PRL 59 (1985) 107, PRL 59 (1987) 1989, PR D35 (1997) 19, PR D35 (1989) 1081, PR D35 (1967) 2747. PF Das (1947) 3533, PR D36 (1987) 680, FR D3E (1987) 1289, PRL

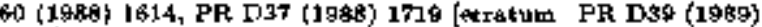
1471) PR D98 (1988) 2679 [erratum PR D40 (1989) 170]|, PL B22s (1989) 470, PL B224 (1989) 445, PL B220 (1989) 192, PL A22A (1989) 401, PEL 62 (1989) 8, PRL 62 (1989) BE3, PFU 62 (1969) 1240, PRL 62 (1939) 2293, PfसL 62 (19k9) 2436, PRL 6a (1989) L687. PR D.3 (1458) 3524, PR De0 (1080) 283. PR D40 (1980) 712 [erratum PR D40 (1989) 3790), PL E243 (1900) 195, PL B261 (1900) 223, PRL 64 (1990) 16. PRL t4 (J9F) 2117 PRL 64 (1800) 2226. PRL 6S (1990) 1184, PRL 65 (1990) 1531, PRL of (1990) 2642, PR Dsl (1996) sus, PR D\$1 (1990) 774, PR 042 (1990) 1401, N1M A302 (1991) 261 PFL 65 (1991) 1592, PRL 67 (1991\} 1696, PR D43 (1991) 65] PR D43 (1991) 1415, PR. D43 (1991) 2836, PR D\$3 (1991) 359\%, rR D44 (1991) 593, PR. D44 (1991) 3383, PR D44 (1991) 3394, NIM A330 (1992) to, PL B263 (1692) 161, PL B291 (J992) 486, PL A294 (1992) 189, PRL 68 (1992) 127S, PRL 6E (1992) 1279. PRL 69 (1992) 2041, PRL 69 (1992) 2016, PRL $\mathrm{EQ}$ (1982) 3278, PRL 69 (1992) 3610 [eratum PRL 7I (1953) 3395], PF D4s

(1992) 1, PR D45 (1992) 21, PR D45 (1992) 752, PR D45 (1992) 2312, PH D45 (1992) 3965, PR D45 (1992) 3978, PR D4E (1992) 4822, WPL AB (J993) 8B9, PL B363 (1993) 37T, PL B317 (1983) E47, PL BS19 (1993) 365, PRL 70 (1993) 139, PRL 70 (1993) 1207, PRL 70 (1993) 2681, PRL 70 (1993) 3700, FFL T1 (1993) 674, FRL T」 (1993) 131 L, PFL 71 (1993) 1680, PRL 71 (1993) 1791, PRL 71 (1093) 1973, PRL 7) (1993) 239], PRL 71 (1993) 3070, PRL 71 (1093) 3265, PRL 71 (1903) $\$ 269$. PRL 71 (1993) 3922, PRL 71 (1993) 41।1, PR D47 (1993) 791, PR D47 (1993) 3671, PR D48 (1998) L007, NIM A345 (1994) 429, NiM 4351 (299d) 19, NiA A341 (1994) 43, PL B323 (1904) 219, FC B324 (19日4) 255, PL E325 (1984) 257, PL E335 (1994) 236 (erration PL B342 (1995) 453). PL B337 (1894) 405, FL B94A (1996) 120, PL 8340 (1994) 194, PFL 72 (1924) 1406, PRL 72 (1994) 
1972, PRL 73 (1994) 2328, PRL 72 (1994) 3762, PFL 73 (1994) 934, PRL 73 (1994) 1079, PRL 73 (1994) 1472, PRL T3 (1994) 3890, FRL 73 (1994) 3503 [erratum PRL 74 (1995) 30901, FR D49 (1994) 40. PR De9 (J194) 5690, PR D49 (1994) 5701, PR DS0 (1994) 43, PR D50 (1994) 1884, PR D60 (1994) 3027, PR DSo (1094) 4285, PR D50 (2094) 54s4, NTht A3ea (1995) 68, PL B34 (1995) 435 [erratum PL B34T (1995) 469]. PL B350 (1995) 256, PL B356 (1905) 580, PRL 74 (1905) 2885, PRL TA (1995) 3113 (erratan PRL 75 (1995) 4155], PRL 74 (1995) 3331, PRL 74 (1995) 3534, PRL 75 (1995) 624, PRL 75 (1995) 785, PRL 75 (1995) 3232, PRL 75 (1995) 7904, PRL 75 (1905) 3009, PFL 75 (1998) 4364, PR D51 (1985) 1014, PH. DB1 (1995) 2053, PR D52 (1995) 2656, PR. D52 (1995) 2661, PR D52 (1905) 4890, PL B38s (1966) 431, PL B369 (1996) 186, PL D373 (1986) 281, PL B373 (1996) \$94, PRL 76 (1996) 1570, PRL 78 (1996) 2637, PRL 76 (1996) 3065, PRL 76 (1998) 9698, PRL 76 (1996) 4L18, PR D6a (1996) L013. PR D53 \{1996) 1039, PR D53 (1906) 4734, PR DS3 (1996) 6033, and PR D53 (J996) 6097

$E$ matl conkuct rapesmase les corsell ed u WWW Home-pege hato $/ /$ Wh las aernell edu/

\section{CESR Future Plans}

The Coratll Election Storage Fung (OESR) p curremly deltvenug pesk lunudoeitisg of $1 \times 10^{32} \mathrm{~cm}^{-2}-1$ Is 1908 , operatione will shut dowa for desertor and machine upgrades that will enable

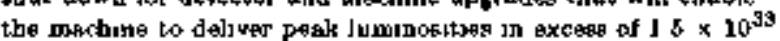

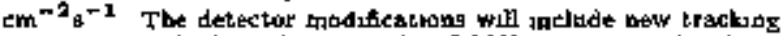
dences and $\mathrm{a}$ bigh performance faat RJCH detector wath $\pi / K$ weparation out to the bunemat,ic limit for $B$ meson daughters When CESR sterts running agann in lale 1998, the physica focus of the upqrate will be rath $B$ decays and $C P$ vinlation $R \& D$ la currenuly uidetway to further intrease the luturioelty of the storage ring to $10^{34} \mathrm{~cm}^{-z_{3}-1}$, and the CESH/CLEO plare promise a rich physics program well inta the dext century 


\section{SUMMARIES OF DESY EXPERIMENTS}

\section{DESY Experiments}

\section{DESY-DORIS-AFGUS}

(Proposed 1978, Approved 1979, Began batantakug \$ep 1962,

Completed dats-tsileng Oct 1992)

\section{ARGUS - A NEW DETECTOR FOR DORIS}

\section{ARGUS COLLABORATION}

DEAY - H Albrecht, T Hamacher, A P Holmang, T Kirchbot,

R. Manke], A Nau, 5 Nowak, D Ressing. H Schroader

( $\checkmark$ Spokespergon), H D Schulz, M Walter, R. Wurth

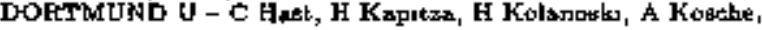

A Lange. A Linduer, ks Schieber, T Slegmund, $\mathrm{H}$ Thurn D Toepler, D Wegenar

DRESDEN, TECH U - C Frankl, M Sethmidter, M Seluramnt,

K R Sthubert, F. Schw'irz, B Spasm, F. Woddı

ERLANGEN U - K Reim, i wagenar

HAMBURG $U$ - F Eekmann, H Kulpers, 0 Mas, R Munde,

T Oest, $R$ Reiner. W Schimudt.Parzefall

HEIDEL BEPS t), IHEP - J \$ti*w*, 5 Werqer

HEIDELEERG, MAX PLANCK INST - K Ehret, W Holmans,

A Huepper, K T Kuoepfle, J Spengler

IPP, CANADA \& MCGLLL U \& TORONTD U - P Kringn,

D B Macparlape, I D Prentice, I A B 9sul], $K$ Trameriudaki, R Van de Watet. T S Yoon

KA.RLSRUHE to - M Scbriendar, S Wealer

GTEEAN INGT, LJUBLJANA \& LJUBLJANA U - A Bracko, G Kernet, P Krizen, E Kriznke, G Medin, T Podobalk, T Zıvbo MOSCOW, ITEP - V Batagirts, $\mathbf{S}$ Barguk, I Betynev, R Chrstor,

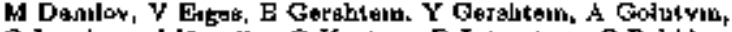

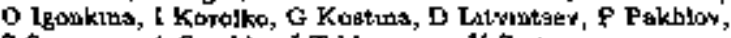
S Semenov, A Sulzkko, I Tikhomirov, Y Zsitgey

Accelerator DESY.DORIS-III Detector ARGUS

\begin{tabular}{|c|c|}
\hline 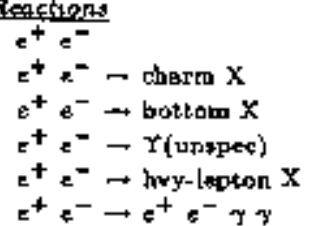 & $\begin{array}{c}\text { B 3-10 } 6 \text { GeV }\left(\mathrm{E}_{\mathrm{CO}}\right) \\
" \\
" \\
n \\
n \\
n\end{array}$ \\
\hline
\end{tabular}

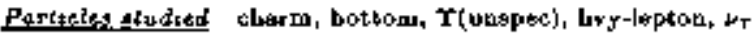
Brtef ditseraption Studles b. and cequask physirs: the $r$ lepton

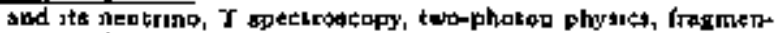
teation of quarks and gluong, and starches for bew partscles The detector conjist; of * slicon ntwi detector agd \& mucrovertex deift chember aurtounding the beam plpe, \& tracking chaunbet. shower tand TOF coupters, solenold rmbs, and a law ge iron yoike Upgradod in 1890/G] Dats anatyya in progrens (July 96)

Jourtiol popets NIMf 163 (1976) 77, NIN 195 (1982) 475 NDM 205 (1923) 126, NIM 216 (1983) 36, NIM 217 (1983) 153, PL B13d (1984) 137, PL B135 (1984) d98, PL B 148 (1984) IIl, NIM A236 (1965) 26, NIM A237 (1985) 464, PL B160 (1985) 235, PL B153 (1985) 343, FL H154 (J985) 452, PL El 58 (1885) J34 ZPHY C28 (1985) 45, PL B157 (1985) 326, PL B158 (1985) 525 .

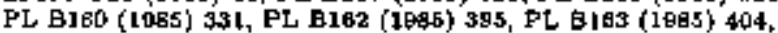
ZPHY C29 \{1985\} 167, NIM A249 (1966) 27\}, NIB A25' (1956) 384: PTE 2 (1986) 36, PAL 56 (1986) 549, PL BLA7 (1986) 369, 2PHY Cs1 (19B6) 181, PL B179 (19B5) 393, PL B170 (1986) 403, PL B182 (1986) 95, ZPHY C33 (1986) T, ZPHY Cs3 (L (1967) 228, PL BIE7 (1987) 425, PL B192 (1987) 245, PL BJe5 (1987) 102, PL B195 (1985) 307, PL B196 (1987) 101, PL B197 (1987) 452, PL B198 (1987) 255, PI B198 (1987) 5i7, ZPHY C35 (1987) 2B3, PL B196 (19B7) 291, PL Bles (1967) 447, FL B199 (1987) 451, PL B199 (1987) 457, PL B199 (1987) 580 PL 6202 (1989) 149, ZPHY C39 (1988) L7T, PL B367 (1958) 109. PL B207 (1888) 349, FL B20日 (1986) 119, PL B2C0 (1988) 380. PL B210 (1888) 258, PL B210 (1988) 263, PL B210 (1988) 285, PL B210 (1088) 273, PL B211 (1988) 489, PL B212 (1988) 528, PL B215 (1988) 424, PL B215 (1986) 629, ZPHY Cd1 (1288) 1, ZPHY C41 (1988) 105, NIM Az74 (1989) 158. NDM A 275 (1989) 1, NfM A283 (1989) 544, NAT WISS 7f (1989) 52 zPHY C41 (1989) 55', PL B217 (1969) 205, PL B219 (1969) 121 PL
B221 (J989) 422, ZPHY C42 (1989) 349, 2PKY C42 (1989) 519 ZPFY O42 (1959) 543, 2PHY CAs (16的) 45, ZPHY C43 (1989) 181, ZPHY C44 (1989) 547, PL B229 (1899) 175, PL B229 (1989) 304, PL B230 (1989) 162, PL B230 (1989) 169, PL B231 (1989) 208, PL B233 (1969) 398, PL B232 (1989) 654, MPL A8 (1990) 73, ZPHY A335 (1990) 231, ZPHY C46 (1990) 9, ZPHY C4B (1900) 15, ZPHY C48 (1900) 158, ZPHY C48 (1990) 543, PL B234 (1990) 409, FL B236 (1990) 302, PL B24L (1990) 278, PL B245 (1960) 315, PL B246 (1990) 278, Pl B247 (1990) 121 PL B249 (1920) 350 PL B260 (2000) 164. Pt 9264 \{1901) 288, PL B255 (1991) 297, PL E265 (199) 834, PL E260 (1991) 259, PL B26 (1991) 148, PL B257 (1991) 535 , PL B269 (1891) 294. ZPHY C49 (1991) 349, ZPHY C60 (1991) 1, ZPHY C52 (1991) 363, PL B2T4 (1992) 239, PL B2T5 (1992) 185, PL B277 (1992) 209, PL B273 (1 192 ) 202, PL B28s (1062) 367. PL B292 (1992) 221. PL B207 (1982) 425, ZPHY C33 (1002) 361, ZPHY CsS (1992) 367, ZPHY C54 (1992) 1, ZPHY C54 (1992) 13, ZPHY C55 (1902\} 25, ZPHY C55 (1992) 179, ZPHY C55 (1992) 357, ZPHY C5E (1992) 1, ZPHY C68 (1902) 7, ZPFY C5E (1992) 339, MPL A8 (1993) 67a, PL B303 (1993) 368, PL B908 (19\%3) 135, PL B316 (1998) 605, PL B917 (1993) 227, PL B318 (1993) 392, ZFHY O5T (1893) 533, ZPHY G5s (1893) 61, ZPtC C58 (1943) 191, ZPHY CW (1983\} J99, ZPFY C60 (1983) 11, NDM A345 (1994) 465. PL B324 (1994) 245, PL B326 (1994) 320, PL B332 (1994) 461, PL B955 (1944) 526, PL B337 (1994) 383 PL B338 (1994) 390, FL E340 (1994) 126, PL B340 (1964) 217, ZPHX CE1 (1994) 1, 2.PHY CE2 (1994) 37), ZPHY C64 (1994) 375, PL E3s1 (1965) 441, PL B342 (1995) 397, FL B349 (1995) 676, FL B353 (1995) 554, ZPHY C85 (1995) 619, ZPHY C86 (1995) 63, ZPHY CER (1995) 25, ZPHY CAB (1995) 215. NIM A 373 (1965) 387, PL B374 (1996) 249, PL B374 (1960) 255, PL BaT4 (1996) 265, and 2 FHY CA9 (1986) 405

Beloted typerintent: CESR-CLEO-II

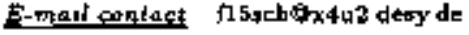
WWW Howe-page

bktp //www phygsk unledortmund de/ARGUS/arzus html

\section{DESY-HERA-HERMES}

(Proposed Jan 1990, Approved Det 1992, Began dace-kaking 1995, In progrets)

MEASUFEMENT OF SPIN DEPENDENT ITRUCTURE FUNCTIONS OF NUCLEONS

HEAMES COLLABORATION

ALEEFTA U - P Green, G Greenisug, P Kitching, K Martent ARGONNE - O llansen, H E JackuOn, C E Jone, N Makus, T O'Nęll, D Potterveld, T Zeule

CAL TECH - B Bray, P Gatler, A Dvaredaky, B W Filuppone, S Jensen, Wr Korsch, $\mathrm{k}$ Mcllbany, F D Mckkeown, M Putt COLORADO U - I Brack, B Fox, E Ktancy, D Mitercer. G Rakge日e, R Ruetisen, R. Smythe, D van Wiastrum

Desר - W Eeckbuseu, S Brons, Ms. A. Funk, Y Holler, H Ihsen, N Meyners, P Oelwein, H.J Plett, V Prabl, P Schaler, K Sinram, $M$ Spenzos, $\mathrm{G}$ Womblo, K Wholler, K Zapfa

DUBNA - A Fechtebenka, N D Gagunatbiril, D Kinlloy, $\checkmark$ Krivolkhijn, V Mutaun, A P Nagaltes, S Plyaghkevich, I Savin, G Yarygın

ERLANCEN U - T Benssch, 5 Derntaucher, B Btoun, M Dueren M Ferstl, K Fledler, A Golendolukhin, C Grosthsuaer, A Gute, N Koch, W Lachuit, F Neuhretther. K Rich, E Steffenn, I sienser, W Hiander

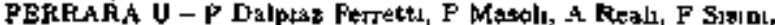
P Blarkh

FRASCATL - It Avakian, N Blanchi, G P Cepptani, E De Senctu,

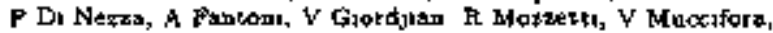
M Nupieri, A R Reolon-Cors, P Rass

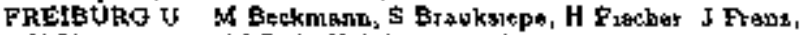

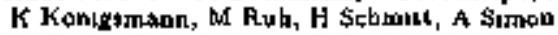

GENT U - E Auchenaetr, D Flyckborach, is Tytgat,

R var de viluer

HEIDELBERG, MAX PLANCK INST - A BOHASOW,

W Brueckmer, A Bruell, P Geiger, W Hoprich, B Pouk, E Volk

ILLTNOIS U, URAAN M Miller, A Natban, e Owter, S E Williamorod

LIVEAPOOL U G R Coust, 3 Morton, J Stewart, 7 Talled

WISCONSIN U .. H J Dtalten, W Heeherl, $T$ W/ge 


\section{SUMMARIES OF DESY EXPERIMENTS}

MIT, LNS - E Bruns, D DeSchepper. J Kelsey, L Kramer.

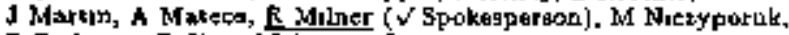
R Fedwine, T Shin, M Sutter, B Tipton

LEBEDEV INST - Y Gashmokny, E Devitan, ₹ Kozlay,

5 Paktaskov, A Terktslor

MUNICH U, EXP PHYS - G Graw, F Herleuberger, H Kolator, A Metz

NEW MEX1CO 9TATE U - P Chumaey, J Has, \& Kyle,

V Papsvacalion, S Psta, G Schnęll

NIKHEF, AMSTERDAM - I Blon, K de Jager.

$P$ de Witt Huberts, M Doets, T Henkex, E Kok, M Kolstmen.

H R. Foolman. F Udo, R van Bomqua, J F I vas don Brand, G van der Steepboven

OREGON STATE U - P Welch

PENJ U - T Fortune, W Lorestoll, A Mast, \& Rudnitaky, R. Zurmitale

ST PETERSBUR, INP .. S I Batostokk, G Gavtlov, A lator. A Y Kabelav, A Kruvclutcb, N Karppatkın, S I Mannenkov, $Y$ Naryahkin, $V Y$ Neluban, $V V$ Vikhrow

DNFN, ROME - E Ciebsint, S Frullani. F Garbaid M Iodwe, G M Urciuol,

SIMON FRABER U \& TEIUMF - E Bels. P J Delhey, O Hausuer R Handeran, R. Kaltar, C A Muller, R Openshaw, M C Vetterli, M Vincter

TOKYO U - H Ogam, Y SBkemt, T A Shibsta

VEREVAF PFCYS EN\$T - A Aitepertian, N Akepor, MA Amorien, R Avakiab, A Avetisgian, G Ejbakıan, V Garibyan, S Tarousn, H Zohrabyran

DESY-IFTI, ZEUTHEN - H Boetseber, S Bruns, Y Gaerber, D Hacb, V Korotkon, F Mersaner, W-D Nowrk, H Rolof,

A Scbwtud, U Sloeggletu

Accierater DESY-HERA Detector Spectrometer

\section{Reactang Polarized target}

$e^{-}$nucleon $\rightarrow \varepsilon^{-} \mathrm{X}$

$$
275 \operatorname{sev} / 6\left(F_{\text {]sb }}\right)
$$

Particles titudied Pit

Bitef diecrotion The it an uternal lorget experment in the HERA alectrou gtarage ring It messutes both inclusw and

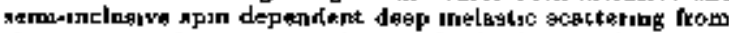
the proton ald atutron, and legts the fundameatal Bjorten oun rule Employs polarized intermal gas targets of bydrogen, deuterium, and ${ }^{3} \mathrm{He}$ luterkel targeta have the advajtage of heing pure atomac species with no dilution factor The angles and the energies of scattered particleg are det.r.cmaned by a magnetic gpsctroaneter Taking datc (July 96)

Jotural papers NIM A (In press)

Related expernments CBEN-NA-047, SLAC-E-142, SLAC-E-143 SLAC:E-154

E mall corfact milner

WWW Hotne fage hrtp/fixhral deyy def

\section{DESY-HERA-H1}

(Proposed Jun 1986, Approved Jul 1986, Besan dats-takidg May 1992, In progster')

\section{H1: A DETECTOR FOR HERA}

H. COLLABORATION

AACHEN, TECH HOCHSCH, I PHYS INST - C Berser,

W Bralroghwos, $\mathbf{H}$ Genzel, T Hsdig. M Harapel 、H Itterbeck,

C Keuker, T Koehlex, H U Martyn, d Niedzbnlls, k Rabbertz,

K Roenenbuter

AACHEN, TECH HOCHSCH, III PHYS INST - H B DTeIB,

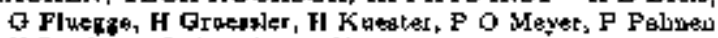

H Pawlelta, R Stetobers, W 5trucanski, I Thessten, P Uelke,

A Wagener, $M$ Wobtsch

BIRMINCHAM U J D Dowell, J Garvey, K Howitt,

V L Hodggon, I R Kenyon, P R Newman, J P Sutton, L R. Wegt

GRUSSSLLS U, thHE - M Enrth, G Bertrand-Coremane,

A. Bunistjan, B Clerbirux, E A De Wolf, D P Johnron,

P Matege, A Psumtch, R Roosen, P Yan Esch, P Van Mechelen

CRACOW - L Goetlıb, K Golec-Blerast, L Hajduk, I Martybuk.

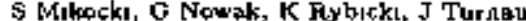

UC, DAVIS - W Dtagow, G Pope, F Roure, S Willard
DESY - R Baracble, W Bartel, H J Behrend, R Beyer, F. Buchuolz, J Buorker, A J Campbesl, M Cherlet, A DeFoeck G Edterin, E Elaces ( $\checkmark$ Deputy Spokerprernon), R Felat, K Flaraw, G Franke, I Gayler, R Gerbards, D Hadk, D toffmand, 34 Kander, M Kaugeb, S Kazariat, C Klengutort, G Kune, V Korbel, H Krehbreh, F Lebnot, F Laebl, B Lut, A Mroyes, J Meyer, G Mueller, C Nisbuhr, J E Oleson, E Panaro S Preil, S Retushoren, A Schoening, V Schragder, V Shekelyao. P Stefiton, J Strer, E Trabariludaki, T Wilkeer, G G Whater, E Wuen:

DORTMUND U - K Borras, J G Contrers, M Dirkming, M Flesscher, D Goldaetr, M Ifopepaer, M Huette, T Kraemerksemper, J Kurzhoefer, D Lueke, H Rack, I Spiekermann, K W Wikkt, D Wegenmi

ECOLE FOLYTECHNIQUE - B Andrieu, V Boudry, S Levoraan, A Auxhori, F Mereau, $Y$ Siros*, A specks, D Vandenglas

GLASGOW U - I O Skilucorn

HAMBURG U - S Add, V Blobel, L Buengener, F W Buekser, A B Pabr, B Heiserason, G Helnzelmson, G Lindatrces, 1 Lrpinak, of C Lopes, 6 Markin, A Meyer, B Naropks.

F Nlebergall, $\mathbf{S}$ Ress, S Schtek, G Schmudt, $\mathrm{H}$ Spltzer, I Stembart, of Weber, $C$ wittak

TEILELBERG, MAX PLANCK INST - M Beck, W Bruecksuer,

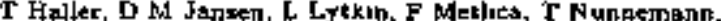
B Powh, R Todenhagen

HETDELDERT U, PHYG INST - A. Brasmer, F Etsate, M Erdmant, J Katzy, O Kaulmsna, P Schleper, B Schwab, U Straumann, T Wentler, M Werner

HEIDELBERG U, IHEP - C Brupe, J Janoth, K Meter, \$ \$cluer, A Stallberger, 3 Stiawe, $S$ Tapprogge, $M$ Weber

MOSCOW, ITEP n A Babarv, A Droutakon, V EFremesko, A Fedotor, I Gurelay, V Lubumov, V Nagovix,m, D Ozerov, A Rcatavte日v, A Semenov, V Solochenkor, V Tchernythov, S T Thetchelputske, A Zhokin

KTEL U - WW D Dau, G Gruber, U Kathage, G Siegmon, U Stewert KOSICE, EFF - d Ben, D Bruncko, J Ferencel, H. Maracek, Purin

LANCASTER U - S Burke, A B Clegt, C L Dbvis, P Dixon, $R \square$ W Henderson, $D$ Newton

LIVERPDOL U - S Cockn, C Cormeck, I B Dqintan, T R Ebert, C Gabatbuler, T Greenshaw, T Holtom, R MartJn, \& J Masktidd 8 J Mcthiabon, D Milatead, M Oskden, G D Patel

LEBEDET TNST - V Aadrcev, P Baranov, A Beloueov,

A Fomenko, N Gupstintre, S K Koteleskoy, A Lebeder,

B Malnoybłk, S Ruakoy, I Sheviskow, L N Shtarkov, P Smimov Y Soloviev, $A$ Volk, Y Vardik

LUND U - L Jcreukign, H Jung, H Kuester, MA Lindstroesm

MANCHESTER U - M Anderan, P Btdduph, F Eispham, MI J Burton, I MI Foster, C D Filton, M Ibboteon, S D Kolya. j W Lamas, \& Markball, B Wald

MARSEILLE U, LUMTNY - D Ca|vet, AS C Coungon

S Kermicho, J Marke, I Nogri, A. Pisuchot, C Vallete

MUNICH, MAX PLANCK INST - T' Carl, M Fleber, F Gaede

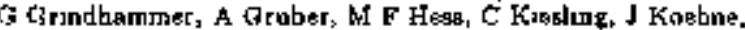
D Kruectsar, M Kublen, J Moddk, H Oberlack, K Ruther, P Scbacht, E Schuhmana, A Wegner

ORSAY, LAL Y Ben. J C Buzot, Y Brusson, A Coujnu,

B Defequart, I Favart, A Jacholkowraks, M Jaffe, G Lobo,

C Fastaud, F Squnathol, 2 Zhang, F Zomer

PARIS, CURE UNIV UT $k$ PARIS, UNIV VII, LFNHE E Barreles, U Babeler, o Bernard, S Degoret, M Coldtuers,

Gonzalez-Finerro, M W Kranny, $\mathbf{H}$ K Ngatyen, P Z 10

PRAKTE, INST PHYS - I Cuach, I Heryote, I Hladky:

P Reimer, M Taseviky

CHARLES Uf - J Formanek, S Valkar, A Valkarowa, J Zacek

PSI, VILLLGEN - K Gabatibulat, R. Hontberger, M W agentr QUEEN MAFY - WESTFIELD COLL - K T Donowa

E Esetubandler, R K Grifitbs, 8 I P Kalmag, D Kant.

M P I Landon, T Msuroidrs, E Rız VI, R Rylko, a Thonpona W vor 5 chlippe

FlITEERFORD - D Clarke, J A Cough]An, D G Custans,

W J Haynes, A Mebta, J V Mocrn, D P C Stokey

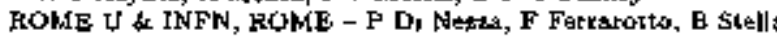

SACLAY - G Cornjkn, M David, I Feltetse, B Labigrge, E Petex, C Foyron, $P$ Yarrecchix, G Vellet, J Zasindery

WUPPERTAL U - K Daum, J Ebert, N Magnubsen, J Matleda H Meyer, D Schmidi

DESY.JFH, ZEUTHEN - J Beehr, H EhrhehmanD, M Gebauer A Glezov, H Hengche], K H Hiller, H H Kaufmann, M Klenn. 


\section{SUMMARLES OF DESY EXPERIMENTS}

H Kolanosk, P Kogtka, T Kurch, W Lanxe, R Nababaner T Norument, E Peppel, if Stoesilein, K Stalve, M Winde

ztJRICH U - H P Beck, C Dolliua, S Egly, D Mualler, P Robnana, F Setrow, $P$ Trined, M surNedden

zURICH, ETH - $\mathbf{F}$ A Echler ( $\checkmark$ Spoleenperman), \& Grab.

U Langenegger, H Niggla, D Pitzl, G Tinpolutis

Acselerafor DESY-HerA Detector H1

Recoctsotas

$c^{-7}$

Brzef descreption Measures energy and diraction of elchtrans. photont, and particle jets Identifies kptons by the shower bape and aentrinos by precul thitsins entercy theasuresthedt: The detactor consuts of a large wuperconducting solenand with tracking chambęr and a ligurd atgon celorimeter iogide An

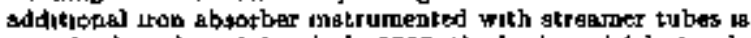

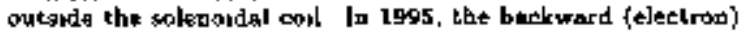
direction EM calorimeter wat teplaced by a SpaCal-lype electromagnetic + hadrontc ralorimeter, and Roman pots were addad in the forward (proton) direction to metronte elactically Bcatiered protonx In 1996 , silicon vertex detectorg wers added w the barrel (CST) and backwand (EST) diretions, and a Spacal-type nowtron counter intalited in the forward direction Taking deas (July 96)

Journal papers NIM A2so (1985) 69, NIM A263 (1987) 467. N1M A267 (1987) 479, NIM A265 (1988) 419, NIM A269 (19B8) 5B0, NIM A2T0 (1988) 334, EEE TNS S6 (1989) 331, NIB A275 (1959) 197, NIM A275 (1989) 246, NIM A277 (1989) 368, NIM A279 (1099) 5\%, WIMC A279 (1989) 227, NIM A283 (1969) 375, NTMf A283 (1989) 167, NIM A283 (1889) 471, NTM A233 (1989) 43', NIM A289 (1989) E37, NIM A283 (1989) B22, NP (PROC SUPPL) 16 (1989) 618, NIM A289 (1900) 44B, NIM A302 (1日81) 277, NIM AS1D (1991) 535, NIMS A312 (1992) 457, NIM A323 (1892) 184, NIM A223 (2992) 401, NMM A323 (1892) 532, NIM A323 (1902) 537 , NIM A336 (1993) 460, NTM A336 (1993) 499 . PL B297 (1992) 205. NIM A 356 (1903) 460, N1MC AGS (1993)

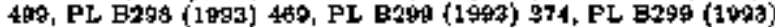
385, PL B314 (1998) 436, NP Ese日 (1993) \$, NP B407 (1993) 515, NIM A3dA (1994) 492, NLM Aa60 (1904) 57, FL B321 (1994) 161, PL B324 (1994) 241, PL B328 (199s) 176, FL E33s (1994) 50T, PL B340 (1994) 205, APP B25 (1994) 319, APP B25 (1094) It5s. NC $107 \mathrm{~A}$ (1994) 210\%, NC 107A (1994) 2345, 2PHY C62 (J994) 59, 2PHV CE3 (1994) 377, ZPHY CEs (1994) 545, NP Bd29 (1994) 177, PL B346 (1905) 416, PL B24B (1925) 681. PL B353 (1995) 578, PL B354 (1995) 494, PL B356 (1995) 118, PL B35e (1905) t12, ZPHY C68 (1995) 529, ZPHY C6? (1995) 56b, ZPHY C69 (1995) 27, NP B435 (1995) 3, NP D436 (1905) 47!, NP B445 (1995) 3, NP B445 (1995) 195, NP B449

(1996) 3, NIM A372 (1996) I89, N1M A372 (1996) 399, NIM A374 (1996) 149, PL B369 (1996) 173, PL B279 (1696) 919 ZPHY DT0 (1906) 17, ZPHY CT0 (1996) 609, NP B463 (1996)

3, NP B468 (1996) 3, and NP BdTo (1998) 3

Refated expertments DESY-HERA-ZEUS

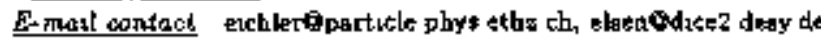
WWW Hame-page htop/fdice2 desy de/

\section{DESY-HERA-ZEUS}

(Fropoed Jun 1905, Mar 19st, Approyed Nov 1986, Began dala thatang May J992, In proxrett)

\section{ZEU日; A DETECTOR FOR HERA}

ZEUS COLLABORATTON

ARGONNE - M Derrek, D Krakauar, S Magull, D Makunas,

D Musgrave, J F, Oksaxinkkt; d Fepond, F Stanck, R L Talaga H Ratag

ANDEEWS US MCK Mattunsly

BOLOGNA U \& INFN, BOLOGNA - P Antongl, G Bari,

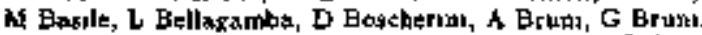

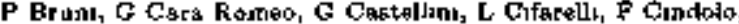
A Contin, M Corradi, I Gialas, P Gıust।, G Lacobuç, G LavfenL, G LevI, A Margott, T Mastem, F Nawa,

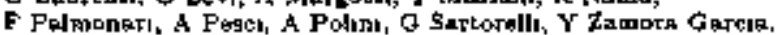
A Zichıck,

BONR U - C Amelung, \& Bornheim, J Critenden, $\mathrm{R}$ Deffuer,

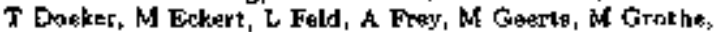

H Hartmana, X Helnoth, L Hemz, E Halter, H P Jakob, U F Kate, $\mathbf{9}$ hasgel, E Paul, M Pfenffex, C Rember, D Schramm, J Stamm, R Wedemeyer

BRISTOL J - $\$$ Camplell Robasa, A Cundy, W N Cotunghan,

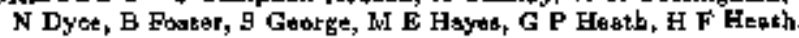
D Piccioni, D G Foft, $R$ J Tapper, $R$ Yoshida

CALABRIA U $b$ INFN, COSENZA - M Arneoda, R Ayad,

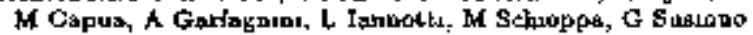

NEVIS LAB5, COLUMB]A U - A Ceddwell ( $\checkmark$ Deputy

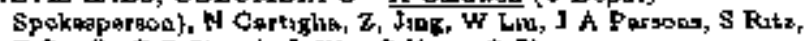
$F$ \$crulli, \& B Straub, I $\mathrm{Wal}_{1}$ \$ Yang, Q Zhu

CRACOW - P Borzemakı, J Chweslowsk, A Eekreys, Z Jacubowski, M $尹$ Przybycien, M Zschars, L 2ariefok CFACOW, INST PHYS NUCL TECH - L Adommyk

B Bednarek, K Jelab, D Kastelewgks, T Konvskat, M Przybyejan, E Fulhkoweska-Zarebska, L Suszyck1, J Zsjoc

IAGELLONIAN U - 2 Dulunkk, A Kolanglk।

DESY - G Abbiendi, I A T Besuerdjak, it Eebren, H Bevet,

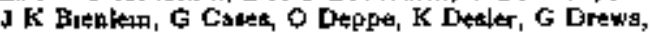

M Plasibekn, D J Gilkungon, C Glasman, P Gotthchnr, J Groses. Knotter, T Hoxa, W Hain, D Hosell, H Hegshng: Y I ga,

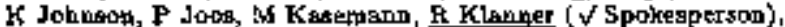
W Koch, U Kotz, H Kowalska, J Laba, A Ledace, E Labr, M Lawe, D Loke, J Manumh, O Marczak, J Blewakl,

T Montero, J S T Ng, D Notx, K Ohreubers, K Plotrelkorsin,

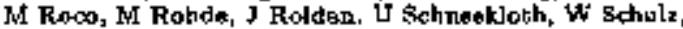

F Seloake, E Surrow, E Taw, T Vote, D Wetphol, G Woll, U Wollmer, C Youngman, W Zeuner

DESY.IFH, ZEUTHEN - H J Graboxch, A Kbarchılava

\$ M Marh A Meyer, S Schlenstedi, N Wulf

FLORENCE U \& INFN, FLDRBNCE - G Eurbal, E Galko, P Palfer

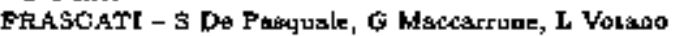

FREIEURG U - A Bamberger, $\mathrm{S}$ Elrenhardi, T Treftget, S wolfie

OLASGOW U - 1 T Bronley, N H Brook, P J Busey, A T Doyle,

D $\mathbf{H}$ Saxon, o E Sicladr, hit L Utlay, A S Wiloon

HAMBURG U - B D Burow, Dagnemann, L Hagge, U Holm.

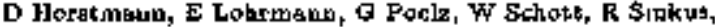

K W Wak, F zetsche

IMPERJAL COLK - T C Eacon, N Brummer, 1 Butterwath,

V L tlerrs, G Jipwetl, I Y H Hang, E Lamberk, K R Long,

D B Miller, N Pavel, a Prınjag, J K Sedgteer, D Sidens,

A Whitfiald

rOWA U - U Mallk, M 2 Wang, S M Wang, J T Wu

JULICH, FORSCHUNGSZENTRUM - P Clath, D Filget

KOREA U - 8 H An, G If Gho, EJ Ko, s B Lee, s W Nam, K 5 Park, 9 K Park

LOLfIS1ANA STATE U - S Kartk, H 9 Kin, R R Mcllal, W Matcalf, $V$ K Nadendla

MADRID, AUTONOMA U - F Barreiro, I F de Troconas,

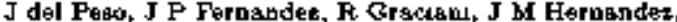

L Heras, L Lobarga, M Martinez, J Puga, J Terron

MOGILL U - F Corrireou, D S Hannt, J Farimank, L W Hung.

I N Larn, C Matthewr, P M Patel, M Alyelıne, M St Laureat,

D 它 Staers, F. Ullminn, G Zavek

MBIJT GAKUNN U - T Tgurün.

MOSCOW PHYS ENG INYT - V Baghturs, B A Dolgoshetn,

A Stofotkin

MOSCOW STATE U - G L Beshindahsgian, P F Ermolov,

L K Gladiln, Y A Golubkov, V D Kobrun, I A Karzhayipa

Y A Kuzmin, O Y Luklod, A S Proskuryakoy, A A Savid.

L M Sheheglove, A N Solomin, N P Zotow

NJKHEF, AMSTERDA.M \& AMSTERDAM U - M BULE,

F Cblebene, M de Kampg, E de Wolf, J Engelen, F Koruman

A Krupc, H Tlecke, A Vab Sighem, R van V'oudenbers,

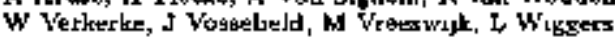

OHIO STATE U - D AcostB, B By]sms, L S Durkin, J Gilmare,

C Ll, T Y Ling, P Nylander, I H Park, $\uparrow$ A Romanowstrı

OXFCRD o - D Bouly, E J Cabmore, A M Cooper-5arkar,

A C E Devenisb, IN Harrew, M Lanciter, L Lindenanb

J MeFal, C Nath, V A Nayes, A Quadt, I R Tetenar,

H Uuterpad, R Walczak. D S Waters, F F Wilgon, T Yip

PADUA U E INFN, PADL'A - A Bertolun, R Brbgners,

I Garlı, F Del Gargo, M De Grorgi, U Dossell, S Limentaml,

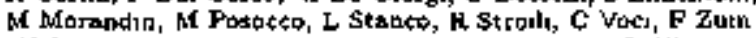

PENN STATE U - J Bulmahn, R G Fejd, B Y Oh, I Whatmere FOlsE U \& INFN, ROLE - G D'Asostin, G Barint, A Nigio RUTHERFORD - J C Hart, N A Mecubbin, T P Sbab 


\section{SUMMARIES OF DESY EXPERIMENTS}

UC, SANTA CRUZZ - E Barbers, T Dubbs, C Heasch, W Lockman, I T Fabn, H F Sadrozintkt, A Stiden, $M$ Van Hook, D C Williama

SIEGEN U - J Bultzinger, O Shwwatuer, R J Selfert, A H Walteta TEL AVIV lo - H Abragpownez, o Grialud, \& Daqan, A Lory

TOKYO U, INS - ] I Fieck, M Inuzuka, T Lshtt, M Kure, S Mine,

M Nakno, J Sinzuks, K Tokushukn, K Umemori, \$ Yamads, Y Yomataki

TOKYO METROPOLITAN U - M Chub, At Hanatsa, 'I' hirate.

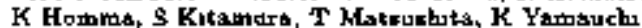

TURIN U \& INFN, TURDN - R CIrI, M Cosis, M 1 Ferrero,

S Masati, C Poroni, R Sacehy, A Solano, A Statane

TURIN U, ALESSANDFIA \& INFN, TURIN - M Dardo

TORoNTo U-D C Bailey, F Benard, M Bthe; C P Fagergtrom, 6 F Hartner, K K Joo, G M Levman, J F M L arten, F S Orr.

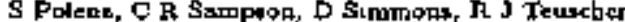

UNIVERSITY COLL, LONDON - J id Butterworth

C D Catterall, T W Jones, F B Kartenwicz: J B Lane,

R L Soundere, J Shulans, M F Sutton

VIROINIA TECH - $\mathrm{B}$ Ln, L W MO

WARSAW U, IEP - $W$ Baguax. J Ciberoprsk, J Gajewtkt,

G Grzolak, M Kasprzak, M Krzyzagowrk,, K Muchorowskk,

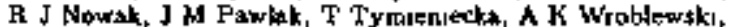
1 A Zaks zewels, A I Zarpeckj

WARSAW, INST NUCL \$TUDLES - M Ad

WEIZMANN INST - C Coldewey, Y Ejsenberg, D Hochmon,

U Karwhon, D Fevel, D Zer-2.

WISCONSIN $\downarrow-W$ F Badgolt, J Ereliweg, D Chap|n, R Cruss,

S Dagu, C Fouda, R. J Lovelees, S Mattingly, D D Reeder,

S Sslverstorn, W H Smath, A Vouclneht, MS Wadarczyk

YORK U, CANADA - 9 Bhenlra, M L Cardy, W Frateren,

M Kholzed, WN Murray. W B Schmike

Accelerator DESY-HERA Detector ZEUS

Eegctwos

$$
\begin{array}{ll}
e^{-} p & 300 \mathrm{GaV}\left(E_{\text {cnu }}\right) \\
e^{+} p & \quad \text { a }
\end{array}
$$

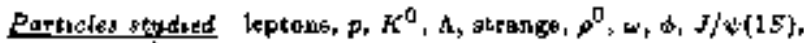
vimeson, chars

Bnef descrostor Measures noutjal apd charged current pos ceske ith electron-protor sud positron-pralon unteractions (27 \& GeV on 820 GeV) and scapches for new unternct pons and

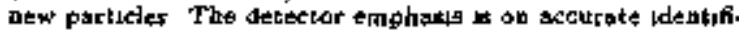
cation and messurement al pets and leptons The to aln delec-

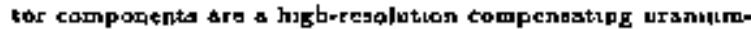
kcintulasor calorimeter and a central tracking detector sur-

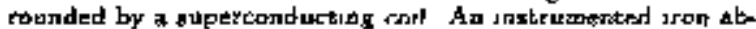

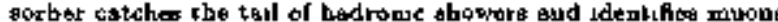
A large fraction of the solid angle is further covered by muron detector! Bending magrets of the modhine are uned xx spec.

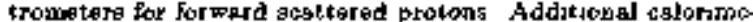
sers masare forward nevtros production and tag flectrons and

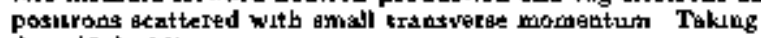
date (July a6)

Jotirtot poperse IEEE TNS 36 (1989) 465, NTM A274 (1989) 134, NIM A289 (1990) 125, NDM A290 (1990) 95, NIM A292 (1990) 259, NIM A900 (1991) 480, NMM A30E (IODI) 495, NIM A309 (1901) 101, NIM A3I9 (1992) 12A: NDM AS21 (1902) 356, PL B297 (1992) 404, FL B293 (1992) 485, NIM A333 (1998) 342, NLA A336 (1993) 23, PL E303 (2993) 183, PL B306 (1999) 148, PL B30日 (1993) 173, FL BS16 (1993) 481, PL B316 (1993) 207, PL 8316 (1903) 412, ZFHY CS9 (1993) 231, NIM A398 (1994) $254 \mathrm{NIM}$ A342 (1904) 260, PL B 323 (1904) 287, PL B332 (1994) 238, PL B339 (1994) 433, NQ 105A (1094) 2123 , 2PHY C63 (1994) 391 PL B342 (1995) 417, PL B345 (1945) 576, PL Bade (1995) 390. PL E948 (1905) 665, PL B 449 (1995) 325. PL B350 (1005) t t20, PL B384 (1896) JE3, PL B336 (1995) 129, PL B956 (19日5) (30L, PL B3B3 (1975) 201, PRL 75 (1995) 1006, ZPHY C65 (1995) 379, 2PHY C C 5 (1995) 627 ZPHY C67 (1995) 81, 2PHY C67 (1998) 98 ZFHY C67 (1990) 22T, ZPHY C68 (1995) 29, 2PHY G65 (1996) 113, ZPHY C68 (1995) 569 ZPHY C69 (1995) 39, PL B369 (1996) 55, ZPHY C69 (1996) 607, ZPHY Cro (1996) 1, end ZPIIY C70 (1906) 301

Retaled expertmethle DBSY-HERA-H1

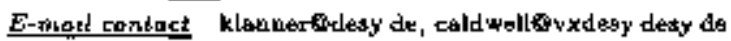
WWW Horne page http / $/ 20$ w00 desy de B000/

\section{DESY-HERA-B}

(Propored May 1994, Approved Jun 1994, Feb 1995, In preparar. tov)

AN EXPERIALNT TO STUDY CP VIOLATION IN THE $B$ SYSTEM USING AN INTERINAL TARGLT AT THE HERA PROTON RING

\section{HERA-B COLLABORATION}

\section{Accelerptor DESY-HERA Detector Spectrometer}

Brief defcription The mann goal is to measure the volotion of $C P$ aydmetry in the decay channet $B \rightarrow K_{S}^{0} J / \psi$ Uoes the $820 \mathrm{GeV}$ HER a proton thit and an inkernal fixed target The ter get conerats of a of of movable wites whob act like colluplaters in the hale of the protap beam The detertor provides a soad $B$ decay vertex retolution: leptan and kaon

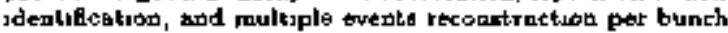
crobuas A 2 m long veplex detector consiots of layers of doubic eident inlwon with perpendicular onertation to the bedint It is followed by tratking chambers instide the spectrometer inagaet which provides a field Integral or $22 \mathrm{Tm}$, by FIClI sod TRD countert Ior patticle identsfication, and by the electromannetic

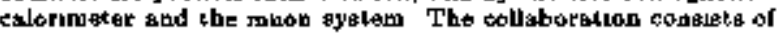
about $\mathbf{2 4 0}$ physicasts from thirseen countries and more than 30 tasticution: Bxper,ted to begin data takros in Junt 1998 for mort daformation, pteses contsat the Spokgapereon, Dr Andreas Schwarz [DESY]

Retated Esponments CERN-WA-099, FNAL-771, FNAL 789

E-mad contact thwargatelerab dosyde WWW Home-page bttp //wwe-hera-b degy de/

\section{DESY Future Plans}

The HEDA acoclerator experturents teraen th the focus of particle physice research at DESY in the coming years $\mathrm{HI}$ and ZEUS, the tiko collosen expenments tumg the $27 . \mathrm{GeV}$ electron/positron beam and s20-GeV proton beano will continue to study the proton etructure functions at vęry low $x$, (test the QCD and electrou'esk interactions and search for neu phenomens HERMES, whych utes a longitudindly palarised olectren/passtron besm and a

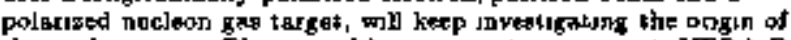
the nnelead upun The ser.nnd beam-target experiment, HEFA-B

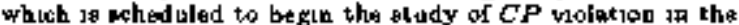
B mesen system in 1998, will vas the proton beas and an unternal slumulum wire target la mischine phyyke; the etlorts are foeubed on the Noxt Accalerstor project, an election-posttron linese colliter at $500 \mathrm{GeV}$ c m Toternat|enal collaboratwons based at DESY are studying two posssible psthe One ta the 8-Band Lintear Collider (SELC) which nermally-oonducterg copper respastore opershag in the frequestcy region uf $3 \mathrm{GHz}$, and tipe other to the TeY-Energy Superconducting L-mear Accelerator

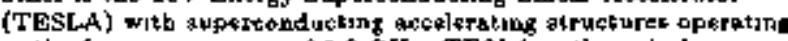
in the frequentcy reglon of a 3 GHE TESLA te the only linese

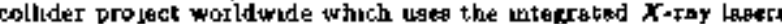
Tegt farlities fír both propectc are andęr cenctruction at DESY 


\section{Selected FNAL Experiments}

\section{FNAL-665}

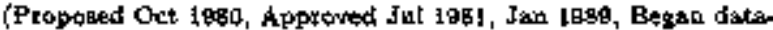
talang 1987, Campleted dats-takmg Jan 1992)

\section{MUON BCATTERING WITH HADEON DETECTION} AT THE TEVATRON

PREIBURG L L T Dreyer, M Erdmanon, J Hass, M Lenakl, W Mohr, G Sergert, H E St,er, $M$ O Wilbelm

AFGONNE - D F Geesamsin, R Gitman, M C Green

H E Jatknon, 8 Kaufran, E R Kinsey, T B W Kirk,

D H Potterveld, 5 Tentindo-ktepond, H I Tront, A. Zighuche

UC. SAN DLEGO - R D Kennedy, H G E Kobrak, P Madden, I R Osinck, A Salt'arami, F A Swaneon

FERMILAB - B R Ealker, G B Crutrakon, J E Haolon

S Kroyordzusts, H Mfelensen, H E Montgomery, J G Morfin. C Splanatio, s a Wolbers

HARVARD U - IM Conrad, G Y Fang. A V Kotwal,

D G hishael, F B Nickarson, F M PJpkın, M H Schmutt, R Wilton

ILLINOJE $\mathrm{v}$, CIUCAGO - $\mathrm{M}$ K Adama D A A varill, $\mathrm{T}$ J Carroll,

R \& Guo, C Heلliwall, D E J日ffe, S R Magill, D W Meleod, T Mckibben

CRACOW - A Eakieys, J Figlel, P Malecki, K Olknewacz,

B Pawlik, P Stopa

CRACOW, INST PHYS NUCL TECH - K Drunnkowsk

LIVERMORE - P L Anthony, F S Dietrkeh

MARYLAND U - 5 Aad, 5 Kunor, \& C O'Oxy, E I Rambera,

A Sknja, G A Snow, P H Stembers, R Talaks

MIT - M Bakek, W Busea, L S Osborne, I J Ryap

MUNICH, MAX FLANCK INST - M Aderholz, F Borterveck,

I Derado, V Erkardt, to 1 Gebanar, D Hentke, is Jeneso,

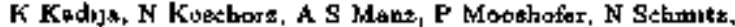

H I Seyeriein, S Soldrer-Rembold, M Vidst. W Wittek

NOFTHWESTERN U - H M Schellman ( $\checkmark$ Spakespersod),

P spentpours:

OHIO U - H L Clark, At W Fualay, K H Hıcks

PENN U - A Banerjee, K Girffloe

WASYTINGTON U, SEATTLE - A A Bhatt1, U Eratzlet,

R Davisoun, W M Dougherty, D M Jansen, $z$ Jin, J I tord,

H J Lubatt 1 , M J Mftchelt, R S Percy, B Yenems, R J Wilkss, T C Zhat

WUPFERTAL U - H M Gran, H Brendung, U Ecker, $\mathrm{k}$ Othen, A poester

YALE U - S K Dheneg, V W Haghes, V Papevasenlout,

K P Schucler, H Venkataramania

Accelerator FNAL-TEV Detector CCW

Reactions Polstixed betw

munn $t^{-} \rightarrow$ muon $e^{-}$

muon $\mathrm{p} \rightarrow$ muon badtont

tutor $p \rightarrow$ moton $\rho X$

tauen $y \rightarrow$ numa of $x$

muron dent $\rightarrow$ molon hadrong

muon daut - muou $\rho \mathrm{X}$

meon deut $\rightarrow$ muen $\phi \mathrm{X}$

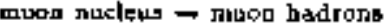

$$
\text { nuclintura }
$$

mavas nucleus - תituch hadrons $x_{0}$

muon nucleus $\rightarrow$ muoh ox

muon nucleus $\rightarrow$ muor $\phi X$

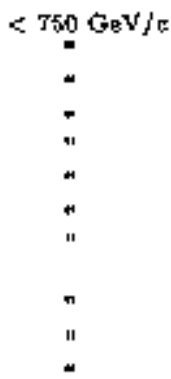

Bnef deacrotion studjes (1) the properties of hadron ayatems recajling from deep melastic mun colligrone, (2) the bucleon structure functions, and (3) exclusive vector meson production Ube日 the flaptreand uttugg vertex magnet fram CERN The first rus was completiod th 1986, the gocosod run with a burabest of dufiereul sargets $\left(\mathrm{H}_{2}, \mathrm{D}_{2}, \mathrm{C}, \mathrm{Cz}, \mathrm{Ke}\right.$, and $\left.\mathrm{Pb}\right)$ and an upkrade of the vertex spectrameter tracking cystem in 1890/91 Data analysis tA prograse (July 96)

Jotrinal paptrg 1REE TNS 3a (19R6) 20S, NIMt A291 (1990) 633, PL E272 (1991) 163, PL B287 (1992) 373, FRL 68 (1992) 3266, PFL 69 (1992) 1026, PL B30E (1993) 413, PL B309 (19A3) 477, PH D48 (1993) 5057, PRL 72 (j994) 464, PL B335 (1994) \$34,
ZPHY CB1 (1994) 179, ZPHY C61 (1969) 539, PR DEó (1994) 1836, PRL 74 (1905) 1525, PEL 7t (1995) 5198, PRL 75 (1995) 1466, ZPHY C65 (1995) 735, ZPHY C67 (14日6) AOJ, ZPHY CT1 (1996) 391, and PR. D54 (1906) (in prent)

Related experements FNAL-09B

E-matl cothect achellmantsinad goy

WWW Home-page hotp ///njouxd frak gov/

\section{FNAL-672A}

(Fropded Feb L98), Approved Jul 1981, Beran datartaking J987. Complited dats-taking Jan 1992)

A STUDY OF HADRONIC FINAL STATES PRODUCED IN ASSOCIATION WITH HIGH-MASB DIMUONS

FERMTLAB - J C Knder

ILLINOIS $\mathrm{U}$, CHUCAGO - $\mathrm{A}$ S Galdberg, R L Jeik, H Mendeg,

J Solounon, $F$ Vaca

INDIANA E- - R. R Cottanden, A R Dererba, A M Gribusbun,

S Kartık, I Ll, R Li, T R Marshall, H J Mart;n, P T Smith,

T Sulanke, A Znemulask, (Spolresperion)

LOUTSVRLE T- C I R Davis

MICHIGAN U, FLINT - L I Daumre

SERPUKHOY - V V Abramor, Y M Antipar, B Baddin,

S P Denıspv, Dyshkant, V Glebov, Y Gor, V J Kareshev,

A Kriniceyn, A A. Pettukbun, V Podelesv kov, V I Sirotenko,

R Sulayev

Accelerator FNaL-TEV Detector Spoctrometer

Reactionz

$$
\begin{array}{ll}
p \text { ninclevs } \rightarrow \mu^{+} \mu^{-} X & 500, B 00 \mathrm{GeV} / \mathrm{C} \\
x^{-} \text {nocleus }-\mu^{+} \mu^{-} \mathrm{X} & 500 \mathrm{GeV} / \mathrm{s}
\end{array}
$$

Particies stodaed $N / \omega(15), \psi(2 S), x_{e r}(1 P), \chi_{c 2}(1 P), \rho, \omega, \phi_{1}$ bottorn

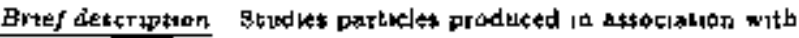

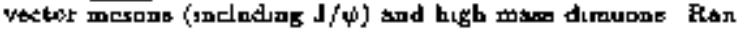
with $\mathrm{H}, \mathrm{Be}$ and Cu targets Collected apprormately $2 \mathrm{M}$ fully linked dispuon events (rwer $30 \mathrm{~K}$ \&'s) witb duferent beams (Igex

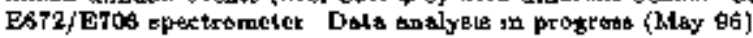

Journal papers N'IM A270 (1988) 99, PR Dd1 (1990) 1, PRL 74 (1906) 485, and PF D53 (1996) 1723

B-mest conlast reminakitinal gov,

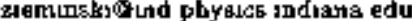

\section{FNAL-687}

(Propoeed Jan 1951, Approsied Dec 1989, Began data-tsking 1987, Completed dotatalung Jan 1992)

HIGH-ENERGY PHOTOPFODUCTION OF STATES CONTAENING HEAVY QUARKS AND OTHER RARE PHENOMENA

ERET COLLAEORATION

BOLOGNá v-V Giordato, G Molimerı

COLORADO V - C W Bogart, H W K Cheung, P Coteut,

8 W Cuty, J P Cumalat ( $\sqrt{ }$ Spokerpergon), $\mathrm{C} J$ Dollaptecols.

D P Edmonds, J Ginkel, V Greene, G Intrond, W E Jabns, R L Lartbury, M Nehtlug, \& E Schults

FER,MILAB - M E Burkley, J N Butler ( $\checkmark /$ Spokesperson),

5 Cibsngur, I Gaines, P H Garbenciux, L Garted: AN F Gosmley.

$s$ A Gourlay, D J Harding, P H Kaxper, A E Kreymes

P L G Lebrun, J Peoplss, Jr, \& Shukle, J T volk

FRASCATI - 3 Bianco, \& Camponaschi, F Colsnt, o Digrovanni,

M Enorsn, F Fabbrs, G Ferrelti, $M$ Gisrdoni, $G$ Gerauda,

A Maccart. L Pastamonti, D Fiondino, G Rtvelıni, Y Rueso,

S Sarwar, A Seatti Di Ucria, \& Zaj]o

ILLINOIS U, URBANA - F D Cogewell, H L Culbertusi, R. W Gardner R Groeno, Q R Jarose, T' Kroc, K L Lrigge], T A O'Hallokas, JT, T Protiman, H G Scott, P D Sheldod

V J Siment Js, T E Trumpubski, J R Wilnoli, J E Wiss KOREA U - G G Chợn, J 5 Kang, $K$ Y Kıग

MILAN U \& INFN, MILAN - G Alimont, G Bacchiocchi,

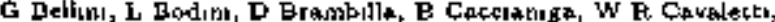




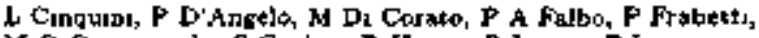
M G Ginammarebu, S Grabar, D Haran, P Inxanı, F Leveraro, E Macavero, S Msalvezzi, P F Manfredt, G Masıpiliabo, M Mazzant1, D Mfenasce, S Meseghinu, E Meroul, L Moroni, S Moron, of Onant, F Palombo, D Pedrun, L Peragno,

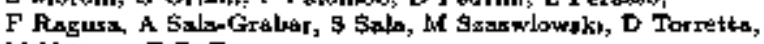
$M 1$ Vitcone, F Zutis

NOFTHWESTERN $\mathrm{U}$ - M Attuto, T N Boblos, D A Buctibals. D R. Claes, B Gobb, B O'Relly, S-W Park, $F$ A Schluter, F Yoshodis

NOTRE DABE U - J M Bubop, N N Buawae, J K Bugenitz,

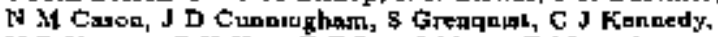
V P Keviney, G N Kim, T F Lib, J Maver, E Mannel.

A P Mtinanue, R J Mountaun, D L Puad,juc, R. C Ruclutl,

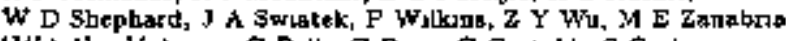

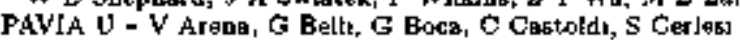

F Diaferas, G Ferrettl, O Foptann, G Fontana, G Giament,

S Rabu, S P Fatti, S Rescla, C Ruccard, V Sptaldi, P Treapj, L Vole, P Vitulo

UC, DAVTS - G P Giाm, V Peolone, $P$ M Yaget

PUEFTO FUCO U, RIO PIEDRAS-A Loper: I Mendez,

J C Palsthungal

MEXICO. IFN - $\mathbf{B}$ Mendes

NORTH CAROLINA Џ - T F Davenport

TENNESSEE y - \& Elackett, w Buge, $K$ Denyo, T Fandler,

O. Kondo, M Phollexody

Accelerator FNAL-TEV Detector Spectrometer

Resactiong

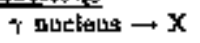

$\tau$ nucieas $\rightarrow$ chorm $X$

$\tau$ nucleus $\rightarrow$ charm $\overline{\operatorname{char}} \bar{x}$

$\tau$ suclens $-\mu^{+} \mu^{-} \mathrm{X}$

$\rightarrow$ Muclean $\rightarrow$ lepton ${ }^{+}$lepinan- $X$

$<\$ 50 \mathrm{GeV} / \mathrm{c}$

$n$

n

11

Partigles atudesed charmed-mezon, chmrmed-baryou

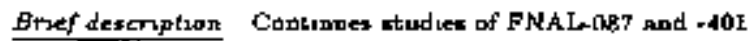

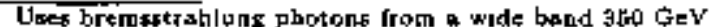
( $\pm 15 \%$ ) eleciron beam, a news large-aperture mulliperticlo pectromntw, a berythura targot, and a slecos mierastrip decay-vertex decectar sludies the dynamirs of hesry quark pholoproduction, and decsys of charm particles Data analysis in progrose (July 96)

Journat papers IEEE TNS no (1983) 3798, NIM A225 (1986) 由19 NIM मं2A (1985) 107, NM A251 (1986) 40, NIM A2H2 (198B) 368, PL B261 (1990) 639. PL B2f3 (1991) 5B4, NIM A 05 (1991) 48, NP (PROC SUPPL) 27 (1992) 20". PL B300 (1993) 190, PL B307 (1993) 282, PL B308 (1993\} 193, PL B313 (1993) 253, PL E314 (J日93) 477, PL B315 (1993) 203, PL BJ16 1993) 197, PRL 70 (1998) 1381, PRL 70 (1993) 1755, PRL 70 (1993) 20K8. PRL 71 (1903) 827, PL B321 (1991) 395, PL B323 (1994) 459, FL B32B (1894) 187, PL B928 (1994) 193, PL BJ31 (1964) 217, PL B338 (1994) I06, PE B340 (1994) 254, PAE, 72

(1995) 324, PRA T2 (i994) 981, NC 107A (1994) 2025, PR D50

(1900) 2853, PL B346 (1945) 1996, PL B351 (1945) B91, PL B354

(1995) 486, PL B357 (1998) 378, PL E859 (1995) 403, PL ES63

(1995) 259, PL ES64 (1895) 127, PI. BS65 (1998) 491, and PL

B370 (1956) 222

Related expetaments FNAL-g\$1

E-mast contact jeutralactifnal zov, pumalstepiotolb colorado edu. butlerefind gov WWW Hame-prge bt.p//wWw hep wer ad11/e687/

\section{FNAL-690}

(Proposed Feb 1981, Approved Jnl 1981, Now 1987, Apr 1997, Begau date tokug 1990, Completed data-taking Jan Xeg2)

STUDY OF HADRONIC PRODUCTION AND SPECTHOSCOPY OF STRANCE, CHARM AND BOTTOM PARTICLES AT THE TEVATRON

COLUMBIA U - A G Gara, B C Kuapp (V/Spokesperson) ILLTNOIS U, LFAANA - E Golkochalk

FERLILA B - D C Clussilat, G Gutjerrez. A A Wehmsin GUANAJUAT 0 - J Felax, G Moreno, M Reyos, of Sobs
MASSACHUSETTS U - M C Berrmo, E F Hartound,

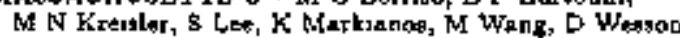

Accelermior FNAl-TBV Detectos Spectrometer

Reactsars

$$
P \mathrm{P}-\mathrm{P} \mathrm{K}
$$

$000 \mathrm{geV} / \mathrm{c}$

Particles sfudied atrange, charm, bolton

Artef degcriptoof Initial goals ipcluds (1) * bystematic study of exclusive resctions, partjcularjy dufrection drgacciatson,

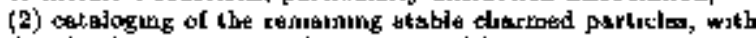
detalk of production and decky, and (B) deterfininak the acde

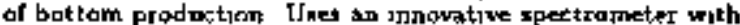
a batdware praceseor Current bocus as on the bindy of the chame production crods-gextion in difiraction dissordation and epin / parity analyrgs of comcrally produced light mabous Data andyaśs in progress (July 96)

fotmat papers NC 107A (1994) 1847

Related experiments BNL 7 GB

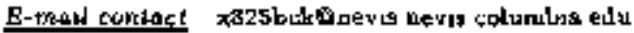

FNAI-740 (DZERO)

(Propared Sep 1983, Approved Seb 1984, Befan detaetakıng May 1992, In progress)

STUDY OF $\bar{P}$ COLL1SIONS USINO A LARGE DETECTOR AT DO

\section{DO COLLABOFATHON}

ANDES U, BOGOTA - B Gornez, R Gomes, B Hoeneisen,

D Mendors, $P$ Mooney. $P$ Nechtev, J $P$ Negrel, J M R Rolden,

A Serno YS E Zanabria.

ARTZONA U - K P Days, D K Fen, G E Farsten, I A Guids,

B. Jeyanti, K A Johne, A M Naraysmad, J P Rutharfoord,

$M$ A Shupe, $D$ Vititoe

BOSTON U - I M Butder

BROOKHAVEN - B Gibbard, H A Gordon, N Grat, 5 \& Kabn,

J R. Kotcher, S D Protopopencu, S Snyder

EROWN $[1-J$ Wantly, D Cullen-YIdw], D CutL; T Fahtand

J M Guide, I S Hottun. F Naug, $R$ A Pattridge, $G$ T Wacts BUENOS AIRES U - S Guinatein. R Piegan

VC, DAVIS - P C Bloom, R Bcoedon, Y V Fusyak, S M Giens,

G Grum, C B Klopfensteın. W Ko, R L Lander, S Masu,

D B Fellatt

UC, IFVINE - J Drjnkard, G Gnfth, R E Hail, A J Lankford.

D Stoker, J N Trraz!

UC, RTVERGIDE - J H Cochran, Jr, J A Eلliswn. P Gartung,

A $P$ Heinson, M Msoon, S J Wimperny

RIO DE JANEIFO, CBPF - G A AIves, W P Caralbo,

H Da Molts Filha, J hi De Mranda, A K A Maciel, Mi Sendes,

J is Mirands, I J P Moreirg, M Necla, A F D S Sabtoro,

M H G Souzs, A Szatjder, W Var

MEXICO, IPN - H Cagtulla-Veldex, J L Gonzeder-Solis,

F. Herandez-Mantoys, L Magsab-Miendozs, A Sapchea-

Hermandex

COLUMEIA U - IM Adam, A Kotwa!, PM Tuts, $B$ Wngr

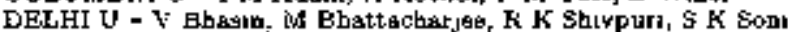

DUBNA - G D klexeov, V I Dadonov, A V EIremov

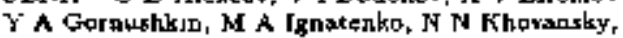

Z V Krumstern, L K Lytkı, V L Malyghey, A A Nozdrin,

I L Pubarey, T O Rudeuko, N A Rusiakoyich, B M Shbirov,

A B Sadarsky, $Y$ Y Sedykh, A N Sigzikian, $L$ G Thistcher,

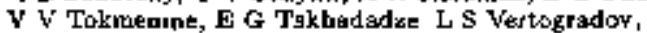

$Y$ A Yetgunesko, A I Zinchenko

FER MILA B - S C Abn, B Ealdın, J F Eartlest, P C Bhat,

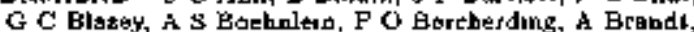
A D Brosg, J H Christangon, W E Cooper, 1 Dermartegu, D \$ Densnov, H T Dielıl, M Dxesburg, S Feber, 11 E Fisk, S C Fueas, K Genter, C E Gerber, D E Green H B Greenlee, W X Gu, H F Haggerty, S Habed, U Heluts, J D Habbo T HW, A 5 Ito, M E Johoson, A M Jonckbere, H Jostletn, B Kluma

S Kroywdensks, G Landsberr, Q H Liblemarlẹau, R J Lipton,

$Q$ Liv, L Lueking, H S Sto, M I Martin, H L Melanoon,

K W Merrict, OS Musbra, N Mokhoy, $\mathrm{H}$ E Monkzomery

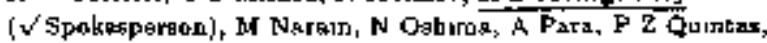




\section{SUMMARIES OF FERMILAB EXPERIMENTS}

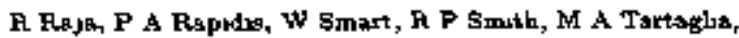
W J Womeroley, R Yamadia

FLORIDA U - G Muteelmakber

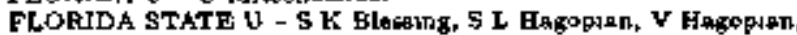

T C Heurng, R I Hirosky, $\mathbf{S} 1$ Lian, F W Maddten,

J McDonsd, B Piekarz, H B Prosper, C Shaffer, H D Wahi, G L Wang, S Yousuer

HAWAll ț - I Balderston, MA Cunmmigs, M D Jonea, M W Peterd, C Y Yasblkawa

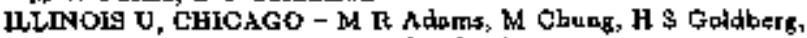
T M MeKibben, II , C R Murshy, J solomon

INDIANA U - G Alverax, T Hu, R L Jeglk, T R Matahsll,

D Ziempiles, A ziemonakı

IOWA STATE U - E W Anderson, J M Hauptman, B Lauer, J A Wichtmen

KOFEA It - 1 S Kang, C L Kim

KYUNGSUNG U - Y Patk

CRACOW - A Eskreyt, J Figel, B Pawik, P Stogx

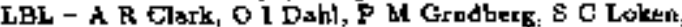

R I Mudarn, M I Steventon, M W Gtravink, T G Trtppe. $\mathrm{E}$ W Verneb

MARYLAND U - A R Baden, W C Coban, $\$$ C Eno, G Gomes, N I Hadley, \& Kupori, A L Lyon, F Tamburello, J A Thompen

MICHIGAN U - N A Amos, S Chopra, K W Del signor, T-

C F Hawh, D W Lucoln, H A Neal, L Otach, J hisisn

MICHIGAN 5TATE IJ - M A Abolums, A \& Brock, $G$ DtLokato,

D L Edmund, E M Flattum, K C Frama, T L Sold.

R. J Geolk, I1, S A Jerger, F Landry, J T Junnemann.

J MeKinky, D P Owen, B f Pope, T D Rockwell, N Yarelas,

B J Werts

MOSCOW STATE U - A Belyat, E E Baoa, L V Dudko,

P F Ermolov, A K Leflat, M Morkin, A Pukbov, E K Shabajina

E Sirotenka, N sotmikava, E G Zvtrey

NEBRASKA U - J Krane, G P. Snow

NEW YORK U - B K Abbott, A mincer, M Mudan, P Nemethy, J Scull, K R. T Streets

NOFTHEASTERN U E Amid, S M Chong, J H Mlorumbato, $\$$ Reucrolt, \& vod Goeter, D R Wood, T Yasads

NOETHERN ILLINOIS U - M R Fortner, J M Gran, D R Hedin, R Misurcelofi, VI Sirotenke, S E Wلس

NOATHWESTERN U - I Bertram, D A Bwchhols, B Gabb,

T Jofie Mindr, S Y Jan, B Mby, P Rubrooy, F M Sched]man. R M snihnr, T L Thylor

WOTTRE DAME U - J BLhop, N N Bıwas, J Jaques, $R$ L Kehoe, M I Kally, $\mathrm{F} C \mathrm{Ruch}$

OKLAHOMA U - G M Gughelmo. P Gutserrez, G K Kalbeterh, $T$ M McMrbon, J M Snow, M Strauss

PANJAB 1! - s Ber, V Bhatnagar, J X Koblı, H Singh, J Siagh, P M Sood

ST PETERß日LRG, INP - V Golortsov, V Kım, P V Neustraev, N K Terenter, L Uyaror

\$ERPUKJIOV - Y V Bebintsov, V A Bezzubav, N I Bagko,

Y $S$ Burkavol, $\$$ V Chekulacv, S P Danuxav, O V Eroshm,

Y N Evitokinow, A N Galyaew, P I Gondarow, 8 N Gurzhiey,

Y E cotnikov, B ] Klachkov, V [ Klyukhın, V [ Kochetkov,

A V Kostribaku, A V Kozolov, E A Koxlorsikl, A A Mayoruy,

V M Podjtavkov, D A Storapovt, A A Volkov, A P Vorobiev

PURDifE U - S Carabello, D S Koltuck, I Levine.

$Y$ M Pkchalpjkow

ECE U - D L Adams, G W Eppley, H E Mettugen, B P Padley, E Platner, P P Yopes

IRIO DE JANEIRO STATE $\mathrm{U}-\mathrm{J} \mathrm{R}$ T de Mella Neto, J G R Lams, $\vee 0 \mathrm{gur}$

ROCHESTER U - D P Esgey, O E Cretsmger, R K Fatyga,

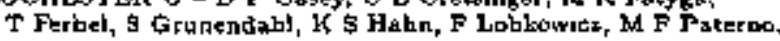
$B$ Won, $\mathbf{z}$ H Zhu, M Zuelınskı

SACLAY Y Ducroe, J F Labrat, Zy]barstej

GEOUL NATION.AE U S Char 9 K Kur, Y S Yu

SUNY, STONY BROOK - M M Bsarmand, Z Casalum, D Chakraborty, W d Chen, S Cn+r, D R Claes, V D Elvus, R J Engelmann, G Fipociphiso, A Gaupwo, P D Grannig ( $\checkmark$ Spolkeperson), T Hu, C K Jung, H L L, A L McCartby.

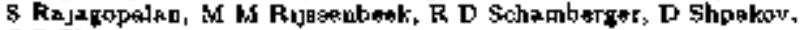
2 Z zhang

TATA INST - B S Acharya, 9 Enoetjee, S R. Dugad. A Gupta

M R Krugnaswamy, N K Mfondal, V S Naragimbam, N Parub.

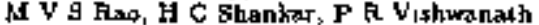

TEXAS U, ARLINGTON - K De, P A Draper, E J Gallas. J Lr, I Ferkinx, C Sowyer, S Shis, M D somebe, F W'stephens. A P White
TEXAS A AND M - L T Goes, F H Hegon, D M Norman,

J T Whice, o V D Wirjawn

Aacelerator FNAL.COLLIDER Detactor Do

Reactions

$\bar{p} p \quad 2000 \mathrm{GaV}\left(\mathrm{E}_{\mathrm{cm}}\right)$

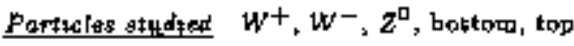

Brief descriplton The experwent studies the properties of 2-TeV Fp collieions wilh parlicular emphass on mexeturemant and jentification of jets, leptons and muand trensverse momentan The detector jncarporstes three main aystems a ceńtral deteccor, uramium lmud argon calorimetry over nearly 4 solyd angla, and a magnecred uros muon spectrometc Dațe was taleed from 3992 ta early 1986 (Ron J), with physicg covering the top quirk propertrea, prection elextroweak mesuruments, studues of QCD, b-quark production and wercher tor new particles and phenopnens The detector is being upgraded for operation an 3899 with the bither lumbosicy upgraded Collyder Trackung detectors will be replaced with

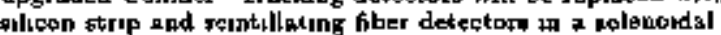
magnetic field, and ather detectora upgraded for hrgher rate operatuon Upgrade tn prograsa (July 06)

Journal popers [EEE TNS 32 (1945) 14t3, NIM A24d (1985) 956, NIX' A247 (1986) 107, CPC 45 (1987) 246, IEEE TNS 34 (1807) 710, NIM A246 (1987) 305, NIM A257 (1887) 556 , Nrut A261 (1987) 420, NCM A263 (198B) 78, NIM A265 (1988) 157. NIMf A269 (128B) 492 [artstum NI41 N273 (1989) 453) NIM A277 (3909) 401, NIM A279 (1899) 107, NIM A27\% (1989)

243, NIM A279 (1989) 310, NIM A279 (1989) S31, NIM A279 (1999) 359, NTM A280 (1969) 36, JEEE TNS 36 (198B) 384, NIM A2s9 (1900) 434, NIM A289 (1990) 543 , NIM A290 (1990) 122, NIM A290 (1900) 346, NIM A29) (1900) 125, NIM A.BOT (18日0) 121, EEEE TNS 35 (1901) 266, JEEE TNS 39 (I991) 398, NP (PROC SUPPL) 23B (I 991) 402, NIM A324 (1995) 53. NEM A.325 (1993) 393. NF (PROC SUPPL) 32 (1993) 29, NP (PROC stPPPL) 32 (1963) Ba, NIM A338 (1904) 185, NIM A342 (1994) 33, NDM A351 (2904) T2, NIML A351 (1964) 77, PEU 72 (1994) 985, PRU 72 (1994) 2138, PRU 72 (1994) 2332, NTM A368 (1905) 263, NP (PROC sLPPL) 44 (1995) 12, NP (PROC SUPPL) 44 (1095) 1 Ks, PL E357 (1995) 500, PL B958 (1905) 40, PRL 74 (1995) 2422, PRL 74 (1905) 2632, FRL 74 (1905) 3548, PRL 75 (1995) 618, PRL 75 (1995) 1023, PRL T5 (1995) 102B, PRU 75 (1905) 1034, PRU 75 (1905) 2466, PRL 76

(1905) 3226, PRL 75 (1995) 3618. PR D68 (1995) 1877, PL B370

(1996) 239, PRL TE (1998) 734, PRL 76 (1996) 2222, PRL 76

(1996) 2228, PRI 76 (1956) 9271, abd PR D5s (1996) 6000

Related expertiments FNALEB3

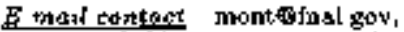
grancustisblutep physucs sunysb adu

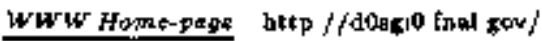

\section{FNAL-741 (CDF)}

(Fropowed Aug 1951, Approved Apr I952, Bercon data-kaking 1987, In progrege'

STUDY OF $\bar{P}$ P COLLISIONS USING A LARGE DETECTOR AT BO- THE CDE DETECTQR

CDF COLLABORATION

AFGOTNED - R E Etar, K Brrum, D Crane, \& Kuhlmann

T LeCompte, L J Noduluan, J Proudloot, R G Wagner, A B Wieklond

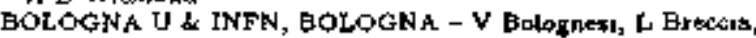

M Deniлno, G Farinell,, I Fion, L Malferrart, P Mazzant],

N Mloges, G B. Pracentuno, F Rimondi, F Semoris, S Zucchall

BRANDEIS U - 9 Bebrendy, J f. Benpager, C Elocker,

L E Kirwhb, I Lamouteux

CAICAGO U - A Amadon, J Barryhill, M Contretss

A. Culberton, H I Fuseh, C Groaso-Pilcher, M J sbochet, D Tobsck. I Wabl: F Wilson

DUKE U - D Crono-Henneses, J R Dittment, L Fortnky,

A T Goohaw, $\$$ A Hauger, W Kowald, S II Oh, T Plichips, W J Robertson, W Walker, C H Wang, C Wel

FERMILAB - $M$ ' $\mathbf{C}$ lbow, id Atac: A Barntvas, J P Berge, K Bıry, h1 E Bunkley, E Buckley-Gerr, A Byon. Waguer. 


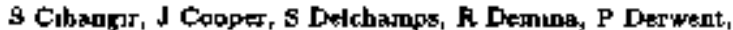
F DeJorgh, J E Elisa, W Erdmand, B Flaugher, G W Prster,

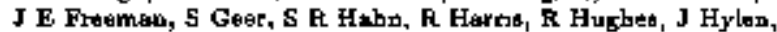
J Incesudeta, H B Jeasen, D Jobb, D Joysnovic, H. D Kephart, W Koska, I J Kroll, S Lammel, I D Lewis, P Limon, $P$ Lukene, K Moeshims, I P Mrruner, T Miad, A Mukberjee, C A Neloon,

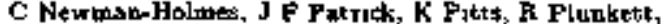
P Sebiabach, E E Schmidt, S L Seg]er, J Spaldıg, L Splegel, J Streat, D Stust, 5 Thacayk, A V Tollostrup, R Vided, A. L Wagner, W Webter, E Wicklund, A Yagil, G P Yeb, J Yob. J C Y Y

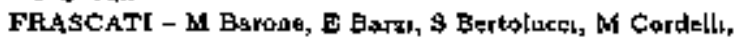
8 Dell: Agnello, F Donno, P Giromini, L Ketble, E Meschn, S Mrsetti, A Parri, $A$ Sangon,

GENEVA U - A Clark, C Conyountzels, H Kвmbara, T speer, $X$ Wh

HARVARD U - T P Baumann, J F de Trocomz, M Franklin, C Gry, A Gordon, R Hamilton, I E Huth, D Kastenbaum,

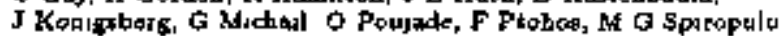
mRoshiMA U \& OSAkA $\mathrm{U}$ - $Y$ Iwala, T Ohmoto, T OhsugI, R Trkeabima, N Tasmurs

ILLDNOIS U, URBANA - L Christefek, D Errede, S M Errede, L E Hodloway, B M Keup. B Kharedia, T Lies, A J Martin, R u Routar

IFF, CANADA \&s MCGIIL U 2 TOFONTO U - B Hinnichaen, A'Holucher, H S Kim, K Kortax, K Ragan, o Signow, P sinervo, K Strahl, W I Taybor, W Triacbuk, A Worburton, $Y$ Yo JOHNS HOPKINS U - B A Earnett, J Cammeraten $Z$ Feng, D Gerdes, J Gumarbes, J Slarks, C Smutb, F Suder, J Tseng KEK - Y Fuker, S Mikrino, Y Morita

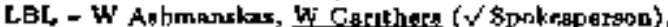
W Chmowaky, K Elnewtler, R P Ely, A B Galtrati, M Gares Seweres, $C$ H Betar, R Kadel, Y K Kun, M Lancaster, J Lyb, M Padina, M D Peters, D Reber, M Staptro, I L Suegrist, Fl Wengel, W Yoo

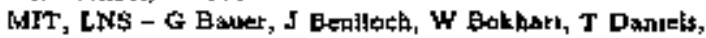
1 Friedmen, E Hafen, K Kelley, A Korytoy, P Maksimovic, C.Y P Nkan, L Roseriton, T Shah, P Sphucas, \$ \$ umarok, $S$ Tether, D Vuckic

MICHICAN STATE U - B Bromberg, J Haston, R Matker, 5 Mursta

MICHIGAN U - D Amader, K Buxkett, M Campbell, J Chspman, N Eddy, E Gullian, E Japes, s-G Kim, M Krawberg, C.C Miso, R Thun. S Trutt, S Vepeuk, D Winn, D Wolinuk,

NEW MEXICO U $M$ Bajley, N Bruner, M Gold, J Matthewg, E Moore, 5 Sadel, T L Thamas; S Worm, L Yu

OSAKA CITY U - Y Kald, T Okugaws, T Takahash,

Y Taramoto, H Toyads, T Yoshida

PADUA U d INFN, PADUA - P Azzi, N Bawchetta, D Birello, G Butetto, A Castro, T Dorıgo, M Gallinarn, Y Gotrk. M Loret, F Manda, L Pezebrs, A Ribon, I stanco, J Wyos

PEIN U - F Azfar, D Benton, B Bevenate, L Gladpey, B Hatral, J Heinrich, C Holck, R J Hollebeek, G Houk, N Loctyer, O Long, S D Metzler, R Oluvara, $F$ Ukegawa, G Una R Wilkrpasa, $\mathbf{H}$ H Williaphs

INFN, PISA \& PISA, SCUOLA NORLSALE SUPERIORE \&

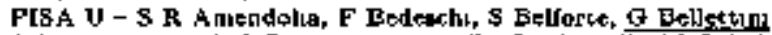
(V'Spokepperyon), \$ Betteth, F Cervelli, G Chiarelih, M Cobal, E Cocea, Mf Dell'Orgo, B Denby, S Donet], C Ferrestı, G Gaglinard, 3 Galnott, P Gianneth, M Incagl, N Labanca, S Lami, S Leone, M I Misangato, A Menzione, P Murat, R Panletti, N Parashar, A Peraxza, G Punzi, I F fustan, A Scribano, F spluells, A Stefanini: G F Tartarall, N Tutubs, $G$ Velov, $F$ Zętr

PITTsBurgh $v$ - I Boudreau, E Engek, Ir , T Hufiman, P F shopard, P F Stagh, g van deru Brwh

PURDUE U - V $E$ Barnes, D Bortoletto, M Fabluse A F Garfinkol, A Hardman, K Hoffinan, T Keaftaber, A T Lataber, $\mathbf{N} \mathbf{M}$ Staw, $Q$ Stet

ROCHESTER, U - S Blukk, A Bodnk, H S Budd, J Cassada, o de Earbate, Q Fap, B Kim, P Koehm, M Kruse, J Lu, M Pillat, W K Sakumoto, P Tipton, $K$ Tolleftan

ROCKEFELLER $U$ A Alcopisa, G A pollinaki, S Bazdasarov, A Bhatt, L Demortier, N D Gioksres, K Goultanos, D Kbazink,

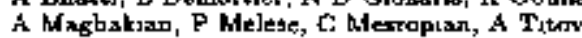

RUTGers U - J Consway T J Devlew, L Groer, C [faw'h, $\checkmark$ Jacobs, R D Kennedys. E W Kuns, C Loemis $I_{\mathbf{r}}, M$ Walsh, T l Watts
TANWAN, INST PHYS - P Chang, P Chang, H Y Cha, M$T$ Chenk, C-N Ghou, R Goo, Y-C Liu, A Soumbrokoy,

F $\mathrm{K}$ Tong, e-H Wagk, M-J Wang, $P$ Yeh

TEXAS A AND M - J DOHE, T Kamon, P M Mclatyre, B Tannertheum, RC Webb

TEXAS TECH - D Bobjamin, M Fraotach, $O$ Ganiel, $w$ klas, Q Litu, V Papadmitrion, A Sill, R Wigmans

TSUKUBA U - S Aot3, T Abrkawa, T Chukmmaten, S' Funak, K Hara, E Flryabli, 5 Tkeda, T Iro, T Kapeko, 3 Kin,

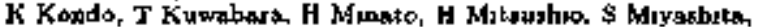

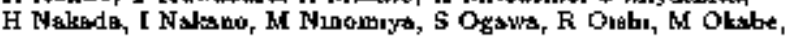
H Sato, Y Selye, M Shimojuma, J- Suzuit, T Taleada, M Takano,

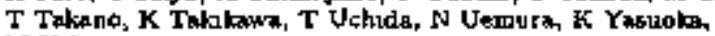
$M$ Yokoyama

TUFTS U - K Kart, K Stro, M Tumko

UCLA - Y Boumghtein, H Dshoke, L Dworkin, J Hauger,

F Keyvan, M Ludgred, T Maller, D Nenberger

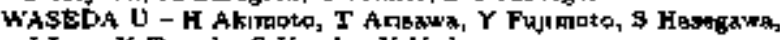
J Iwal, $K$ Teradb, $\mathbf{G}$ Leasles, Y Yods

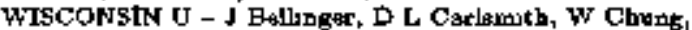

a M Hapdler, 8 Lean, J Otsen, I G Pondrom, J Steels, L Zbang

YALE U - R G Fenld, H Katha, K E OhI, S Pappat, A Setheffer, M P Schmadt.

Aceterntor FNAL-COLLIDER Detextor CDF

Reactean

$\overline{\mathbf{p}} \mathbf{y}$

600-2000 GiV (Erm)

Partictes studized $\mathrm{W}^{+}, \mathrm{W}^{-}, Z^{0}$, higgt, top

Brief degcription The flrat physice results were obtanted durmg 1987, in an engunoring rod, and th 1988/69, in a yoar-long run Uptrates for the 1991 rub are deacribed in the FNALT75 proposal, and another major toproviment wos ptoposed for the 1993 rus CDF a a general-purpose detector deargaed to study the pbysicg of $p \bar{p}$ colliarous Is has both azimuthat and forwnt-backward symmetry A superconductung solenold of

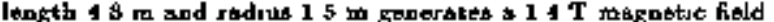
ond conkialns tincking chambery ured to detect charged particles and mestore thetr moratenta Sarrounding the solebond are aempling ealorimeters used to measuxe tha oloctzamarnctic and Jisdronic enerey of jels and electranks outande the calorimeters ste drilt chambers used for muton detection Surrounding the besm pips if a 4-layer plicion micrastrip vertex detector, and a vertex dejt chanber, both 1096 alled th 1292 The Colluder rua ended in Spring 96 with a total lumtnomsy of $110 \mathrm{pt}^{-1}$ recarded on tepe The detectot will be upgraded with rew trectesg. vertex derector, scint trgget electranics, and exhended muon eaverage lot the aext Collider run with the Main Injector, bow seheduled for Aprl, 1999 Upgrade in progress (July 96 )

Jaurnal puper\% NIM 204 (1983) 951, NIM 204 (1983) 361, NMT 205 (1988) 113, N1M 216 (1983) 127, NTM A21\& (1981) 172 . JdAP 45 (1984) 333, NIM A238 (1985) 18, IEEE TNS 34 (1987) 865. NIM A2ES (198B) 199, NIM A.267 (1958) 240. NIM A267 (IOBg) 257, NIMC A267 (1988) 272, NIM A267 (1986) 280, NMM A267 (1989) 301. NIM A2BT (1988) 315, NIM A267 (1988) 350, NIM A26\% [1988) 451 . NDM A268 (1988) 24: NIM A268 (1988) 33, NIM A26B (1988) 41, NTS A2B8 (1988) 46, NFM A2BS (1988) 50, NIA A26B (198B) 75, NLY A2ES (1989) 92, NIMS A269 (1989) 33. NIM A2669 (1989) 40, NIM A269 (1988) 51 Ni1M 2269 (1988) 83, NIM A269 (1988) 68, NIM A269 (1968) 82. NIM A269 (198B) 93, N[M A27] (1988) 387, PRL 6] (1988) 1619. PRL 62 (1989) 613, PRL 62 (1989) l005, PAL 62 (1988) 1525, PRL 62 (1099) 3020, PRL es (1989) 724, PRE 63 (1999) 1447, NTM A274 (1969) 443, NIM A281 (1969) 485, PR D40 (1969) 3791, NP A498 (1989) 193c, IEEE TNS 36 (1989) 35, IEEE TNS 36 (1989) 347. IEEE TNS 36 (1989) 40, TEEE TNS 36 (1989) 75., NP (PROC SUPPL) 12 (1990) 18, NP (PROC SUPPL) 12 (1990) 254, FRL 64 (1990) 142, PRL 64 (1990) 147. PRL 6d (1290) 152, PRL 64 (J的o) 157. PRL Es (1900) 348, PRL 65 (1990) 968, PRL \&s (1990) 2243, PT D41 (1990) 1717, PR D41 (1990) 1722, PR D41 (1990) 2340, PRE 68 (1901) 2951. PRL 67 (1991) 1502, PRE 67 (jg91) 2418, PRL 67 (1901) 2609, PRL 67 (1991) 2957, PFL e7 (1991) 3381, PF. D43 (1991) 664, PR. D43 (1981) 2070, PR D44 (1991) 29, PR D44 (1991) 601, NHA A315 (1992) 125, NP (PROC SUPPL) 25B (1992) 19, NP (PROC SUPPL) 27 (1952) 240, NP (PROC SUPPL) 27 (1992) 246, MPL A7 (1992) 2669, PRL 68 (1992) 417, Pfut 68 (1982) 1458, PHL 的 (1962) 1463, PFL 68 (1992) 2734, PFL 69 


\section{SUMMARIES OF FERMILAB EXPERMMENTS}

(1962\} 3398, PRL 的 (1992) 3403, PFL 69 $\{1902)$ 28, PRL 69 (1925) 2160, PRL 69 (1992) 2896, PRL 69 (1992) 3439, PRL 69 (1992) 3704, PR DAS (1992) 1448, PR D45 (1992) 2249. PR D45 (1992) 3921, PR D46 (1992) 1889, NIM A331 (3995) 57, NTM A3\$3 (1995) 200, NP (PROC GUPPL) 11 (1993) 189, PRL 70 (1903) 713, PRL 70 \{1993\} 1376, PRE 70 (1993) 2232, PRL 70 (1993) 4012, PRL 71 (1993) 500, PRL 71 (1993) 679, PRL 71 (1993) 1635, PRL 71 (1993) 2396, PRL 71 (1993) 2537, PRL 71 (1985) 2542, PRL 7] (1903) 3421, PR D47 (1403) 2639, FR D47 [1993) 4857, PR D4s (1993) 098, PR D48 (1963) 2998, PE D48 (1983) 3939 , NIM A 450 (1994) 73, NDM A35I (1994) 59, NIMI A351 (1994) 68, PRL 72 (1994) 1977, PRL 72 (1994) 3004, PRL T2 (1094) 3456, PeL 73 (1994) 220, PRL 73 (1994) 225, PRL 73 (1960) 2296, PRL 75 (I994) 2662 [erratun PRL 74 (1995) 1891], PFL T3 (1094) 208T, NC 107A (190d) 2085, PR. D49 (1094) L, PR DSO (1994) 2906, PR D50 (1994) 4252, FR D50 (1994) 551B, PR D50 (1964) 5535, FR D50 (1994) 5550, PR D50 (1994) 5562, NP (PROC SUPPL) \$9BC (1995) 348. NP (PROC SUPPL) 44 (1895) 20, NIM A359 (1095) 90, PRL 74 (1995) 341 FRI 74 (1996) \$60, PRL T4 (L905) 855, PRL T4 (1985) 1938 . PRL 74 (1995) 1941, PRL 74 (1695) 2626, PRL 74 (1995) 2900, PRL 74 (TO25) 3638, PRL T4 (J996) 40\$8, PRL 75 (1905) 11, FRL 75 (1995) 608, 户KL T5 (1995) 613, PRL 75 (1985) 1012, PRL 75 (1995) 1017, PRL 75 (1995) 1451, PRL 75 (J995) 3068, PRL 74 (J905) 3097, PRL 75 (1995) 4358, PR D51 (1995) 849, PR DSI (1995) 4623, PR D5s (1995) 2605, FR D52 (1995) 2624, PR D52 (1995) 4784 JJMP All (1996) 2045, IJMP A11 (1906) 2233, PRL TS (1996) 2006, PRI TE (1996) 2015, PRL 76 (1996) 2852, PFI 70 (1996) 3070, PRL 76 (1996) 4307 , PRL TO (1998) 4462, PFL 76 (1996) 4675, PR. D53 (1996) 1051, and PR D53 (1996) 3496

Reloted experimterts FNAL-T75, FNAL-830, FNAL-876

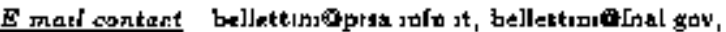
caritheteforinal gơ

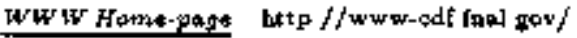

\section{FNAL-760}

(Proposed Max 1986, Approwed Jun 1985, Begen datp-takidx 1990, Completed dete-taking Jan 1992)

INVESTIGATION OF THE FORMATION OF CHARMO. NJUM STATES USING THE $\vec{p}$ ACCUMULATOR RING

UC, IRVINE - D F Broemralsuk, J E Fast, K E Gollwitzer, M A Mandelikern, J $\tau$ Marques, J Schults, A Stpith, M F Weher, C Zuoules

PERMILAB - L Bartosiek, V K Bharsdwaj, M D Church, A A Habs, 5 Y Hemeh, W L Marsh, J Peopler, Jr , S H Fardea $P$ A Feprdis, $s$ Werkemr

FERRARA U - D Bettonl, R Calabrest, V Carasulu, F Dalpias. M Fabbri, P Ferrett-Dalpuar, A Gianol, E Luppi, Mí Martinu. $F$ Petrucel, M Sarrle

INFN, GENDA - A Buzzo, M Mack, as M Marnell, M Palavietnt. S Passaggto, C Patrignami, M G Pia, A Pozzo, A Santront, A Scoliss, M Zito

NORTHWESTERW [f - D A Dunterovands, C M Girgburg H Whavzers, F. E Fsy, Jr, J L Roman, M Sermienla, K K Sarh, S Trokonheld, J L J Thao

PENN STATE L - T A Armglfong M A Hasth, $R$ A Lewlo, A M MajewskB, 3 Passnmexl, I D Red, G A Smith, Y Zhang TURUN U .. C Buno, G Barreani, A. Cecrucç, R. Certer ( $\sqrt{ }$ Spokesperson), F. Dibenedeteo. G Girsudo, F isarchtto,

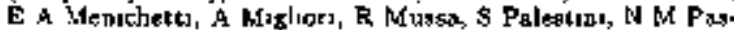

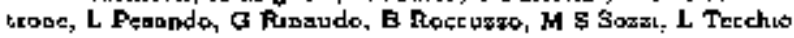

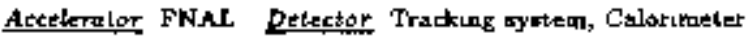
React:otas

$$
\begin{aligned}
& \bar{p} p \rightarrow \text { cbarm charar } \quad 3-T \text { GeV/c } \\
& \overline{\mathrm{P}} \rightarrow \mathrm{e}^{+} \mathrm{e}^{-} \\
& \text {р p-a's } \\
& \bar{p} p \rightarrow \bar{p} p \\
& \text { + }
\end{aligned}
$$

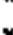

Particles aturied charmonium

Brtef descrapten Studies charmonum statey formed exclustwely in op collighodr, and their decaya to electromagnetic final states U\$क् a gas jet bydrogen touget in tho Fermilab p вource The detector consusts of a tradking system, hodoscopes. and Cerenkow couplers turrounded by a central load gtans eliettomsemetic calorimeter and a planar forward calorimeter Data analygis completed

Jourtuad papers NIM A271 (1986) 417, NIM A27T (1999) I16. NIM A2OD (1990) 73, NTM A301 (1991) 47, NLM AFOT (1991) 254, NIM A317 (1992) 135, SJNP 55 (1992) 792, 5.JNP 55 (1992) 811, SJNP 55 (1992) 865, PRL 68 (1902) 1468, PRL 69 (1992) 2337, NP Bs73 (1992) 35, FL Bsat (1993) 391, PL BSOt (1993) 399. PRL 70 (1943) 1212, PRC 7D (1903) 2983, NP A558 (1993) 2094, PR D4T (1993) 7T2, PR D48 (1993) 3037, NC 20TA (1094) 2013, PAN 57 (1994) 1513 - Y5 57 (1994) 1587, PAN 57 (1994) $1722=$ YF 57 (1064) 1763, NIMI A36s (1995) 308, and PR D52 (1965) 1839

Related GxpGrimente FNAL-835

E-mall contact captor WWW Home-page hotp //Www-e835 fral govf

FNAL-7\%1

(Pcoposed Fob 1986, Approved Apr 1987, Eegan deta-takeng 199I, Completed dext-trking Jan 1\$92

BEAUTY PRDDUCTION AND OTHER HEAVY QUARK PHYSICS ASSOCIATED WITH DIMUON PRO DUCTION IN \$0D (935) GFV/C SI INTERACTIONS

SOUTH ALABAMA L - R. K Clark, S M Jenkıa

UC, BERKELEY - H C Eatlogh, Jr, H H Bengham, J E Lyu,

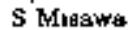

UCLA - A F Eoden, D B Cline, 8 Ramachadran, J M Rhoodes

DUKE U L R Fortne, W R Kowald, C Wta, B T Zou

FERMILAB - P O Mazur, O T harphy, R F \$mith, L Splegel, W Yants

HOLJSTON UJ - K H Lau, \& H Rí

DUBNA - I Budsgon

LECCE U P Greti, V Blıs, E Gortnt, F Grancagnola, M Parareo

MCGILL $U$ - J M Trobuk

NANJING U - T Y Clen, N G Yao

NORTHWESTERN U * M M Block

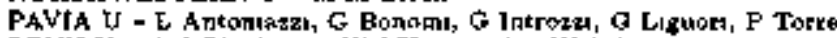

PENN U - A J Blankman, W J Kononaukb, W Selowe

PRATIE VIEW A AND M - M L Haire, D J Judd, L Turnbuk, D E Wogoner

SHINSHUU - M He, C H shep, C Warg: C Wer, N zhang

VANER COLL - M 5 Cooper

VIRGINIA U - M W ATwion, 2 L C 20 , S Conett

( $\checkmark$ Spokesperson), G Cort1, B B Cox ( $\sqrt{\text { Spohteaparesn }), ~}$ E Q Dukes, C M Ducandet. V Golovatyuk, K Hagan-Ingtam, P M Haulet, A A Ledavikoy, A P Memshus, K 9 Nelton,

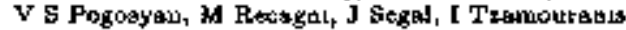

WtşQNSIN U - T Alexcopulow, A R ErwI, J R Jenning Accelertilor ENALTEV Detector Spectromeder

Reactogrs

$$
\begin{aligned}
& \text { p St } \rightarrow \mu^{+} \mu^{-X} \quad \text { squ GeV/c } \\
& 5, \rightarrow \text { muor } X \\
& \text { P } 5, B \overline{\mathrm{D}} \mathrm{X} \\
& p \mathrm{St} \rightarrow J / \psi(1 S) \mathrm{X} \\
& \text { pSi } \rightarrow \text { Xc(unspex) } X
\end{aligned}
$$

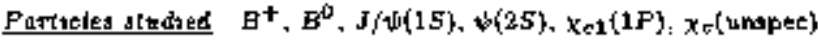
Eirtef dectiptadn Uses the FNAL-TOS spectrometer angmenled by $\$ 10,000$-channel aluron dotoctor and a saw zengle mnon and

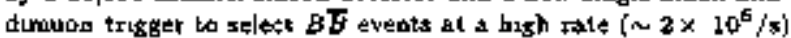
Data anslyals th progrta (July 06)

Journel papers NP (PROC SLIPPL) $23 \mathrm{~B}$ (1991) 249, NIM A314 (1992) 56S, NIM A315 (1992) 92, NIM A333 (1993) 142, NIM A337 (1903) 350, NIM AS40 (t994) 401, NIM A355 (1995) 320 , NIM A360 (1995) 334, PL B374 (1096) 271, and PRL 77 (1996) (In pres)

E-masl cortact cox\&luvahep phys virginis edu, caxtinal sov, serk1000virginis edu, gergiodial gov

WWW Home-page

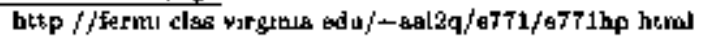




\section{FNAL-T'3}

(Prapaned Mar 1986, Approved Jul L988, Jun 1989, Degan data taking Jul 1991. Completed datsataking Sep J99L)

MEASUREMENT OF THE PHASE DIFFERENCE BETWEEN ?

CHUCAGO U - A R Barker, R A Erter, E Cheb, L K Gibbons, $D$ Harns, O D Makaf, K S Melsrland, is Roodmen, B Schwingenheuer, $Y$ W Wha, B D WJoste1n, R Winston ELMHURST COLL - E C Swallow

FERlMILAB - G J Back, R N Colomari, M Cruter, J Eangono, R Ford, Y B Hsurd, D Jengen, E Rambers, $R$ S Techiehert, $T$ Yamanake

ILLINOIS U, URBANA - E Cotline, G D Gollen ( $V$ Spokeppenon)

RUTGERS U - P Gu, P Hass, W P Hogan, S K Kum,

J N Matthews, \& S Myune, \& R Schnetzer, S Y Soroslwar,

G B Thomsan, Y Zau

Acceleratar FNALTEV Datector Spettramolet

\section{Brecteons}

$\begin{array}{lc}K_{\mathrm{L}}-\pi^{+} \pi^{-} & 60-160 \mathrm{GeV} / c \\ K_{\mathrm{L}} \rightarrow \pi^{+} \pi^{-} & " 1 \\ K_{\mathrm{L}}-\pi^{0} \pi^{0} & n \\ K_{S}-\pi^{+} \pi^{-} & " 1 \\ K_{S} \rightarrow \pi^{+} \pi^{-} & " \\ K_{S} \rightarrow \pi^{0} \pi^{0} & n\end{array}$

Particies studted $K_{\boldsymbol{L}}, K_{S}$

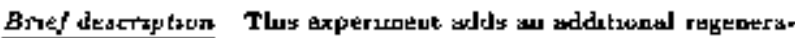
tor to the FNAL 73] spectrometer A druble $K_{I}$ heaps 1 1991dent on the spectrometex, which has 804 lead glase blocks and four drift chambert One beam posses throurh a tbin rerepoeretor at the start of the fiducies dersy volume, the other (rs. verses a thick resemerator 11 melers lurther upatresm The ro generators switch beams betweso manthe putias Neutral besm 6 praduced by 800 GrV protass on a 36-cm beryllium target

The experiment test $C P T$ Inwarnes Deta andyge completed (July 98)

Jourtal papers PRL 71 (1955) 4376, sad PFL i5 (1996) 2809

Retated expermerts FNAL-7g1, FNAL-796

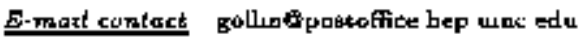

\section{FNAL-781}

(Froposed Mar I9B7, Approved Oct 18BB; In preparation)

GEGMENTED LARGE-X BARYON GPECTHOMETER (SELEX)

BEJJING, IHEP - K L He, F F Lang, $\mathrm{C}$ Z L1, Y S LI, Z G L1, C S Mro, Z L Mo, F K Tang, D R, Wang, Y G X, I Q Zhang, W H Zhas $\mathrm{S}$ C Zheng

BOGAzJCI U - E Gulme:

BRISTOL U - Y J S

CARNEGTE MELLON U - A MI Edt]ttenn, D G,bant,

E E Gotkecbalk, A Kushurenko, D Mao, P Mathew, M Mattgon,

D is Potter, M P Proceno, LS Evisa (Spokeeperson), S Yeng

RIO DE JANEIRO, CBPF - E C De Olivelrs, A A F Endle? L C G Olivers, M C Pormmol Mala

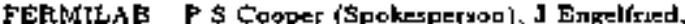

I R Kulmer, s Korad, J Luch, G Oleymik, E J Romberg.

D D Skuw, L G Stutte

HAWAIl U - C J Kenney, \$ 1 Farker

IOWA U - N A hecherin, M Aykat, K R Barger: M Kays, U Mashin, E R Meclunent, J M Mepterzon K D Neloon, C R Newgom, Y Onel, E Ozel, S Ozkorjacukly, L Pasqual 1

MUNICH, MAX PLANCK INST U Dersch, I Exchnch,

K Koebigamasu, I Konotov, H Krvegar, s Masosochi,'s M Paul, B Povh, J Simon, K Vorwather, fo Werdisg

MOSCOW STATE U - I Flımanov, E M Leukın. A Nemikkın $\checkmark$ riud

MOSCOW, JTEP - M Balats, G Devidenko, A Dolgolenko, G Dayutenter, V Evdokierow, P A Gontehev, V M Guahavin,

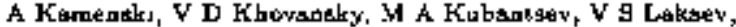
V Matveyev, A P Nulow, V A Pratskol, V K Seroyachkon, A L, Sitnikav, V Verehryusov, VE Vuhnyakav
PARAIBA $v$ - M LukByB

ST PETERSELRG, JNF - A Atsmantchouk, N Bondat, A 5 Dennov, A Golysh, L Golovtsov, V T Gratchey, A V Khangadectr, V T KIm, L M Kotchendn, A G Krivsbrch, N P Karopatkın, V Maleey, P V Naugtresv, VM Semeanov, V A Schogeleky. N N Smirnov, V L 9tepenor, M Srorahı, N K Terentiey, L N Uvaroy, A P Yorobsev

SERPUKHOV - R Elockld, Y Gonchakenke, O A Grathor, Y Koubarowskı, A Kozheyalkos, N Kulyaytzey, V F Kursbetsoy, l G Laadoberc, V Moltchenow, B A Mukhın, $\vee$ Mukhin. $S \mathrm{G}$ (B) Nurushev, A N Vatilvey, D V Vovslow, V A Victorav ROCHESTER U - T Farbel, G E Guther, $J_{r}, \mathrm{C}$ Hammer,

P F Slattery, M Zıelıbak

INFN, ROME M IOH

SAN LUIS POTOSI U - A MI Moreloe-Pineda

SAO PAULO U - O P Ebolt. L Emodlato, C O Eacobar, F Garcis,

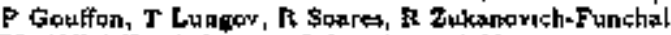

TEL AVIV U - S Getaod, J Grunhant, \$ Kanagoy,

M A Motneter, A Deherashvili, $Y$ glenbet

INFN. TRIESTE - A Bravar, D Dreosat, A Lamberto, A Penzo, G F Rsppazzo, F P Schravon

WhSHINGTON U, \$EATTLE - v Cbalonpka, T Zhoo

Acexieratar FNAL-TEV Detecter spectrometor

Parterles studyed charmed-baryon

Bruef deseriptuan Stodues both charmed baryon production and decays Trigen is baod on Impact perameter The opectrometer deploys \& aumber of exishing detectors as wedl as the new silicon attrip and pixel devices and a rug imsang Corankov sount.er Berag installed (May 9e)

E.maticaniact peooperofind gav, rusormphys phys cmu edu WWW Horne-page btip $/ /$ /n781 a mal gov/

\section{FNAL-789}

(Proposed Nor 1987, Appraved Oct 1998, Began datantoking 1890, Completed dasa-saking Jan 1992)

MEASUREMENT OF THE PRODUCTION AND DECAY INTO TWWO-BODY MODES OF b-QUARK MESONS AND BARYONS

ABIEENE CHRISTLAN U - L D toenhower, M E Sadler, R. Ot Schnothorst

TAIWAN, INST PHYS - Y C Chen, G K Kang, P K Teng

CHICAGO LT - L M Lederman, M H gchub

PERMLAB - G N Bronsn, W E Coopor, H D Glass.

K N Gounder, C S Muhra

LBL - G Gidal, F M Ho, M S Kowitt, K B Luk, D Psupstein

LOS ALAMOS - T A Curey, D M Jaubeu, R G Jeppesen,

J S Kapustinsky, D W Lans, M J Lejteh, J W LIJberz,

P E Mckanghny, J M Man, J C Pepg ( $/$ Spokeperson)

NORTHERN ILLINO]S $U-\mathrm{D}$ M Kaplan ( $/$ Spoke日perbon).

W R Lusbke, V M Mertin, R \& Preaton, I J Sa, V Talkelts SOUTH CAROLINA $\mathrm{W}-\mathrm{R}$ L Childerrs: C W Durden, J R Wron Acacieratar FNAL-TEV Detectar spectrometer

Regrivers

$$
p \text { nucleus } \quad 900 \mathrm{GeV} / \mathrm{c}\left(\mathrm{P}_{\text {女b }}\right)
$$

Partuctes studied bottom, charm

Braff descreption Studick low multuplicaly decsys of b. and oquark hadrons Easentinl to evaluating the autiabulity af dinsdroule besuty decaya for the otudy of CP vitolstion Is the $B$

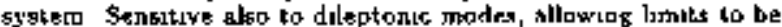
atet on theu branching ratides Uos the exibting PNAL-60s/772 apectrometer whth guitably upsraded tngger processor s\}:tem Datb ans]yajs in progreas (July 96)

Jourmal popers IEEE TNS as (1991) 46L, IEEE TNS 39 (1892) 758, NP A544 (1992) 197c, PRL 72 (1994) 1318, PRL 72 (1904) 2542 FK C50 (1994) 9, PKL T4 (1995) 3118, PR D52 (1985) 13ơr, and PR D52 (105) 1251

Related experiments FNAL-7J1, DESY.HFRA-B

E-mad contace pebgotlanl gov, leplauglnal gov WWW Home-page butp //p2hpt lart gov/e789/e789 btmt 


\section{SUMMARIES OF FERMUAB EXPERIMENTS}

\section{FNAL-791}

(Propoed Nor 1987, Approved Jun 1988, Began data-taking 1991, Completed datontakisg 360 [892)

\section{HADROPRODUCTION OF HEAVY FLAVOAS AT THE}

\section{TAGGED PHOTON LABORATORY}

\section{ETO1 COLLABORATION}

RIO DE JANERTO, CBPP - S F Anato, I Bedraga

I Costa, J M De Birandm J C Dos Anjos, I Mello, Neto,

F Motta, Filho, A Reti, A Saptora, I Solano, M H G Souzs

UC, SANTA CRUZ - \& Blaylock, F Gagpop. J Loglus.

K O'Shaughatesy, $\mathbf{K}$ Sugano

cinclinkati U - 5 Devmel, B Meadows, L P Pertera,

A K Sentbe, M D Sokoloff

FERMILAB - I A Appel ( $\sqrt{ }$ Spokmerson). 5 Bonerjes,

S B Bracker, T G Carter, L Chen-Tokbrek, K Denuswnko,

A $M$ Hedhug, $O C$ James, $S$ Kwan, $B$ o Lundberg, $K$ A Therne

ILliNOIS TECH - R A Burnstein, P A Kasper, K C Pebs. H A Rubin

KANGAS STATE U - M Aryal, A Nguyen, N W Eleay

R A 81dwell, N F Stanton, A Tripsth, N Witchey, S Yashudo, C 2 bang

MEXICO, IPN - A Gogo, G Herrera-Carral

MISS[SSIPP] U - E M Aitsla, L M Crematdi, K N Gounder,

A Rafatiar, J J Raidy, D I Suramers, D Y Y,

DHIO STATE $\mathbf{U}-\mathrm{K}$ Repbet

PRINCETON U - D Ladiga, A Schurarts, 3 Wlegte

PUEDLA U, MEXICO - E Cantiral, A B D'Olvairs, A Firnsider RIO DE JANEIRO U - H D S Garvalho, A J Ramallio

SOUTH CAROLINA U - N Copty, 11 V Pninhut \{ $V$ Spokespersaú

STANFORD U - F R Burchat, $\mathbf{R}$ Zalornyak

TEL AVTV U - D Aebery, S Gerzon, G Hurvits, J Lichtenstadt, 3 MayTal-Beck, R Weire-Babay

TUFTS U - R H Milbum, A Napare

WISCONSIN U - \$ A Radeztsky, M C Shętf, K Stenam, 5 Watengbe

YALE Ut - C L Dafling, A J Slanghtert, S F Takknch, E J Wodin Accelematar FNALTEV Detscites TPS

\section{Eleactions}

$$
\begin{aligned}
& x^{-} \text {nuclevs } \rightarrow \text { charm } X \\
& x^{-} \text {nuclevs } \rightarrow \text { botton } X
\end{aligned}
$$

Particter tuadiad charm, bothom

Bref diescription Conkipues studnes of FNAL-T69 Emphasues charm phy"日lcs and a first look at brottom hadroprodiction Targets zre $\mathrm{Pt}$, and $C$ fols some 20 bullipn events are collected More than 200,000 charm decays are fully reconstructed Data analysis in progresa (July 96)

Jpurtat prpers NIM A324 (1993) 535, Pl B571 (1996) 157, PL

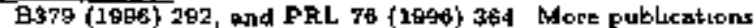
expected

Related experinerits FNAL-653, FNAL-687, FNAL-691, PNAL769, CERN-WA-082, CEFN-WA-0gB

E.mati contact sppelafual gov, putahitofial gow WWW Home-pisge

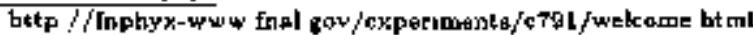

\section{FNAL-799}

(Proposed Jan 1989, Approved Jun (999, Began dats-takjing Oet 1991: In progrens\})

\section{SEARCH FOR THE DECAY $K_{\iota} \rightarrow \pi^{\mathrm{N}} \mathrm{e}^{+} e^{-}$}

UCLA - K AJjsoke, D Chen, 5 M Field, J R Jennwgs, I Kubnc, D Roberts, W E Slater, $M$ Weawer

UC. SAN DHEGO - H G E Kobrak, E Potter, R A Bronotor, $G$ A. White

CHICAGO I - E C Blnchor, G C Bowm, F. A Brince, 5 Rrught. E Chev, C E Grahari, J Grabam, D Harris, is S Kisolor, G D Makon K Mcferland, V Fragad, \& B Qurnn, of Rondman, g Schwingenteuter, P S Sbawbon, N Solomey, Y W Wah

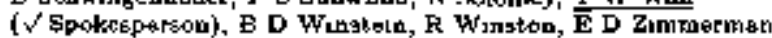

COLORADO U - A R Batker, D Filhwore, P L Mikslaons, U Nasubberg, J-Y Wu

ELMHURST COLL - E C SWE llow

FER,MILAB - R Ben-Dayd, G I Back, $\$$ R Chaldreat

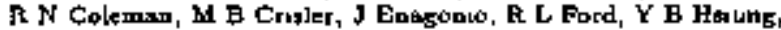

D A Jenten, T Kobularelk. H Neguyen, V O'Dell, R Porde日,

S A. Pordeg, D R. Puahks, E I Rambarg, R E Ray, JT,

A I Ronzhin, K C stanileld, Af \& Techichart, $K$ Vaxur,

H B While, J Whitmore

ILEINOIS $U$, URBANA - E Collins, G D Goll

OSAKA U -K Hanagak, M Hazum, T Hezumi, S Hudala,

F KBto, T Nakayr, M Sadsmoto, M Soro, M Yar, T Yamsantas ( $\sqrt{\text { Spoketperson) }}$

RICE U - J Barnes, In, B D Conoran, B P Psidley

RUTGERS U - I F M Albuquergue, I W Eelz, F M Hans,

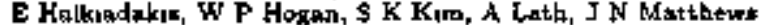

S S Myurg, G Fing, S R Schnetzer, S V Somelwar, R L Stond, R Tesurek, $\mathrm{G}$ I Thomson, $Y$ Zou

VIRGINLA if - $M$ w Arenton, B B Cox, F Duap, $K$ Hagan.

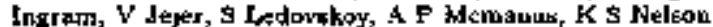
WISCONSIN U - A. Alert-Harath, T Alexapoulos, A R Erwin Acceleratgr FNALr'TBV petector Spectrometer, Colorimeter

Reactions

$p$ tuckeou $\rightarrow A X$

p aucleon $-\overline{\mathrm{A}} \mathrm{X}$

$K_{\mathrm{L}} \rightarrow \pi^{0} e^{+} e^{-}$

$K_{L} \rightarrow \pi^{0} \mu^{+} \mu^{-}$

$K_{L} \rightarrow \pi^{0} v_{k} I_{*}$

$K_{t} \rightarrow e^{+} e^{-} e^{+} e^{-}$

$K_{L} \rightarrow e^{+} e^{-}+\gamma$

$\kappa_{L}-\mu^{+} \mu^{-}{ }^{-}$

$K_{L} \rightarrow \pi^{\circ} \pi^{\circ} \mathrm{\gamma}$

$\pi^{0} \rightarrow e^{+} e^{-}$

$\pi^{0} \rightarrow e^{+} \mu^{-}$

$x^{0} \rightarrow e^{-} \mu^{+}$

Particles studutat $K_{L}, \pi^{0}, A$

Bref descroption The sopl is ti use rare $K_{L}$ decayt as a probe for the $C P$ volation Phase-I modtles the existing apparatus of FNAL-731 to handle increated $K_{L}$ flux and an Extended docay ragion, spd to provide o better makn derifification Stufise various multibody r8re $K_{L}$ decays, and $\pi^{0}$ decess Phese1 complated data takiag in Jeduary 92 Phapo-ll user a new

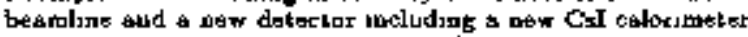

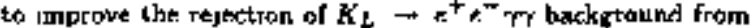
whe $K_{I} \rightarrow \pi^{0}{ }^{+} e^{-}$elgotal It also bete a new lranastion radiation detector (TRD) to achieve a bettex $\pi / 2$ rejection The senetivity is expocted to upproach the $10^{-11}$ level far many rafe $K_{\mathrm{L}}$ decays See also the efopely related FNAL- $83 z$ experiment Phego-II is pow teking date (July 96 )

Jourzal paptrs PFL 71 (1893) 31, PRL 73 (1993) 3914, PRL 71 (1953) 3918, PL B320 (1994) 407, PL B338 (2004) 403, PRL 72 (1994) 3000, PRL 72 (1994) 3758, PRL 73 (1994) 2l69, PR D50 (1904) 1874, and PRL 71 (1905) 3323

Reialed expertments FNAL791, FNAL-773. FNAL-832

E-tnat! contact wahohep uchicago edu, takufifuat gov

\section{FNAI-8DD}

(Proposed Mar 1988, Approved Oct 1988, Completed datstakug Jan 1992)

MEASUREMENT OF THE MAGNETIC MOMENT OF THE "? HYPERON

ARUZONA U - D K Fein, E B Jamen, K A Johos (Spokesperson) DEPAUW $U-V$ A Decarlo, $P$ M Tynan

FERMILAB - C Allan, R A Ramejka \{5 pokerperson\}

MICHIGAN U - $Y T$ GOO, 1 LONB

MINNESOTA U - J A AYala-Merçado, P M Border, D P C Cartipa.

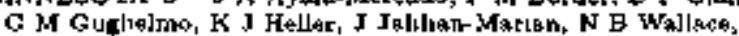

D is Woodte

Acasismigr FNAL-TEV Datestor Spectrordeter 


\section{SUMMARIES OF FERMILAB EXPERIMENTS}

Reactrons

$\begin{array}{lc}p \mathrm{~B} \rightarrow \Omega^{-} \mathrm{X} & 300 \mathrm{GeV} / c \\ \Lambda \mathrm{Cu} \rightarrow \Omega^{-} \mathrm{X} & 300-500 \mathrm{GeV} / \mathrm{t} \\ \Lambda \mathrm{Cu} \rightarrow \Xi^{-} \mathrm{X} & - \\ G^{0} \mathrm{Cu}-\Omega^{-} \mathrm{X} & +1 \\ \Xi^{0} \mathrm{Cu} \rightarrow \Xi^{-} \mathrm{X} & -\end{array}$

Earticlesptadiad $\Omega^{-}, \vec{b}^{-}$

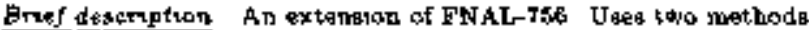

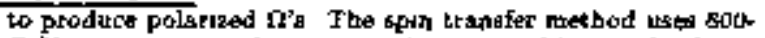
GeV protosa to produce a secondary netutesl besm of polarned $A^{\prime} g$ and $\Xi^{0} \mathrm{~s}$, which in then used to produce a tertiary beam of polarized $\Omega^{\prime} \mathrm{s}$ at $0 \mathrm{mr}$ The neutral production method uses

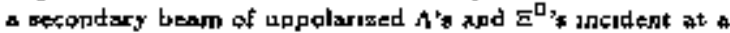
prodpetion sacle iq produce polartzed $\Omega^{\prime} \mathrm{B}$ The spectrometer

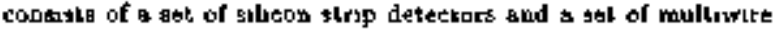
proportional chembers

taprnal papers PRL T4 (1986) 3732

Atioted expertinents FNAL 756

E.matl cortact johne inal gov, rameska

\section{FNAL-803}

(Propoed 1990, 1993, Approved Nov 1993, lo prepssstion)

$v_{\mu}$ TO ${ }_{2}$ OSCILLATIONS

COSMOS COLLABOFATION

AICHI U OF EDUCATION - K Kodame, N Ushada

ATHENS V - G S TranekoB

UC, DAVIS - V Paodore, P M Yager

CHANGWON NATIONAL U - O H Fahn

CHONISAM NATIONAL U - J Y Kum

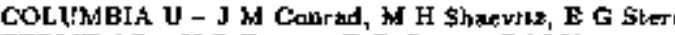

FERMLLAB - V D Boger. T G Dscter, G M Kolsuml,

B \& Litadberg, A J Molenxek, I G Morfa, R A Rameıks

GIFU U - K Nakarawa S Tragka

GYEONGSANG NATIONAL U - I G Park, I S SOME

TtROSAKI U - S KuTamata

ILLINOIS TECH - F. A Burogte]n, H A Rubin

[NDIANA U-C Bewer, R M Hetra, L Ma]ler, s Mufoon, J Murecr

KANSAS STATE

N W Resy ( $\checkmark$ Spokesperion), R A Bdwell, E Smith,

M R Startan, DM Woods, \& Yoabuda

KINKI U, OSAKA - M ChIKaWr

KOEE U - S Aokd, T Hara

KOHEA INST SCI - J K Kim

KOFEA U - J S Kang, $C$ O KIm

MICHIGAN U - R C Ealt, S Couty K Graen, D Levin,

3 Mallhews, S McKee, D F Ntts, S Nutter, J K Rtles, E F Roe,

G Tarle, R P Thun: J O Vander Velde, C Weaverdyck

MINNESOTA U - J K Nolson, F, W Rlusack, v M Singh

MOSSOW, ITÉ - A Asratym, M Belot, 6 Davidenka,

A Dolkolenko, G Diyubertko, A Evdoksmov, A Garsestmar,

V 3 Ksttanov, H A Kuhantegv, V K Semyarhkin, A L Sitnikov,

$\checkmark$ Verebryusov, $V E$ Vinhnyalsov

NAGOYA INST TECH - Y Isokane, Y Tauneolk

NAGOYA U - K Hoghino, H Kitamura, M Kobsyaght,

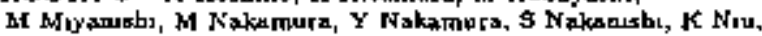

K Nrwas M Nomuta, K Salto, H Tajums, K Teraoka

OKAYABA Af - K Monynma, H Shbola

OSAKA CITY U T Okwawa, M Teqrabake, T Tomunaga,

$T$ Watsmabe, $T$ Yogbuds

OSAKA PREFECTURE U - H Okabe, I Yokota

OSAKA U OF COMMERCE - G Fujloka, Y Takabash

SEOUL NATFONAL U -J W KM

SOAI U O Kusumoto

SOUTH CAROLLNA U - F T Avigand, A Kulık, \& Rosenfeld

TBCHNION - J Goddherg

TOHO U - M Adachi, MS Kaguno, Y Kobaynabi, E Nuv, S Ono

I Shibuya, Y Umezaw

TUFTS L - T Kanta, A Napler. Wi P Olivet, J Schaeps

UCLA - M Atac, D B Clune, w Houg, J Fark, J M Rhoader,

J K Woo

UTSUNOMIYA $\mathrm{U}-Y$ SALD, I Teguka

YOKOHAMLA NATIONAL D - Y Mtędz
Afrealerator FNAL Detector Emulason, Spectrorater

Eeactions

$\omega+$ nucletap $\rightarrow+X$

Partaclest giudised $v_{\mu}, \nu_{T}$

Brief description This is a sbort-bastlitio neurrino oecillation experımenl which searches for the $v_{a} \rightarrow v_{\tau_{1}} v_{s} \rightarrow v_{r}$, and $v_{\mu} \rightarrow 1 / t_{r}$ transibiobs Uner the COSHOS detector, a bybrid

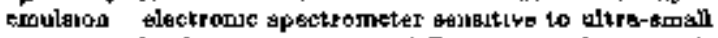
muxing anglas for nevtrino mass differences in the range iarger than $01 \mathrm{eV}^{2}$ Resndes an a wide band, 10-70 GeV, nequtnn: beam from Fermulab'a bew Masn Injector In preparanon (July 96)

Fetated expertmert, FNAL-531, CEFN-WA-095, CERN-WA-096 B-trand contact rasybep phys ksu edu, reaytaInal gov WWW Home-poge

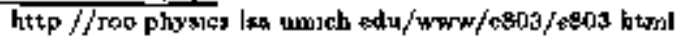

\section{FNAL 811}

(Propoed Mar 1001, Approved Jul 1992, Brgan dete-takubg Jan Je96, Completed datarlaking Feb (996)

\section{$\bar{p}_{p}$ ELASTIC SCATTEFING}

CERN - R. DoSatvo, MC R Aroodardim

CORNELL $U-C$ Ayils, $C$ M Gusa, J Orear ( $\sqrt{ }$ spokesperson)

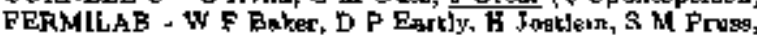

F. Rubidetten, F Turkot

decelerutor FNAL-COLLIOER Defector Sesmilletor

Reactions
$\bar{p} p-\bar{\nu} p$

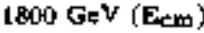

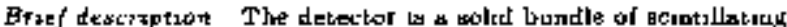
fibers The fibers are parallel to the beam, insude the beam pipe Cau be wemotely movod clase to the beam Measures at and y coordinates of ecstleted protong to 50-mecron securecy seattering sngles gre amoll enough to observe Coulomb interference and to we the opticai theorem to eet total ctoso-section Data sualysig L progress (July 96)

dourtal paperis NIM A323 (1992) 419, NP (PROC \$UPPL) 25B (1902) 281, and NP (PROC SUPPL) 25B (1992) 204

Retated experimerts FNALro 10

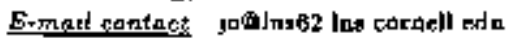
WWW Home page beto //freq811 fatal gov B000;

\section{FNAL-815}

(Ploposted Oct Igeo Approved Jul 1992, Begad date-takıng May 1906, It progres')

PRECIBION MEASUREMENTS OF NEUTTENO NEU. TRAL CURRENT INTERACTIONB USING A SIGN. SELECTED BEAM

\section{NUTEV COLLABORATION}

ADELPHI U R V Stoiner

CINCINNaTi U - R Johmson, L P Perere, o W Trohn, M Vekil

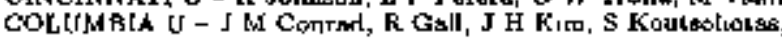

S Lammers, C keNutty, A Romosob, P C Rowrou,

M H Shotvitz ( $/$ Spokesperson), F Spentrour]3, E G Stern: A Vaitnitis

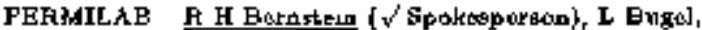

G Kolzum, MI J Lamm, W L Maceh, K S MeFartand, J Yu

KANSAS STATE U T \& Bolton, J Goldman, S MePetk,

D L Nopjes, J Norres

NORTHWESTERN U - D A Buchholz, L Debarbara

H Mt schelttrasn, G Zeiler

OREGON U - J E Bralu, R B Dtucker, R E Frey, D Matars ROCHESTER U P S Aucbuncloxs, 8 Ayvaknmor, A Bodek,

H S Budd, P Debarbaro, D Harris, W K Sakumnto, U K Yang $X$ Aviek Ut $P$ Nienaber

Accelerator FNAL-TEV Detectar LAB-E 


\section{SUMMARIES OF FERMTLAB EXPERTMENTS}

\section{Reactrang}

$$
\begin{array}{lc}
\nu \pi \rightarrow \nu^{-} \mathrm{X} & 250 \mathrm{GeV}\left(\mathrm{E}_{\mathrm{lab}}\right) \\
\nu n \rightarrow \nu \mathrm{X} & \|
\end{array}
$$

Partigles atadied 2

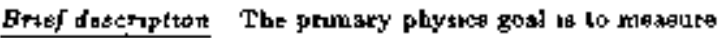
$\sin ^{2} \theta_{W}$ to a preanion of $\pm(0002-0,003)$ The bigh precistod s. achered by makeng use of a bew high-intensity sign-selected neutrino beam The new bean desjgn permits chasn separation of $v$ fropd $\bar{v}$ whylo providmg enough uthensity to mauntsun small utstuptics errors Other zoald upclude the stady of the QCD acale parmetter $A$, the charm mats, the CKN matrux elament $V_{c d}$, and the effecta of than gtrenge querk wa and charm quark sea on proton Approwed for a two-year run Taking data (Jaly 96)

Reloted experiments FNAL-744, FNAL-TTD

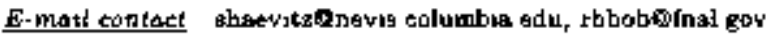
WWW Home-page hutp //condelse foal gow/NuTeV htm

\section{FNA L-831}

(Proponed Uct 1990, Approved Dex 1992, In preparation)

HIGH \$TATTSTIES STUDY OF STATES CONTAINING HEAVY QUARKS USING THE WIDE BAND PHOTON BEAM

\section{POCUS COLLABORATION}

UC, DAVIS - J Link, Y Paolon, P M Yager

RIO DE JANEIRO, CBPF - A L Barbroes, I Bediage

J C Dos Apjos, C Gobel, G Guedes, I M Pepe, A Re1 Corres, P Simas

MEXICO, IPN - S Carrillo, E Cagmuro, G Hermats-Corral, H Mender C țnie

COLORADO U - L Cumquid, J P Cumedet (V Spokesperacn), E S Erdos, E O:Relliy, B Vaunderang

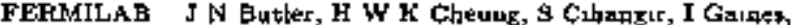

P 8 Garbmciug, L A Garren, S A Gourlay, D J Harding,

P H Kaspar, A E Krojmer, P L C Lebrun, S Shukla

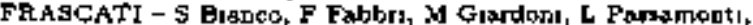
$\checkmark$ Ruz9o, S Sarwar, A Zallo

ILLINOIS U, UREANA - C Carlfald, F D Gogawall, R Gardiner,

K I Park, L Peak, A M RahıमI, J E Wh,

KOREA U - B G Cheor, Y S Churs, J S Kang, K Y Kinh K B LeB, S S My

DNFN, MIL AN - M B Boschin, P D'Angelo, P Inzand \$ Malvezzl,

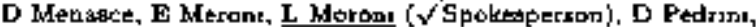

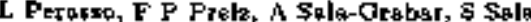

MILAN U - G Alimopt b, G Beltun: B Caccianiza, M1 Droorato,

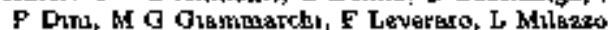

NORTH CAROLITNA U - T F Davenpart, JII

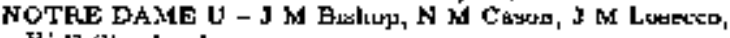
Wi D shephard

PAVIA U - V Arena, O Bararba, G Braca, G Boncmi, 8 Bricola C Cesella, E D'Usan G Guantn1, E lolurte, G Jovius, G Liguran, 1. Locatefl, M Marcberottt, S P Ratt, C M Ructard. P Totre, F Vertellati, I Viala, P Vitulo

PUEBLA U MEX]CO - E Cavioral: A Fêrnaudex, g Hernundeq, G Munot, J Ramiraz

PIJERTO RLOO U, MAYAGUEZ - I Alemar, A M Lapex,

L Mendes, A Wirles, E Montrel, H Mourad, J Raunies,

C Ruvera, W Rolke Y l Zharg

SOUTH CAROL INA U - N Copty, W Jobre, M V Purohu, I R Wilson

TENNESSEE U G T Condo, K Danyo-Blacketr, $T$ Hendlet

VANDEREILT U - J W Can, M Hoteck, M Nehriag P D Sheldon, us $\mathrm{S}$ Webgiet

WISCONSLN U - M C Sheaff $K$ Giengon

\section{Accelefator FNALTEV Detector Spectrometer}

\section{Angiglong}

$\rightarrow$ Ducleus $\rightarrow$ tharm megon $X$

Tr nucleus $\rightarrow$ charm baryon $K$

$$
<250 \mathrm{GeV} / \mathrm{c}\left(\mathrm{P}_{\mathrm{lsb}}\right)
$$

Parbicles gtudzed $\dot{\psi}$ (nuspec) charm

Druef descrtptioti Coutiouea studiee of FNALregr Uges

bremeatrablants photoing from a wide baud $250 \mathrm{GeV}( \pm 15 \%)$

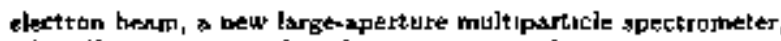

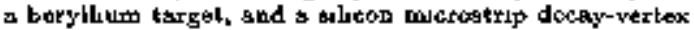
detector Stadies the photoproduction and decay of heavy quarks Betuq installeri (July 96)

Related trpertmerts FNAL-687

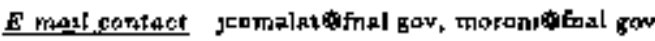
WWW Home-page http //da831 [nal gov/

\section{FNAL-832}

(Proposed Oet 1990, Approved Jun 1982, Begac dationtakıg Jul 1998, In progress)

SEARCH FOR DIRECT CP VTOLATION IN THE DECAYS OF THE NEUTRAL KAON

KTEV COLLABORATION

UCLA - K Arlaska, \& M Field, J R Jengings. J Kubic, W E Slater UC, SAN DIEGO - H G E Kobrkk, E Poller, R A Swanton, G A white

CHICAOO D - E C Blucher, G C Bown, S Braght, E Cheu,

g E Graharm, J Graham, R \& Kertler, V Prosad, \& 8 Quunn A I Foodmall, P S Shawhin, N Solomey, Y W Wall,

B D Winstien ( $\checkmark$ Spokesperson), R Winston, E D Zummermen

COLORADO U - A R. Barker, D Fillmore, P L Mikalgont, $\checkmark$ Nacenbers, J $Y$ Wu

ELMHURST COLL - E C Swallow

FEPLMILA B - L Bdlanton,, R Een.David, G J Bock,

S R. Chldzese, R i Colemsn, M B Cnsler, R L Ford,

Y B Hxiung (V Spokexperson\}, D Jenen, T Kablarck,

T Naknya, II Nguyen, V OיDell, al Fans, R Pordes, S A Pordes,

L R P usbate, E J Ramberg, R E Ray, Jr A I Ronzbip,

P Shanahan, $k \subset$ Stanfiald, R. S Tuchurbart, K Vozur,

HI B Whute, J Whitmote

OSAKA U K Hanagaki, M Hszum, T Haximi, S Hidaks,

$F$ Kato, Y Matanmiya, M Sadamato, M sogo, is Takits.

T Tsujs, $M$ Yagl, $T$ Yamanalks

RICE U A Bellantance, M D Corcoran, B P Padley

RUTGERS U - I F M Albuquerqac, s Avetstte, J W Bele,

E Halkiadakis, A Lath, 5 R schmetzer, S V Sonnalwar,

R. L Stone, R. Tagsrek, G B Thomos

VIRGINIA $\mathrm{U}-\mathrm{M}$ W Arorton, B $\mathrm{B}$ Cux, K Hagan-lngram,

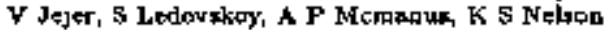

WISCONSIN U - A Alswi-Haratl, T Alexopoulos, A R Erwia

Acceterator FNAL-TEV Detector Spactrometar, Calorimeter

Reactans

$K_{L} \rightarrow \pi^{+} \pi^{-}$
$K_{L} \rightarrow \pi^{0} \pi^{0}$
$K_{L} \rightarrow \pi^{+} \pi^{-} \gamma$
$K_{L} \rightarrow \pi^{0} \gamma \gamma$
$K_{L} \rightarrow \pi^{0}{ }^{2} \bar{\nu}$
$K_{S} \rightarrow \pi^{+} \pi^{-}$
$K_{S} \rightarrow \pi^{0} \bar{r}^{0}$
$K_{S} \rightarrow \pi^{+} \pi^{-} \gamma$

Partacles tiudited $K_{L}, K_{S}$

Brad descropteon Mtewsures the direct CP yiolation pargereter Fee(e'fe) to the precision of $10^{-4}$ The new aeutral kgon boam facility, KTeV. is constructed to give five times monre flux, with raduced muon beckground and scaldobled rate Tbe spparatus gave twice as long a decay regron, higher rate capability, and more bermelie photon veto covernge againet the 3x backgraund The positurn and energy repolution of the electromsgnetic colonmeter (C $\mathrm{C}_{\mathrm{BI}}$ ) for etectron and pholon are improved UЈge日 a beryllium target Takıng data (Juty 96)

Jaurict papers NM A.967 (1995) 252, and NIM A369 (1998) 6.53

Relisted erpertments FN/LL-731, -773, 799, CERN-NA-048

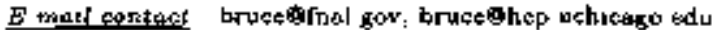
hatungorlast gov

WWW Home.page

hstp //faphyx-wwow inal gov/experiments/ktev/ktcy html 


\section{SUMMARIES OF FERMILAB EXPERIMENTS}

\section{FNAL-895}

(Propoged 1992, Approved Dec L993, I preparalian)

\section{STUDY OF CHARMONIUM SPECTFOSCOPY IN} PROTON-ANTIPROTON ANNIHILATION

\section{CHARMONIUM COLLABORATION}

UC, IRVINE - K E Gallwitzer, M A Mandelkern, 3 sochultz, $M$ Thorppon, G Zhoulas

FERMILAB - M D Chuteh, A A Habr: W L Barsh,

J Peoplea, Jr, 5 A Pordes, P \& Fapidis J Stretts, 5 Werkerna

FERRARA U - M Ambrograan, W Beldiu, D Bettom,

M Barpangol, D Bons, $\mathrm{K}$ Calabrese, F Dappiaz, E Lappi,

R Msubes, M Ssyrié, G Stancan

INFN, GENOA - A Bizzos, M Lorvelate, M Marer, M M Martuelli, M Pallsticini, C Patrisanar, M G Pta E Robutti, A Santiand

NORTHWESTERN U - X I Fan, $S$ Jin, P Mans. T K PAdlar,

I L Ruern, K K Seth

TURIN U - G Bortasmi, R Oesler ( $V$ Spokesperson), F Marchetto, E A Mentchett], N M Pagtrobe

Arceicrator FNAL Deteglar Tracking system, Calorumetor

Reactions

$$
\begin{aligned}
& \text { p p } \rightarrow \text { charw charm } \\
& \overline{\mathbf{p}} p \rightarrow e^{+} e^{-} \\
& \overline{\boldsymbol{p}} \boldsymbol{p} \rightarrow \boldsymbol{十}^{\prime} \mathrm{\varepsilon} \\
& \overline{\boldsymbol{p}} \boldsymbol{p} \rightarrow \overline{\boldsymbol{p}} \mathrm{p} \\
& \overline{\mathbf{p}} \boldsymbol{p} \rightarrow \boldsymbol{\phi} \phi \\
& \begin{array}{c}
\text { 3-T GeV/C }\left(P_{3 a b}\right) \\
n \\
" \\
n
\end{array}
\end{aligned}
$$

Particies strdited $n_{c}(1 S), n_{\infty}(2 S), h_{4}(3 F), x_{e}(1 P), D$

Bruf descraption Continted the protram of the FNAL-760 expetiment in order to cumplete the study of clot masede, widthe and brasching fractiong of chamonum states Adds a search Ior trypidexolics and a study of $\mathrm{J} / \mathrm{y}$ formation to mellesr mattet Improvement; in the spparasus include mereaved denijty of the tuterbal torgez, better tharged particle detecuon, ob upgrarte in thr central celorimetry snd a now data raginution atchindectute Thit to sh Aceumulstor Rogs axpersmont Boing installed (July 9 )

Reigated experzments FNAL-760

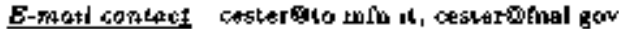

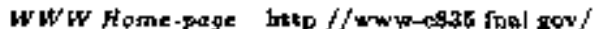

\section{FNAL-866}

(Fropowed Sep J日92, Approved Des 1992, In pjeparation)

MEASUREMENT OF THE RATIO OF ANTIQUARK DISTAIBUTIDNs $d(x) / \vec{u}(x)$ IN THE PROTON

NUSEA COLLABORATION

ABILENE CHRLSTIAN V L D Isenhower, XI E Sadler,

R S Towel, $D$ Whe

ARGONNE - K G Balley, D F Geesennar, H E Jackson, Jr

C Jones, \$ Kavfrasn, R Kowrkezyk, N Makins, T O Neall,

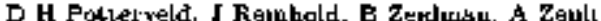

FERMILAB - $Q$ N Erowa, W E Copper, C $g$ Mebre

GEORGIA STATE U XCHe, WM L $\mathrm{L}_{\text {, }}$ \& Petitt

ILLtWOIs TECH D Mi Kaplan

LOS ALAMOS - M L Brooks, T A Corey, F Federspenl.

G T Garvey, D M Jansern, D M Lee, M J Leith,

J B McClelland, P E MeGaughey ( $\sqrt{ }$ Spokeapersou), C I Morrs,

J M Moue B K Perk, J C Peng, W Sondbeim. T Thompaon

LOUISIANA STATE $\mathrm{U}$ - P N Kryk, Y C Wang, Z F Wiag

NEW MEXICO 5TATE U - W Beddo, G Eurkeson, T H Chang.

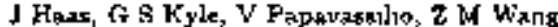

OAK RIDGE - T Awes H Kur, P Obealhsin, F Platil, S Saul.

P Stonkus, G R Young

TEXAS A AND M - C A Oaghagd E Hawker, R E Trubble

Valparulso U, INDIANA - D D Koetke, P Nord S Stonmlatae

Acccleralor FNALTEV Betector Spectrorneter

Fiterctotits

p nucleon $\quad$ Ron (ieV $\left(\mathrm{T}_{\mid, \mathrm{b}, \mathrm{b}}\right)$

Partucles attratieri p
Brief desctiptor The axpersment is a precision measurement of Drefl-Yan yulds from hydrosen and deutarium The ratio of shese $\gamma$ relda can be noed to inter the ratio $\bar{z}[x) / d(x)$ in the proton, over the $x$ unterval batwert 0 as and 03 Messares afio the $\mathrm{J} / \phi, \phi^{t}, \mathrm{Y}, \mathrm{Y}^{t}$, sad $\mathrm{Y}^{\mathrm{fl}}$ yjelde from both targets Unen she Magnetic Dikpton \$pectromitter, with 3 dipolsos, 3 stations of wir. chamhers, 1 xtation with prop-tubes, 4 hodoscope ststrost, and high rate capobitily with better thas 100 MeV realution af the $J / p$ Beam produces $10^{17}$ protons/apill Targets are LH and L. Beng unstailed (Jaly 00 )

Related experinerts FNAL-772, CEFN-NA-051

Esmand contack plmalatil gov

WhW Home-page ht1p //p2hp2 lanl gav/e866/e866 htm

\section{FNAL-868}

(Propoed Sep 1992, Approved Mar 1993. Began dats-takıg Apr 1995, complected data-takong Jul 1090)

SEARCH FOR ANTIPEOTON DECAY AT THE ANTIPROTON ACEUMULATOR

APEX COLLABORATION

UCLA - C D Bucbenen, B Corbun, M A Ludgran, T Mviler

FERMILAB - S Geer ( $V$ Gpokotperson), I P Marruer,

M Martens, R E Ray Jr I Streta, W C Wester

MICHIGAN U - H R tmostafion

NEBRASKA U - M J Hu, G R Sndw

PENN STATE U - T A Arastrong

Accebrator FNAL-TEV Detector Calurianeter

Reacting:

$$
\bar{D} \rightarrow e^{-} \mathbf{X} \quad \text { \&9 Gov/e }\left(\mathrm{P}_{\text {lab }}\right)
$$

Particies studted $\overline{\mathbf{p}}$

Bref descroptor Tle desechor conserts of a calorimeser, fiber tracker, pre-radintor, DEDX, ipd vetos IJse the Ant iprotan

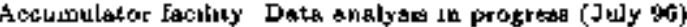

Aclated erperisents FNAL-8El

E-mal cantact $\$$ gerortifal gow WWW Hoinerage http //Foapxl fhal gov/

FNAL-871

(Proposed Oct 169\$, Approved Jul 1894, In preparation)

SEARCH FOR CP VIOLATION IN THE DECAYG OF $\Xi^{-} / \bar{B}^{+}$AND $A / \bar{\Lambda}$ HYPERONS

HYPERCP COLLABORATION

AAIWAN, INST FHYS A Cbat, Y C Chen, $\mathrm{K} C$ Cheng, $C \mathrm{~L}$ Ho, M Hutis, 8 C Lee. P K Teng

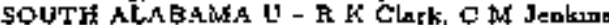

LeL \& UC, EEFKELEY W S Choong, $A$ Fuzesy, $Q$ Gidel

P Gu, K B Luk ( $/$ Spokeeperson), B Turko

FERhill AD -. T Girter, Mi Cneler, C C Jamen, I T Volk

GLANaJUATO U - J Felix-Velde, G Moreno, M A Sose Aquno

ULLNOIS TFCH - S Biedron F A Butngtein, J A Drapala,

D M Kapjan, L M Lederman, W' Lueblc, A Oaturk, H A Rubur,

D Sowjngla, $C$ White, $S$ white

CAI!SANNE U - Ñ Leros, I P Prrroud

MICHIGAN U - H R Gustarson, M J longo

VIRGINLA U - K Elodor, S Conets], E C Dnke

( $\checkmark$ spakexpersog') C Durandet, $K \leqslant$ Nelson, D Fajarart.

N Badeh, H-C Zhu

Aeselarator FNAL-TEV Detectar Spectromaler, Spectrameter

Reactions

$$
\begin{aligned}
& \text { p nuclem } \rightarrow \bar{\Xi}^{-} \mathrm{X} \\
& \text { p nucleon } \rightarrow \overline{\bar{c}}^{+} \mathrm{X}
\end{aligned}
$$

Partictes stadied $\Xi^{-}, \bar{\Xi}^{+} \Omega^{-}, \bar{\Omega}+$

Brafef description topalaruxed $\Xi^{-}$and $\bar{\Xi}^{+}$hyperonx are produced by prolons and momentiom selected by a mogactic 


\section{SUMMARIES OF FERMILAB EXPERIMENTS}

chanbel The decay sequences $\Xi^{-} \rightarrow B \pi^{-}, \Lambda \rightarrow p \pi^{-}$, and $\overline{\bar{S}}^{+} \rightarrow \overline{\mathrm{A}} \pi^{+}, \bar{A} \rightarrow \bar{p} x^{+}$, are detected with $\mathrm{a}$ wre charaber spectremeter wik high-rate appebiluty By gtudyos the sogular distribution of $p(\bar{p})$ with respect to the belichty sxis on the A

$(\bar{\Lambda})$ rent frame, the decky partameter $\alpha$ ( $\alpha$-bat) can bo axtractad The $C P$ tymmetry is violated if there is any difference between the products $\left(\alpha_{h} \alpha_{I}\right)$, and $\left(\alpha_{\bar{\pi}} \alpha_{\bar{J}}\right)$ The apparatus, 80 m kn, consests of a hyperon maznel, nine wire chamber statjoul, momentum abelysing maguet, twe planes of hodobcopes for tmulas and triggering purpoese, and 1 hadronac celorrmeter For triggering A muon detechipd aysilem th the rear of the spectrometer allows swarches for fare and forbydden hyperos dacaya Under construction (3uly 96)

E-then contact luke fual gov, dukes WWW Home-page bitp //beautyl lod gov/estl htmi

\section{FNAL-872}

(Proposed Ja 1994, Approved Jun 1994, In preparation)

MEAGUREMENT OF + LEPTON PRODUCTION FROM THE PHOCESS $\alpha_{\tau}+N \rightarrow++X$

EST2 COLLAEORATION

AICHI U OF EDUCATION - K Kodama, N Ushida

ATHENS U - N Ginkart, G S Traitakoe

UC, DAVIS -. V Panlane ( $/$ Spoktepereon), P M Yagot

KANGWEON NATION $A L$ U - CH Hahn

CHONNAM NATIONAL $\mathrm{d}$ - J.Y KIm

FERMILAB B R Bsllex, D Bochphen, W E Frection,

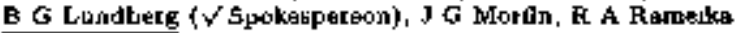
GYEONGSANG NATIONaL U - S H Chung. 1 G Parte, J-S Song KOEE U . S MOkI, T HarE

KON-KUK U - J T Rhot

KOREAN NATIONAL EDUCATION U - S N Km

MINNESOTA U - P M Eardar, D P Clampa, K J Hellar,

F W Rusack, J Stelafi, J J Tramsand, JO Wulcox

NAGOYA t] - N Hashizume, K Hoshino, $\mathrm{H}$ lınums, k lto,

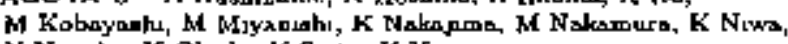

N Nonaks. K Okada, K Salto, K Yemamor

OSAKA PREFECTI1G,E U H Okstbe

PUSAN NATIONAL $U-W$ H ChUTE

SOUTH CAROLINA U - A Kulik, CErusenfeld

ToHO U - M Adschı, M Katuno, Y Kobsyathi, E Nın, S Ono,

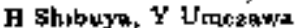

TUFT' U-T Karka, A Napier, W F Oliver, I schreps,

M Skender

UTSUNOMIYA U Y Sata, I Tegulas

WONKWANG $v$ 5 Y Bahk

Acosteratar FNAL-TEV Detecter Spectrometer

Bref descripion Measures the prodaction of $\%$ loptopse in the charged-current interactions of $r$ neutrinos The neutrinos are produced in a besin dump by the 800 GeV proton beam,

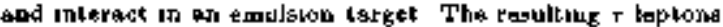
are anbsequently delected in a bugh renolutuba hybrid embltian spectrometer, prowiding a disect confirwation of the existence of tho T meutnino Urder conskraction (July 9 )

Rolated experments FNAL-603, CEAN-WA-095

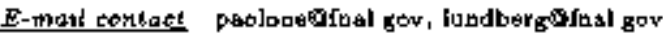
WWW Home page http //for872 Inal gov/

FNAL-875

(Propend Jes 1995, Approved Way 1998, In preparation)

CONG BASEUNE NEUTRJNO OSCILLATION EXPERIMENT

YINDS COLLAEDRATJON

ARGONNE 1 Ambsts, D S Ayreg ( $\checkmark$ Depuny Spokesperson), L J Balks, R E Bloir, D A Crand. J W Dawson, $T$ H Fueldy, M C Goodman, V J Guarnßo, N F Hill, D J Jantowski,

E N Msy. S Sreasia, L E Proce, P Schoesgow, R. V Seldlein,

R Tslags, K i Thompeon J Thron, $L$ R Tumer
BOSTON COLL - T E Tónhe

CAL TECIT - B C Bariah, D L Burke, J Hanson, N P Longley, D G Michatel, C w Peit

COLUMBIA U - Y Ho, W Lee

FERMILAB - R H Berngl atn, V D Bogert, D Corsest,

W 8 Freeman, J Hylen, D E Johason, G M Koszu mu,

$G$ otityelmakber, $J \&$ Morfin, F A Nernck, SCODay;

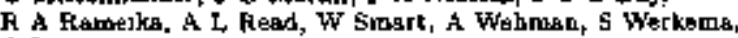
C. P Yun

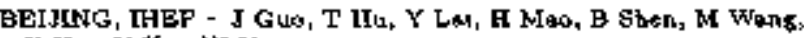
$X X_{12}, Y X_{1 e}, W$ Yar

INDIANA U - C Bowrt, M Gethatd, R W Hetchar, a M Herrz,

L. Mullin, 8 Mulwon, I A Musser

DUBNA - S Afanasjer, G D Alexser, S Borodin, V Eradenin,

C. Chelkor, Y A GornughkLa, M Ignatankso, IN Khoragakı,

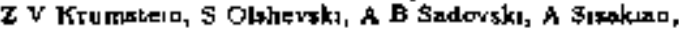

V Timofeev, L T'katehev, A I Zunchenko

LIV'ERMORE - E Ables R Bipnts, R Corpey, $E$ of Hortonn,

T Ladran, 14 Lablend, F Olenn, L Ott, E Parker, J Swan,

D Wight. C Wuet, T Yokota

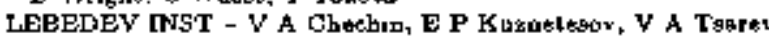

MINNESOTA D - T Berg, P h Border, T Chisge, D P Guampe

H W J Courant, P Cukhman, K J Hoilor, M L Marabak,

D E Marsin, J Merer, a Miller, J K Melkon, E a Peteraon,

K Rudduck, $R$ w Rusack, M H Schub

MOSCOW, ITEP - V Srootrlasv, I Trostın

OAK RUDGE - C L Beitcon, W Bryan, 0 Jagadıb

OXFORD U - W W WS Albaot, C B Brooke, J H Cobb, S Cooper,

H Gallagther, A R Holme日, D H Perkmg, D A Potyt. P D Shield,

J Thomas, F. L Waster, w' Weat

R.UTHERFORD - G J Alner, R. J Catcon, Fl Edreconk, G Grayer,

P. J Litch ficld, G F Payrice

STANFOFD U - G Itwin, S Q Wojchtkt (V/Spokesperson)

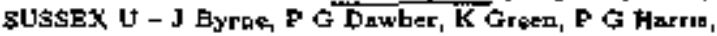

5) White

TEXAS U - K Laug

TEXAS A AND $\mathbf{~}$ - A D David, N Dibrenke, M Drow, H-

I Trost, A. C Webt

TCFTS U - T kaske, W A Mann, R Ë Mulburn, A Napuser,

W P Oljver, J Shrieps

WESTERN WASHINGTON U - W L Bsrret1

Accsigrator FUAL Detestor Calorimeter

Pgrticiles studiend $v$

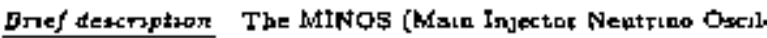
letion Search) colltbroration proposeng to conduck a search fox $v_{\mu} \rightarrow v_{\tau}$ and $v_{k} \rightarrow v_{e}$ cochllations using a new whde bend (15 GeV) Va beam from the Wisun Inpector wak enargies well sbove the $r$ production threghoold The ogcillatious will be de. tected by the cormperiogn of stgrals ma a 'Dear' detector at Fermulab acd a 'far' derector situated $730 \mathrm{kmm}$ thray in the Soudan undergrougd iaboratory A wew 10 kton detector wilk be bult at Soudan to allow the atudy af the asciliat jon potenneters down to $\Delta\left\{m^{2}\right\}=0002 \mathrm{AV}^{2} \Delta u d \mathrm{sin}^{2}(2 \theta)=001$ The exustuss. muth finer graned bot smaller, SOUDAN-2 detectar (see the UNDEAGROUND-5OUDAN-2 expeIJment) will provide an inde-

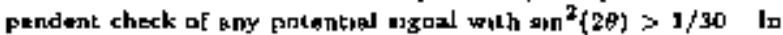
preparation (July 96)

Related experment UNDERGROUND-ICAFほS, KEK J日2

E-mazh contact egwegorslac utenford odu, wegehep anl gov WWW Howte-pags

blep/wwe hep and gov/NDK/Hypertext/gumt batrl

\section{FNAI-876}

(Propoted Jan 1995, Approved Aug 1995, Began data-takug Dec 1995, Completed dato-taktug Feb 1996)

\section{CDF HARD DIFFGACTION GTUDIES}

CDF COLLABORATION

Accelerater FNALCOLLIDEF Detector ODF Orber

Renctrots

$\bar{p} p-\bar{p} \mathrm{X}$ 


\section{SUMMARIES OF FERMILAB EXPERIMENTS}

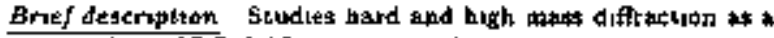
part of the CDF QCD program The physics god la a better understanding of the natove of the pomeron's constitusats

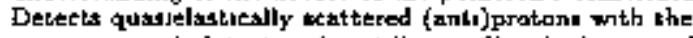

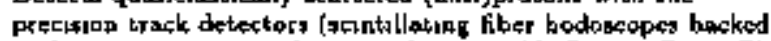
up by a trigger counter), jngerted in movable Romen Pots The track measnrecient to of ber with the vertex information from the CDF vertex detectror can be used to select the high-mass

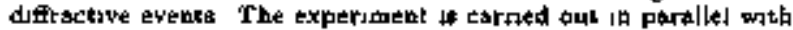
the okber CDF gtudies, and with the particpation of the fuil CDF Collaboration Dals anslysa in progress (July 96) To bearn nore about the program, phease contact the Spokespereon for the Hard Diffoct garoug, Dr Machasl Albrow [Formilab)

Ralated Axperstretrits CERN-UA.ODE, FNAL-741

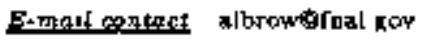

\section{Other FNAL Experiments}

Lwied here are come ocber Fermulab experimepts of intefect to the perticle phyojes commontty Find more detesle about theye projects opluas, on the SLAC" EXPERIMENT's dotabase (bee p 3), at contaci the opotespertions Check atso a detsuled dencripting of the masing progects at the Lab in the anutal report 'Fermilab Rerearch Program - Workbook', or visit the Wab site http //www fabl gov/faw/fermlab_st_ work html

\section{FNAL-00s (Completed date-1akJng Jan J992)}

PHOTOPRODUCTION OF HGH $p_{\perp}$ JETS

Spokeaperaon Marjorie D Corcorba [Ruce U]

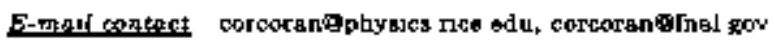

FNAL-T06 (Completed detataking Jan 1992)

A COMFREHENSIVE STUDY OF DIRECT PHOTON PRODUCTION IN HADRON INDUCED COLLISIONS

Ey 宝706 Callaboratton

Spokesperaon Paul F Slattery [Rochester U I

$\underline{E}$ masl contect altery

FNAL-70s (In proparation)

EMULSION EXPOSURE TO 1000 GeV, OR HIGHEST ENERGY PROTONS

Spokepersind Jere J Lord [Wastunglon U, Seattle]

E-most coxtect Iorditobys washington edu

FNAI_802 (Completed deta-1akung Dec 1991)

DEEP JNELASTLC MUON INTERACTIONS WVTH NUCLEAR TARGETS USING THE EMUESION TELE SCOPE TECHNIQUE

Spakespersons Lall Chatterjec, Dipak Ghosh [Jadavpur $\mathrm{V}$ ]

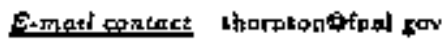

FNAL-645 (Completed date-1sking Jul 1991)

INTERACTIONS OF B0, 100, AND 490 GeV MUONS

WITH EMULSION NUCLEI

Spoleeperion C O Kim [Kores U]
FNAL-85s (Completed destakung Feb 1996)

TEST DF LOW INTENSITY EXTFACTION FROM THE TEVATRON USING CHANNELING IN A BENT CRYSTAL

Spokespersons Gerry P Jacken, C Tbornton Murphy [Fermulab] E. mazl contact thorntonginal gor, gPJelnal gov

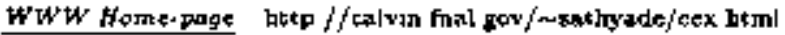

FNAL-856 (Compkted dota-cekking Doc 1991)

TEST BEAM REQUEST TO DLRECTLY MEASURE dE/dY OF HIGH ENERGY MUONS FROM 150 TO ALO GQY/C IN THE MUON IABORATORY

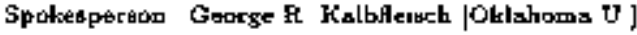

E-mati contuet grkefrad gov

FNALA61 (Complated datb-takjng Oct 1092)

BEAFCHING FOR ANTIPROTON DECAY AT THE FERMLLAE ANTIPROTON SOURCE

By APEX Cullaburatus

Spohesporion Steve Geer [Ftrmlab]

Emazt centact 1 geortinald fnal gov

WWW Homerpage butp $/ /$ fnam l fal gov/

FNAL-B82 (In preparstion)

DETECTION OF RELATIVISTIC ANTI-HYTROGEN ATOMS PRODUCED BY PAIR PRODUCTION WITH POSITfoN CAPTURE

By ANTIHYDROGEN Collaboration

Spokesparson David C Chrugtean |Fermulab|

E.matt contret doctinal guy

WWW Homerpage

hitp //fophys-wrw fual gow/experdments/e862/e862 htwl

FNH-BBd (Completed data-taking Feb 1996)

MINIMAX, A TEST / EXPERMTENT EOR THE FERMILAE COLLIDER

\$pokespersonf Cyrus C Taylor [Cope Weatera Festerva U ],

Jandea D Bjorkan [SLAC]

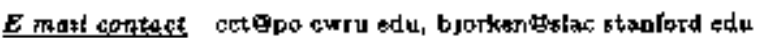
WWW Home-page butp $/$ /inmue fual gow/

\section{FNAL Future Plans}

The 800 -GeV fixtd target rug will contunise unt.l early 1998 Typecal intentitues of $20 \times 10^{13}$ per Teverion sycle art anticipated, with the experimental progiam incloding $r^{r} / \epsilon$, rate kath decays,

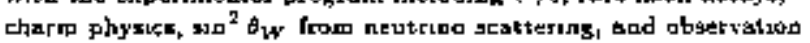
of $\mathrm{k}_{\mathrm{r}}$ Completion of commisatontng of the Basn Injector and Rncyc|er is expected by mid 1999, when the npgraded CDF aud

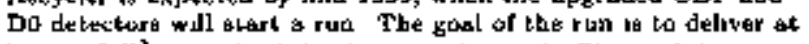
lenkt $2 \mathrm{~h}^{-1}$ to each of the detectors by 2003 The peak luminedity goat is $2 \times 10^{32} \mathrm{~cm}^{-2} \mathrm{~s}$ Construction of the NuMl fachlides at

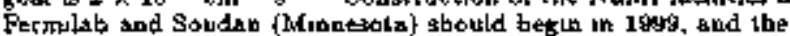
(wo neluteno obcillation experiment6 uill etart data taking non completion Slow spll $120 \mathrm{GeV}$ pretors from the Bak Injector wil be wred for Exed target experdneats couclartenily with the Tevalran collider prograni 


\section{Selected DAPHNE Experiments}

\section{FRASCATI-DAPHNE-KLOE}

(Propoed 1992, Approved Mar 1993, In preparation)

A. LARGE GENER AL PUPPOSE DETECTOR FOR CP VIOLATION STUDIES IN $e^{+} e^{-}$ANNJHILATIONS AT

\section{KLOE COLLABORATION}

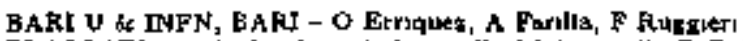

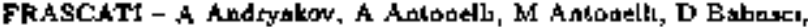

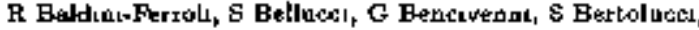

C Blorse, F Bosal, A Calcaterra, P Cempanz, 17 Capon,

M Carhoni, M Cardalle, R De Sangro, P De Sumome, F Donnal

G Feluci, M L Ferrar, E Funoccharo, A Geddh, E Gero,

W Grandegger, $F$ Haydar, L Keeblo, P Lautelly, A Martınt.

S Migcatt], S Mockiz, F Murtas, 6 P Murtar, E Pare, V Patara

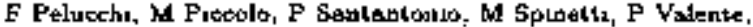

X L Wang, S Wolfle, A zello

PRASCATI \& SUNY, STONY BROOK - J LE-PremzInI

KARLSRUHE U - K Earth, A Denig, M Inhor, $\mathrm{C}$ Joram,

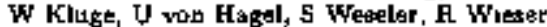

LECCE O \& INFN, LECCE - G CAtedd, P CTat1, v Elie,

E Gorin, F Grancagaolo, G F Palanu, in Panares,

M Primavera, S Spasaolo

NAPLES U, IFS \& INFN, NAPLES - A Akosia, Y Baturin,

N Cavallo, F Ceverual, G Chrefan, E Drago, D Fuare, C Gatto,

L Mercla, M Napolutsno, A Smblo

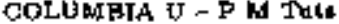

PISA U \& INFN, PISA - V Bolognebl, F Cervelb, A Feryari, $M$ Incagh, M M Maspan, $G$ Vepangoni

ROME L \& INFN, ROME - F Anull, C Bacel, M Barone,

It Beker, C Binl, L Bucci, R Calob, M Ceru, G De Zorm,

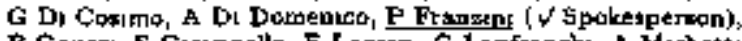

P Gauzz, S Gimanesla, F Lacava, G Lanfranchi, A Micbetti,

A Parn, M Passusco, E Petrolo, A C Petratci, $L$ Pontecarwo,

E Valente, $S$ Yentazıno

ROME U, TORVERGATA - V Boce, L Certito, S D'Angela,

R Mesos, L Paolusz, E Patqusiluces

ROME, ISS - P Branchipt, E Grafiant, A Pasteri, E Spirits,

C Itantsicu, L Tortars

SUNY, STONY BROOK - W K.m, M Pollsck, R D Schamberger

TRIESTE U, IST F1S \& INFN, TR[ESTE - G Barbuellım,

A Mertınis, $F$ Scun

Accelerator FRASCATT-DAPHNE Detector KLOE

Pertictes sfudied $K_{5}, K_{L}$

Brtef descripttan Studes rare kaon decays, meagures fiefe $\left.\epsilon^{\prime} / \epsilon\right]$

to the preciswu of $10^{-4}$, analyzes other $C P$ violatiug parame ters in the $K K$ sybters KLOE conbets of a 2-m diameter, i 35 long beluum-filled central diff chimber, a $4 \pi$ electromagnet/c

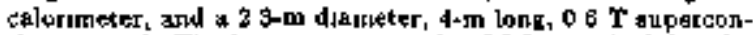
ductink $c 01$ The beam pipe 14 made of $05 \mathrm{~mm}$ thek berylliam to minimixe multiple testteng and energy lows for charkod

kaons In praparation Expected to ran un 1997

Journal papers FL B287 (1902) 259, NP (TROC \$UPPE) 2TA

(1994) 43, WFM A360 (1095) \&8, NIM A360 (1995) 2Nh, NIM

A367 (1006) 104, NIS A3ET (JPOS) 108, NIM A354 (I995) 352, and NiM A $A 6 B(1996)$ 自: B

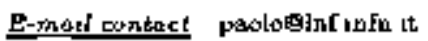

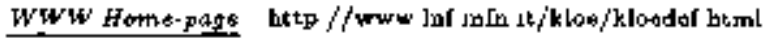

\section{Other DAPHNE Experiments}

Lusted lore us another intereatung DAPHNE experment Find more detalls sbout thig projects online, in the ILAC's EXPERI. MENTS dotabaxe (sea p 3), or contact the spokesporan

FRASCATI-DAPHNE-FINUDA (In preparatjon)

FINUDA: A DETECTOR FOR NUCLEAR PHYSICS AT DA

Ey FINUDA Collataratipn

Spakesperson Tullo Brasaran |Tutw U I

E-trnat contact bresbanislo into it

WWW Home page

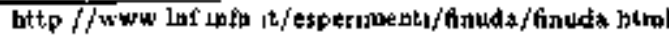




\section{Selected ITEP Experiments}

\section{ITEP+896}

(Propoaned 196a, Approwed 1989, Began date-tskung 1991, In progrezs)

DETAILED GTUDY OF SPECTRA OF A PAFTICLES AND OF THE POLARIZATION IN THE NUCLEAR FRAGMENTATION REGION IN HADRON-NUCLEON INTERACTIONS

\section{LAMEDA-I] COLLABDRATION}

MO5OOW, TTEP - V A Akumoy, Y D Beyrukov, I M Belyesev,

M P Besuglov, B M Eobihenko, E A Dorosblevich, \$ V Froloy, Y G Grubuk, Y V Kantsertor, M M Katz, S M Kuelev,

$Y \vee$ Korchagtn, S V Kulesboy, L N Kuleshows, A I Kurzentrov, A A Lebedev G A Letsin ( $\sqrt{ }$ Spokesperson), N A Plvsyuk, N K Sergeov, V S Gerov, s M Shnvalav, $A$ V Smumitaky

( $\checkmark$ Spokesperson), A V Stavinaky, V P Surb, A V Vlasov,

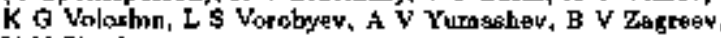
$\checkmark \vee$ zhurkw

KURCHATOV INST, MOSCOW - S L Fokin, M 8 Jppoltoy,

A L Lebedav, V I Manko, G M Mgatirubral, P I Sheharbachev, M A Vasilyev

\section{docetergiter ITEP Reieckor LAMBDAMETER}

Eencteons

$$
\begin{array}{lc}
p \mathrm{Al} \rightarrow \Lambda \mathrm{X} & 75 \mathrm{GeV} / \mathrm{e} \\
\mathrm{p} \mathrm{Pb} \rightarrow \Lambda \mathrm{X} & -
\end{array}
$$

Bref description The ktnetic anergy range of the detector is 10अं0 Ma'V Takınc dats

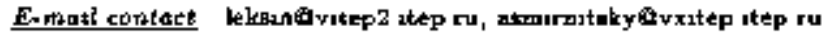

\section{ITEP-918}

(Propobed 1990, Approved 1990, Began dusentakung 1990, Completerd datixotakme 1993)

STUDY OF THE INTERACTION OF LOW-ENERGY ANTIPRCTONS WITH NUCLEI USING THE XENON BUBELE CHAMBER DLANA

\section{DIANA COLLABORATION}

MOSCOW, ITEF - V V Bartain, $V$ a Berylor, o V Davndenko,

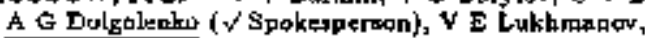

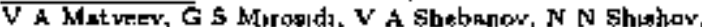

S Volkow, H K Zomplokpvakays

OSLO U - KM Dable]gen, T Jatobyen

FRASCATI \& BUCHAREST, IAP - F Nibutie, C Petratel MOSCOW, INR - E 5 GolubevB, A S पymow, I A Psbenichnow BERGEN U - K Myklebost, J M Olger

FRASCATI - C Quataldo

Acteternter ITEF petector HLBC-DIANA

$$
\begin{aligned}
& \text { Acacterars } \\
& p \text { Xie } \rightarrow K_{S} \mathrm{X} \\
& \bar{p} \mathrm{X}_{\mathrm{t}} \rightarrow \Lambda\left(\Sigma^{0}\right) \mathrm{X} \\
& \overline{\mathrm{F}} \mathrm{Xe}-\mathrm{\Sigma}^{\mathbf{0}} \mathrm{X} \\
& \bar{\nabla} \mathrm{X}_{t}-\kappa_{S} \kappa_{s} \mathrm{X} \\
& \nabla \mathrm{Xe} \rightarrow K_{s} \wedge\left(\varepsilon^{0}\right) \mathrm{X} \\
& \bar{P} \mathrm{X}_{e} \rightarrow K^{+} \mathrm{A}\left(\Sigma^{0}\right) \mathrm{X} \\
& \bar{\nabla} \mathrm{X} e-K^{+} \Sigma^{+} \mathrm{x} \\
& \bar{x} x_{e}-K^{+} \Sigma^{-} X \\
& \bar{p} \mathrm{Xe}_{\mathrm{e}} \rightarrow K^{+} K^{+} \mathrm{x} \\
& \bar{p} \mathrm{X}_{\mathbf{5}} \rightarrow K_{S} K^{-} \mathrm{X} \\
& \bar{p} \mathrm{X}_{\mathrm{e}}-K^{+} K^{-} \mathrm{X} \\
& \bar{p} \mathrm{X}_{e} \rightarrow K_{S} \Sigma^{+} \\
& \bar{p} \mathrm{X}_{e} \rightarrow K_{S} \Sigma^{-} \\
& \bar{p} x_{0} \rightarrow \omega \mathrm{X} \\
& \bar{p} x_{e} \rightarrow \pi x
\end{aligned}
$$

Ertef deacrotion Uats the 700-liter Xenon bublok chamber DLANA Studite the production of itrange particles and $p$, mesons in low-anergy $\bar{p} \mathrm{X} e$ anphlitations

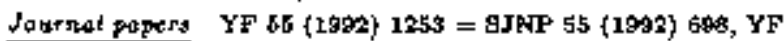
DE $(1992) 1268=$ SJNP 55 (1992) 705, NP A556 (1993\} 409, NP A55B (1093) $36 \mathrm{LC}, \mathrm{YP}$ E7 (1094) 1724 = PAN 57 (1994) 1656, YF 57 (1994) J日05 = PAN 67 (1994) 1335, and PL B370 (1996) $23 \times$

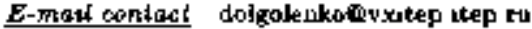

\section{FTEP-122}

(Propoad 1992, Mpproved 1992, Begen data-taking 1996, Io progretsis)

SEARCH FOR H-PARTICLE AND RESONANT STATES IN $M A$ \$YSTEM

MOSCOW, ITEP - E G Baguanav, V \& Demudoy

( $\sqrt{ }$ Spokesperron\}, N D Galanma, K E Guser, N A Khalderva, V N Mlarkisov, A A Nedobokin, VA Sodykov, ME vishnoveky, M O Vlasove

Acceterator ITEP Detector Spectronetar

Renctiotis

$$
\begin{aligned}
& \text { nt wueleus } \rightarrow \text { A X } \\
& n \text { mpcleas } \rightarrow \text { dibaryou }(S=-2) \mathrm{X}
\end{aligned}
$$

Bruef descripton Suarch for the B-duberyon through the $\pi^{-}$ph dacay channat Takung date (Juby as)

$\underline{E}$ mant contect demidavowxitep lep tu

\section{Other ITEP Experiments}

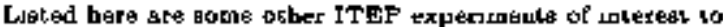
the particle physucs community Fund morn detaiks about these projotet onlthe, it the SLAC's EXPERDMENTS datsbste (bee p 3), or contact the spokespersons

ITEP-8s2 (Takung data)

A TRACKING EXPERIMENT FOR STUDY OF DOV. BLE BETA DECAY IN ${ }^{136} \mathrm{Xe}$ and ${ }^{160} \mathrm{Nd}$

Spokespersons V A Lyubmooy, O Ye Zeldowlch [X Iobecm, ITEP]

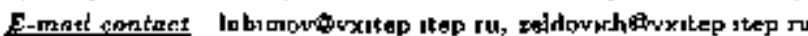

ITEP_Bss (Completed deta-taking 1991)

STUDY OF PIONIC NUCLEAR DEGREES OF FREE DOM IN $(\pi, \pi \pi)$ REACTIONS

Spakeapersons MV Kowsov, G A Leksin [Motton, [TEP]

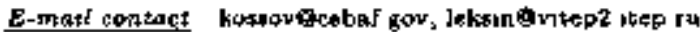

ITEP-881 (Completed dats-takipq 1891)

SEARCH FOR ${ }^{16} \mathrm{Ge}$ DOURLE BETA DECAY

Spokespergon IV Kirpleluakay [Mtoвcow, ITEP)

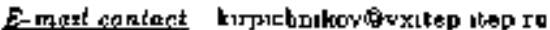

ITEP.865 (Completed deta-taking J992)

ANTIPROTON-NUCLEI ANNLHILATION CROSS.

SECTIONS WITH Ee, C, Al, $\mathrm{Fe}$ Cd, Cu, AND Pb TAR. GETS AT 0.70, $0.95,1.26,1.53,1.70$ AND $2.60 \mathrm{G} * \mathrm{~V} / \mathrm{c}$

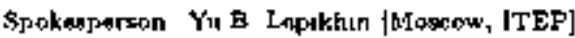

B-rearl cortact smarnitsky 


\section{SUMMARIES OF ITEP (MOSCOW) EXPERIMENTS}

[TEP-B73 (Completed dats-taking 19B1)

HIGH-ENERGY CUMULATIVE PARTICLE PRODUCTION AT $10 \mathrm{GoV}$

Spokespertons Yu T Kıselav, Yu V Terekbow [Moscent, ITEP]

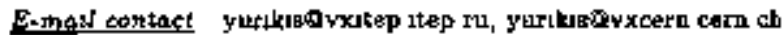

TTEP-876 (Corapleted datrotakıng 1992)

SEARCH FOR ${ }^{2}$ AND $Z_{r}$ DOUBLE BETA DECAYS IN PHOTOGRAPHLC HMULSION

Spokesperwop A S Barabask [Moecow, ITEP]

Emant contact Barabahalivittep leep ru

ITEP \&897 (Completed dats-takag 1984)

STUDY OF ${ }^{4} \mathrm{KF}$ F INTERACTIONS

Spakesperson V E Grectho [Moscow, [TBP]

E-matil contacaf arechlobvitepl itep in

ITEP \&894 (Completed data-takınx J991)

QUASIPARTICLE VELOCITY MEASUREMENTS

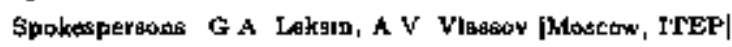

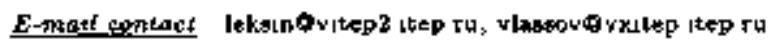

ETEP-g9o (Takmg daca)

TWO-NEUTRINO DOUACE AETA DECAY OF ${ }^{1 \mathrm{M}} \mathrm{MO} \mathrm{TO}$ THE FIRST EXCITED 0 ${ }^{+}$STATE IN ${ }^{10} \mathrm{Ru}$

Spokespersons a \$ Barsbash pMokcow, ITEP], F T Avignode [South Ceraline U] ]

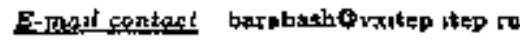

ITEP-897 (Completed deta-laking 1991)

STUDY OF ${ }^{4} \mathrm{H} / \pi^{-}$INTERACTIONS

Spokeperson V B Grachko [Moscour, ITEF]

E-mail contact Erachkoliviteps atep ru

ITEP-901 (Taking data)

GUASIELAGTLC $\left\{{ }^{-}\right.$- d) BACKWARD SCATTERING ON NUCLEI AT 0.7-1.3 GeV

Spokespersor A P Krutenkpor |,Mokcow, [TEP|

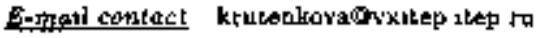

ITEP-Q11 (Completed data-taking 1994)

PORWARD DIRECTION CUMULATIVE PARTICLE PRODUCTION ÁT $10 \mathrm{GeV}$

Spolesperæons Yu T Kevles, YA Sheinkman [Morcow, ITEP]

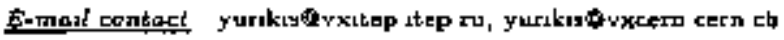

ITEP-gב2 (TakınR datk)

SEARCH FOR NEUTRINOLESS DOUBLE EETA

DECAY OF ${ }^{76} \mathrm{Ge}$

Spotrtsperesus IV Kutpucloblkox |Moycaw, ITEP], R L Irodzin\&k! [Bstrelle Memorjal Inst, Northwett], F T Avigrene [Stuth Carohus U|, A Meralet [Zinragoso U]

E mati contact kirpichnikovoryaltep step ru amoralesolgae unizzr et
JTEP_gl4 (Taking dsta)

MEASUREMENT OF SPIN ROTATION PARAMETERS R AND A IN ELASTIC * $p$ BCATTERING

By FTEP-PNPI Collaboration

Spokespotrong V P Kentwet, [Morcow, ITEF], S P Knuglow [St [etersbura, INP]

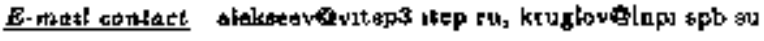

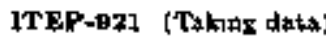

INVESTIGATION OF QUASICOHERENT AND DEEP INELASTIC INTERACTIONS OE PIONS AND PROTONS WTTH NUCLEI

Spokedperiens V S Demidow, ] V Kirpucbukep [Moscow, ITEP]

Hamat contact demudovavxitep step ro,

kirpichnitrova ysilep itep ro

ITEF-923 (Completed dics-tsking 1994)

INCLUSTVE PION DOUBLE CHARGE EXCHANGE ON LIGHT NUCLEI AT 0.7-1.3 GeV/c

Spotosperson A F Krutenkwa [Mowcow, [TEP]

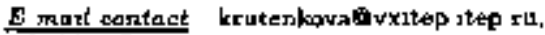

kuhkonswatep ikep ru

ITEP-941 (Takug data)

SUDTHRESHOLD $K^{+}$PRODUCTION IN PROTONNUCLEUS COLLISIONS

Spokespersons Yu T Kiselev, VA Shelekrato (Mogctow, ITEP)

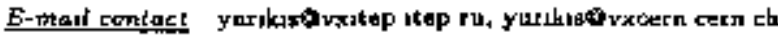

TTEP-943 (Taking data)

SEARCH FOR $D^{\prime}$ DIBARYON IN WINTERACTIONS

Spokesperton A [ sutormin [Mogcow, ITEP]

E-rnal contact khanovgesteps itep iw, kulikovayutep jtep ru

ITEP $\leadsto 51$ (Takung data)

SUBTHRESHOLD $K^{-}$PRODUCTION IN PROTONNUCLEUS COLLISIONS

Spokepersons Ya T Kinelev, VA Stenrkman [Moscon, ITEP]

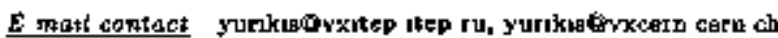

NEMO-3 (Taktug dala)

DOUBLE BETA DECAY EXPERIMENTS WITH THB

TRACKING DETECTOR NEMO-Z

B) NEMO Collaboration

Spokespotaton 9 Julluan [Otasy, [AL]

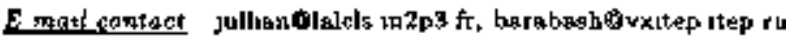

\section{ITEP Future Plans}

The ITEP's U-16 proton gyuchrotron will contubue inveitugatiang of particle interactions with rucles The proxraro includes experiments an the subthreghold product ton of partucles, studie日 of cumblative eflects, and a teaseh for muiti-quarh atasee in nucles The syochrotion will also be used to continue polarization

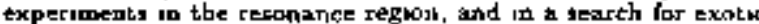
resonences Tegt besms will be utrlized in a developmet and testing of equipment for expendiente at CERN, DESY, FNAL and ot ber facilitien specinl proton besm will be devoled to the medical trestmeat of cercer patients The otudy of douthe bets

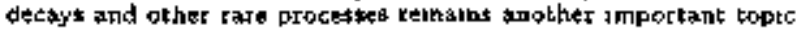
ta the ITEP'в experimental pringtam 


\section{Selected JINR Experiments}

\section{JINR-LHE-0941-1B}

(Proposed Sep 1992, Apsroved Nov 1992, Elegan datt-taking Sep ip9s, [n progreas)

STUDIES OF POLARIZATION CHARACTERISTICS OF INELASTIC SCATTERING AND BRRAKUP OE RELATIVISTIC POLAFIZED DEUTERONB ON PROTONS AND COMPAHISON WITH DATA OBTAINED WITH NUCLEAR TARGETS

DUBNA - L S Ashgirey, Y T Bortunoy, E V Chernykh, L B Oplowedov, A D Karilor, V P ladygin, L V Adunas, P F M Manyakov, N M Plokunay ( $\checkmark$ Spokesperson),

P A Rukoyalku, I M StIuk, G D Stoletor, E A Strokoraky (V'Spokesperson), A L Svetov, A P Tuyney, S A Zaporozhets SAMARA AIFSPACE U - P P Katovn WLLLAM AND MARY COLL - C F PerdrneAt SOFIYA, LYST CHEM TECH - $\$$ Nedey KIEV, ITF - A P Kobnghk10, A I Syameomor NORFOLK STATE 0 - $V$ PuIJab

Acceterzuar JIRH Detector ALPHA

\section{Repeftoons}

deut $p \rightarrow$ dent $X$

deut $p \rightarrow p X$

deut nudesus - deut $X$

45 - $9 \mathrm{GeV} / \mathrm{c}$

$$
\text { Ir }
$$

Particles atudited N(1940 $\left.P_{11}\right)$

Braf description Ont of tha gerale of the exparisient ts to aturly

the mechanum of the resction $p\left(N, N^{\prime}\right) X$ with excilstion of $\Delta$ and $N^{*}(1240)$ tegonabces $T$ The $p\left(d, d^{\prime}\right) X$ reaction is chosen becsume the excitstion of the Foper rasonance is enhanced due to the istapin seloction ruke $A$ seudy of the axpected mterfereace effects between $\Delta$ and $N^{*}(1440)$ resonasuces in prilarizstion abservables of thus resction ti planted The dats will provide valuable informarion ebout propertser of the Roper reson ance and alio help rewotve the existing theorelical wictertantieg It slso measuree the tensor analyzung power $T a$ w inclusivg deutaton brealkug at $B \mathrm{GeV} / c$ and $0^{\circ}$ on bydrogen and carton targets

Journal payers NIM A357 (1895) 384, add PL 9361 (1995) 21

Retated expersmetits JINR-LHE-0941-1C, SATURNE-260, SATURNE-276

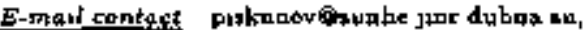
scrokbunbe jut tubas gu

\section{Other JINR Experiments}

Lubed bere are some ather Dubos experimenis of imter ost to the particles phy'siçs communtty Find pare detals about the prosects ovlide, in the SLAC's EXPERIMENTS

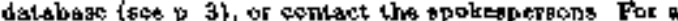
complete dectriptopp of the curretil Lab's expetimental re aestch protisn, viglt the JINR exparmentar Wob page Bt

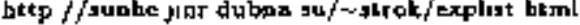

\section{JINR-LHE-0996-3 (Takjg data)}

SEARCH FOR NARROW HADRONIC RESONANCES PRODUCED IN NEUTRON-PROTON IMTERACTIONS Spokesperans Yu A Proyas [Dubse, JINR]

Femast contenci troysa troyangavahe ytor dubns eu
JINR-LHE-DPC1-1A (Completed datatuking ro9d)

MEASUREMENTS OF TENSOR ANALYSING POWER IN BACKWARD ELASTIC $\not p$ SCATTERING AND BREAKUP AT $180^{\circ}$ (CM) BETWEEN 9.5 - $6.5 \mathrm{GeV} / \mathrm{c}$ Spokespereore N M Plskunov, I U Sitnik, E A Strokovsky [Dubns. JINR]

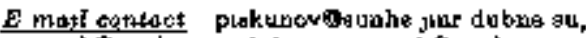

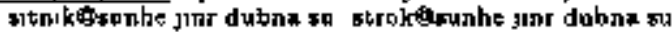

JUNR-LHE-0941-1C (Takng data)

SEAFCH FOR AA DIBARYON PRODUCTION USING SPIN DEPENDENT INELASTIC DEUTERONDEUTERON SCATTERING

Spokespersons NM Pıkunou [Dubna, JINR], F W Hersmab |New Fampshise to

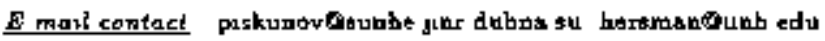

JLNE-LHE-0941-1D (Completed datatsking Oet 1993)

MEASUREMENTS OF THE POLARIZATION TRANS PER COEFFICIENT IN BHEAKUP OF RELATIYISTIC POLARIZED DEUTEFONS INTO PROTONS EMITTED AT $\sigma^{0}$

Spokespersans N M Piskunay, I MS Situlk [Dubns, JINR]

E-mart contact prakuaoy

ontonkfornhe dubnt ton

JINR-LHE-0041-1E (Gompleted data-taking Nov I904)

MEABUREMENTS OF THE POLARIZATION TRANSFER COERFICIENT AND TENSOR ANALYSING POWER IN BREAKCUF OF RELATIVISTIC POLAR. JZED DEUTERONS INTO PROTONS EMITTED AT $0^{\circ}$

Spokeapernans LN šsunav. A V Zaruben [Dubas, JiNR]

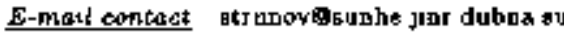

JINR-LHE-Ged1-s (In prepatation)

STUDLES OF SPIN-\$PJN CORRELATIONS IN BACKWARD ELASTIC DEUTERON-PROTON SCATTERNS \$pokespersons L 9 Azlugirey, IM Silnik [Dubna, JNR], M P Rekalo [Kharkoy, FTI)

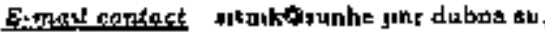
azbgiteyocv junr dubns au

JINR-LHE-0041-4 (Tatung dats)

SPIN DEPENDENT TOTAL CFOSS-SECTION DIFFER ENCES IN $n p$ SCATTEFING

Spakedperson B A Khachaturev, N M Pskwoov. VI Sharov, L N Strunow [Dubas JINR], H if 9purka [Argonne], F Lehar |Sacley|

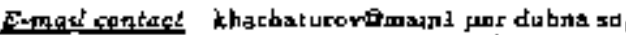

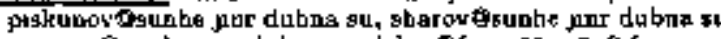

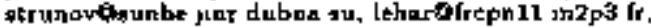
bmothep an gor

JINR-LHE-0041-5 (Completed datso-takug 1992)

INVESTIGATION OF FSI AND INTERMEDIATE IBO. BAR EFFECTS IN A GEOMETRY dp INTERACTIONS

Spokeapersong VV Glagolev [Drbas, J[NR], G Martintks [Koalce V], M S Nioradze [Thilili State U l, T \$lemtarcizuk

[Warkaw, INR]

E-mati contact glagolew frunhe jenr dobnt sh,

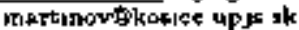


JLNR-LFE-0959 (Takıng data)

INVESTIGATION OF PION-NUCLEON AND NUCLEON-NUCLEON INTERACTIONS

Spokexperson A B Kurepun [Moscow, DNR]

E.matid contact kurepingal20 tar troutsk ru

JINR-LHE-0971-1 (Takng dala)

MEABUREMENTS OF JNCLUSIVE, QUASJELASTJC AND INET.ASTIC ANALYZING POWERS IN PROTONCARBON SCATTERING

Spokespextans L I Sarycheva |Mogeov State U], A I Malakbiov [Dubne, JINE]

E-melt contunt leoginlex apa mau su,

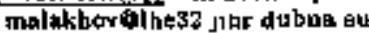

JINR-LHE-0OT1-2 (In preparation)

MEASUREMENTS OF THE A DEPENDENCE OF THE LEADING PAFTICLES YIELD FROM NUCLEI

Spokespersons LI Sarycheva [Moscow State U!, A I Malakhov [Dubua, JINR

E-that contact lugselex npI тsu su, matathorfolhe3z jur dubas su

JINR-LHE-0oss-4 (TBking dala)

THE STUDY OF THE TENSOR ANALYZING POWEI IN CUMULATIVE PARTICLES PROODUCTION ON A POLARIZRD DEUTEROM GEAM AT THE DUBMA SYNCHFOPHASOTRON

Spokerpersons A Litvipeako, L Zalin [Dubna, JINR]

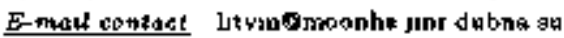

$$
\text { JINE-LHE-1010 (In preparation) }
$$

INVESTIGATION OP GPLN PHENOMENA IN THE EN ERGY RANGE CORRESPONDING TO TRANGJTION FROM NUCLEON TO QUARK-GLUON DEGREES OF FREEDOM IN NUCLEI

Spokespergons A A Bakdın, A S Vodopranor [Dubse, JINR]

Eratraid conterct baldingenube jus dubua su,

wodopunosunte pur dubss su

JINR-LHE- 1011-1 (Taking datb)

MEASUREMENTS OF INCLUSIVE AND CORRELATION CHARACTERISTICS IN CUMULATTVE KINE. MATICAL REGION IN do AND dd INTERACTION WITH POLAFIZED DEUTERON BEAM

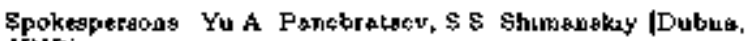
JiNFi]

E-matl anterct parebratosunite her dubre su, shumsnsittignobe jur dubsa sn

JINR-L WP-OO ('Takıng dats)

SEARCH FOR NARROW DIBAFYON RESO

NANCES IN THE DOUBLE PROTON-PROTON BREMSSTHAHLUNG REACTION AT THE ENERGY BELOW THE FION THRESHOLD

Spokesperson A 5 Khrykud Dubna JINR]

E-mast mentact khrykip Oinutur juet dubas su
JINR-LNP-39 (Taking dats)

THE EXP ERIMENTAL STUDY OF RP ELASTIC SCATTERING AMPLITUDES AT $16 \mathrm{MeV}$

Spokespersoine Ya A. Uson [Dubns, JINR], I Whlhelon [Charles U]

E-mat? conteet ugov 4 rosul jine dnban su, jvap wilhetmotuk cunt c\%

\section{JINR Future Plans}

The 1996-98 JINR Seientific Progran showt a tlear comnoutment to contintation of bath the theoretrcai (porticularly related to plewonnenology), and the expenmental research The experiments groups will be working at Dubas and at otber inatjtuthons (IHEF, CERN, DESY, BNL, ; The ot-horee Epenipents sacludt

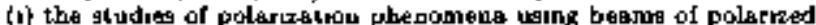
deuterons and nucleon: from the LHE tcceleration fomplex, and (13) נnvantigations of the color degrees of freedom in the aucles mateat with (he Nuclotron relativistic meles beasis, at the transition energles regron wher the non-perturbative QCD effects are considered sippifictot The phoritiet in the machere plysises are the completian of the slow extracison system for the Nuclotion, sad further development of the extracted bostn infrastrueture at the LHE complex (Nuclotron is to bew supericonduct ive synchrotron, butl Iar acceleration of protony up to the binetic energy of $128 \mathrm{GeV}$, snd deuterone sud otber nucles, Ineluding uranium, up to 6 GEV $/ A$ (for $Z / A=1 / 2\}$ 
SUMMARIES OF KEK EXPERIMENTS

\section{Selected KEK Experiments}

\section{KEK-TE-001}

(Appravad Mar 1983, Begau datertaking Now 1686, Complated data-takung May 1905)

\section{TRISTAN $e^{+} e^{-}$EXPERIMENTS BY THE VENUS COL} LABOFATION

VENUS COLLABORATION

TOKYO METROPOLITAN Џ - 2A Cbuba, T Hirost, N Howoda,

TT Ortms, F Ysbiaki

TOHOKU $\mathbf{U}-\mathbf{K}$ Abe, $\boldsymbol{J}$ MacNeughton

KEKS - K Amako. Y Ars, M Fukswa, Y Fukushims, F Hunode,

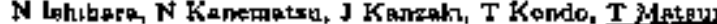

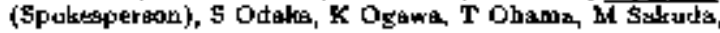

N Sato, J Shirai, T Sumiyusht, F Talessaki, T Teuboyama,

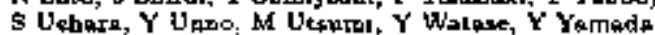

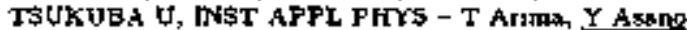

(Spokespergon), H Hamasaki. M Miura. S Mori, Mi Sturaliata,

Y Takade, K Yuse

YASLDA WOMENS JR COLL - Y ChibA

WAKAYAMA MEDICAL COLL - M DALGO

OSAKA U - J Habs, H Hans, N Kamdb, A Krager, Y Nagashima,

A Suzukn, H Takaks, MI Takıle, D Tatsuml, $Y$ Yamenote

KYОTO U - Y Hemmı, R Kıkuch,, H Kurashıze, K Miygke,

A Oknmolo, H Sakamblo

TOHOKU GAKUIN U - M HIEnthl, Y Hoshi, M Sato

KOBE U - Y Homma, A OLO

FItROOSHIMA U - Y Jwakn, T Ohgugi, 1$]$ Ohyama

KEK \& HELS[NK] U - T T Korbonen

OKAYAMA U E K Msuuda, K Obabe, N Tsmora

TOKYO, INTERNATIONAL CHRISTIAN U - Y Naksgawa,

is Yamagata

MSIYAZAKT U - T Nstomurt

I\$UKUBA U - I Nakand

IBAFAKI COLL TECH - M Shoden

KOGAKUFN U - K Tobmateu, T Watangbe

TSUKUBA COLL TECH - Y Yonetawe

NARTTO U OF FHOUCATION - H Yoshids

Acceicrulor KEK-TRISTAN Detector VENUS

Reactions

$$
\mathrm{e}^{+} \mathrm{e}^{-} \quad<70 \mathrm{GtV}\left(\mathrm{B}_{\mathrm{cm}}\right)
$$

Bnef descroblen Physics topacs ubclude seevaluation of the

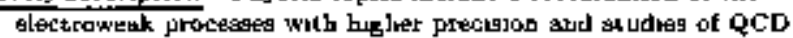
through quark-psir production in photon photon collisions The integrated lamindesty for the expenment resched 400 inverto preobaros Data snalysta iv progreas (June 96)

Jourtad papets NLM 21T (1963) J81, JJAP 23 (1984\} 697, NTM A228 (1985) 309. NIM A238 (1985) 328, NIM A243 (1986) 58, NLM A253 (1986) 27, LEEE TNS 33 (1986) 73, JJAF 25 (1886) 1049, NLM A264 (1307) 35, NIM A254 (198T) 317, NLM A259 (1967) 430, NIM A259 (1967) 438, JJAP 26 (1987) 982, JPSI 56 (Je87) 37e3, J[SJ 56 (1987) 37a7, PL B198 (1887) 570, PRL 89 (1887) 2915. NMM A265 (1088) 457, NMM A269 (1988) 171 NIM A269 (1998) 622, NIM A270 (1988) 319. NIM A27L (198R) 132, NIM A272 (1988) 687, IEEE TNS 35 (1988) 300, PL B207

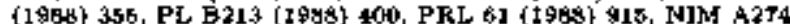
(19B9) 453, NIMA A2Bi (1989) 462, IEEE TNS 36 (19R9) E65, IEEE TNS 36 (1969) 670, JJAP 28 (1998) 1081, JPSJ 58 (1969) 3937. FL B232 (1969) 425, FL E232 (J989) 431, FRL 63 (1989) 1776, ZPHY G45 (1999) 175, PR. D39 (1889) 3524, PL B234 (1990) 203, PL B234 (1000) 382, PL B240 (1990) 282, PL B246 (1990) 297, ZPHY C48 (1900) 13, NLM A801 (1991) 497, NEM A303 (1991) 348, NTM A305 (1991) 71, FL B284 (1991) 212, FL B266 (1991) 188, PL B267 (1991) 309, PRL 66 (1981) 290 NIM A311 (1952) 67, NIM A322 (1992) 211, KIIM A323 (1992) 47], PL B278 (1992) 39. Pl B278 (1992) 499, NIM A330 (1993) 64, PL ESOs (Ins) I19, PL 1373 (1923) 245, PL BsIs (1963) 288, FRL 71 (1993) 38, N1M A340 (1994) 501, PL B331 (1994) 21L, PRL 72 (1994) $3313,2 P H Y$ Ces (1094) 213, JPSJ 64 (1965)

135, and ZPHY Cकs (1996) 60?

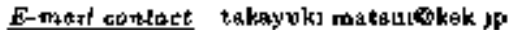
WWW Howe page batp //venurux $\mathbf{l}$ kok jp/

\section{KEK-TE-002}

(Approvod Mar 1983, Begad dats-takıng Nuy 19286, Comploted data-takung Nay 1905)

\section{BTUDY OF $\varepsilon^{+}{ }^{-}$ANNIHILATION PHENOMENA BY A} DETHCTOR WITH PARTICLE IDENTIFICATION

\section{TOPAZ DOLLABORATION}

NARA WOMENS U - K Adach, S Awa, N Fujwwara,

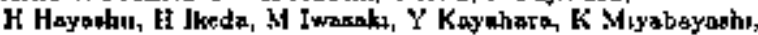
K Muramalju, T Nagja, M Nalkojima, H Nighioke, N Njahrwakd, S Noguchi, M Obkurs, E Ssker, MI Takerooto, N Toorm, A Yenagucbi, Y Yosks

TOKYo U OF AUkIC TECH - K EmI, N Pujı, K Hargee,

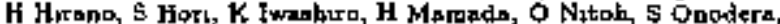

T Shooharb, K Takshasby, $O$ Teumuta, I Yobhazawa

N.AGOYA U - IK Aba, T A be, M Aob, E Kajtawa

(Spokesperson), K KurstB, H Msouda, K Matsughita,

K Nakabaysohi, N objghi, T Obshump N \$asayame,

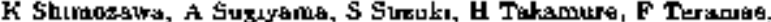
$M$ Tomoto, T Toyams

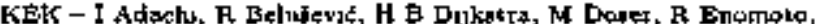
H Fujn, K Fuñ, J Fulumoto, N I I ds, H Ikeds, R Itwh.

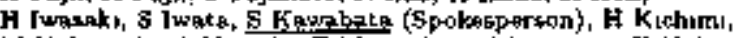

M Kabsyanth, \$ Kuroda T Matanda, A Mlyasoto, K Nakamura.

Y Ohweht, H Oeskl, T Sato, R Sugathars, T SuwgdB, T Taucbi

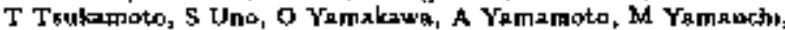

$\gamma$ Yoxhımura, in Youholes

TOKYO U - H Aıhars, T Kamse, T Kuhids, N Kueuki, F Sa,

A Shrahashi, S Yamawoto, \& Yamaghite

OSAKA CITY U - K Furte, S H,gaebr, Y loue, Y Kato.

A Natuyamb, M Nitesmurs, E Nobano, M Nateaysuss,

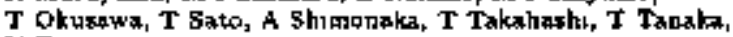

$Y$ Teramolo

PURDUE V - B Howell, D Koltick, I Leviad, E Shibsta

TOK'YO INST TBCH - M Aakı, K Kaneyaki, S Minnhra,

N Nekaraws, A Ohhi, Y Ohehime, N Sugryama, T Tachibana,

T Tanlirott, K Watanabe, $Y$ Watanabe

TOKYO U, INS - A Lmanash, T Ishin, S Kato, K Maruyamb,

T Morımotro, $\mathbf{H}$ Okun口

KOBE U - T Fuju, K Fujwarts, K Nagat

TEZUKAYAMA U - F OChuB

Acceterator KEK-TRISTAN Detector TOPAZ

Reschions

$$
\mathrm{e}^{+} \mathrm{e}^{-} \quad<70 \mathrm{GeV}\left(\mathbf{E}_{\mathrm{tm}}\right)
$$

Bryef description Searchen tor new prgticles sach as havy quarks, hesvy legtone; and vanous buperaymmetnc particteg, and studjes in detol electroweak os well as OCD phenomenn The detector bas large ooled angle coverage with very good

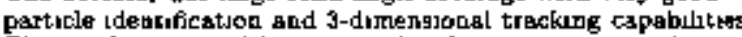
The total integrated luoninosity for the experimest is about $\$ 30$

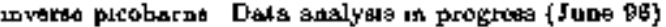

Jotrital papers WIM A220 (1984) 23, NIM A230 (1985) 50, NIM A252 (1966) 123, NDM A256 (J987) 149, N]M A2BQ (1998) 507 NIM A269 (1988) 513, NtM A.270 (1986) I1. NM A 271 (1988) 404, PL B200 (1988) 391, PL B2DS (1988) 319, PRL 60 (1988) 07, PR D37 (1988) 1339, PL E218 (1980) L05, PL B227 (1989) 495, PL B228 (1989) 553, PL B229 (1989) 427, NIM A287 (1990) 148, FL B234 (1990) 185, PL B234 (1990) 197. FL B234 (1990) 625, PL B240 (1990) 513, PL B244 (1990) 352 , PL B249 (1990) 336, NIM A300 (1991) 555 FL B255 (1991) 613, PL B268 (1991) 457 NIMS A312 (1092) 440, NI34 A316 (1992) 202, PL 8279 (1992) 506 PL B279 (1992) 422, FL B294 (1992) 144, PL B261 (1992) 206, NIM A334 (1993) 367, FL B304 (1953) 379. PL B313 (1993) 475, PL B314 (1993) 149, PL B314 (1993) 471, PL B325 (1994) 635, PL, B322 (1994) 477, PL B340 (1994) 135, PL B34L (1894) 98, PL E341 (1996) 238, PR D50 (1924) 1B79, PL B345 (1905) 335, PL B347 (1905) 171, PL B347 (1925) 179, PL B351 (1905) 199, and PL B368 (1986) 299

E-mati contact kejukwagkekvax trek JP

wWW Horre-page http//tophpt kek Jp/ 


\section{SUMMARIES OF KEK EXPERIMENTS}

\section{KEK-TE-00S}

(Proposed 1983, Approved Nov 1983, Began data-takıg Nov 1986, Completed dete-teking Jan 1994)

\section{AMY - A HIGH RESOLUTLON IEPTON DETECTOR} FOR TRISTAN

AMY COLLABORATION

ROCHESTER U - A Bodek, B J Kım, T Kumita, Y K L, C Velis:ass

SOUTH CAROLINA U - C Rpsenteld, s Wilson

KOREA U - J S Keng, D Y KIm

LOUISLANA STATE U - P Kırk

BEIJHNG, IHEP - N H Ye, Z P Zbeng

VIRGINTA TECH - A AbxhhIa, K Gotom, D HaIm,

Mt E Mattwan, I Pulanon

UC, DAVTS - R E Brţędon, W Ko. R L Lauder, J Rowe,

J F Smith, D Stuart

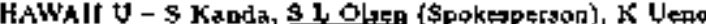

KEK - $\mathbf{K}$ Abe (Spokesperson), Y Fuju, Y Kunbats, F Lit,

A Maki, T Nozals, T Omorb, F Sarewn, Y Saks, T Sagak)

Y Summoto, Y Takmiwa, S Terada

GYEONGSANG NATIONAL L - S K Cbo

KONAN U - F Kajmo

MINNESOTA U - T Thoroes

NIJGAT及 U - T Aso, K Mryano, H Myyka, N Taknshimizy

NIHON DENTAL COLL - Y Yomahitx

RUTGERS U - F Sauuet, S Schnetzer, R Stout, J Vingon

SAGA U, JAPAN - \$ Behan, S Kabsyad, A Marakami,

K S Sarb

STEOUL IFATIONAL U $\mathbf{3}$ K Kım, M H La, S S MYUng

KYUNGPCOK NATIONAL U - D SOL

CHUO $\mathrm{U}$, TOKYO - S Miatsumato

SAITAMA U - T Lehezaté

Acedcrator KEK-TPISTAN Detcotar AMY

Reditions

$$
\mathrm{e}^{+} \mathrm{e}^{-} \quad<70 \mathrm{GeV}\left(\mathrm{E}_{\mathrm{mm}}\right)
$$

Bryef descrtption Dane enslyas in prograss (June got)

Jotarnal prapers IEEE TNS 23 (1987) 520, N]BA A260 [1987) 361 .

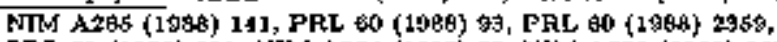
PFI 51 (1988) 911, NIMI A274 (1989) 85, NTM A283 (1989) 665 PL B218 (1989) 112. PL B218 (1089) 496. PL B223 (1909) 175,

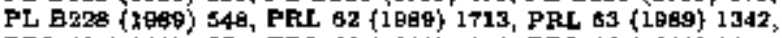
PRL 63 (1989) 1773, PRL 63 (1989) I910, PRL 63 (1989) 234, PL B234 (1960) 534, PL B240 (1900) 243. PL B244 (1980) 573 , PL B362 (1990) 491, PRL 64 (1890) 984, PR. D4l (1890) 2675. PR D42 (1960) 737, PR D42 (1990) 944. PR D\$2 (1050) 1339, IJUP AB (1991) 2583, NIM A3B7 (1991) 62. NEM A317 (1992)

T8, NIM A323 (1962) 601, FI. B277 (1962) 215, PL B303 (1923) 385, PL B313 (1993) 469, PL 自325 (1994) 248, and PL Bad6 (1065) 208

E-matil contact abegotselivax trek JP.

tolveginubeph phys hawal edu

WWW Home-page bttp / / amywww kek Jp/

\section{KEK-162}

(Proposed 1987, Approwed Oct 1087; Began datataking J996, In progress)

MEASUREMENT OF THE CP-VIOLATING DIRECT AMPLITUDE IN $K_{L}^{-0} \rightarrow \pi^{0} \mathrm{e}^{+} e^{-}$DECAY

KYOTO U H Kurasbige, T T Nakamura, T Nomuta,

it Sikamoto, N Sasao (Spokesperson), MI Suehteo

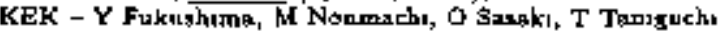

Alacederotot KEK.PS Detector TOKIWA

Rerctions

$K_{L} \rightarrow \pi^{6} e^{+} \leftarrow^{-}$

Barticites strdited $\boldsymbol{K}_{L}$

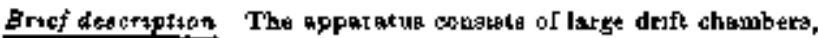
a UV-sengutive Cerenkow count.er for delection of electrons, tod in electromagretic CsI talortmeter whth good energy redolution The drift thambert ude argan and $\boldsymbol{C F}_{4}$ gap Sentitive eo bradehug ratios atmaller than $10^{-10}$ Taking data (July :6) Jourst popper NIM A270 (J968) 106, NTM A283 (1969) 709 . and NTM A $\$ 17$ (1902) 213

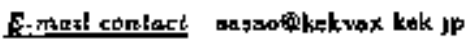

\section{KEK-231}

(Proposed 1900, Appraved Jul 1900, Berap dzto-taking I98d Cumpleted daca-taking 1904)

STUDY OF VIOEATJON OF TIME REVERSAL INVAFI ANCE IN NEUTRON REACTIONS

KEK - 'L' Adach, S Ishmoto, Y Mithdt (Spoketoernon), Y Mon,

K Morimoto, H M Shemieu

KYOTO U - M IInpsan, \& Mrasuke, Y Matsuda

TOKYo INST TECH - K Aash, M Harada, H Sato

TOHOKU U - K Sala, S Tanaka, A Yamaguabl

Accelerator KEK.F' Detector Connter

Rezctons Polanzed besm and target

${ }^{139} \mathrm{~L} \mathbf{s} \rightarrow \mathrm{nX}$

$n^{81} \mathrm{Er} \rightarrow \mathrm{n} X$

Jotrmal paper: NJM A264 (J987) 169, NP As0s (1989) 269, and HF」 $7 \$(1992) 148$

E-matt cunlact rasoudaksokvax kak JP

\section{KEK-246}

(Approved Jul 1891; In progrets)

SEARCH FOR T.VIOLATING MUON POLARIZATION IN $K^{+} \rightarrow \pi^{0}{ }^{+} v$ DECAY USYNG STOPFED KADNS

EAA COLLABORATLON

KEK - I Imszelo (Spokesperson), Y Kuno, H M Shtmaxu, K. H Tranka

TOKYO U, WNS - MI Aols, Y Fupta, H Onta, S Stgumoto,

$\uparrow$ Yamatalkı

TOKYO U - R 5 Hвyauto, T Ishikawe, H Tamura

MOSCOW, INR - D V Dementyey, M Grigorey, A P lvashkin,

M M Khathbullın, Y G Kudenko, V M Lababbev, O V Manesv, $\checkmark$ Popar

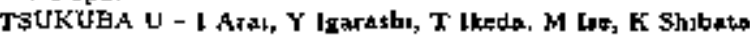

TSUKUEA U, INST APPL PHYS - M Abe, Y Aabno

IBAFAKF U, HITACHI - T YokOH

TOKYO INST TECH - S Sbums

SASKATCHEWAN U - T Baker, C Rongacharyulu, Y M B Shin

YONSEI U - E J Ktm, J M Lee, Y H Shin

KYUNGSUNG U - Y M Pork

BKITLSH COLUMELA ti P Gumplinger, M Hagnof, E Stattler

TRIUKAF - I Doornbos, R Hepderson, J A Mactonsld,

N Stevenoon

MONTREAL U - P DEPomalér

VIRGINLA TECH - M Elocher

KAWAGAWA U - A KBgB

Accelerotor KEK-PG Detector Spectrontéter

Resctronts

$$
K^{+} \rightarrow \pi^{0} \mu^{+}=\quad \text { GeV/c }\left(\mathbf{P}_{\text {lab }}\right)
$$

Purticles atudied $\mathrm{K}^{+}$

Brief description Uges the Superconducting Toroudal Spectrome-

tor A prowed for 150 shufte Taking dats (July 95)

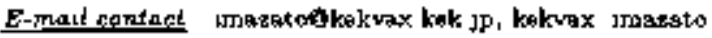


KEK-\$04

(Approved 1996, Began dake-takug 1998, In progress)

STUDY OF $K^{+} \rightarrow \pi^{B} t^{+} v$

Acceleratar KEK-PS betector *

Partaclesstudtaft $k^{+}$

Bref deseription A collaboration of KEK, Tokyo lnst Tech, and Tokyo U Taking dale (July 9B) For furthor informateion, please contart the Spokesperson, DT Taksyosh Ohshima [KEK]

E-matil cotatect obslutisokakrax kak Jp

\section{KEK-\$62}

(Apptovel 2995, lo prejaration)

A LONG BASELINE NEUTRINO OSCLLLATION EXPEREMENT USING KEK-PS AND SUPERKAMIOKANDE

Atecterator KEK-PS Detector ?

Partecies sividad $V$

Bref defcrigtaph An international collaboration of scionkuts

Iram Japan and USA Le praparation (July 99) Fof further wformation, pleass contact the Spokesperson, Dr Kotchure

Nushikawa [KEK]

E-mazil conturet mohtkawgkekvax kek Jp

WwW Hores page attp //pnahp kets jp/

\section{KEK-BF-BELLE}

(Propased 1994, Approved Mar 1994, In preparation)

A STUDY OF CP VIOLATION IN a MESON DECAYS BELLE COLLABORATION

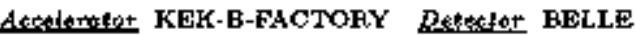

Bnef description KEK B-lactory is expected to bo capable

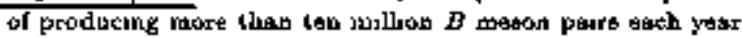
When the faclity becomes operntional, the proposed expertment

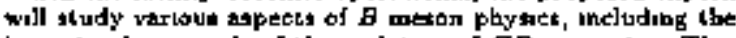
loog-atajding pnzale of the violation of CP symanelry The

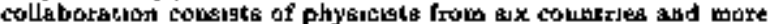
then 36 tast,itution. The Letter of Intent has been acceptad w March Bd Expected to begro data takjng in FY 1998/99 For more information, plesese contact the Spokespersone,

Dr Shiro Suzukı [Naroys J], Dr Stephen L Olsen [Harral V], or Dr Fumbera Tabasaka |KEK!

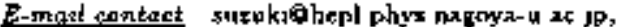

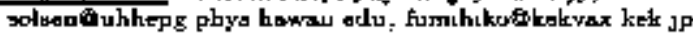
WWW Homespoge http / bannarw kek Jp/

\section{Other KEK Experiments}

Litted here are some ather KEK expertuents of minerest to the psiticle physecs community Fund raore detasls sbout these profects online, on the SLAC'6 EXPERIMENTS databse日 (see p 3), or contact the spokexpersars Check also the KEK's yearly publicatean 'Anous' Report' for s completa list of active experiments

KEK-140A (Conpleted date-(stang Mar 19\%3) STUDY OF HEAVY HYPERNUCLEI VIA THE $(x, K)$ REACTION USING THE \$KS DETECTOR.

Spokegperbon o Hatktmoto [Takyo U , INI]

E-mont contract hashimotinge u-tokyo aC JP
KEK-318 (Completed data-takung Feb 1993)

STUDY OF THE FORMATION OF COINCIDENCE

Spokoperson H Tymura |Tokyo U)

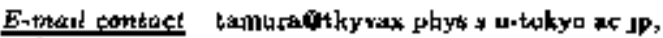
thovex tsmura

KEK-724 (Completed datsotakikg Jan 1992)

SEAFCH FOR THE $H$-DIBARYON WITH A SCINTLL LATING FIEER TEACK DETECTOR

spokespersou K Imal [Kyoto t!

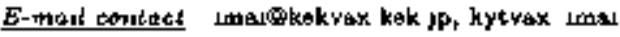

KEK-328 (Complated date-takivg 1996)

PRECISE MEASUREMENT OF THE STRONG INTERACTION SHIFTS OF KAONIC HYTROGEN $X$-RAYS

Spokesperion M Iwesenkt (Tokyo UI, INS)

KEK-255 (Completed date-takıg Apr 1993)

DIFFERENTLAL CROSS-SECTION FOR $p(n, r) d$

5pokesperson M A Kavash [Kertucky U]

E-math confect kovashore pe uky edu

KEK-24: (Taking data)

SEARCH TOR $H$ PARTICLES IN THE pP $\rightarrow K^{+} K^{+}$

FEACTION

Spakespersan II Kamsi [Chiba U]

KEK-251 (Completed dato-taking Dee 1992)

HYPERON-NUCLEON SCATTEHING EXPERIMENT I: $\Sigma^{+}$SCATTERING

Spokesperaon M [estr [KEK, Toultubs]

Esmaticontact retrikekpra kek JP

KEK-287 (Corapleted data-taking May 1992)

SUETHRESHOLD ANTIPROTON PRODUCTION IN d A REACTLONS

Spakesperson 5 ChIba |K(HK, Tsukabs|

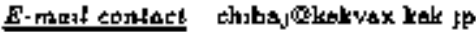

KEK-292 (Contpletod dsta-takng Nor 1993)

BEHAVIOR OF PIONIC HYDFOGEN ATOMS. PART 1 Spokespergon A Shinohara [Nacoya U]

KEK-289 (Corpleted dats-takung 1995)

DIFFERENTIAL $\pi C$ CROSS-SECTION ABOVE THE $\triangle$ RESONANCE REGION

Spokesperson Harutaka Sakaguchu [Kywto U]

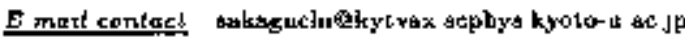

KEK-278 (Coropleted dols-tsking Dec 1998)

ASYMMETRY OF NON-MESONIC WEAK DECAY OF FOLAFIZED ${ }_{\mathbf{A}}^{3} \mathrm{He}$

Spokesperson Teriafami Kishimoto [Dreka U]

E-mant montant kuımatoaphys so cosktalu ar JP 


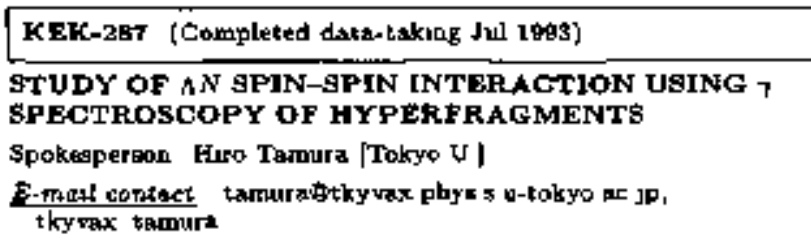

KEK-289 (Takink data)

HYPRON-NUCLEON SCATTERING EXPERIMENT:

$\mathbf{2}^{-}$p AND hP SCATTERING

Spokesperson Masaharu Lerre [KEK, Truknba|

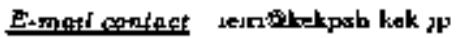

KFK-90T (Conaploted dalantakung 1995)

LIFETIMES AND WEAK DECAY WIDTHS OF LLGHT AND MEDIUM HEAVY A HYTERNUCLEI

Spokstperton He Bhang [Seoul Natjonal ij]

E-mogk contect hbangatorknx kek jp

KEK-sas (Taking dats)

NUCEEAR MATTER EFFECT ON MESON MASS IN DECAY

Spokesperson Hideto Enyo |Kryote U |

E-mard contact buyogktkvax kek JP.

enyoepn sephys kyoton ac up

KEK-\$20 (Completed data-caking t994)

STUDY OF HYPERFRAGMENT PRODUCTION IN ABSORPTION OF STOPPED $\mathrm{K}^{-}$

Spakeperson H Tamura [Toky'o U]

p-matl contacl tarourstatky yax Dhys 3 w-lokyo ac JP,

thyvax tamura

KEK-3\$s (Taking date)

SPECTROBCOPIC INV DSTIGATION OF LIGHT $A$

MYPEANUCLEI BY THE $\left(x^{+}, K^{+}\right)$REACTIDN

Spokesperbon O Hashunoto [Kyola U)

Ermat costact hasbmoterise v-tokyo at JP

\section{KEK-352 (Takug data)}

QUASIELASTIC $x$ SCATTESING

Spokebperton Roy J Peteksou [Colorada U]

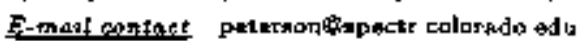

\section{KEK Future Plans}

The unkediscipluary regearch with the 12.GeV Proton Syn.

chrotion will continue utilising the protou acrl light now beaw

'he particle physcs itidies will focus on the long baselian nes-

trtno oscillation experiment with the SuperKnmickande detectar,

and the tume-teveral mysriagec experinent on the kaon aystem

Butb expenmented will seed twice (or more) rntentet prolon beamba

than prebently arsitable, and an accelerator study group is bejng

formed to denl with the new requramente The TRISTAN colleder

bas been clostod, and its tubnel ta now used for two rings of the

KEK-8 (B Factory) asymmetric elertron-position col]jder (A $x 3$ is

Gov) The machlod is acheduled to be camplisioned in FY 1998-

99 , wad the design Iumbotity is $10^{34} \mathrm{~cm}^{-2}-1$ 


\section{Selected LAMPF Experiments}

\section{LAMPF-969}

(Proposed Jul 1965, Apprownd Apg 2988, Begen dsta-taking Jun 1992, Completed data-taking i9g5)

MECA - SEARCH FOR THE RARE DECAY $\mu^{+}-*^{+}$ MEĢA COLLABORATION

UCLA - D Barkw, B A K Nofkans, B Tippess

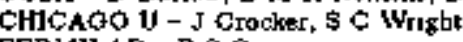

FERAMLAB - P $\$$ Cpoper

HARIPTON U - L Tang

HOUSTON U - M Barakat, Y Chen, M Dzemdane, A Emph,

3 Fhek. E V Hupgerford, K Jehostan, K Lan, B W Mayes,

R Phejpu, L Pinaky, W vou Wutloch

INDLANA U - J Knott, K M Stantz, J Szymangk

LOS ALAMOS - J F Amaga, K Black, R D Eolton, M Brook*

S Cartus, M D Codper ( $V$ Spoksaperash), W Fordmak,

C M Hofman, G E Hogan, T Koalowakt, M Kraupe, D Lite,

G Miths, $\mathrm{E}$ E Miachke, F J Najvar, M A Oothoudt, C Plllal,

R. D Werbeck, D Whitebause, o Wilkinkon

QUEENS U, KINGSTON - A Hálun

STANFORD U - E B Hugbes, C Jw, J N OtıB, M W Ritter

TEXAS A AND M - C Gagliardı, G Kım, F Liu, R E Trbble,

$X \mathrm{Tu}, \mathbf{L}$ Vart Ausdelth, X Zinou

VALPARA]SO U, [NDIANA - R Fibk. D D KoetjeB,

F. W Manyelelar, S Stanalau*

VIRGLNIA U - R Marthadl, B Wight, $K O \mathrm{H}$ Zock

VIRGIN]A TECH - D Faım, F Lee, L E Pulonen, Y Zheng.

Whou

WYOMTNG U - A R Kunsalman

YALE V - K HBhD, J Miarkey

Accelerator LAMPF Detector MEOA

Reactions Folarized beam

$$
{ }^{+} \rightarrow e^{+} Y \quad \text { o SoV/c }\left(\mathrm{r}_{\text {lab }}\right)
$$

Paricles studied is ${ }^{+}$

Brief descriptwon Algo sestebes for a $V+A$ contribation to radratuve decay Approved for 1000 hours Looke for $\mu^{+}-e^{+} \gamma$ al a level of $6 \times 10^{-13}$ a factor of 80 becter thas tbe Geystst Box detector Took data 10 1992/93 and in 1984/95 Data analyss in preqren (July 98)

Jowrnal paysors NIM A.03 (199]) 298, NIM A323 (1992) 198,

NIM A349 (1994) 118, and NLM A372 (1996) 196

Related expersinerts PSI-R-67-05

E-that comtact mocopereland gow

\section{LAMPF-1054}

(Proposed Dec 1086, Approved Feb 1987, Began dats-takung 1991, Completed dats-takubs $19 \%$;

ULTRAHIGH PRECISION MEASUREMENTS ON THE MUONIUM GROUND S'TATE: HYPER FINE STRUCTURE AND MUON MAGNETIC MOMENT

LOS ALAMOS C PIllan, O vac Dyck

HEIDEL GERG U, PKYS [NST - K Jungmann, I Resnhard,

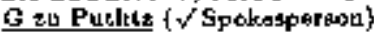

YAle V-M G Boshier, \& Dhawan, X Fet, V W Iraghes

( $V$ Spokesperson), D Kewall, W Lin

YALE U \& PSI, VULLIGEN - M Janouych

BROOKHAVEN F Mariam, $K$ Woodle

LIVERMORE P EgR

Accelerator LAMPF Detector Othas

Partaches studied moonump

Bryef descriptopis An uhrohigh preciaron measurement of the mutanion byperfine atructure latef sal $A \nu$ and of the macrowave

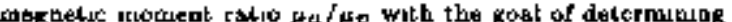
$\Delta \nu$ to $10 \mathrm{ppb}$ and $\mu_{\mu} / \mu_{p}$ to $B 0 \mathrm{pph}$ life the microwate magaetic rexanagce spertroseopy methad with an intengse ind prese subaurface $\mu^{\circ} \div$ beam, B large supercond acting howogeneoub tolemond, sad a lise.asrrowing method involving a ebopped $\mu^{+}$ besm Nata andygus w Hrógriqus (July 96)

Jowrnal papere NIM A333 (1943) 280, nod PF AB2 (1990) 1948 Retoled eptraments BWL-s21

E-moed conkact vernon hughew ty yals ed a

\section{LAMPF-1173 (LSND)}

(Proponed Jal 1989, Approved Jar 1890, Began data-takiag Sep 1903, In pragrad)

\section{SEARCH FOR $\bar{\nu}_{\mu} \leftrightarrow \bar{\nu}_{e}$ OSCILLATIONS WITH HIGH} SENSITIVITY

LNND COLLABORATION

UC, RIVERSIDE - R M Gugasmgha, K McIlbany, 1 Stanç,

W Strosaman, G J VanDalen

UC, SAN DIEGO - W Vernon

UC, SANTA BARBARA - D O Caldwell, M Gray, S Yellı EMBRY-RIDDLE AFFONAUTICAL U - D Smbth, I Wals UCIIFPA, SLAC - A Fisner, Y Hang

LINFIELD COLL OREGON - 1 Caha

LOS ALAMOS - F Burman, J Donabue, F J Foterspel,

G T Garvey, W C Lonis $(\sqrt{ }$ Spokenperkat, G $\mathrm{g}$ Mills,

V Gandbarg, $\mathbf{R}$ Tayloe, $\mathbf{D}$ H Whate

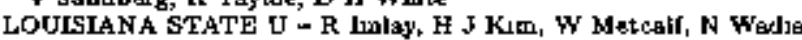
LOUISLANA TECH $\mathrm{U}$ $\mathrm{K}$ Jobnston.

NEW MEXICO U - B B Dielot to, R Reeder

SOUTHERIN U - A FBzely

TEMPLE Uं - C Athapasyopoulas, L B Auerbach, R Majk1c,

J Margulies, D Worke, Y Xiaso

Acceleralor LAMPF Detector LSND

Reactions

$$
\begin{aligned}
& \bar{v}_{t+t} \rightarrow \bar{v}_{t} \\
& v_{u_{0}} \rightarrow x_{s} \\
& v_{e} \mathrm{C} \rightarrow \mathrm{e}^{-} \text {Duclecen } \\
& v_{\mu} \mathrm{C} \rightarrow \mu^{-} \text {nucleon } \\
& v \mathrm{C} \rightarrow v \mathrm{C}^{+} \\
& \nu_{\mu} P \rightarrow \nu_{\mu} P \\
& \nu_{e} e^{-} \rightarrow \nu_{e} e^{-} \\
& <53 \mathrm{MeV}\left(\mathrm{T}_{\mathrm{lab}}\right) \\
& \text { < } 250 \mathrm{MeV}\left(\mathrm{T}_{\mathrm{Jab}}\right) \\
& <53 \text { hie } \forall\left(\mathrm{T}_{\text {lab }}\right) \\
& <200 \mathrm{MeV}\left(\mathrm{T}_{\mathrm{lab}}\right) \\
& 53 \mathrm{MeV}\left(\mathrm{T}_{\mathrm{bab}}\right)
\end{aligned}
$$

Brief description A seateh for mentrono ogcillatsona to the level $\sin ^{2} \theta=3 \times 10^{-4}$, where $\theta$ represents the maxing angte of ther were a two-gennration mixing Uses neutrinon prodnced by both

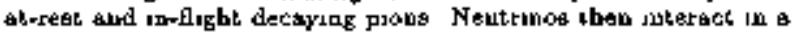

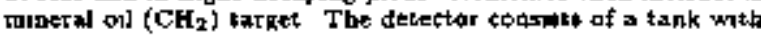
1 的 cons of iquad santillator and with 1220 pbotomultipley

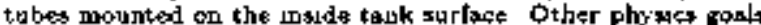

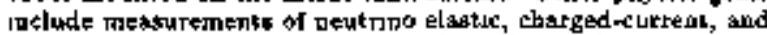
neutral-current acettering Takjnc dats (July gi) Will contisue the date taking fore st lasest ons more yenr, and possibly lar Ancther 5-10 yetrot

Jotsrnel papers NIM A334 (1943) 353, PR C51 (1995) 1065, and PRL $75(1995) 2850$

Relaled expentinetzts ISIS-KARMEN

$B$ mati contac! loussision zov

WWW Hown-page

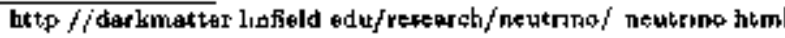

\section{LAMPF-1188}

(Propored 1987, Approved 1987, Eegar datatakiag On 1987, In prograsts

SEARCH FOR TIME REVERSAL SYMMETFY VIOLA TION AND PARIT' VIOLATION AT THE PROTON STORAGE RING

LOS ALAMOS - J D Bowman (Sprokeperbon), C M Frenkle,

J N Kribdeon, 5 Pentelis, 5 J Seestrom, $V$ Yuan 


\section{SUMMARIES OF LOS ALAMOS EXPERIMENTS}

DUKE U - B Crswiord, N A Robeteon

TRIUMF' - P P I Deaheu

NORTH CAROLINA STATE U - C $\mathrm{f}$ Gould, D G Hane,

G E Mitahell, \& Fatterson

DUBNA - Y Popoy, E Elarspon

Atcolerifor LAMPF Detroctor Counter

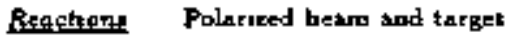

to nucleas.

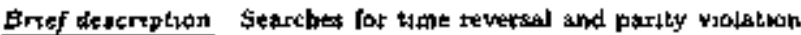

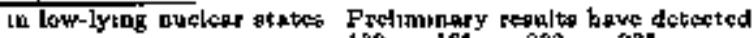
parity vialstion in states of ${ }^{138} \mathrm{Le},{ }^{169} \mathrm{Ho},{ }^{232} \mathrm{Th},{ }^{234} \mathrm{U}$, and

${ }^{238} \mathrm{U}$ The nentron bearp is $57 \%$ polargyed fram 1 eV to 20 keV Continues taking date (Jume 66)

Jotrmal popers PR C39 (ID80) IT21, PRL t5 (I990) 1192, PRL ET (1981) 564, PR. C44 (1961) 21BT, PR C45 (1992) 214\$, PR Cu6 (1592) 76s, PR C46 (1992) 778, PR C46 (1992) 1542, PR

C48 (1993) 11]6, and PR C48 (1993) 1601

E mant contact bow manglsul gor

\section{LAMPF-1240}

(Propoted Jal 1991, Approved Aug 1991, Begen datortaking Aag 1992, Comploted dela-takiag Aug 1993)

MEABUREMENT OF THE MICHEL PARAMETER $a$ WITH THE MEGA POSLTRON SPEGTROMETER

MEGA COLLABORATION

CHICACO V - S C Wught

FERMILAB - P\$ Cooper

HOUSTON U - Y Chen, M Dzemudac, E V Hungarford, K Lan,

B W Mayes, L Pinaky: W van Wituch

INDLANA $\mathrm{U}-\mathrm{J}$ Kavts, K M Stante, J J Szymanokı

LOS ALAMOS - JF Amagת, T. D Bolton, MI D CoOper

( $\checkmark$ Spakespersan), W Foreman, R Harruan, G Hart, G E Hogan,

T Kozlowskl, M A Kroups, E. E Muchks ( $V$ Spoksoperbod),

C Pillo, S Schillige, D whitehobute

TEXAS A AND M - C Gaglard, F Liv, R E Trubte, X L Tu,

L A Van A urgdeln

VALPARAJSO IT, INDIANA - D D Knetke, R W ManNeIkT, S Stamislaut

VIRGIN]A UI - E Wright, K O H Z

VIRGINIA TECH D Haim, F Lee, 1 E Pulonem

( $\checkmark$ Spokepergan), Y Zhapg, w Zhom

Accelerntor LAMPF Detecior MEGA

Reactions Polsrized beams

$$
\mathrm{\mu}^{+} \rightarrow \mathrm{e}^{+} \mathrm{V}_{*} \overline{\mathrm{V}}_{\mu} \quad 2 \mathrm{MeV} / \mathrm{c}
$$

Brtef descrigforn As improved measurcmant of the Michel paraweter o Rad for 336 hours Dats analybis an progrease (July $\oplus 6$ )

Relater expertments LAMPF-ge9

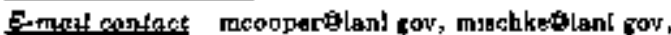
pulonotiosing phys vt do

\section{LAMPF-1268}

(Proposed Nov 1992, Approved Jal 1943, Begau data-laking Jun i993, Complered date-taking Sep 1003)

$\pi^{-} p \rightarrow \pi^{0} \pi$ CROSS-SECTIONS IN THE REGION OF THE $\triangle$ RESONANCE

ABILENE GHRISTIAN 0 - L D Lsenhow*e, J Redmou,

MI E Sidler ( $v^{\prime}$ Spokegperson)

AREONA STATE U - I R Comfort, C Gaulard

BOSKOVIC INST, ZAGREE - A Marubic, I Supek

CATHOLIC U - H Crapnell, L Nguren

GEORGE WASHIIVTON 0 - W J Bntede, J Connelley, 5 Matthews

LOS ALAMOS - J Amann R Boudre, J Knudson, C Morrs,

B Park, M Rawool, R M Whitton

PENN U - F F HUI, D Smith

Aeceiteratar LAYPF Detector NMS $\begin{array}{cc}\frac{\text { Rasettotisg }}{\pi^{-} p \rightarrow \pi^{0} n} & 138,166,190,215,23 \%, 263 \mathrm{MeV} \\ & \left(\mathrm{T}_{\mathrm{lab}}\right) \\ \text { Parteclef otudied } & \left.\text { A(1232 } \mathrm{P}_{33}\right)\end{array}$

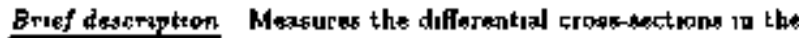
reppon of the $\Delta_{1}(1232)$ resonance Uye elleraents of tbe Neutral Meton Spectrotneter (NMS) to messure the two p-rays from the

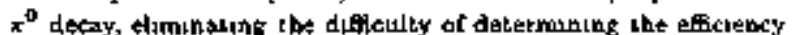
of neutron counterg The gosis are to provite socurste data for suput to charge-dependent part1al wase andyaes, and to study the charde splutting of the $\Delta$

Retated experment: LAMPF.1178

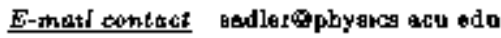

\section{Other LAMPF Experiments}

Labled bere sre some ather LAMPF experiments of unteress to the particle physks corrarumity Fund more detals about these projects onlute, in the SLAC', EXPERIMENT' databste (Bee P 3), or contsct the spokesperang

LAMPF-87t (Completed dats-takeng Oet 1992)

SPIN TRANSFER MEASUREMENTS FOR NEUTRONPROTON ELASTIC SCATTERING

Spokesperson Mrchael W MeNaughoon |Los Alamoel

E.mand ophtstat manauthtolanl gov

laMPF-1178 (Completed data-takng Nov 1805)

POLARURATION ASYMMETRY MEASUREMENTS

FOR $\left.{ }^{1} \mathrm{H}_{\{} x^{-}, \pi^{\mathrm{D}}\right\} \mathrm{n}$ BETWEEN 45 AND $100 \mathrm{MeV}$

Spakespersous Joseph R Comfork (Arizora State U ], Beorca $R$ Burlagon [Now Maxico Statc U]

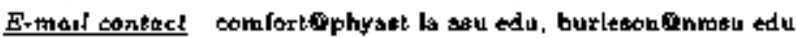

LAMPF-1179 (Completed data-takıng Jul 1993)

REACTION $x^{+}-\pi^{+} \pi^{0} p$ NEAR THRESHOLD

Spakesperson Dinto Föanaé [V'trganja U]

E-minileartact pocanicevirginga adu

WWW Home.page

bthp //helena phys vergana edv/ p1pin/E1179/ E1179 html

LAMPF-1180 (Completed dsta-takıng Jnl 1882)

PION-PROTON INTEGRAI CROSS-SECTION MEA SUREMENTS

Spokerpereons Rabert A Rbatmen [Colorado U], C L Morris intat Alstopos

$E$-matl contact retinentsepertr coloredo edu

LAMPF-1200 (Taking dsts)

NEUTRON-PROTON BREMSSTRAHLUNG

Spokemperson Stephen A Wender [Las Alamoss]

E matl cortact wanderolan gor

LAMPF-121s (Completed data-taklang Nor 1985)

MEASURELENT OF THE NEUTRINO CAPTUFE

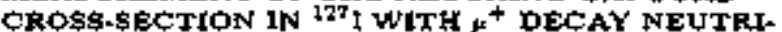

NOS

Spuknaperaon Kenoeth Lsade [Pen U|

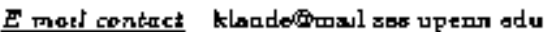


LAMPF-1231

LASER POLARIZED MUONIC ATOMS AND BPIN DEPENDENCE OF NUCLEAR MUON CAPTURE

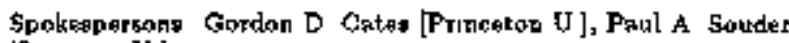
Syracuad U

E-mat cortact tateobpuce prinction edu, sondertionubep phy syr edu

LAMPF-1234 (Completed dacarkking Sep 1901)

$K_{L L}$ AND F FOR np ELASTJC SCATTERINC Spokexperfop Mechael W Mctiaughton [Los Alampa]

E-phas tothlect meneughtoleal gov

LAMPF-1256 (In proparation)

$\pi^{t_{F}}$ ANALYZNG POWERS AT AS AND GT MQV

Spokespotzong Joeeph R Comfort (Arrone State U!, George R Burieson fNew Mexpe Stente U I

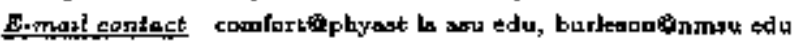

CAMPF-1207 (Completed deta-taking Oct 1993)

ELAGTIC SCATTERING OF $x^{+}$FROM POLAFLED ${ }^{3} \mathrm{H}_{\mathrm{C}}$ AT $T_{\pi}=100,143,180$, AND $256 \mathrm{M} / \mathrm{V}$

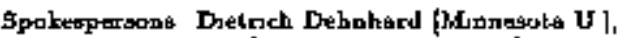

Goorge R Burleson [Naw Mexico State U], Dtto F Haepraser

|TRIUNAF and Simon Frasert U |

E.mazl conlect debuhardaphysics spa uron edu,

burkeontama edu, hausseroterumI ch

LAMPF-1286 (Completed dabstrakng Sep 1993)

MEASURING THE NEUTRON-NEUTRON SGATTER. ING LENGTH AND FFFECTIVE RANGE USING THE ${ }^{2} H_{\left(x^{-}\right.}$: 2n') REACTION

Spokeaperood Ahmed H Huesein [Northern Brytigh Columbes $v$ ]

Esmant cantact bumengurbe edu

LAMPF-1393 (Completed dsts-taking Oct 1983)

thp ELASTIC ANALYZING PQWER

Spokesperson Micheel W McNagahton [Los Alamios]

E. matt contact menaughtolaal gov

LAMPF-1809 (Corapleted date-takıng Det 1983)

ANALYZJNG POWER AMD SPIN TRANSEER MEA-

SUERMENTS IN

Spokesperson Reorge Glass [Texns U]

E mat contact mplegolsmpf lanl gov

\section{LAMPF-1S10}

MEASUREMENT OF THE DOUBLY DHFFERENTIAC CROSS-gECTION FOR $\pi^{-} \rightarrow \pi^{+} \pi^{-} n$ AT 190 AND 2ub MOV AND SOFT PION THEORY

Spokespersons Glen A Rebka, Jr Wyoming U! Pe.

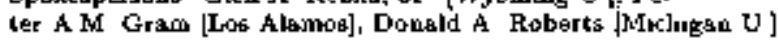

E-matl contact physeloturyo edu, grameilampf lasl gov, drobertilimichl physuce las umich edu

\section{LAMPF Future Plans}

TDe LAMPF nuclear and particle physicg user program has offerally ended The facility, now called LANisCE (Loa Mlamacs Neutron \$cience Center) contunues to operate with she purpuse

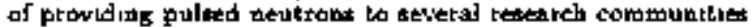
Howevar, partucte phyesce retsure ito presence at Los Alanos The LSND neutrino expenment. wil continue to ren for at texat severa] mors years A number of tandamental physick expenments uning cold and ultracold nontront are plabned at IANSCE 


\section{SUMMARIES OF NOVOSIBIRSK EXPERIMENTS}

\section{NOVOSIBIRSK Experiments}

\section{NOVOSIBIRSK-CMD-2}

(Propaned 1984, Approved 1985, Begson datzolakung 1981, In progratu)

\section{THE CRYOGENIC MAGNETIC EXPRRIMENT}

NOVOSIBLSK, TYF - R R Alkhmetshow, G A Akbanov,

E V Apsshken, V $M$ Aukhenka, B O Belhuxinov, V S Benganoy,

L M Barkov, 5 E Baru, iN \$ Barhiopor, A E Bundar,

D V Chernysk, S ] Eidel mad, G V Fodotowitch, N I Gobythev,

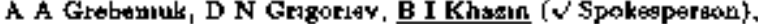

I A Koop, A S Kuzmun, I B Logashenko, F A Lukın,

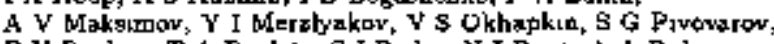

E V Poptor, T A Purlede, S I Reduc, N I Rost, A A Rubat.

N hi Rrakulov, $Y$ is Shatunov, A I Shekbtman, A E Shex,

M A shubin, B A strwartz, V A Sidorow, A N Skrinsky

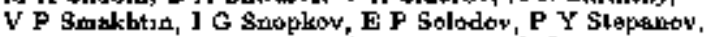

A I Sukbason, V M Titov, Y V Yudin, V G Zsvaryin,

SG Zverev

BOsTON U - D H Brown, L B Rokerta, W Woretell

PITTSBURGH U - J A Thompsor

YALE U - S K Dhewan, VW Hughe

Acselereior ROVO-VEPP.2M Detentor CMD.2

Reactabats

$$
\begin{aligned}
& e^{+} e^{-} \rightarrow \text { chnrgend }^{+} \text {,harged }{ }^{-} \quad 0 \mathrm{St}-14 \mathrm{GeV}\left(\mathrm{E}_{\mathrm{cer}}\right) \\
& \text { (chargedn) (noutrals) }
\end{aligned}
$$

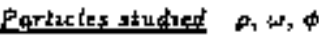

Bruf descriptsoti Messures the badrome part of the snomalons misignetic srament of the mnob Studaes the dyosmies of multihadrou production and jate deseys of vector mesons The magnetic detectopt conelals of a 15 teala superconducung echenord, drict dhember, Z-chamber, mus idemetilleption syatem,

CsI barrel calorimeter: and BGO endasp calvrumeter

Journat papers NIM A252 (1986) 289, NIN A265 (1989) 137, NIM A353 (1989) 752, NIM A329 (1962) 178, and PL B364 (1995) 199

Feluted experminents BNl-821

$\underline{E}$-mati contact khazuginp nak au

WWW Harne-puge http //Wwin inp nxk xis/cod 2/

\section{NOVOSIBJRSK-KEDR}

(Froposod 1986, Approved 1987, In preparation)

\section{THE MAGNETLC SPECTROMETER EXPERIMENT}

NOVOSLBIRSK, TYF - $\$ 2$ A khmadgliey, $v$ v Anashn, V N Auteheako, B D Babuanov, L M Barbov, A A Barladyan,

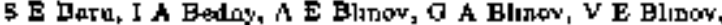

A E Bondar, A D Bukı, A G Chulınarov, 5 I Exdejman,

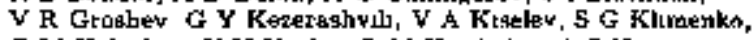

G M Kolacher, V N Kozloy, L M Kurdadse, A 5 Kuzulu,

A $\zeta$ Lelthuk, $V$ M Matrsher, A A Maslennikov, A A MLlov,

G D Minakov, 51 Mushnev, N A Muchroo, V' P Nagaslaev

E I Nakbane rith, A B Nomeroteky' A P Onuchin, V S Pabun,

S V Felexsmehyrak, V V Petrav, G E Pakpelor, Y V Pral,

I Y Protopopov, T A Purliatiz, V A Podyaktu. L V Rom snov,

N I Root, A V Fylu, G A Savinov, B A Schwarky, A G Shamov,

A1 I ghubin, A I Shushero, V A Sidoroy, Y I Skovpen,

A IN Skrineky, V P Smakhtis, A I Sokolow, iv A Tayurgkı,

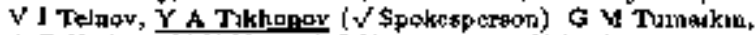

A E Undrus: Y V Utow, A I Vorobter, N I Yakevlev,

I A Zagorodnjoy, VN Zhılich, A A Zholents

BOLOGNA U \& INFN, BOLOGNA - P L Frsbett

MILAN U \& INFN, MILAN - F Palombr a gala

PAVTA D \& INFN, PAVIA - P F Malrexd, V Fe, V Spesiali

Acceterotor NOVO-VEPP-4M Detector KEDR

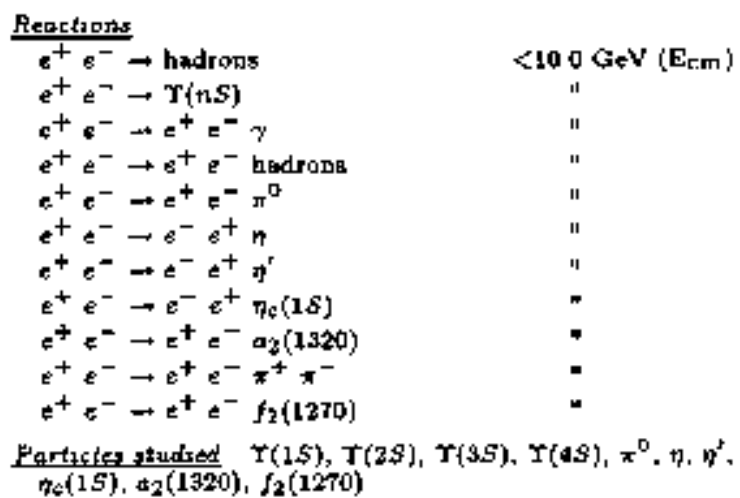

Bryef descrtptan Studie日 apectroscopy of $\mathbf{Y}$ mesons Proticular

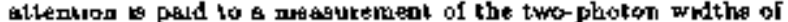
particles The dotector KEDR consests of a vertex detector, dnft chembers, aerogel counter, roF, barrel LKr calonmeter, endcop

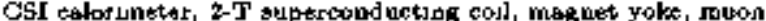
gystem, and a tagging gyetem for detection of $e^{+} e^{-}$in the two. photon procesece Seheduled to run is 1997

Joumal popers NIM A289 (1990) 48S, N1M A3J5 (1992) 491, and NIM Aslo ( 1998 ) 8

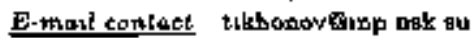

WWW Horne-poge bttp //WW up nak su/kedr/

\section{NOVOSIBIRSK-\$ND}

(Froposed 1986, Approyed 1987, Elakn datz-taking 1995, Is progres6?

THE NEUTRAL-SPECTROMETER EXPERIMENT

NOVOSIBIRSK, TYF - M N Achawo, T Y Baner, M O Deck, F M Beschastingv, A V Bozbetiok, A D Bukin, D A Bulk1, 5 v Burdin, V P Druzhmm, M 8 Dubrovin, I A Gaponentro, V B Golluber, A V Gritasa, Y N Iranchenko, A A Korol,

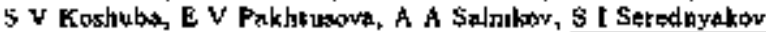
(V Spakeeperzon), V V Sbary, Y M Shetunow, V A Bdoroy, Z K Slaged2e, A N Skrinsky, Y Y Uaov, Y S Velikshanom Acactenator NOVO-VEPP-2M Detector SND Reactions

$e^{+} e^{-} \rightarrow \pi^{0}$

$e^{+} e^{-} \rightarrow+\gamma$

$e^{+}+e^{-}-\omega \pi^{0}$

$e^{+} e^{-} \rightarrow \phi \pi^{0}$

$e^{+} e^{-} \rightarrow+\pi^{+} \pi^{-}$

$e^{+} e^{-} \rightarrow d \gamma$

$e^{+} e^{-}-5 y$

$e^{+} e^{-\ldots} e^{-} e^{+}$

$e^{+} e^{-} \rightarrow e^{-} e^{+}$ar

$e^{+} e^{-} \rightarrow 2 e^{-} 2 e^{+}$

$e^{+} e^{-} \rightarrow 2 e^{-} 2 e^{+} \gamma$

$e^{+} e^{-}-$proas

$e^{+} e^{-} \rightarrow 2$ pion

$\epsilon^{+} \epsilon^{-} \rightarrow K^{+} K^{*}, K_{S} K_{L}$

$<14 G e V\left(E_{e m}\right)$

4

11$$
11
$$

Partickes gtudted $K^{+}, K^{-}, K^{-t}, p_{1}$ is, $J_{0}(975)$ : $x_{0}(980), \phi$ Briaf defortg toon Studieg radiative and rare decays of vector mesons, nonrebonant hadronc production, sad dẹucrak haon

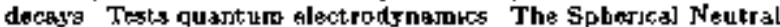
Detector (SND) censisty of $18 B 0$ Naf(TI) conpters and provider a good tood to atudy multiphoton svente The inegrsted Iumenanty resched $2 \mathrm{pb}^{-1}$ in the $\phi$ regian af of Jane 96

Joturnat papers YF s6-11 (1999) 75, NIM A342 (1994) 47T, and NTM $A 361\{1990) 138$

Rnlated appernerits NovosibinsK-No

E-mat contact beradnyakowkinp ngk gu

WWW Horretpage htcp //Www Inp ask su/snd/ 


\section{Selected PNPI Experiments}

\section{PNPI-SC-147}

(Prepoxed Jun 1990, Approved Jun 1990, Begen dake-taking Nor 1992, In progress)

STUDY OF BINARY $\pi^{-}$, REACTIONS WTTH NEUTRAL PARTICLES IN THE FINAL STATE IN THE REGION OF $N\left(1410 P_{11}\right)$ AND $N\left(1535 s_{11}\right)$ RESONANCES

PNPI-UOLA-ACU COLLABOFATION

ST PETERSEURG, INP - V V Abat, V S Bakrendv,

E A Filimonow, A B Gridnes, M F Kan, N G Kozlanko,

\& P Krnglav, L V Lapochking, I V Lopetin ( V Spotesperson),

D V Noylnsty, A B Statoedr, V V Sumachar

UCLA - R M Clsjug, B M K Nofksns

ABLENE CHRISTIAN U - L D Lrenhower. M E Sadkr

Acoelestotor PNPI Defictor Counter

Reactions

$x^{-} p-q \eta \quad$ T

$\pi^{-} p-\pi 7^{0} \quad 500-750 \mathrm{MeV} / \mathrm{c}$

Bricf defcription Meadrements of DCS for the raction $x^{-} p-$

${ }^{0}{ }_{n}$ in the reglou of $N\left(14 \$ 0 P_{11}\right)$ and $N\left(1536 s_{1}\right)$ resonences, and at the in production threshold are anderwy. If tot four neutron counters is colmcldence with tu'c lotsl sbeorption t?

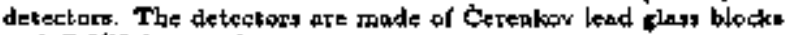
and $\mathrm{CaI}(\mathrm{Na})$ tryotals

pournat papers FTE 1 (1995) Ls, and FES SUPPL of (1995) 241

E-mail cantact lopatinalnpt.epb.eu

\section{Other PNPI Experiments}

Llsted here ark tome other PNPI axperlmente or iuterest to

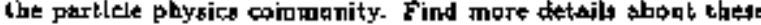
projects ontlue, in the SLAC'x EXPERIMENTS datsbate (see p. 3), ar contact the opoknopernons.

PNPI-SC-124 (Completod date-tak tog Aug 1901)

MAEASUFQMENT DF THE SPIN ROTATION PARAM. ETERS $R$ AND A IN $\pi^{-} p$ ELASTIC SCATTERTNG IN THE REGION OF LOW-LYING PION-NUCLEON RESONANCES

Spokesperson: S.P. Krugko [St. Peterstrarg, LNP]

Expait cantact. kruglovolopi.spb.sn

PNPl-SC-120 (Completed data-taking 190z)

MEAGUREMENT OF VECTOR ANALYZING POWEA IT $T_{11}$ IN REACTION $\pi^{-}$a $^{\rightarrow} \rightarrow$ py AT THE PION KINETIC ENERGY RECION $350-450 \mathrm{MOV}$

Spokesperion: A.N. Prokofler [St. Pelersblarg, ]NP]

E-maif cortact proksoginpi.spb.su 


\section{Selected PSI Experiments}

\author{
PSI-R-87-01 \\ (Proposed Nov 1986, Approved Jan 1967, Begran datatakng Dec \\ 1988, Completed dals-(aking 1993)

\section{PFECISION MEASUREMENT OF THE MUON MO-} \\ MENTUM LN PION DECAY AT REST

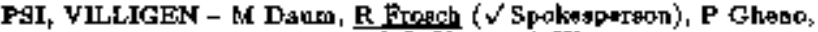

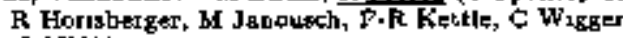
VIRGINIA U - K ALANAAgan
ZUFICH. ETH - H Forrar
ZURICH $U$ - C Broenamana, T \$pirzz
Accalaritior PBI Detecter Spettroweter
Revitante

$$
\pi^{+} \rightarrow \mu^{+}{ }_{\nu \mathrm{S}} \quad \mathrm{OHEV} / \mathrm{C}
$$

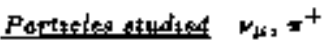
Breef destrtption Utes a surface muon besm and a magnetrc spectrameter Fan for approxthost fily 10 weeks sind mestured the muon mowentum to better than 4 pom The measurement glyos an uppor limit on the $\mathrm{k} / \mu$ mage and determines the $\pi^{+}$ mass
Joumal paptrs PL B265 (I991) 425, PL B934 (1094) 221, and
PR D63 (J096) 6045
Exprati conlact manfred dsumpos ch

\section{PSI-R-87-03}

(Froposed Nov Lo86, Approvad lan 1987, Began finta-takinc 1989, in progress)

SEARCH FOR $\mathrm{a}^{-} \rightarrow \mathrm{e}^{-}$CDNVERSION WITH SINDAUM II

\section{STNDRUM-II COLLABOFATION}

AACHEN, TECH HOCHSCH, III PHYS INST - G Cahnor,

J Kaulard, I Knth, G Otter, A Soluengul

PS1, YULIGEN - W Bertt, J Exrer, D Ronker, I Z1chy SWIERK, INST ATOMLC ENERGY - $\tau$ Katbowte ZURICH U - R Engfer, E A Hersmer, fI S Pruy7, F Riepenohousen,

If Ruluche, A van der Schats (V Spoketpersan), P Winte Accelenutor PSI Detecior SINDRUM-II

ㄱesactiony

$$
\text { H- nuclens }-e^{-} \text {nuclens } \quad 0 \mathrm{MoV} / \mathrm{c}\left(\mathrm{P}_{\text {|ah }}\right)
$$

Particilsa studzted $\mu$

Braef descrtpttoti The gosl as to study the aeutrinolese $\mathrm{p}^{-} \rightarrow \varepsilon^{-}$ converaion is a muonsc atom, which is a leat of lepton havor canservatjan Date were taken on $T$ is $J 98 \theta$ (reanltas fre publeghed), on Pb in 1982, and on T1 again in 1928 With the new beamlige which will become avalable hy $199 \%$, the sersitivity 19 expecied to reach 10 $0^{-14}$ Active (July 90)

Jourthal papers NIM A927 (1903) 37\%, PL B317 (1993) 631, and PRL 76 (1996) 200

Fietated expermeghs LAMAPF.968

$E$ matl contact vanderschanfopil th

WWW Home-page

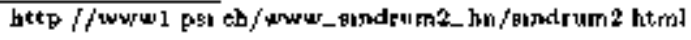

\section{PSI-R-89-01}

(Propaged Ja I989, May J991, Appraved Jan 1892, I preparatoon)

A PRECISE MEASUREMENT OF THE $\pi^{+} \rightarrow \pi^{0} e^{+}$ DECAY RATE

PIBETA COLLABORATION
V'LRGINIA U - E Frlež, I E Koglan, I \$ MeCarthy, R C Mupehart, B E Norum, D Počsme ( $\checkmark$ Spokerpersos), S Ritt, P L Slacum. L C Smıth, W A Stephens, B K Wnght, KO H Zinck

PSI, VILLIGEN - M Deus,, T Flugel, $\mathrm{R}$ Froch, R Horseberger,

B R Kratrea, D Renker, R Schnydar

PSI, VILLIGEN \& ZURICH U - C BroenDImenn

BOLTAN INST, SWIERK - T Koalowsk!

ARIZONA STATE U - D W Lsurence, B G Flitche

DUBNA - V A Boranov, V V Karpukhed, N V Khomutow,

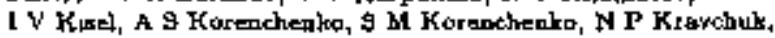

N A Kuchingky, A Motseatko

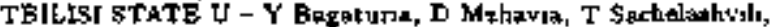
$z$ Tsamalauder

BOSKOVIC INST, ZAGREB - N Sonc, I Supek

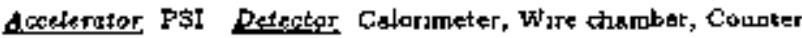

Reactrons

$$
\begin{array}{lc}
\pi^{+} \rightarrow x^{\circ} e^{+} & 0 \mathrm{MeV} / \mathrm{C} \\
x^{+}-e^{+} & \text {- } \\
x^{+} \rightarrow e^{+} &
\end{array}
$$

Particles gtudited $\pi^{+}$

Bruf descriptior The sim it to determine the branching ratio for the $x^{+}-\pi^{0} e^{+} \nu$ decay to about 0 b\% gecuracy The apparatut is a alopped-pion detectar system degignad to observe the two $\gamma$ ' finm the $x^{D}$ docky, an well as the $e^{+}$Uaes

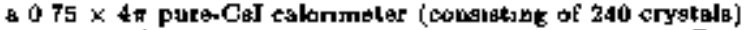
with a good exargy resolution, MWPC's, avd coubtorg Target

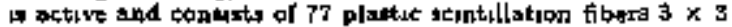
m $^{2}$ Developmert ғuम" are scheduled for 1996 Dats ksking is expectid in late 1896

Reiated expermenty LAMAPF-DS2

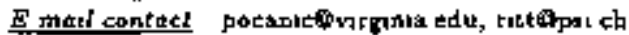
WWW Hortre.poge bttp//ptbeta p*l ch/

\section{PSI-P-89-06}

(Froponed Mar 1990, Approwed Apr 1980, Began dats-taking Jul 1992, Iv progrtas)

SEARCH FOR SPONTANEOUS CONVERSION OF MUONIUM TO ANTEMUONIUM

FETEL BERG U, PHYG INST - A Grobsimana, D Hubl, K Jungmana ( $\checkmark$ ' Spokespersoa), J Merkel, Y Meyes, I Rernhard,

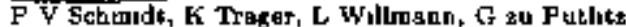
2URICl1 ti - R Engitr

PSI, VILLIGEN - fi Abels, D Renker, H K Walke

DUBNA - V Baranov, V Kerpucbu, I Kıgek, A S Korthchenko,

S M Korenchenko, N P Kravahuk, N Kuchansky, A Mousentoo

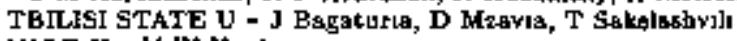

YALE U - V Wughes

Aoctienter PSI Detector SIlNDRUM

Feactions

$$
\mu^{+} e^{-} \rightarrow \mu^{-} e^{+} \quad 25 \mathrm{MeV} / \mathrm{C}\left(\mathbf{P}_{\text {lab }}\right)
$$

Parltciles atidted $\mu^{+}$, wuonium

Anef degareption Studies lepton mumber viatation The $\mu^{-}$ treson 15 deterted by jts decay eloctron, the atomic $e^{+} \leq$ dirtetly detectod anot accoleration by $8 \mathrm{kV}$ The reection

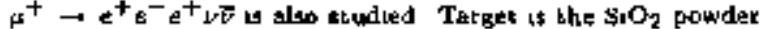

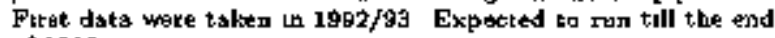
of 1996

Related expertinents LAMPF 1073

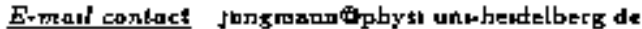
wW Home-page

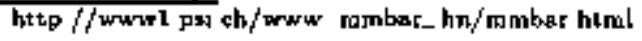

\section{PSI-R-94-01}

(Propored Dec 1993، Approved Dec 1983, Began datertakigg Jun 1994, In progress)

FEASLELLITY STUDY TO DETERMINE THE * MASB FATIO 
IOANNINA U - D F Ansernostopoultas

JULICH, FORSCHUNGS2ENTTUM - G Borchert, f Gork, D Gotla $(\sqrt{ }$ Spoktaperton $), \$$ Lanz, o w E schnlt

PARIS, CURIE UNIV VI, LPAN - P EJ Khoury, P Iadelicato

PSI, VLLIGEN - M Danm, R Fronch, P Hauser, K Kirch,

L M Sumons

NEUCHATEL U - M Aukaburcter, D Chatellard, d P Exrer,

E Jo日nnet

Acceleritor PSI Detectior Spectrorbeter

Particies atudied $=-, \mu^{-}$

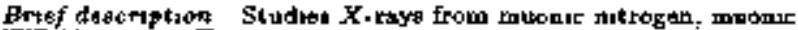
oxyged, and proble nitrogen Unea a beol cryatal spectrometer Aams to determino the $\pi^{-}$mass to $\pm \mathbf{l}$ perr Tatung dots (July 90)

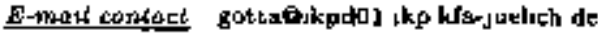

\section{PSI-E-94-10}

(Proposed May 1905, Approved Jun 1995, In preparations)

MEASUREMENT OF THE TRANEVERGE PQLARIZA TION OF POSITRONS FROM THE DECAY OE POLAK. IZED MUONS

ZURICH, ETh - I Bernett, C Bee D Cont, Wr Fetrcher ( $\checkmark$ Sproketpersort), M Hadru, S Kutryn, J Lang, O tVavilat, J Gromicks, E Stephar

JAGELlonian U - K Bodel, L Jarcayk, J Smyrakı, A Stezo|knw:k1, J Zmyora

Acreterutor PSI Petectar Drift thanaber, Counter

Reactures Poltarized botal

$$
\mu^{*}-e^{+}{ }_{\omega_{e}} \overline{\mathrm{F}}_{\mu} \quad \quad 125 \mathrm{MeV} / \mathrm{c}\left(\mathrm{P}_{\mathrm{lab}}\right)
$$

Partectes_tydised +

Brtef descropteon Masgures two transverse polangation corrponents, $P_{T 1}$ and $P_{T 2}$ of the postron, as a function of the e ${ }^{+} \in n^{*}$ exgy, with an expected precispon of $3 \times 10^{-3}$ Thas conld improve by sa arder of maghirude the results of a preylous PSI measo cement of the two components, and provide a tughter trmit both for the yolation of ture reveris] sand on the value of the low-energy paramoter $\#$ which is used in the determinetion of

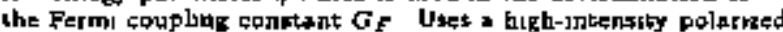
muon besm, and $s$ set of drift chembers, plasic counters. and a Bc⿴囗十 aाтау

E-mat eontact woll fotecheropse eth

\section{PSI-R-05-03}

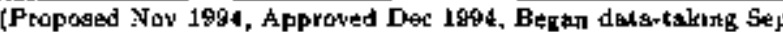
jegs, tn program)

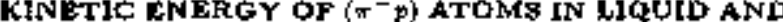 CASEOUS HYCROGEN}

PSI, VILLIOEN - M Daum ( $V$ Spokesperion). W Hajdas

F-R Kettie, V Mfartuahin J Schottmueller

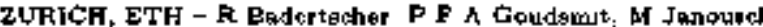

Z G Zlow

Atcekerator PSI Betector Countek Cstorimeler

Retact+orss

$$
\begin{aligned}
& \pi^{2} p-\pi^{0} n \\
& \tau^{--} p \rightarrow \gamma n \\
& 0 \mathrm{MeV} / \mathrm{c}\left(\mathrm{P}_{\text {lab }}\right)
\end{aligned}
$$

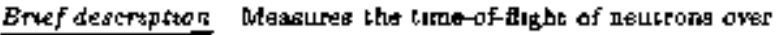
varique fight-path leaglthe (from 1 to 12 w, and poperbly mors) to determine the kunetic energy datnbusion of pionac asoms under vaxying prataure condutiong The same dasa wrll also be noed to deteribua the mask difference between $x^{-}$and $x^{0}$ with umproved preciston The detection sppesatns conelats of coututer and a NaI a e orimeter

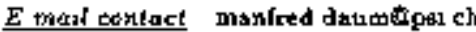

\section{PSI-R-96-05}

(Propazed Apr 1996, Appruved Jen 1996, Beran data-takure L996, In progress)

SEARCH FOR A NEUTRAL PARTICLE OF MASS = \$3.9 MEV IN PION DECAY

P5I, VILLJGEN - M Dam ( $\checkmark$ 'Spokespersan). P-R Kettle,

B Kraute, I Schotemuellar, o willetim

PSL, VILLJGEN \& VIRGINIA U - 3 R,tL

TUBINGEN $U-K$ Foeh!

ZURICH, ETH - M Jorough, Z G Zhao

Acctierator PSL Delecior Sewtillator

Reactions

$$
\mathrm{x}^{4} \rightarrow \mathrm{A}^{+} \mathrm{X} \quad 100-150 \mathrm{MeV} / \mathrm{c}\left(P_{\mathrm{lab}}\right)
$$

Brief descroption Meseures the muon momebcum distribution in cherged pran decey in flught, in order to sebsch for a pmall branching Eraction ti of pron decays $\pi^{+} \rightarrow \mu^{+} X$, in which a heavy neutral parkicie $x$, with $x$ mass of $339 \mathrm{Mg}$, mondd be onslled Such a particle may have been observad recenlly by the KARMEN Collabotation

Journal papers PL E3el (1995) 179

Related experamenss ISIS-KARMIEN

E-matl confect manfred daumopsi ch

\section{Other PSI Experiments}

Listed here are some other PSI oxperments of unteregt to the particle physits comproupity Find more detall abont there projects online, in tha SLAC'a EXTERUMENTS dalabuse

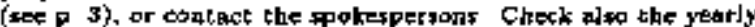
publreatica 'PSI Annual Feport - Abuex I'

PSI-R-86+18-3 (Completed datar-(atcong Aug 1990)

MEASUFFMENT OF ELASTYC $x \neq n$ SCATTERING BELOW 100 MEV

By LEPS Gollвboration

Spokepperwons W Kluge [Koslsrube V, IEKP], $\mathbf{H}$ Clement [Tubungen ㅂ]

E-thatl contact wolfgeng kluge日pbyak un-kerlssuhe de

PSI-R-86-08 (Completed dato-taking Aug 1994)

CRYSTAL DIFFRACTION OF PIONIC HYDROGEN AND DEU TERIUM $X$-RAYS

Spakesporatone I P Eggor [Noutbakel U h, A Badertatber [Zurich, ETH]

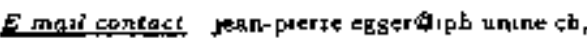
andrevs badertscherfips] ch

PSI-F-67-12 (Completad dats-takıng 1996)

n F ELASTIC SCATTERING. AN EXPERIMENT WITH POLARIZED NEUTRONS

Spoke日person H Scbmit: [Fralburk U]

E-moil contact hechmiteGumi-freburg dc

PS]-R-B9-03 (Taking data\}

PIONIC DOUBLE CHARGE BXCHANGE AT LOW ENERGIES

Spokenpareon H Clement \{Tubungeu $\mathbf{U}$ \}

E-trast confact tlementoput phyalk unt tuebingen de 


\section{SUMMARIES OF VILLIGEN EXPERIMENTS}

PSI-R-01-08 (Completed riata-taking Jul 1993)

MEASUREMENT OF THE STOPPING POWER FOR MUONG $\left(\mu^{-}, \mu^{+}\right)$AT ENEFGIES BETWEEN 2 AND 40 kov

Spokenpersong FI Hartruann [Munch, Tech U]: D Trqqu |PSI, Villigen]

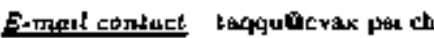

PSI-R-02-DB (Conopleled dass-takitit Sop 1992)

MEASUREMENT OF THE PRODUCTION OF THERMAL MUONIUM IN VACUUM FROM SILICA ABHOGELS

Spokesperbons K Jungmann, B Nathtan |ffefldefbars U, Phy, Loot ]

PSI-R-03-06 (In preparation)

MEASUREMENT OF THE $3 d-3$ TRANSITJON IN MUONIC HYDROGEN WTTH A COMPACT WAVEGUIDE FFBE-ELECTRON LASER

By MUH Collabotation

Spoktæpergons E Zayattini [Trisste U/

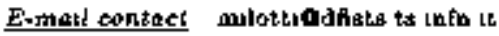

P\$1-RL-8S-08 (Takıng dnta)

SPIN DEPENDENCE OP PION FROUUCTION IN NEUTRON-PROTON COLLISIONS

Spoknotergone H Schmit: [Freiburg $v$ ], M Finger [Cher]ed $v$ and Dubna, JLNFI

E-manlepofact hechmittoum-Irelburg de

FS1-R-95-09

MEASUREMENT OF THE DESTRUCTIVE INTERFERENCE OF \& AND WAVE IN $\pi{ }^{\circ}$ ELASTIC SCATTERING AT $180^{\circ}$

Spokmperson a Janowich [Zurich, ETH]

Ement contact matkut jonousch Gipat ch

PSI-Z-89-02 (Completed dats-laking Juח 199N)

NEUTRRON MAGNETIC FORM-FACTOR

\$pokfepersoú Juerg Jpurdar [Bakel V]

E-rinati contedet pourdanburz uabas ch

PSI-Z 89-0e (Completed dats-takide 1991)

SPIN DEPENDENT TOTAL CROSS-SECTION $A O_{L}$ IN n\# SCATTERUNG

Spokpperson $F$ fratter [Basel v']

E mapl cotitact jourdandurz umbra ch

PS1-2-89-0T (Completed deta-taking 1961)

HP RADIATIVE CAPTURE

Spakeaperiou G S Masou |Basal U |

E.mart contact masonenurz unibas oh

PSI-Z-20-0 (Takung dato)

SEARCH FOR EXTENSIONS OF THE STANDARD

MODEL BY A RELATIVE B-POLARIZATION MEA-

SUREMENT FROM POLARIZED NUCLEI

Spukeaperson Oscar Navlast-Co qс15 [Zurich ETH]

E-matl contact aavilialoump phose etho ch
PSI-Z-90-12 (Takıng date)

DEYELOPMENT OF A SUPERCONDUCTING NEU. TRINO AND DAFK MATTER DETECTOR

Spokesperoph Klaus Prelal |Bern U|

E matd conlact pretzlacentaven teth ch

PSI-Z-91-02 (Conpleted dats-takkng J日92)

MEABUREMENT OF THE NEUTRON-PROTON SPIN CORRELATION PARAMETER AT FORWAFD ANGLES

Spokssperson Benny Zlblmann [Besel U]

Eampit costact ziblmanourz uribas ch

\section{PSI Future Plans}

PSI plans to cantunue its development and operation of large, complex accelerstor Iacilitieg The Spa]letwon Nautron Source

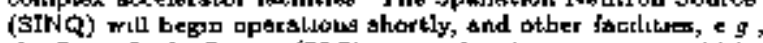
the Swibs Light Source (SLS) are in the planning 6tege Although

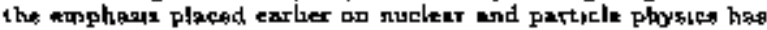
shifted more tawards solud alste phyaica and materials sciences over the last few years, the muctear/psricle physucs programs can heaeth Largely form the diversfication of accelerator ysage Actordugly, new large partucle plysuce experimatate ( $\mathrm{g} g$, the rare decay expertirents guch of $\mu-e y$ ), are envisared in the long torm plang at PSI The near-future plang include scontinujag Etudy of

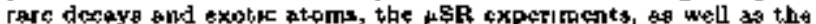
explostescon af very slow muous 


\section{Selected SATURNE Experiments}

\section{SATURNE-1B6}

(Fropowed Oct 1987, Approved Dec 1997, Brgan deta-takung 1988, Cowpjeted datartaking 1993)

STUDY OE HEAVY MESON PRODUCTION IN REAC. TIONS pd $\rightarrow{ }^{3} \mathrm{He} X$ AND dd $\rightarrow{ }^{4} \mathrm{He} X$

STRA5BOURG, CRN - $O$ Bing, F Hibou

SACLAY - I ATvकux, M ENvn, I M Durapd, F Plous

ORSAY, IPN - 1 Bimbet, M P Comet, $P$ Courtat, $Y$ Le Bornec

(Spolkmperop), E Loiralikux, F Resde, B Tatischeff. N Willes

diteleralot SATURNE-II Petretar SPES-IIf

fiencliont

$p$ deut $\rightarrow{ }^{3} \mathrm{He} X$

deut deitt $\rightarrow$ He $\mathrm{X}$

$900-2700 \mathrm{MeV}\left(\mathrm{T}_{\mathrm{tab}}\right)$

$1150-2150 \mathrm{MeV}\left(\mathbf{T}_{\mid \mathbf{x b}}\right)$

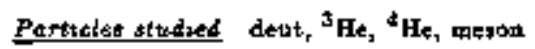

Brte/ description Studier the beary moson production al protan

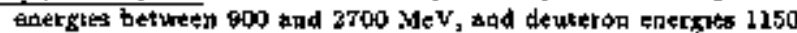
and $2150 \mathrm{MeV}$ Measures the sngular duttribution

Journal paperst Paper to be publisher toon

Related expertments SATURNE.253

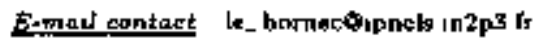

\section{SATURNE-198}

(Propobed Mar 1988, Approved Dec J988, Begap datsotikking Nov 1990, Completed dstarlaking 1991)

\section{MEAGUREMENTS OF SOME RARE DECAY MODED} OF THE +

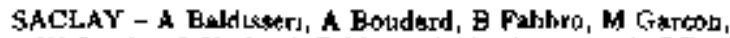

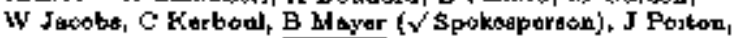
J Saudtuos, E Tomath, S Visdor. F Wellers

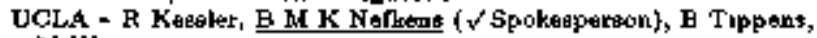
M Wanc

ZUEICH U - E A Hermex, C Niebuhr, A vas der Schasaf

GEORGE WASHINGTON U - W Bracos, A Petrow

TRIUMF R Abegg, W T H vas Oetra

DUBNA - L LTtkin

Aecelerator SATURNE-II Detectar SPQis-t1

Reactions

$$
p \text { dewi } \rightarrow{ }^{3} \mathrm{Ke} \mathrm{n} \quad>\operatorname{meg} \mathrm{MeV}\left(\mathrm{T}_{\mathrm{lab}}\right)
$$

Eartecten choutied o

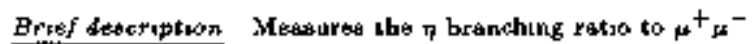
with $12 \%$ securecy The muons are detected by a tro-rogge teleacepe Events are identified by using canptraints like coplenarity, anguise cotrelation. tots anergy conerersation, and the javarisnt mass of the two muons

Jonrsat papers PRL TO (1993) 892, PR D5i) (1994) 82. PR C53 (1995) 2069, and PR D53 (1909) E658

falated experiments SATURNE-25B

E that contact mayeropluxt aecloy ten fI, bnefkenstouclapp physes ucla odu

\section{SATURNE-209}

(Froposed Mar 1989, Appraved 1989, Bogan dats-takung 1960, Complated dstartakng Nor 1961)

CROSS-SECTION AND ASYMMETRIES FOR THE $p p \rightarrow p^{p \pi^{0}}$ REACTION FROM THRESHOLD TO 1 GaV

BONN $\mathbf{U}-G$ Azton, J Arends, M Bzeuer, $K$ Bucbler,

F Hoffmern-Rathe, G Norldiek
SOUTH CAROLINA $U$ - G Blanpled ( $V$ Spokesperson), B Pradom

ORLAY, IPN - G Berrier-konsin, J P Didekz ( $\checkmark$ Spokesperson)

A Eloys, R. Frasciria, E Hongend (V/Spokerperson).

$G$ Rappontecker, M Pugaey, L Rosker

SACLAY - J M Lagel, B Sagha

Accelerator SATUFNE-II Detector SPES-0

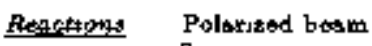

$$
P \text { p } \rightarrow \text { p x } x^{0} \quad 325-1000 \mathrm{MeV}\left(\mathrm{T}_{\mathrm{lab}}\right)
$$

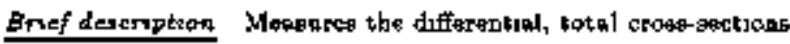
and beam axymmatges for the $\pi^{0}$ emittad in the reaction, from threphold to 1000 MeV Uses the SPEFa $2 \pi$ Nequtral Meson Spectronoter and a linguld $\mathbf{H}_{2}$ lsiges whth polarized proton as propectiles

Journal papert NP A590 (1995) 768

Fefated esperiments SATURNE-129, SATURNE-132, SATUP'WE-134, SATURNE-165

E motl cortact didelorenpocie w2ps ir

SATURNE-213

(Propoud 1989, Approved 1989, Began datalaklag 1904, Lil progress)

MEASTUEMENT OF SPIN DEPENDENT OESERV. AgLES IN THE REACTION $\rightarrow p^{*} \mathrm{Y}^{*}$

DHSTO COLLABORATION

INDIANA U - L C Elend, S Chol, i4 Daemodes, W W Jacobat, $S$ E Vigdor

TURIN U \& INPN, 'TUFIN - F Bsalettra, S Bobsolasco,

$M$ Gasse. L Fava, L Fercero, R Ostragridi, A Grasm,

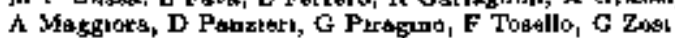

DUBNA - I Falomkin, V Frolow, V Ivanov, G B Pontecorwo,

A Popov, V Tehaboher, B Zalukbanav

SACLAY - $\gamma$ Bedfer, R_Bertind ( $\gamma$ Spokenperton): F Brochard, J C Fsiyre, A Manara

DARMSTADT, GSI - M Debumsu, P genger

CRACOW \& JAGELLONIAN $v$ - I Forytiars, P Salabura

GIËSSEN U - A BTenachede, W Kuhn, H PIaff

FRANKEIJRT U - I Stroth

ROSSENDORF, FORSCHUNGSZENTRUM - E Groge TRIUMF - D G山

Accelemetor \$NTURNE-II Detector DIsTo

Reactione Polsartod bram

$$
\begin{aligned}
& p p \rightarrow p K^{+} A \\
& p p \rightarrow p K^{+} \mathcal{L}^{0} \\
& p p \rightarrow p K^{+} Y^{+}(\text {unepes) } \\
& p p \rightarrow p \mathrm{p} K^{+} K^{-} \\
& p p \rightarrow p \mathrm{p} K^{+} K^{-} \phi
\end{aligned}
$$

20.25.285 GeV \{ $\mathbf{T}_{1 \mathrm{lab}}$ \}

.

$285 \mathrm{GeV}\left(\mathrm{T}_{\mathrm{eb}}\right)$

$\pi$

-
Brief description The experiment mescures differential crowgectiona and spu-dependest observables (apalyang power, polatization and depolarfintion) between the threbhosd and the highest energy asollable at SATURNE.ll Studien a corretation between the measured obserybles and $\mathrm{N}^{*}$ and $\mathrm{Y}^{-}$reacuancet Uges a lequid bydrozen tariget The decector, DISTO, cossista af a magrel, gcintillating fiber detectors, MWPC's, sontiltation bodrascope and $\mathrm{s}$ water Cerenkow hodousope in the next year's run, a replacement of the liqud bydtogen terget by a nuclear targel is enysarged in order co study the interaction of $\gamma$ and $Y^{*}$ with nuslear Juatert Taking deta (July De)

Jowrinat papers NP A386 (1995) $268 \mathrm{c}$

E-mast ansiact bortinfortrepnll in2p3 ir WWW Home-page

ht tp //www to infn it/experment//deto/dueto html 


\section{SUMMARIES OF SACLAY EXPERIMENTS}

\section{SATURNE-2Z0}

(Froposed Jan 1990, Approved Dec 1990, Began datartakung Mar 1891, Comploked dakp-taking 1981)

SEARCH FOR THE EXCITATION OF THE ROPER RESONANGE (1\$ㅇ) BY INELASTIC SCATTEFING OE a. Particles

SACLAY - M Bodvin, H P Morach (Spokespergon), F Plown, B Sogbay, $J$ Yonnet, P Zuprandk

ORSAY, IPN - J F Didelez, R. Fragcatele (Spokesperaon), M Motlet, E Siebert, E Warde

JULECH, FORSCHUNGSZENTRUM \& STOCKKOLM U P E Tegner

Acceterator SATURNE-II Defitctor GPES-JV

Reactrans

$$
{ }^{4} \mathrm{H}_{*} \mathrm{p} \rightarrow{ }^{4} \mathrm{He} \mathrm{X} \quad 42 \mathrm{GeV}\left(\mathrm{T}_{\text {lah }}\right)
$$

Pattecte, studied $N^{*}$ (unfpec), $N\left(1410 P_{11}\right)$

Brtef description Studies the baryon excitation is the $\alpha p$ ayratem, from the parn threshold up to the Roper reswusnce Uaes the a beam up to 7 GeV/c [nelestically scottered alphe particles are detectent by the SPES.[V npectsometer Uwe LHs terrget

Journal paptro FRL 69 (1962) 1338, snd ZPHY Aat8 [1994) t5 Related experiment, \$ATURNE.251

E.mat contact morbchofrepsil in2p3 fr, frascarlaipipncs in2 $\mathrm{p}^{3}$ if

\section{SATURNE-222}

(Proposed Now 1999, Approved 1989, Bersn detsersking 1990, Comploted dsta.taking 1993)

MESON PFODUCTION NEAR THRESHOLD FROM THE $\phi$ TO THE $f_{1}(1285)$

ORSAY, IFN - I P Dhdeles, M A Duval, R Francona, M Marlet, R. Siebert (Spokesperson), E Warde

SACLAY - J Arvieux, F Ploijn

BONN U J Buptinghaf, J Einst, F Hinterberger, R Jabn (Spokeaperson), F. Joosten, U Lebr, C Lippert, A Marx,

R Wussuger

Accelerator SATURNE-1I Detector SPES-IV

fiscacteons

p deut $\rightarrow{ }^{3} \mathbf{H}_{e} \mathrm{X}$

Partecles studiad $\phi_{1} f_{1}(1286)$

Brief description Extends and refines exsting messureserln of the threshot exaitstion curve of meson production An tacreasing degste of exclutirity is achibyed by addog scrntillator srrays to the SPES-IV detector in the serond phass, during $1992 / 93$, the remols arpund the $\kappa^{+} \kappa^{0}$ threhold, and above 19 GeV. are explored in 100-MeV stepts

Jotrofat pepers FR C51 (1995) 443

E-thent contact sebertaipnets in2p3 ft

\section{SATURNE-22:}

(Proposed Dee Ios9, Approved Jun 1991, Bagan data-taking Now r991, Completed datataking Apr 1995)

DETERMINATION OF THE NUCLEON-NUCLEON SCATTEFING AMILITUDES IN THE ENERGY FEGION FKOM 1.1 TO 2.7 G\&V AND A SEARCH FOR A. STRUCTURE ARDUND $T_{\mathrm{k} I 0}=2 \mathrm{f}$ GoV

WLCLEON-NUCLEON COLLABOFATION

SACLAY J Atvitux, J Bast ( $/$ Spotsesparson) J Bystacky, P A Charrouard, M Combet, A de Legquen, M de Mad, J M Fontaune ( $V$ Spokesperson), R. Kunne J M Lsgate] F Lebar J L Lemaltia, J L Sarla
GENEVh U - P Demarre, R Hess, Z F Jamout, Jt , D Rspun (5/Spokeapersoub), A Teglta, B Vuandel

ARGONNE - C Allgover, M Eeddo, D Grosnick, T Kasprzyk, D Loplato, H Sputhe (V'Spokesperson)

DUBNA - L S Bargbatb, V A KNlınıkov, Y K Kezeribav, B A Khachaturov (V Spoketperton), V N Matafotor,

1 L PJgarev, A A Popoy, $Y$ A Usoy

DUBNA \& PRAGUE, TECH U - Z Jamout

GT PETERSEURG, INP - A N Ptokatily, v Vubror, A A Zhdanov

UCLA - A Bouternoucbet, C M Dulya, V Gha्यkhanנar,

$\$$ Trentalange, CA Whatter

MIIT, LNS - E L Lamon

Accctitrator SRTURNE.II Detetor Combunsion

Afertitionz Polatized beam and target

$$
\begin{array}{ll}
p p \rightarrow p p & 11-28 \mathrm{GeV}\left(\mathrm{T}_{\text {lab }}\right) \\
p n \rightarrow p+n & 11-24 \mathrm{GeV}\left(\mathrm{T}_{\text {lsb }}\right)
\end{array}
$$

Particles atodted pin

Brsef descripltots Ues a polarrzed proton beam and polanzad protan and deuterap targota Mesurure the complete tose of apid-dependent obgarveblea in ph quagelagtsc acautering betwren 11 and 24 GeV Derickted po spin-dependant observablex ares mesoured between 18 and $2 \mathrm{~B} \mathrm{GeV}$ w inall stepu of thetsy The messung spparatus consists of a two-bem spectrometer.

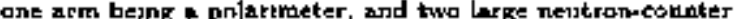

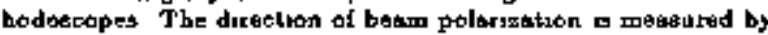
three additjonat beam polorimeter, Dath andysis in proffens (July at)

Jowrial paperr NlM Aiz' (1993) 3Dg, abd PL Bazo (1994) 206 Refated experiments SATURNE-144, SATURNE-216

E-mat' contact ballofrcpn11 unps fr, fulontofrephl1 in2ps if, hrastshep anl gov, khachfturoyemainl jint dubns au

\section{SATURNE-237}

(Propored Now 1980, Appoved Jan 1991, Bigan deta-takjng Jul 1991, Completed date-taking 1995)

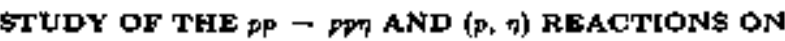
NUCLEI AT $T_{p}>12 B$ GeV

PINOT COLLABORATION

TURIN U \& INFN, TUTREN - E Chiarasea, N DE Marco

\{5pokebperson\}, C De Olyelra Martuna, G Dellatesta, F Ferseto,

M Gelina, P Guelte, A Musso, A Ficcort, E scomparin,

E Vercellin (Spokenpekion)

SACLAY - J M Durand, G MLlieret

Accelerutor SATLRNE-II Defector PINOT

Reactwerg

$$
\begin{aligned}
& \text { จ } \\
& P \text { nucleug } \rightarrow \text { + } X \\
& >126 \mathrm{GgV}\left(\mathrm{T}_{\mathrm{lbb}}\right) \\
& \Rightarrow \text { dent } \rightarrow \text { in } X \\
& \text {. }
\end{aligned}
$$

Parcicles studied in

Erief deecretion The ofm 18 to study the firtt wo reactions nest the thregbold by detecting on nesons with the two-ann neutral menor spectrometer, PINOT For the first reaction the total and doubly differentsal croes gectson $d^{2} v / d i n d T$ IB metasured The $(p, \eta)$ retation op nuclet if st ud

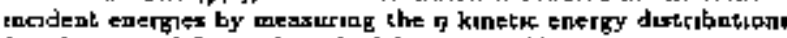
for n's emoltted forward in the laboratory Alao investigateg the pod $\rightarrow \pi X$ reaction IA order to extract Jnformokion an the $p m \rightarrow{ }_{n} X$ ekementary process, by companis gd and pp udduced resctsona U日as the follokjng targets Loqud $\mathbf{H}_{2}$ and $\mathbf{D}_{2}, C_{1}$ Al.

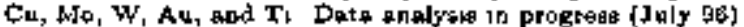

Journat popers NP RS3 (1992) 121c, ZPFY A342 (1902) 107. JP'HY GJQ (1993) L51, NC 106.4 (1993) 861, ZPHY AS44 (J993) 345, NC 107A (1994) 1195, PL B322 (1894) 270, and PL B337 (LOA) 192

Related stperiments SATURNE 125

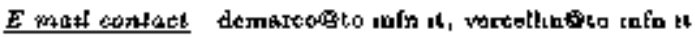




\section{SATURNE-25:}

(Froposed Now 1092, Approved Dec 1992, Besan datsotaking Mar 3993, Completed data-takıng Oct 1903)

SEARCH FOR THE EXCITATION OF THE ROPER RESONANCE (1440) IN NUCLEI

SACLAY - AI Botvo, J L Boyard (Spokeperson), F Fuchs,

R Kunns, H P Morgch (Spokesperson), F Pluuin, P Radvanyl, W 5 prax

ORSAY, IPN - T Heanjno, 1 C Jourdan, B Rametoun, M RayStephan, 5 Frasteau

JULLCH, FORSCHLNGSZENTRUM - V JBOckle

WARSA W t $-P$ Zupransk?

STOCKHOLM U - P E TEXner

RENSSELAER POLY - L Mutphy, P Stokr

Acceitratar SATUFNE-II Delfator SPES-IV

Fitact toms

${ }^{4}$ He dernt $\rightarrow$ Hie $X$

$42 \mathrm{GeV} / \mathrm{c}$

${ }^{4} \mathrm{He}_{\mathrm{C}}{ }^{12}{ }^{4} \mathrm{HeX}$

-

Partacles studued N(1440 Pu)

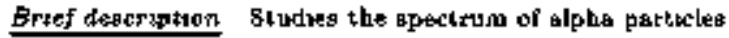

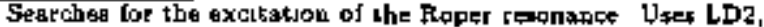

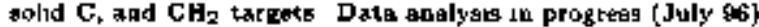

Rictated experments SATURNE.220

E-mazl cortact boyatdofrepn1l in2p3 Ir, morach ircpn 11 is $2 \mathrm{p} 3 \mathrm{Ir}$

\section{SATURNE-258}

Proposed Nov 1982: Appraved Dec 1992, Began data-tekjng 1993, Completed dsta-taking 1993)

\section{DIRECT MEASUREMENT OF THE BRANCHING} RATIO IN THE $\eta \rightarrow \neg \gamma$ DISINTEGRATION

\section{ETA COLLABORATION}

SACLAY - A Doudard, I M Dursad, B Fabbro, M Gencan

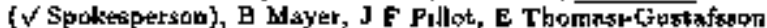
DUBNA - A Elendiev, L Lytiun ( $\checkmark$ Spolituperion)

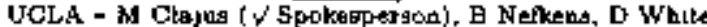
P'Sl, VILLTEN - R Abelo

TRIUltiff - R Abegg, P Fuchs, W T H var Oers

GEORGE WASHINGTON U - W EtLstae, T Motretan

ST PETERSEURG, INP - V Nikul

Aocelerator SATURNE.II Detector Gelonmeter, SPES-IJ

Reactions

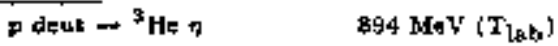

Particles ifudted ti, $r$

Braf' descroption The firsi dereft messurement of the brapching

Fatso $\Gamma(\gamma-\gamma \gamma) / \Gamma_{f o k}$ Expected to schiteve an tecturaty or 1

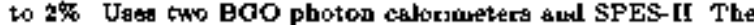

torzet if LD2 Data anslysts complested

Journat paper\$ PF D53 (1996) II No other papers expected

Reiated axperiments 5ATUkNE-196, SATURNE-284

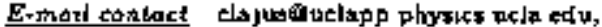
garcontophox 7 usclay ces fr, lythenematel junr dubnt su

\section{SATURNE-2T:}

(Approved Jun 1993, Begen dats-takeng Dec 1995, In progreay) STUDY OF BARYONIC RHSONANCES WITH THE NEW DETECTOF AROUND THE SPES-IV TARGET POINT

SPESA-PI COLLABORATION

COPENHAGEN y R Dahi, C Eltegard, C Gaarde, J Jensen. IS Larsen, M Skausen
DUENA - Y T Bersunov, L P Golovanov, V P Ladygn, L V Málimins, N M Puskunov, I M Situik, E A Strokesvsky ( $\checkmark$ Spokesperson), A P T Briney

ST PETERSBURG, INP - G D Alkhazov, A V Khanzadeyev, V A Mylmbov, A N Prokoter, V M Sambonor, I V Tkach,

V N Vikhrow, A A Vorobyov, A A thdenov

JULJCH, FORSCHUNGSZENTRWM - H P MOISA

NOFPOLK STATE U - Y Punjab

ORSAY - L Bımbot, J L Boyacd ( $/$ Spokeaperson), L Furbl

J C Jourdaw, B Ramitein, M Roy.Stephan

GACLAY - M Borvin, T Hennuno, M Kagatla, A.A Kunne

( $\checkmark$ Spokesperson), P Radvanyy, E Tomatg-Gutiafison

WATSAW, INST NOCL STUDIEg - W AUgutyoubh, P Zupransks WLLIAM AND MARY COLL - M Jones, C F Perdrat

Acceterator SATURNE-II Detertor SPBSA-PI

\section{Renctions}

$$
\begin{aligned}
& \text { dout } p-\text { dent } \boldsymbol{x} \\
& { }^{3} \mathrm{He} \text { nocleur } \rightarrow \operatorname{trit} \pi^{+} X \\
& { }^{4} \mathrm{HI} p-{ }^{4} \mathrm{He} p X \\
& <38 \mathrm{GeV} / \mathrm{c} \\
& 11 \\
& { }^{12} \mathrm{C} \text { nacians }-{ }^{12} \mathrm{~N}-\mathrm{X} \\
& <70 \mathrm{GeV} / \mathrm{c} \\
& \text { c } 3 \text { 日 G } \mathrm{G} V / \mathrm{c}
\end{aligned}
$$

Eartacles ntwdted $N^{*}\left(\right.$ urspoc), $\left.N(1440) P_{11}\right)$

Brief descrtptanis Thu ta a threo-port experiment (278, $278 \mathrm{~b}, 278 \mathrm{c}$, carried out with $\mathrm{a} \mathrm{n}$ w detector at the target point of SPES-IV The delector consisty of the SPES- IV high renolution spoctrompter to datect the scattered basm particte日 in coincidence with the aecondary pratons and pions wheh

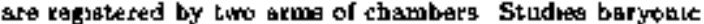
resonances Useg polarized and unpolstreed borams, and C. Ca and Pb, targets The tapics covered by the three parts kre (1) coherent pion production from charge excbange reaction6, (2) prodnction of the $N^{-1}$ (1440) resonance with alpha particlex, and (3) study of apeis obraervablea in the production of the $N^{*}(1440)$ resonance with deateron. lo progreas (July B6)

Retated experments SATURNE-250, SATURWE-291, SATURNE-305, JINR.LHE-4941-1B, JINR-LBE-0941-10

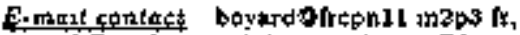

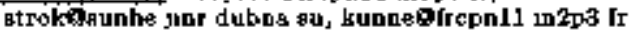

\section{Other SATURNE Experiments}

Lelwed hore are nome other Saclay experimenth of untorest to the particle phyasca commusity Find more detals about these projects onlens, in the SLAC's EXPERIMENTS detabase (aed p 3), or contact the tpotresparsotit

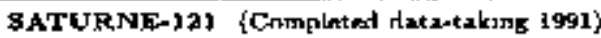

SEARCH FOR DIAARYONE OF STRANGENESS \$ = BETWEEN THE AN AND $2 N$ THFESHOLDS

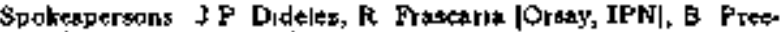
dont [South Caroluas U]

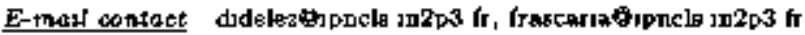

\$ATU RNE-144 (Copripleted dararalung Dec 1960) NUCLEON-NUCLEON PROGRAM (PART ET): np SCATTERING UP TO $1.2 \mathrm{GOV}$

Spokemersons F Lehar, F Perrol [Sac|ayl, et Hess [Geneva $\Psi$ ]

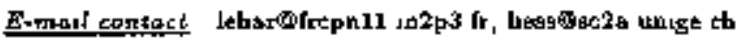

gATURNE-105 \{Completed dals-laking 1990)

MEASUREMENTS OF $A_{M X}$ AND $P_{2}$ BOR THE REAC TtON $\vec{d}_{p}-\overrightarrow{p p m}$ IN COMPLETE KINEMATICS

Spokesperzong S L Beloatotgky (St Patersburg, INT), A Boudard [Sheleyl]

Emad conlarit hrodardiaphnxt naclay ceth fr 
SATURNE-18B (Completed dsta-t8kung J991)

REACTION H(d, 2p)n WITH POLARIZTO DEUTERONS AT $300 \mathrm{MeV}$

Spoketperson $\mathbf{S}$ Kox [Grenable U |

E-mintl cantect koxolrepa11 in $2 \mathrm{p} 3 \mathrm{ft}$

SATURNE-174 (Completed dela-taking)

PRODUCTION OF LIGHT MESONS $X$ IN pp $\rightarrow$ pp $X$ AT THRESHOLD AND IN NUCLEAR MATTER

Spokeqperson O Buge (Strosbourg, CRN]

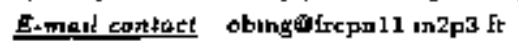

SATERNE-190 (Compkted data-taking Misy 1891)

SPIN STRUCTURE OF THF $\triangle$ EXCITATION

Spokespergone C Caarde [Coponhagen U I, J L Boysard |Orgay], P Zupransk] [ [Soltan Inst, Swerk]

smart cantací gasdegabuax nb, dk, zupradoluw edu pl

SATURNE-202 (Completed data-takking Jp92)

STUDY OF THE PROTON POLARIZATION IN $\overrightarrow{d A} \rightarrow \vec{p} X$ REACTION AT $0^{\circ}$ AND $2.1 \mathrm{GEV}$

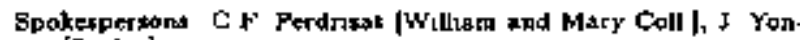
net [Spelay]

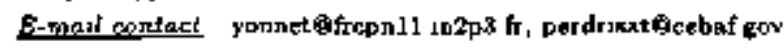

SATURNE-29S (Corapleted detarakugg 1998)

CALIBRATION OF POLDER, A NEW DEUTERON TENSOR POLARIMETER AT INTERMEDIATE ENERGlEs

Spukeaperton Alerge Kox [Srenoble Џ।

E-meit confagt koxpefepall inzps fr

SATURNE-244 (Completed datotakidx Sep 1991)

STUDY OF THE REACTION $\overline{p D} \rightarrow \pi^{-} \pi^{-} X$

Spokesperson D Tatischert [Orasy, JPN]

E-mani contect tatıofrepsil in2po fo

SATURNE-246 (Completed data-taktng 1992)

${ }^{0}$ PRODUCTION IN THE FEACTION $\mathrm{dp} \rightarrow{ }^{2} H_{4} \pi^{0}$ NEAR THEESHOLD

Spokespertons B Mryer [Seclay], \& M K Nefkens [UCLA]

E-mattepntact mayertephox 7 saclsy cea Ir,

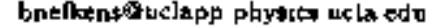

SATURNE-249 (Completed dato-taland 1993)

POlarization taANSFER IN ELAstic BACK. WAFD DEUTERON PROTON SCATTERING

Spokesperdons C F Pardrisat [Willegm apd Mary Coll), V Pudjebl [Norfolk State U], I Sttmik [Dubnb, JINR]

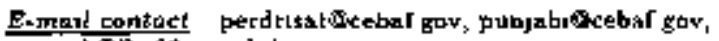

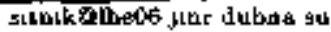

SATURNE-353 (Craptalend dake-knkigg 1992)

MEASUREMENTS OF THE POLARIZATION TENSOR AND THE PROBAEILITY OF THE SPIN-FLE IN THE REACTION ${ }^{12} \mathrm{C}\left(d, d^{\prime}\right)^{12} \mathrm{C}$ AT $400 \mathrm{MOV}$

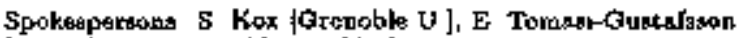
[Saclayl, M Morlst |Otsay, IPN]

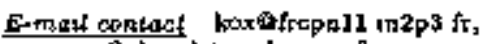

tomasi wishatelet stalay tes fr

SATURNE-280 (Tabong data)

\$TVDY OF THE FEACTION $d d \rightarrow$ ON CLOSE TO THE THRESHOLD OF $\uparrow$ PRODUCTLON

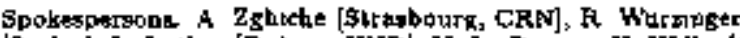
|Saclay], L Lytkin [Dubna, JINR , Y Lo Botnec, N Wills |Ortay, IPN|

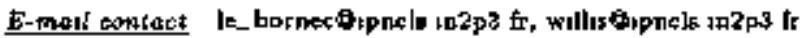

\$ATURNE-290 (Completod data-taking L98d)

MEASUREMENT OF TENGOR OBSEAVABLES

RELATED TO THE POLARIZATION OF RECOLL

DEUTERON IN THE REACTION $p p \rightarrow d \pi^{+}$

Spakesperronn C Furget, 3 Kux [Grenoble U]

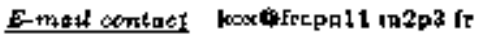

\section{SATURNE Future Plans}

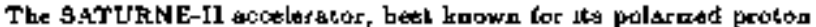
and deuteron besms, will be clored by the end of 1997 The strong

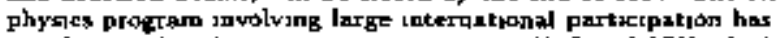
bow beell raduchd to just two oxperiments (213 and 270) whok sre studying the baryoule rotopances and a productpon of coberent prons in the charge exchange reactions othe axperiments and important R\&D projecta have ether been completed of capcelled Thete tre $\mathrm{TQ}$ plans for direct experimental Tefearch at the alte beyond 19a7, but thr Lab will be involved y many intermediateand bugh-energy phyeses projects at other facilties 


\section{SUMMARIES OF SERPUKHOV EXPERIMENTS}

\section{Selected Serpukhov Experiments}

\section{SERPUKHOV.128}

(Proposed 1977, Approvad 1984, Began dalartakkpg 1987)

\section{SEARCH FOR NEW \$HORT-LIVED PARTICLES IN} NEUTRINO INTERACTIONS

SERPUKHOV - V V Ammogov, V I Baramov, A A Jvadlov,

P V Irenoy, V M Korsblev, V A Korotkov, V V Mateev,

A G Myskoy, P V Pitukhin, A Y Polyarush, A A sokoloy

MOSCOW PHYS ENG INST - E Goshehin, A I Lebedev,

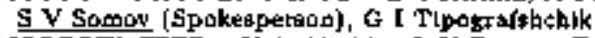

MOSCOW, ITEP - Y A Ateshin, OK Egorov, E D Knlganova

A N Makeimor, I A Melsicbanko, E A Pozharove, V I SHlacy,

$V$ A smirnitsky, V A \$motryatr, I S Trostin

LEBEDEV INST - S I Kateloikry, E P Kuxnetan,

B I Lomonasow, I I Pervow, V A Tyeknov, P S Vasilley

MOSCOW STATE U - P F ETmokr, V S Mirzin, \& I Sivotclakov

DUBNA - Y A BatuBor: S A Eunyator, O M Kunnetsov,

Y V Lyukou, V I Tretyak

Accelerator SERPUKHOV Detertor Combination

\section{Renctions}

$\nu_{\mu}$ nucloon $\rightarrow \mu^{-}$charm $\mathrm{X}$

$\nu_{\mu}$ vucteon $\rightarrow \Lambda_{c}^{+} p^{-} X$

$v_{\mu}$ nuclear $\rightarrow \Sigma_{c}(2455)^{+} \mu^{-} X$

$\Sigma_{\mu}$ nndeon $\rightarrow \Sigma_{\mathrm{c}}(2455)^{++}{ }_{\mu}-\mathrm{X}$

$\nu_{\mu}$ nucleon $\rightarrow \mathrm{H}^{-}$cherwed-moson $\mathrm{X}$

$\forall_{\mu}$ uucteon $\rightarrow D_{*}^{ \pm} \mathrm{H}^{-} \mathrm{X}$

$3-30 \mathrm{GgV} / \mathrm{c}$
$=$
$=$
$=$

Particicf skpdief tharm

Brief degcriptipn The detector is a wide angle spectromater with a streamer chamber apd क्याओu|sion. $2 \times 10^{1 E}$ protons on target Ware taked.

\section{SERPUKHOV-136}

(Propogted 1878, Approved Apr 1878, Began dotantakink 1989, It progrees)

\section{NEUTFINO DETECTOR}

SEEPUKHOV - A A Borisov, N I Bathte, S K Cherbichenko, G L ChukLn, V N Goryachev, M M Kituabov. A I Kononoy. A s Korhin, V I Kravtsov, A V Ktalikos, A I Mukhin,

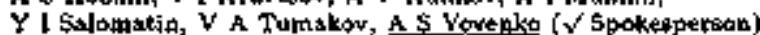
DUBNA - L S Barabash, Y A Batugov, 9 A Bunjats

(V Spokesperson), O L Klimov. V V L Yukov, Y A Nefedov,

B A Popov, V J Snyetkov, V Y Veluev

Aeceterator SERPUKHOV Detector Celorimeter

firactions

\begin{tabular}{|c|c|}
\hline puclean $\rightarrow$ charm $X$ & 70 Qev/o \\
\hline$p$ nucleon $\rightarrow e^{ \pm} \mathrm{X}$ & $n$ \\
\hline$\nu_{\mu}$ nucleon $\rightarrow \mu^{-} \mathrm{X}$ & $5-30 \mathrm{GcV} / \mathrm{c}$ \\
\hline$w_{\mu}$ nIteloms $\rightarrow \mu^{+}{ }_{S^{-}}-\mathrm{X}$ & * \\
\hline $\begin{array}{l}\nu_{\mu} \text { nucleon } \rightarrow \text { charrt } X \\
\bar{\nu}_{\mu} \text { nudeos }-\mu^{+} \mathrm{X}\end{array}$ & $\ddot{n}$ \\
\hline $\bar{\nu}_{\mu}$ sucleon $\rightarrow \mu^{+} \mu^{-} \mathrm{x}$ & $"$ \\
\hline$\overline{\boldsymbol{\nu}}_{\mu}$ gurleon $\rightarrow$ tharn $\mathrm{X}$ & " \\
\hline$\nu_{e}$ Duclen $\rightarrow e^{ \pm} \mathrm{X}$ & $n$ \\
\hline $\begin{array}{l}\bar{\nu}_{\text {e nucleod }} \rightarrow e^{ \pm} X \\
\text { charm } \rightarrow \mu+\mathrm{X}\end{array}$ & ". \\
\hline charm $-e^{ \pm} \mathrm{X}$ & - \\
\hline
\end{tabular}

Particlea situdied charm

Erief description \$exrches for $v_{a} \rightarrow v_{x}$ arcillation. Ruaning (April 9 B)

Jotarnal popers YF 30 (1979) T02 = 8JNP 30 (1979) 362, YF 33

(1981) $715=\$$ JNP $\$ 3$ (1981) 371 , YF $\$ 0$ (1984) $739=\$$ \$NP 49

(198d) 475. YF 49 (1989) 172, ZPUY C51 (1991) 341, JJAP A.7

(1992) 3935, YF 55 (1992) 2092, PL B279 (1992) 405, PL B295
(1992) 154, FL Ba02 (1993) 336, YF 57 (1994) $2050=$ PAN 57 (1904) 1974, PL 9369 (1906) 39, and ZPHY C70 (1906) 39.

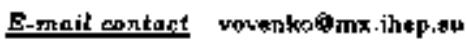

\section{SERPUKHOV-145}

(Propased 1981, Approved 1984, Eegen data-taking 1987, Campleted dats-tosking 19日2)

STUDY OF THE PAORUCTION AND DECAY PEOP. ERTIES OF THE CHARMED BARYONS IN NEUTRINO INTERACTJONS WITH THE BUBBTE CHAMBER SKAT

SERPUKHOV - V V Ammoson ( $V$ Spokegperboo), E N Andaabev,

$Y \vee$ Bardin, A P Burornky, N A Chabrow, V I Erraolapv,

V S FHelpor, A A Irańtlor, V I Khleborad, V ] Kunyusiltso,

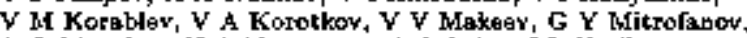

A G Mysatov, N A Netyaga, A A Sakplav, I I Variliev

Actelerator SERPUKHOV Detector FLBC-SKAT

Reastions

$$
\begin{array}{lc}
v_{\mu} p \rightarrow \Sigma_{c}(2455)^{++} \mu^{-} & 5-20 \mathrm{GeV} / \mathrm{c} \\
v_{\mu} p \rightarrow \Sigma_{4}(2530)^{++} \mu^{-} & 11 \\
\nu_{\mu} n \rightarrow \Lambda_{c}^{+} \mu^{-} & 11
\end{array}
$$

Perticits \$tudied $\Sigma_{c}(2455)^{++}, \Sigma_{c}(2530)^{++}, \Lambda_{c}^{+}$

Brief description The chamber fill is a light freon-propane mox. $4 \times 10^{18}$ protons on tarket were taken.

Journal papers ZETFF 58 (19日3) 24ג.

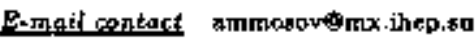

\section{SERPUKHOV 147}

(Fropored 1942, Approved Mar 1982, Bagad data-taking 1964. Completed dakta-taking 1991)

STUDY OF REACTIONS WTTH STRANGE FARTICLE PRODUCTION IN THE $\pi^{-}$AND $K^{-}$MESON BEAM OF THE IHEP ACCELERATOF

MOSCOTi, ITEP - B P Barkov, B V Bolonkin, 1 A Erofeev,

ON Erafeevs, V K Grigoriev, A F Grishin, Y V Kotinov,

1 Y Korolhov, V N Luath, V V Mttler, V N Nozdractrev,

Y P shkurenko, V V Sokalovsky (V Spokesperkon), A I Su-

cormln, G D TLkiromirow, V V Vladimiraky

Accelemeter SERPUKHOV Detector MIS

Reactions

$$
\begin{aligned}
& \text { म }^{-}-n \Delta \bar{\Lambda} \\
& \bar{A}^{-} p \rightarrow n \Lambda \bar{\Lambda} \pi^{D} \\
& \pi^{-} p \rightarrow p \Lambda \bar{n} \pi^{-} \\
& { }^{\prime \prime} p \rightarrow n 2 K_{S} \\
& g^{-} p-n K_{S} K_{L} \\
& \pi^{-} p-n 2 K_{S} x^{0} \\
& \pi^{-} p-n K_{S} K_{L} \pi^{0} \\
& x^{-} p \rightarrow p 2 K_{S} *^{-} \\
& { }^{-} p \rightarrow K_{S} K_{L} \pi^{-} \\
& { }^{-} p \rightarrow n \Sigma^{d} \vec{E}^{\prime} \\
& r^{-}, \text {Flueball } X \\
& \pi^{-} p \rightarrow f_{2}(1720) X \\
& \square^{-} \mathrm{p} \rightarrow \mathrm{X} C(1480) \\
& K^{-} \nu-\Lambda \bar{\Lambda} Y^{*} \text { (unepec) } \\
& K^{-} p-K_{S} K_{L} Y^{*} \text { (unepec) } \\
& \text { पlitectanl -- } 2 K_{S} \\
& J(1720) \rightarrow 2 K_{S} \\
& C(1480)^{-} \rightarrow K_{S} K_{L} \pi^{-}
\end{aligned}
$$
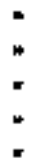

Particles atudied $f_{0}(97 b), a_{0}(980)^{0}, f_{2}(1720)$, glueball: $C(1480)^{-}, Y^{2}$ (unspec),$S_{2}^{\prime}(1526)$

Brief destription Requeated 2400-3000 hous 


\section{SUMMARTES OF SERPUKHOV EXPERIMENTS}

Jourtad popers YF 43 (1986) 1211, YF 43 (1986) 1487 - \$JNP 43 (1986) 956, YF 46 (1987) 799, NF B309 (198B) 426, and YF 49 (1998) $1213=$ \$1NP 48 (1989) 770

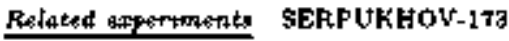

E-mal contect soleolovakyônvitep ttep ru

\section{SERPUKHOV-152}

(Proposed 1983, Approved Aug 1964 In preparation)

\section{NEUTRINO EXPERIMENT USING A TAGGED NEU. TFUNO BFAM}

\$ERPUKHOV - V V Ammasev, V B Anylayev, A A Bel'hov,

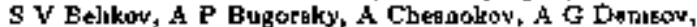
S P Deplsov ( $\checkmark$ Spokesperson), A $Y$ Dushkj, N N Fedyakn,

A N Galyarer, N A Galyatv, S S Gershtein, Y V Giltsly.

$5 \mathrm{~N}$ Gurzhev, V I Kochetson, V I Kotow, A V Koralev,

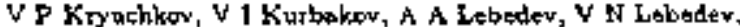

V V Lepsjew, A Y Malow, S A Medved, V N Mikbsilin,

$Y$ V Mikhorlow, V A Onuchin, Y M Pighchalmikov, A V' Schukin,

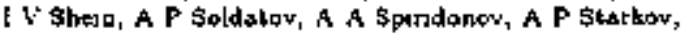

D A Stoyanova, A V Uzubyan

INFN, PISA - C Cerr, G Genrar, F Sergiampietri, G Spendre

INFT, FLOEENCE - G Conforto, A Machionnı

BEFLIN-ZEU'THEN ADW - J Bacht, G Bohm, R. Nahnhauer,

5 Nowak, A Sebwind

DUINA - J Cvech, V K Dodokhow, N G Fades, V Gencher,

I A Golutwn, J Hladky, V G Kadykoy, V Y Karzhavin

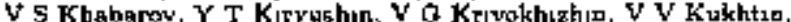

V N Lyyyatery, P K Markoy, 5 Namoctk, A A Popoy, D Pooe,

A Proker, P Bemor, S Rıman, I A Bswı, G I Smarmow,

D A Smolın: J Strachorn, G Sulsmov, 1 V Svetow, V A Sviridov,

P Taulorov, d Vinde, J Znoek, N 1 Zamystu

Accelerator sERPUKHOV Detector Combinstion

\section{Rouctiotss}

$$
\begin{aligned}
& \nu_{e} e^{-} \rightarrow e^{n} v_{s} \\
& \nu_{\mu} \mathrm{e}^{-} \rightarrow \epsilon^{-} \boldsymbol{\nu}_{\mu} \\
& \nu_{*} \text { uucleon } \rightarrow e^{-x} \\
& v_{n} \text { nucleon } \rightarrow v_{z} \mathrm{X} \\
& \nu_{e} \text { pucleon } \rightarrow r^{-} X \\
& \nu_{e} \text { oucleon } \rightarrow e^{-} \mathrm{Ns}^{+} \mathrm{X} \\
& \omega_{\mu} \text { tucleon } \rightarrow \mu-\mathrm{X} \\
& v_{\mu} \text { nucleon } \rightarrow v_{\mu} X \\
& v_{\mu} \text { nucleon } \rightarrow \mu^{+} \mu^{-} X \\
& \text { charmed-meson }-\mathrm{A}^{+} \mathrm{X} \\
& \begin{array}{c}
<\pi 0 \text { GeV }\left(E_{1 \times b}\right) \\
= \\
= \\
= \\
= \\
= \\
=
\end{array} \\
& \text { - }
\end{aligned}
$$

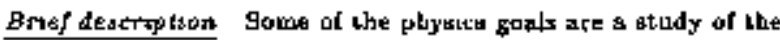

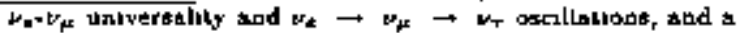

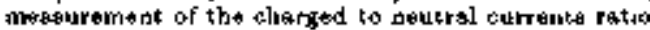

lowrtat papers YF 52 (1990) 10d0

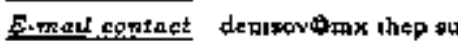

\section{SERPUKHOV-157}

(Froposted 1983, Approved Mar 1983, Beran data-lakint 1986, In progrees]

NEW RADIAL-EXITED RESONANCES SEARCH IN DIPFFACTIVE PROCEŞฺฺS ON NUCLEI WITH DETECTOR MIS-3

DUBNA M A Ananjeva, Y V Aatipoy, Y I lvanshin,

I G Kogarey, V A Moneenko, V A Petrov, Y P Petulkboy,

\$ Y Sychloov, A A Tyepkin ( $V /$ Spokeepergon), I M Vesulevsky,

V V Vishnyakov, OA ZBymudotoga

MILAN US - P L Frabetti, F F Bfanfed, F Palonbo

Acceterator SERPUKHOV Defector MIS-2

Rescittons

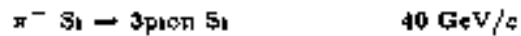

\author{
प्र $\mathrm{Be} \rightarrow$ अpक्ष $\mathrm{Be}$ \\ $40 \mathrm{GeV} / \mathrm{s}$ \\ Partaten abudien meson \\ Er7ef descrtp5toTe Useg the modified spectroneter MIS whth

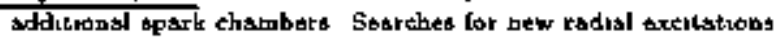 \\ of $\pi_{1} a_{3}(1260), a_{2}(1320)$, snd $\pi_{2}(1670)$ mesons Requested \\ runaing time ix 720 hours \\ Joturnat paperg YF 43 (1986) $917=5 J N P 43(1986)$ 585, and \\ NC A.107 (1994) 2856 \\ Retated expermetres FNAL.706, SERPUKHOV-164

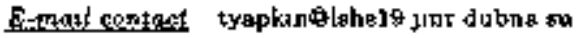

\section{SERPUKHOV-159}

(Proposed 1983, Approved May 1986, Began deta-taktng 1992, In progress)

INVESTIGATION OF CHARMED PARTICLES AND SEARCH FOR MULTIQUARK STATES USIMG RXCHAFM SPECTROMETER AT THE SEFP UKHOV AC. CELERATOR.

DUBNA - A N Aleev, V P Batandin, I I Evoskoy, P Z Hrigtos,

I M lvanchanko, $\mathbf{z}$ id Ivancherko, M N Kapishun, N N Karpenko,

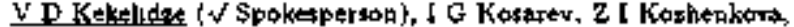

Y A Kozhernikov, Y A Kretov, N A Kuz'mın, A L Lyubinow,

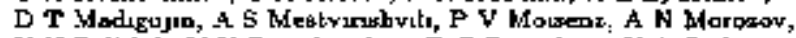

Y V Pol'chik, Y K Potrebenikoy, T G Progulovs, V A Sasber,

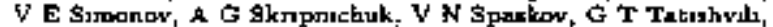

A I Thachev, I P Yudin, O I Yuldarlesv, MI B Yuldachoba,

A I Zigchenko

ALMA ATA, PHYS INST - A A Loktionov

KAZAKH STATE U - G K Potrabentkery

LEBEDEV INST - S P Baradow, of V Below, $V$ A Kotolov,

S $Y$ Fotsahev

UTNSK, INST NUCL PROELEMS - A. S Knrلم

BUCHAREST, INST PHYS - A Eragadiranu, L Grozs,

M Ilexku, T Ponta

SERPUKHOV - A P Bugorak

SOFIYA, INST CHEM TECH - V Zayakt:

SOFIYA, INST NUCL RES - 1 M Gebhkoy, 5 Pıpero4

TEILISI \$TATE U - 1 N A beap|ahvili, N S A maglobeb,

B G Chitadze, M V Kopadze, A. A Kuatsine. N L lomides,

$G 1$ Nikobadze, T o Pitokhelaur

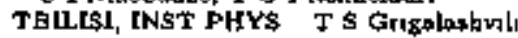

Ackederator GERPUKHOV Detector EXCHARM

Rencteriss

sopclew $\rightarrow X\{3100\} X$

7 nocleus $-X(3260) X$

$\rightarrow$ aucleus $\rightarrow$ No(1950) $X$

it nuclaus $\rightarrow D^{0} \mathrm{X}$

s. naclew $\rightarrow \mathbf{E}_{t}(2 \mathrm{d55})^{++} \mathrm{x}$

ת naclew $\rightarrow \mathbf{E}_{t}(2455)^{0} \mathrm{X}$

$\pi$ neclaun $\rightarrow \Lambda_{c}^{+} \mathrm{X}$

7 nacleus $\rightarrow \Xi^{+} \mathbf{X}$

t) nuclaus $\rightarrow \underline{-}_{\mathrm{t}}^{0} \mathrm{X}$

tr nucleus $\rightarrow \phi x$

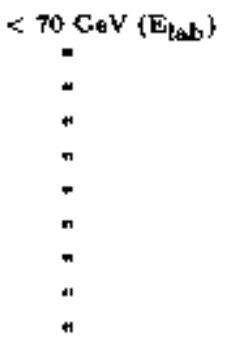

Partacied atudzed $X(3100), X(3250), N \phi(1950), D^{0}, A^{+}$, $\Sigma_{x}(2455)^{++}, \Sigma_{c}(2455)^{0}, \Xi_{c}^{+}, \Xi_{c, \phi}^{D}$

Btef descrition Uta the EXCHARM detector

Jaurral ynpers FTE \& (1995) 6, JINR PAPID COM 3(7T) (1996)

E-mint cortact kekelorgunge jonr dubne st

\section{SERPUKHOV-164}

(Propoed 1980, Approved Msy I986, Began datamakun 1988, Completed datsoteliag Mar 1996)

INVESTIGATIONS OF THE $x^{-} p \rightarrow n \pi^{+} \pi^{-} \pi^{+} \pi^{-}\left(\gamma^{\prime} \mathrm{a}\right)$ RE: ACTION AT AO C-V/s. USING THE VRRTEX SPECTROMETER 
SERPUKHOV - D V Amelia, E B Berdajkon, \$ I Bityukrov, G V Bortsov, V A Dorofeev, R I Debelyad, L, Y P Gom, Y M Ivasyoshenkow, I A Kathace, A N Karyulshen, $Y$ \& Khokhlov, I A Klyuchnzkrv, Y F Konetentimov, S V Kaplkav, M E Kmegtnkow, V V Koetyuthin, A A Kruubin, M A Kulagin, S A Iukhoded, V D hatveev, A P Ostankow, D I Rysbehikow, O V Solovisuov, E A Starchenko,

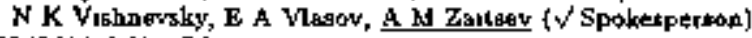

TBtLISI, INST FHYS - T A Lombadse, G G Sekhnuad2e,

B G Trkhad adzo

\section{Accetitrator SERPUKHOV Detertar VES}

\section{Reactions}

$$
\star^{-} p \rightarrow n 2 z^{+} 2 \pi^{-}\left(\gamma^{\prime} s\right)
$$

$x^{-} p \rightarrow \pi 2 x^{+} 2 \pi^{-}$

$x \rightarrow n \rightarrow p^{0}$

$x^{-} p \rightarrow \pi 2 \eta^{\prime}$

$\pi^{-} p \rightarrow+f_{1}(\mathbf{1} 286)$

* $p \rightarrow n+n^{\prime}+$

$x^{-} p \rightarrow n$ w

$\pi^{-} p \rightarrow$ it moson (stagons)

$x^{-} P \rightarrow P$ metos (meroms)

$x^{-} p \rightarrow \pi \pi^{+} x^{-} n$

$\pi^{-} \not \rightarrow \pi^{-} \eta \pi$

$\mathbb{X}^{-} \mathbf{p} \rightarrow \mathrm{n} \omega$

$x^{-} \rightarrow \rightarrow K^{+} K^{-}{ }^{-}$

$K^{-} p \rightarrow p K^{-} \pi^{+} \pi^{-}$

$K^{-} p \rightarrow K^{-} \pi^{+} \pi^{-} \pi^{0}$

$37 \mathrm{GeV} / \mathrm{C}$

$n$

*

ต

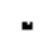

"

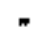

$+$

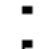

.



ต

$\omega$

-

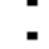

Partiales studied $\theta^{D}:$ si, $\eta^{\prime}, w_{1} f_{1}(1286), \times(1770)$, oxotic, gluebull, moson

Briff descreption Vos VES (VErtex Spectrometer) - a inde

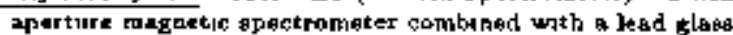
ganimas detector and Oerenkw ideatifiers In addition to the reactions listed aboye, also otudies decays of the means produced in the rexctions

Journal paport FL B268 (1991) 197. ZPHY C54 (1992) 235. ZPHY C54 (1992) 367, YF 6S (1992) 2460, ZPFY C5T (1992) 13, PL B313 (1993) 276, PL P337 (1994) 219, ZPEY C66 (1995) 71, PL B960 (1995) 595, ZPBY Cot (1920) 71, and YF 59 (1996) 1021

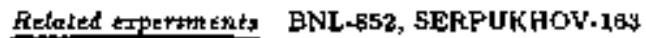

E.mul cortact satsev'ors shep so

WWW Home-gage bitp $/ /$ dxbect shep su;

\section{SERPUKHOV-168}

(Proposed 1967, Approved 1987, Began dets-taking 1987) STUDY OF RARE DECAYS WTTH THE ISTRA-M DETECTOR

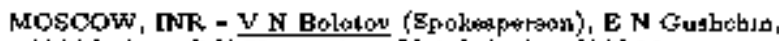
$\checkmark V$ lakkov, ov Kararkhey, $V$ A Lebeder, $V$ N Mard, Y V Mudenko, A A. Poblaguev, V E Poetosv, G N Semenuk, SA Valkov

SERPUKHOV - Y F Konetentınov

DIIBNA - C Kolmar, A Z Kitıkyan, E V Kombnarm, V S KurbsLov, V Z Sordyuk, V V Sudoro, A D Volkov, B Z Zadjkhamav Accelerator SERPUKHOV Defigtor ISTRA-M

Eeactions

$$
\begin{aligned}
& \boldsymbol{K}^{-}-\pi^{-} \nu_{e} \bar{\nu}_{*} \\
& K^{-} \rightarrow \pi^{-} \nu_{\mu} \bar{\nu}_{\alpha} \\
& K^{-}-e^{-V_{0}} \bar{v}^{-T} \\
& K^{-}-\tau^{-} e^{-}+ \\
& K^{-} \rightarrow \pi^{-} \mu^{-} \mu^{+}
\end{aligned}
$$$$
25 \mathrm{GeV}_{\mathrm{O}} \mathrm{C}
$$$$
\text { - }
$$

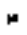$$
\text { - }
$$

Paritciesatydted $\pi^{-}, \mathrm{K}^{-}$

Emanl sontact bolotoptoint mak a

\section{SERPUKHOV.167}

(Propoeed J日55, Approved 1987, Began hata-takung 1987, In progreas]

\section{STUDY OF RARE KAON DECAYS}

SERPUKHOV - A M Blick, V N Kologor, V M Knt]19, V N Shelikhow

DtfeNA - Y Y Batusay, Y A Budagow, I E Churikoy-Zorn, Y I Davydow, Y B Fly'ton (Y' 8pokssperson). V V Glagolev,

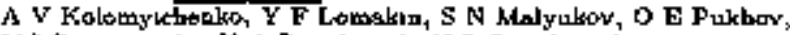
vi Romenovgky, is A Rusekowkch, N L Rueskoyıch, A A Serrenov, A N Shaljugia, A S Soloviev, V o Virogradov, A $G$ Volodko

TBILISI STATE $U$ - G A Dblachndze, I A MLbaghnt SOFIYA $U$ - A B Jordxnoy, $L$ Litov, o V Velev MINSK, INST PHYS - Y A Kakchtsky, A \$ Kwrilta Accelerator SERPUKHOV Detector HYPERON-II Rexcinons

$$
\begin{aligned}
& K^{+} \rightarrow \pi^{+} 2 \pi^{0} \\
& K^{+} \rightarrow \pi^{+} \pi^{0}+ \\
& K^{+} \rightarrow \pi^{+}{ }^{2 \gamma} \\
& K^{+} \rightarrow \pi^{0} e^{+} \nu_{e} \\
& K^{+} \rightarrow 2 \pi^{0} e^{+} v_{e} \\
& K^{+} \rightarrow \pi^{0} e^{+}{ }^{+}+ \\
& K_{S} \rightarrow e^{-} e^{+} \\
& K_{S} \rightarrow 3 \gamma \\
& K_{S}-e^{-} e^{+}+ \\
& K_{S}-\pi^{0} e^{+} e^{+}
\end{aligned}
$$

Particles studief $K^{+}, K_{5}$

Brief descripton The setup conbuts of a past of anearm spectrounelety on in messure the bess momentum, and the socond, a large aperture Apectromater, to regstar the becondory charged particles moments all coordjuster in the epectromerere are messured by proportitanal chambers (about 10,000 chanaels)

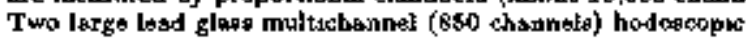
spextrometer6 are nesed to det.ect p energres Tskıлs dath (Jully D6)

Journal paperg PL B259 (1991) 226, PL B334 (1994) 234, and FेTE 3 (3994) I3

Roloted 6apertitents SERPUKHOV-166, BNL-791, BNL-846

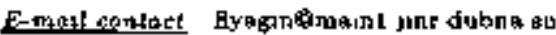

\begin{tabular}{|c|c|}
\hline$\underset{\text { vuclasu }}{p \text { vucleon }} \rightarrow \mathrm{DD}<p K^{+} K^{-}>$ & To GeV $\left(\mathbf{E}_{\text {lab }}\right)$ \\
\hline$p$ nucleon $\rightarrow D D<p \phi>$ nuclean & " \\
\hline p nacleon $\rightarrow \mathrm{DD}<\mathrm{A} \mathrm{K}^{+}>$pucleon & n \\
\hline 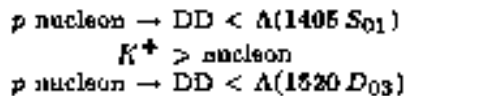 & $\pi$ \\
\hline $\begin{aligned} K^{+} & >\text {nucleous } \\
\text { p nuclasn } & \rightarrow \text { DD }<\Sigma^{D} K^{+}>\text {nuclemn }\end{aligned}$ & " \\
\hline
\end{tabular}

\section{SERPUKHOV-168}

(Propoeted 1977, Approved Jul 1977, Btgev dateteking 1985, Completed dats-teking 1993)

\section{INVESTICATIONS OF HADRONIC BPECTROSCOPX} WTTH THE DETECTOR SPHINX

SEFPUKHOV - 3 V Golowku, A P Koxhernikgv,

Y P Kubaraysky. A I Kulyaytesy, V F Kurshetsoy,

$A$ E Kustuserenka, $L$ G Landuberg ( $\checkmark$ Spokeaparson).

V V Molehanton $V \vec{A}$ Mulsbin, I IN Nikutin, A V Sklesnev, $V$ I Salyzmik, D V Vavlow, VA Viktorav

MOSCOW, ITEP - M Y Baledz, O B Dryubenko, G K Kliger,

V Z Kolkanov, Y S Lakasv, G S Lonkatt), A F Nilov,

V T Smalyanter, V E V'tshuyaker

Acreterator SERPUKHOV Detector SPHIUX

\section{Feactiong}




\section{SUMMARIES OF SERPUKHOV EXPERIMENTS}

$$
\begin{aligned}
& \text { p uticleon } \rightarrow D D<\Sigma\left(1306 F_{3}\right)^{0} \\
& K^{+}>\text {uucleon } \\
& p \text { nucleon } \rightarrow \text { DD }<\mathrm{p}^{+}{x^{-}}^{-}\left(r^{\prime} \mathrm{s}\right)> \\
& \text { sucheon } \\
& \text { p nucleon } \rightarrow \text { DD }\langle p \omega\rangle \text { mucleon } \\
& \text { D nucleon } \rightarrow D D\langle p \eta\rangle \text { nuclean } \\
& p \text { uucleon } \rightarrow \text { DN }\left\langle p \eta^{\prime}\right\rangle \text { vuclecn } \\
& \text { p nucteon } \rightarrow \text { DD }\langle p p \bar{p}\rangle \text { nucleon } \\
& p \text { uucteon } \rightarrow \text { (reutrals) } \mathrm{X} \\
& \text { g auckess } \rightarrow \mathrm{DD}\left\langle\boldsymbol{H} \mathrm{K}^{+} \mathrm{K}^{-}\right\rangle \\
& \text {Duclew } \\
& p \text { uucteus }-\mathrm{DD}\langle\xi \phi\rangle \text { mucleun } \\
& \text { p uucless } \rightarrow D D<A K^{+}>\text {nucleus } \\
& \text { p nuclous } \rightarrow \mathrm{DD}<A\left(1005 S_{01}\right) K^{+}> \\
& \text {aucleus } \\
& \text { mucleus } \rightarrow \mathrm{DD}<\mathrm{A}\left(1520 D_{03}\right) \\
& \mathrm{K}^{+} 3 \text { aucleus } \\
& \text { D nucless } \rightarrow \mathrm{DD}<\Sigma^{\prime \prime} K^{+}>\text {nucleus }
\end{aligned}
$$

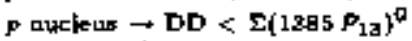

$$
\begin{aligned}
& \mathrm{K}^{+}>\text {nucleus } \\
& p \text { uxcleus } \rightarrow \text { DD }<p \pi^{+} \pi^{-}\left(\gamma^{\prime} \omega\right)> \\
& \text { meleus } \\
& \text { p auckeus }-D D\langle p \omega\rangle \text { nacleus } \\
& p \text { uxcleus } \rightarrow \mathrm{DD}<p \eta\rangle \text { nucleus } \\
& p \text { ancleus } \rightarrow \mathrm{DN}\langle p \bar{p}\rangle \text { nucleus } \\
& \phi \rightarrow \mathrm{K}^{+} \mathrm{K}^{-} \\
& x \rightarrow \pi^{-} \\
& A\left(1405 S_{01}\right) \rightarrow \Sigma^{+} \pi^{-} \\
& \Delta\left(1405 S_{01}\right) \rightarrow \Sigma^{0} \pi^{0} \\
& \Sigma^{+} \rightarrow p \pi^{0} \\
& \mathrm{M}\left(1520 D_{0 \mathrm{~S}}\right) \rightarrow \mathrm{PK}^{-} \\
& \Sigma^{0} \rightarrow A+ \\
& \Sigma\left(1385 P_{13}\right)-A x^{D} \\
& \omega \rightarrow \pi^{+} x^{-} \pi^{4} \\
& \text { i) } \rightarrow \pi^{-} \pi^{-} \pi^{0} \\
& n^{\prime} \rightarrow \pi^{+} \pi^{-} \\
& 70 \text { GcV (S }
\end{aligned}
$$
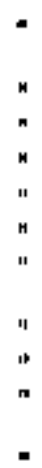

Portacles stadied baryon. $N \phi(1950), \Sigma\left(3170\right.$ B) ${ }^{+}$

Eref defcraptian Stadiak batyon diffactive penduction, woherent reactions on nucles, and searabes for axotse baryons

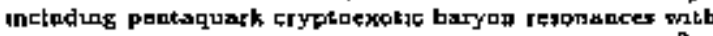
hidden ctragremens in the maks regron up to $+5 \mathrm{GeV} / c^{2}$ Uaes Be and $C$ as maclear targets SPHINX countats of a wide eperture maxnetic npectrometer azth proportienal and drift. chambers working in conbmatron with a mulk|chsontl gamma spectromeler and a syatan of Gerenkov deloctors for the

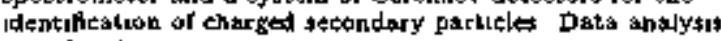
completed

Journal paperg YF 52 (1990) d9d, YF 57 (1994) 47, YF 57 (1994) 24t, YT $5 T$ (1994) 203, ZPHY CB1 (1904) 223, ZPITY CБ1 (1994) 390, NC Alor (1464) 2441, YF 5 T (1904) 1376, YT \$7 (1994) 2030, YF 5T (1994) 2042, UFN 164 (1994) $1129, Y F$ 58 (1905) 1426, and ZPHY C68 (1996) Б95

Related apertmants SERPUKHOV-120, SERPUKHOV-179

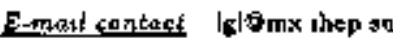

\section{SERPUKHOV-172}

(Proposed 1958, Approved Mny 1983, 自xan datn-takjng Dec L981, In progresei)

STUDY OF MESONS WITH AN ENHANCED GLUON COMPONEINT (GLUEBALLS INCLUDED) ANL MESONS WITH HIGH APINS USING THE MULTIPHOTON $4 \pi$ SPECTROMETER

SERPIJKHOV - A V Dolkopolow, $\$$ V Dantkbv, A V Ibytkin,

C V Khapstov, A A Koudaohov, A K Konoplyandolor,

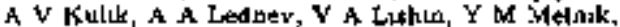

9 A Polowejkov: VA Polyakow, YD Prokoshk!n

[V \$pokespersun], S A. Sadibuky, V D Sarjoylenko, PM Shasan,

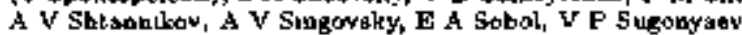
TBILISI, INST PHYS - A K DJsyrskbvth, T A Lomtadze.

G G Sektniaudae, E G Tskhadadse

LOS A LAMOS - D Aldq, E A Krapp, T Lopex

BRUSTSELS U, JUSN - F Brmon, I P StToot

ANNECY - J P Peigreanx, M Poulet

KEK - S Insba, M Kobayasbi, T Taura

MrAaZAKI U - T Nakamura, K Taksmatteu

Acookengor SERPUkHoV Detester OAMSSAPS

Bentitians

$$
\begin{aligned}
& \pi^{-} p \rightarrow n 2 \pi^{0} \\
& \pi^{-} p \rightarrow \pi 27
\end{aligned}
$$

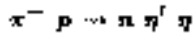

$$
\begin{aligned}
& x^{-} p \rightarrow n+x^{0} \\
& x^{-} \rightarrow \pi \eta 2 \pi^{0} \\
& \pi^{-} p \rightarrow+2 w \\
& \pi^{-} p \rightarrow n K^{a} \vec{K}^{0} \\
& \pi^{-} \rightarrow n 2 \pi+\pi^{2}{ }^{\circ} \\
& \pi^{-} p-\operatorname{mesco}{ }^{\circ} \mathrm{X} \\
& \pi^{-} p \rightarrow \text { gluebsall } \mathrm{X} \\
& \pi^{-} p \rightarrow J i t(1 s) \mathrm{X} \\
& \pi^{-} p \rightarrow \psi(2 s) X \\
& \pi^{-} p \rightarrow \pi_{c}(1 s) \mathrm{X} \\
& x^{-} p \rightarrow x_{c} \text { (unspec) } x \\
& K^{-} \rightarrow \rightarrow n^{\theta} \times \\
& \text { gluebsall } \rightarrow 4 \mathbf{r}^{\circ} \\
& \text { glueball } \rightarrow 2 n \\
& \text { glusta|d - } n^{2} \text { क } \\
& \text { glueball } \rightarrow 2 \pi^{\prime} \\
& \text { meson } \rightarrow 2 \pi^{0} \\
& \text { 世esod } \rightarrow \text { in }
\end{aligned}
$$

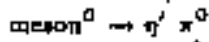

$$
\begin{aligned}
& \operatorname{mc}(1 S) \rightarrow \text { i } 2 \pi^{\circ} \\
& \nabla^{\circ} \rightarrow 24 \\
& \rightarrow 2 y
\end{aligned}
$$

$32 \mathrm{GeV} / \mathrm{c}$

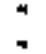

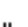

$$
\begin{aligned}
& " \\
& \text { " } \\
& \text { " } \\
& 4 \\
& \text { - }
\end{aligned}
$$

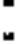

$$
\begin{aligned}
& \text { - } \\
& \text { - }
\end{aligned}
$$

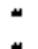

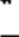

$$
\begin{aligned}
& \text { - } \\
& \text { - } \\
& \text { - } \\
& \text { - } \\
& \text { - }
\end{aligned}
$$

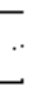

Paricteg otudiet Eluctiall, moson"

Brof description Data taking and data anolyat are in proziess (May 96)

Jasrna popers NIMI A.68 (1988) 112. NIM A27B (1989) B52.

PTE I (1990) as, PTE 2 (1990) 90, PTE 5 (1991) sk, and NTM A.302 (1901) 143

Related experwinent DNL-Bs2

E-trat confuc! prokosblanibux thep to

\section{SERPUKHOV -173}

(Frapored 1981, Approved 1892, Began dateretnkng 1997.

L protoses)

STUDY QF BTRANGE PARTICLE RESQNANT STATES USING HADRON BEAMS WITH MOMENTA OF $40-70$ GOV/C AT THE IHEP ACCELERATOR

MOSCOW, ITEF - B P Barkor, I A Erutev. O N Eroterva

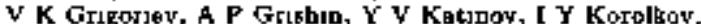

V I Lem, V N Lnejn, V N Nordrachey (V Spakexpersan),

Y P Shkurenko, V V sokolowk, G D Tikmorouror,

$\checkmark$ v' Vledumest

Acreicrator 5EtPPUKHOV Delectar, MIS

Reactrons

$$
\begin{aligned}
& x^{-} p-n 2 K s \\
& x^{-} p \rightarrow \pi 2 K_{s} q^{0} \\
& \pi^{-} \boldsymbol{p} \rightarrow \mathrm{n} K_{\mathrm{s}} K_{\mathrm{L}} \boldsymbol{\pi}^{+} \pi^{-} \\
& x^{-p}=\mathrm{n} \boldsymbol{K}_{\mathrm{S}} K_{\mathrm{L}}{ }^{\mathrm{r}} \\
& x^{-} p \rightarrow K_{S} \pi^{+} \pi^{-} \gamma^{-} \text {(unapes) } \\
& { }^{-} p \rightarrow p K_{5} K_{L} \pi^{-} \\
& x^{-} p \rightarrow n \Lambda \bar{\Lambda}
\end{aligned}
$$




\section{SUMMARIES OF SERPUKHOV EXPERTMENTS}

$$
\begin{aligned}
& K^{-} p-2 K_{S} Y^{-} \text {(unspec) } \\
& K^{-} p \rightarrow n K_{S} \pi^{+} \pi^{-} \\
& K^{-} D-4 \pi \gamma^{-} \text {(nTsper) }
\end{aligned}
$$

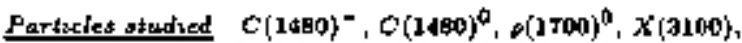
$\phi(1680), \phi_{3}(1850), f_{3}(1720), f_{2}(1810), f_{3}(2010), f_{4}(2050)$, $f_{1}(2220), K_{2}^{*}(1450)^{-}$

Bref degerption Extends an earher $K_{S} K_{S}$ and $\Lambda_{\bar{\lambda}}$ finsl atateg study (SERPUKHOV-147) to the range of masees betweed 18 and 25 GeV Investigaten the $K_{5} K_{L}$ system usung $\pi^{-}$and $\mathrm{K}^{-}$bearax with the monentum of $40 \mathrm{GeV} / \mathrm{e}$ Studieg baryon-

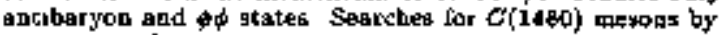

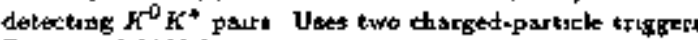
Requested 2100 hours

lotrinat papers PTE 3 (1994) 43, YF 58 (1995) $60=$ PAN 58 (1995) 46, atd YF 58 (1995) 1628

Related experiments SERPUKHOV-14?

E mad' contact rozdrachevelmx ibep su, nozdracheviovxitep itsp ru

\section{SERPUKHOV-177}

(Proposed 1990, Approned Jul 1963, Iv prepatation)

\section{MEASUREMENT OF THE MASS OF THE $\Sigma^{-}$} HYPERON

ST PETERSBURG, INP - A 5 Dennacy, O L Fectu, M P Gurlyey,

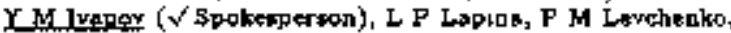
A A Petrlatil, Y P Platobon, V M Suvoror, A V Zhelambav ANesermotar SERPUKHOV Detector QUARTZ Feartrotes

$$
\begin{aligned}
& \boldsymbol{C} \rightarrow \boldsymbol{\Sigma}^{-} \mathrm{X} \\
& p \mathrm{Mg} \rightarrow \mathrm{\Sigma}^{-} \mathrm{X} \\
& p \mathrm{Ca} \rightarrow \Sigma^{-} \mathrm{X} \\
& p \mathrm{~Pb} \rightarrow \Sigma^{-} \mathrm{X} \\
& 70 \mathrm{GeV} / \mathrm{c} \\
& \text { " } \\
& 11
\end{aligned}
$$

Particles gitudiad $\Sigma^{-}$

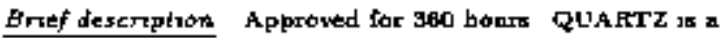
crystal diffraction apectrometer for hadrone $X$-raya with a Bemiconductor delector Walsug for the rub time [July 96)

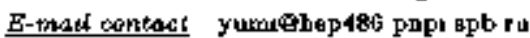

\section{SERPUKHOV-178}

(Proponed 1994, Approved 1994, In preparation)

SEARCH FOR EXOTIC BARYON STATES WITH THE MODIFJED DETECTDR SPHINX

SERPUKHOV - S A Akımenko, Y M Antspoy, V A Beterid,

V A Berrubov, A Derevshchikev, s V Erid, o V Eroshi, 3 V Golowkw, Y I Gorm, V N Govorun, A N Istev',

V A Kachianoy, Y D Karpekoy, A \& Konstentıroy,

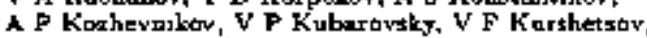

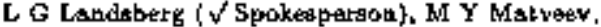

V \& Mfedavikay, V V Molehabav, V A Nukhit, I N Nikutit o V Orel, A I Parlinow, \$ V Petrenko, V S Petrov,

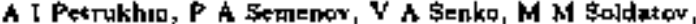

V L Solovisrov. V I Solyenik, A N Sytun, V S Yentew

D V Varion, V A Viktorov, is A $Z_{\text {trmin }}$

Mó̦CoW, ITEP - G K Klıger, V 2 Kolganor, \& 5 Lomkatzz,

A F Niloy, V T Smolyantron

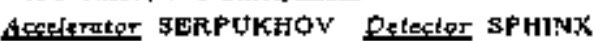

Resictions

$$
\begin{aligned}
& p \text { nucleon } \rightarrow \mathrm{DD}\left\langle\mathrm{p} \mathrm{K} \mathrm{K}^{+} \mathrm{K}^{-}\right\rangle \quad 70 \mathrm{GeF}\left(\mathbf{E}_{\text {tab }}\right) \\
& \text { ก나검ㅁ } \\
& \text { * nucleon } \rightarrow \mathrm{DD}<\mathrm{p} \phi>\text { nucleon } \\
& \text { D nucleon }-D D<A K^{+}>\text {nucleon } \\
& \text { D nucleon } \rightarrow \mathrm{DD}<\mathrm{A}\left(1405 s_{03}\right) \\
& \mathrm{K}^{+}>\text {nucleon } \\
& \text { p nuckeon } \rightarrow \mathrm{DD}<\mathrm{A}\left(1520 \mathrm{D}_{03}\right) \\
& \mathrm{K}^{+}>\text {nuelear }
\end{aligned}
$$

$$
\begin{aligned}
& \text { P auclean } \rightarrow \mathrm{DD}<\mathrm{\Sigma}^{+} \mathrm{K}^{+}>\text {nucken } \\
& P \text { aucleon }-D D<\Sigma\left(1386 F_{1 s}\right)^{\circ} \\
& \mathrm{K}^{+}>\text {nuelean } \\
& \text { p nucleon - DD } \angle D \pi^{+} \pi^{-}\left(\gamma^{\prime} \theta\right)> \\
& \text { aucleon } \\
& p \text { nuclesn } \rightarrow \text { DD }<p \text { w }>\text { nuclean } \\
& p \text { nucleon } \rightarrow \mathrm{DD}\langle p \text { i }>\text { aucleon } \\
& p \text { nuclean }- \text { DD }<p \eta^{\prime}>\text { nucleon } \\
& p \text { uucleon } \rightarrow \text { DD }\langle p p \bar{p}\rangle \text { nuclean } \\
& p \text { nucleus }-D D<p K^{+} K^{-}> \\
& \text {Huckint } \\
& p \text { nucleus } \rightarrow \text { DD }\langle p \phi\rangle \text { nucleus } \\
& p \text { nucleux } \rightarrow \text { DD }<\Lambda K^{+}>\text {muclieus } \\
& p \text { nucleus } \rightarrow \mathrm{DD}<\Lambda\left(1 \mathrm{~d} 0 \mathrm{~S} S_{0 \mathrm{~J}}\right) K^{+}> \\
& \text {puckevs } \\
& p \text { nucleus } \rightarrow D D<A\left(1520 D_{03}\right) \\
& \mathrm{K}^{+}>\text {nuclem } \\
& \text { p nucleus }-\mathrm{DD}<\bar{\Sigma}^{0} K^{+}>\text {nuckesk } \\
& p \text { nucleus }-\mathrm{DD}<\Sigma\left(1395 \mathrm{P} P_{33}\right)^{0} \\
& K^{+}>\text {unclaus } \\
& \text { p uucleur } \left.-\mathrm{DD}<\mathrm{p}^{+} \pi^{-}\left(\gamma^{\prime}\right)\right\rangle \\
& \text { Ducleus } \\
& p \text { nucleut } \rightarrow \text { DD }\langle p \omega\rangle \text { tuckeut } \\
& p \text { nuclous }- \text { DD }<p \text { i }>\text { naclous } \\
& \nabla \text { nucleus }- \text { DD }\langle\nabla, \bar{D}>\text { nucleus } \\
& \phi \rightarrow K^{+} K^{-} \\
& \Lambda \rightarrow p \pi^{-} \\
& A\left(1405 S_{01}\right)-\Sigma^{+} \pi^{-} \\
& \Lambda\left(1406 S_{01}\right)-E^{0} \pi^{0} \\
& \mathbf{\Sigma}^{+} \rightarrow \boldsymbol{p} \boldsymbol{\pi}^{0} \\
& \Lambda\left(1520 D_{03}\right) \rightarrow \mathrm{F}^{-} \\
& E^{D} \rightarrow h+ \\
& \Sigma\left(13 \text { 的 } P_{13}\right\} \rightarrow A \pi^{0} \\
& \text { w } \rightarrow \pi^{-} \pi^{-} x^{0} \\
& \pi \rightarrow \pi^{+} \pi^{-} \pi^{0} \\
& \pi^{\prime} \rightarrow \pi^{+} \pi^{-}
\end{aligned}
$$

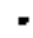

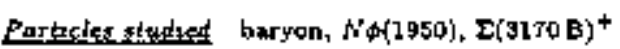

Brtef dascription Studie日 the baryon difiractive production.

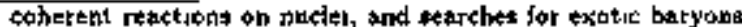
includug pentaguark cryptoexot be haryon regonenseg wath hidden otrangeness in the mase region up to $4 \mathrm{~b} \mathrm{GeV} / \mathrm{c}^{2}$ leses $C$ and nuclesc tarzets SPHiNd ronssgk of a wide aperture

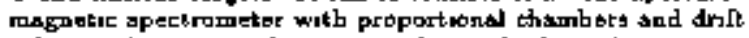
tubes workng in combination with a multichannel gamma apectrometer and a system of Cerenkoy detextrirs (including FICH counters) for the idantilestion of charged eecondary particles

Reluied experimerts SERPUKHOV-169

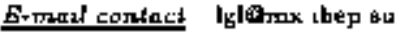

\section{SERPUKHOV-LNNK-(M]}

(Proposed 1988, Approved Ape 1988, Ju prapsration)

ITUDY OF SPIN EFFECTS AT 100 TO 000 GeV USING AN INTERNAL JET TARGET AT UNK

SERPUKEOY - G A Alekseey, Y I Arestov, N I Belikoy,

V V Borag, B V Chouken, V \& Datsto, A M Davideako,

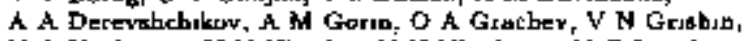

$\checkmark$ A Kachanow, Y V Kharlow, $V$ Y Khodyrev, V G Lapkhn,

V M Leontuev, I V Monojlov, Y A Matulenko, V A Mtodvedev,

Y M Molurk, A P Mogchanu, N o Minaer, V V Mocbeloy,

A I Mysmik, S E Nurusher, D 1 Fotalak ha, A M Pavlıgov,

$\checkmark$ A Polyakor, A F Prudkaglyad, V I A ylalın, V V Rykalın.

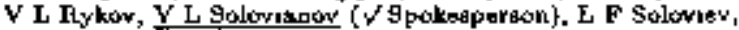

$S$ M Troshin, OD Tsay, M N Ukhanov, A N Vasiliev,

A E Yekutis, 5 it Yerip

DUBNiA - L 9 Barabasti, S I Bleskays, N S Borraw,

$\checkmark$ A Budilow. V V Fimushken, 14 Finger. N V Gorbunov, 


\section{SUMMARIES OF SERPUKHOV EXPERHMENTS}

N L Gorshkovs, V A Kalınalkoy, A G Karev, B A Khachiturov, $V$ i Kaelev, B Z Kapthoritb, of I Kullkov, R. H Kutuev,

E A Ladykith, A B Leykowich, M Y Liburg, V N Matefonow,

A. B Negabev; V A Nikstin, P V Nomokonov, V P Obudkovsky,

Y K Putuento, I L Puarev, Y A Plins, A A Popor,

I K Potashnskoya, M S Shafrangoy, V V Shutery, V I Sayalkbv,

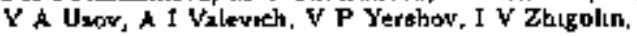

N K zhydkor, R. Y Zulkarnoser

TBILLSI STATE U - N 9 Amaglobed, Y S Bageturte

B G Chiladra. G A D-hambarabrib, I N Glopty.

G G Macharashvil, A I Ocherashvih, FM Sakandelidze

T M Sakbelaghrij!

MOSCOW STATE U - L I Belarer, A I Dounlamov.

A pA Gribasblem, N A Kruglov, A S Probkuryakov.

L I Sarychewa. N B Sinev, A A Yershow

MICHIGAN U - L V Akexeeva, V A Anferov, B B BLpDy,

J A Bywater, C M Chu, D G Crubb, D B Craudell,

Y S Derbenter, $S$ E Glsdychers, S-Q Hu, $S$ V Koutin,

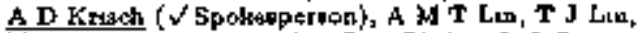

V G Lappor, D C Poarder, E A Pbelpg, L G Ratiner,

R S Raymand, M A Stealey, J A Sterart, S M Varzar,

Y $\mathbf{K}$ Wong

MFT - G R Court, D Kleppner

Accetgrator SERPUKFIOV.UNK Detector NEPTUN

\section{Aesecturs Polerized target}

$p p \rightarrow p p$

$p D \rightarrow \mathrm{X}$

$p p \rightarrow e^{-} a^{+} x$

$p p \rightarrow n^{-} h^{+} X$

$p \rightarrow \operatorname{pros} X$

$p p \rightarrow K^{*} \mathrm{X}$

$p p \rightarrow+\mathrm{X}$

$p p \rightarrow \eta^{\prime} X$

$p p \rightarrow \infty x$

$\rightarrow \rightarrow f_{2}(1230) x$

$\Delta \rightarrow \rightarrow$ Jar X

$P P \rightarrow \uparrow$ yet $X$

$\nabla D \rightarrow \Lambda X$

$\Rightarrow D \rightarrow \bar{A}$

$p p \rightarrow p X$

$p \mathrm{p} \rightarrow$ 芦 X

$p p \rightarrow$ hygeren $X$

$p p \rightarrow \Sigma^{*} X$

$p \rightarrow \Sigma^{-} x$

$p_{p \rightarrow a^{-}} \mathrm{X}$

$p p \rightarrow \Lambda_{c}^{+} X$

p nucleve $\rightarrow \rightarrow X$

$p$ vuckets $\rightarrow t^{-} e^{+} X$

D nuckeds $-\mu^{-} \mu^{+} \mathrm{X}$

p nucleve - paon $\mathrm{X}$

p nucleve $\rightarrow K^{ \pm} \times$

$p$ nucke $\rightarrow \eta \mathrm{X}$

$p$ vuekes $\rightarrow \eta^{r} X$

p nuclevs $-i \mathrm{X}$

p nuclevs $-f_{2}(1270) x$

$p$ uncle te $\rightarrow$ Jat $X$

$p$ Duteleus $\rightarrow$ r jet $X$

$p$ racleus $\rightarrow \mathrm{nX}$

Doucleus $-\bar{\Lambda} \mathrm{X}$

$p$ vuclevs $\rightarrow p \mathrm{X}$

$p$ quck

p aucleus - hyperon $X$

$p$ buck to $\rightarrow \Sigma^{+} \mathrm{X}$

$p$ pucle us $-\Xi^{-} \mathrm{X}$

$D$ sucleus $\rightarrow \Lambda_{c}^{+} \mathrm{X}$

Brtef descriptson Studies spun efiects when the 600 OeV protons in the UNK riugy collide wixh a spin-polartuted ultra-cold acomic hydroxen internad jet targel Five diferent spectromelers on ll obeerve rgid pheporqehs in varions bartrou-hadron reachoms at

arosh, inedinm, and latge transyerge momente Jourat popers PTE a (1991) 52, and PTE 4 (J992) 57 Retated expriment: BVL-794

E-mat contact solovianow kTrochtimiphys physics las umirh edv

\section{Other Serpukhov Experiments}

Listed here sre gons othet Serpukhov experquenti of interest to the partiele physpes continatity Find mors detale sbout the projects oulue, in the SLAC's EXPERJWIENTS datsbage (aee $p$ a), of eontact the spokepersons

SERPUKHOV-149 (Completed data-tahing 1996)

STUDY OF ASYMMETRY IN INCLUSIVE REACTIONS

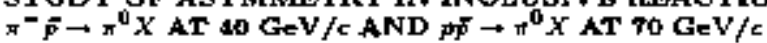
Spokebperson A N Vasuley [5erpukhow, IFVE]

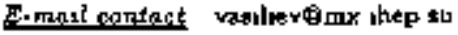

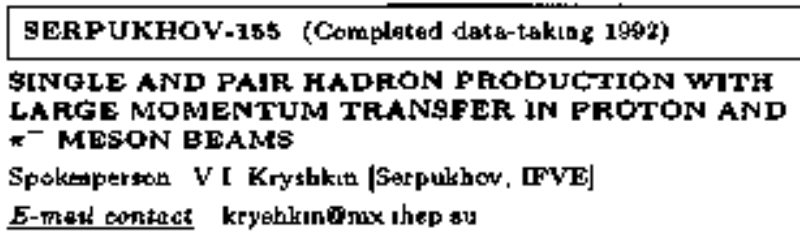

gERPUKHOV-161 (Taking data)

\$TUDY QF CHARMED PARTICLE PRODUCTION AT IHEP ACCELERATOR ENERGJES

\$pokeapertohs A M Monster [Serpukhow, IFVEJ, F F Ermolow [MIoscose State U], I V Boguedsvaky (Dubna, JINR)

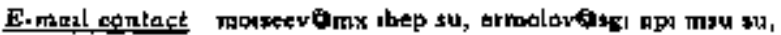

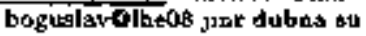

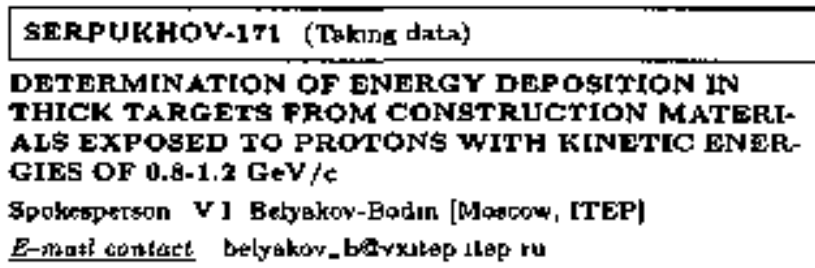

SERPUKHOV-174 (Completed data-taking 1991)

PHYSICS OE RELATIVISTIC DIMESON ATOMS

Spokeeperion L L Nemenow [Dubne, 31NR]

E-mati contact nemenovtiousun jus dmbna su

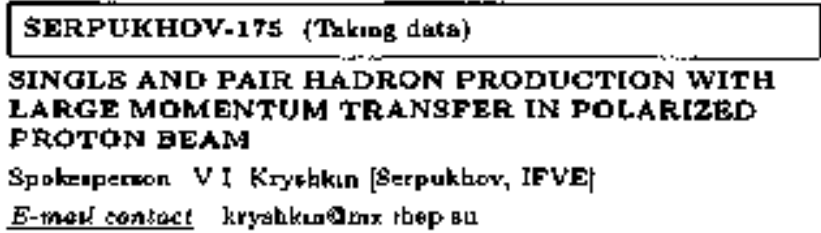




\section{Selected SLAC Experiments}

\section{SLAC-E 142}

(Proposed Oct 1989, Approved May 1990, Began dati-taking Nov 1992, Completed dats-taking Dec 1892]

MEASUREMENT OE THE NEUTRON SPIN DEPEN. DENT \$TRUCTURE FUNCTION

AMERICAN U - R G Armold, P E Bosted, J Dunne, C E Keppe], S E Rock, M Spengos, Z M Sigalate, J C Whte

BON:N $v-w$ W $M$ -

CLERLONT.FERRAND U - V Bretor, H Fonvidle

HARVARD U - A K Thompan

LBL - G Sbspto

LIVFFMORE - P L Anthony, F Detruch, K van Babber

MIOHIGAN U - T E Cloup

PRINCETON U - G Cates, H L Muddleton, N Newroury

\$ACLAY - H Bored, R Lomberd-Neleen, J Marrocke,

J Morgenstern, $\boldsymbol{F}$ \&s Stedey, $Y$ D Ternen

SLAC - R A Gearbate, E W Hnghes ( $\sqrt{ }$ Spokenperson),

T Maruyane, of M Petratos, F Pithan, L 5 Rochester,

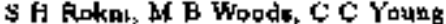

STAWFORD U - D B Kawall, S Kuhd, 2 E Mexiar

SYRACUSE U - R Eolmes, F A Sonder, J Xu

WISCONSIN U - H Beand, J F Jobnson, R A Mas, F Prepast

G H Zapalac

Acceietutor SLAC Detector Double-arm apectrometer

Reactions Polarized beam and target

$$
e^{-3} \mathrm{He} \quad 2266 \mathrm{GeV} / \mathrm{C}\left(\mathrm{P}_{\mathrm{lah}}\right)
$$

\section{Porticles atyinetin $n$}

Ertef description Studies a polarized clection beom acatticing

of a polaraed ${ }^{3}$ He gax target The scattered electrons zre detected by a two-arm fled spectrometer Terta the Bjorken podarization sopr rule and nucleor foin models Run for d00 bolars Dats analysts id progress (Jaly 96)

Journal papart: PRL T1 (1993) 959, and NC 107A (1004) 1197

Related appertments SLACLE-154

$\underline{E}$ mot cotitet emlyotolac uanford edu

\section{SLAC-E-143}

(Prapased Noy 1991, Approved Dec 1981, Begen dasa-tekkag Nov 1993. Complated data-taking Fob 1994)

MEASUREMENTS OF THE NUCLEON SPIN STRUCTURE IN END STATION A AT SIAC

E143 COLLABOAATION

AMERUCAN U = R G Aruold ( $\checkmark$ Spokebperson), P E Bouted,

I Dưne, I Fellbsum, D Reyad, S E Ronck, M Spengos,

z M Saratato, J L Whito

BASEL U - A Feltham, I Suck, F Stemne, B Zıhtmang

CLERMONT-FERRAND U - V Ereton, C Comptout,

II Fonvitalla, Y Roblin

CEBAF - I Gomes, J H Milchell

DAPNIA, SACLAV - H Boral, P Grentes, R, Comberd-Netsen,

I Marronde, J Morgenatern, F M Sialey, Y D Terren

LIV' GFMORE - F Dretrich

MASSACHUSETTS U, AMLHERST - J Bsuer, I Button-Shafer

MTCHIGAN U - T E Chupp, K P Coulter, T B Smuth

ULD DOMINiON U - C E Hyde Wraht, A KIeın, \& Kuhn, B Rave

PENN U - R Aútonov, $K$ A Gratioen, $F$ Puntry

SLAC - P L Antbony, J Clendentin, MI Deoudi, H Dutz,

R Erbecher, R A Gesrhert, E W Hurhes, T Meruyama,

W Meyer, o U G Petratar, E PLthan, C Prescott.

L S Rochester, S I Gt Loranl, L M Stunt, H Tane, T Uther.

D R Wilz, KW,te, C C Youns, B Youngmas

STANFORD U - D B Kawal]

TEMPLE U - 2 E MtEisa!

TOHOKU $\mathbf{Z}^{-}$- K Abe, T Aksg, M Kurka, P Sluksú, H Ylts
VIRGTNA U - T Averet, I C Chen, D C Crabb, D B Day,

E Frlet, $\mathbf{R}$ A Lindgreb, T J Luu, J S McCortby, $\mathbf{R}$ C Mpchart,

D Pocansé, O A Rondon ( $\sqrt{ }$ Spokegptronk), L C Smith

D Zimmerman

WiscolsiN U - H Band, J R Jobmean, R Prepost. \& H Zapalac

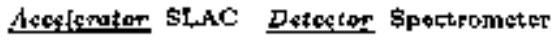

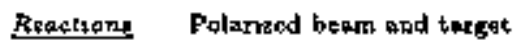

$E^{-}$deut $\quad \theta 7,162,291 \mathrm{GaV}\left(\mathrm{s}_{\mathrm{Jab}}\right)$

$e^{-}$p

H

Parkictes stadied $p_{1} n$

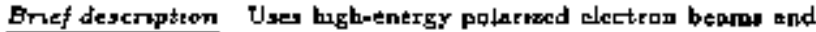
a set of ammonis baged polarized proson and denteron targets Eoth parallet and perpendicular bear-tnrget upin arsentintiong

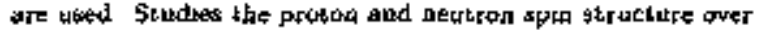
the range $003 \leq x \leq 0$ \& at momentum tranglers greater than

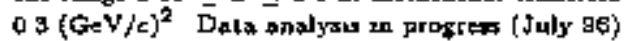

Jowrnal papers PRL TA (1395) 346, PRL 75 (1985) 25, PL Babl (1905) 61, sad PRL 76 (1996) 687

Retaled erpertments SLAC.E-142, SLAC-E.15d, SLAC.E.155, CERN-NA-037, CERN-NA-O4T

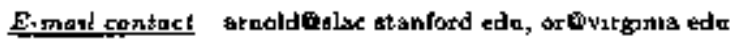
WWW Home- pape

bttp / / wwow lac atanford edu/exp/s143/home html

\section{SLAC-E-154}

(Proposed Oct J993, Approved Nov 1993, Begara dationtakink Oct i905, Complered datstaking Nov lirgs)

PRECISION MEASUREMENT OF THE NEUTRON SPIN STRUCTURE FUNCTION USING A POLARIZED ${ }_{H E}$ TARGET

AMERICAN U - R G Amald, P E Bated, I N Follboum,

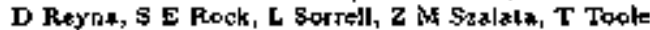

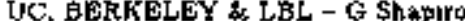

CAL TECH - T A verat

CLERMONT FERFAND $\mathbf{u}-\mathbf{V}$ Ereton, H Fonrieb]le, 9 [ncert?

TFFNDBLE $v$ - M J Bnenerd

KENT 9TATE U - B D Andersok, M Kbayai, M D MoDley, M Oluon, G G (M) Folraton, J $w$ Watson, WaM 2 hang

MASSACHUSEI'T U - C M Berıto, S Cburchwell.

$Y$ Kolomenstry, th Petertotin

MICHIGAN U - T E Chupp, $\mathbf{K}$ Coniber T E Sralb, E Welsh

MIT, LNS - B BTan

MIST, WASH, DC - A K Thompeor

OLD DOMINION U - S F Kuhn, B Fave, F Wesselmann

PENN U -. P Raines

PRINCETQN Y - P Bogarad, G D Ceter, K Kumar,

H Middieton, M1 Romolus

FUHA U, BDCHURA - W Mayer

SACLAY - H Eoral, $\mathbf{R}$ Lombrif Neluen, I Marroncle, F Sebatie, $\mathbf{F}$ Ştaley, $X$ Terruen.

SLAC - T Akagk, P I Anthong, M Droudi, R Erickson,

R Gearbart, R Hocks, E W Hughos $(\sqrt{ }$ Spokesperson),

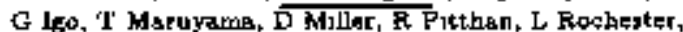

5 H Rokmi, J shour, \& St Lorast, L M stuart, T Usher, K Witte.

C C Young, B Youngman

SMITH COLL - P Decowat]

SOUTHEIRN OREGON STATE COLL - T Marin

SYRACUSE U - R Halme, P A Souder, XJ (J) Wa Was

TEMPLE U - J Martof, Z.E Maztani, P Zyla

TOHOKU U - K Abe, M Kurkk, F Suekase, H Yuta

UCLA $V$ Ghazkhemad

WILLIAM AND MARY COLL - K Grıfroen

WISCONSIN U - H R Band, J R Jolnson, G Mitchell, R Prapost.

Axceleriztor SLAC Dezector Spectrometer

Reactrons Folarszed beam and target

$$
e^{-3} \mathrm{H}_{5} \quad \text { Ho } 6 \mathrm{GeV}\left(\mathrm{B}_{\mathrm{ln}}\right)
$$

Particter sttudited $n$

Braj desctiption Mesaures the neutron spin serusture functien $g_{1}^{\mathrm{n}}$ ovar $x$ rangang frow 0015 to $0 \mathrm{~T}$ and $Q^{2}$ rangıng from $]$ 


\section{SUMMARLES OF SLAC EXPERTMENTS}

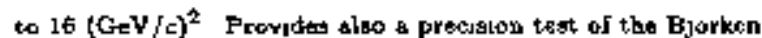
sura rulte at bigh average $Q^{2}$ at about $5(\mathrm{GeV} / \mathrm{e})^{2}$, and allows at extrection of the guart partop model parameters, $\Delta$. nnd $\Delta$ q Wres the $82 \%$ polanged electron beam it the upataded Aline The target polarnalion is achueved by way of the optical

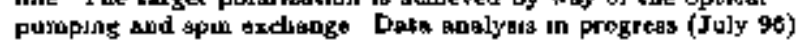

Aetated expartmente SLAC-E-142, SLAC-E-14S, SLAC-E-155, CERM-NA-047, DESY-HERA-HERMES

E-masl sontact emplyasolace oustord edu WWW Home-poige

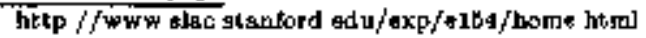

\section{SLAC-E-155}

[Proposed Oet 1993, Appraved Nov 196s, In prepartiont)

MEASUREMENTS OE THE NUCLEON SPIN STRUCTUAE JN END STATION A AT SLAC

AMEFJCAN U - R G Arnold (Spokespergon), P E Eobtad,

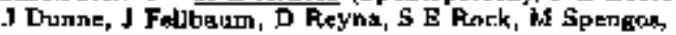

2 M Sxalata, J L Whit:

BASEL U - A Folthsm, 1 Sick, P stteiner, B Zahlmann

BONN U - W MeYer

CEBAF - J Gomaz

CLERMONT-FERRAND U - V Brelan, C Complous,

H Fonyulle, Y Rohlın

LIVERMORE - P L Ambbony, F Duetrich

MASSACHUSETT'S ot, AMHERST - I Bauer, J But

MICHIOAN U - T E Chopp, K P Coulter, T E 5 mith

NAVAL POSTGRADUATE SCHOOL - D Garvey,

$\mathrm{X} K$ Maruysms

OLD DOMLNION y - CE Hyde-Wingbt, A Klem, B Rąue

PENN US - $R$ Antonov, $K A$ Cnfionn, $P$ Raspes

DAPNIA, SACLAY - T Alagi, H Barel, R Erbacher, $\mathbf{P}$ Grenter,

R Lombart-Neleses, J Marroncto, I Morgeastera, F M Steley, Y D Tetried

SLAD - J Thendenin, G Court, M Droud, H Duts, R A Gothar, E W Hughes, T Maroyams, $G$ M G Petratot, 证 Pitthan,

C Prescott, A. Rujlart, L S Rochester, 5 J St Lotant,

L M Stust, H Tang, T Uehes, D R Walz, $\mathrm{K}$ Whu, $\mathrm{C} C$ Young,

B Yotungrann

STANFORD U - D M Kawall, I Kuhn, 2 E Mezlan

TOHOKU U - K Abe, M Kurity, $F$ suckeve, If Yute

VIRGINIA $\mathbf{U}$ - T Averect, J F Chen, $D$ G Crabb, D B Day

E Frlez, S Horbresles, $\mathbf{R}$ a Ltndgren, T J Lu, J S MeCarthy

(Spokesperson), R C Minehart, J H Mitchell, D Pocknid,

O A Fondon, L C Szrnteb, D Zamanternan

WISCONSIN U H Band, J R Johnson, R Prcpost, G H Zapadac

Acteterator SLAC Detector Spactrometer

Reacteraf Polanzed beam and tarxet

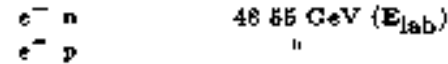$$
1
$$

Particlet standied to p

Bnef descripteon Measures the deep inclastic seatiteriog of polarized electrons from polartzed amonoms targets, $\mathrm{NH}_{3}$ and $\mathrm{ND}_{3}$, to determine tha opm thrbeture fugctsons $g_{1}$ and 92 over 1 rakging from 0015 to 085 and $Q^{2}$ ranging from 1 to $17(\mathrm{GeV} / c)^{2}$ The date will double the $Q^{2}$ renge of precueron measurements and sllow a starch for gonscring bugher twist contributions to the spin attucture functions Uted a new palr of focuaring magnetic apectrometers subtrumented with ebowar counters, Ceerenkov coupkerk, and \$cinkillator hodoscopes to measute teattered eder.trons aud raject plong In preparztion (July 96) Scheduled to run is Jonuary 97

Related trpermerts SLAC-E-142 SLAC-E-143, SLAC-E.J54, CERN.NA+03T, CERN.NA.047

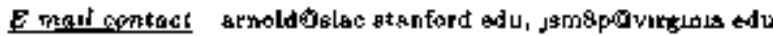

\section{SLAC-SLC-SLD}

(Proposed 1983, Approved Way 1964, Begar, datiolakint Apr i99I, In progress)

THE SLD DETECTOR FOR THE SLC

\section{SLD COLLABORATION}

BOSTON U I A Coller, S Hedqes, J T Shank, J \& Whitaker BRUNEL U - N J Allep, P Dervan, E Elaion, A Hasan,

A K MfoKemey, C Ward, o J Watts

COLORADO U - S Fabey, U Nauenberg. D Wagaer

COLORADO STATE U - M Dimi, J Flarton, M \$ny, H Stoengk, H. J Wilson

COL,UMIBIA U - P C Rowsom

FEARARA U \& INFN, FERRARA - G Mangzath, L Prewontege FFASCATI - A Gakcaterrs, R De Sangro, 1 Permzต, M Piccolo

LEE - G Shapiro, if Steinex

LOUSIANA TECII U - B Barakat, X. JaAR, K Jubriston

MASSACHUSETTS U, AMHERST - 8 Blaylock, S S Hertzbach,

R R Kafler, A Traodali, J W/ttluk

MIT - P N Burrowa, R F Cowad, \$ Dagraca, D Dong, MC I Fero,

H W Kendall, V Lis, L \& Osborke, J Quigley, F B Taylor,

E Tarreencte, R Vardier, $\mathrm{R}$ it Yasmamolo

MISSISSIFPI U - B Bolen, R. Kronger, J Ready

MOSCOW STATE $v-G$ Bashindzhakyar

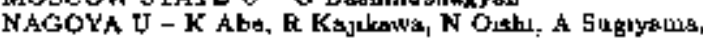

S Struble

OKEGON U - A Arodzerd, 9 E Brau, R Frey, I Huber.

$M$ Langrton, N B Sinov, X. Yang, J- Zhou

PERUGIA U \& TNFN, PERUGIA - D Falci\$1, G Napamelli:

G Martovam

RUTGERS U - K O Beitd, P Jocques, M Kalelker, R J Pleno,

P Stance

HUTHERFORD - C J 5 DAmErill, D J J tokson, F J Wickems

SLAC - T A kagn, D Astom, T L Barklow, J R Bogart, G R Bower,

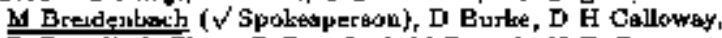
R Gaesell, A Chou, G Crawford, dS Daoudi, N Degroet, R Dubols. A E Hufles, J A Jarob, J Jask, A S Jobseon, H Kowahars, M E Kiog, D W Q Leth, H L Lyach,

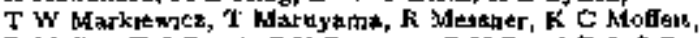
D Mfuller. T J Psivel, C Y Preocott, B N Ratchif, I \& Pochester, J J Roxjell, O H Suxton, I Schwianog. D Su, M Swstlt,

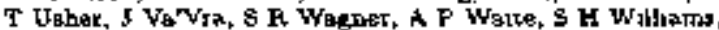
$s$ Wllocq, W J Wurievak, MA B Woods, C Q Young SOGANG U - Y- Km, C F Park.

TENNESSEE $V$ - B Burg, H O Coha, E L Hart, L Kamychkpr,

U Oropnenko, K Sbrogkew, A W Wentemang

ToHokU U .. K Abe, K Haiuko, T Nagamane, 5 Nartto

F Sueksne, J Yashima, $\mathbf{f}$ Yute

UC, SANTA BARBARA SJ Yellin

UC, SANTA CRUZ - D G Coybe, J Fernatodo, X Lu u, PL Reinertwen, T Schalk.

VANDERBILT U - R S Panviar, T

WASHINGTON U, SEEATTLE T H Burnet, $E$ Ghurch, $V$ Cook, $P$ M Xockets, E H Weas,

WISCONSIN U - H R Basd, J R Johnson, R Prepost, V Serto, T witught.

YALE U - C Baltay ( $\checkmark$ Sprokerperison), M Lau, S Marly, T Moore, I A Snydet

Accelarifar SLAC-SLC Petector \$LD

Renctiotse Polanzed besm

$$
e^{+} e^{-} \quad<100 \mathrm{Gov}\left(\mathrm{E}_{\mathrm{em}}\right)
$$

\section{Partictes studized $Z^{0}$}

Erief descriptean Studies includd (i) precisian terts as the

Stabdard Model of the electrowerk interactsoos and the $Z$ partial width to boliem itatex, particularly by mezstring the left-rught polarization saymmetry $A_{L R}$, (2) heavy quark phyracs of the $b$ system, (3) $B$ B mixug, with polarized basms, (4) losti or QCD in multe-jets, sind (5) a sastch for new

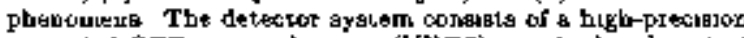
upreaded CCD vertex detector (VXDS), a cylındrical central daft chamber with lour cireulat endesp drift thambers, a Cerenkov ring imaging detector, finely segmented projective tower reorpetry calotimetry, and a muon trackins system Taking data (July 86) Expected to tun tsll Match 98

Jouranl ympers NIM A2sE (1955) 49B, IEEE TNS 33 (1086) 46 IEEE TNS 93 (1966) 65, IEEE TNS 33 (1956) 81, IEEE TNS 33 (1986) 113 IEEE TNS 33 (1986) 167. IEEE TNS 33 (1986) 178, TEEE TNS 33 (198E) 194, IEEE TNS 23 (1896) 187, IEEE TNS 33 (1896) 201, IEEE TNS 33 (1986) 25 h. NIM A262 (1966) 295, NLM A257 (1987) L39, NIM A25T (3987) \&25, LEEE TNS 35 (1968) 231, [EEE TNS 55 (1998) 282, IEEE TNS 35 (1988) 311, IEEE TNS 36 (1986) 308, NIM A264 (1088) 219, NIM A285 


\section{SUMMARIES OF SLAC EXPERIMENTS}

(1988) 99, NIM A2T3 (1688) 858, TEEE TNS 3E (1989) 23, JEEE THS 36 (1989) 276, IEEE TNS 36 (1989) 339, IEEE TNS 36 (1969) 595. IEEE TNS 36 (1989) 675, IEEE TNS 36 (1969) 751, IEEE TNS 36 (1989) 822, IEEE TNS 36 (1989) 165T, NIM A275 (1989) 454, NIM 1278 (1989) 94, NIM A277 (1969) 222, NIMI A283 (1989) 582, NLM A283 (1989) 590, NIM A284 (1989) 359, IEEE TNS 37 (1900) 1132, IFEE TNS $\$ 7$ (1990) 1191. NIM A286 (1990) 236, NIM A289 (1990) 449, NIM A289 (1990) 463 . NIM A269 (1990) BT, NIM A290 (1990) 363. NIM A293 (1990) 139, IEEE TNS 38 (1991) 348, NP (PROC SUPPL) 23B (1991) 219 , NP (PROC SUPPL) 23E (1991) 227, NIM A300 (1991) 501, NIMI A32E (1933) 472. MIPL A8 (I993) 2237. PRL 70 (1993) 25L5, PRL 71 (1993) 2528, NP (PFOC SUPPL) 37B (1994) 28, PFL 72 (1994) 3145, PEL TS (1904) 25, FR D\$0 (1984) 55Bo, NP (PHOC SUPPL) 39EC (1985) 121, PRL 74 (1995) 1512, PRE 74 (1995) 2830, PRL 74 (1998) 2890, PRL 74 (1995) 2894. PRL 75 (1995) 3609, PRL 75 (1995) 3624, PRL 75 (1995) 4173, PR. D51 (T455) 962, PE D52 (1995) 4240. PF D52 (1695) 4828, PL B371 (1906) 149, PR D59 (1996) 1023, and PR D43 (1908) 2271

Related expertrents CERN.LEP.ALEPH, CERW-LEP.DELPHI, CERN-LEP-L3, CERN-LEP-OPAL

E-thend tontact jubbraloc Blanford edu, battayoyalph2 phy61ca yale edn WWW Horre-page

betp $/ / w^{\prime} w^{\prime} w-a k d$ slac glanford edu/sldwww/sld btgl

\section{SLAC-PEP2-BABAR}

(Propered Jun 1994, Jun 5994, Approved Jas 1996, Jn prepasatwon)

\section{THE B FACTORY DETECTOR}

\section{BABAR COLLABORATION}

Aceedratar SLAC-PEF2 Dateator BABAR

Briej de\$cription BABAR i\$ a detector dewigned to explote the angle sotaraction region in the PEP2 facdty The primary Btm is a detalled atudy of CP volatung aeymmetrites in $B^{0}$ mogon docsy, but a nde vanety of other $B$, charm, $r$, sad 1 wophoton physks will also be accessible The delector compross

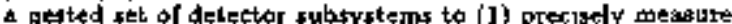
the collegion pount and decay points of the $B$ meecon palrs produced, (2) measure the momentum and energy of the decay particles with s heb resolution, and (3) prectsely identify)

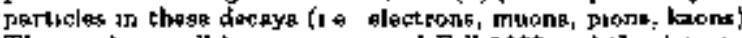

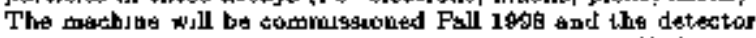
should be ready to begin the atudies in early 1999 Under construction \{July 96\} The collibaration consigle of more than 500 scientigts, angincers, and graduate students fram 10 countries For tarther detals, plense contact the Spoterperson, Prol David G Hitlin [Gal Tech| The Deputy Spokesparson us Di Roy Alessan [Secley]

$E$ mal contact bitheoslac kaniord edu WWW Hotne page

bitp $/ / w w w$ slac stonford ede/EF/doc/wgrw/bfHome html

\section{Other SLAC Experiments}

Lusted hete are gone other SLAC expermiments of Jbleregt to the particle physics community Find more detalls abaut these projects onluee, th tbe SLAC's EXPEFIMENT'S databses (ase p 3) or contact the epokespersobs

SLAC-E-140X (Cokppleted datariakıng Sep 1991)

MEASUREMENT OF THE $x, Q^{2}$, AND HYDROGENDEUTERIUM DEPENDENCE OF $R=\sigma_{t} / \sigma_{j}$

Spokespersong Stephen E Rock |American U J, Arle Bodek [Rocheatert U]

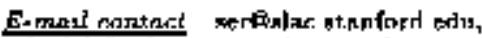

bodelefiurbep pos rochester edu
SLAC-E-144 (Taking data)

STUDY OF QED AT CRITICAL FIELD ATRENGTH IN INTENSE LASER - HIGH-ENERCY ELECTRON COLLISIONS AT SLAC

By El44 Collaboration

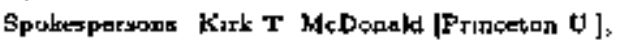

Adtian C Me]issinos |Rochenter U], Dand L Borke [SLAC]

$\boldsymbol{E}$-matl contact medonoldepupbep princeton td

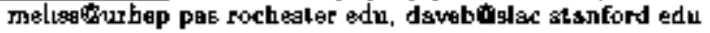

WWW Home-pege

hitp //www slac etanford edu/axp/e1 14/e144 htmal

SLAC-E-140 \{Completad dats-tsking Apr 19g9)

STUDY OF THE INTEAFERENCE BETWEEN MULTIPLE SCATTERING AND BREMSSTRAHLING (LPM EFFECT)

Spokesperoon Spencer \& Kienn [LBL, Berkalty]

$E$-mast contact srtelemollbl gor

SLAC-NE-17 (Corspleted detr-taking Oct 1991)

TWO-BODY PHOTODISTNTEGRATION OE THE DEUTERON AT FORWARD ANGLES BETWEEN 1.0 AND 3.0 GeV

Spokesperaon Roy $\mathbf{l}$ Holt [Asxonne]

$E$-mast cuntact 3 -holtounuc edu

SLAC-NE-18 (Completęd data-tukeng Oct 1981)

MEASIJREMENT OF THE NUCLEAF DEPENDENCE AND MOMENTUM TEANSFER DEPENDENCE OF QUASIELASTYC $\left(c, e^{\prime} p\right)$ SCATTERING AT LARGE MOMENTUA TRANGFER.

Spokesperione Rrehard G Mulyer [MIT, LNS], Beadley W FJ] ppout [Cal Tech]

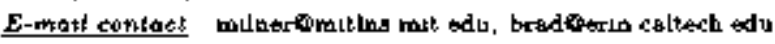

\section{SLAC Future Plans}

In the next Iew years SLAC will continue to aupport a Beries of complementary high-enerty physict prosrams (1) scudtes of the Bpin etructure of sucleong using the high current, high energy, polarized slection-beam scattering fram poiartzed peutron and proton targets, (2) 6tudy of the ejectro-wreak interbection with the SLD delector through precisson messurements at the $Z$ pole, wing palarized electron collusonk pn unpolarjzed positrong at. the energy of the $Z,\{3\}$ accelerstor RinD towads a resl liness collider (NLC) (4) preparations to study time-depentent $X$-ray sources wath the USA expenmont to be launched in 1997 , sad FeteD tow rayx $-\mathbf{x}$ follow on to the very successful EGRET experument how orburing tortb, and (6) actsity relsted ta tho dovislopment of the far-fature higb-losdreps accelerstion The spun struct are and SLD experiments are tebednled to run through kiarth 1998 The aryminctre $B$ Factory (PEP2) ap expected to bo comminsioned tefore the end of thal year, and the BaBar detector yhould begur ste atudy of the CP.viatation phenoment in early 1 eg9 


\section{SUMMARIES OF TRIUMF EXPERIMENTS}

\section{Selected TRIUMF Experiments}

\section{TRIUMF-497-287}

(Propoed Ott 1997, Appraved Dec 1987, Beger date-taking 1995, In progrose)

\section{MEASUREMENT OF THE FLAVOR-CONGERYING} HADRONIC WEAK INTERACTEON

PARITY COLLABORATION

MANITOBA U - J Birmall ( $V$ Spokesperront), I R Campbell,

A A Groen, A Hamuab, L R Lbe, 5 A PBge ( $\sqrt{ }$ Spokeaperbob),

W D Romaray, 5 D Rentener, V Sum, W T H van Gers

( $\sqrt{\text { Spokesperson). R Woo }}$

LOS ALAMOS - J D Bowman, R E Much ke

TRJUMF - C A Davis, D C Healey, R Helmer, F Levy,

$\mathrm{P}$ W Sthmor

ALEERTA U - P W Green, E Korkmas, G Ray, 3 Sonkup,

G) M Stugson

CARNBGIE MELLON U - A Berdor

MOSCOW, INR - Y Kuznetzov, N Titov, \& Zadormesy, A Zelenskı

Accelerator Trivutp petector lonusation

Roactiont Polarimed beam

$$
p p \rightarrow p p \quad 221 \mathrm{MeV}\left(\mathrm{T}_{\mathrm{lab}}\right)
$$

Ertef descriptuon Bessure日 the panty voluting (PV) lonzitudie nol pingly consisot $h_{p}$ p

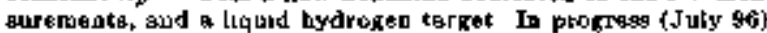

Jowinat popert PR. D37 (1985) 1760, NIM A307 (1991) 26, and NP A563 (1993) 823c

E-manl contact burchall iphywes tumsoltobs co,

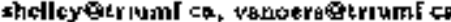

\section{TRIUMF-537}

(Fropaned May 1985, Complated datmetaking Jus 1991)

\section{RADIATIVE DECAY OF THE $\triangle$ RESONANCE}

BRITISH COLUMBIA U D F ileasidy (Spokesperson).

$S$ Sranieleus, $P$ Waber

KENT TCKY U M A Kovagh

NEW MEXICO U - B Bassalleck

BOSTON L3 - E C Elooth, I P Miller

Atceterator TRIUMF Detector Phaton spectrometer

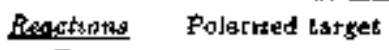

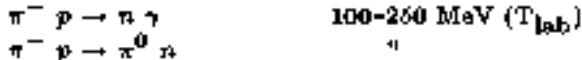

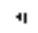

Brief description Messurea $\Delta^{0}$ radiative decay moltipolod and differential crose-bectionit A polerused target has been successfulty ued in phase-1I of the experiment Date onolyou in progress (July 66)

E-mial coniuct measdaybitriumf ta

\section{TRIUMF -614}

(Fropoes Jun 1990, Approved Dec 1993, In preperation)

PRECTSION MEAJUPEMENT OF THE MICHEL PARAMETERS IN $\mu^{+}$DECAY

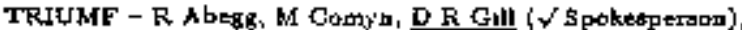

$P$ Guraplinger, E Helmer, J Macdonald, J-M Pouttegou,

R Poutiason, G Wat, D Wrieht

TEXSS A AND X - 0 Gogliard ( $\checkmark$ Spoketperson), R Thbale

( $\checkmark$ 8pokesperson)

BRTISH COLUNABIA U - M Hannaf

VALPARAISO U. DNDIAWA - D Koelké, R Mapwejler

KURCHATOY TRST, LOSOOW - A Khruchnyky, Y Lecbun,

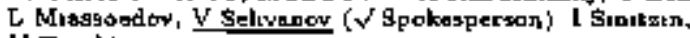

$\checkmark$ Toroktion
ALBERTA U - N ROdDIAK

SASKATCHEWAN U - Y M ShID

RBGINA U \& TRIUMF - R Tacik

Aczelerytor TRIUNF petector Spectrometer

Brsef destrifttort Tosts the rucht-lefit models of Weak interac

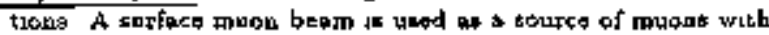
polarization slmart compitetely (bet ter thain 29 98\%) ophosite to the muon momentum The expersmental abparatus is contzined is a eupereonducting otenold with p maxum ficld of $23 \mathbf{T}$, collinear to the mum polarisatipan Buons olop in a thin planser aluminum tarset loreted it the center of the magnet, and the resulting poeatrons are recorded by one of the two planar drilt chamber (PDC) assemblies located on both ydes of the target Uader construction Date toking expected in Fall 1953

Related experments LAMPF-1240

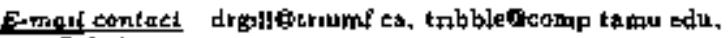
avistolv kiee ay

\section{TRIUMF-6A5}

(Froposed Jon 1991, Approved Jun 1991, Beran detz-takıng May 1992, Completed datn-taking Jun 1992)

A 9 SOLUTE DIFFERENTLAL CROSS-SECTIONS IN THE $\pi^{ \pm} \rightarrow \pi^{ \pm} p$ GEACTION AROUND THE $\triangle$ RESONANCE

PISCAT COLLABORATION

BFITISH COLUMBIA U - F Duncan, A Foltbam, G Jones,

J Lapge, $M$ M Paven ( 4 Spakespersan), K Rayuraod, $M$ E Sevior

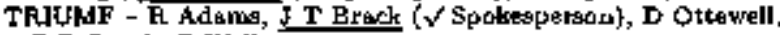
G R Smitb, B Walls

REGINA U - E I Mathie, R Trat:

COLORADO U - R A Ristiden

KAFUSFUHE U - H M Gtaudentrasar

ST PETERSBURG, CNP - I I strakawky

STMON FRAGFR U - R Helmer

Acesterator TRIUMF Detestor Scintulator

Reactione

$$
\begin{aligned}
& x^{+} p \rightarrow \pi^{+} p \\
& x^{-} \mathrm{z} \rightarrow \mathrm{x}^{-} \mathrm{D} \\
& \text { 1dl-267 MeV (T } \left.T_{\operatorname{lnb}}\right)
\end{aligned}
$$

Brtef descreption Uot flet, sold $\mathrm{CH}_{2}$ (polyetbylent) serget" as well as a supercooled ftat-window liquid hydrogen torget Scuncullator telacong RTe used for colperidence detection of prony and protong Covere angular range between $35^{\circ}$ and $160^{\circ}$

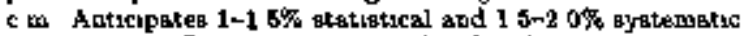
uncortisinties Data analysis completed atd a pablugatron being prepared (July 96)

Retated experiments TRUUMF-322, TRIUMF-47

E-marl cortact pavantimatlas nat edu, mpavand $\$$ bun mit edv, bracksepecti rolor ado edv

\section{TRIUMF-703}

(Proposed May 1903, Approved Jul 1963, Began datartaking Dec 1993, [n progrem)

\section{PION LIFETIME MEASUREMENT}

TFUUMF - D A Brymsn, M Fujurars, I A Macdonsld, \& Marebel, T Numag $(\checkmark$ spokeaperson $\}$. A Olun

Azcelerator TRUUMT Detector Contur

Reactson

$$
\pi^{+} \rightarrow e^{+}
$$

P4rtoctas siludied $\pi^{+}$

Brtef description The pron leteture ts meastred by observing the time dependence of the surface muba yold Takeng dato (July 96)

Journd papers PR D52 (1895) da55

Retated expenments TRIUMF-248

E trant contact toophoutrium $\mathrm{CA}$ 


\section{Other TRIUMF Experiments}

Luted here are some other TRIUMF experimenta of interest to the perticle pbysaca communaty Fidd more detanls aboul then projects onlize, in the SLAC's EXPERMLENTS databste (see P 3), of eaptact the spokespersone Chech aleo the yestly

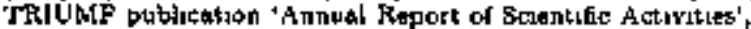
which lutts all the Lab's ongoing mxpariments

TRIUMF-3t9 (Completed dotantakeng Har 1893)

CHARGE SYMMETRY BREAKING IN ${ }^{p}$ EILASTIC SCATTERJNG AT 350 MEV

Spakespersons Willem T H van Oers [Mantaba U],

L Gordon Gresnisus [Alberts U]

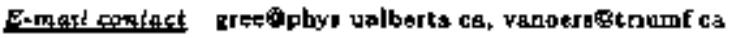

TRJUMF-373 (Completed tiats-taking Feb 1991)

SINGLE PION PAODUCTION IN $n p$ BCATTERING

Sppkesperyon Nerman E Davison [Manitobs U]

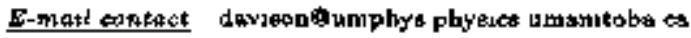

TRIUMF-145 (Completed dotatakteng Dec 1993)

Polarization MEASUREMENT IN THE ${ }^{3} \mathrm{He}\left(\pi^{+}, \bar{p} p\right) p$ REACTION

Spoleseptroans D Ashery, S MayTal-Beck [Tel Avrv U ]

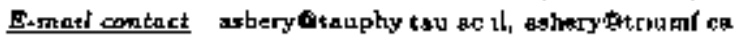

TRIUMF-45z (Completed dota-takedas Fet 1894)

RADIATIVE MUON CAPTURE ON HYDFOGEN

By RMC Dollahoration

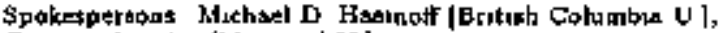

George日 Aruelo日 [Montreat $\mathbf{U}$ ]

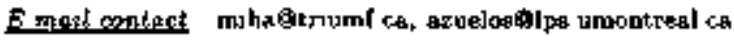

TRIUMF-482 (Completed data-taklag Sep 1991)

MEABUREMENT' $\% F$ \$PIN TRANSFER COEFFI-

CIENTS IN pI ELASTIC SCATTERING

Spatzerspa Rudalf Abegg [TRTUMT]

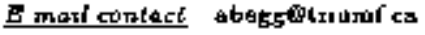

TRJUMF- 49B (Completed dota-taking L993)

ANALYZING POWER ZERO CROSSING ANGLES IN $n p$ ELASTIC SCATTERING BELOW $300 \mathrm{MeV}$

Spolespergon Cherlea A Dans [TRIUMF]

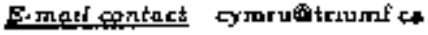

TRJUMF-503 (Completed dats-takung 1992)

MEASUREMENT OF ANAZYZING POWERS IN LOW

ENERGY

Spokmparion NR Stbvaboul |Baskatcbewan U|

TRIUMF.5O8 (Compleked data-taking l99l)

LOW ENERGY $5 d \rightarrow$ pp ANALYZING POWERS

Spoketpetson Edxisd I Matbje [Regros U]

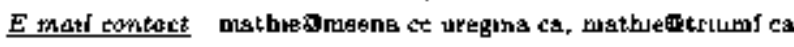

TRIUMF-SOB

STUDY OF THE $\pi^{+} d \rightarrow \pi^{-} \pi^{+} p p$ REACTION AT $T=240$ MeV

Spokexpernas Punsido Ruj (Trtarte U)

E-meti contect ruothtement ca

TRIUMF-557 (Completed dats-tsking 1992)

ELASTIC SCATTERING OF $100 \mathrm{MEV} \pi^{+}$FROM A POLAFUZED ${ }^{3}$ He TARGET

Spokeapersone Otto F Hotuager [TRIUMP and Sumon Fraser UI, B Latson (Simon Fraser U)

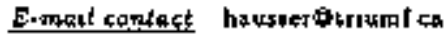

TRFUMF-580 (Completed dals-(akmg 1994)

LOW ENERGY ${ }^{+}$, ANALYZING POWERS WTTH

CHAOS

Spoketperson G $\mathbf{E}$ smith [THUMF]

E.mant contect smitboterich triuml ea

TRJUMF-561 (Completed data-takıne Feb le9l)

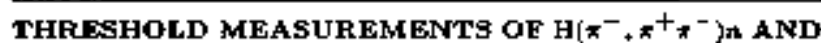
$H\left(x^{+}, x^{+} x^{+}\right) n$

Spokesperton Martın E Seviar [Briteh Columbin U]

E-mati contect msevorgitrumf ca

\section{TRIUMF-586}

ELASTIC PROTON SCATTERING FROM POLARIZED ${ }^{3} \mathrm{He}$

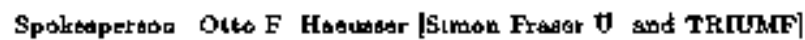
E.mant contact haubseritrumi is

TRIUMF-570 (Completed date-takisg Ita j992)

GAMMA-NEUTRINO ANGULAR COKRELATION IN MUON CAPTURE ON ${ }^{25^{\circ}}$

Spokexpernan David s Armatrang [LBL, Berkeley]

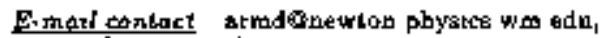
armdGreg trumf c*

TRUUMF-be2 (Completed datartatuag 2065)

RADIATIVE MUON CAPTURE ON ${ }^{3} \mathrm{He}$ By RMC Collaboration

Spokesperson Dendis H Wiright [TRUUNF]

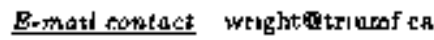

TRIUMF-5Bs (Completed date-taking Aug 1922)

INTEGRAL CROSS-5ECTIONS FOR THE $\boldsymbol{\pi}^{\boldsymbol{*}} \boldsymbol{p}$ INTER ACTION IN THE 3, 3 AESONANCB REGION

Spokesperaon El, Frodaran [Hebrew t]।

Esmarl contact elifnedfivms huj act th

TRIUMF-812 (Completed datp-teking 1900)

HYPERTINE DEPENDENCE OF EXCLUSIVE MUON

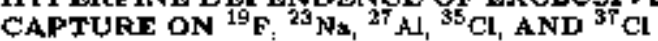

Spokespetacon Tum P Gotrenge [Keotilcky U]

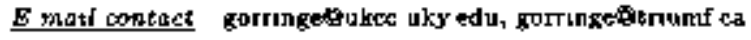




\section{SUMMARIES OF TRIUMF EXPERMENTS}

TRIUMF-624 (Completed datartaking Aug 1994)

THE $(\pi, 2 \pi)$ REACTION, A TOOL TO DETERMINE SCATTEBING LENGTHS AND COUPLING CGKBTANTE

Spakesperaons Rechard R Johngan, Martis E Savor [Butiah

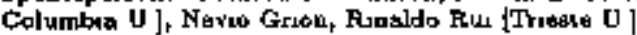

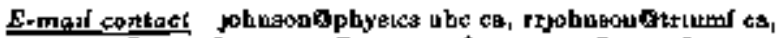
moeviotoriumf es, gnongtrieste info tt, grionetrinmf cs, ratotrumete

ThIUMF-630 (Comploted data-tateing 1996)

ELASTIC PROTON SCATTERUNG FROM SIDEWAYS AND LONGITUDINALLY POLAFIZED ${ }^{3} \mathrm{He}$

Spalcespertion B M Whittal [Sumod Fraser U]

\section{TRUUTHF-689}

MEABUREMTNT OE pD $\rightarrow$ gN, ${ }^{+}$AT 420 AND 600 MeV

Spokesperton Kenbeth H Hıcke [Ohpo U]

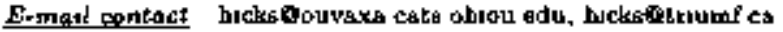

TRIUMP-649 (Completed dale-taking 2902)

TEST QF THE LOW ENERGY THEOREM FOR RADJATIVE PION CAPTURE

Spokesperans David A Hotcheou [TRIUMF], Michoel A Kovash [Kentucly U I

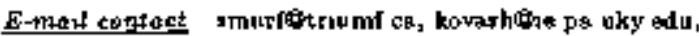

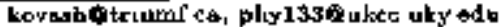

TRIURAF-659 (Completed date-kakug Aug 1903)

MEASUEEMENT OE THE $\pi^{+} x^{-}$INVAFLANT MASS IN NUCLEI AG A TOOL FOR DETERMINING THE MASS DISTRIBUTION OF THE a MESON

\$pokespersoge Nevo Griop, Fupaldo Atu |Truale Џ।

E-mard contecf gronotrumf es, grionatrieste infin th,

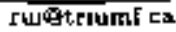

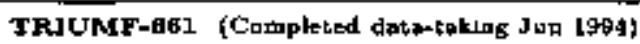

NEUTRON-NEUTRON SCATTERING LENGTH V1A.

$\mathbf{x}^{-} d \rightarrow$ ynn

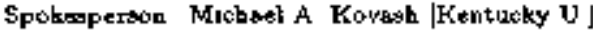

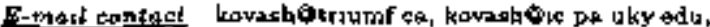

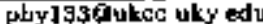

TFIUMF-68s (Completed dats-sking Sep 1904)

ASYMMETFUES FOLLOWING MUON CAPTURE BY POLARIZED MUONIC ${ }^{3} \mathrm{He}$

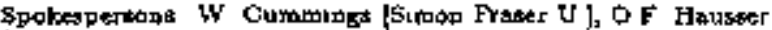

[Gimon Frager $U$ and TEIUMF

E-tmal comficte hausser bitrumf ca

TAUUAF-70- (ID preparadion)

CHARGE SYMMETRY BREAKING IN $\pi p \rightarrow$ d $\pi^{0}$ CLOSE TO THRESHOLD

By SASP-CSB Callaboration

Spokespersong Allens K Opper Alberts Up, Elie Korkman [Nort bers Bntulb Columbes to ]

E.mal contact opperaphys ualberte ca; loorkmaa aunbe adu
TRLCMF-T19 (Completed dats-takung 1995)

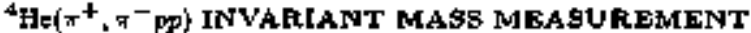
WITH CHAOE

Spokfoperson Grag $\mathbf{F}$ Snuth |TRTUMF|

$E$-mal contact amithotnumf cB 


\section{Nonaccelerator Experiments}

\section{UNDERGROUND-BOR.XNNO}

(To prepsiratien)

\section{BOREXINO: A SOLAR NEUTRINO EXPERTMBNT AT} GRAN SASGO

\section{EOREXINO DOLLABORATION}

Brief descrption BOFEXINO w one of the 'next generstion' solar neotrino projects at the Gran Saseo Laborstory Uses a Patudocumene based [inuid seintillator as netive modivm Measures the ${ }^{7}$ Be lune neutripo flux (energy $=0861 \mathrm{MeV}$ ) It is vary gensitive to the poutrinc oecllatione in both the scepariog of varumin awcillations and the MSW eftect Teste of the feastbility of the project bave beet suctestully completed For further in Formotion, plepan contect the Spokeeperson,

Frof Granpaola Gellin! [Milan U and INFN, Milom]

Aeloved eaperaments SAGE, GALLEX, SUDBURY,

KAMIOKANDE, SUPERKAMIOKANDE

E-traw contact bellnugm infin th, bellanglngs win it WWW Hornegage

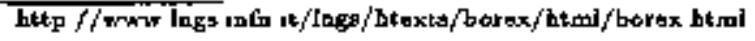

\section{UNDERGROUND-GALLEX}

(Froponed l9g3, Appraved Ape 1985, Began datsotsking May 1991, lo progretei)

\section{GALLIUM EUROPEAN EXPERIMENT}

\section{GALLEX GOLLABORATION}

HEIDELBERG, MAX PLANCK INST - W Hoinpel, G Hetgrer, I Kiko, T Kifytan ( $\checkmark$ 'spokespanon', M Laubenaten,

E Pernicks, W Rau, U Roenn, M Wojcuk, Y Zakbscov

FORSCHUNGSZENTFUM, KARLSRUHE - K Ebert, T Fntoch.

D Heldt, E Henrweh, L, Stieglit:, R. von Ammon, F Weirkeh

GRaN 5Asso - M Batale, F X Hartmand, M Sann

MILAN U - E Beltott], C Catador, O Cremoned, N FartarI, E Fronm, L 2 anatt:

MUNICH, TECH U - M Altmann, R hosebsuer, F won Fultitzech

NICE OBSERVATORY - G Berthomeu, E Schatzman

ROME U \& INFT, ROME C Bzcsi, Bellt, R Bermaber, S D'Angelo, L Paoluzi

SACLAY - A Bevilatinta, M Cribuer, L Goeged, J Fuch, M Spuro, C Tro, D Vignerud

GRODKHAVEN - J Boger, R L Hahn, J K kowley, A W stoenner, I Weneter

Accicterator NONE Detector Gounter

Reacitotis

$\nu_{e}{ }^{71} \mathrm{Gu}_{\mathrm{x}} \rightarrow e^{-71} \mathrm{Ge}$

Particiter studuted be

Brief descriptian Tbil on tadiocheracal geutrino experiment Giet 30 tons of gallium th the 8 2-molar GaCly solution Inoskalled tn the South Wing of Hall A of the Gran Santo Laboralory Has an overbead shyelding of absut $3400 \mathrm{~m}$ of wrater

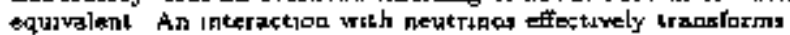
galloum chlotude into GeOl, which is then extracted froms the eolulion wh th ap apropropte gas purging gyatcm Counted in extremely dow-level proportionsl counters Sersitive to the luw energy mettrimus prodiced by pp fusion in the Sun Deshgned for su ordet of one avent per day Taking data (July 96)

Journet popers NIM A2TA (1889) 203, PL B285 (1992) 3T6, PL B265 (1992) 390, NIM A.329 (1493) 541, PL E314 (18日3) 145, PL B327 (1994) 377 , NP (PROC SUPPL) 35 (1904) 418, PL B342 (1995) 446, PL B 347 (1996) 237, and ASPF \& (1998) 23

Related experiments SAOE, BOFEXINO

E-matl corfact kirntokgamo mpr-hat mpg de WWW Home-page

http //kosmogc mpl-hd mapg de/gallex/gallex him

\section{UNDERGROUND-HOMESTAKE- CHLORINE}

(Proposod 1965, Approwed 1965, Began datataking 1970, In propress]

\section{THE HOMESTAKE CHLORINE SOLAR NEUTRINO} EXPERIMENT

PENN U - B T Cleveland, T Durly, R Davis, Ir

( $\checkmark$ Spokesperson), K Lande ( $\checkmark$ Sposesperson), C K Loe,

P Wildenhain

LEHMANN COLE - J VUman

Acsclemator NONE Detector Counter

Feactaons

${ }^{u_{e}{ }^{77} \mathrm{Cl}} \rightarrow e^{-37} \mathrm{AI}$

Paricler. Ifulined $\nu_{e}$

Brtef description The ${ }^{\text {sT }}$ Cl bolar neutrino detector in the

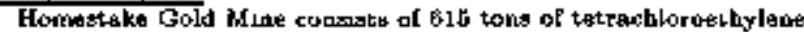

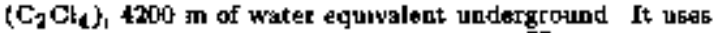
radiochement techsiques to determine khe ${ }^{37}$ Af production rate The delector whs built at BNL in 1963-67 and operaled by Brookhaven untll 1 的 4 At tbat towe the laboratory was

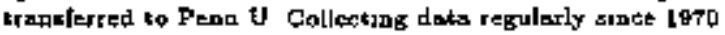

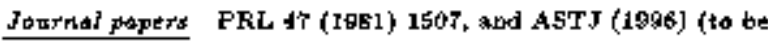
published)

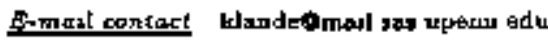

\section{UNDERGROUND-HOMESTAKE-IODINE}

(Propaned 1998, Approved J994, In prepartion)

THE HOMESTAKE IODINE SOLAR INEUTFINO EXPERIMENT

PENN U - a T Clevclatad, T Dally, R Dava, Jr, K Lands

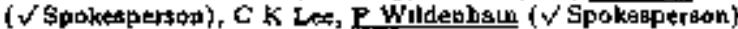
LEHMANN COLL - J Ultasan

LOS ALAMOS - R Barman

WASHINGTON U, SEATTLL - W Haxton

MOSCOW, INR - V N Gevetn

Acrelerator NONE Defiector Countar

Reactions

$v_{t}{ }^{127} \mathrm{I} \rightarrow t^{-127} \mathrm{Xe}$

Porlicies stadted ve

Bnef deycraptwon The ${ }^{127}$ I golor Deutrino expenmegt in the Homertake Gold Mine uses a detector medium of soduath codide degeolved ill wster The total detector mare of the unt nop under construction will be 235 kpnl of whych 100 tmms

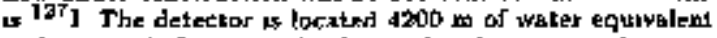

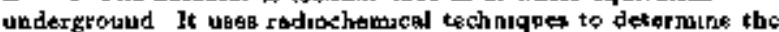
${ }^{127} \mathrm{Xe}_{\mathrm{e}}$ production tate The detector. which has an extroction the constant of 11 minutes, will bo fully rotionated ajd will opefals under eamplete compular control Twa excriations will be carrsed aut per day to besich for a Dxy/Night affect for olectron neutrinos from the dexay of ${ }^{7}$ Be Expected to besun dats tokung at che eud of 1996

Reloted etpertinents LAMPF-1213, JUCF-E-373

E-mart remitact tlandegraml sas upenn edv. psullogoodeckles sotro upenn edu

\section{UNDERGROUND-ICARUS}

(Approved 1995, In preparatjoa)

ICARUS: IMAGING COSMIC AND RARE UNDER. GROUND SIGNAL

CERN - P Gennis, 5 Gnttolu. G Manin, A Placa, I P Revol,

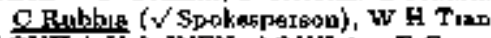
AQUILA U \& INPN, AQUILA - F Cavanna, E Olcjarcyk,

G Piano Mortari. M Verdecchis 


\section{SUMMARIES OF NONACCELERATOR EXPERIMENTS}

BEIJNAG, IHEP - L K Ding, Y Lu, F Lu, J M Ms, J F Qu.

H Y Sheng, $K$ L Tung, J Y Zong, B Z bang

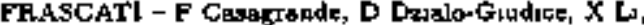

G Minunocehu, \& Mntto, P Piechi

UCLA - D Clint, W Hogg, Q Muratori, 5 Otwnewaks, J Patk, H G Wang, M Zhoir

PADUA Uf \& INFN, P\&DUA - A Betlin, C Carpenter, S Centro

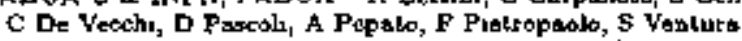

PAVIA U \& INFN, PAVTA - P Benett 1 , E Gallegarich, R. Dolfini,

A Gigl-Berzotari, F Manri, L Mazzone, C Montenari,

A Pinseoli, A Rappoldh, G L Rarefli: M Robeclla, D Scannochio, C V ISnol

PISA U \& INFN, PISA - F Serpogmptri

TURIN, CO\$MO-GEOFL\$ICA LAB - L Perrale, S Sarukı

TEXAS U, DALLAS - E FEynytas

MILAN U \& INFN, MILAN - D Carall, 5 Cebsus, A Fatrar],

$P$ Gibattb, 8 Rescont, $P$ Sala, M Terran?

Accelenator NONE Detector ICARUS

Porticles aftidied pin

Bref dexcripteon The Rrst phate of the project waludes the centrityctson of a s00-ton detector at Gran Srano Laboratory ThJs could bo done wathen the next three years In the second phese, a 5,000 -ton detector will] be built The ICARUs dintartor represents a jew geptration of bobble chambers, and will uas the ulcra-pute liqud argon and the rasdout technique (LArTPC) of ionizstion dats it is designed to prexide three-

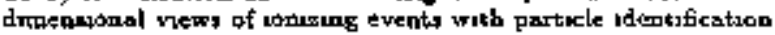
from $\mathrm{d} E / \mathrm{d} x$ and rang trosurements It 18 aleo a homogeneous calorimeter of wery fine granulatity $\Delta$ nd higb accuracy, and thut a good tool for rare evept searches, such to proton decays

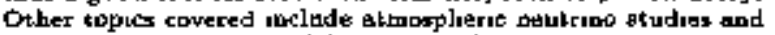
golar neutrino detection (aboye $5 \mathrm{MeV}$ ) It so expected that

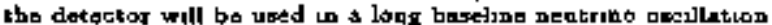
experimest with the $v$ beam from CERN (T32-km to the North) Under congtaction (July (66)

Jaurnal papers NIM AS15 (18日2) 223, N1M A327 \{1993\} J73, NIM A332 (1993) 395, NIM A333 (1903) 587, NIM A345 (1994) 230, NIN A346 (1994) 550, NTM A355 (1985) 680, NIM A356

(1905) 507, and NUMl A3B6 (1995) 528

Retated experyments SUPERKAMJOKANDE

$\boldsymbol{E}$-mati contact carlo rubbiagcera ch

WWW Home.page hetp //Www aquila wof it/ccafuy/

\section{UNDERGROUND-KAMIOKANDE-III}

(Began dala-taking Nov 1985; Campleted dat;o-takıg hpr (996)

\section{THE KAMYOKANDE EXPERIMENT}

TOKYQ U, tCRR - Y Pukada, T Hayakawn, K Inowe, T IBluds,

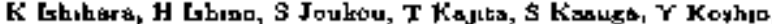

T Kumila, K Matsamioto, M Nabegbata, K Nakaraura

K Okutrore, A Sstal, M Shlozsurs, I Suzuks, Y Suzuks,

$T$ Tomoeds, $Y$ Totsukn ( $V$ spokesperson)

TOKYO U, INS - E Ithıbers, S Miyamoto, K Nighikswa

KEK - K 8 Hirata, K Kuhere, Y Oyame

TOKAI U, gHIBUYA - T Hokuch, N Korbuba, K Nishyıms KOBE U - T SudA, A T Suzukı

NITGATA U - T Ishizula, K Myymo, H Obaxama

OSAKA U - T Fora, Y Nagathema, N Takta, T Yomaguch

TOKYO TNST TECH - Y Hayalo, K Kaneyuls, T Sinzulı,

$Y$ Takeucbi, T Themor.

GIFU U - 5 Tasaks

TOHOKU U - K Fujito, \$ Hatakeyama, M Kaga, 9 Marayams,

A suzvkı

MIYAGI 0 OF EDUCATION - $\$$ Mark

Anceterator NONE Deteritor Counter

Reactions

$\nu \epsilon^{-} \rightarrow \boldsymbol{r} e^{-}$

$\bar{v}_{e} p \rightarrow n e^{+}$

Partrates studized $P$ n. monopole, minon, $p$

Bref description A 3000-ton water Ceteskow detactar, $2700 \mathrm{~m}$ of water equuvslede anderaround The KAMIOKANDE-I detector has been upcraded with new electronics, TDC'B, aud one thousend 20knch phototvbes surrounded by alumwared

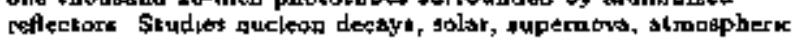
and high-epergy cosmic neutrituos, high-epergy vuwons, etc The eecond phase was completed in April B0, the third phase atsred in Qctober 90 Date taking offinally ended in Aprl 96,

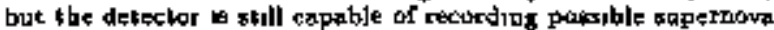
qeutinost Dats andyst in progreas (July ob)

Jowinal papers NIMI 20S (1983) 443, JPSJ 54 (1986) 3213, JPSJ 54 [1985) 1065, PRL 58 (1986) 991, JPSJ 55 (1986) 711, JPSJ 55 (1986) 3786, PR D3A (1986) 902, PRL S8 (1987) 1490, PIL $59\{1987\}$ 2604, PR D3e (1987) \$537, PL B205 (1988') 4]E, PRL 81 (1888) 385, FRL E1 (1988) 2653, PR DS8 (198B) 4AB, PL E220 (1989) 308, PRI 63 (1969) 16, PF D39 (1989) 1481, ASTJ 359 (1999) 57., PRL 65 (1990) 1297, PRL 65 (1990) 1301, PL B270 (1981) 89. PRL 66 (1991) \&, JP5J 60 \{1981) 280a, PR D44 (190)) 2843, PR D44 (1991) 617, PR Des (1901) 2220, PR D44 (1991) 2241 [9tralum PR D45 (1902) 2170], NMM A320 (1992) 310, PL B278 (1992) 2)7, PL B280 (J992) I46, PL E283 (1992) 446, PL B289 (1992) 463. PR D45 (1992) 3355, NIM A329 (1903) 290, FL B371 (1993) 36T, NP (PROC SUPPL) 31 (1993) J05, PR D48 (1993) 5505. NJM A340 (1994) 612, TL B335 (1994) 23\%. ASTJ 435 (1994) 226, and PL B37 4 (1396) $2 \$ 8$

Redated experzosents KEK-261A

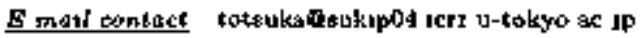
WWW Hame page

http / / www-sk rar v-tokyo ac Jp/dec/ksm/tadex htral

\section{UNDERGFOUND-SUPERKAMIOKANDE}

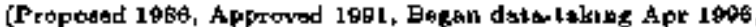
In progras)

THE SUPEF-KAMIOKANDE SOLAR NEUTRINO AND MUCLEON DECAY DETECTOR

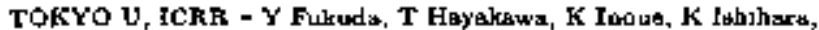

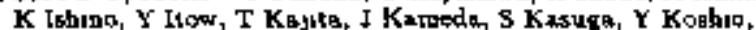
K Marken, M Murr, M Naknhata, M Obeta, K Okumus M Oba, N Sakura, M Shlozawa, Y Sueuk, Y Takeucbi. Y Tolsuks ( $\sqrt{ }$ Spokesperson)

KEK - JKanakk, $K$ Nekscaurb, $Y$ Oyoma, $M$ sakude, $O$ Sarakı

TOHOKU U - K Fuுts. A. Hasegatra, T Haqegawd,

S Hatskeyams, T Iwamoto, T Kınebucht, M KogB, T Maruysma. H Ogawn, M Seito, A Suzuk1, F Trusburo

TOKAI U, HIRATSUKA - M Eto, M KonbibB, K Nushıma OSAKA U - A Kusano, Y Nagsehime, M Tikits, T Yamaguch

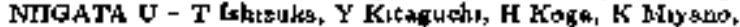

H Oksrsws, M Thkahsi:

TOKYO INST TBCH - Y Haysto, Y Kanays, K Kaney Llkt, $\checkmark$ Watanabe

GIEU U - S Taraks

MIYAGI U OF EDUCATION - M Mot

KOBE U - S Echtgo, M Kobnms, A T Surak

TOKYO U, INS - E Ichhark, T Inggaki, K Niahıkama, A Sahk

BOSTUN if - M Etri, E Kearns, S B KIm, M Mesar, J L Sitone. L R galak, K Yoaheds

BRQDKILAVEN - M Goldbaber

UC, IRVITE - T Earkezak, W Gajeretkl, P G Halvetson, J Hsn,

W R Kropp, L R Proe, F Reines, H W Sobel

CAL STATE, DOMINGUEZ HDLIS - K Gencer, W Keg GEORGB MASON V - R W Ellawarth

HAWAIl 0 - J Flanggan, J Loorned, S Matiuno, V Slonger LOS ALAMOS - T J HвйeB

LOUISIANA STAPE LS E Blonfust, A Sanford, F Svoboda, M R Vognt

MARYLAND $\mathbf{u}$ - M I Chep, $\mathbf{z}$ Conner, J A Goodman, G Sullıvan SUNY, STONY BROOK - J Hıll, C K Jugg, C Mauger,

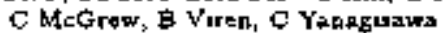

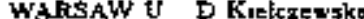

WASHINGTON $U$, SEATTLE - $V$ Chaloupla, J George, A Stochyra, L ל2, J Wilke6, K Young

Aceelerator NONE Datectior SEPFR-KAMIOKAWDE

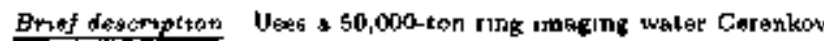
detactor ax a depth of $2700 \mathrm{~m}$ of water equivelent (mwe) in the Kamrok Mozum: tolne in Japan The detector consists of 


\section{SUMMARIES OF NONACCELERATOR EXPERIMENTS}

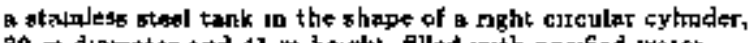
39 ar diameter and $41 \mathrm{~m}$ keugbt, fllled with porified water It a optically mgmeuted wito an נnner rolume, and au ouler (ant-concideace) region The inner regob at unwed by 11,200 photomultiplete tubes (PMT'B) The onater susulas is usid to teg enterng muont as will as to attenuste lopenerg; $\gamma$ 's and neintrous The outer region as vewed by 1,B00 PMT's Takin: dats (July ge)

Related axperments KBK.962

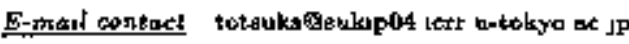
WWT Horte-poge bttp / / www-sk ICrr u-tokyo bc JP/

\section{UNDERGROUND-KGF}

(Began datz-takıng Ott 1980, Conppleted date-tsking 2982)

\section{THE KOLAR GOLD FIELD EXPERIMENT}

TATA INST - H Adarkar, S R Dugad, s D Kalmant,

Y R KTohnagramy, J D Kuikerm, h G K Menon, It K Mondal,

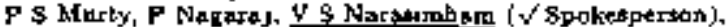

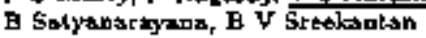

OSAKA CITY U - Y Haysaht. N Ito, S Kawstam, T Mtiudyrm,

$T$ Nakamura, $K$ Tagrka

KANAGAWA U - S Siyste

Accelefotor NONE Defector Calortmeter

Partictes stirdied $\mathrm{P}, \mathrm{th}$

Brfef descriptron Phase-I of the experiment was cormpleted in 1985 The phego-jl detectior ix a 280-ton Iron trackug calonuter with 60 layers af pruporlignal coupter tubs, $\theta 600 \mathrm{~m}$ of water equisiont undarpround A monopole detector has been added in phase-III Siudies vuteleon decays and nemiches for macrotic monopoles and poinl sources of high-energy $v_{\mu}{ }^{\prime}$ * Completed in 1892

Jowinal papers PL B106 (1981) 339, FL B115 (19k2) 349, FL

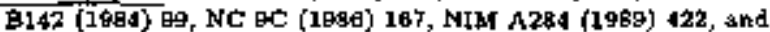

PL B257 (1991) 138 No further papers expectech

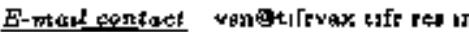

\section{UNDERGROUND-LVD}

(Froposed 1984, Appraved Apr 1985, Beran dataskakıng Jun 1992, In prograss)

SEARCH FOR STELLAR-COLLAPSE NEUTFINOS WITH THE LARGE VOLUME DETECTOR

\section{LVD COLLABOFATION}

ASHIKAGA JNST 'IECH - K Salloh

BologNA U - F Anloniol, G Bar,, M Basla, F Berbenl,

G Bondl, G Brusa, G Cark Rompo, 1 Cifarell, F Cindolo,

A Conbn, L Emald, C Gbett, P Gusut, F Griapti, G Iacobuccs,

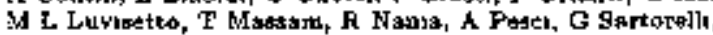

Id Selvi, A Zlchechl ( $\sqrt{ }$ Spoktapernon)

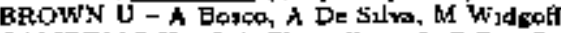

CAMPINAS U - J A Cbucellato, L G Doe Sautos, E Kerap.

N Mengottp Sulva, A TuJtell,

FRASCATI - F L Fabbar, G Uacearrone, L Vat tno

GRAN 5 ASSO \& INFN, ASSERTI - N Tsborgns

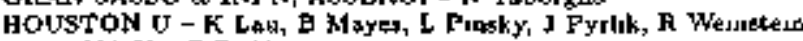

INDIANA U - E D AlYEA

MIT, LNS - M Deutach, E S Hefen, $P$ Handse, I A Plexx, I Tang

NORTIIEASTERY U - J Moromtsato, E Von Goelar

MOSCGW, INR - V S Berrzınky, V L Dadykin, R. I Euskeev,

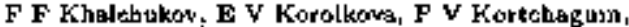

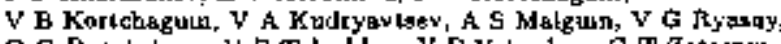

O G Ryazbskoya, V P Talochkin: V F Yakushev, G T Zatiepin

OKNYAMA U - S Ttujl, T Wada

OKAYAMA UNTV SOI - I Yenomoto

PERUGIA U \& INFT, PERUGIA - B Alpat, I Uman

SAITAXIA U $N$ InouC

TURIN, COSMO-GEOFISICA 1AB \& TURTN U \& INPN,

TURIN - C Agluetta, G Badino, M Bertatna, R. Berton,

C Ceptaguol, $A$ Certallina, A Chiavases, W Fulgione,
P Gateott, P Ghia, $R$ Grandla, C Meleqrads. C Morello, G Navarsa, L Panpin, L Perisle, P Piech, 0 Sapvadra,

GC Trinchera, P Vallania, 5 Veraetly, C Vagorito

UREINO U \& INEN, PLORENCE - G Conforto, P Domenda. G God, R Monlovani, A Maght, 5 Swalıbl, F Vetrapo

Accelefutor NONE Deteclor Seintillator, Stztamer eharabet

Reactions

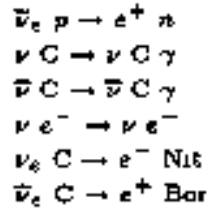

Parfacles sfudted $p_{1} \boldsymbol{n}_{\text {; }}$ nuton,

Bruef description The experiment is located in the Gran Sasoo Laburatory al a minimini depth of about $\mathbf{3 0 0}$ mwe The apperatus contists of a atresmer tube trackung aysteon intarlagved with a large valume of lequad tentillator and its support structure whtch acts al a pasive absorber lt 15 a high praciston trackeg ctlorimeter anth the mejor patt of ita volun

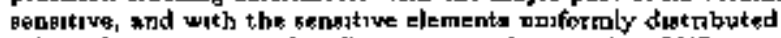
of the flve topwers whwh will congtitute the complete LVD. the firgt one is operstrongl sures Juse 92 and the eecond one

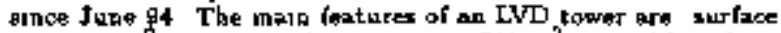

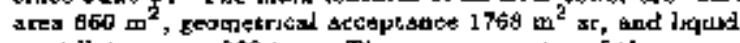
scutillator mass 368 tons The mejor purpose of the experiment

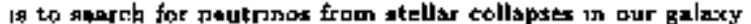

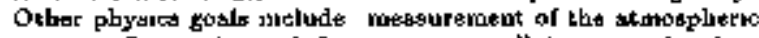
neutnno flux and eesch Ior netring ptallitions, stody of the spectrud and arteractions of cogmic rsy motons and muon bundles, and inygatigation of everts detected in time colacidence weth the EASTOF experiment at the surface of the nountsta Tokeg dete (July $0 \%$ )

Jotargal papete NC C9 (1806) 237, NIM A26d (1988) B, NIMI A274 (1989) 177, NTM A27T (1989) 11, NIM A27T (1989) 17, NIA A295 (J990) 469, NC 105A (1992) 179s, NC 105A (1992) 1815. NMI A329 (1093) 521, NP (PQDC SUPPL) 31 (1998) 400, NP (PROC SUPPL) 35 (X994) 24D, NP (PROC SUPFL) 35 (3094) 243, NF (EROE SUPPL) 30 (1994) 259, NP (PROC SUPPL) 30 (1994) 267, ASPP 2 (1994) 103, ASPP 3 (1995) 311, and NC 18C $\{1906\} 628$

Retoted expesmentr MACHO, KAMIOKANDE, SUPERKAMIOKANDE

E:man? confact gartorellinbo infn it

\section{UNDERGROUND-MACRO}

(Proposed 1884, Approved Apr 1985, Bectat dstombtkent Feb 1989, (s) pragress)

MONOPOLE, ASTAOPHYSICS, AND COSMUE RAYS OBSERYATQRY

MACRO COLLABORATION

BARI U b INFN, BAFI R Bellatth, F Cafagna, M Calicchn, $M$ Castellano, L We Eentadietis, G De Catsldo $C$ De Marzo. O Brrliquex, C Fryurat, P Fusco, N Giglietico, P Guarnaccls,

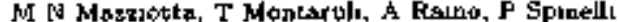

BOLOGNA Uf \& INFN, BOLOGNA - S Cuachin, H Dekhussi, R Fantini, G Glacomelly (V/Spolesperson), F bickarouh,

G Mandrlali, S Manzoor, A Margotts-Kerı, L Patruzl, V Popa.

P Serra-Lucareal, M Spufio, V Togo

BOSTON U-E Keards, C Okads, C Orth, J L Stone, L F Sulak

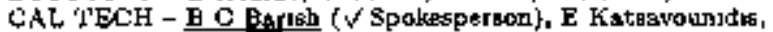

S Kyrlaropoulou, N Longlay, D G Mrchael, R Nolty, C W Feck, $\mathrm{K}$ Scholberg, $\mathrm{C}$ w Walker

DREXEL U - C Lane, R Stewober

FEASCATI - G Batiatoni, H Bilokon, C Blonse, M Carbon,

V Chisalla, C Forci, E taroç, A Alarını, Y Patera, F Ronga.

L Satta A Sciubba, M Spritets

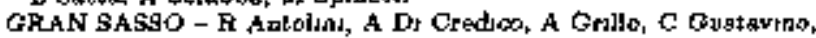

S Mulhayew, S Pardach. J Reynoldon, E scapparone

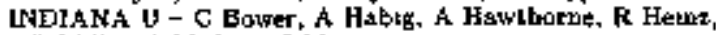

1. Miller, s Mutson, J Musser 


\section{SUMMARIES OF NONACCELERATOR EXPERIMENTS}

AQUICA U - 1 De M|kTI, P Magacali,

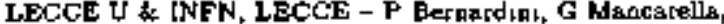
D Martello, O Palamata, 5 Petrers, P Pittill, A Surdo

MICHIGAN U - R Baltat, \& Coutu, K Hamoon, D Levin, M Longo, G Tarle

NAPEES U, TFS * INFN, NAPLES - M ALrbbrobo

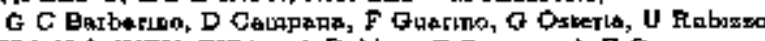

PISA U \& INFN, PISA - A BoldinI, C Eemperat, F Ces,

G Gixpman, M Grassi, D Nitolo, R Pazzi

FOME U - G Aurremms, S Buseino, M De Vurcenzi, E Lemanos, $F$ Lipari, C Setruano, M Seven

TEXAS A AND M - A Sarzart, R. Wobb

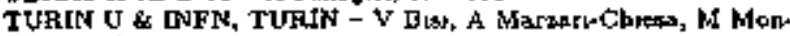
teno, M Sitts

Accelerator NONE Detector MACRO

Particles studicet monopole, muran, w

anef descroptur The MACRO detector hat been primatily deagned to conduct a search for superoaseave geand unfied manghtetic monapolea It it a general porpant detectot, wheh 14 also sesrchtng for nuclearikes, WIMP'6, frsctionsl charge pasticies, $\overline{v_{e}}$ from atellar qravitationd collepses, heghenergy

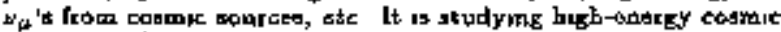
ray muone (vertictl intenenty, geabonal variation, moon shadow,

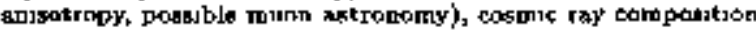
at bigh onergics, atmosphorec neutrinos, ete Operstes in cojncrdence with an atr shower erray (EASTOP) to study the

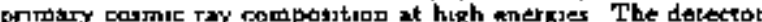
bes six oupermodules in two lavels, esch instrumented ta operate endepeidently of the pthers Each lawer sppermotule constgte of a borrental array of two layera of bquid acentillation countsis, ton leytats of limited strosimer bubas, one layer of CR39 abclest track detectorg aul weyed layera of abrorbecs The upper pars (Attueo) bas faur kerigantal layers ol shrepper tubes abd obe tayer of gctatilfators The eldes are covered with one layer of sremtillators and 6 layors of streaner tubes The CR39 detector w also mounted on the east verkical ande and on the sorth lower yide The globat dimedsions are $12 \times 78 \times 9 \mathrm{~m}^{2}$ and it contalts

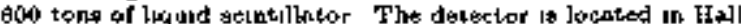

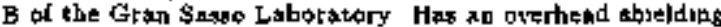
of sbout 3800 w of water equrvalent Taktng dats us the Intl configuration (July 为的)

joumal papars NC $9 \mathrm{C}$ (1996) 281, NIM A23) (1989) 213, PR D42 (1890) 1396, FL B245 (1890) 149, NIM A 300 (1991) 5B NIM Aan (1901) 275, NP (PROC SUPPL) 24B (1991) 101, NIM A321 (1992) 609, PRL 69 (1992) 2660, ASPP 1 (1982) 11, PR. D46 (1992) 895, PR D46 (1992) 4839, NP B370 (1992) 432, NIM A32s (1903) 337, ASTJ 412 (1993) 30. PL As37 (1994) 376, PRL 72 (1994) 60A, PRL 73 (1994) 1308, PR DSO (1994) 3098, FL Ba57(1905) 481, ASPP (1055) 93, and PR D52(1998)

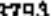

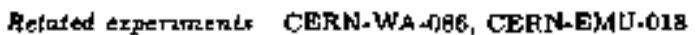

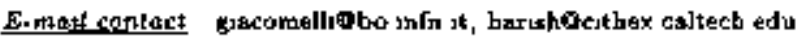

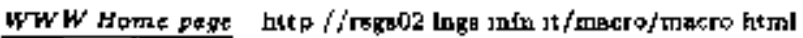

\section{UNDERGROUND-SAGE}

(Ptopones 1984, 1984, Approved 1985, Begen dataklaking May igse, In progrest)

THE RUSSIAN.AMEFICAN GALLIUM GOLAR NEU. TRINO EXPEATMENT (SAGE)

SAGE COLLABOFATION

MOSCOW, INR - J N Abduranhitow, $V$ N Gnvrin

( $Y$ Spoke A V Kalikhoy, N O Khalrnasoy, $T$ V Kaodel, I N Mirmov,

A A Shakhw: E P Vereteakin, V M Vermul, V B Yanta,

G T Zatsepun

LOS ALAMOS - T J Bowled (V Spoke6pergon), J S Nroo,

W A Teacdole, D L Wark

WASHING TON U, SEATTLE - \$R Ellott, J F Walkerson

PENN U - B T Cleveland, T Darly, R Dave, K Lande, C K Lee,

$P$ W Wildenbain

LOUISLANA STATE U - M L Cbercy

Acceifrater NONE Detectar GCNT
Reatront

$$
\nu_{e}{ }^{T^{2}} \mathrm{Gt}_{\mathrm{t}} \rightarrow e^{-71} \mathrm{Ge}_{\mathrm{s}}
$$

Partictes studteri V*

Bref descraptor Ute日 the Galtum-Germabium Neutruo Telescope (GGNT) ust naled in Bn underground laborstory butk un the Bakgan Neutrino Observatory, Northern Caucesur. Rucsas Has an overhead ablelding of about $4700 \mathrm{~m}$ of wotas equjuatent Sespstive to the kow enefay neutrinas produced by the pp fuswn to the Sur Explots the radichencal proctedure

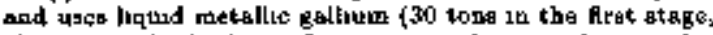
57 tors in 1991). A purification procedure, implemented begtnojug with the Jaminzy 90 extrartion, resalted th a significant bockgreund reduction The SAGE-II phose tog in Sejpleruber 92 Counts the $K$ and $L$ peaks in ${ }^{T}$ Oe decay, w7th 57 vons of $\mathrm{Ga}$ and low background A calbrration with \& ${ }^{51} \mathrm{Ct}$ artificial peuterwo source of Bbout $05 \mathrm{mC}$ ectivity was carrod out w 1996 Takjng data (Tuly 96)

Journat papers PRL 67 (1991) 3332, and PL BS1B (1995) 234

feploted experments GALLEX, BOREXINO

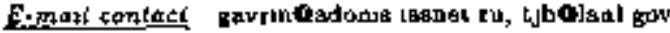

\section{UNDERGROUND-SOUDAN-2}

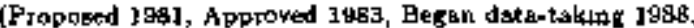
In progrese)

\section{THE SOUDAN-2 PROTON DECAY EXPERIMENT}

ARGONNE - D 5 Ayren, $T$ F Field, dS C Goadman, E N May, L E Price, \& V Sidleın, I L Thron, 3 L Uretsky

MINNESOTA U - C R Bode, F M Bordet, H Courant,

D 41 Deknth, R Gran, 5 M 8 Kaeabara, N P Longtey.

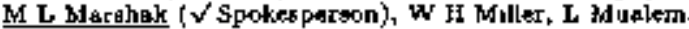

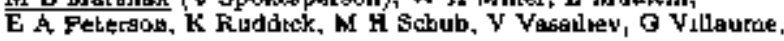
5 Wakely'

OXFORD U - W W M Allison, C B Brooks, J H Cobb

H M Gallegher, D E Porkms. A 5tassingkas, N Weat, U Wielgosz RIJTHERFOFD - O J Alper, D I A Cockertll, R J Cokton, P J Lutchield, G F Pestce

TUFTS U - B Ewrn, T Kafke, W Leesan, W A Mand,

R H Nuburn, A Napier, W Oliver, J Gibuaps, N Sundaralingam WESTERN WASHINGTON $-W$ L BArrett

Accetentor NONE Detector Cajortmeter

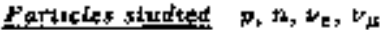

Bref detcription A. 860-ton ton tracking calorineler uges drift projection tubos arranged in s hexagoned arrsy The tubos are $15 \mathrm{~mm}$ is dismetsr e日pacated by $16 \mathrm{~mm}$ of gteel Trigger threxholds are 100 MeV kinetie entrgy for muns and 150 WieV for electrons The matn detectar us orapletely eutrounded by a $1700 \mathrm{~m}^{2}$ actsve pheld of proportioul tube whach udentries avente associated wath coemic ray muoss $A$ charged perticle test.beata calibration of the d 3-ton calorumeter modules has boser completed A surface array and an anr C̈erenkav detector are operaled a coincidenet with the SOUDAN.2 detector to provide informasion about the ayr showers which prodede

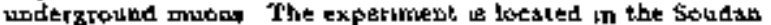
tune, Mumnerots, $2090 \mathrm{~m}$ of water equisitent underground The data taking began in mich-1983 when 275 tons of the detector were dnstalled The detector was coraplated In late 1993 Physucs topres include studies of nuckenn decay, stmosphersi neutrings and neutrono dacillatians, casmic ray cpmpasition, and eastches for masoetrc monopoles and pornt aources of canme rays Takjne data (Juby 9 ) The collaboration has Elbs praposed the use of the SOUDAN-3 detectar lax a long baseline neutrins ocillation experiment MINOS (see FNAL-875)

Noutna' papers NIM A27B (1989) 371, NMM A283 (1989) 642, PR D42 (1990) 2987, JPHY \$17 (1991) S393, PL. B269 (1991) 220, NP (PEOC SUPPL) 28A (1982) 377, FR D46 (1992) 4646, PE D52 (1995) 2760, and NIM A37B (1996) 36

Reiated rxperaments FTAL-822, FNAL-B75

Emat contact mowshak omnhepl hep umn edu WwW Home-poge http //bepwew rd ac uk/goudan2/indox htm 


\section{UNDERGROUND-SUDBURY}

(Propoted 1995, Approyed 1990, ls preparation)

\section{THE SUDBURY NEUTRTNO OBSERVATORY (SNO)}

\section{SNO COLLABORATION}

QUEENS U, KINGSTOA - E P Bouvin, I Erhards, H C Evads,

G T Ewsn (V Spokesperson), R J Ford, A L Hallu, A Hamet,

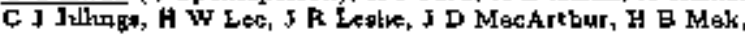

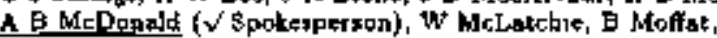

T J Radchif $\theta_{1}, B$ C Robertaon, P Shensutd, R L Steventan

CHALK RIVER, AECL - E D Earle, J D Jlepbarn, G Jonkmana, B Sur

CRPF, OTTAWA - W F Deridkon, $F$ Delpokr Verens,

C K Hargrove, $K$ MeFaslane, T Nobje, V M Noviknv, M O'Nell, M Statkay, D Sinelair

GUELPH U - T Anderean, $M C$ Chon, $P$ Jagan, J Law, F. Ollethead, J J 5 tmpuran, $1 \times$ wang

LAURENTIAN UT - I Bigu, E D Hollman, A U Hand

J G Hyksway, A Roberge, E Sapteler. C I Virtue

BRITJSTH COLUMBIA U - R Kelmer, R Komar, A Poon,

C Wojtham

PENN U - E W Beser ( $\checkmark$ Sppkesperson), D Cowen, W Frsti,

J R. Klem. D MlcDorald, $F$ M Newomer, $R$ V Vap de Water.

f. Vas Barg, 3 Waug, $P$ witt $10 \mathrm{~h}$

LOS ALAMOS - T J Brwleg, S J Erck, M M Fowler,

A Goldschmidt, A Hurne, P Thornewell, J B Wilhelmy.

J S Wauter:

LEL - Y D Ghen, MI P IEeac, K T Leako, M E Moortead,

E $B$ Norman, A R. Simuth, $R$ is Stakatad

WASHINGTON U, SEATTLE - Q R Abmad, J Back,

M C Erewne, P J Doe, C Duba, 5 k Elliolt, J V Germam.

F Menjes-Drees, A G H Robertion, T D Stelger, J F Wilkerson

OXFOFD U - J O Barton, 8 Biller, M G Bawler, $X$ Chen,

0 Dopcas, H $D$ Heron, $N$ i Jelley ( $V$ Spokesperson), A B Knox,

W J Lotko, J J Lyoa, N W Tsnaer ( $\checkmark$ Spaketportant)

F. K Taplun, AI D Thortes, F T Trent, D L Wark, N We日t

BROOKHAVEN - J Bager, R L Hahu, I K Rowley

Accelerintor NONE Defoctor Conunter

Besations

$$
\begin{array}{ll}
v e^{-} \rightarrow \nu e^{-} & - \\
v_{s} \text { deut } \rightarrow p p e^{-} & - \\
\nu \text { dout } \rightarrow \nu n \nu & - \\
\bar{v}_{*} \text { deut } \rightarrow n n \mathrm{e}^{+} & - \\
\bar{v}_{e} p^{+} \rightarrow \mathrm{ne}^{+} & --
\end{array}
$$

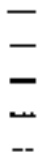

Earficter studied v

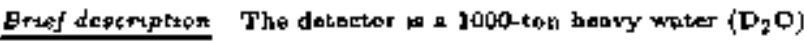
Cereakov detertor dasenred co study nevtrinos from the Sun and other satrophyscal solarete The use of hobyy wister allow boch electron neutruors and all athar types of noutrinos to be otarerved by three r.mmplementiry reactiank The detector will be senajtive to the to flux and snergy spectrum shepe and to the cotal beutrino flux urrespestive of neutrino typ- These mpinturemeats will provide jofarmation on hott vacuum neutsine

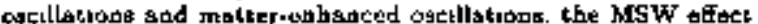
In the event of a superyova 3 will be very benalstye to $\nu_{a}$ and $v_{-}$

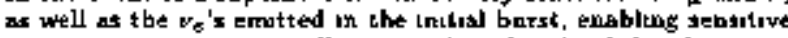

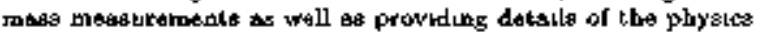
of stellar collapere The underground cav'ty is curculete and equipront ts being installed The detector is scheduled to be filled with hopvy water I Spring 1997 In preparation (July \%6)

Journal papers No $9 \mathrm{C}$ (1886) 308, PL B194 (1887) 221, NIM A314 (1902) 373, and NIW A.970 (J996) 579

E. mal caniact ewangmlper phy qutepsy ca,

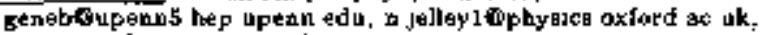
$n$ tooger i Ophysics oxford ac uk

WWW Hope page htip //saodung phy queensu ca/sNo/spo ht mal

\section{UNDERICE-AMANDA}

(In preparation)

\section{ANTARCTIC MUON AND NEUTRINO DETECTOR:} AMANDA

\section{AMANDA COLLABORATION}

UC, BERKELEY - D Luwder, T Miller, P B Price, A Puchard, LBL - D Nygren

UC, IPW'INE - \$ Garwsck ( $V$ Spokesperson), P C Mock,

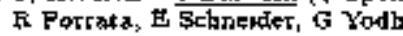

WIgConsIN U - A. Coultbard, K Engel, L Gray, F Halzer

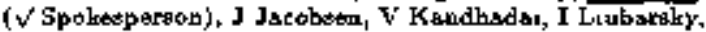
i. Morew, J C Spang, S Trlav

STOCKHOLN U - P AnkebjeT, L Bergatrom, A Bouchts,

E Dahlberg, B Er]wadteron, A Goobar, P O Hulth, S Johtsutbon, Q Sun, C Walck

UPPSALA U - \$ Canus, A Hsllgren, H Rubingtein

DESY-IFH, ZEUTHEN - H Hevkenksmp, \$ Hundertpasrk,

A Karla, C Spsering, O Streicher, T Thon, R Wuchuewakt

BARTOL RESEARCH INST - T C MJ]ler

Atcelemator NONE Detector PMT

Partictes stadied v

Emef description AMANDA it a bjgh-energy neutrina observa-

lory whych uge the deep Sauth Pole we as a target and a track.

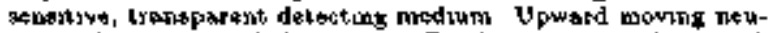

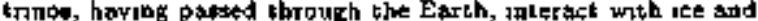
produce muong The mosons ganerate Cerenkov radistion which cpn bo recorded by ghotomiltipleer tubes (PMT) The tubes are placed in verticel ghafts melied into che icacsp to the depth of $1720 \mathrm{kn}$ The date sequettion ta beadled in a countiog tactity at the surfoce As of July 86 , a tolsl of 173 operatung PMTe has been metalled The plan ig to have at lesst sob opticad modulex npernting before the end of the tentuty Under construction (July on)

Joumat papers NATU 353 (1991) gon, and SCl 267 (1905) 1147 Aelatad agpremerts BATKAL, DUMAND, NESTOR

Eman' antact tbarwictanci edu,

hatzon iptenxk physics wisc adu

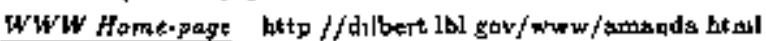

\section{UNDERWATER-BAIKAL}

(Proposed 1964, Approwed Jan 1987, Nor 1992, Began datzor takeng 1993, Ja progrtas)

THE LAKE BAIKAL DEEP UNDERWATER NEUTRINO TELESCOPE + NT-300

\section{BATKAL GOLLABORATION}

MOSCOW, INR - L B Bexrukur, 9 A Borisorets,

I A Danilcbenko, Z A M Djukibaev, $G$ V Domogataky

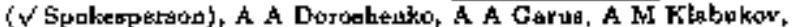
SI Klimuabin, B K Lubkandorzbsev, A I Fanfilov, D P Pelukbou, P G Pokhil, I A Solklaks

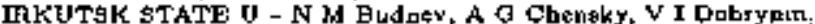

O N Geporenko, T A Gress, A P Koshechkin, F . M Mrrgazon,

A. $V$ Morra, $S$ A Nikiforov, $Y V$ Pstfenov, A A Pavlov,

K A Pockojkin, P A Pokojev, V Y Rubaov 5 I Sunegoritky,

$V$ a forthansky

MOSCOW STATE U - S B lgat'ev, L A Kuzmuthes

N [ Moselka, E A Onipara

NI2HIVII NOVGOROD \$T'T'E U - S V Fualloorstry, V F Kulepor M B Mflenin

ST PEIERS自URG, MARANE TECH U - M 1 Razanov

KURCHATOV INST, MOSCOW - A I KlımoN

DLBNA - I A Belolaptikov

DESY, ZEUTHEN - A Karle, T Mikolajaki, D Fandel, C Spioriag. O Strescher, T Thon, C Wiebuach, R Whechnewski

Acceletritur: NONE Detector Covnter

Partzitey gtudied $\quad \forall$, muon, nionopole

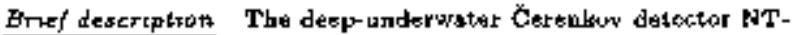

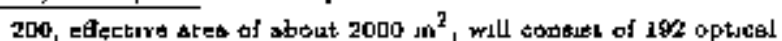




\section{SUMMARJES OF NONACCELERATOR EXPERIMENTS}

modules srranged on $B$ gtragt at 1100 m depth The modules are exutpped with $37 . \mathrm{cm}$ QUUASAR phototubes The experument otudieg muppx geperatod in nevtrino interactions or in repction of primary cotinuc rays in the attposphere, and searchen for GUT magnetse monopoles Messures the rentrino fiux from the Earth atsosphere, eesrebor for neuthnot from tosmic sources and from WIMP antutulations in the center of the Eisth A Part of the delector conststyng of 36 optier-al mondulek hat operaled in 1993/94 \& 72-medule veraion toak dats ftom Apr 95 to Mar 95, and the so-modile detecter (hall of the NT-200) begsn taking dete In Apr ge

Journal gagets NP (PROC SUPPL) 14B (1900) 51, NP (FROC) 3џPFL) 28 (1991) 386, YF 52 (1990) $80=3 J N F \$ 2$ (1990) 54, NP (PROE SUPPL) 281 (J992) 491, NP (PROC SUPPI) 35 (1994) 290, NP (FROC SUPPL) 35 (1994) 301, NP (PROC ȘUPFL) 49 (1985) 2d1, and NP (FROC sUPFL) 48 (1995) 463

Retated expersnerts AMANDA, DUMAND, NESTOR

E-mad conlace domogatsiof phalo lpa rak su, crapoifh de

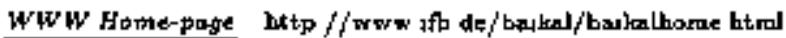

\section{UNDERWATER-DUMAND}

(Fropored 1983, Appoved 1990, In preparalivi)

\section{DEEP UNDERWATER MUON AND NEUTRINO} TELESCOPE

DUMAND.II COLLABORATION

AACHEN, TECH HOCHSCH, JII PHYS INST - P Boctt,

D Ssmon, C Wriebusch

BERN U - P K F GJeder, P MLnkowakn, E Totrente-Lugan

BOSTON U - S T Dye, E Hsant, A Meviatie

UC, BERKELEY - H Crowford, C Kod, G Shapura, L Stevenupp

UC, SAN DIEOO - H Brsdatet

HAWAf1 U - I Boleota, P W Gorhaw. S Kondo, J G Learned

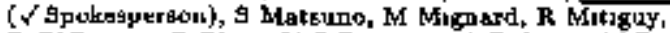

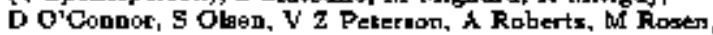

$\checkmark$ J Stenger, D Takemon, o Wilkms

IOWA STATE Uf - E W Anderson, J M Hsupumsa, K Mourttz

KIEL U T KituLa, P Kogke

KOBE U - K Kobayakaws

KINKI U, IIZUKA - T KIt:RTUI

LOUTISIANA STATE U - R Clark, R Svobods. M Vaginu

OKAYAMA LNNW \$CI - I Yarámoto

SCRIPPS UNST OCEANOGRAPHY - H Bradner

TOHOKU U - H Habsda, T Hayashino, M Ito, Y Kawarporitn,

Il Knwamoto, T Mateburoto, T Taksyama, S Tanaks.

A Yamaguctu

TOKYO D, ICRR - T Aok, K Mitsul, Y Ohesbl, A Okada

KEK - M Fuknow, M Saknda, s Uobara

VANDERBILT U-K Mtther. M Webater

WASHINGTON U, SEATTLE - H Berns, P Bognton,

V Chaloupka, B Egaas, I George, I J Lord, R, I Wilkes,

$K$ Youta

WISCONSIN U - U Camarnm, w Grogad, M Jaworeki, R March, $T$ Narita, $D$ Nicklaus

Acosierator NONE Dettector Countat

Particles studied now, $w_{\text {, roonopole }}$

Bryef description In the firat stage of the experiment

(DVIMAND-I), sest of the aperation of 7 phatolube मnadules

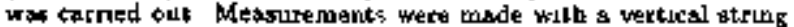
of modulas suspended from a this phase-IF was pppopved in 1980 The plans called for an octegonal s-string array, 24 tubet per stzing, to be bullt by 1995 The array called DUMAND-I was to be located at $B$ depth of $4760 \mathrm{~m}, 25 \mathrm{~km}$ of the coant of

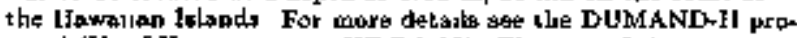
posal (J of Hawall report, HDC-1-88) The alm of the exper3. ment is to build a gystom capable of searching for point sourcen

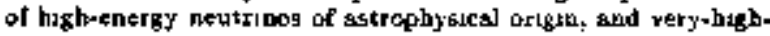

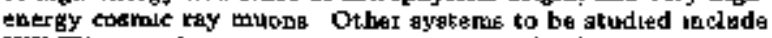
WikP"g, quark nuggets, and manopoley The deteckor in 72 megaton Cerenkoy counter, with a muon Bres of $20.000 \mathrm{~m}^{2}$, and

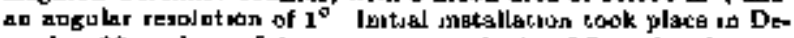
embar 43, and proof date wha acqured As of Jung 94, three grong are ready to be ingtalled Hawever, a SAGENAP report of June g6 recommende cencetlation of funding for the pruject, despte endoraement of its gonls and feasubjlyty Other gources of anpport afe being sought (July 96)

Jourral paptrs NIM A2T6 (1989) 359, and PR. D42 (1990) 3813 Fiteted arpertmsats BAIKAL, AMANDA, NESTOR

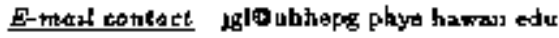
WWW Home-pege http / / whb plyse wabinglod edu /kocal whb/tumandfasa dumsnd home btml

\section{UNDERWATER-NESTOR}

([ta preparation)

NESTOR: A NEUTRINO PARTICLE ASTROPHYSICS UNDEKWATER LABORATORY EOR THE MEDITER. RANEAN

\section{NESTOR COLLABOFATION}

Asceleraior NOHE Detector Caunter

Parfictes atudied $N$

Erfef descripton NESTOR, is a detector For undersater neutruno antiophysucs It in located in the uterasutuonal watara of the enuthwest const of t5reect The first phsed of the project calla For the construction and deployment of a bexagonal towter with Jes optical spodules and son effectuve area of $20,000 \mathrm{~m}^{2}$ suitatile For the delection of ToV neutmos When completed, the fuli NESTOR array will bave bxe additional toware in a hexagonal configuration, providing a tensitive ares bogger than $100,000 \mathrm{~m}^{2}$ for I Tey ntutrunos, and an overall angular resolution better than $1^{\circ}$ The frat toner ut ander conetruchion and ks axpocted to be deployed un logr For further information about the provect please contact the \$poke日perton, Prof Leobudas K Resvabla [Athens U]

Retasted ctperthentg BAIKAL, DUMAND, AMANDA

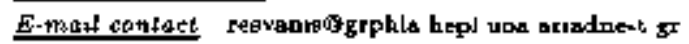
WWW Homerpage hetp / /abyst hepl uon aridinet gr 